\title{
Dynamics Modeling and Loads Analysis of an Offshore Floating Wind Turbine
}

Technical Report NREL/TP-500-41958

November 2007

J.M. Jonkman

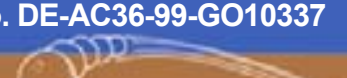




\section{Dynamics Modeling and Loads Analysis of an Offshore Floating Wind Turbine}

Technical Report NREL/TP-500-41958

November 2007

J.M. Jonkman

Prepared under Task No. WER7.5001

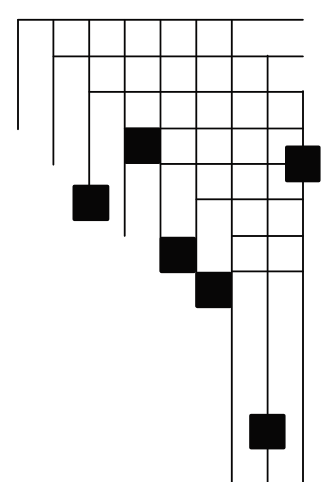




\section{NOTICE}

This report was prepared as an account of work sponsored by an agency of the United States government. Neither the United States government nor any agency thereof, nor any of their employees, makes any warranty, express or implied, or assumes any legal liability or responsibility for the accuracy, completeness, or usefulness of any information, apparatus, product, or process disclosed, or represents that its use would not infringe privately owned rights. Reference herein to any specific commercial product, process, or service by trade name, trademark, manufacturer, or otherwise does not necessarily constitute or imply its endorsement, recommendation, or favoring by the United States government or any agency thereof. The views and opinions of authors expressed herein do not necessarily state or reflect those of the United States government or any agency thereof.

Available electronically at http://www.osti.gov/bridge

Available for a processing fee to U.S. Department of Energy and its contractors, in paper, from:

U.S. Department of Energy

Office of Scientific and Technical Information

P.O. Box 62

Oak Ridge, TN 37831-0062

phone: 865.576 .8401

fax: 865.576 .5728

email: mailto:reports@adonis.osti.gov

Available for sale to the public, in paper, from:

U.S. Department of Commerce

National Technical Information Service

5285 Port Royal Road

Springfield, VA 22161

phone: 800.553.6847

fax: 703.605.6900

email: orders@ntis.fedworld.gov

online ordering: http://www.ntis.gov/ordering.htm 


\section{Acknowledgments}

I would like to thank many individuals for the successful completion of this project. Without their advice and help, I could not have completed a work of this scope.

First, I would like to thank my Ph.D. committee for evaluating this work: Professor Mark Balas, formerly of the University of Colorado and now with the University of Wyoming; Professors Carlos Felippa and Lucy Pao of the University of Colorado; Dr. Michael Robinson of the National Renewable Energy Laboratory; and Professor Paul Sclavounos of the Massachusetts Institute of Technology. Special thanks go to my advisor, Professor Mark Balas, for his guidance and support of this work, and to Professor Paul Sclavounos, for educating me in marine hydrodynamics.

Thank you to Dr. Robert Zueck and Dr. Paul Palo of the Naval Facilities Engineering Service Center for giving me insight into the dynamics and modeling of mooring systems.

I would also like to thank Dr. Jon Erik Withee of the U.S. Navy for initiating the study of offshore floating wind turbines at the Massachusetts Institute of Technology, and Kwang Lee for continuing in that effort and verifying the output of SWIM. I am also grateful to Libby Wayman for modifying SWIM to output the frequency-dependent solutions of the radiation and diffraction problems, for developing a floating platform concept, and for providing me with data that I could use to validate my own models.

Thank you also to Torben Larsen of Risø National Laboratory and the Technical University of Denmark for introducing me to the importance of the role that a variable blade-pitch-to-feather control system can play in offshore floating wind turbines.

I would also like to thank Ian Edwards of ITI Energy for sponsoring the loads-analysis activities and Professor Nigel Barltrop and Willem Vijfhuizen of the Universities of Glasgow and Strathclyde for developing the ITI Energy barge and mooring system concept.

Big thanks go to several of my colleagues at the National Renewable Energy Laboratory's National Wind Technology Center. I thank George Scott for processing the reference-site data from the Waveclimate.com service, and Bonnie Jonkman for assisting me in developing the scripts needed to generate the WAMIT ${ }^{\circledR}$ geometric-data input files. I thank Marshall Buhl for developing the scripts used to run the loads analysis and for assisting me in processing the loadsanalysis data. Thank you to Dr. Gunjit Bir for assisting me in examining the system instabilities and to Lee Jay Fingersh and Dr. Alan Wright for their guidance and advice in my controlsdevelopment activities. Thanks also to Kathleen O'Dell, Rene Howard, Janie Homan, Bruce Green, and Bonnie Jonkman for editing this work to make it much more readable. Thank you also to Walter Musial and Sandy Butterfield for leading the offshore wind energy program and to Dr. Robert Thresher and Dr. Michael Robinson for directing the National Wind Technology Center and for giving me the time and resources needed to work on this project.

I would like to thank my family and friends for their gracious support and encouragement throughout this effort-I couldn't have completed the project without your help. 
This work was performed at the National Renewable Energy Laboratory in support of the U.S. Department of Energy under contract number DE-AC36-99-GO10337 and in support of a Cooperative Research and Development Agreement (CRD-06-178) with ITI Energy. 


\section{Acronyms and Abbreviations}

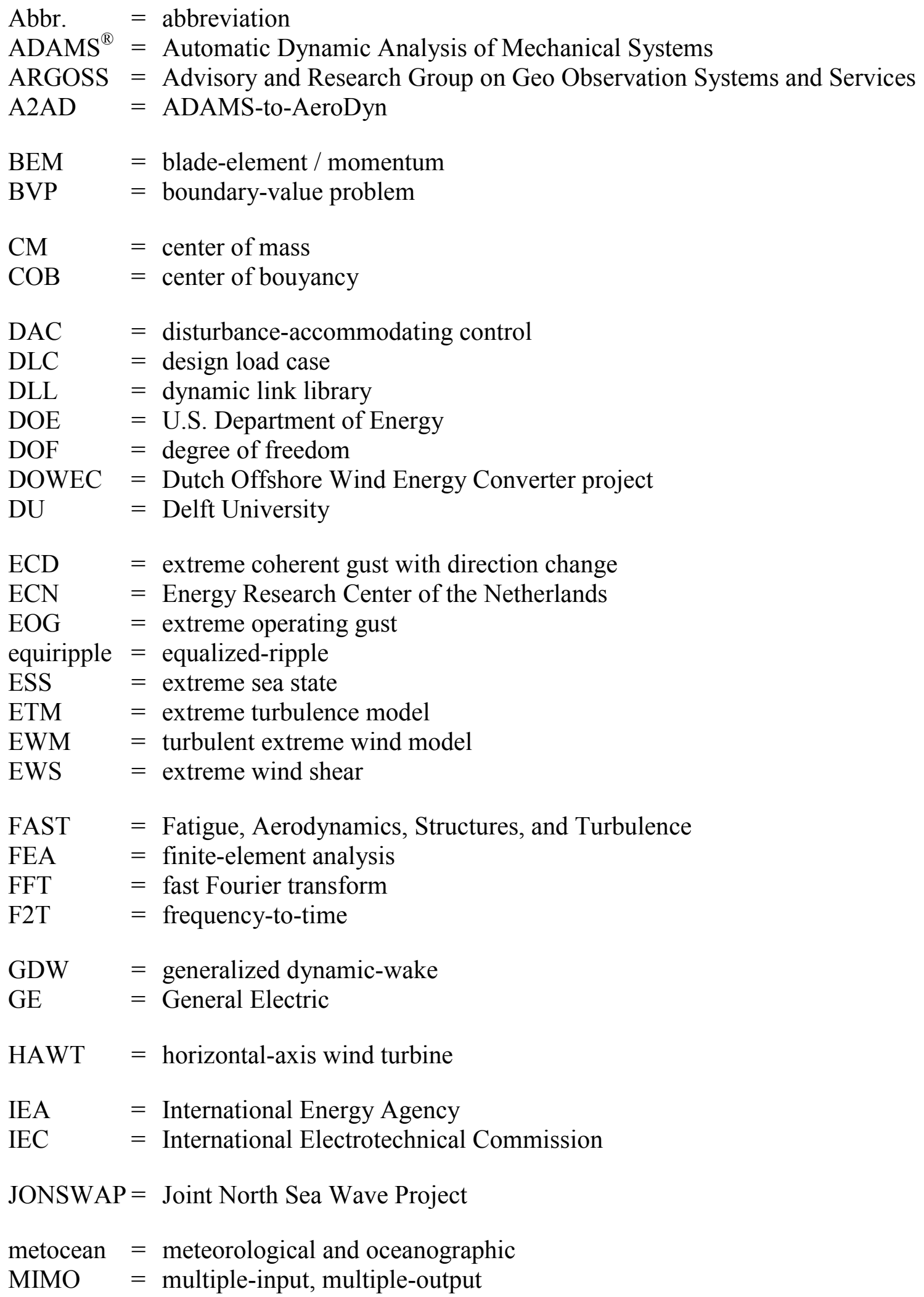




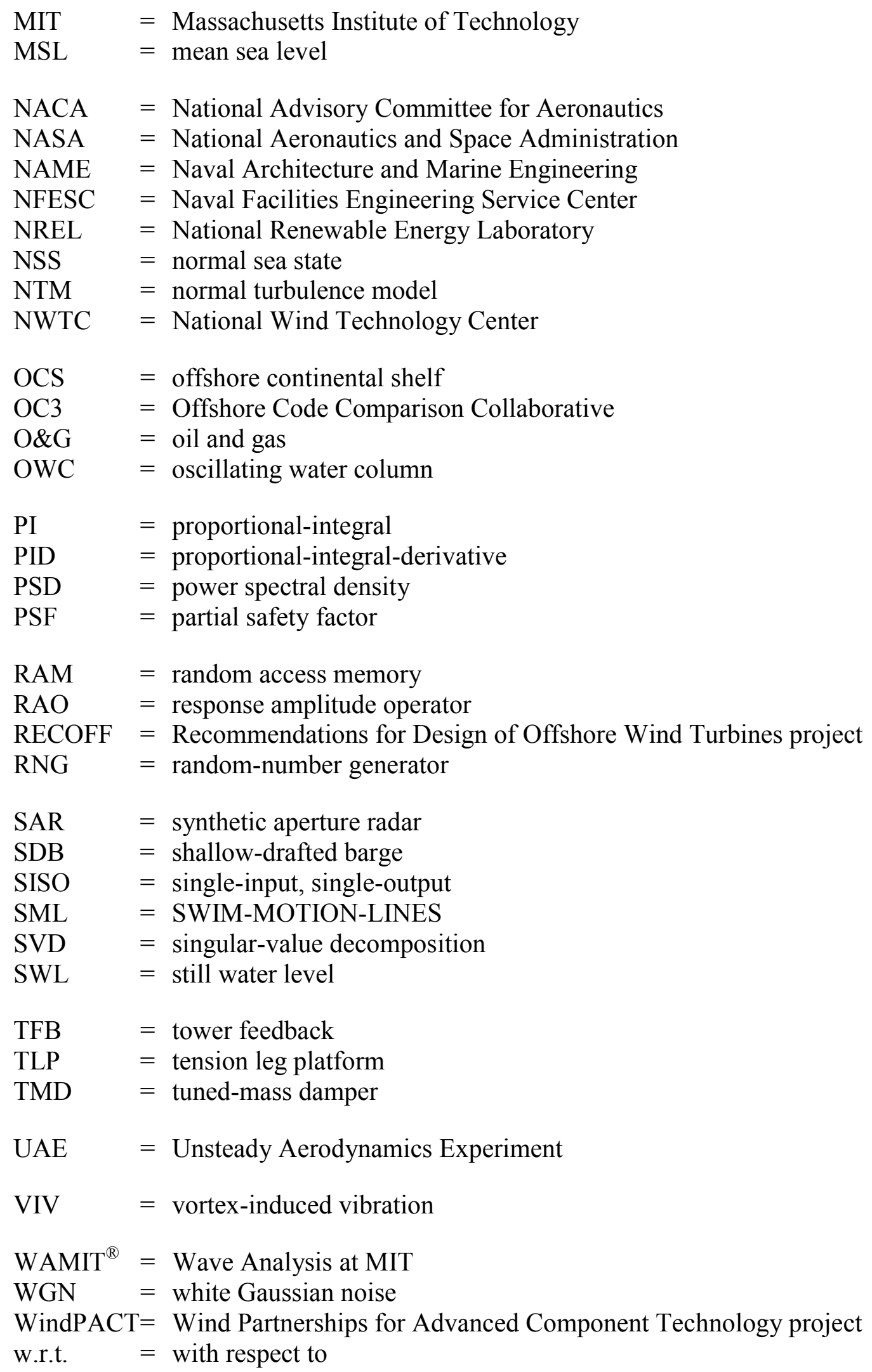




\section{Nomenclature}

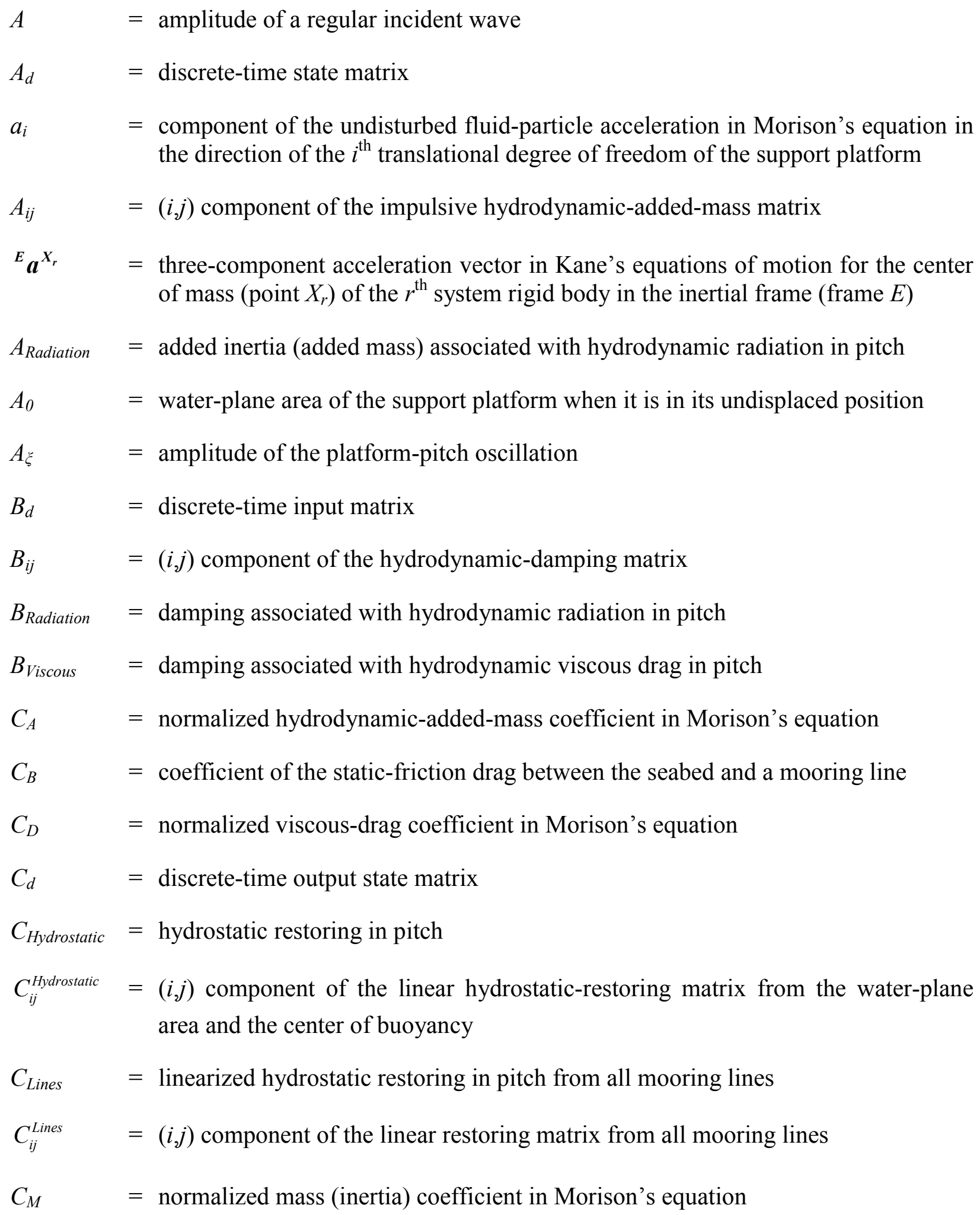




$$
\begin{aligned}
& C_{x} \quad=\text { effective damping in the equation of motion for the platform pitch in terms of the } \\
& C_{\varphi} \quad=\text { effective damping in the equation of motion for the rotor-speed error } \\
& D \quad=\text { diameter of cylinder in Morison's equation } \\
& D_{c} \quad=\text { effective diameter of a mooring line } \\
& D_{d} \quad=\text { discrete-time input transmission matrix } \\
& d F_{i}^{\text {Platform }}=i^{\text {th }} \text { component of the total external load acting on a differential element of cylinder } \\
& d F_{i}^{\text {Viscous }}=i^{\text {th }} \text { component of the viscous-drag load acting on a differential element of cylinder } \\
& \text { in Morison's equation } \\
& d z \quad=\text { length of a differential element of cylinder in Morison's equation } \\
& E\left[H_{S} \mid V_{h u b}\right]=\text { expected value of the significant wave height conditioned on the mean hub-height } \\
& \text { EA } \quad=\text { extensional stiffness of a mooring line } \\
& f_{c} \quad=\text { corner frequency } \\
& f_{i} \quad=\text { component of the forcing function associated with the } i^{\text {th }} \text { system degree of } \\
& \text { freedom } \\
& F_{i} \quad=\text { generalized active force in Kane's equations of motion associated with the } i^{\text {th }}
\end{aligned}
$$




$$
\begin{aligned}
& F_{i}^{\text {Platform }}=i^{\text {th }} \text { component of the total external load acting on the support platform, other than } \\
& F_{i}^{\text {Viscous }}=i^{\text {th }} \text { component of the total viscous-drag load acting on the support platform from } \\
& \text { Morison's equation } \\
& F_{i}^{\text {Waves }}=i^{\text {th }} \text { component of the total excitation force on the support platform from incident } \\
& \text { waves } \\
& \boldsymbol{F}^{\boldsymbol{X}_{\boldsymbol{r}}} \quad=\text { three-component active-force vector in Kane's equations of motion applied at the } \\
& \text { center of mass (point } X_{r} \text { ) of the } r^{\text {th }} \text { system rigid body } \\
& g \quad=\text { gravitational acceleration constant } \\
& G K=\text { gain-correction factor } \\
& h \quad=\text { water depth } \\
& H_{A} \quad=\text { horizontal component of the effective tension in a mooring line at the anchor } \\
& H_{F} \quad=\text { horizontal component of the effective tension in a mooring line at the fairlead } \\
& H_{F}^{0} \quad=\text { starting value of } H_{F} \text { used in the Newton-Raphson iteration during the initialization } \\
& \text { of the mooring system module } \\
& { }^{E} \dot{\boldsymbol{H}}^{N_{r}} \quad=\text { three-component vector in Kane's equations of motion representing the first time } \\
& H_{s} \quad=\text { significant wave height } \\
& H_{s 1} \quad=\text { significant wave height, based on a 3-h reference period, with a recurrence period } \\
& \text { of } 1 \text { year } \\
& H_{s 50} \quad=\text { significant wave height, based on a 3-h reference period, with a recurrence period } \\
& \text { of } 50 \text { years } \\
& I_{\text {Drivetrain }}=\text { drivetrain inertia cast to the low-speed shaft } \\
& I_{\text {Gen }} \quad=\text { generator inertia relative to the high-speed shaft } \\
& I_{\text {Mass }}=\text { pitch inertia associated with wind turbine and barge mass } \\
& I_{\text {Rotor }} \quad=\text { rotor inertia } \\
& j=\text { when not used as a subscript, this is the imaginary number, } \sqrt{-1}
\end{aligned}
$$




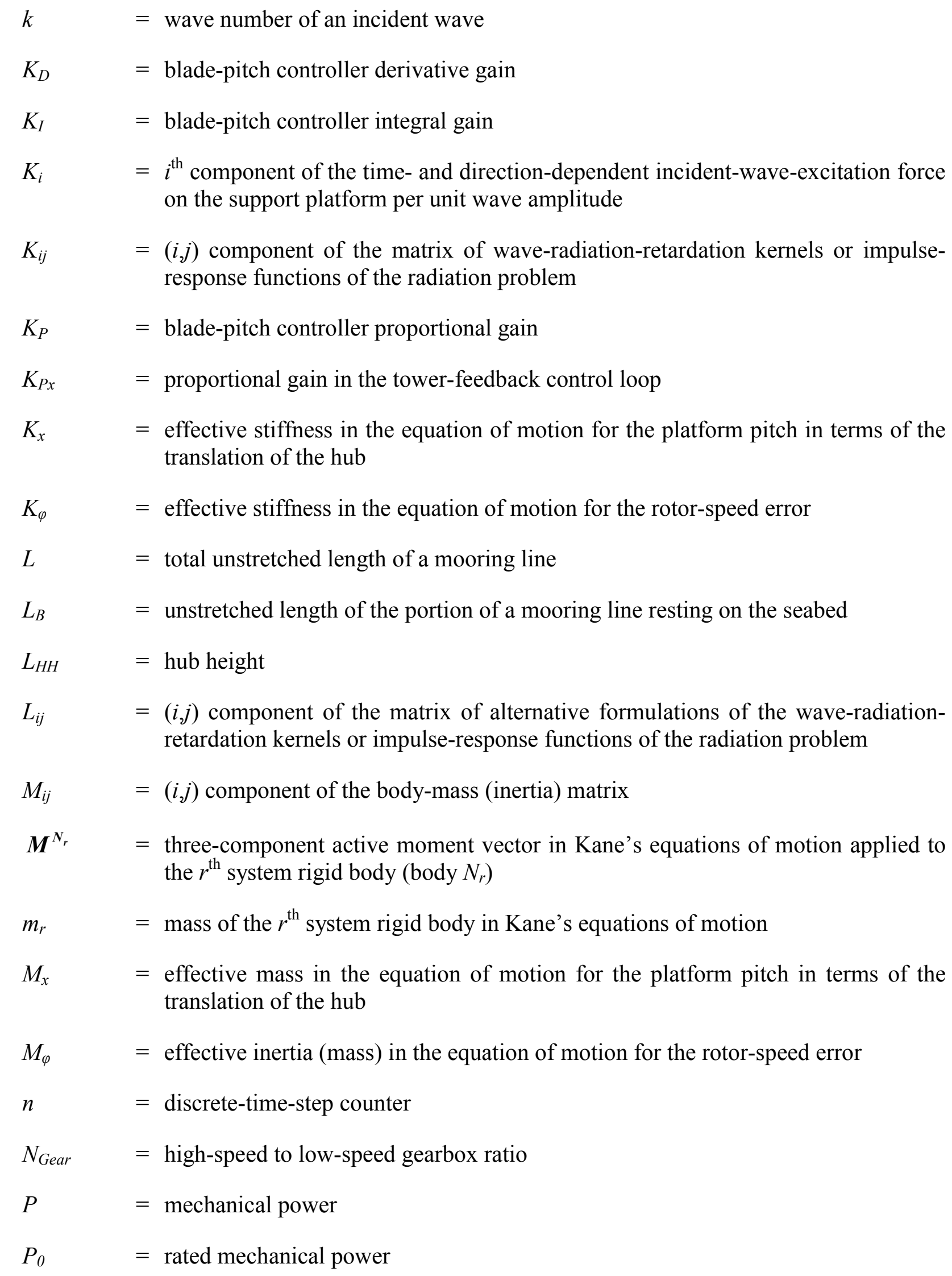




$$
\begin{aligned}
& \partial P / \partial \theta=\text { sensitivity of the aerodynamic power to the rotor-collective blade-pitch angle } \\
& q_{j} \quad=\text { system degree-of-freedom } j \text { (without the subscript, } q \text { represents the set of system } \\
& \text { degrees of freedom) } \\
& \dot{q}_{j} \quad=\text { first time derivative of system degree-of-freedom } j \text { (without the subscript, } \dot{q} \\
& \ddot{q}_{j} \quad=\text { second time derivative of system degree-of-freedom } j \text { (without the subscript, } \ddot{q} \\
& \text { represents the set of second time derivatives of the system degrees of freedom) } \\
& s \quad=\text { unstretched arc distance along a mooring line from the anchor to a given point on } \\
& \text { the line } \\
& S_{\zeta}^{l-\text { Sided }}=\text { one-sided power spectral density of the wave elevation per unit time } \\
& S_{\zeta}^{2 \text {-Sided }}=\text { two-sided power spectral density of the wave elevation per unit time } \\
& t \quad=\text { simulation time } \\
& T=\text { aerodynamic rotor thrust } \\
& T_{\text {Aero }}=\text { aerodynamic torque in the low-speed shaft } \\
& T_{e} \quad=\text { effective tension at a given point on a mooring line } \\
& T_{G e n} \quad=\text { generator torque in the high-speed shaft } \\
& T_{p} \quad=\text { peak spectral period } \\
& T_{s} \quad=\text { discrete-time step } \\
& T_{0} \quad=\text { aerodynamic rotor thrust at a linearization point } \\
& u \quad=\quad \text { for the control-measurement filter, the unfiltered generator speed } \\
& u \quad=\text { for the system equations of motion, the set of wind turbine control inputs } \\
& U_{1} \quad=\text { first of two uniformly-distributed random numbers between zero and one } \\
& U_{2} \quad=\text { second of two uniformly-distributed random numbers between zero and one } \\
& V \quad=\text { rotor-disk-averaged wind speed } \\
& V_{A}=\text { vertical component of the effective tension in a mooring line at the anchor } \\
& V_{F} \quad=\text { vertical component of the effective tension in a mooring line at the fairlead }
\end{aligned}
$$




$$
\begin{aligned}
& V_{F}^{0} \quad=\text { starting value of } V_{F} \text { used in the Newton-Raphson iteration during the initialization } \\
& \text { of the mooring system module } \\
& V_{\text {hub }} \quad=\text { hub-height wind speed averaged over a given reference period } \\
& v_{i} \quad=\text { component of the undisturbed fluid-particle velocity in Morison's equation in the } \\
& \text { direction of the } i^{\text {th }} \text { translational degree of freedom of the support platform } \\
& { }^{E} \boldsymbol{v}_{r}^{\boldsymbol{X}_{r}} \quad=\text { three-component partial linear-velocity vector in Kane's equations of motion for } \\
& \text { the center of mass (point } X_{r} \text { ) of the } r^{\text {th }} \text { system rigid body in the inertial frame } \\
& \text { (frame } E \text { ) } \\
& V_{\text {in }} \quad=\text { cut-in wind speed } \\
& V_{\text {out }} \quad=\text { cut-out wind speed } \\
& V_{r} \quad=\text { rated wind speed } \\
& V_{0}=\text { displaced volume of fluid when the support platform is in its undisplaced position } \\
& V_{l} \quad=\text { reference 10-min average wind speed with a recurrence period of } 1 \text { year } \\
& V_{50}=\text { reference 10-min average wind speed with a recurrence period of } 50 \text { years } \\
& W \quad=\text { Fourier transform of a realization of a white Gaussian noise time-series process } \\
& \dot{x} \quad=\text { translational velocity of the hub } \\
& \ddot{x} \quad=\text { translational acceleration of the hub } \\
& x_{F} \quad=\text { horizontal distance between the anchor and fairlead of a mooring line } \\
& X_{i}=i^{\text {th }} \text { component of the frequency- and direction-dependent complex incident-wave- } \\
& \text { excitation force on the support platform per unit wave amplitude }
\end{aligned}
$$


opposite gravity along the centerline of the undeflected tower when the platform is undisplaced)

$x, y, z=$ set of orthogonal axes making up a transformed reference frame (when applied to the support platform in particular, $x, y, z$ represents the set of orthogonal axes of a body-fixed reference frame within the platform, with the $x y$-plane designating the still water level when the platform is undisplaced and the $z$-axis directed upward along the centerline of the undeflected tower)

$y \quad=$ for the control-measurement filter, the filtered generator speed

$z \quad=$ for mooring systems, the vertical distance between the anchor and a given point on a mooring line

$z_{C O B} \quad=$ body-fixed vertical location of the center of buoyancy of the support platform (relative to the still water level and negative downward along the undeflected tower centerline when the support platform is in its undisplaced position)

$z_{F} \quad=$ vertical distance between the anchor and fairlead of a mooring line

$\alpha \quad=$ low-pass filter coefficient

$\beta \quad=$ incident-wave propagation heading direction

$\gamma \quad=$ peak shape parameter in the Joint North Sea Wave Project (JONSWAP) spectrum

$\Delta \zeta_{x} \quad=$ effective increase in the platform-pitch damping ratio

$\Delta \theta=$ small perturbation of the blade-pitch angles about their operating point

$\Delta \dot{\theta} \quad=$ blade-pitch rate

$\Delta \Omega \quad=$ small perturbation of the low-speed shaft rotational speed about the rated speed

$\Delta \dot{\Omega}=$ low-speed shaft rotational acceleration

$\delta_{i j} \quad=(i, j)$ component of the Kronecker-Delta function (i.e., identity matrix), equal to unity when $i=j$ and zero when $i \neq j$

$\zeta \quad=$ instantaneous elevation of incident waves

$\zeta_{x} \quad=$ damping ratio of the response associated with the equation of motion for the platform pitch in terms of the translation of the hub

$\zeta_{\varphi} \quad=$ damping ratio of the response associated with the equation of motion for the rotor-speed error

$\theta \quad=$ for the blade-pitch controller, the full-span rotor-collective blade-pitch angle 
$\theta_{K} \quad=$ rotor-collective blade-pitch angle at which the pitch sensitivity has doubled from its value at the rated operating point

$\theta_{1}, \theta_{2}, \theta_{3}=$ set of orthogonal rotations used to convert from an original to a transformed reference frame (when applied to the support platform in particular, $\theta_{1}, \theta_{2}, \theta_{3}$ represent the roll, pitch and yaw rotations of the platform about the axes of the inertial reference frame)

$\lambda_{0} \quad=$ dimensionless catenary parameter used to determine the starting values in the Newton-Raphson iteration during the initialization of the mooring system module

$\mu_{c} \quad=$ mass of mooring line per unit length

$\xi=$ platform-pitch angle (rotational displacement)

$\dot{\xi}=$ platform-pitch rotational velocity

$\ddot{\xi} \quad=$ platform-pitch rotational acceleration

$\pi \quad=$ the ratio of a circle's circumference to its diameter

$\rho \quad=$ water density

$\sigma \quad=$ scaling factor in the Joint North Sea Wave Project (JONSWAP) spectrum

$\sigma_{\zeta}^{2}=$ variance of the instantaneous elevation of incident waves

$\tau \quad=$ dummy variable with the same units as the simulation time

$\varphi \quad=$ the integral of $\dot{\varphi}$ with respect to time

$\dot{\varphi} \quad=$ small perturbation of the low-speed shaft rotational speed about the rated speed

$\ddot{\varphi} \quad=$ low-speed shaft rotational acceleration

$\Omega \quad=$ low-speed shaft rotational speed

$\Omega_{0} \quad=$ rated low-speed shaft rotational speed

$\omega \quad=$ for hydrodynamics, this is the frequency of incident waves or frequency of oscillation of a particular mode of motion of the platform

$\omega=$ for mooring systems, this is the apparent weight of a line in fluid per unit length of line

${ }^{E} \omega_{r}^{N_{r}} \quad=$ three-component partial angular-velocity vector in Kane's equations of motion for the $r^{\text {th }}$ system rigid body (body $N_{r}$ ) in the inertial frame (frame $E$ ) 
$\omega_{x n} \quad=$ natural frequency of the response associated with the equation of motion of the platform pitch in terms of the translation of the hub

$\omega_{\varphi n} \quad=$ natural frequency of the response associated with the equation of motion for the rotor-speed error 


\section{Executive Summary}

The vast deepwater wind resource represents a potential to use offshore floating wind turbines to power much of the world with renewable energy. Many floating wind turbine concepts have been proposed, but dynamics models, which account for the wind inflow, aerodynamics, elasticity, and controls of the wind turbine, along with the incident waves, sea current, hydrodynamics, and platform and mooring dynamics of the floater, were needed to determine their technical and economic feasibility.

This work presents the development of a comprehensive simulation tool for modeling the coupled dynamic response of offshore floating wind turbines, the verification of the simulation tool through model-to-model comparisons, and the application of the simulation tool to an integrated loads analysis for one of the promising system concepts.

A fully coupled aero-hydro-servo-elastic simulation tool was developed with enough sophistication to address the limitations of previous frequency- and time-domain studies and to have the features required to perform loads analyses for a variety of wind turbine, support platform, and mooring system configurations.

The simulation capability was tested using model-to-model comparisons. The favorable results of all of the verification exercises provided confidence to perform more thorough analyses.

The simulation tool was then applied in a preliminary loads analysis of a wind turbine supported by a barge with catenary moorings. A barge platform was chosen because of its simplicity in design, fabrication, and installation. The loads analysis aimed to characterize the dynamic response and to identify potential loads and instabilities resulting from the dynamic couplings between the turbine and the floating barge in the presence of combined wind and wave excitation. The coupling between the wind turbine response and the barge-pitch motion, in particular, produced larger extreme loads in the floating turbine than experienced by an equivalent land-based turbine. Instabilities were also found in the system.

The influence of conventional wind turbine blade-pitch control actions on the pitch damping of the floating turbine was also assessed.

Design modifications for reducing the platform motions, improving the turbine response, and eliminating the instabilities are suggested. These suggestions are aimed at obtaining costeffective designs that achieve favorable performance while maintaining structural integrity. 


\section{Table of Contents}

Chapter 1 Introduction................................................................................................................................. 1

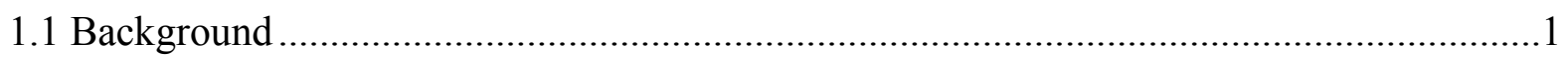

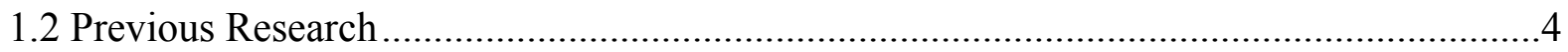

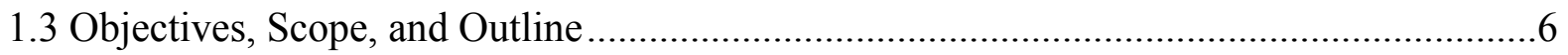

Chapter 2 Development of Aero-Hydro-Servo-Elastic Simulation Capability .......................... 8

2.1 Overview of Wind Turbine Aero-Servo-Elastic Modeling ...............................................10

2.2 Assumptions for the New Model Development.............................................................11

2.3 Support Platform Kinematics and Kinetics Modeling ....................................................13

2.4 Support Platform Hydrodynamics Modeling...................................................................18

2.4.1 The True Linear Hydrodynamic Model in the Time Domain..................................19

2.4.1.1 Diffraction Problem ......................................................................................19

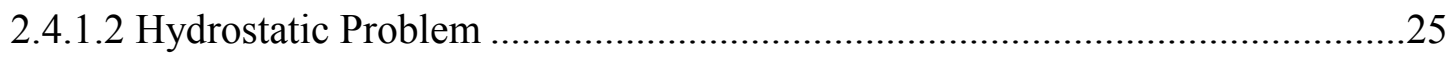

2.4.1.3 Radiation Problem .................................................................................26

2.4.2 Comparison to Alternative Hydrodynamic Models ................................................28

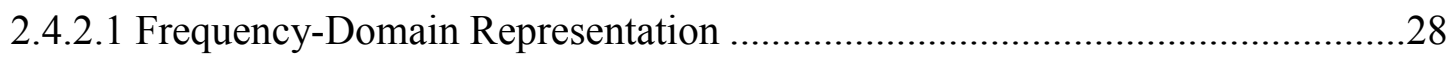

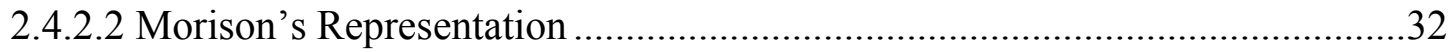

2.4.3 HydroDyn Calculation Procedure Summary ……………………...............................34

2.5 Mooring System Modeling ..............................................................................................

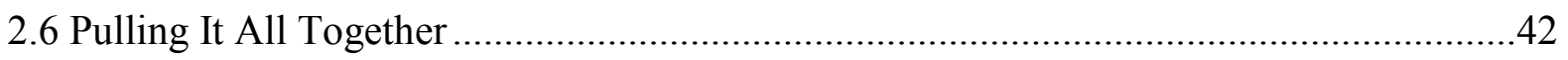

Chapter 3 Design Basis and Floating Wind Turbine Model.......................................................... 45

3.1 NREL Offshore 5-MW Baseline Wind Turbine............................................................45

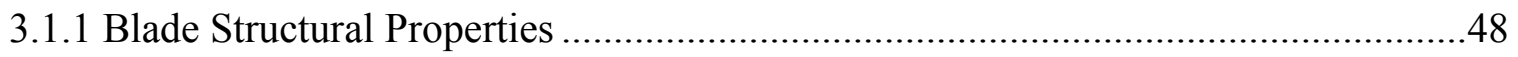

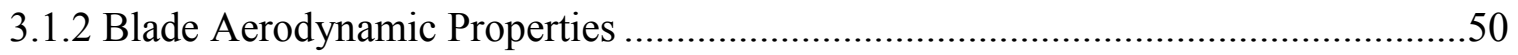

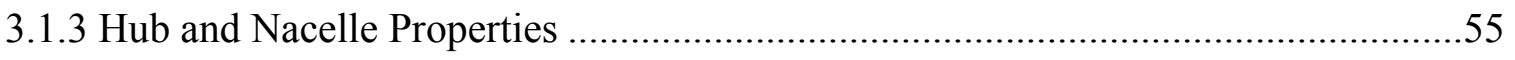

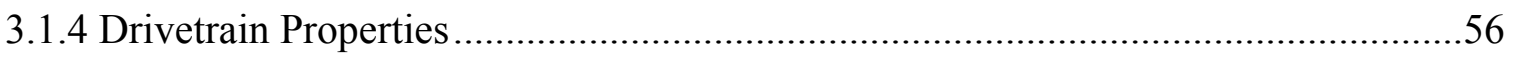

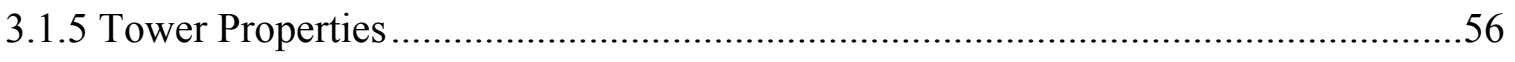

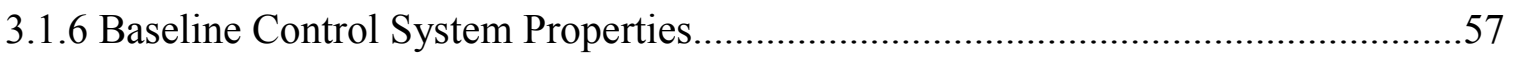

3.1.6.1 Baseline Control-Measurement Filter..........................................................58

3.1.6.2 Baseline Generator-Torque Controller ..........................................................60

3.1.6.3 Baseline Blade-Pitch Controller .......................................................................61 
3.1.6.4 Baseline Blade-Pitch Actuator ...................................................................67

3.1.6.5 Summary of Baseline Control System Properties...............................................67

3.1.7 FAST with AeroDyn and ADAMS with AeroDyn Models.........................................68

3.1.8 Full-System Natural Frequencies and Steady-State Behavior ...................................69

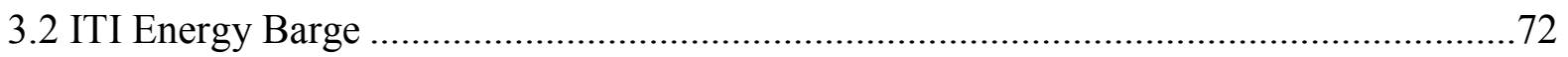

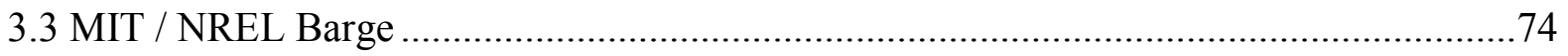

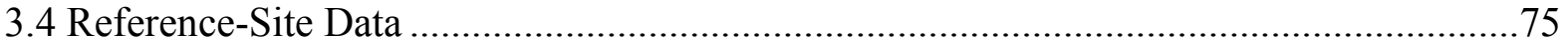

Chapter 4 Verification of Simulation Capability ............................................................................... 79

4.1 Verification of the Hydrodynamics Module ..................................................................79

4.1.1 Wave Elevation versus the Target Wave Spectrum..................................................79

4.1.2 WAMIT Output / HydroDyn Input...................................................................... 80

4.1.3 Computation of Radiation Impulse-Response Functions .........................................84

4.2 Verification of the Mooring System Module....................................................................

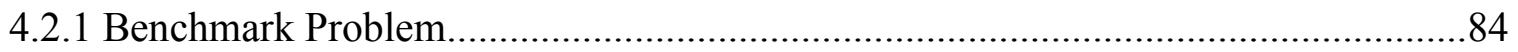

4.2.2 Nonlinear Force-Displacement Relationships ........................................................86

4.3 Time Domain versus Frequency Domain Verification......................................................8

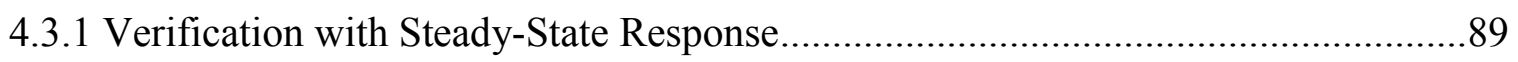

4.3.2 Verification with Stochastic Response ...............................................................91

Chapter 5 Loads-Analysis Overview and Description ................................................................... 93

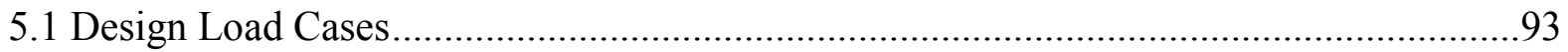

5.2 Postprocessing and Partial Safety Factors ……………...............................................100

Chapter 6 Loads-Analysis Results and Discussion .............................................................. 102

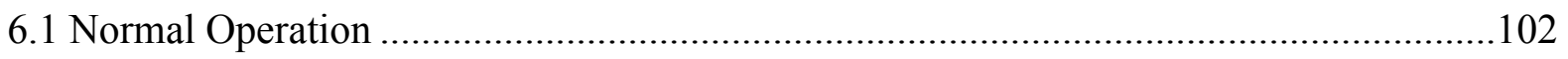

6.1.1 Characterizing the Dynamic Response ……………........................................103

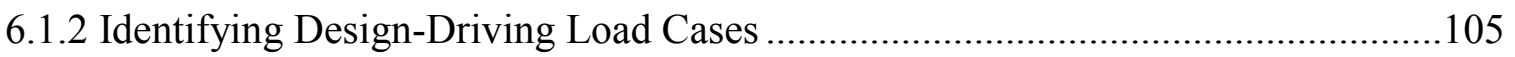

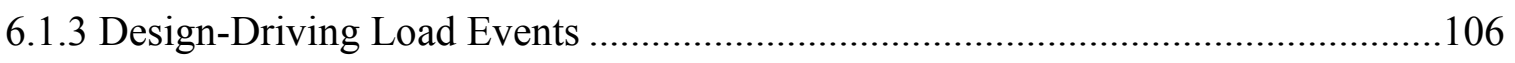

6.1.4 Comparing Land- and Sea-Based Loads............................................................112

6.1.5 Drawing Conclusions about Responses in Normal Operation..................................115

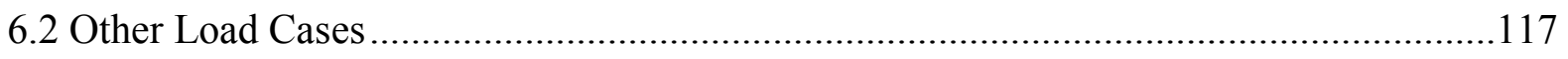

6.2.1 Tower Side-to-Side Instability of Land-Based Wind Turbine................................118

6.2.2 Platform-Yaw Instability of Sea-Based Wind Turbine............................................119

6.2.3 Excessive Barge Motions in Extreme Waves ..........................................................121 
Chapter 7 Influence of Conventional Control on Barge-Pitch Damping . 123

7.1 Overview of the Platform-Pitch-Damping Problem .123

7.2 Influence of Conventional Wind Turbine Control Methodologies 127

7.2.1 Feedback of Tower-Top Acceleration 127

7.2.2 Active Pitch-to-Stall Speed-Control Regulation .131

7.2.3 Detuning the Gains in the Pitch-to-Feather Controller 137

7.3 Other Ways to Improve the Pitch Damping with Turbine Control...... .142

Chapter 8 Conclusions and Recommendations 144

References 148

Appendix A FAST Input Files for the 5-MW Wind Turbine. 159

A.1 Primary Input File 159

A.2 Blade Input File - NRELOffshrBsline5MW_Blade.dat .161

A.3 Tower Input File - NRELOffshrBsline5MW_Tower_ITIBarge4.dat 162

A.4 ADAMS Input File - NRELOffshrBsline5MW_ADAMSSpecific.dat. .163

A.5 Linearization Input File - NRELOffshrBsline5MW_Linear.dat.. .164

Appendix B AeroDyn Input Files for the 5-MW Wind Turbine 165

B.1 Primary Input File - NRELOffshrBsline5MW_AeroDyn.ipt .....................................165

B.2 Tower Input File - NRELOffshrBsline5MW_AeroDyn_Tower.dat .............................165

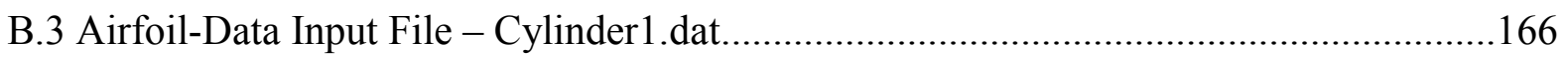

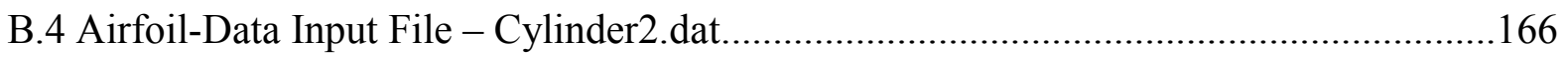

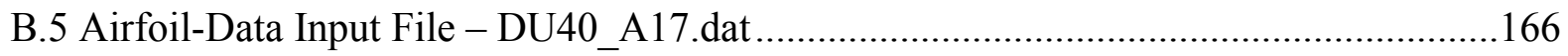

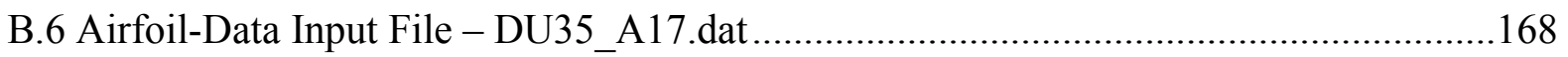

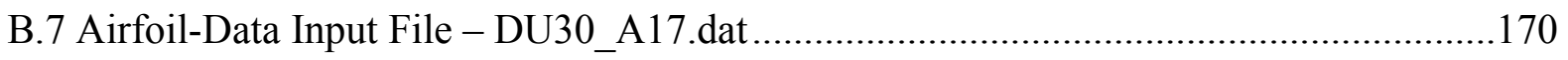

B.8 Airfoil-Data Input File - DU25_A17.dat ............................................................. 172

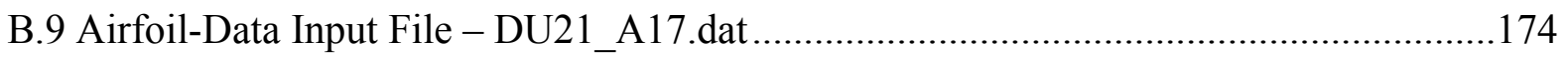

B.10 Airfoil-Data Input File - NACA64_A17.dat ........................................................176

Appendix C Source Code for the Baseline Turbine Control System DLL........................... 178

Appendix D Input Files for the ITI Energy Barge......................................................... 185

D.1 FAST Platform / HydroDyn Input File ................................................................ 185

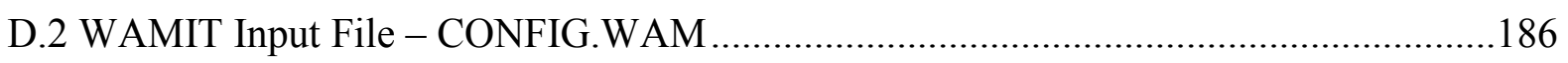

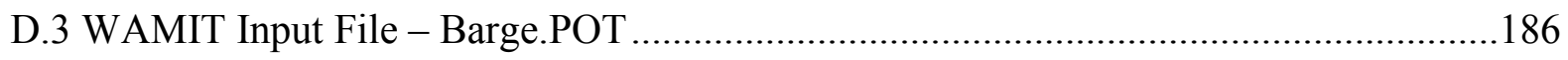




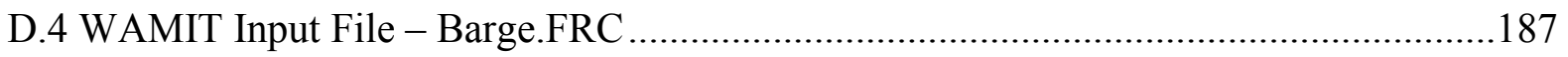

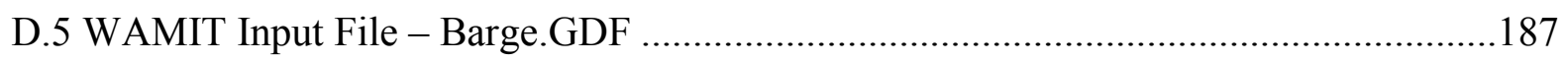

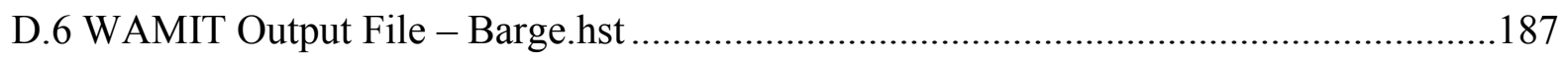

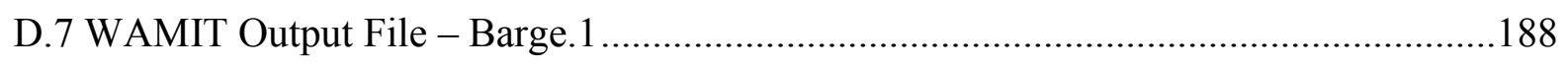

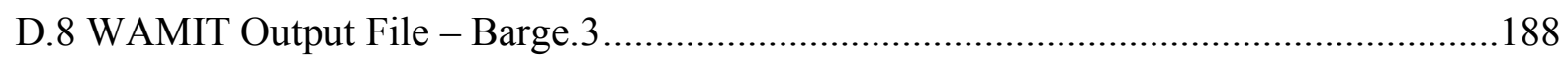

Appendix E Input Files for the MIT / NREL Barge........................................................................ 197

E.1 FAST Platform / HydroDyn Input File ……………...................................................197

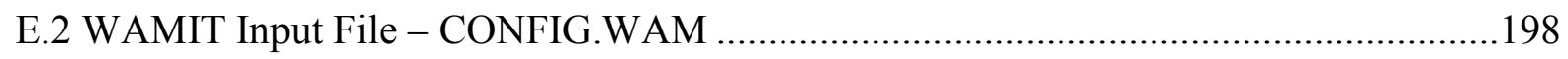

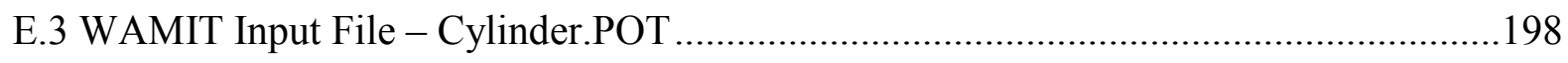

E.4 WAMIT Input File - Cylinder.FRC ……………….................................................198

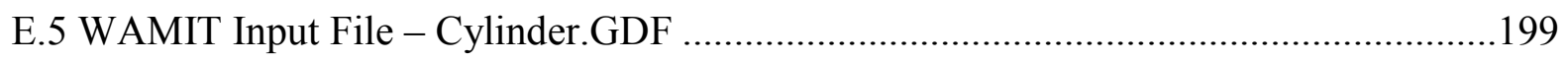

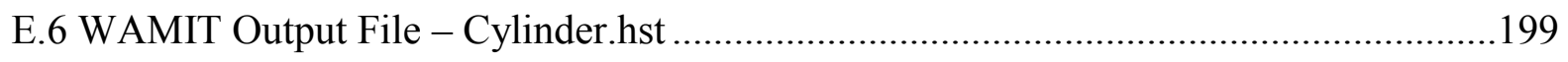

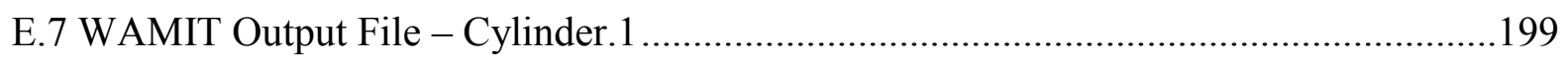

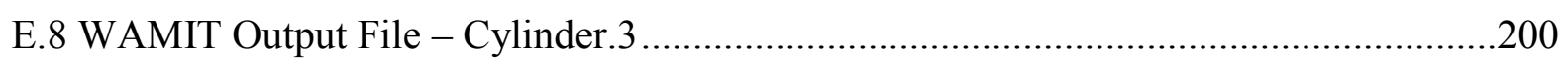

Appendix F Extreme-Event Tables for Normal Operation ........................................................ 201

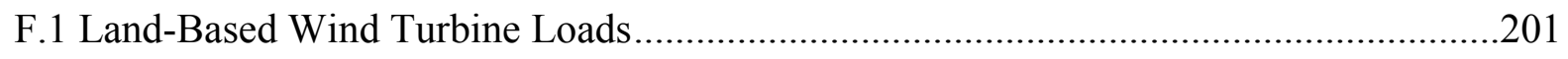

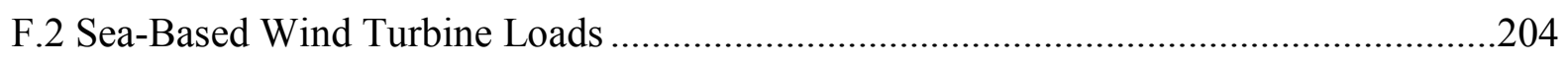




\section{List of Tables}

Table 3-1. Gross Properties Chosen for the NREL 5-MW Baseline Wind Turbine................... 47

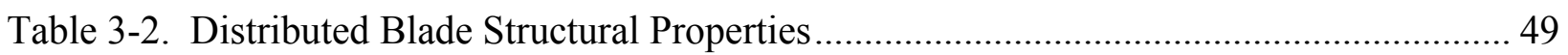

Table 3-3. Undistributed Blade Structural Properties............................................................ 50

Table 3-4. Distributed Blade Aerodynamic Properties.......................................................... 51

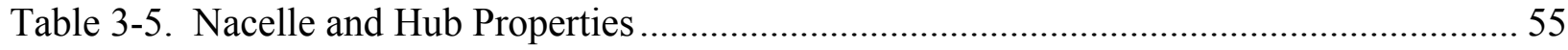

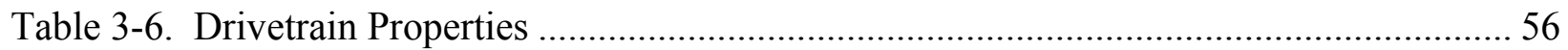

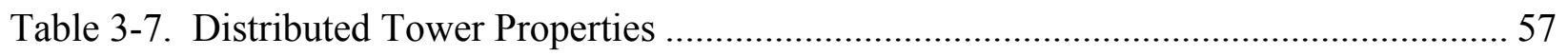

Table 3-8. Undistributed Tower Properties .................................................................. 57

Table 3-9. Sensitivity of Aerodynamic Power to Blade Pitch in Region 3 ............................... 64

Table 3-10. Baseline Control System Properties ....................................................................... 68

Table 3-11. Full-System Natural Frequencies in Hertz .................................................... 70

Table 3-12. Summary of ITI Energy Barge Properties......................................................... 73

Table 3-13. Summary of MIT / NREL Barge Properties..................................................... 74

Table 5-1. Summary of Selected Design Load Cases ........................................................ 94

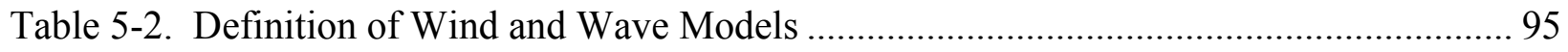

Table 6-1. Extreme Events for the Blade 1 Root Moments - Land ...................................... 105

Table 6-2. Extreme Events for the Blade 1 Root Moments - Sea ........................................ 105

Table 6-3. Extreme Events for the Tower-Base Moments - Land ...................................... 105

Table 6-4. Extreme Events for the Tower-Base Moments - Sea.......................................... 105

Table 7-1. Pitch-to-Feather Sensitivity of Aerodynamic Thrust to Wind Speed..................... 126

Table 7-2. Pitch-to-Feather Sensitivity of Aerodynamic Thrust to Blade Pitch...................... 129

Table 7-3. Sensitivity of Aerodynamic Power to Blade Pitch (to Stall) ................................ 132

Table 7-4. Pitch-to-Stall Sensitivity of Aerodynamic Thrust to Wind Speed ......................... 135

Table 7-5. Pitch-to-Stall Sensitivity of Aerodynamic Thrust to Blade Pitch ......................... 137 


\section{List of Figures}

Figure 1-1. Natural progression of substructure designs from shallow to deep water ................ 3

Figure 1-2. Floating platform concepts for offshore wind turbines ...................................... 4

Figure 2-1. Support platform degrees of freedom ............................................................. 14

Figure 2-2. Comparison between Pierson-Moskowitz and JONSWAP spectra ....................... 24

Figure 2-3. Summary of the HydroDyn calculation procedure ............................................ 35

Figure 2-4. Summary of my mooring system module calculation procedure ........................... 37

Figure 2-5. Mooring line in a local coordinate system ................................................... 38

Figure 2-6. Interfacing modules to achieve aero-hydro-servo-elastic simulation .................... 43

Figure 3-1. Corrected coefficients of the DU40 airfoil........................................................ 52

Figure 3-2. Corrected coefficients of the DU35 airfoil......................................................... 52

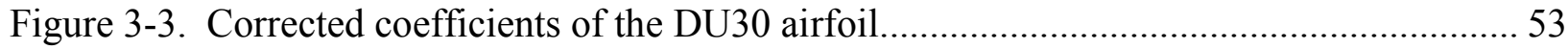

Figure 3-4. Corrected coefficients of the DU25 airfoil....................................................... 53

Figure 3-5. Corrected coefficients of the DU21 airfoil..................................................... 54

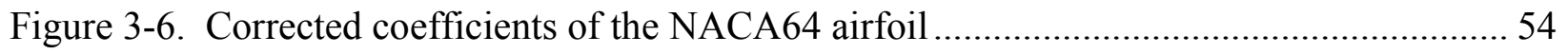

Figure 3-7. Bode plot of generator speed low-pass filter frequency response.......................... 59

Figure 3-8. Torque-versus-speed response of the variable-speed controller .......................... 61

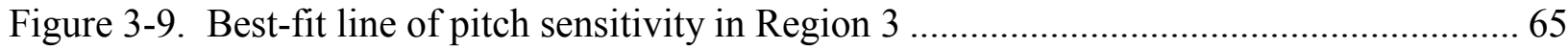

Figure 3-10. Baseline blade-pitch control system gain-scheduling law ................................ 66

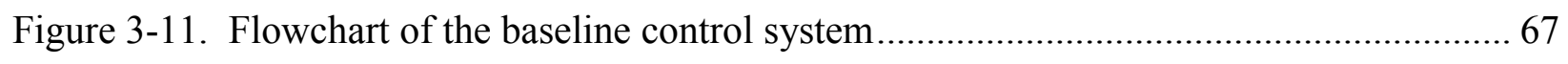

Figure 3-12. Steady-state responses as a function of wind speed........................................ 71

Figure 3-13. Illustration of the 5-MW wind turbine on the ITI Energy barge.......................... 74

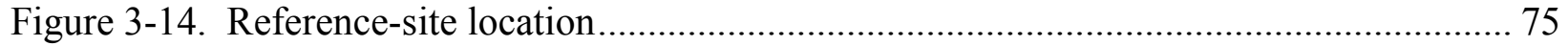

Figure 3-15. Normal sea state conditions at the reference site .......................................... 77

Figure 4-1. PSD of wave elevations versus target wave spectrum ..................................... 80

Figure 4-2. Wave-elevation probability density .............................................................. 81

Figure 4-3. Panel mesh of the ITI Energy barge used within WAMIT .................................. 82

Figure 4-4. Panel mesh of the ITI Energy barge used by NAME......................................... 82

Figure 4-5. Hydrodynamic added mass and damping for the ITI Energy barge ...................... 83

Figure 4-6. Radiation impulse-response functions for the ITI Energy barge ......................... 85

Figure 4-7. Benchmark problem for a suspended cable ................................................ 86 


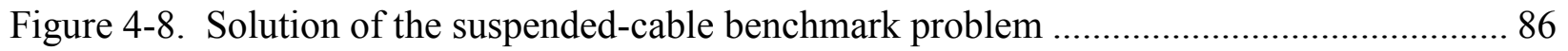

Figure 4-9. Force-displacement relationships for the ITI Energy mooring system ...................8 87

Figure 4-10. RAO comparisons for the MIT / NREL barge ................................................ 90

Figure 4-11. Probability density comparisons for the MIT / NREL barge ............................. 92

Figure 6-1. Statistics from each simulation in DLC 1.1 ..................................................... 104

Figure 6-2. Time histories from sea-based simulation number 164 in DLC 1.1 .................. 108

Figure 6-3. Time histories from sea-based simulation number 101 in DLC 1.4 .................. 110

Figure 6-4. Sea-to-land ratios from DLCs 1.1, 1.3, 1.4, and 1.5 …..................................... 113

Figure 6-5. Sea-to-land ratios for variations in significant wave height............................... 116

Figure 6-6. Time history of the tower side-to-side instability ........................................... 119

Figure 6-7. Time history of the platform-yaw instability ............................................. 121

Figure 7-1. Pitch-to-feather barge-pitch damping ratios.................................................. 127

Figure 7-2. System responses with and without a tower-feedback control loop .................... 130

Figure 7-3. Steady-state pitch-to-stall responses as a function of wind speed ....................... 133

Figure 7-4. Comparison of pitch-to-feather and pitch-to-stall system responses .................... 134

Figure 7-5. Pitch-to-feather and -stall barge-pitch damping ratios ..................................... 136

Figure 7-6. Detuned blade-pitch control system gain-scheduling law ................................. 138

Figure 7-7. System responses with and without detuned blade-pitch control gains ................. 139

Figure 7-8. Sea-to-land ratios with and without detuned blade-pitch control gains ................. 141 


\section{Chapter 1 Introduction}

\subsection{Background}

Nonrenewable resources such as coal, natural gas, oil, and nuclear power are the primary sources of energy for many parts of the world. Burning fossil fuels, however, is harmful to the environment, and fossil fuel supplies are limited and subject to price volatility. And the safe storage and disposal of radioactive waste, the potential for radioactive contamination from accidents or sabotage, and the threat of nuclear proliferation are serious challenges to the success of nuclear power. Renewable resources such as wind possess great potential because they are indigenous, nonpolluting, and inexhaustible.

Land-based wind power has been the world's fastest growing energy source on a percentage basis for more than a decade [99]. In the United States, most of the wind energy development has taken place in the West and Midwest, where the wind is strong but the land is sparsely populated. The major barrier to increased development of this wind resource potential is insufficient transmission-line capacity to major urban population (and load) centers near the coastline [71].

To power coastal load centers, wind turbines can also be installed offshore. In Europe, where vacant land is scarce and vast shallow-water wind resources are available, more than $900 \mathrm{MW}$ of offshore wind energy capacity has been installed in and around the North and Baltic seas [72]. Although offshore wind turbines are not currently in use outside Europe, worldwide interest is growing because the global offshore wind resource is abundant. The U.S. potential ranks second only to China [70]. For instance, the wind resource potential at 5 to 50 nautical miles off the U.S. coast is estimated to be more than the total currently installed electricity-generating capacity of the United States (more than 900,000 MW when accounting for exclusions), and much of this offshore potential lies close to major coastal urban populations [99]. Additional advantages of installing wind energy offshore include the following [30,71]:

- The wind tends to blow more strongly and consistently, with less turbulence intensity and smaller shear at sea than on land.

- The size of an offshore wind turbine is not limited by road or rail logistical constraints if it can be manufactured near the coastline.

- The visual and noise annoyances of wind turbines can be avoided if the turbines are installed a sufficient distance from shore.

- Vast expanses of uninterrupted open sea are available and the installations will not occupy land, interfering with other land uses.

These advantages are offset by several disadvantages of placing wind turbines offshore [30,71]:

- A higher capital investment is required for offshore wind turbines because of the costs associated with marinization of the turbine and the added complications of the foundation, support structure, installation, and decommissioning. 
- Offshore installations are less accessible than onshore installations, which raises the operations and maintenance costs and possibly increases the downtime of the machines.

- Not only do offshore wind turbines experience environmental loading from the wind, but they must also withstand other conditions, such as hydrodynamic loading from waves and sea currents. As a result, the complexity of the design increases.

Much of the offshore wind resource potential in the United States, China, Japan, Norway, and many other countries is available in water deeper than $30 \mathrm{~m}$. In contrast, all the European offshore wind turbines installed to date are on fixed-bottom substructures. These turbines have mostly been installed in water shallower than $20 \mathrm{~m}$ by driving monopiles into the seabed or by relying on conventional concrete gravity bases. These technologies are not economically feasible in deeper water. Instead, space-frame substructures, including tripods, quadpods, or lattice frames (e.g., "jackets"), will be required to maintain the strength and stiffness requirements at the lowest possible cost. The Beatrice Wind Farm Demonstrator Project in the North Sea near Scotland, where two 5-MW wind turbines are being installed on a jacket structure in $45 \mathrm{~m}$ of water, is a good example of this technology. ${ }^{1}$ At some depth, however, floating support platforms will be the most economical. This natural progression is illustrated in Figure 1-1 [71]. Without performing a dynamic analysis, Musial, Butterfield, and Boone [70] have demonstrated the economic potential of one floating platform design.

Numerous floating support platform configurations are possible for offshore wind turbines, particularly considering the variety of mooring systems, tanks, and ballast options that are used in the offshore oil and gas $(\mathrm{O} \& \mathrm{G})$ industries. Figure 1-2 illustrates several of these concepts, which are classified in terms of how the designs achieve static stability. The spar-buoy concept, which can be moored by catenary or taut lines, achieves stability by using ballast to lower the center of mass (CM) below the center of buoyancy (COB). The tension leg platform (TLP) achieves stability through the use of mooring-line tension brought about by excess buoyancy in the tank. In the barge concept, the barge is generally moored by catenary lines and achieves stability through its water-plane area. Hybrid concepts, which use features from all three classes, are also a possibility [14].

Because the offshore O\&G industries have demonstrated the long-term survivability of offshore floating structures, the technical feasibility of developing offshore floating wind turbines is not in question. Developing cost-effective offshore floating wind turbine designs that are capable of penetrating the competitive energy marketplace, though, will require considerable thought and analysis. Transferring the offshore O\&G technology directly to the offshore wind industry without adaptation would not be economical. These economic challenges impart technological challenges [14], which, in turn, must be addressed through conceptual design and analysis.

The International Electrotechnical Commission (IEC) 61400-1 design standard [33] specifies the design requirements for land-based wind turbines. The upcoming IEC 61400-3 design standard [34] supplements the 61400-1 design standard with design requirements for sea-based wind turbines. Both design standards require that an integrated loads analysis be performed when a machine is certified. Such analysis is also beneficial for conceptual design and analysis,

\footnotetext{
${ }^{1}$ Web site: http://www.beatricewind.co.uk/home/default.asp
} 


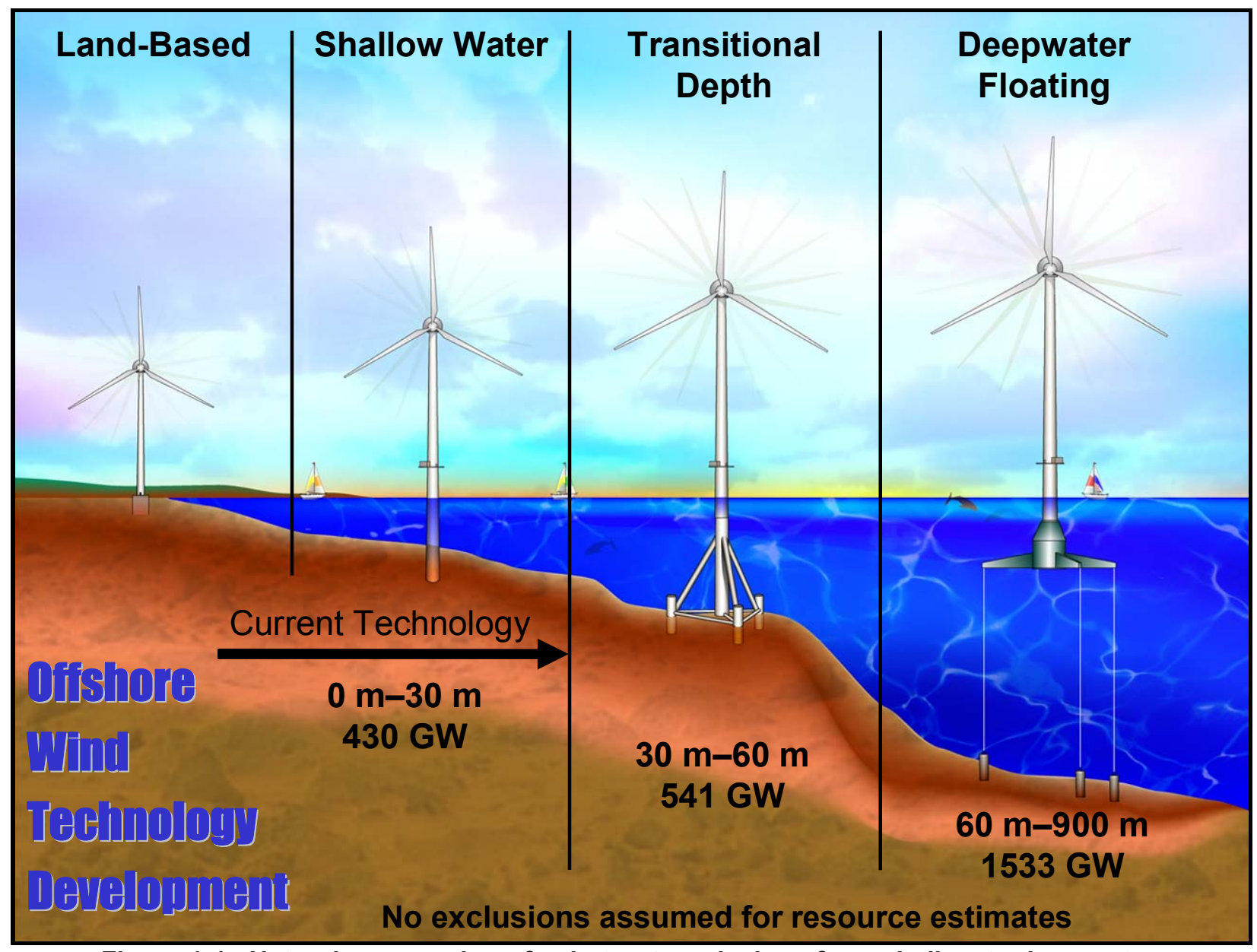

Figure 1-1. Natural progression of substructure designs from shallow to deep water

allowing designers to conceptualize cost-effective wind turbines that achieve favorable performance and maintain structural integrity.

Integrated loads analyses are carried out with comprehensive simulation tools called design codes. For land-based wind turbine analysis, these design codes are labeled as "aero-servoelastic" tools, meaning that they incorporate aerodynamic models, control system (servo) models, and structural-dynamic (elastic) models in a fully coupled (integrated) simulation environment. More precisely, these simulation tools incorporate sophisticated models of both turbulent- and deterministic-wind inflow; aerodynamic, gravitational, and inertial loading of the rotor, nacelle, and tower; elastic effects within and between components and in the foundation; and mechanical actuation and electrical responses of the generator and of the control and protection systems.

In the offshore environment, additional loading is present, and additional dynamic behavior must be considered. Wave-induced (diffraction) and platform-induced (radiation) hydrodynamic loads, which are the most apparent new sources of loading, impart new and difficult challenges for wind turbine analysts. Additional offshore loads arise from the impact of floating debris or sea ice and from marine growth buildup on the substructure. The analysis of offshore wind turbines must also account for the dynamic coupling between the motions of the support platform 


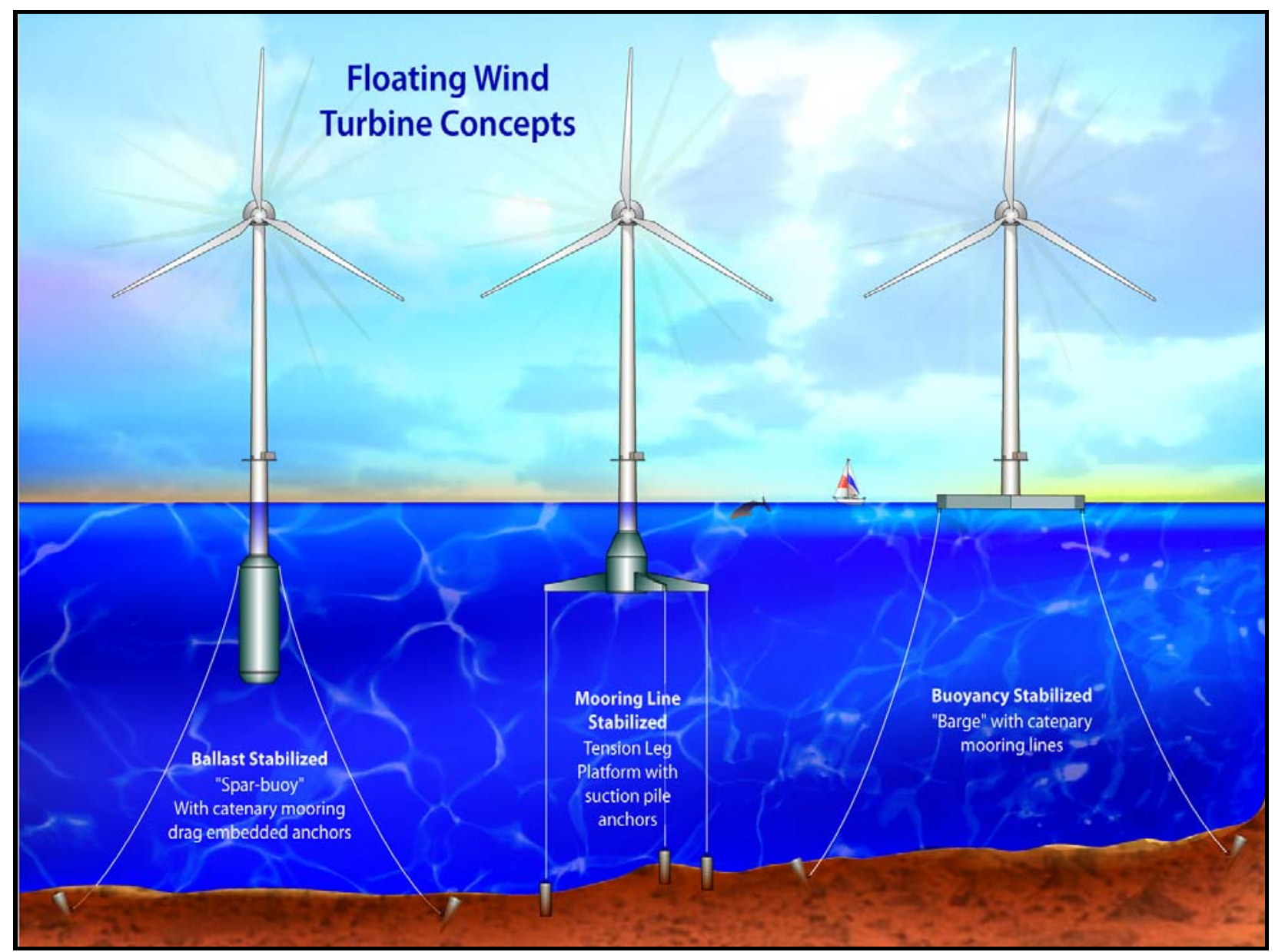

Figure 1-2. Floating platform concepts for offshore wind turbines

and the wind turbine, as well as for the dynamic characterization of the mooring system for compliant floating platforms.

\subsection{Previous Research}

In recent years, a variety of wind turbine aero-servo-elastic simulation tools have been expanded to include the additional loading and responses representative of fixed-bottom offshore support structures $[4,15,19,52,61,77,97]$. For the hydrodynamic-loading calculations, all of these codes use Morison's equation [22,74]. The incident-wave kinematics are determined using an appropriate wave spectrum together with linear Airy wave theory for irregular seas or one of the various forms of nonlinear stream-function wave theory for extreme regular seas. The effects of sea currents are also included. Morison's representation, which is most valid for slender vertical surface-piercing cylinders that extend to the sea floor, accounts for the relative kinematics between the fluid and substructure motions, including added mass, incident-wave inertia, and viscous drag. It ignores the potential effects of free-surface memory and atypical added-massinduced couplings between modes of motion in the radiation problem [16,76], and takes advantage of G. I. Taylor's long-wavelength approximation [16,76,85] to simplify the diffraction problem. These neglections and approximations inherent in Morison's representation limit its 
applicability for analyzing many of the proposed support platform concepts for offshore floating wind turbines.

A number of studies have also assessed the preliminary design of offshore floating wind turbines. Many of these studies used linear frequency-domain analysis, which is commonly employed in the offshore O\&G industries. For example, Bulder et al [13] used linear frequencydomain hydrodynamics techniques to find the response amplitude operators (RAOs) and amplitude standard deviations of the six rigid-body modes of motion for the support platform of a tri-floater design for a 5-MW wind turbine. Lee [59] used a similar process to analyze a TLP design and a taut-leg spar-buoy design for a 1.5-MW wind turbine. Wayman, Sclavounos, Butterfield, Musial, and I [100,101] also used a similar process to analyze multiple TLP designs and a shallow-drafted barge (SDB) design for a 5-MW wind turbine. Most recently, through frequency-domain analysis, Vijfhuizen [98] designed a barge for a 5-MW wind turbine, which was also a platform for an oscillating water column (OWC) wave-energy device. In these studies, the attributes of the wind turbine were included by augmenting the body-mass matrix with the mass properties of the turbine. The hydrodynamic-damping and -restoring matrices were also augmented with damping and restoring contributions from rotor aerodynamics and gyroscopics. Additionally, the linearized restoring properties of the mooring system were derived about a mean offset displacement of the support platform caused by the aerodynamic thrust on the rotor. The elasticity of the wind turbine was ignored. All of the studies demonstrated the technical feasibility of offshore floating wind turbines by showing that, through proper design, the natural frequencies of the floating support platform could be placed where there was little energy in the wave spectrum to ensure that the overall dynamic response was minimized.

One limitation of these linear frequency-domain analyses is that they cannot capture the nonlinear dynamic characteristics and transient events that are important considerations in wind turbine analysis. Several other offshore floating wind turbine studies have addressed this limitation. Using what they termed a "state-domain" technique, Henderson and Patel [31] used RAOs to prescribe the motions of a $700-\mathrm{kW}$ wind turbine to determine the effect that platform motions have on turbine fatigue loads. They showed that platform motions have little effect on power capture and rotor loads; instead, these were dominated by the aerodynamics of the rotor. They also showed, though, that platform motions have a substantial effect on the nacelle and tower loads, which are dominated by inertia. As a result, the tower would have to be strengthened if the platform motions could not be reduced. The same conclusions were drawn independently by Fulton, Malcolm, and Moroz [23] and by Withee [103]. These researchers used different time-domain aero-servo-elastic wind turbine simulators that had been adapted to include the effects of platform motion and hydrodynamic loading of TLP designs for a 5-MW and 1.5-MW wind turbine, respectively. In a more recent analyses, Nielsen, Hanson, and Skaare [75,87] and Larsen and Hanson [57] drew similar conclusions. These researchers used a combined aero-servo-elastic, hydrodynamic, and mooring program to design a deep-drafted spar buoy (called "Hywind") to support a 5-MW wind turbine and develop its corresponding control system. This study, in particular, was important because the computer program simulations were verified by the response of a scaled-down model in a wave tank experiment. Finally, Zambrano, MacCready, Kiceniuk, Roddier, and Cermelli [105,106] demonstrated the technical (but not economic) feasibility of smaller floating wind turbines. They used a time-domain model to determine the support platform motions and mooring tensions for a semisubmersible platform 
that supports three wind turbines of either $90 \mathrm{~kW}$ or $225 \mathrm{~kW}$ each and a TLP that supports a single $1-\mathrm{kW}$ turbine.

These studies have other limitations that must also be addressed. For instance, the time-domain dynamics models employed by Fulton, Malcolm, and Moroz [23], and Withee [103] used Morison's equation to compute the hydrodynamic loading on the TLPs, which, as I mentioned earlier, ignores potentially important hydrodynamic effects. Although the hydrodynamics model was more sophisticated in the time-domain dynamics program employed by Zambrano, MacCready, Kiceniuk, Roddier, and Cermelli [105,106], their aerodynamics and structuraldynamics models were unsophisticated, consisting only of a single horizontal drag force for the aerodynamics model and the six rigid-body modes of motion of the support platform for the fullsystem structural-dynamics model. Also, the concept analyzed by Nielsen, Hanson, and Skaare [75,87] and Larsen and Hanson [57] had such a large draft $(120 \mathrm{~m})$ that it would be difficult to construct and be deployable only at sites with very deep water. Moreover, the findings and conclusions drawn by all of the researchers mentioned in this section must be verified through a rigorous loads analysis.

\subsection{Objectives, Scope, and Outline}

In light of the limitations of the research studies described in the last section, I set three objectives for my work: (1) develop a comprehensive simulation tool that can model the coupled dynamic response of offshore floating wind turbines, (2) verify the simulation capability through model-to-model comparisons, and (3) apply the simulation tool in an integrated loads analysis for one of the promising floating support platform concepts.

My first objective addresses the foremost problem with the prior research studies - that the dynamic models developed previously were not general enough to allow analysis of a variety of support platform configurations and were also limited in their capability for the configurations they could model. My model development activities also address the primary need dictated by the upcoming international design standard [34] for offshore wind turbines and fulfill the leading recommendation from the study by Fulton, Malcolm, and Moroz [23] about the design of a semisubmersible platform and anchor foundation system for wind turbine support. My offshore floating wind turbine simulation tool was developed with enough sophistication to address the limitations of the previous time- and frequency-domain studies. In addition, it has the features required to perform an integrated loads analysis for a variety of wind turbine, support platform, and mooring system configurations. The simulation tool I developed is a fully coupled aerohydro-servo-elastic model based in the time domain. By "aero-hydro-servo-elastic," I mean that aero-servo-elastic models and hydrodynamic models are incorporated in the fully coupled simulation environment. The "fully coupled" nature of this capability is important for possible follow-on design optimization projects, which would be difficult to carry out without taking the integrated dynamic response into account. I describe the development of this simulation capability in Chapter 2. I have previously published some of this material in papers coauthored with Sclavounos [40] and Buhl [41].

Chapter 3 presents the input data used for the model verification exercises and loads analyses discussed in the subsequent chapters. These data include the specifications of a 5-MW wind turbine, of two floating platforms, and of environmental conditions at a reference site. Although 
I developed the specifications of the wind turbine, I did not develop the basic designs of the two floating support platforms used in this work. Butterfield, Musial, Scott, and I have submitted the material in Chapter 3 for publication [42] and Buhl and I have already summarized parts of the information $[41,43]$.

In fulfillment of my second objective, Chapter 4 presents a verification of the simulation capability covered in Chapter 2 using the input data given in Chapter 3. The verification exercises were important because they gave me confidence in the simulation capability that led me to pursue more thorough investigations into the dynamic behavior of offshore floating wind turbines. Again, I have previously published some of this material in work with Buhl [41].

My third objective addresses the secondary problem with the previous research studies - that their results were demonstrated through only a handful of simulations. To carry out my integrated loads analysis, I applied the simulation capability using the analysis requirements prescribed by the IEC design standards as my guide. Chapter 5 contains an overview and description of the loads analysis, and Chapter 6 presents the analysis results. Buhl and I have published some of this material elsewhere [41,43]. I ran loads analyses for a 5-MW wind turbine supported both on land and offshore by a floating barge with slack catenary moorings. The loads analysis allowed me to characterize the dynamic response of the land- and sea-based systems. In addition, by comparing both responses, I was able to quantify the impact brought about by the dynamic coupling between the turbine and the floating barge in the presence of combined wind and wave loading. The results of comprehensive loads analyses for some of the other promising offshore floating wind turbine configurations are left for future work.

My loads analysis quickly demonstrated that the pitching motion of the barge brought about load excursions in the supported wind turbine that exceeded those experienced by the equivalent turbine that was installed on land. One possible avenue for improving the response of the floating wind turbine is to apply active wind turbine control. To this end, Chapter 7 addresses the influence of conventional wind turbine control methodologies to the pitch damping of the floating wind turbine system analyzed in Chapter 6. I have submitted some of this material for publication [44].

In Chapter 8, I summarize the work, present my conclusions, and suggest directions for further research.

My work does not address system economics; manufacturing, installation, or decomissioning considerations; or optimization of the floating wind turbine system or wind farm. Nonetheless, the work I present here is fundamental to determining the most technically attractive and economically feasible floating wind turbine design as outlined in Section 1.1. 


\section{Chapter 2 Development of Aero-Hydro-Servo-Elastic Simulation Capability}

Limitations with previous time- and frequency-domain studies on offshore floating wind turbines motivated me to develop simulation capability for modeling the fully coupled aero-hydro-servoelastic response of such systems. In developing this capability, I found it beneficial to combine the computational methodologies of the land-based wind turbine and of the offshore O\&G industries.

Over the past decade, the U.S. Department of Energy's (DOE's) National Renewable Energy Laboratory (NREL), ${ }^{1}$ has sponsored the development, verification, and validation of comprehensive aero-servo-elastic simulators through the National Wind Technology Center (NWTC). ${ }^{2}$ These simulators are capable of predicting the coupled dynamic response and the extreme and fatigue loads of land-based horizontal-axis wind turbines (HAWTs). The U.S. wind industry relies on two primary design codes: (1) FAST (Fatigue, Aerodynamics, Structures, and Turbulence) [39] with AeroDyn [55,67] and (2) MSC.ADAMS ${ }^{\circledR}$ (Automatic Dynamic Analysis of Mechanical Systems) with A2AD (ADAMS-to-AeroDyn) [20,54] and AeroDyn. FAST and MSC.ADAMS are separate programs that can be run independently to model the structuraldynamic response and control system behavior of HAWTs. FAST is a publicly available code distributed $^{3}$ by the NWTC that employs a combined modal and multibody structural-dynamics formulation in the time domain. I wrote most of the code in its present form (while employed at NREL / NWTC), but I based much of it on previous development efforts at Oregon State University and the University of Utah. The more complex MSC.ADAMS code is a commercially available and general-purpose code from MSC Software Corporation ${ }^{4}$ that employs a higher fidelity multibody-dynamics formulation in the time domain. It is adaptable for modeling wind turbines through the set of A2AD modules Windward Engineering LLC ${ }^{5}$ and I developed. This set of A2AD modules is distributed ${ }^{6}$ by the NWTC.

The complicated HAWT models possible within MSC.ADAMS can be generated through a preprocessor functionality built-into the simpler FAST code. To enable the fully coupled aeroservo-elastic modeling of wind turbines in the time domain, both FAST and MSC.ADAMS have been interfaced with the AeroDyn aerodynamic subroutine package for calculating wind turbine aerodynamic forces. AeroDyn was developed by Windward Engineering LLC and is distributed 7 by the NWTC. Note that I use the term "ADAMS" to mean "MSC.ADAMS with A2AD" in this work.

\footnotetext{
${ }^{1}$ Web site: http://www.nrel.gov/

${ }^{2}$ Web site: http://www.nrel.gov/wind/

${ }^{3}$ Web site: http://wind.nrel.gov/designcodes/simulators/fast/

${ }^{4}$ Web site: http://www.mscsoftware.com

${ }^{5}$ Web site: http://www.windwardengineering.com/

${ }^{6}$ Web site: http://wind.nrel.gov/designcodes/simulators/adams2ad/

${ }^{7}$ Web site: http://wind.nrel.gov/designcodes/simulators/aerodyn/
} 
For the offshore O\&G industries, the Center for Ocean Engineering at the Massachusetts Institute of Technology (MIT) ${ }^{8}$ has sponsored the development, verification, and validation of comprehensive hydrodynamic computer programs capable of analyzing the wave interaction and dynamic responses of offshore floating platforms in both the frequency and time domains. SML (SWIM-MOTION-LINES) $[47,48,49,50]$ from MIT is a publicly available suite of computer modules for determining the hydrodynamic properties and responses of floating structures operating in wind, waves, and current in waters of moderate to great depth. SML's SWIM module [48] analytically solves the linear- and second-order frequency-domain hydrodynamic radiation and diffraction problems for platforms composed of simple geometry, such as arrays of vertical surface-piercing cylinders. The MOTION module [49] finds solutions of the largeamplitude, time-domain, slow-drift responses and the LINES module [50] determines the nonlinear mooring-line, tether, and riser reactions with the platform. The computer program WAMIT $^{\circledR}$ (Wave Analysis at MIT) [58], a commercially available product from WAMIT, Inc., ${ }^{9}$ uses a three-dimensional numerical-panel method in the frequency domain to solve the linearized hydrodynamic radiation and diffraction problems for the interaction of surface waves with offshore platforms of arbitrary geometry.

This chapter presents my efforts to develop an upgrade of the land-based wind turbine simulation tools, FAST with AeroDyn and ADAMS with AeroDyn, to include the additional dynamic loading and motions representative of offshore floating systems. Also in this chapter, I discuss how the SML and WAMIT codes are used in the overall solution.

Before I describe the additional formulations needed to incorporate offshore dynamic responses within FAST with AeroDyn and ADAMS with AeroDyn, I take a step back and outline the general class of theories employed for modeling a wind turbine within the simulation tools (see Section 2.1). Then, in Section 2.2, I discuss the assumptions inherent in, and the implications of, the new formulations relating to floating support platforms for wind turbines. The remaining sections of this chapter cover the addition of support platform kinematics and kinetics modeling (see Section 2.3), the incorporation of support platform hydrodynamics modeling (Section 2.4), and the inclusion of mooring system modeling (Section 2.5) into FAST and ADAMS. I then summarize this information in Section 2.6.

I call my newly developed time-domain hydrodynamics module "HydroDyn" because it is to hydrodynamic loading what AeroDyn is to aerodynamic loading in the system.

I make extensive use of equations to describe the hydrodynamic and mooring system formulations as they relate to floating support platforms for offshore wind turbines. For conciseness and clarity, I have not included the derivations of these equations; it is the form of the equations and the physics behind them that I want to emphasize. (Please refer to the associated references for many of the derivations.) In this chapter, I also emphasize the distinctions between my model and others used in the offshore wind turbine industry. These distinctions are important because the approach I have taken to implement offshore dynamics into wind turbine design codes is substantially different than the approach taken by other

\footnotetext{
${ }^{8}$ Web site: $\underline{\text { http://oe.mit.edu/ }}$

${ }^{9}$ Web site: http://www.wamit.com/
} 
simulation specialists who have analyzed fixed-bottom offshore turbine support structures $[4,15,19,52,61,77,97]$. Finally, my approach is more comprehensive than that taken by others who have performed preliminary dynamic analyses of floating wind turbines $[13,23,31,59,98,100,101,103,105,106]$. I discuss these dissimilarities at greater length in this chapter.

\subsection{Overview of Wind Turbine Aero-Servo-Elastic Modeling}

The FAST code is a nonlinear time-domain simulator that employs a combined modal- and multibody-dynamics formulation. Although FAST has a limited number of structural degrees of freedom (DOFs), it can model most common wind turbine configurations and control scenarios, including three-bladed turbines with a rigid hub, two-bladed turbines with a rigid or teetering hub, turbines with gearboxes or direct drives, turbines with induction generators or variablespeed controllers, turbines with active blade-pitch regulation or passive stall regulation, turbines with active or passive nacelle-yaw control, and turbines with passive rotor or tail furling.

In FAST, flexibility in the blades and tower is characterized using a linear modal representation that assumes small deflections within each member. The flexibility characteristics of these members are determined by specifying distributed stiffness and mass properties along the span of the members, and by prescribing their mode shapes as equivalent polynomials. FAST allows for two flapwise and one edgewise bending-mode DOFs per blade and two fore-aft and two side-toside bending-mode DOFs in the tower. Along with one variable generator speed DOF, torsional flexibility in the drivetrain is modeled using a single-DOF equivalent linear-spring and -damper model in the low-speed shaft. The nacelle (or at least the load-bearing base plate of the nacelle) and hub are modeled in FAST as rigid bodies with appropriate lumped mass and inertia terms. All DOFs can be enabled or locked through switches, permitting one to easily increase or decrease the fidelity of the model. All DOFs except the blade and tower bending-mode DOFs can exhibit large displacements without loss of accuracy. Time marching of the nonlinear equations of motion is performed using a constant-time-step Adams-Bashforth-Adams-Moulton predictor-corrector integration scheme. More details can be found in other works $[37,38,39]$.

Not only can FAST be used for time-domain simulation, but it can also be used to generate linearized representations of the complete nonlinear aero-elastic wind turbine model (not including the influence of the control system). This analysis capability is useful for developing linearized state matrices of a wind turbine "plant" to aid in the design and analysis of control systems. It is also useful for determining the full-system modes of an operating or stationary HAWT through the use of a simple eigenanalysis. More information can be found elsewhere $[38,39]$.

The structural-dynamics model in ADAMS is more sophisticated than the one in FAST. ADAMS is a nonlinear time-domain code that employs a general-purpose multibody-dynamics formulation, which permits an almost unlimited number of configurations and DOFs. It is not a code specific to wind turbines and is routinely used by engineers in the automotive, aerospace, and robotics industries. ADAMS represents a mechanical system as a series of six-DOF rigid bodies with lumped mass and inertia interconnected by joints (constraints). Flexible members, such as the blades and tower of a wind turbine, are modeled in ADAMS using a series of rigid bodies interconnected by multidimensional linear stiffness and damping matrices (i.e., six-DOF 
joints). As in FAST, the nacelle and hub are typically modeled using rigid bodies with lumped mass and inertia properties. ADAMS incorporates a time-marching scheme similar to the one in FAST, except that the ADAMS scheme incorporates a variable-time-step algorithm.

It is often necessary to use the more complicated ADAMS code in place of FAST because ADAMS has many features that FAST does not. These include torsional and extensional DOFs in the blades and tower, geometric (mass and elastic offsets from the pitch axis) and material (asymmetric composite ply lay-up) couplings in the blades and tower, built-in prebend in the blades, and actuator dynamics in the blade-pitch controller, among others. I also find ADAMS useful for verifing the dynamic-response predictions obtained from FAST when I add new functionality to FAST, especially new DOFs. This is because the equations of motion in ADAMS are not defined by the user and because its dynamic-response predictions are well verified (see Ref. [39] for more information).

Both FAST and ADAMS allow analysts to include control system logic for actively controlling nacelle yaw, generator torque, and blade pitch, among other actuators. The controller outputs can be based on inputs that can be developed from the feedback of any number of previously calculated model states or other derived parameters (see Ref. [39] for more information).

Both FAST and ADAMS interface to the AeroDyn aerodynamic subroutine package for computing aerodynamic forces. This aerodynamic package models rotor aerodynamics using the classic quasi-steady blade-element / momentum (BEM) theory or a generalized dynamic-wake (GDW) model, both of which include the effects of axial and tangential (rotational) induction. The BEM model uses tip and hub losses as characterized by Prandtl. Dynamic-stall behavior can be included using the optional Beddoes-Leishman dynamic-stall model. The element motion and position are included in the calculation of the instantaneous relative wind vector at each blade element, making the codes fully aero-servo-elastic. More details can be found in Ref. [67].

\subsection{Assumptions for the New Model Development}

When adding models for floating wind turbine simulation; including the support platform kinematics, kinetics, and hydrodynamics, as well as the mooring system responses; I invoked a number of assumptions in addition to those that were previously inherent in the land-based aeroservo-elastic simulation tools.

For the support platform kinematics and kinetics, I assumed that the floating support platform is represented well as a six-DOF rigid body with three small rotational displacements. As I discuss in Section 2.3, the implications of the small-angle assumption are not thought to be critical. Like the load-bearing base plate of the wind turbine's nacelle, the support platform was modeled as a rigid body because it is considered to be so strong and inflexible, at least in relation to the wind turbine's blades and tower, that direct hydro-elastic effects are unimportant. Additionally, I assumed that the tower is rigidly cantilevered to the support platform. Also, the CM (not including the wind turbine) and $\mathrm{COB}$ of the support platform were assumed to lie along the centerline of the undeflected tower.

I had originally planned [40] to include mooring system behavior in my offshore upgrades of FAST and ADAMS by interfacing the dynamic mooring system LINES module of SML. 
Because I discovered that LINES is numerically unstable when modeling the slack catenary mooring lines of interest in some of my analyses, I developed my own quasi-static model for mooring systems instead. I present the development of this model and the implications of its quasi-static characteristic in Section 2.5.

My fundamental assumption in the development of the HydroDyn hydrodynamics module was linearization of the classical marine hydrodynamics problem. In the field of marine hydrodynamics, the assumption of linearity signifies many things, three of which I discuss next.

First, linearization of the hydrodynamics problem (i.e., linearization of the nonlinear kinematic and dynamic free-surface boundary conditions) implies that the amplitudes of the incident waves are much smaller than their wavelengths. This permits the use of the simplest incident-wavekinematics theory, which is known as Airy wave theory. This assumption necessarily precludes me from being able to model steep or breaking waves and the resulting nonlinear wave-induced "slap" and "slam" loading. Linearization is a reasonable assumption for most waves in deep water and for small-amplitude waves in shallow water. When waves become extreme or propagate toward shore in shallow water, however, higher-order wave kinematics theories are required, but neglected in my model.

Second, linearization implies that the translational displacements of the support platform are small relative to the size of the body (i.e., the characteristic body length). In this way, the hydrodynamics problem can be split into three separate and simpler problems: one for radiation, one for diffraction, and one for hydrostatics. I discuss the details of these problems in Section 2.4. As is often misunderstood, linearity of the hydrodynamics problem does not imply that the characteristic length of the support platform needs to be small relative to the wavelength of the incident waves. When the characteristic length of the support platform is small relative to the wavelength of the incident waves, the hydrodynamic scattering problem (part of the diffraction problem) can be greatly simplified, but I did not invoke this simplification in my analysis. I did, however, simplify the diffraction problem by ignoring incident-wave directional spreading and by assuming that all irregular sea states were long-crested. In other words, I modeled irregular sea states (stochastic waves) as a summation of sinusoidal wave components whose amplitude is determined by a wave spectrum, each parallel and described by Airy wave theory.

Third, linearization suggests that one can take advantage of the powerful technique of superposition. I discuss how superposition relates to the hydrodynamics problems in Section 2.4.

I have augmented the linearized hydrodynamics problem with the viscous-drag term from Morison's equation. I included this effect because it was relatively easy to add, it allowed me to incorporate the influence of sea current, and it can be an important source of hydrodynamic damping in some situations.

Naturally, linearization of the hydrodynamics problem implies that second- or higher-order hydrodynamic effects are not accounted for in my model. Although this follows directly from the definition of linearity, it is important to discuss its implications. Second- or higher-order nonlinear hydrodynamics models more properly account for the loading about the actual instantaneous wetted surface of a floating body and may be important when the support platform motions are large relative to their characteristic lengths. For example, I neglected second-order 
mean-drift forces in my hydrodynamics calculations even though they can be derived from the linear hydrodynamics solution. Just like the wind-induced thrust loading on the turbine rotor or sea-current-induced loading on the platform, mean-drift forces can bring about a mean offset of the support platform in relation to its undisplaced position. And for compliant floating systems, the mooring system resistance is often related nonlinearly to its displacement; thus, the effects of mean-drift forces on the mooring system loads and resistances may be important for some designs. Second-order slow-drift forces, which result from the difference among the components of multiple incident waves of varying frequency, were also neglected in my linear formulation of the classical marine hydrodynamics problem. Mean- and slow-drift forces are sometimes important for wave-induced loading on support platforms with small drafts, large water-plane areas, and mooring system configurations that impose little resistance to surge and sway, such as in barge designs with catenary mooring lines. Likewise, second-order sum-frequency excitations, which result from the summation among the components of multiple incident waves of varying frequency, were also neglected in my linear problem. Second-order sum-frequency excitations are sometimes important when analyzing the "ringing" behavior in support platforms with mooring systems that impose a strong resistance to heave, such as in TLP designs.

In my models I also ignored the potential loading from vortex-induced vibration (VIV) caused by sea currents. When the VIV frequency nears a natural frequency of the system, a resonance phenomenon known as "lock-in" can occur. VIV is also known to be critical for the stability of some designs. The ancillary effect of the sea current on the radiation and diffraction problems, such as the Doppler-shifted frequency-of-encounter effect [22], was ignored as well.

Finally, I ignored the potential loading from floating debris or sea ice. Sea ice can be a significant source of loading if the support platform is intended to be used where sea ice is present. In the continental United States, this may be of particular concern when designing offshore wind turbine support platforms for installation in the Great Lakes.

Also note that the classical marine hydrodynamics problem takes advantage of unsteady potential-flow theory to derive the governing equations of fluid motion. This theory assumes that the fluid is incompressible, inviscid, and subject only to conservative body forces (i.e., gravity), and that the flow is irrotational.

\subsection{Support Platform Kinematics and Kinetics Modeling}

The first step required in upgrading existing land-based wind turbine simulation tools to make them useful for analyzing offshore systems is to introduce DOFs necessary for characterizing the motion of the support platform. For floating systems, it is crucial that all six rigid-body modes of motion of the support platform be included in the development. These include translational surge, sway, and heave displacement DOFs, along with rotational roll, pitch, and yaw displacement DOFs, as shown in Figure 2-1. In this figure, $X, Y, Z$ represents the set of orthogonal axes of an inertial reference frame fixed with respect to the mean location of the support platform, with the $X Y$-plane designating the still water level (SWL) and the $Z$-axis directed upward opposite gravity along the centerline of the undeflected tower when the support platform is undisplaced. 
Because most of the support platforms that have been proposed for floating wind turbines are more or less axisymmetric, and because there is no hydrodynamic mechanism that will induce yaw moments on such floating bodies, one might question whether the support platform yaw-rotation DOF is necessary. The wind turbine, however, induces yaw moments that are primarily the result of (1) the aerodynamic loads on the rotor when a yaw error exists between the rotor axis and nominal wind direction; and (2) the spinning inertia of the rotor combined with pitching motion (whether from support platform pitching or tower deflection), which induces a gyroscopic yaw moment.

As implied by item (2), then, the dynamic coupling between the motions of the support platform and the motions of the supported wind turbine are crucial in the development of the equations of motion. In fact, I use the term "fully

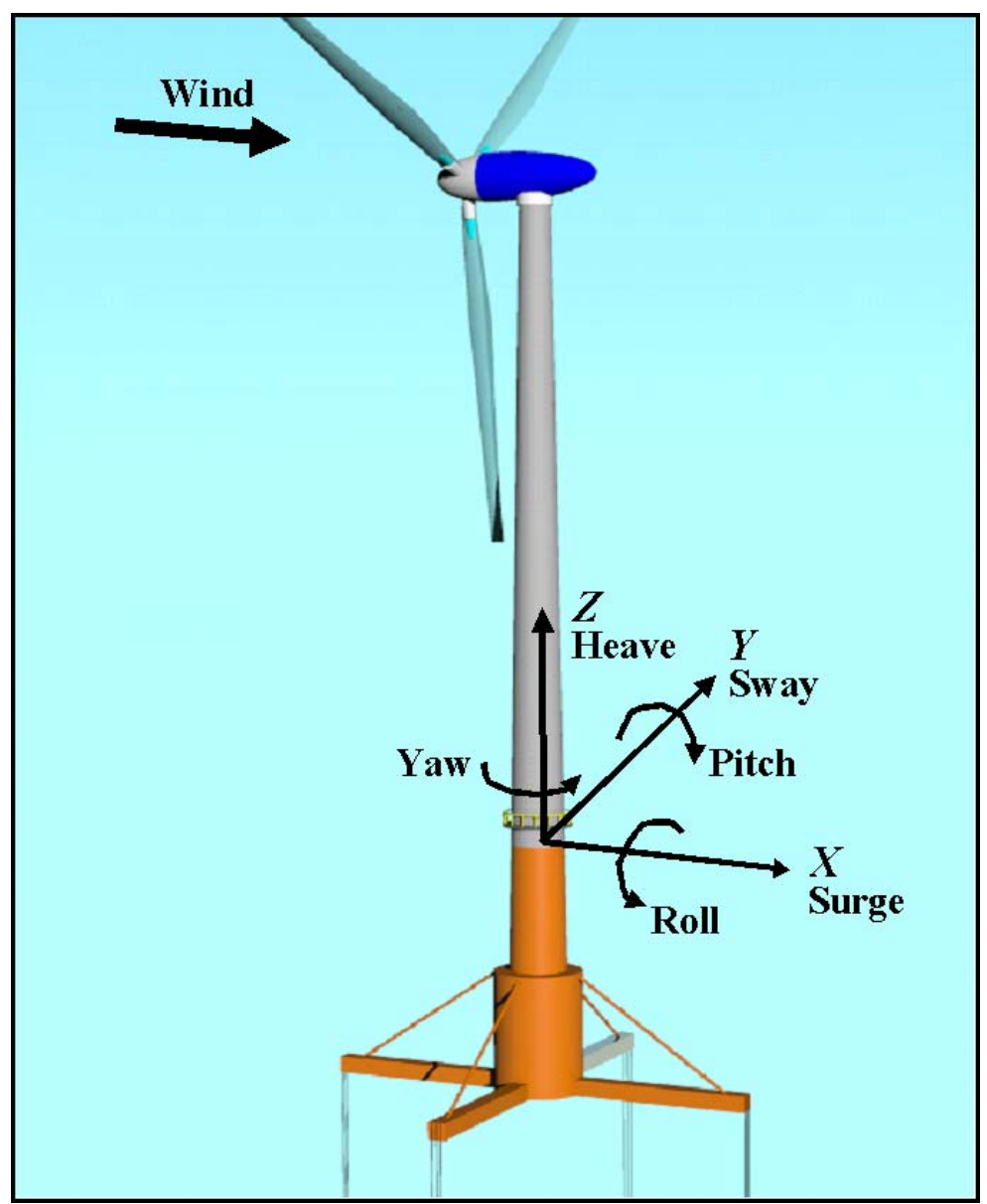

Figure 2-1. Support platform degrees of freedom Source: Modifed from Ref. [103] coupled" throughout this work, partially to imply that the wind turbine's response to wind and wave excitation is fully coupled through the structural-dynamic response. I do not use the term to imply that the wind-inflow and sea state conditions need to be correlated. I am not attempting to model the air-sea interface, which is a very complicated, multiphase fluid-flow problem.

In ADAMS, I obtained all the dynamic couplings between the motions of the support platform and the motions of the supported wind turbine by simply introducing the six-DOF support platform rigid body in the ADAMS model. In FAST, however, I obtained these couplings by introducing the six rigid-body support platform DOFs into the system's equations of motion. While rederiving the equations of motion, I incorporated all appropriate terms in the derivations of the kinematics expressions for the points and reference frames in the system. For example, before I added the six support platform DOFs in FAST, the kinematics expressions for the position, velocity, and acceleration vectors of a point in the nacelle depended only on the tower bending-mode and nacelle-yaw DOFs (because the tower-base reference frame was the inertial frame). Once the six support platform DOFs were added, the tower-base reference frame moves with the support platform, and thus, the kinematics expressions for a point in the nacelle also depend on the support platform DOFs. Indeed, the kinematics expressions for all of the points and reference frames in the system are affected by the support platform DOFs. 
With the assumption that all rotations of the support platform are small, rotation sequence becomes unimportant. Consequently, I could avoid all the complications of using Euler angles (or the like), where the order of rotation is significant, when I derived and implemented the equations of motion in FAST. Take $x, y, z$ to be the axes of the reference frame resulting from a transformation involving three orthogonal rotations $\left(\theta_{1}, \theta_{2}, \theta_{3}\right)$ about the axes of an original reference frame $X, Y, Z$. Using the first-order small-angle approximations for the sine and cosine functions, and neglecting terms of higher order in the Taylor series expansion, the standard Euler-angle transformation [25] relating the original and transformed reference frames simplifies to

$$
\left\{\begin{array}{l}
x \\
y \\
z
\end{array}\right\} \approx\left[\begin{array}{ccc}
1 & \theta_{3} & -\theta_{2} \\
-\theta_{3} & 1 & \theta_{1} \\
\theta_{2} & -\theta_{1} & 1
\end{array}\right]\left\{\begin{array}{l}
X \\
Y \\
Z
\end{array}\right\} .
$$

In this equation, the approximation $\operatorname{sign}(\approx)$ is used in place of an equal symbol $(=)$ because the resulting transformation matrix is not orthonormal beyond first order when the small-angle approximations are used. This implies that the transformed reference frame is not made up of a set of mutually orthogonal axes. (All transformation matrices relating sets of mutually orthogonal axes must be orthonormal.) Because using axes that are not mutually orthogonal can lead to inaccuracies that propagate in the dynamic-response calculations, I invoked a correction to the transformation matrix in Eq. (2-1) to ensure that it remained orthonormal. From matrix theory [32], I knew that the closest orthonormal matrix to a given matrix, in the sense of the Frobenius norm, ${ }^{10}$ was $[U][V]^{T}$ where $[U]$ and $[V]$ are the matrices of eigenvectors inherent in the singular-value decomposition (SVD) of the given matrix and the symbol "T", represents a matrix transpose. By performing these operations, the correct transformation expression was found to be

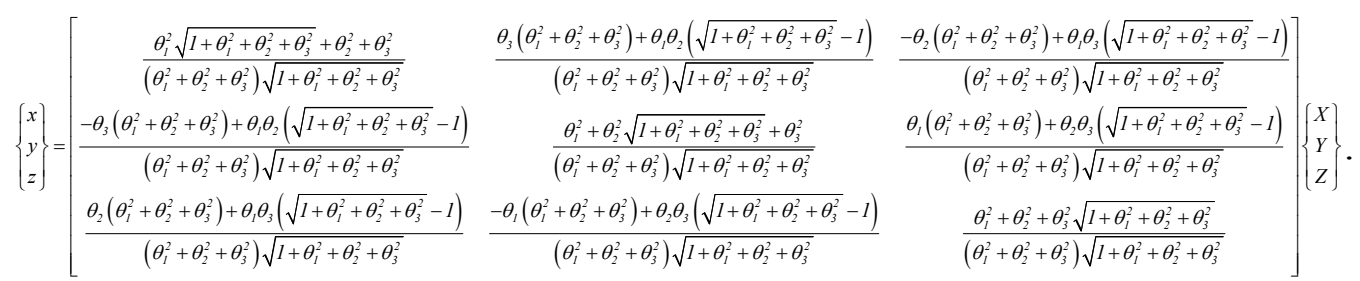

Showing that the transformation matrix in Eq. (2-2) is orthonormal beyond the first-order terms is a trivial exercise.

When applied to the support platform, $x, y, z$ represents the set of orthogonal axes of the bodyfixed reference frame within the support platform and $\theta_{1}, \theta_{2}, \theta_{3}$ are the roll, pitch, and yaw rotations of the support platform about the axes of the inertial reference frame (i.e., $X, Y, Z$ ). The origin of $x, y, z$ is called the platform reference point and is the location in the platform about

\footnotetext{
${ }^{10}$ The Frobenius norm, also known as the Euclidean norm, $l_{2}$ norm, Schur norm, or Hilbert-Schmidt norm, of a real $n \times n$ matrix $[A]$ is $\|[A]\|_{2}=\sqrt{\sum_{i=1}^{n} \sum_{j=1}^{n} a_{i j}^{2}}$, where $a_{i j}$ represents an element of $[A]$.
} 
which the support platform DOFs are defined. It is also the point at which the external load on the support platform is applied.

Similar labeling of $x, y, z$ and $X, Y, Z$ is used when applying Eq. (2-2) to relate a reference frame that is oriented with an element of a deflected blade (or tower) to the reference frame fixed in the root of the blade (or tower) - in this case the rotations are the flap, lag, and twist slopes of the blade (or tower) element.

In FAST, I have implemented Eq. (2-2) instead of Eq. (2-1) for all transformations relating the support platform to the inertial frame, all transformations relating the deflected tower elements to the tower base, and all transformations relating the deflected blade elements to the root of the blade. Although these results are not shown here, I have demonstrated that incorporating Eq. (2-2) in FAST instead of Eq. (2-1) leads to dynamic responses that are in much better agreement with responses obtained from ADAMS, which uses Euler angles, especially as the magnitude of the angles increases. The dynamic responses are more accurate when Eq. (2-2) is used in place of Eq. (2-1) because such transformation matrices get multiplied in series when determining the orientation of subsystems far along the load path away from the inertial frame, such as in a tower or blade element. Errors in a single transformation matrix are compounded when multiplied together. If the wind turbine were very rigid, the correction would not be necessary.

The transformation expression of Eq. (2-2) still loses considerable accuracy when any of the angles greatly exceed $20^{\circ}$. This threshold, though, should be adequate for support platform designs suitable for floating wind turbines because (1) the floating platform must be stable enough to enable access for maintenance personnel at regular intervals; and (2) the energy capture from the wind is proportional to the swept area of the rotor disk projected normal to the wind direction. (This projected area greatly diminishes with increasing angular displacement of the support platform, particularly in pitch.)

I used Kane's dynamics [45] to derive the equations of motion used in FAST. By a direct result of Newton's laws of motion, Kane's equations of motion for a simple holonomic system with $P$ generalized coordinates (DOFs) can be stated as follows:

$$
F_{i}+F_{i}^{*}=0 \quad(i=1,2, \ldots, P),
$$

where for a set of $W$ rigid bodies characterized by reference frame $N_{r}$, mass, $m_{r}$, and CM point $X_{r}$, the generalized active forces, $F_{i}$, are

$$
F_{i}=\sum_{r=1}^{W}{ }^{E} \boldsymbol{v}_{i}^{X_{r}} \cdot \boldsymbol{F}^{X_{r}}+{ }^{E} \omega_{i}^{N_{r}} \cdot \boldsymbol{M}^{N_{r}} \quad(i=1,2, \ldots, P),
$$

and the generalized inertia forces, $F_{i}^{*}$, are

$$
F_{i}^{*}=\sum_{r=1}^{W}{ }^{E} \boldsymbol{v}_{i}^{X_{r}} \cdot\left(-m_{r}{ }^{E} a^{X_{r}}\right)+{ }^{E} \omega_{i}^{N_{r}} \cdot\left(-{ }^{E} \dot{\boldsymbol{H}}^{N_{r}}\right) \quad(i=1,2, \ldots, P)
$$


In these equations, it is assumed that for each rigid body (body $N_{r}$ ), the three-component active force and moment vectors, $\boldsymbol{F}^{\boldsymbol{X}_{r}}$ and $\boldsymbol{M}^{\boldsymbol{N}_{r}}$, respectively, are applied at the CM location (point $X_{r}$ ). The three-component acceleration vector of the CM point $X_{r}$, is given by ${ }^{E} a^{X_{r}}$, and the first time derivative of the angular momentum of rigid body $N_{r}$, about $X_{r}$, in the inertial frame (frame

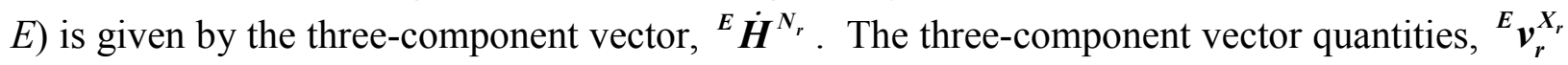
and ${ }^{E} \omega_{r}^{N_{r}}$, represent the partial linear velocities of CM point $X_{r}$ and the partial angular velocities of rigid body $N_{r}$ in the inertial frame, respectively. The symbol "." represents a vector dot product.

Although it was a long and tedious process, I had no particular difficulty in deriving the FAST system's equations of motion (which I do not present here). First, I derived kinematics expressions for the position, velocity, and acceleration vectors for all of the key points and reference frames in the system, taking into account all appropriate DOFs I described previously. These derivations were manageable when expressing terms relative to an appropriate reference frame, taking advantage of transformation relationships like Eq. (2-2). For example, with the tower assumed to be cantilevered to the support platform, it is fairly straightforward to write an expression for the angular velocity of a tower element relative to the support platform. The absolute angular velocity of the tower element is then just the vector sum of the angular velocity relative to the support platform and the angular velocity of the support platform relative to the inertial frame. The angular velocity of the support platform relative to the inertial frame, in turn, is just the vector sum of the first time derivatives of the roll, pitch, and yaw DOFs.

Once I derived the kinematics expressions, I established the partial velocity vectors utilized by Kane's dynamics. These, along with expressions for the generalized active and inertia forces, established the kinetics and led systematically to the complete nonlinear time-domain equations of motion of the coupled wind turbine and support platform system.

The kinetics expressions for the support platform included contributions from platform mass and inertia, gravity, hydrodynamics, and the reaction loads of the mooring system. I used an implementation that assumed that the $\mathrm{CM}$ of the support platform (not including the wind turbine) is located along the centerline of the undeflected tower; a point mass and all three principal inertias of the support platform (roll, pitch, and yaw) were included in this model. The effects of marine-growth buildup on the support platform can then be modeled through a suitable adjustment of the platform mass and inertia.

Once derived, the complete nonlinear time-domain equations of motion of the coupled wind turbine and support platform system are of the general form:

$$
M_{i j}(q, u, t) \ddot{q}_{j}=f_{i}(q, \dot{q}, u, t),
$$

where $M_{i j}$ is the $(i, j)$ component of the inertia mass matrix, which depends nonlinearly on the set of system DOFs $(q)$, control inputs $(u)$, and time $(t) ; \ddot{q}_{j}$ is the second time derivative of DOF $j$; and $f_{i}$ is the component of the forcing function associated with DOF $i$. The forcing function, $f_{i}$, depends nonlinearly on the set of system DOFs and their first time derivatives ( $q$ and $\dot{q}$ respectively), as well as the set of control inputs $(u)$ and time $(t)$, and is positive in the direction 
of positive motion of DOF $i$. I am employing Einstein notation in Eq. (2-6), in which it is implied that when the same subscript appears in multiple variables in a single term, there is a sum of all of the possible terms. In FAST, for example, subscripts $i$ and $j$ range from one to the total number of DOFs in the model (i.e., up to 22 for a two-bladed floating wind turbine or up to 24 for a three-bladed floating wind turbine).

Naturally, when hydrodynamic loading is present on the support platform, hydrodynamicimpedance forces - including the influence of added mass - are important. The added-mass components of these forces are present because the density of water is of the same order of magnitude as the density of the materials that make up the primary structure. This is in contrast to aerodynamic loading on the wind turbine, in which one generally ignores the influence of added mass because the density of air is much less than the density of the materials that make up the primary structure. To ensure, then, that the equations of motion were not implicit (i.e., I wanted to avoid $f_{i}$ depending on $\ddot{q}$ ), the total external load acting on the support platform (other than those loads transmitted from the wind turbine and the weight of the support platform) was split into two components: an impulsive added-mass component summing with $M_{i j}$ and the rest of the load adding to $f_{i}$. In other words, the total external load on the support platform, $F_{i}^{\text {Platorm }}$, was written as follows:

$$
F_{i}^{\text {Platform }}=-A_{i j} \ddot{q}_{j}+F_{i}^{\text {Hydro }}+F_{i}^{\text {Lines }},
$$

where $A_{i j}$ is the $(i, j)$ component of the impulsive hydrodynamic-added-mass matrix to be summed with $M_{i j}, F_{i}^{H y d r o}$ is the $i^{\text {th }}$ component of the applied hydrodynamic load on the support platform associated with everything but $A_{i j}$, and $F_{i}^{\text {Lines }}$ is the $i^{\text {th }}$ component of the applied load on the support platform from the contribution of all mooring lines. I then included both $F_{i}^{\text {Hydro }}$ and $F_{i}^{\text {Lines }}$ with the rest of the forcing function, $f_{i}$, in Eq. (2-6). In Eq. (2-7), subscripts $i$ and $j$ range from 1 to 6 ; one for each support platform DOF $(1=$ surge, $2=$ sway, $3=$ heave, $4=$ roll, $5=$ pitch, $6=$ yaw). I discuss the forms of the hydrodynamic impulsive-added-mass and hydrodynamic-forcing terms in Section 2.4, and the term associated with the mooring lines in Section 2.5.

My implementation of the kinetics was not specific to the dynamic response of offshore floating systems. It can also be used as the basis for modeling land-based foundations and fixed-bottom sea-based foundations. With any type of foundation, the contribution to the kinetics expressions from the mooring system must be replaced with contributions from soil added mass (if any), elasticity, and damping. For land-based foundations, the effects of hydrodynamic loading would be ignored.

\subsection{Support Platform Hydrodynamics Modeling}

Hydrodynamics are included within computer simulation programs by incorporating a suitable combination of incident-wave kinematics and hydrodynamic loading models. Hydrodynamic loads result from the integration of the dynamic pressure of the water over the wetted surface of a floating platform. These loads include contributions from inertia (added mass) and linear drag 
(radiation), buoyancy (restoring), incident-wave scattering (diffraction), sea current, and nonlinear effects.

I discuss the true linear hydrodynamic-loading equations in the time domain in Section 2.4.1, taking advantage of the assumptions outlined in Section 2.2. By "true linear hydrodynamicloading equations," I mean that these equations satisfy the linearized governing boundary-value problems (BVPs) exactly, without restriction on platform size, shape, or manner of motion (other than those required for the linearization assumption to hold). In Section 2.4.2 I compare and contrast these with alternative hydrodynamic formulations, which are routinely used in the offshore industry but contain restrictions that limit their direct application to the analysis of offshore floating wind turbines. As I present in Sections 2.4.1 and 2.4.2, I have brought together parts of all the formulations in developing my HydroDyn support platform hydrodynamics module for offshore floating wind turbines. I summarize how these formulations are organized within HydroDyn in Section 2.4.3.

\subsubsection{The True Linear Hydrodynamic Model in the Time Domain}

In linear hydrodynamics, the hydrodynamics problem can be split into three separate and simpler problems: one for radiation, one for diffraction, and one for hydrostatics [22,74]. The radiation problem seeks to find the loads on a floating platform when the body is forced to oscillate in its various modes of motion and no incident surface waves are present. The resulting radiation loads are brought about as the body radiates waves away from itself (i.e., it generates outgoing waves) and include contributions from added mass and from wave-radiation damping. The diffraction problem seeks to find the loads on a floating platform when the body is fixed at its mean position (no motion) and incident surface waves are present and scattered by the body. The diffraction loads are the result of the undisturbed pressure field (Froude-Kriloff) and wave scattering. The hydrostatics problem is elementary, but is nevertheless crucial in the overall behavior of a floating platform.

In Section 2.3, I discussed how the total external load on the support platform of an offshore floating wind turbine - other than those loads transmitted from the turbine itself - is in the form

of Eq. (2-7). In the true linear hydrodynamics problem, the term $F_{i}^{\text {Hydro }}$ in Eq. (2-7) is of the form shown in Eq. (2-8) [16,76]. I discuss the terms of this equation separately in the subsections that follow.

$$
F_{i}^{\text {Hydro }}=F_{i}^{\text {Waves }}+\rho g V_{0} \delta_{i 3}-C_{i j}^{\text {Hydrostatic }} q_{j}-\int_{0}^{t} K_{i j}(t-\tau) \dot{q}_{j}(\tau) d \tau
$$

\subsubsection{Diffraction Problem}

The first term on the right-hand side of Eq. (2-8), $F_{i}^{\text {Waves }}$, represents the total excitation load on the support platform from incident waves and is closely related to the wave elevation, $\zeta$. As background, Airy wave theory $[22,74]$ describes the kinematics of a regular waves, whose periodic elevation is represented as a sinusoid propagating at a single amplitude and frequency (period) or wavelength. (Airy wave theory also describes how the undisturbed fluid-particle velocities and accelerations decay exponentially with depth—see Section 2.4.2.2.) Irregular or 
random waves that represent various stochastic sea states are modeled as the summation or superposition of multiple wave components, as determined by an appropriate wave spectrum. Expressions for $\zeta$ and $F_{i}^{\text {Waves }}$ are given by [21]:

$$
\begin{gathered}
\zeta(t)=\frac{1}{2 \pi} \int_{-\infty}^{\infty} W(\omega) \sqrt{2 \pi S_{\zeta}^{2-\text { Sided }}(\omega)} e^{j \omega t} d \omega \\
\text { and } \\
F_{i}^{\text {Waves }}(t)=\frac{1}{2 \pi} \int_{-\infty}^{\infty} W(\omega) \sqrt{2 \pi S_{\zeta}^{2-\text { Sided }}(\omega)} X_{i}(\omega, \beta) e^{j \omega t} d \omega .
\end{gathered}
$$

Equations (2-9) and (2-10) are inverse Fourier transforms, where $j$ is the imaginary number, $\sqrt{-1} . \quad S_{\zeta}^{2-\text { Sided }}$ represents the desired two-sided power spectral density (PSD) of the wave elevation per unit time, or the two-sided wave spectrum, which depends on the frequency of the incident waves, $\omega . W(\omega)$ represents the Fourier transform of a realization of a white Gaussian noise (WGN) time-series process with zero mean and unit variance (i.e., the so-called "standard normal distribution"). This realization is used to ensure that the individual wave components have a random phase and that the instantaneous wave elevation is normally- (i.e., Gaussian-) distributed with zero mean and with a variance, on average, equal to $\sigma_{\zeta}^{2}=\int_{-\infty}^{\infty} S_{\zeta}^{2 \text {-Sided }}(\omega) d \omega$. The same realization is used in the computation of the wave elevation and in the computation of the incident-wave force. $X_{i}(\omega, \beta)$ is a complex-valued array that represents the wave-excitation force on the support platform normalized per unit wave amplitude; the imaginary components permit the force to be out of phase with the wave elevation. This force depends on the geometry of the support platform and the frequency and direction of the incident wave, $\omega$ and $\beta$, respectively, and I discuss it further in Section 2.4.2.1. I have made the incident-wavepropagation heading direction, $\beta$, which is zero for waves propagating along the positive $X$-axis of the inertial frame, and positive for positive rotations about the $Z$-axis, an input to the model. This allows me to simulate conditions in which the wind and wave directions are not aligned.

In my HydroDyn module, the realization of the WGN process is calculated using the Box-Muller method [83], which considers not only a uniformly-distributed random phase, but also a normally-distributed amplitude. The normally-distributed amplitude ensures that the resulting wave elevation is Gaussian-distributed, but causes the actual variance to vary among realizations. This is why I refer to the variance of the resulting wave elevation as "on average" in the previous paragraph. (To ensure that the variance remains constant for every realization requires that one consider only random phase variations among the individual wave components - but then the instantaneous wave elevation would only be Gaussian-distributed with an infinite number of wave components.)

The Box-Muller method turns two independent and uniformly-distributed random variates into two unit-normal random variates stored as real and imaginary components (see Ref. [83]): 


$$
W(\omega)= \begin{cases}0 & \text { for } \omega=0 \\ \sqrt{-2 \ln \left[U_{1}(\omega)\right]}\left\{\cos \left[2 \pi U_{2}(\omega)\right]+j \sin \left[2 \pi U_{2}(\omega)\right]\right\} & \text { for } \omega>0 \\ \sqrt{-2 \ln \left[U_{1}(-\omega)\right]}\left\{\cos \left[2 \pi U_{2}(-\omega)\right]-j \sin \left[2 \pi U_{2}(-\omega)\right]\right\} & \text { for } \omega<0\end{cases}
$$

where $U_{1}$ and $U_{2}$ are the two independent and uniformly-distributed random variates (random numbers between zero and one) chosen for each positive-valued incident-wave frequency $(\omega)$. $W(\omega)$ is set to zero at zero frequency to ensure that each WGN process, and resulting wave elevation, has zero mean. The use of random variates requires that a seed be specified for the pseudo-random number generator (RNG). I have made these seeds inputs to the HydroDyn module.

Equation (2-10) for the incident-wave-excitation force is very similar to Eq. (2-9) for the incident-wave elevation - the only difference is the inclusion of the normalized wave-excitation force complex transfer function, $X_{i}$. This follows directly from linearization of the diffraction problem. Superposition of the diffraction problem implies that (1) the magnitude of the waveexcitation force from a single wave is linearly proportional to the wave amplitude and (2) the wave-excitation force from multiple superimposed waves is the same as the sum of the waveexcitation forces produced by each individual wave component. In the limit as the difference between individual wave frequencies approaches zero, this sum is replaced with the integral over all incident-wave frequencies, as exemplified by Eq. (2-10). These same properties can also be seen, perhaps more clearly, when Eq. (2-10) is expressed in an alternative-but equivalentform. Equation (2-12), which was derived by applying the basic properties of bilateral transforms [66], shows this form:

$$
F_{i}^{\text {Waves }}(t)=\int_{-\infty}^{\infty} K_{i}(t-\tau) \zeta(\tau) d \tau
$$

In this equation, $\tau$ is a dummy variable with the same units as the simulation time, $t$, and the time- and direction-dependent incident-wave-excitation force on the support platform normalized per unit wave amplitude, $K_{i}$, is given by

$$
K_{i}(t)=\frac{1}{2 \pi} \int_{-\infty}^{\infty} X_{i}(\omega, \beta) e^{j \omega t} d \omega .
$$

The integral over all frequency-dependent incident-wave-excitation forces from Eq. (2-10) has been replaced in Eq. (2-12) with a convolution over all time-dependent incident-wave-excitation forces. Regardless of which formulation is used, the floating support platform should be designed with minimal structure near the free surface to minimize the wave-excitation forces.

In HydroDyn, I have implemented Eq. (2-10) instead of Eq. (2-12) because the former requires fewer calculations. I implemented the inverse Fourier transforms using computationally efficient fast Fourier transform (FFT) routines [92]. 
The incident-wave-excitation force given by Eq. (2-10) or Eq. (2-12) is independent of the motion of the support platform. This demonstrates how the diffraction problem has been separated from the radiation problem and reveals how the linearization assumptions would be violated if the motions of the support platform were large.

It follows that Eq. (2-9) for the wave elevation is valid only at the mean position of the support platform. For other locations, Eq. (2-9) can be expanded to

$$
\zeta(t, X, Y)=\frac{1}{2 \pi} \int_{-\infty}^{\infty} W(\omega) \sqrt{2 \pi S_{\zeta}^{2-\text { Sided }}(\omega)} e^{-j k(\omega)[X \cos (\beta)+Y \sin (\beta)]} e^{j \omega t} d \omega,
$$

where $(X, Y)$ are the coordinates in the inertial reference frame of a point on the SWL plane and $k(\omega)$ is the wave number, which is $2 \pi$-times the number of waves per unit distance along the wave-propagation direction, $\beta$. For water of depth $h$, the wave number is correlated to the incident-wave frequency, $\omega$, and the gravitational acceleration constant, $g$, by the implicit dispersion relationship [22,74]:

$$
k(\omega) \tanh [k(\omega) h]=\frac{\omega^{2}}{g} .
$$

In HydroDyn, this implicit relationship is solved using the numerical approach adopted in the SWIM module [48] of SML; that is, a high-order initial guess is used in conjunction with a quadratic Newton's method for the solution with an accuracy of seven significant digits using only one iteration pass. This solution method is attributed to Professor J. N. Newman of MIT. I have implemented Eq. (2-14) in HydroDyn for animating the wave surface around the floating platform.

Because the inverse Fourier transforms require a distinction between positive and negative frequencies, the frequency-dependent terms in the previous equations have several characteristics that ensure that the total wave-excitation force on the support platform is a real function of time. The requirement for this is that the real components of the integrands be an even function of frequency and the imaginary components of the integrands be an odd function of frequency [91]. Thus, the realization of the WGN process has the property that $W(-\omega)=W^{*}(\omega)$, where the symbol " "" is used to denote the complex conjugate. The normalized wave-excitation force has the same property: $X_{i}(-\omega, \beta)=X_{i}^{*}(\omega, \beta)$. Similarly, I set $k(-\omega)=-k(\omega)$ to ensure that $e^{-j k(-\omega)}=\left[e^{-j k(\omega)}\right]^{*}$. The relationship between the two-sided wave spectrum used in the inverse

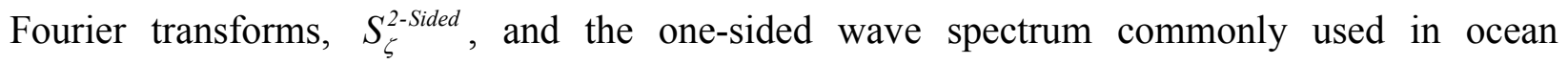
engineering, $S_{\zeta}^{l-\text {-Sided }}$, follows standard practice [80]: 


$$
S_{\zeta}^{2-\text { Sided }}(\omega)=\left\{\begin{array}{ll}
\frac{1}{2} S_{\zeta}^{l-\text { Sided }}(\omega) & \text { for } \omega \geq 0 \\
\frac{1}{2} S_{\zeta}^{l-\text { Sided }}(-\omega) & \text { for } \omega<0
\end{array} .\right.
$$

Equation (2-16) ensures that the variance of the wave elevation, or the area under the PSD curves, is the same for both the one- and two-sided spectra, as in $\sigma_{\zeta}^{2}=\int_{-\infty}^{\infty} S_{\zeta}^{2 \text {-Sided }}(\omega) d \omega=\int_{0}^{\infty} S_{\zeta}^{I \text {-Sided }}(\omega) d \omega$.

In HydroDyn, I have included three options for prescribing the wave spectrum. I have included the Pierson-Moskowitz and the Joint North Sea Wave Project (JONSWAP) spectra as they are defined by the IEC 61400-3 design standard [34], and I have included an option for a userprescribed site-specific wave spectrum. The Pierson-Moskowitz wave spectrum is routinely used to describe the statistical properties of fully developed seas and the JONSWAP spectrum is routinely used in limited fetch situations [22]. From the IEC 61400-3 design standard, the onesided JONSWAP spectrum is defined as

$$
S_{\zeta}^{l-\text { Sided }}(\omega)=\frac{1}{2 \pi} \frac{5}{16} H_{s}^{2} T_{p}\left(\frac{\omega T_{p}}{2 \pi}\right)^{-5} \exp \left[-\frac{5}{4}\left(\frac{\omega T_{p}}{2 \pi}\right)^{-4}\right][1-0.287 \ln (\gamma)] \gamma\left\{\exp \left\{-0.5\left[\frac{\omega T_{p}-I^{2}-I^{2}}{\sigma(\omega)}\right]\right\},\right.
$$

where $H_{s}$ is the significant wave height, $T_{p}$ is the peak spectral period, and $\gamma$ is the peak shape parameter of a given irregular sea state, and $\sigma$ is a scaling factor. The IEC $61400-3$ design standard recommends that the scaling factor and the peak shape parameter be derived from the significant wave height and peak spectral period as follows:

$$
\begin{aligned}
& \sigma(\omega)= \begin{cases}0.07 & \text { for } \omega \leq \frac{2 \pi}{T_{p}} \\
0.09 & \text { for } \omega>\frac{2 \pi}{T_{p}}\end{cases} \\
& \text { and } \\
& \gamma= \begin{cases}5 & \text { for } \frac{T_{p}}{\sqrt{H_{s}}} \leq 3.6 \\
\exp \left(5.75-1.15 \frac{T_{p}}{\sqrt{H_{s}}}\right) & \text { for } 3.6<\frac{T_{p}}{\sqrt{H_{s}}} \leq 5 . \\
1 & \text { for } \frac{T_{p}}{\sqrt{H_{s}}}>5\end{cases}
\end{aligned}
$$


In Eq. (2-19), $H_{s}$ and $T_{p}$ must have units of meters and seconds, respectively.

When the peak shape parameter of Eq. (2-19) equals unity, the one-sided JOWNSWAP-spectrum formulation of Eq. (2-17) reduces down to the one-sided Pierson-Moskowitz spectrum, as given in Eq. (2-20). This simplification occurs in all but the most extreme sea states. Figure 2-2 compares the Pierson-Moskowitz and JONSWAP spectra for an extreme sea state with a significant wave height of $11.8 \mathrm{~m}$ and a peak spectral period of $15.5 \mathrm{~s}$, which corresponds to a peak shape parameter of about 1.75 in the JONSWAP spectrum. For spectra with the same total energy, the JONSWAP spectrum, in general, has a higher and narrower peak than the PiersonMoskowitz spectrum.

$$
S_{\zeta}^{l-\text { Sided }}(\omega)=\frac{1}{2 \pi} \frac{5}{16} H_{s}^{2} T_{p}\left(\frac{\omega T_{p}}{2 \pi}\right)^{-5} \exp \left[-\frac{5}{4}\left(\frac{\omega T_{p}}{2 \pi}\right)^{-4}\right]
$$

I have implemented the one-sided JONSWAP spectrum formulation of Eq. (2-17) into HydroDyn with only one modification - to avoid nonphysical wave forces at high frequencies (i.e., at short wavelengths), I truncate the wave spectrum above a cutoff frequency. I have implemented the method proposed by Massel [65], in which the cutoff frequency is chosen to be proportional to the peak spectral frequency. I used a proportionality factor of 3.0 in all simulations.

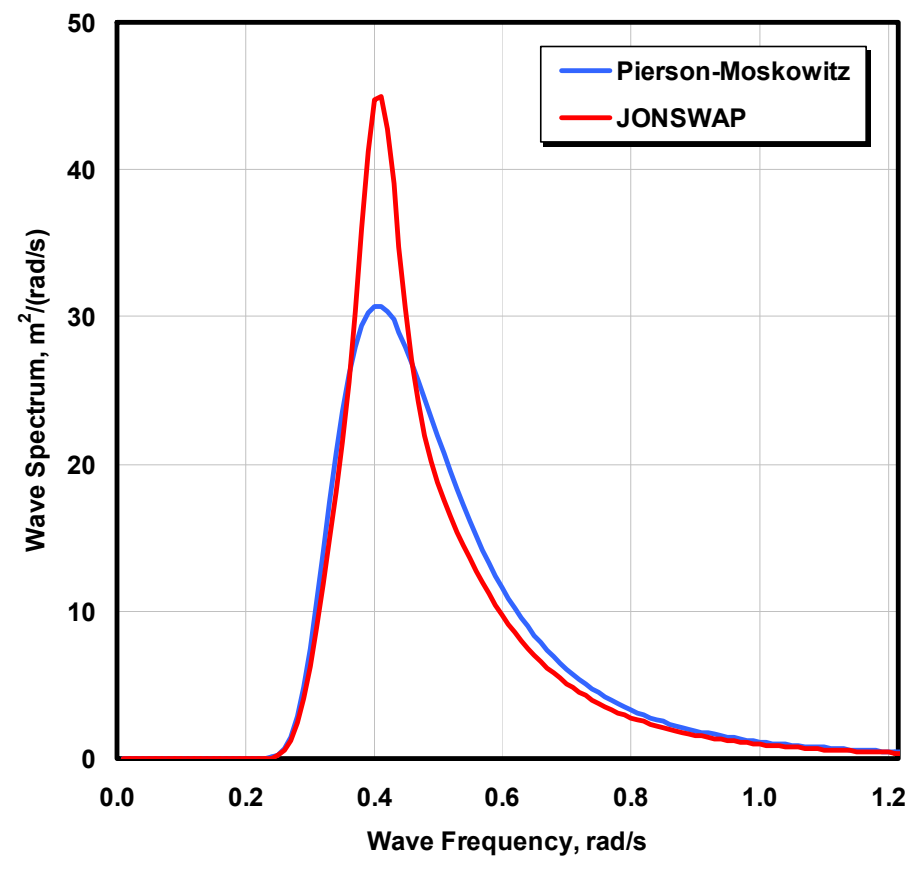

Figure 2-2. Comparison between Pierson-Moskowitz and JONSWAP spectra 


\subsubsection{Hydrostatic Problem}

The second and third terms on the right-hand side of Eq. (2-8) combined, $\rho g V_{0} \delta_{i 3}-C_{i j}^{H y d r o s t a t i c} q_{j}$, represent the load contribution from hydrostatics as I have implemented them in HydroDyn. Here, $\rho$ is the water density, $g$ is the gravitational acceleration constant, $V_{0}$ is the displaced volume of fluid when the support platform is in its undisplaced position, $\delta_{i 3}$ is the $(i, 3)$ component of the Kronecker-Delta function (i.e., identity matrix), and $C_{i j}^{\text {Hydrostatic }}$ is the $(i, j)$ component of the linear hydrostatic-restoring matrix from the effects of water-plane area and the COB. The hydrostatic loads are independent of the incident and outgoing waves from the diffraction and radiation problems, respectively.

The first of these terms, $\rho g V_{0} \delta_{i 3}$, represents the buoyancy force from Archimedes' principle; that is, it is the force directed vertically upward and equal to the weight of the displaced fluid when the support platform is in its undisplaced position. This term is nonzero only for the vertical heave-displacement DOF of the support platform (DOF $i=3$ ) because the COB of the platform is assumed to lie on the centerline of the undeflected tower (or z-axis of the platform). If this were not the case, the cross product of the buoyancy force with the vector position of the COB would produce a hydrostatic moment about the support platform reference point (i.e., the origin of the platform DOFs). In the field of naval architecture and in the analysis of large offshore O\&G platforms, the term $\rho g V_{0} \delta_{i 3}$ is not often found in the equations of motion because it cancels with the weight in air of the floating body and the weight in water of the mooring system. Because the location of the $\mathrm{CM}$ of the floating wind turbine continually changes as a result of wind turbine flexibility, however, it was important to separate out the individual contributions of gravity. These contributions are wind turbine and support platform weight, weight in water of the mooring system, and buoyancy. The weights of the wind turbine and support platform are inherent in the $f_{i}$ term of Eq. (2-6).

The second of the hydrostatic terms, $-C_{i j}^{\text {Hydrostatic }} q_{j}$, represents the change in the hydrostatic force and moment resulting from the effects of the water-plane area and the COB as the support platform is displaced. The water-plane area of the support platform when it is in its undisplaced position, $A_{0}$, affects the hydrostatic load because the displaced volume of the fluid changes with changes in the support platform displacement $\left(q_{j}\right)$. Similarly, the body-fixed vertical location of the COB of the support platform, $z_{C O B}$, affects the hydrostatic load because the vector position of the $\mathrm{COB}$ also changes with platform displacement and because the cross product of the buoyancy force with the vector position of the COB produces a hydrostatic moment about the support platform reference point. ( $z_{C O B}$ is, in general, less than zero because the $z$-axis is directed upward along the centerline of the undeflected tower.) The only nonzero components of $C_{i j}^{\text {Hydrostatic }}$ are $(3,3),(4,4),(5,5),(3,5)$, and $(5,3)$ when the body-fixed $x z$-plane of the submerged portion of the support platform is a plane of symmetry [22]: 


$$
C_{i j}^{\text {Hydrostatic }}=\left[\begin{array}{llllll}
0 & 0 & 0 & 0 & 0 & 0 \\
0 & 0 & 0 & 0 & 0 & 0 \\
0 & 0 & \rho g A_{0} & 0 & -\rho g \iint_{A_{0}} x d A & 0 \\
0 & 0 & 0 & \rho g \iint_{A_{0}} y^{2} d A+\rho g V_{0} z_{C O B} & 0 & 0 \\
0 & 0 & -\rho g \iint_{A_{0}} x d A & 0 & \rho g \iint_{A_{0}} x^{2} d A+\rho g V_{0} z_{C O B} & 0 \\
0 & 0 & 0 & 0 & 0 & 0
\end{array}\right] .
$$

If the body-fixed $y z$-plane of the submerged portion of the support platform is also a plane of symmetry, the $(3,5)$ and $(5,3)$ components of $C_{i j}^{\text {Hydrostatic }}$ are also zero. Equation (2-21) clearly demonstrates how hydrostatics provides restoring only for roll, pitch, and heave motions; restoring in the other modes of motion must be realized by the mooring system. In classical marine hydrostatics, the effects of body weight are often lumped with the effects of hydrostatics when defining the hydrostatic-restoring matrix; for example, when it is defined in terms of metacentric heights [22,74]. For the same reason given in the previous paragraph for the $\rho g V_{0} \delta_{i 3}$ term appearing in the hydrostatic-loading equations, though, it was important to separate out the contributions of body weight and hydrostatic restoring. So to reiterate, $C_{i j}^{H y d r o s t a t i c}$ really is the hydrostatic contribution solely from the water-plane area and the COB.

\subsubsection{Radiation Problem}

The wave-radiation loads include contributions from hydrodynamic added mass and damping. Because the radiation problem has been separated from the diffraction problem, the waveradiation loads are independent of the incident waves.

In Eq. (2-7), the impulsive hydrodynamic-added-mass components, $A_{i j}$, represent the force mechanism proportional to the acceleration of the support platform in the time-domain radiation problem. In particular, the $(i, j)$ component represents the hydrodynamic force in the direction of DOF $i$ resulting from the integration (over the wetted surface of the support platform) of the component of the outgoing-wave pressure field induced by, and proportional to, a unit acceleration of the $j^{\text {th }}$ DOF of the support platform. Like the body (inertia) mass matrix, the impulsive hydrodynamic-added-mass matrix is symmetric. Unlike the inertia mass matrix, and depending on the shape of the support platform, the impulsive hydrodynamic-added-mass matrix may contain off-diagonal components that couple modes of motion that cannot be coupled through body inertia.

The final term in Eq. (2-8), $-\int_{0}^{t} K_{i j}(t-\tau) \dot{q}_{j}(\tau) d \tau$, is a convolution integral representing the load contribution from wave-radiation damping and also represents an additional contribution from added mass that is not accounted for in $A_{i j}$. In this expression, $\tau$ is a dummy variable with the same units as the simulation time, $t$, and $K_{i j}$ is the $(i, j)$ component of the matrix known as the wave-radiation-retardation kernel. In the radiation problem, the free surface brings about the 
existence of memory effects, denoting that the wave-radiation loads depend on the history of motion for the support platform.

The meaning of the wave-radiation-retardation kernel is found by considering a unit impulse in support platform velocity. Specifically, the $(i, j)$ component of the kernel, $K_{i j}(t)$, represents the hydrodynamic force at time $t$ in the direction of DOF $i$ resulting from a unit impulse in velocity at time zero of DOF $j$. The wave-radiation-retardation kernel, consequently, is commonly referred to as the impulse-response functions of the radiation problem. An impulse in support platform velocity causes a force at all subsequent time because the resulting outgoing freesurface waves induce a pressure field within the fluid domain that persists for as long as the waves radiate away. As in Eq. (2-12) for the diffraction problem, the convolution integral in the radiation problem follows directly from the assumption of linearity. Superposition of the radiation problem implies that if the support platform

experiences a succession of impulses, its response at any time is assumed to be the sum of its responses to the individual impulses, each response being calculated with an appropriate time lag from the instant of the corresponding impulse. These impulses can be considered as occurring closer and closer together, until finally one integrates the responses, rather than summing them [76, p. 33].

Using what I would label as "convolution by parts" (instead of "integration by parts") and assuming zero-valued initial conditions, the convolution integral in the radiation problem can be rewritten as follows [102]:

$$
-\int_{0}^{t} K_{i j}(t-\tau) \dot{q}_{j}(\tau) d \tau=-\int_{0}^{t} L_{i j}(t-\tau) \ddot{q}_{j}(\tau) d \tau,
$$

where the convolution kernels, $K_{i j}$ and $L_{i j}$, are related by

$$
K_{i j}(t)=\frac{d}{d t} L_{i j}(t)
$$

Equation (2-22) highlights the elusive nature of the memory effect in the radiation problem - that both acceleration-dependent (added-mass) and velocity-dependent (damping) forces are captured by the convolution term. To minimize the wave-radiation loads, the floating support platform should be designed with minimal structure near the free surface, regardless of which formulation of the convolution integral is applied. The mooring system should also be designed to limit the motion of the support platform. I discuss the impulsive hydrodynamic-added-mass matrix and retardation kernels from the radiation problem further in Section 2.4.2.

In the HydroDyn module, I have implemented a numerical convolution in the time domain to capture the memory effect directly. I chose to implement the velocity formulation from the lefthand side of Eq. (2-22) because it is more convenient than the acceleration formulation from the right-hand side. The latter would lead to an implicit formulation of the time-domain equations of motion for the coupled wind turbine and support platform system. As demonstrated in Section 4.1.3, the memory effect, in general, decays to zero after a certain amount of lapsed time. 
Because of this, I have enabled HydroDyn to truncate the numerical convolution after a userspecified amount of time. This allows for faster calculations of the memory effect.

\subsubsection{Comparison to Alternative Hydrodynamic Models}

I discused the true linear hydrodynamic-loading equations in Section 2.4.1. Alternative hydrodynamics formulations, however, are routinely used in the offshore industry. The two most common alternatives are the frequency-domain representation and Morison's representation.

\subsubsection{Frequency-Domain Representation}

The frequency-domain representation is most aligned with how marine hydrodynamics is taught in the classroom and presented in textbooks. For instance, the frequency-domain representation is the hydrodynamics formulation most emphasized in Refs. [22] and [74], which are popular textbooks in ocean-engineering education. The presentation here summarizes these references.

In the time-domain representation of the frequency-domain problem, Eq. (2-7) for the total external load acting on the support platform, $F_{i}^{\text {Platform }}$, is replaced with

$$
F_{i}^{\text {Platform }}(t)=-A_{i j}(\omega) \ddot{q}_{j}+\operatorname{Re}\left\{A X_{i}(\omega, \beta) e^{j \omega t}\right\}-\left[C_{i j}^{\text {Lines }}+C_{i j}^{\text {Hydrostatic }}\right] q_{j}-B_{i j}(\omega) \dot{q}_{j}
$$

where $A$ is the amplitude of a regular incident wave of frequency $\omega$ and direction $\beta$; $C_{i j}^{\text {Lines }}$ is the $(i, j)$ component of the linear restoring matrix from all mooring lines (discussed in Section 2.5); and $A_{i j}(\omega)$ and $B_{i j}(\omega)$ are the $(i, j)$ components of the hydrodynamic-added-mass and -damping matrices, which are frequency dependent. $\operatorname{Re}\{\}$ denotes the real value of the argument; the only complex-valued terms in Eq. (2-24) are the normalized wave-excitation force, $X_{i}$, and the harmonic exponential, $e^{j \omega t}$.

The frequency-domain hydrodynamics problem makes use of the same assumptions used in the true linear hydrodynamics formulation. There are additional requirements, though. The incident wave must propagate at a single amplitude, frequency, and direction (i.e., the incident wave is a regular wave), and the platform motions must be oscillatory at the same frequency as the incident wave. To reiterate this point, when Eq. (2-24) is incorporated in Eq. (2-6), the resulting differential equations are not true differential equations in the proper sense. This is because the time-domain representation of the frequency-domain problem is valid only when the platform motions are oscillating at the same frequency as the incident wave $(\omega)$. In other words, Eq. $(2-24)$ is valid only for the steady-state situation, and not for transient-response analysis. When used within the system's equations of motion, Eq. (2-24) also requires that all additional loading in the system be linear in nature. This prevents me from being able to apply the frequencydomain hydrodynamics formulation to the direct analysis of offshore floating wind turbinesexcept under steady-state conditions - because nonlinear characteristics and transient events are important considerations for wind turbines. Nevertheless, others have applied this approach to the preliminary analysis of several offshore floating wind turbine concepts $[13,59,98,100,101]$.

The solution to the frequency-domain problem is generally given in terms of an RAO, which is the complex-valued amplitude of motion of a floating platform normalized per unit wave 
amplitude. Imaginary components indicate that the response is out of phase with the wave elevation. In the frequency-domain problem, the support platform's response to irregular waves can only be characterized statistically because the frequency-domain representation is not valid for transient analysis. Specifically, the motion of a linearized floating body will have a response that is Gaussian-distributed when it is excited by a sea state with a Gaussian-distributed instantaneous wave elevation [85,101]. (Irregular sea states are, in general, Gaussiandistributed.) The standard deviations of the motion response are dictated by the WienerKhinchine theorem.

Just as in the true linear hydrodynamics model, the radiation and the diffraction problems can be solved separately in the frequency-domain representation. In the radiation problem, six BVPs are solved independently to find six velocity potentials, one for each mode of motion. By substituting these velocity potentials into the linearized unsteady form of Bernoulli's equation, the resulting pressures, when integrated over the wetted surface of the floating platform, yield the added-mass and damping matrices. Similarly, in the diffraction problem, two BVPs are solved independently to find two velocity potentials, one for the incident wave and one for the scattered wave. By applying Bernoulli's equation and wetted surface integration again, one arrives at the normalized wave-excitation force.

The formulation for the radiation and diffraction BVPs, and hence the resulting hydrodynamicadded-mass and -damping matrices, $A_{i j}$ and $B_{i j}$, and wave-excitation force, $X_{i}$, depend on frequency, water depth, and sea current, as well as on the geometric shape of the support platform, its proximity to the free surface, and its forward speed. Additionally, the waveexcitation force depends on the heading direction of the incident waves.

The frequency dependence of the hydrodynamic-added-mass and-damping matrices is of a different nature than that of the wave-excitation force. The frequency dependence of the hydrodynamic-added-mass and -damping matrices means that the matrices depend on the oscillation frequency of the particular mode of support platform motion. In contrast, the frequency dependence of the wave-excitation force means that the force depends on the frequency of the incident wave. In Eq. (2-24), however, both frequencies are identical because the platform is assumed to oscillate at the same frequency as the incident wave.

Analytical solutions for the hydrodynamic-added-mass and -damping matrices and waveexcitation force are available for bodies of simple geometry such as cylinders and spheres. Usually, approximations are employed to find these analytical solutions. For example, if the characteristic length of the body is small relative to the wavelength, G. I. Taylor's longwavelength approximation [85] can be used to simplify the diffraction problem. Morison's equation (discussed next in Section 2.4.2.2) uses G. I. Taylor's long-wavelength approximation $[16,76,85]$ to simplify the diffraction problem for the case of slender vertical surface-piercing cylinders. For bodies with complex geometrical surfaces, like the hull of a ship, numerical-panel method techniques are required.

Even though the frequency-domain formulation cannot be directly applied to the transient analysis of offshore floating wind turbines, where nonlinear effects, transient behavior, and irregular sea states are important, the solution to the frequency-domain problem is valuable in determining the parameters used in the true linear hydrodynamic-loading equations. For 
instance, the solution to the frequency- (and direction-) dependent wave-excitation force, $X_{i}(\omega, \beta)$, is needed not only in the frequency-domain solution, but also in the time-domain formulation of the linearized diffraction problem in Eq. (2-10). Equally important is the relationship between $A_{i j}(\omega)$ and $B_{i j}(\omega)$ from the frequency-domain solution and $A_{i j}$ and $K_{i j}(t)$ from the time-domain formulation of the linearized radiation problem. By forcing a particular mode of motion of the support platform to be sinusoidal in the true linear hydrodynamics formulation, and comparing the resulting expression to the time-domain representation of the frequency-domain problem, Ref. [76] shows that

$$
\begin{gathered}
A_{i j}(\omega)=A_{i j}-\frac{1}{\omega} \int_{0}^{\infty} K_{i j}(t) \sin (\omega t) d t \\
\text { and } \\
B_{i j}(\omega)=\int_{0}^{\infty} K_{i j}(t) \cos (\omega t) d t .
\end{gathered}
$$

The $A_{i j}$ term on the right-hand side of Eq. (2-25) represents the impulsive hydrodynamic-addedmass matrix from Eq. (2-7). Note that Eq. (2-26) is valid only when the ancillary effects of sea current or forward speed are ignored in the radiation problem (as assumed, see Section 2.2); though not given here, a slightly different expression exists when these effects are important.

Equations (2-25) and (2-26) highlight the interdependence between the hydrodynamic added mass and damping. Section 2.4.1.3 alluded to their relationship, which is discussed more in Ref. [76].

Because the radiation-retardation kernel, $K_{i j}(t)$, may be assumed to be of finite energy, application of the Riemann-Lebesgue lemma to Eq. (2-26) reveals that the infinite-frequency limit of $B_{i j}(\omega)$ is zero. Similarly, the infinite-frequency limit of Eq. (2-25) yields

$$
A_{i j}=\lim _{\omega \rightarrow \infty} A_{i j}(\omega)=A_{i j}(\infty)
$$

Thus, the appropriate impulsive added-mass matrix to be used in the true linear hydrodynamicloading equations does not depend on frequency, but is the infinite-frequency limit of the frequency-dependent added-mass matrix, represented here as $A_{i j}(\infty)$. This limit does, in general, exist for three-dimensional bodies.

Through application of Fourier-transform techniques and Eq. (2-27), Eqs. (2-25) and (2-26) can be rearranged to show that

$$
K_{i j}(t)=-\frac{2}{\pi} \int_{0}^{\infty} \omega\left[A_{i j}(\omega)-A_{i j}(\infty)\right] \sin (\omega t) d \omega
$$




$$
K_{i j}(t)=\frac{2}{\pi} \int_{0}^{\infty} B_{i j}(\omega) \cos (\omega t) d \omega,
$$

and from Eq. (2-23) in Section 2.4.1.3 that

$$
\begin{gathered}
L_{i j}(t)=\frac{2}{\pi} \int_{0}^{\infty}\left[A_{i j}(\omega)-A_{i j}(\infty)\right] \cos (\omega t) d \omega \\
\text { or } \\
L_{i j}(t)=\frac{2}{\pi} \int_{0}^{\infty} \frac{B_{i j}(\omega)}{\omega} \sin (\omega t) d \omega .
\end{gathered}
$$

As a corollary to the interdependence between added mass and damping discussed previously, Eqs. (2-28) and (2-29) show that the radiation-retardation kernels depend on both added mass and damping. Once the solution of the frequency-domain radiation problem has been found, any of these expressions can be used to find the wave-radiation-retardation kernels to be used in the true linear hydrodynamic-loading equations. When the velocity form of the radiation convolution is used, the sine transform of Eq. (2-28a) should be applied if the solution accuracy for the frequency-dependent hydrodynamic-added-mass matrix is greater than the solution accuracy for the frequency-dependent hydrodynamic-damping matrix. Similarly, the cosine transform of Eq. (2-28b) should be used if the solution accuracy for the frequency-dependent hydrodynamic-damping matrix is greater than the solution accuracy for the frequency-dependent hydrodynamic-added-mass matrix. If the solution accuracy is the same for both matrices, Eq. $(2-28 b)$ is generally a better choice when the integrals are computed numerically because, without a correction for truncation error, the accuracy of Eq. (2-28a) is poor near $t=0$, where $K_{i j}(0)$ is, in general, not zero [even though $\sin (0)$ is]. Similar to the inverse Fourier transforms, I have implemented the cosine transform of Eq. (2-28b) using a computationally efficient FFT routine [92] in my HydroDyn module.

Because the frequency-domain approach is so often employed in analyses in the offshore O\&G industries, many computer codes are available for solving the frequency-domain hydrodynamics problem. For instance, the SWIM module [48] of the SML computer package can be used to analytically solve the frequency-domain problem for support platforms of simple geometry. For platforms of more complicated surface geometry, the numerical-panel WAMIT code [58] can be employed.

My hydrodynamics formulation in HydroDyn is applied identically regardless of how the frequency-domain radiation and diffraction problems are solved. This is because I simply made the frequency-dependent hydrodynamic-added-mass and -damping matrices $\left(A_{i j}\right.$ and $\left.B_{i j}\right)$ and wave-excitation force $\left(X_{i}\right)$ inputs to HydroDyn. 


\subsubsection{Morison's Representation}

Morison's representation is widely used in the analysis of fixed-bottom offshore wind turbines $[4,15,19,52,61,77,97]$. Though somewhat misapplied, it has also been used directly in the analysis of offshore floating wind turbines [23,103]. Morison's representation, in conjunction with strip theory, can be used to compute the linear wave loads and nonlinear viscous-drag loads in a straightforward manner, mostly for slender vertical surface-piercing cylinders that extend to the sea floor. In hydrodynamic strip theory, as in BEM theory for wind turbine aerodynamics, the structure is split into a number of elements or strips, where two-dimensional properties (added-mass and viscous-drag coefficients in the case of Morison's hydrodynamics) are used to determine the overall three-dimensional loading on the structure [22].

The total external load acting on the support platform, $F_{i}^{\text {Platorm }}$ in Eq. (2-7), is thus found by integrating over the length of the cylinder the loads acting on each strip of the cylinder, $d F_{i}^{\text {Platform }}$. In the relative form of Morison's representation, Eq. (2-7) for the surge and sway modes of motion ( $i=1$ and 2$)$ is replaced with Morison's equation [22,74]:

$$
\begin{aligned}
d F_{i}^{\text {Platform }}(t, z) & =-C_{A} \rho\left(\frac{\pi D^{2}}{4} d z\right) \ddot{q}_{i}(z)+\left(1+C_{A}\right) \rho\left(\frac{\pi D^{2}}{4} d z\right) a_{i}(t, 0,0, z) \\
& +\underbrace{\frac{1}{2} C_{D} \rho(D d z)\left[v_{i}(t, 0,0, z)-\dot{q}_{i}(z)\right]|v(t, 0,0, z)-\dot{q}(z)|}_{d F_{i}^{Y_{\text {sicous }}}(t, z)} \quad \text { for } i=1 \text { or 2 }
\end{aligned}
$$

where $D$ is the diameter of the cylinder, $d z$ is the length of the differential strip of the cylinder, $C_{A}$ and $C_{D}$ are the normalized hydrodynamic-added-mass and viscous-drag coefficients, $d F_{i}^{V i s c o u s}$ is the viscous-drag load acting on the strip of the cylinder, and $v_{i}$ and $a_{i}$ are the components of the undisturbed fluid-particle velocity and acceleration in the direction of DOF $i$. $\left(v_{i}\right.$ and $a_{i}$, including their arguments, are discussed below.) The symbol "|.|" denotes the magnitude of the vector difference of $v$ and $\dot{q}$; it is implied in Eq. (2-30a) that only the vector normal to the strip of cylinder is included in this magnitude. The term $\left(\frac{\pi D^{2}}{4} d z\right)$ is the displaced volume of fluid for the strip of the cylinder. The term $(D d z)$ is the frontal area for the strip of the cylinder. Please note that Morison's equation is often written in terms of the normalized mass (inertia) coefficient, $C_{M}$, in place of $C_{A}$, where $C_{M}=1+C_{A}$.

Using strip theory, expressions similar to Eq. (2-30a) can be written for the roll and pitch moments $(i=4$ and 5). Because a cylinder is axisymmetric, the yaw moment $(i=6)$ is zero, and because Morison's equation is strictly valid only for bottom-mounted cylinders, the heave force $(i=3)$ is also zero. These expressions are all given in Eq. (2-30b): 


$$
d F_{i}^{\text {Platform }}(t, z)= \begin{cases}0 & \text { for } i=3 \\ -d F_{2}^{\text {Platform }}(t, z) z & \text { for } i=4 \\ d F_{1}^{\text {Platform }}(t, z) z & \text { for } i=5 \\ 0 & \text { for } i=6\end{cases}
$$

Consistent with Eq. (2-14) and Airy wave theory, the undisturbed fluid-particle velocity and acceleration in the direction of DOF $i, v_{i}$ and $a_{i}$, respectively, at point $(X, Y, Z)$ in the inertial reference frame (where $Z \leq 0$ ) are, in the absence of sea currents

$$
\begin{aligned}
& v_{I}(t, X, Y, Z)=\frac{\cos (\beta)}{2 \pi} \int_{-\infty}^{\infty} W(\omega) \sqrt{2 \pi S_{\zeta}^{2-S i d i d e d}(\omega)} e^{-j k(\omega)[X \cos (\beta)+Y \sin (\beta)]} \omega \frac{\cosh [k(\omega)(Z+h)]}{\sinh [k(\omega) h]} e^{j \omega o t} d \omega, \\
& v_{2}(t, X, Y, Z)=\frac{\sin (\beta)}{2 \pi} \int_{-\infty}^{\infty} W(\omega) \sqrt{2 \pi S_{\zeta}^{2-S i d i d e d}(\omega)} e^{-j k(\omega)[X \cos (\beta)+Y \sin (\beta)]} \omega \frac{\cosh [k(\omega)(Z+h)]}{\sinh [k(\omega) h]} e^{j o t} d \omega,
\end{aligned}
$$

and

$$
v_{3}(t, X, Y, Z)=\frac{j}{2 \pi} \int_{-\infty}^{\infty} W(\omega) \sqrt{2 \pi S_{\zeta}^{2-S \operatorname{Sided} d}(\omega)} e^{-j k(\omega)[\cos (\beta)+Y \sin (\beta)]} \omega \frac{\sinh [k(\omega)(Z+h)]}{\sinh [k(\omega) h]} e^{j o x} d \omega
$$

and

$$
\begin{aligned}
& a_{l}(t, X, Y, Z)=\frac{j \cos (\beta)}{2 \pi} \int_{-\infty}^{\infty} W(\omega) \sqrt{2 \pi S_{\zeta}^{2-S \operatorname{sided}}(\omega)} e^{-j k(\omega)[X \cos (\beta)+Y \sin (\beta)]} \omega^{2} \frac{\cosh [k(\omega)(Z+h)]}{\sinh [k(\omega) h]} e^{j \omega t} d \omega, \\
& a_{2}(t, X, Y, Z)=\frac{j \sin (\beta)}{2 \pi} \int_{-\infty}^{\infty} W(\omega) \sqrt{2 \pi S_{\zeta}^{2-S \operatorname{sided}(}(\omega)} e^{-j k(\omega)[X \cos (\beta)+\sin (\beta)]} \omega^{2} \frac{\cosh [k(\omega)(Z+h)]}{\sinh [k(\omega) h]} e^{j \omega t} d \omega,
\end{aligned}
$$

and

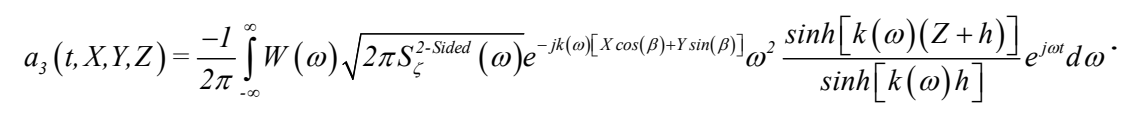

By comparing Eq. (2-30) with the true linear hydrodynamic-loading equations, it can be seen that Morison's representation assumes that viscous drag dominates the damping such that waveradiation damping can be ignored. This assumption is valid only if the motions of the cylinder are very small (i.e., it is most appropriate when the cylinder is bottom-mounted and very rigid). The viscous-drag load is not part of the linear hydrodynamic-loading equations because the viscous-drag load is proportional to the square of the relative velocity between the fluid particles and the platform. Nevertheless, I did augment the linear hydrodynamic-loading equations in HydroDyn by including the nonlinear viscous-drag term from Morison's equation. I include the viscous-drag term by assigning an effective platform diameter $(D)$ and by integrating $d F_{i}^{V i s c o u s}$ over the draft of the support platform to find the total viscous-drag load, $F_{i}^{V i s c o u s}$. I included this effect because (1) it was relatively easy to add, (2) it allowed me to incorporate the influence of sea current, and (3) it can be an important source of hydrodynamic damping in some situations. To include the influence of sea current generated by winds, tides, and thermal gradients in 
HydroDyn, I have vectorally combined a steady, depth-varying current velocity with the surfacewave-particle velocity [Eq. (2-31)] when computing the viscous-drag term from Morison's equation.

By comparing Eq. (2-30) with the true linear hydrodynamic-loading equations, it is also seen that Morison's representation ignores off-diagonal terms in the added-mass matrix other than those that directly couple the motions between surge and pitch and sway and roll. It may do this because a cylinder is axisymmetric, which ensures that there is no other added-mass-induced coupling between modes of motion. Morison's representation also takes advantage of G. I. Taylor's long-wavelength approximation $[16,76,85]$ to simplify the diffraction problem (i.e., the cylinder must be slender). This approximation is how the second term in Eq. (2-30a) for the wave-excitation force can be expressed in terms of the normalized added-mass coefficient and the undisturbed fluid-particle acceleration along the centerline of the cylinder. In the linear hydrodynamics problem, $C_{A}$ theoretically approaches unity $\left(C_{M}=2\right)$ in the infinite-frequency limit. In practice, however, $C_{A}$ (or $C_{M}$ ) and $C_{D}$ must be empirically determined and are dependent on many factors, including Reynold's number, Keulegan-Carpenter number, and surface roughness, among others. The assumptions inherent in Morison's representation explain why it is applicable to the analysis of bottom-mounted monopile designs for offshore wind turbines. The asumptions also explain why Morison's representation is not applicable for the analysis of many of the proposed platform concepts for offshore floating wind turbines (except for the viscous-drag term).

One useful feature of Morison's equation, and strip theory in general, is that the hydrodynamic loading is written in terms of the undisturbed fluid-particle velocity and accelerations directly, instead of velocity potentials, which are inherent in the hydrodynamic-added-mass and -damping matrices and the wave-excitation force of the linear frequency-domain problem. This feature allows Morison's equation and strip theory to take advantage of nonlinear wave- and sea-current kinematics models. Nonlinear wave theories account better for the mass transport, wave breaking, shoaling, reflection, transmission, and other nonlinear characteristics of real surface waves. Various forms of nonlinear stream-function wave theory-including Dean's theory, Fenton's theory, and Boussinesq theory - are the most widely used when these characteristics are required [17]. Researchers have also developed a new nonlinear wave-kinematics model that does not require the solution to the nonlinear potential-flow free-surface BVP [86]. This new model can be used as input to an extended Morison formulation to evaluate the wave loads on slender vertical cylinders in steep and random shallow-water waves.

\subsubsection{HydroDyn Calculation Procedure Summary}

The presentation of a variety of hydrodynamics formulations creates a virtual forest of concepts and formulas. In Sections 2.4.1 and 2.4.2, I investigated each tree in detail as I made my way through that forest, but sometimes "it's hard to see the forest for the trees." To help you see that forest, Figure 2-3 draws together the information I have presented.

In summary, HydroDyn accounts for

- Linear hydrostatic restoring

- Nonlinear viscous drag from incident-wave kinematics, sea currents, and platform motion 


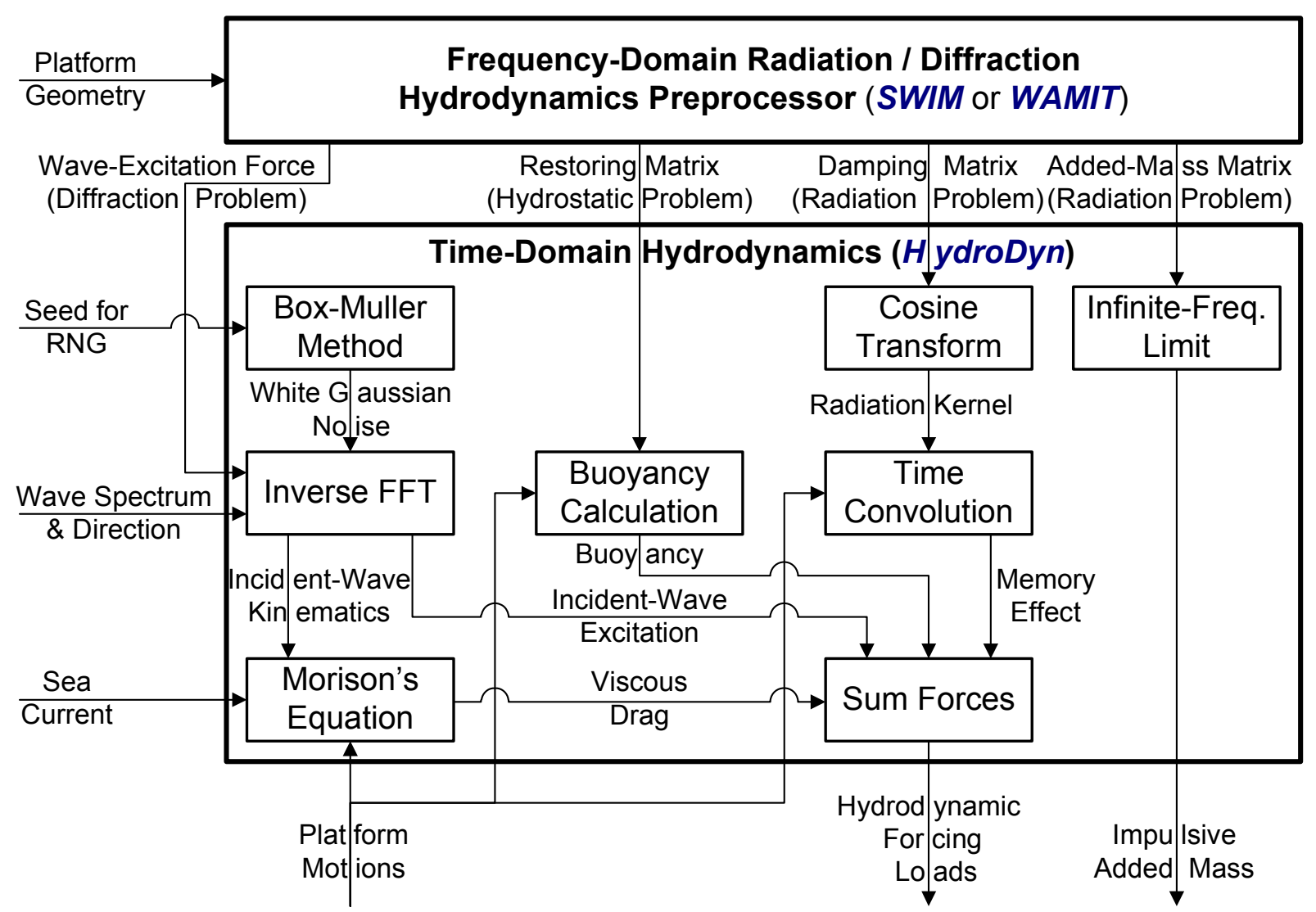

Figure 2-3. Summary of the HydroDyn calculation procedure

- Added-mass and damping contributions from linear wave radiation, including freesurface memory effects

- Incident-wave excitation from linear diffraction in regular or irregular seas.

Just as aerodynamic loads depend on the shape of the rotor-blade airfoils, hydrodynamic loads depend on the support platform's geometry. To this end, I developed HydroDyn such that the hydrodynamic coefficients for platforms of arbitrary shape are imported from SWIM, WAMIT, or an equivalent hydrodynamic preprocessor.

HydroDyn does not account for the effects of nonlinear steep and / or breaking waves, VIV, and loading from sea ice. It also does not account for the second-order effects of intermittent wetting and mean-drift, slow-drift, and sum-frequency excitation.

\subsection{Mooring System Modeling}

Mooring systems are used as a means of station-keeping - holding a floating platform against wind, waves, and current. In some support platform designs, such as in a TLP, they are also used as a means of establishing stability. A mooring system is made up of a number of cables that are attached to the floating support platform at fairlead connections, with the opposite ends anchored to the seabed. Cables can be made up of chain, steel, and / or synthetic fibers and are often a segmented combination of these materials. Restraining forces at the fairleads are established 
through tension in the mooring lines. This tension depends on the buoyancy of the support platform, the cable weight in water, the elasticity in the cable, viscous-separation effects, and the geometrical layout of the mooring system. As the fairleads move with the support platform in response to unsteady environmental loading, the restraining forces at the fairleads change with the changing cable tension. This means that the mooring system has an effective compliance $[22]$.

If the mooring system compliance were inherently linear and mooring inertia and damping were ignored, the total load on the support platform from the contribution of all mooring lines, $F_{i}^{\text {Lines }}$, from Eq. (2-7), would be

$$
F_{i}^{\text {Lines }}=F_{i}^{\text {Lines }, 0}-C_{i j}^{\text {Lines }} q_{j},
$$

where $C_{i j}^{\text {Lines }}$ is the $(i, j)$ component of the linearized restoring matrix from all mooring lines [as included in Eq. (2-24)] and $F_{i}^{\text {Lines, } 0}$ is the $i^{\text {th }}$ component of the total mooring system load acting on the support platform in its undisplaced position. For catenary mooring lines, $F_{i}^{\text {Lines }, 0}$ represents the pre-tension at the fairleads from the weight of the cable not resting on the seafloor in water. If the catenary lines were neutrally buoyant, $F_{i}^{\text {Lines, } 0}$ would be zero. For taut mooring lines, $F_{i}^{\text {Lines, } 0}$ is the result of pre-tension in the mooring lines from excess buoyancy in the tank when the support platform is undisplaced, including the contribution of the weight of the cable in water. $C_{i j}^{\text {Lines }}$ is the combined result of the elastic stiffness of the mooring lines and the effective geometric stiffness brought about by the weight of the cables in water, depending on the layout of the mooring system.

In general, however, the mooring system dynamics are not linear in nature; instead, nonlinearities are generally evident in the force-displacement relationships. The mooring dynamics also often include nonlinear hysteresis effects, where energy is dissipated as the lines oscillate with the support platform around its mean position.

Because I discovered that the dynamic LINES module [50] of SML was unsuitable for my general use, I developed my own quasi-static module to simulate the nonlinear restoring loads from the mooring system of floating platforms. Instead of interfacing with LINES, I have interfaced my mooring system module to FAST and ADAMS.

My module can model an array of homogenous taut or slack catenary mooring lines. It accounts for the apparent weight in fluid, elastic stretching, and seabed friction of each line, but neglects the individual line bending stiffness. But because my quasi-static module is fully coupled with FAST and ADAMS, it also accounts for the nonlinear geometric restoration of the complete mooring system. By "quasi-static," I mean that with the fairlead positions known for a given platform displacement at any instant in time, my mooring system module solves for the tensions within, and configuration of, each mooring line by assuming that each cable is in static equilibrium at that instant. Using the tensions and additional loading on the platform from hydrodynamics and loading on the turbine from aerodynamics, FAST or ADAMS then solves the dynamic equations of motion for the accelerations of the rest of the system (platform, tower, 
nacelle, and blades). Next, FAST or ADAMS integrates in time to obtain new platform and fairlead positions at the next time step, repeating this process.

Clearly, this quasi-static approach also ignores the inertia and damping of the mooring system, which may or may not be important in various situations. To justify using this approach, I used the system-mass data presented in Chapter 3 to calculate that the mass of a typical mooring system is $8 \%$ of the combined mass of a typical wind turbine and floating support platform. According to conversations with Dr. R. Zueck of the Naval Facilities Engineering Service Center (NFESC), about one-quarter of the inertia of a mooring system is important to the dynamic response of a floating platform. One-quarter of $8 \%$ is only $2 \%$, which justifies ignoring mooring system inertia in the analyses for these turbine / platform configurations. Ignoring mooring system damping is also a conservative approach.

Figure 2-4 presents a layout of the calculation procedures in my quasi-static mooring system module. Each line of the mooring system is analyzed independently. The user must specify the fairlead locations of each mooring line relative (and fixed) to the support platform and the anchor locations of each mooring line relative (and fixed) to the inertial reference frame (i.e., the seabed). For each mooring line, the total unstretched length, $L$, apparent weight in fluid per unit length, $\omega$, extensional stiffness, $E A$, and coefficient of seabed static-friction drag, $C_{B}$, must also be assigned. Because a mooring line is buoyant, $\omega$ is related to the mass of the line per unit length, $\mu_{c}$, by

$$
\omega=\left(\mu_{c}-\rho \frac{\pi D_{c}^{2}}{4}\right) g,
$$

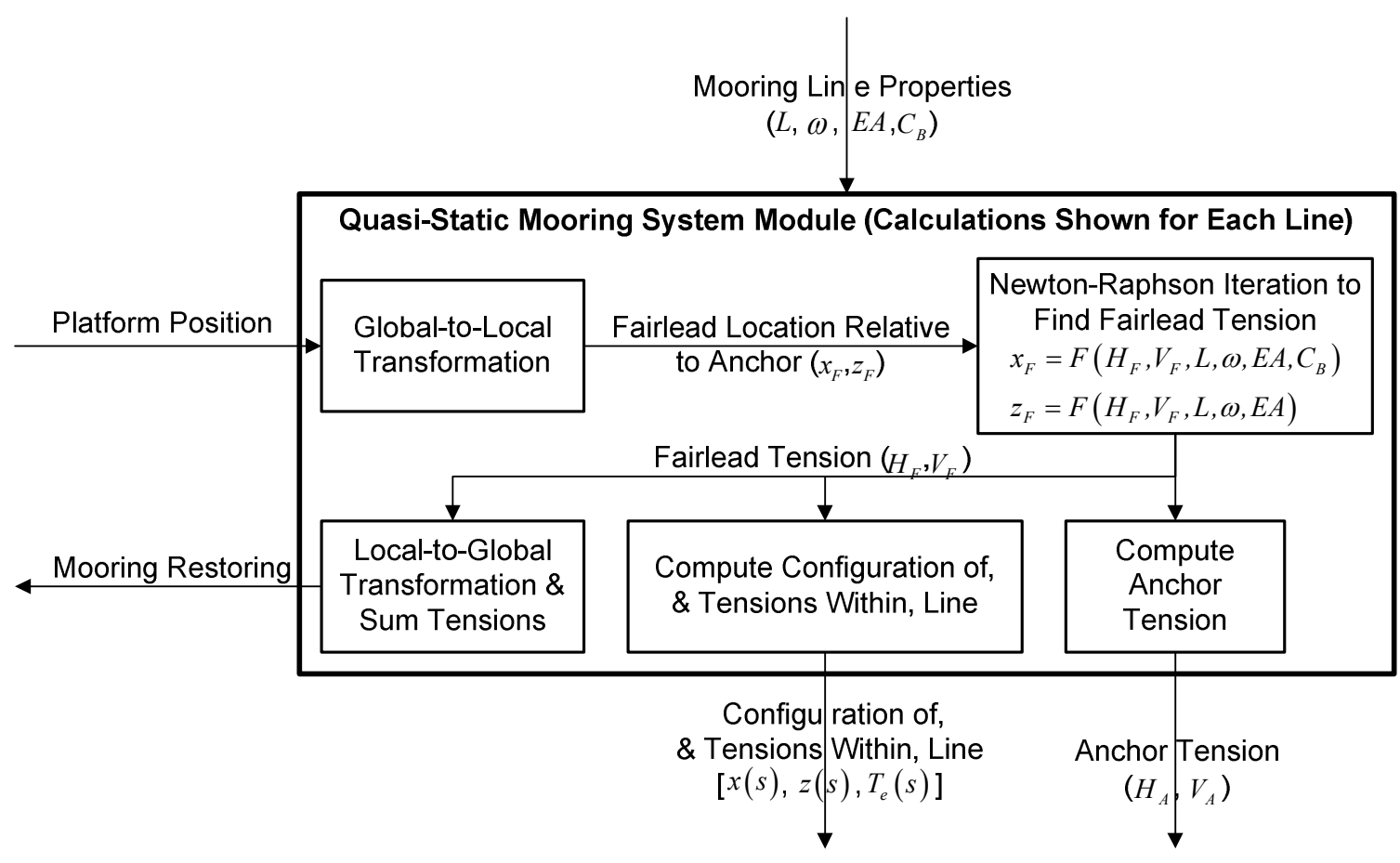

Figure 2-4. Summary of my mooring system module calculation procedure 
where $\rho$ is the water density, $g$ is the gravitational acceleration constant, and $D_{c}$ is the effective diameter of the mooring line. Because I have limited the model to simulating only homogenous mooring lines, I handle multisegment lines (i.e., chain plus wire plus chain segments in series) by using an equivalent line with weighted-average values of the weight and stiffness (weighted based on the unstretched lengths of each segment).

Each mooring line is analyzed in a local coordinate system that originates at the anchor. The local $z$-axis of this coordinate system is vertical and the local $x$-axis is directed horizontally from the anchor to the instantaneous position of the fairlead. Figure 2-5 illustrates a typical line. When the mooring system module is called for a given support platform displacement, the module first transforms each fairlead position from the global frame to this local system to determine its location relative to the anchor, $x_{F}$ and $z_{F}$.

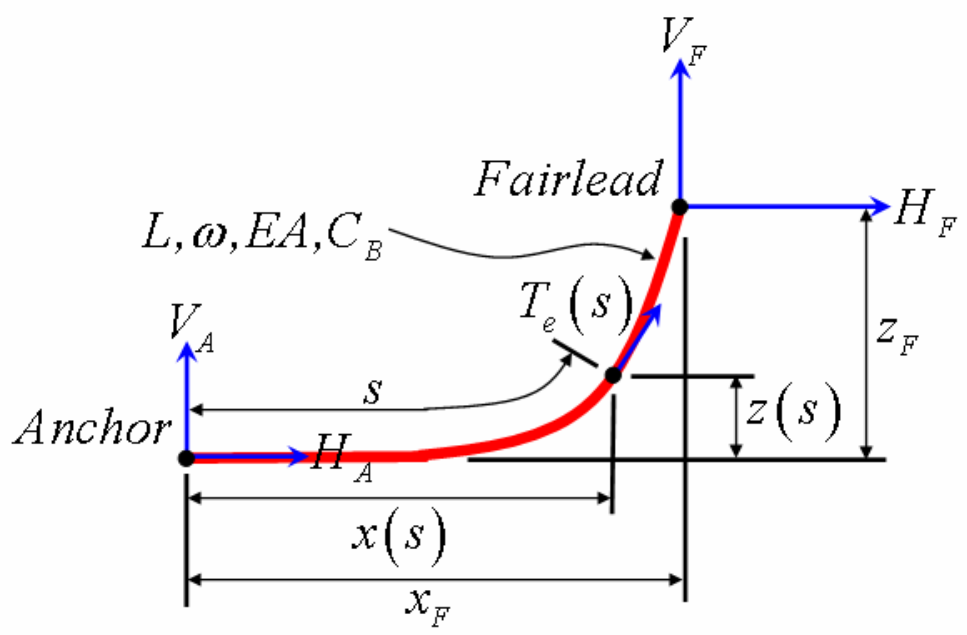

Figure 2-5. Mooring line in a local coordinate system

I took advantage of the analytical formulation for an elastic cable suspended between two points, hanging under its own weight (in fluid). I derived this analytical formulation following a procedure similar to that presented in Ref. [22], which I do not give here for brevity. (The derivation was not exactly the same because Ref. [22] did not account for seabed interaction nor did it account for taut lines where the angle of the line at the anchor was nonzero). The derivation required the assumption that the extensional stiffness of the mooring line, $E A$, was much greater than the hydrostatic pressure at all locations along the line.

In the local coordinate system, the analytical formulation is given in terms of two nonlinear equations in two unknowns - the unknowns are the horizontal and vertical components of the effective tension in the mooring line at the fairlead, $H_{F}$ and $V_{F}$, respectively. (The effective tension is defined as the actual cable [wall] tension plus the hydrostatic pressure.) When no portion of the line rests on the seabed, the analytical formulation is as follows: 


$$
\begin{aligned}
x_{F}\left(H_{F}, V_{F}\right)= & \frac{H_{F}}{\omega}\left\{\ln \left[\frac{V_{F}}{H_{F}}+\sqrt{1+\left(\frac{V_{F}}{H_{F}}\right)^{2}}\right]-\ln \left[\frac{V_{F}-\omega L}{H_{F}}+\sqrt{1+\left(\frac{V_{F}-\omega L}{H_{F}}\right)^{2}}\right]\right\} \\
& +\frac{H_{F} L}{E A}
\end{aligned}
$$

and

$$
z_{F}\left(H_{F}, V_{F}\right)=\frac{H_{F}}{\omega}\left[\sqrt{1+\left(\frac{V_{F}}{H_{F}}\right)^{2}}-\sqrt{1+\left(\frac{V_{F}-\omega L}{H_{F}}\right)^{2}}\right]+\frac{1}{E A}\left(V_{F} L-\frac{\omega L^{2}}{2}\right) .
$$

Equivalent formulations of Eq. (2-35) are sometimes cited in terms of the inverse of the hyperbolic sine; that is:

$$
\ln \left(x+\sqrt{1+x^{2}}\right)=\sinh ^{-1}(x) .
$$

The first terms on the right-hand side of Eq. (2-35) characterize the arc length of the catenary, projected on the $x$ - and $z$-axes. (Even taut mooring lines have a catenary-shaped sag.) The second terms on the right-hand side of Eq. (2-35) represent the horizontal and vertical stretching of the mooring line.

The analytical formulation of two equations in two unknowns is different when a portion of the mooring line adjacent to the anchor rests on the seabed:

$$
\begin{aligned}
& x_{F}\left(H_{F}, V_{F}\right)= L-\frac{V_{F}}{\omega}+\frac{H_{F}}{\omega} \ln \left[\frac{V_{F}}{H_{F}}+\sqrt{1+\left(\frac{V_{F}}{H_{F}}\right)^{2}}\right]+\frac{H_{F} L}{E A} \\
&+\frac{C_{B} \omega}{2 E A}\left[-\left(L-\frac{V_{F}}{\omega}\right)^{2}+\left(L-\frac{V_{F}}{\omega}-\frac{H_{F}}{C_{B} \omega}\right) M A X\left(L-\frac{V_{F}}{\omega}-\frac{H_{F}}{C_{B} \omega}, 0\right)\right] \\
& \text { and } \\
& z_{F}\left(H_{F}, V_{F}\right)=\frac{H_{F}}{\omega}\left[\sqrt{1+\left(\frac{V_{F}}{H_{F}}\right)^{2}}-\sqrt{1+\left(\frac{V_{F}-\omega L}{H_{F}}\right)^{2}}\right]+\frac{1}{E A}\left(V_{F} L-\frac{\omega L^{2}}{2}\right) .
\end{aligned}
$$

The first two terms on the right-hand side of Eq. (2-37a) combine to represent the unstretched portion of the mooring line resting on the seabed, $L_{B}$ :

$$
L_{B}=L-\frac{V_{F}}{\omega}
$$

In Eq. (2-35), $L_{B}$ is zero. 
The last term on the right-hand side of Eq. (2-37a), which involves $C_{B}$, corresponds to the stretched portion of the mooring line resting on the seabed that is affected by static friction. The seabed static friction was modeled simply as a drag force per unit length of $C_{B} \omega$. The $M A X$ function is needed to handle cases with and without tension at the anchor. Specifically, the resultant is zero when the anchor tension is positive; that is, the seabed friction is too weak to overcome the horizontal tension in the mooring line. Conversely, the resultant of the $M A X$ function is nonzero when the anchor tension is zero. This happens when a section of cable lying on the seabed is long enough to ensure that the seabed friction entirely overcomes the horizontal tension in the mooring line.

The remaining terms in Eq. (2-37) are similar in form to, and typify the same information as, the terms in Eq. (2-35). They are simpler than the terms in Eq. (2-35), though, because a slack catenary is always tangent to the seabed at the point of touchdown.

My mooring system module uses a Newton-Raphson iteration scheme to solve nonlinear Eqs. (2-35) and (2-37) for the fairlead effective tension $\left(H_{F}\right.$ and $\left.V_{F},\right)$, given the line properties $(L, \omega$, $E A$, and $\left.C_{B}\right)$ and the fairlead position relative to the anchor $\left(x_{F}\right.$ and $\left.z_{F}\right)$. The Jacobian in the Newton-Raphson iteration was implemented with the analytical partial derivatives of Eqs. (2-35) and (2-37). My mooring system module determines which of Eqs. (2-35) or (2-37) must be used as part of the solution process. The equations were implemented in a slightly different form than shown to avoid numerical problems (e.g., a division by zero when $C_{B}$ is zero-valued).

My mooring system module uses the values of $H_{F}$ and $V_{F}$ from the previous time step as the initial guess in the next iteration of Newton-Raphson. As the model is being initialized, I used the starting values, $H_{F}^{0}$ and $V_{F}^{0}$, documented by Peyrot and Goulois in Ref. [79]:

$$
\begin{gathered}
H_{F}^{0}=\left|\frac{\omega x_{F}}{2 \lambda_{0}}\right| \\
\text { and } \\
V_{F}^{0}=\frac{\omega}{2}\left[\frac{z_{F}}{\tanh \left(\lambda_{0}\right)}+L\right],
\end{gathered}
$$

where the dimensionless catenary parameter, $\lambda_{0}$, depends on the initial configuration of the mooring line:

$$
\lambda_{0}= \begin{cases}1,000,000 & \text { for } x_{F}=0 \\ 0.2 & \text { for } \sqrt{x_{F}^{2}+z_{F}^{2}} \geq L . \\ \sqrt{3\left(\frac{L^{2}-z_{F}^{2}}{x_{F}^{2}}-1\right)} & \text { otherwise }\end{cases}
$$


Note that Eqs. (2-39) and (2-40) are slightly different from those given in Ref. [79] because my analytical formulation and notation differs.

Once the effective tension at the fairlead has been found, determining the horizontal and vertical components of the effective tension in the mooring line at the anchor, $H_{A}$ and $V_{A}$, respectively, is simple. (The blue arrows depicting $H_{A}$ and $V_{A}$ in Figure 2-5 are the horizontal and vertical components of the effective line tension at the anchor-they are not the reaction forces at the anchor.) From a balance of external forces on a mooring line, one can easily verify that

$$
\begin{gathered}
H_{A}=H_{F} \\
\text { and } \\
V_{A}=V_{F}-\omega L,
\end{gathered}
$$

when no portion of the line rests on the seabed, and

$$
\begin{gathered}
H_{A}=\operatorname{MAX}\left(H_{F}-C_{B} \omega L_{B}, 0\right) \\
\text { and } \\
V_{A}=0,
\end{gathered}
$$

when a portion of the line does rest on the seabed. Although they do not affect the dynamic response of the floating wind turbine system, the anchor effective tensions are computed by my mooring system module and become available outputs from the simulation.

Next, my mooring system module solves for the configuration of, and effective tensions within, the mooring line. Again, the values of these parameters do not affect the dynamic response of the floating wind turbine system, but they are available outputs from the simulation. When no portion of the mooring line rests on the seabed, the equations for the horizontal and vertical distances between the anchor and a given point on the line, $x$ and $z$, and the equation for the effective tension in the line at that point, $T_{e}$, are as follows:

$$
\begin{gathered}
x(s)=\frac{H_{F}}{\omega}\left\{\ln \left[\frac{V_{A}+\omega s}{H_{F}}+\sqrt{1+\left(\frac{V_{A}+\omega s}{H_{F}}\right)^{2}}\right]-\ln \left[\frac{V_{A}}{H_{F}}+\sqrt{1+\left(\frac{V_{A}}{H_{F}}\right)^{2}}\right]\right\}+\frac{H_{F} s}{E A}, \\
z(s)=\frac{H_{F}}{\omega}\left[\sqrt{1+\left(\frac{V_{A}+\omega s}{H_{F}}\right)^{2}}-\sqrt{1+\left(\frac{V_{A}}{H_{F}}\right)^{2}}\right]+\frac{1}{E A}\left(V_{A} s+\frac{\omega s^{2}}{2}\right), \\
\text { and } \\
T_{e}(s)=\sqrt{H_{F}^{2}+\left(V_{A}+\omega s\right)^{2}},
\end{gathered}
$$


where $s$ is the unstretched arc distance along the mooring line from the anchor to the given point. The similarity between Eqs. (2-43) and (2-35) should be apparent. Similar to Eq. (2-37), the equations with seabed interaction are more onerous:

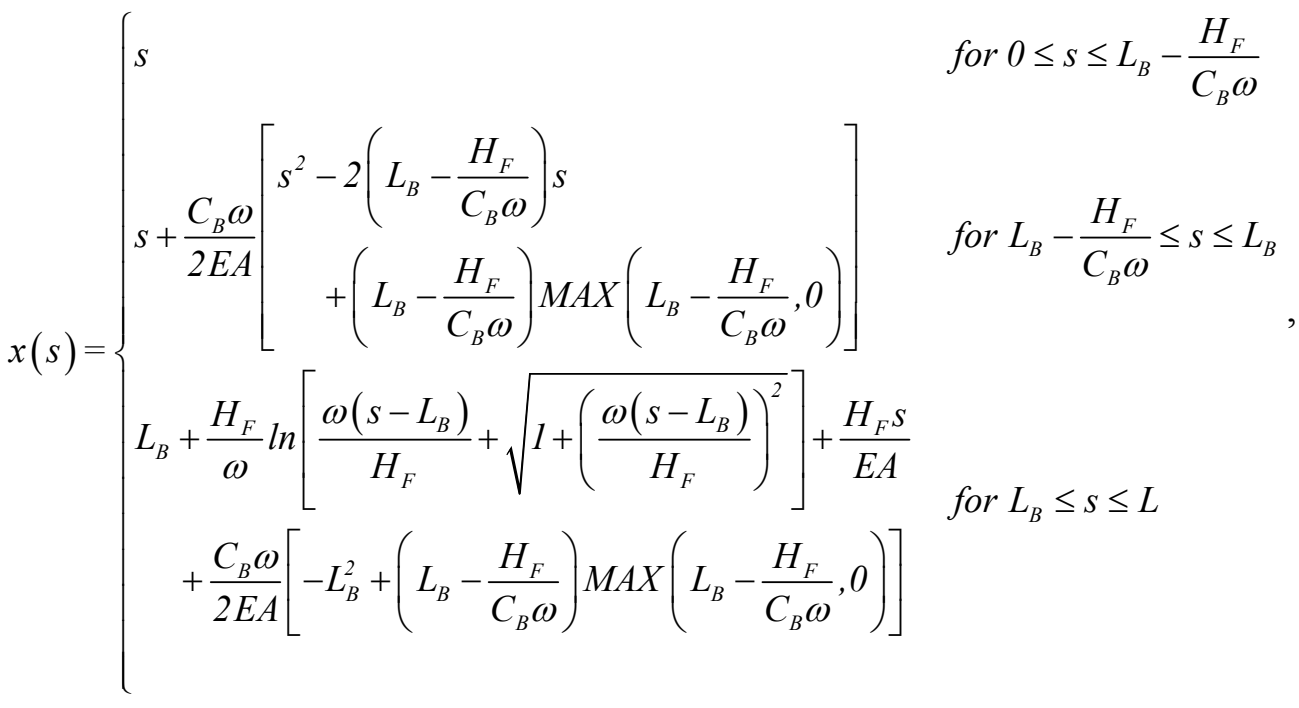

$$
\begin{aligned}
& z(s)=\left\{\begin{array}{ll}
0 & \text { for } 0 \leq s \leq L_{B} \\
\frac{H_{F}}{\omega} \ln \left[\sqrt{1+\left(\frac{\omega\left(s-L_{B}\right)}{H_{F}}\right)^{2}}-1\right]+\frac{\omega\left(s-L_{B}\right)^{2}}{2 E A} & \text { for } L_{B} \leq s \leq L
\end{array},\right. \\
& \text { and } \\
& T_{e}(s)=\left\{\begin{array}{ll}
\operatorname{MAX}\left(H_{F}+C_{B} \omega\left(s-L_{B}\right), 0\right) & \text { for } 0 \leq s \leq L_{B} \\
\sqrt{H_{F}^{2}+\left(\omega\left(s-L_{B}\right)\right)^{2}} & \text { for } L_{B} \leq s \leq L
\end{array} .\right.
\end{aligned}
$$

As shown in Figure 2-4, the final calculation in my quasi-static mooring system module is a computation of the total load on the support from the contribution of all mooring lines; that is, $F_{i}^{\text {Lines }}$ from Eq. (2-7). This mooring system-restoring load is found by first transforming each fairlead tension from its local mooring line coordinate system to the global frame, then summing up the tensions from all lines.

\subsection{Pulling It All Together}

Limitations of previous time- and frequency-domain studies on offshore floating wind turbines motivated my development of simulation tools capable of modeling the fully coupled aerohydro-servo-elastic response of such systems. I have developed this capability by leveraging the computational methodologies and analysis tools of the onshore wind turbine and offshore O\&G industries. The onshore wind-industry-accepted aero-servo-elastic turbine simulation capabilities of FAST [39] with AeroDyn [55,67] and MSC.ADAMS with A2AD [20,54] and AeroDyn have been interfaced with the external hydrodynamic wave-body interaction programs SWIM [48] and WAMIT [58], which are commonly employed in the offshore O\&G industry. I 
established the interfaces among these simulation capabilities by developing modules for treating time-domain hydrodynamics (HydroDyn) and quasi-static mooring system responses. Figure 2-6 summarizes the modules and their interfaces.

Turbulent-wind inflow is prescribed by the external computer program TurbSim [36], and deterministic-wind inflow (not shown in Figure 2-6) is prescribed by the external computer program IECWind [56]. FAST with AeroDyn and ADAMS with AeroDyn account for the applied aerodynamic and gravitational loads, the behavior of the control and protection systems, and the structural dynamics of the wind turbine. The latter contribution includes the elasticity of the rotor, drivetrain, and tower, along with the newly added dynamic coupling between the motions of the support platform and the motions of the wind turbine. ${ }^{11}$ Nonlinear restoring loads from the mooring system are obtained from a quasi-static mooring line module that accounts for the elastic stretching of an array of homogenous taut or slack catenary lines with seabed interaction. HydroDyn is a module that computes the applied hydrodynamic loads in the time domain, as summarized in Section 2.4.3.

By interfacing these modules as described, fully coupled time-domain aero-hydro-servo-elastic simulation of offshore floating wind turbines is achieved. This capability is crucial for analyzing the dynamic response from combined wind and wave loading because both can affect the motions, loads, and power production of the system. The generality of each module also ensures

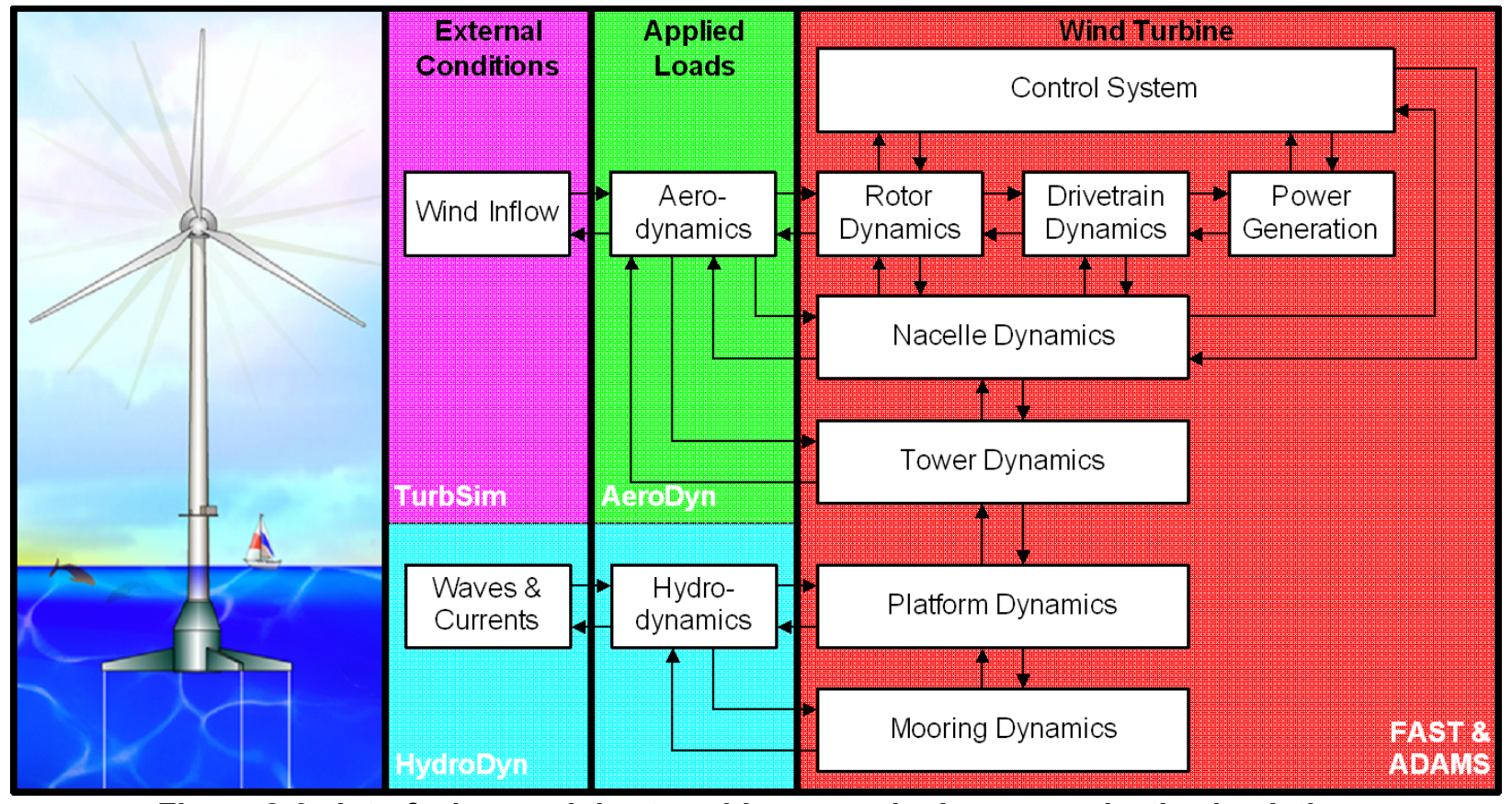

Figure 2-6. Interfacing modules to achieve aero-hydro-servo-elastic simulation

11 FAST and ADAMS are separate programs that can be run independently to model the structural-dynamic response and control system behavior of wind turbines. FAST employs a combined modal and multibody structuraldynamics formulation, whereas ADAMS employs a higher fidelity multibody formulation. They have both been interfaced with AeroDyn to enable the full aero-servo-elastic modeling of wind turbines. 
that the overall simulation tool is universal enough to analyze a variety of wind turbine, support platform, and mooring system configurations. Moreover, the same simulation tools can still be used to model land-based wind turbines by disabling the hydrodynamic and mooring system modules. 


\section{Chapter 3 Design Basis and Floating Wind Turbine Model}

To obtain useful information from this and other concept studies aimed at assessing offshore wind technology suitable in the deep waters off the U.S. offshore continental shelf (OCS) and other offshore sites worldwide, use of realistic and standardized input data is required. This chapter summarizes the input data developed and used in the simulation code verification exercises and in the integrated loads analyses presented in subsequent chapters. A large collection of input data is needed, including detailed specifications of the wind turbine and support platform, along with a design basis. A design basis consists of analysis methods (see Chapter 2); a collection of applicable design standards (i.e., IEC); and the site-specific meteorological and oceanographic (metocean) parameters at a reference site.

In this work, I developed the turbine specifications of what is now called the "NREL offshore 5MW baseline wind turbine," as presented in Section 3.1. Although I put together the specifications of this wind turbine, I did not develop the basic designs of the two floating support platforms used in this work. Instead, I used two platforms that were developed by others through partnerships with NREL. Both platforms were developed specifically to support the NREL offshore 5-MW baseline wind turbine. The first platform, which I summarize in Section 3.2, was a barge with slack catenary moorings from a company called ITI Energy ${ }^{1}$ [98]. The second platform, summarized in Section 3.3, was a barge with a spread-mooring system developed at MIT through a contract with NREL [100,101]. Barge concepts were chosen because of their simplicity in design, fabrication, and installation. For the loads analyses, ITI Energy selected a location in the northern North Sea as the reference site from which to obtain metocean data. These data are described in Section 3.4.

\subsection{NREL Offshore 5-MW Baseline Wind Turbine}

This section documents the specifications of NREL's offshore 5-MW baseline wind turbine and the rationale behind its development. My objective was to establish the detailed specifications of a large wind turbine that is representative of typical utility-scale land- and sea-based multimegawatt turbines.

Before establishing the detailed specifications, however, $\mathrm{we}^{2}$ had to choose the basic size and power rating of the machine. Because of the large portion of system costs in the support structure of a deepwater wind system, we understood from the outset that if a deepwater wind system is to be cost-effective, each individual wind turbine must be rated at $5 \mathrm{MW}$ or higher [70]. ${ }^{3}$ Ratings considered for the baseline ranged from $5 \mathrm{MW}$ to $20 \mathrm{MW}$. We decided that the baseline should be $5 \mathrm{MW}$ because it has precedence:

\footnotetext{
${ }^{1}$ Web site: http://www.itienergy.com/

${ }^{2}$ My NREL colleagues, W. N. Musial and S. Butterfield, assisted me in selecting some of the basic specifications of this offshore turbine. To acknowledge this support, I use "we" in place of "I" and "our" in place of "my" where appropriate.

${ }^{3}$ A single 5-MW wind turbine can supply enough energy annually to power 1,250 average American homes.
} 
- Feasible floater configurations for offshore wind turbines scoped out by Musial, Butterfield, and Boone [70] were based on the assumption of a 5-MW unit.

- Unpublished DOE offshore cost studies were based on a rotor diameter of $128 \mathrm{~m}$, which is a size representative of a 5- to 6-MW wind turbine.

- The land-based Wind Partnerships for Advanced Component Technology (WindPACT) series of studies, considered wind turbine systems rated up to 5 MW $[64,73,88]$.

- The Recommendations for Design of Offshore Wind Turbines project (known as RECOFF) based its conceptual design calculations on a wind turbine with a 5-MW rating [93].

- The Dutch Offshore Wind Energy Converter (DOWEC) project based its conceptual design calculations on a wind turbine with a 6-MW rating [24,51,60].

- At the time of this writing, the largest wind turbine prototypes in the world-the Multibrid M5000 [18,68,69] and the REpower 5M [62,81,82]—each had a 5-MW rating.

I gathered the publicly available information on the Multibrid M5000 and REpower 5M prototype wind turbines. And because detailed information on these machines was unavailable, I also used the publicly available properties from the conceptual models used in the WindPACT, RECOFF, and DOWEC projects. These models contained much greater detail than was available about the prototypes. I then created a composite from these models, extracting the best available and most representative specifications.

The Multibrid M5000 machine has a significantly higher tip speed than typical onshore wind turbines and a lower tower-top mass than would be expected from scaling laws previously developed in one of the WindPACT studies [88]. In contrast, the REpower 5M machine has properties that are more "expected" and "conventional." For this reason, we decided to use the specifications of the REpower $5 \mathrm{M}$ machine as the target specifications ${ }^{4}$ for our baseline model.

The wind turbine used in the DOWEC project had a slightly higher rating than the rating of the REpower 5M machine, but many of the other basic properties of the DOWEC turbine matched the REpower 5M machine very well. In fact, the DOWEC turbine matched many of the properties of the REpower $5 \mathrm{M}$ machine better than the turbine properties derived for the WindPACT and RECOFF studies. ${ }^{5}$ As a result of these similarities, I made the heaviest use of data from the DOWEC study in my development of the NREL offshore 5-MW baseline wind turbine.

The REpower 5M machine has a rotor radius of about $63 \mathrm{~m}$. Wanting the same radius and the lowest reasonable hub height possible to minimize the overturning moment acting on a floating

\footnotetext{
${ }^{4}$ Note that I established the target specifications using information about the REpower $5 \mathrm{M}$ machine that was published in January 2005 [81,82]. Some of the information presented in Refs. [81] and [82] disagrees with more recently published information. For example, the published nacelle and rotor masses of the REpower $5 \mathrm{M}$ are higher in the more recent publications.

${ }^{5}$ This was probably because the REpower 5M prototype utilized blades provided by LM Glasfiber [62], a company that helped establish the structural properties of the blades used in the DOWEC study.
} 
support platform, we decided that the hub height for the baseline wind turbine should be $90 \mathrm{~m}$. This would give a $15-\mathrm{m}$ air gap between the blade tips at their lowest point when the wind turbine is undeflected and an estimated extreme 50-year individual wave height of $30 \mathrm{~m}$ (i.e., 15 $\mathrm{m}$ amplitude). The additional gross properties we chose for the NREL 5-MW baseline wind turbine, most of which are identical to those of the REpower 5M, are given in Table 3-1. The $(x, y, z)$ coordinates of the overall CM location of the wind turbine are indicated in a tower-base coordinate system, which originates along the tower centerline at ground or mean sea level (MSL). The $x$-axis of this coordinate system is directed nominally downwind, the $y$-axis is directed transverse to the nominal wind direction, and the $z$-axis is directed vertically from the tower base to the yaw bearing.

Table 3-1. Gross Properties Chosen for the NREL 5-MW Baseline Wind Turbine

\begin{tabular}{|l|r|}
\hline Rating & $5 \mathrm{MW}$ \\
\hline Rotor Orientation, Configuration & Upwind, 3 Blades \\
\hline Control & Variable Speed, Collective Pitch \\
\hline Drivetrain & High Speed, Multiple-Stage Gearbox \\
\hline Rotor, Hub Diameter & $126 \mathrm{~m}, 3 \mathrm{~m}$ \\
\hline Hub Height & $90 \mathrm{~m}$ \\
\hline Cut-In, Rated, Cut-Out Wind Speed & $3 \mathrm{~m} / \mathrm{s}, 11.4 \mathrm{~m} / \mathrm{s}, 25 \mathrm{~m} / \mathrm{s}$ \\
\hline Cut-In, Rated Rotor Speed & $6.9 \mathrm{rpm}, 12.1 \mathrm{rpm}$ \\
\hline Rated Tip Speed & $80 \mathrm{~m} / \mathrm{s}$ \\
\hline Overhang, Shaft Tilt, Precone & $5 \mathrm{~m}, 5^{\circ}, 2.5^{\circ}$ \\
\hline Rotor Mass & $110,000 \mathrm{~kg}$ \\
\hline Nacelle Mass & $240,000 \mathrm{~kg}$ \\
\hline Tower Mass & $347,460 \mathrm{~kg}$ \\
\hline Coordinate Location of Overall CM & $(-0.2 \mathrm{~m}, 0.0 \mathrm{~m}, 64.0 \mathrm{~m})$ \\
\hline
\end{tabular}

The actual REpower 5M wind turbine uses blades with built-in prebend as a means of increasing tower clearance without a large rotor overhang. Because, as I mentioned in Section 2.1, the FAST code cannot support blades with built-in prebend, I chose a $2.5^{\circ}$-upwind precone in the baseline wind turbine to represent the smaller amount of precone and larger amount of prebend that are built into the actual REpower 5M machine.

The rotor diameter indicated in Table 3-1 ignores the effect of blade precone, which reduces the actual diameter and swept area. The exact rotor diameter in the turbine specifications (assuming that the blades are undeflected) is actually $(126 \mathrm{~m}) \times \cos \left(2.5^{\circ}\right)=125.88 \mathrm{~m}$ and the actual swept area is $(\pi / 4) \times(125.88 \mathrm{~m})^{2}=12,445.3 \mathrm{~m}^{2}$.

I present other information about this model as follows:

- The blade structural properties in Section 3.1.1

- The blade aerodynamic properties in Section 3.1.2

- The hub and nacelle properties in Section 3.1.3

- The drivetrain properties in Section 3.1.4

- The tower properties in Section 3.1.5 
- The baseline control system properties in Section 3.1.6

- The aero-servo-elastic FAST with AeroDyn and ADAMS with AeroDyn models of the wind turbine in Section 3.1.7

- The basic responses of the land-based version of the wind turbine, including its fullsystem natural frequencies and steady-state behavior in Section 3.1.8.

Although I summarize much of this information ${ }^{6}$ for conciseness and clarity, Section 3.1.6 contains a high level of detail about the development of the wind turbine's baseline control system. These details are fundamental to the controls work presented in Chapter 7.

Beyond its application to this work, the NREL offshore 5-MW baseline wind turbine has been used to establish the reference specifications for a number of research projects supported by the U.S. DOE's Wind \& Hydropower Technologies Program [1,23,84,100,101]. In addition, the integrated European Union UpWind research program ${ }^{7}$ and the International Energy Agency (IEA) Wind Annex XXIII Subtask $2^{8}$ Offshore Code Comparison Collaboration (OC3) [78] have adopted the NREL offshore 5-MW baseline wind turbine as their reference model. The model has been, and will likely continue to be, used as a reference by research teams throughout the world to standardize baseline offshore wind turbine specifications and to quantify the benefits of advanced land- and sea-based wind energy technologies.

\subsubsection{Blade Structural Properties}

The NREL offshore 5-MW baseline wind turbine has three blades. I based the distributed blade structural properties of each blade on the structural properties of the 62.6-m-long LM Glasfiber blade used in the DOWEC study (using the data given in Appendix A of Ref. [60]). Because the blades in the DOWEC study were $1.1 \mathrm{~m}$ longer than the 61.5-m-long LM Glasfiber blades [62] used on the actual REpower 5M machine, I truncated the $62.6-\mathrm{m}$ blades at $61.5-\mathrm{m}$ span to obtain the structural properties of the NREL 5-MW baseline blades (I found the structural properties at the blade tip by interpolating between the $61.2-\mathrm{m}$ and $61.7-\mathrm{m}$ stations given in Appendix A of Ref. [60]). Table 3-2 lists the resulting properties.

The entries in the first column of Table 3-2, labeled "Radius," are the spanwise locations along the blade-pitch axis relative to the rotor center (apex). "BlFract" is the fractional distance along the blade-pitch axis from the root (0.0) to the tip (1.0). I located the blade root $1.5 \mathrm{~m}$ along the pitch axis from the rotor center, equivalent to half the hub diameter listed in Table 3-1.

"AeroCent" is the name of a FAST input parameter. The FAST code assumes that the bladepitch axis passes through each airfoil section at $25 \%$ chord. By definition, then, the quantity (AeroCent -0.25 ) is the fractional distance to the aerodynamic center from the blade-pitch axis

\footnotetext{
${ }^{6}$ Note that some of the turbine properties are presented with a large number $(>4)$ of significant figures. Most of these were carried over from the turbine properties documented in the DOWEC study [24,51,60] — I did not truncate their precision to maintain consistency with the original data source.

${ }^{7}$ Web site: http://www.upwind.eu/default.aspx

${ }^{8}$ Web site: http://www.ieawind.org/Annex\%20XXIII/Subtask2.html
} 
Table 3-2. Distributed Blade Structural Properties

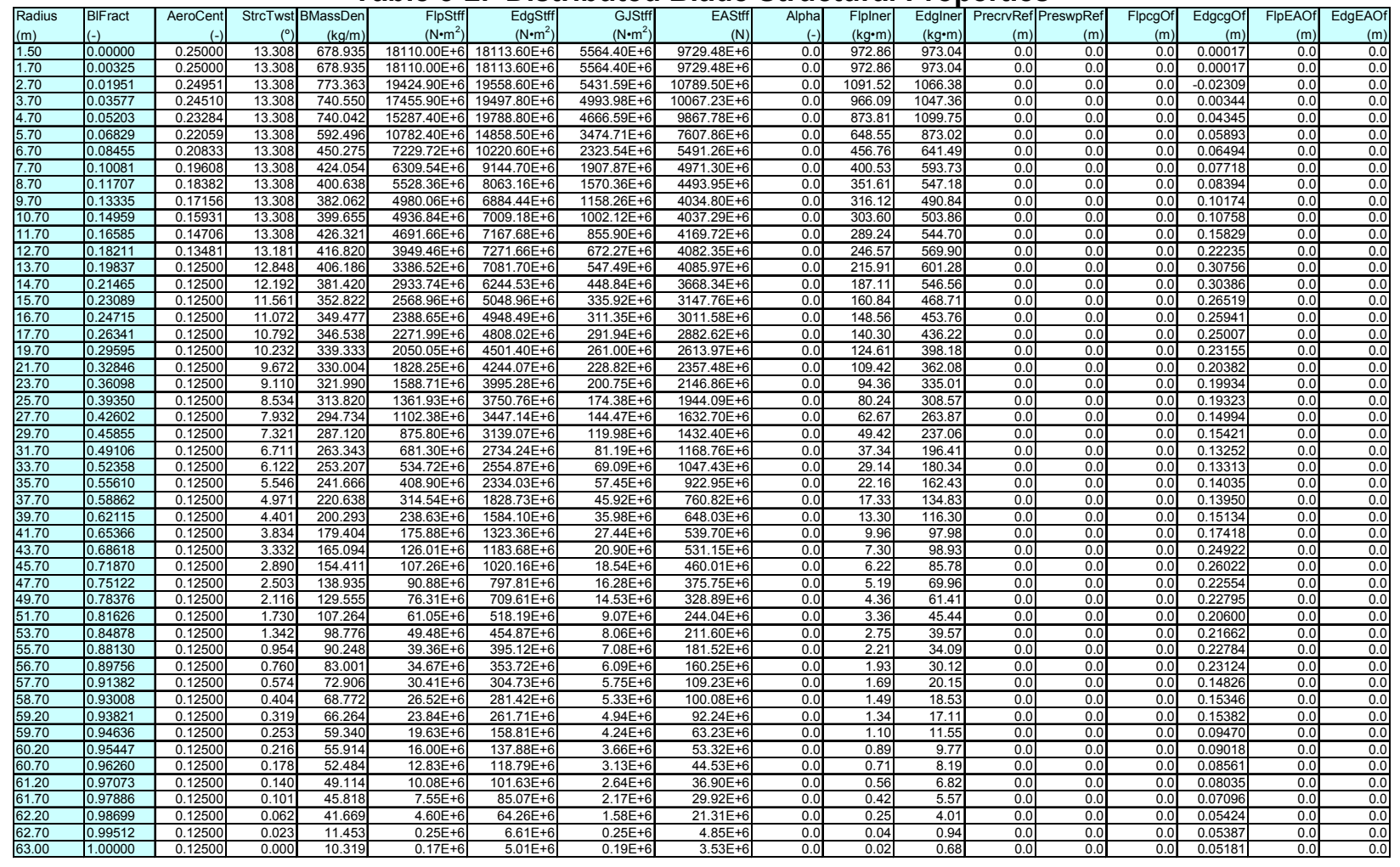

along the chordline, positive toward the trailing edge. Thus, at the root (i.e., BlFract $=0.0$ ), AeroCent $=0.25$ means that the aerodynamic center lies on the blade-pitch axis [because $(0.25-$ $0.25)=0.0$ ], and at the tip (i.e., BlFract $=1.0$ ), AeroCent $=0.125$ means that the aerodynamic center lies 0.125 chordlengths toward the leading edge from the blade-pitch axis [because $(0.125$ $-0.25)=-0.125]$.

The flapwise and edgewise section stiffness and inertia values, "FlpStff," "EdgStff," "FlpIner," and "EdgIner" in Table 3-2, are given about the principal structural axes of each cross section as oriented by the structural-twist angle, "StrcTwst." The values of the structural twist were assumed to be identical to the aerodynamic twist discussed in Section 3.1.2.

"GJStff" represents the values of the blade torsion stiffness. Because the DOWEC blade data did not contain extensional stiffness information, I estimated the blade extensional stiffness values"EAStff" in Table 3-2 - to be $10^{7}$ times the average mass moment of inertia at each blade station. This came from a rule of thumb derived from the data available in the WindPACT rotor design study [64], but the exact values are not important because of the low rotational speed of the rotor.

The edgewise CM offset values, "EdgcgOf," are the distances in meters along the chordline from the blade-pitch axis to the $\mathrm{CM}$ of the blade section, positive toward the trailing edge. I neglected the insignificant values of the flapwise CM offsets, "FlpcgOf," and flapwise and edgewise elastic offsets, "FlpEAOf" and "EdgEAOf," given in Appendix A of Ref. [60]. Instead, I assumed that they were zero as shown in Table 3-2.

The distributed blade section mass per unit length values, "BMassDen," given in Table 3-2 are the values documented in Appendix A of Ref. [60]. I increased these by $4.536 \%$ in the model to 
scale the overall (integrated) blade mass to $17,740 \mathrm{~kg}$, which was the nominal mass of the blades in the REpower 5M prototype. In my baseline specifications, the nominal second mass moment of inertia, nominal first mass moment of inertia, and the nominal radial CM location of each blade are $11,776,047 \mathrm{~kg} \cdot \mathrm{m}^{2}, 363,231 \mathrm{~kg} \cdot \mathrm{m}$, and $20.475 \mathrm{~m}$ with respect to (w.r.t.) the blade root, respectively.

I specified a structural-damping ratio of $0.477465 \%$ critical in all modes of the isolated blade, which corresponds to the 3\% logarithmic decrement used in the DOWEC study from page 20 of Ref. [51].

Table 3-3 summarizes the undistributed blade structural properties discussed in this section.

Table 3-3. Undistributed Blade Structural Properties

\begin{tabular}{|l|c|}
\hline Length (w.r.t. Root Along Preconed Axis) & $61.5 \mathrm{~m}$ \\
\hline Mass Scaling Factor & $4.536 \%$ \\
\hline Overall (Integrated) Mass & $17,740 \mathrm{~kg}$ \\
\hline Second Mass Moment of Inertia (w.r.t. Root) & $11,776,047 \mathrm{~kg} \cdot \mathrm{m}^{2}$ \\
\hline First Mass Moment of Inertia (w.r.t. Root) & $363,231 \mathrm{~kg} \cdot \mathrm{m}$ \\
\hline CM Location (w.r.t. Root along Preconed Axis) & $20.475 \mathrm{~m}$ \\
\hline Structural-Damping Ratio (All Modes) & $0.477465 \%$ \\
\hline
\end{tabular}

\subsubsection{Blade Aerodynamic Properties}

Similar to the blade structural properties, I based the blade aerodynamic properties of the NREL 5-MW baseline wind turbine on the DOWEC blades (using the data described in Table 1 on page 13 of Ref. [51] and in Appendix A of Ref. [60]). I set the FAST and ADAMS models to use 17 blade elements for integration of the aerodynamic and structural forces. To better capture the large structural gradients at the blade root and the large aerodynamic gradients at the blade tip, the 3 inboard and 3 outboard elements are two-thirds the size of the 11 equally spaced midspan elements. Table 3-4 gives the aerodynamic properties at the blade nodes, which are located at the center of the blade elements.

The blade node locations, labeled as "RNodes" in Table 3-4, are directed along the blade-pitch axis from the rotor center (apex) to the blade cross sections. The element lengths, "DRNodes," sum to the total blade length of $61.5 \mathrm{~m}$ indicated in Table 3-3. The aerodynamic twist, "AeroTwst," as given in Table 3-4, are offset by $-0.09182^{\circ}$ from the values provided in Appendix A of Ref. [60] to ensure that the zero-twist reference location is at the blade tip. Integrating the chord distribution along the blade span reveals that the rotor solidity is roughly $5.16 \%$.

As indicated in Table 3-4, I incorporated eight unique airfoil-data tables for the NREL offshore 5-MW baseline wind turbine. The two innermost airfoil tables represent cylinders with drag coefficients of 0.50 (Cylinder1.dat) and 0.35 (Cylinder2.dat) and no lift. I created the remaining six airfoil tables by making corrections for three-dimensional behavior to the two-dimensional airfoil-data coefficients of the six airfoils used in the DOWEC study (as detailed in Appendix A 
Table 3-4. Distributed Blade Aerodynamic Properties

\begin{tabular}{|l|r|r|r|r|r|}
\hline $\begin{array}{l}\text { Node } \\
(-)\end{array}$ & $\begin{array}{r}\text { RNodes } \\
(\mathrm{m})\end{array}$ & $\begin{array}{r}\text { AeroTwst } \\
\left({ }^{\circ}\right)\end{array}$ & $\begin{array}{r}\text { DRNodes } \\
(\mathrm{m})\end{array}$ & $\begin{array}{r}\text { Chord } \\
(\mathrm{m})\end{array}$ & $\begin{array}{r}\text { Airfoil Table } \\
(-)\end{array}$ \\
\hline 1 & 2.8667 & 13.308 & 2.7333 & 3.542 & Cylinder1.dat \\
\hline 2 & 5.6000 & 13.308 & 2.7333 & 3.854 & Cylinder1.dat \\
\hline 3 & 8.3333 & 13.308 & 2.7333 & 4.167 & Cylinder2.dat \\
\hline 4 & 11.7500 & 13.308 & 4.1000 & 4.557 & DU40_A17.dat \\
\hline 5 & 15.8500 & 11.480 & 4.1000 & 4.652 & DU35_A17.dat \\
\hline 6 & 19.9500 & 10.162 & 4.1000 & 4.458 & DU35_A17.dat \\
\hline 7 & 24.0500 & 9.011 & 4.1000 & 4.249 & DU30_A17.dat \\
\hline 8 & 28.1500 & 7.795 & 4.1000 & 4.007 & DU25_A17.dat \\
\hline 9 & 32.2500 & 6.544 & 4.1000 & 3.748 & DU25_A17.dat \\
\hline 10 & 36.3500 & 5.361 & 4.1000 & 3.502 & DU21_A17.dat \\
\hline 11 & 40.4500 & 4.188 & 4.1000 & 3.256 & DU21_A17.dat \\
\hline 12 & 44.5500 & 3.125 & 4.1000 & 3.010 & NACA64_A17.dat \\
\hline 13 & 48.6500 & 2.319 & 4.1000 & 2.764 & NACA64_A17.dat \\
\hline 14 & 52.7500 & 1.526 & 4.1000 & 2.518 & NACA64_A17.dat \\
\hline 15 & 56.1667 & 0.863 & 2.7333 & 2.313 & NACA64_A17.dat \\
\hline 16 & 58.9000 & 0.370 & 2.7333 & 2.086 & NACA64_A17.dat \\
\hline 17 & 61.6333 & 0.106 & 2.7333 & 1.419 & NACA64_A17.dat \\
\hline
\end{tabular}

of Ref. [51]). ${ }^{9}$ In these airfoil tables, "DU" refers to Delft University and "NACA" refers to the National Advisory Committee for Aeronautics. I used AirfoilPrep v2.0 [28] to "tailor" these airfoil data. I first corrected the lift and drag coefficients for rotational stall delay using the Selig and Eggars method for $0^{\circ}$ to $90^{\circ}$ angles of attack. I then corrected the drag coefficients using the Viterna method for $0^{\circ}$ to $90^{\circ}$ angles of attack assuming an aspect ratio of 17 . Finally, I estimated the Beddoes-Leishman dynamic-stall hysteresis parameters. I made no corrections to the DOWEC-supplied pitching-moment coefficients. The resulting three-dimensionally corrected airfoil-data coefficients are illustrated graphically in Figure 3-1 through Figure 3-6. The numerical values are documented in the AeroDyn airfoil-data input files that make up Appendix B.

${ }^{9}$ C. Lindenburg of the Energy Research Center of the Netherlands (ECN) provided numerical values for these coefficients. 


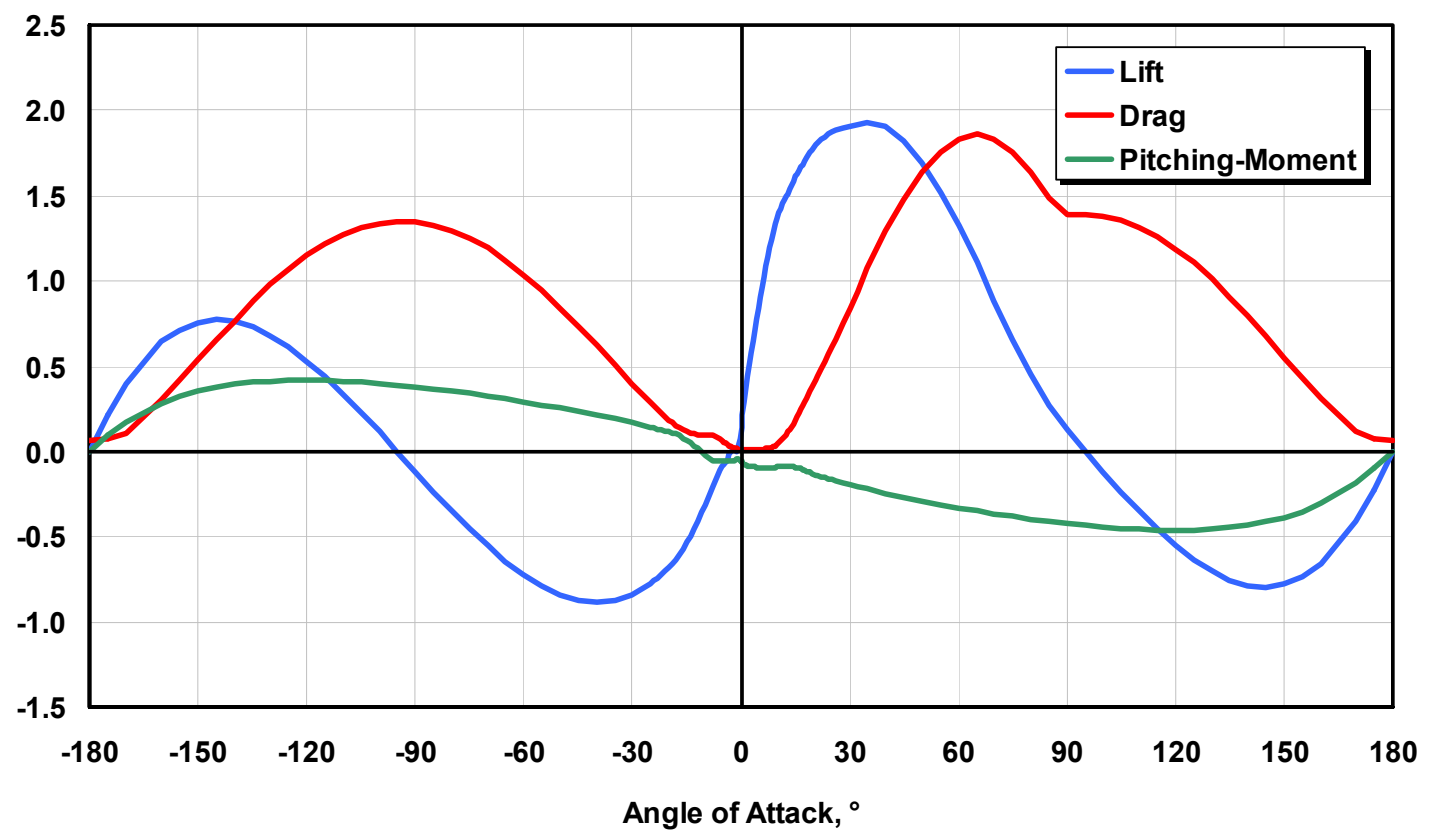

Figure 3-1. Corrected coefficients of the DU40 airfoil

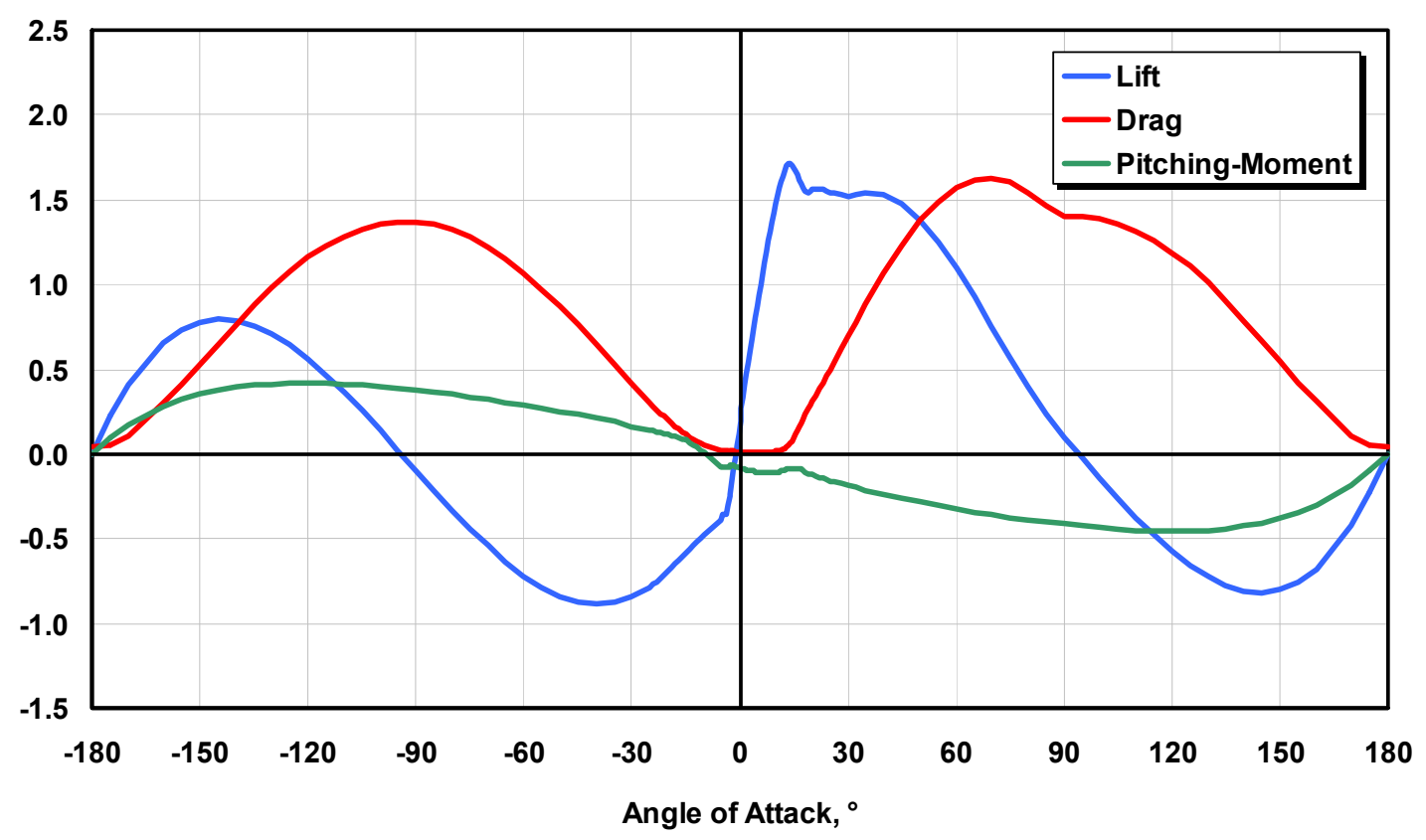

Figure 3-2. Corrected coefficients of the DU35 airfoil 


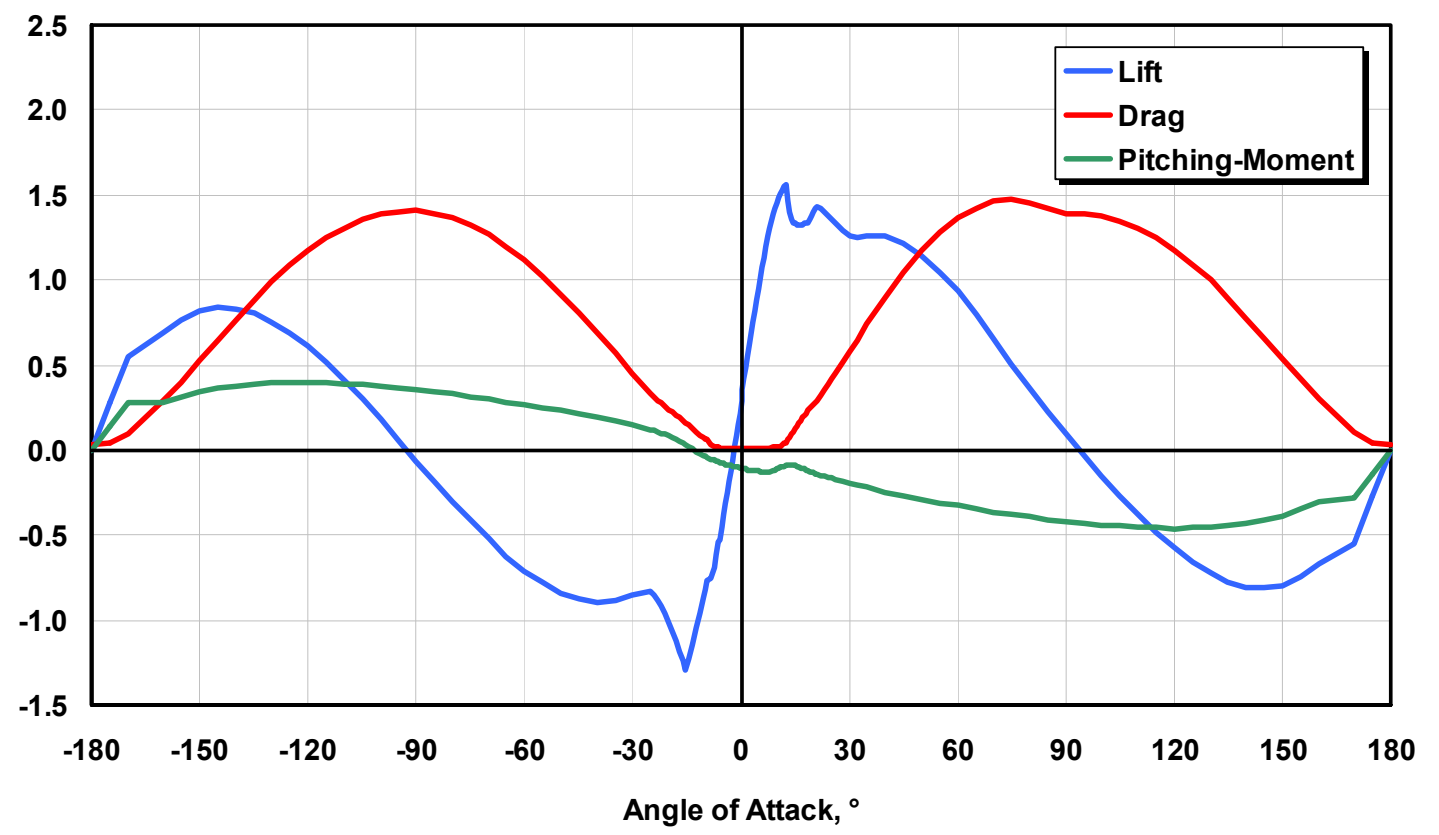

Figure 3-3. Corrected coefficients of the DU30 airfoil

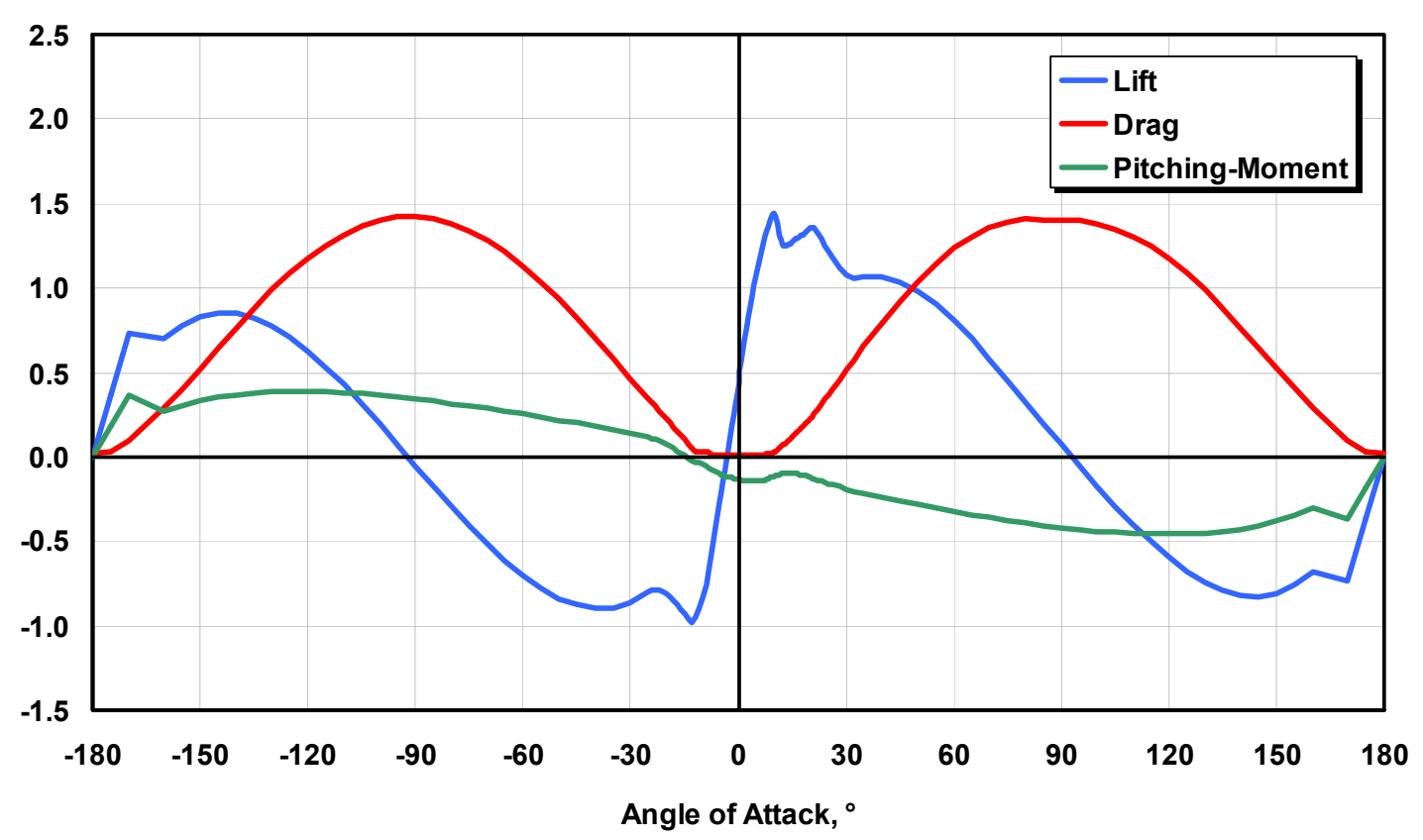

Figure 3-4. Corrected coefficients of the DU25 airfoil 


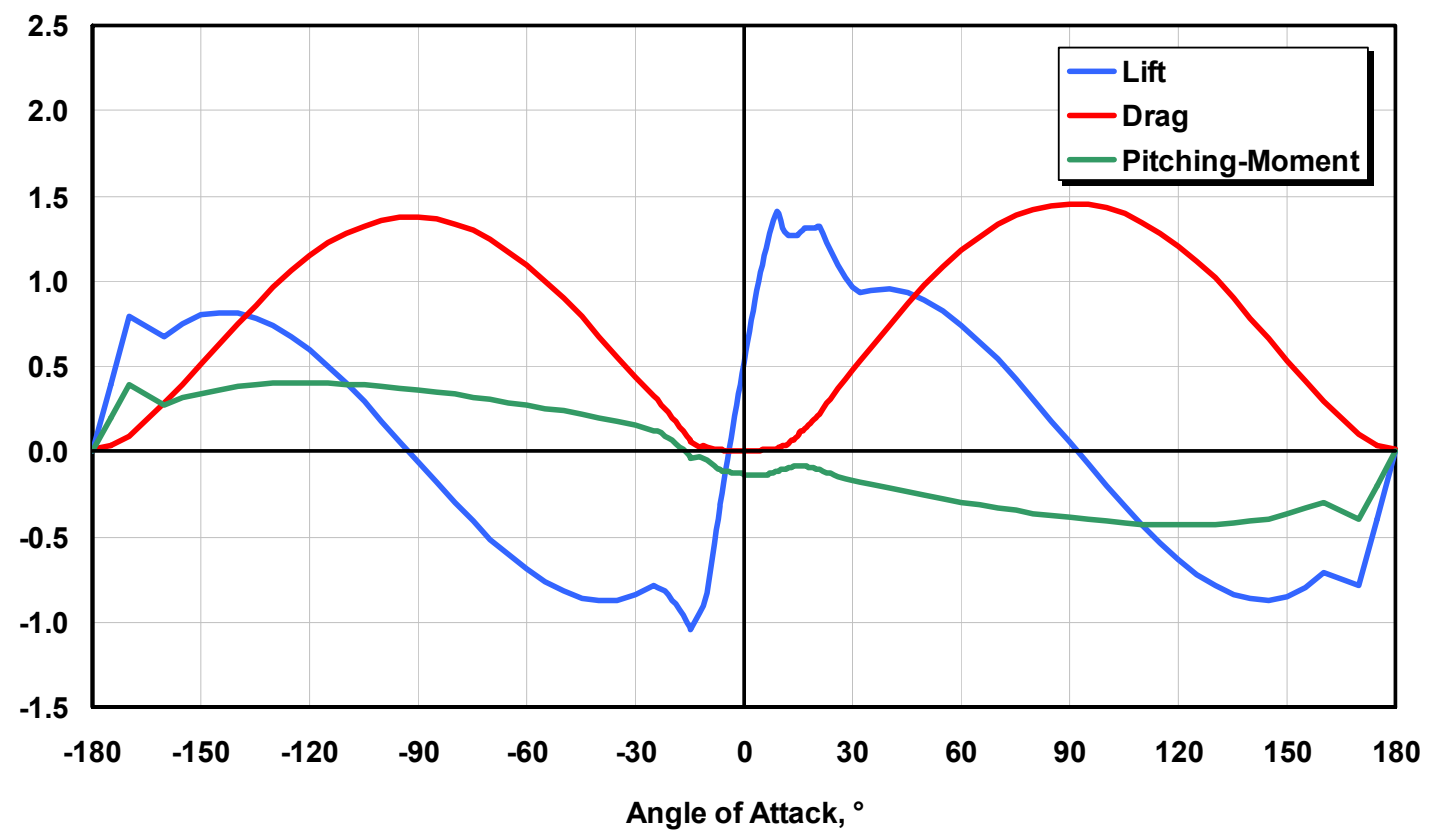

Figure 3-5. Corrected coefficients of the DU21 airfoil

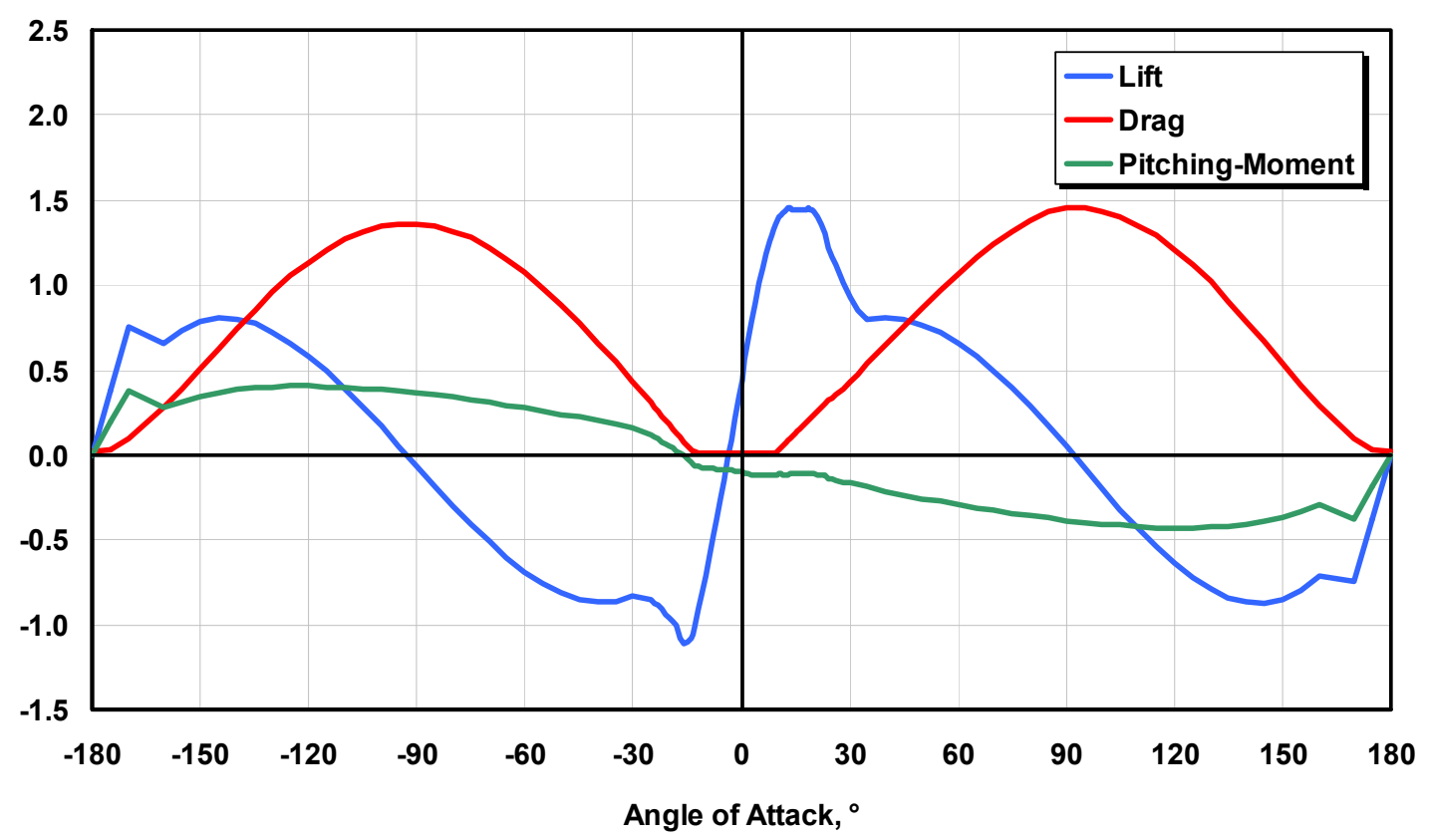

Figure 3-6. Corrected coefficients of the NACA64 airfoil 


\subsubsection{Hub and Nacelle Properties}

As indicated in Table 3-1, I located the hub of the NREL 5-MW baseline wind turbine $5 \mathrm{~m}$ upwind of the tower centerline at an elevation of $90 \mathrm{~m}$ above the ground when the system is undeflected. I also specified the same vertical distance from the tower top to the hub height used by the DOWEC study - that is, $2.4 \mathrm{~m}$ (as specified in Table 6 on page 26 of Ref. [51]). Consequently, the elevation of the yaw bearing above ground or MSL is $87.6 \mathrm{~m}$. With a shaft tilt of $5^{\circ}$, this made the distance directed along the shaft from the hub center to the yaw axis 5.01910 $\mathrm{m}$ and the vertical distance along the yaw axis from the tower top to the shaft $1.96256 \mathrm{~m}$. The distance directed along the shaft from the hub center to the main bearing was taken to be $1.912 \mathrm{~m}$ (from Table 6 on page 26 of Ref. [51]).

I specified the hub mass to be $56,780 \mathrm{~kg}$ like in the REpower $5 \mathrm{M}$, and I located its $\mathrm{CM}$ at the hub center. The hub inertia about the shaft, taken to be $115,926 \mathrm{~kg} \cdot \mathrm{m}^{2}$, was found by assuming that the hub casting is a thin spherical shell with a radius of $1.75 \mathrm{~m}$ (this is $0.25 \mathrm{~m}$ longer than the actual hub radius because the nacelle height of the DOWEC turbine was $3.5 \mathrm{~m}$, based on the data in Table 6 on page 26 of Ref. [51]).

I specified the nacelle mass to be $240,000 \mathrm{~kg}$ like in the REpower $5 \mathrm{M}$ and I located its CM $1.9 \mathrm{~m}$ downwind of the yaw axis like in the DOWEC turbine (from Table 7 on page 27 of Ref. [51]) and $1.75 \mathrm{~m}$ above the yaw bearing, which was half the height of the DOWEC turbine's nacelle (from Table 6 on page 26 of Ref. [51]). The nacelle inertia about the yaw axis was taken to be $2,607,890 \mathrm{~kg} \cdot \mathrm{m}^{2}$. I chose this to be equivalent to the DOWEC turbine's nacelle inertia about its nacelle $\mathrm{CM}$, but translated to the yaw axis using the parallel-axis theorem with the nacelle mass and downwind distance to the nacelle CM.

I took the nacelle-yaw actuator to have a natural frequency of $3 \mathrm{~Hz}$, which is roughly equivalent to the highest full-system natural frequency in the FAST model (see Section 3.1.8), and a damping ratio of $2 \%$ critical. This resulted in an equivalent nacelle-yaw-actuator linear-spring constant of $9,028,320,000 \mathrm{~N} \cdot \mathrm{m} / \mathrm{rad}$ and an equivalent nacelle-yaw-actuator linear-damping constant of $19,160,000 \mathrm{~N} \cdot \mathrm{m} /(\mathrm{rad} / \mathrm{s})$. The nominal nacelle-yaw rate was chosen to be the same as that for the DOWEC 6-MW turbine, or $0.3 \%$ (from page 27 of Ref. [51]).

Table 3-5 summarizes the nacelle and hub properties discussed in this section.

Table 3-5. Nacelle and Hub Properties

\begin{tabular}{|l|c|}
\hline Elevation of Yaw Bearing above Ground & $87.6 \mathrm{~m}$ \\
\hline Vertical Distance along Yaw Axis from Yaw Bearing to Shaft & $1.96256 \mathrm{~m}$ \\
\hline Distance along Shaft from Hub Center to Yaw Axis & $5.01910 \mathrm{~m}$ \\
\hline Distance along Shaft from Hub Center to Main Bearing & $1.912 \mathrm{~m}$ \\
\hline Hub Mass & $56,780 \mathrm{~kg}$ \\
\hline Hub Inertia about Low-Speed Shaft & $115,926 \mathrm{~kg} \cdot \mathrm{m}^{2}$ \\
\hline Nacelle Mass & $240,000 \mathrm{~kg}$ \\
\hline Nacelle Inertia about Yaw Axis & $2,607,890 \mathrm{~kg} \cdot \mathrm{m}^{2}$ \\
\hline Nacelle CM Location Downwind of Yaw Axis & $1.9 \mathrm{~m}$ \\
\hline Nacelle CM Location above Yaw Bearing & $1.75 \mathrm{~m}$ \\
\hline Equivalent Nacelle-Yaw-Actuator Linear-Spring Constant & $9,028,320,000 \mathrm{~N} \cdot \mathrm{m} / \mathrm{rad}$ \\
\hline Equivalent Nacelle-Yaw-Actuator Linear-Damping Constant & $19,160,000 \mathrm{~N} \cdot \mathrm{m} /(\mathrm{rad} / \mathrm{s})$ \\
\hline Nominal Nacelle-Yaw Rate & $0.3 \% / \mathrm{s}$ \\
\hline
\end{tabular}




\subsubsection{Drivetrain Properties}

I specified the NREL 5-MW baseline wind turbine to have the same rated rotor speed (12.1 rpm), rated generator speed $(1173.7 \mathrm{rpm})$, and gearbox ratio $(97: 1)$ as the REpower $5 \mathrm{M}$ machine. The gearbox was assumed be a typical multiple-stage gearbox but with no frictional losses-a requirement of the preprocessor functionality in FAST for creating ADAMS models [39]. The electrical efficiency of the generator was taken to be $94.4 \%$. This was chosen to be roughly the same as the total mechanical-to-electrical conversion loss used by the DOWEC turbine at rated power - that is, the DOWEC turbine had about $0.35 \mathrm{MW}$ of power loss at about $6.25 \mathrm{MW}$ of aerodynamic power (from Figure 15, page 24 of Ref. [51]). The generator inertia about the highspeed shaft was taken to be $534.116 \mathrm{~kg} \cdot \mathrm{m}^{2}$, which is the same equivalent low-speed shaft generator inertia used in the DOWEC study (i.e., 5,025,500 $\mathrm{kg} \bullet \mathrm{m}^{2}$ from page 36 of Ref. [51]).

The driveshaft was taken to have the same natural frequency as the RECOFF turbine model and a structural-damping ratio - associated with the free-free mode of a drivetrain composed of a rigid generator and rigid rotor-of $5 \%$ critical. This resulted in an equivalent driveshaft linearspring constant of $867,637,000 \mathrm{~N} \cdot \mathrm{m} / \mathrm{rad}$ and a linear-damping constant of $6,215,000 \mathrm{~N} \cdot \mathrm{m} /(\mathrm{rad} / \mathrm{s})$.

The high-speed shaft brake was assumed to have the same ratio of maximum brake torque to maximum generator torque and the same time lag as used in the DOWEC study (from page 29 of Ref. [51]). This resulted in a fully deployed high-speed shaft brake torque of $28,116.2 \mathrm{~N} \cdot \mathrm{m}$ and a time lag of $0.6 \mathrm{~s}$. This time lag is the amount of time it takes for the brake to fully engage once deployed. The FAST and ADAMS models employ a simple linear ramp from nothing to full braking over the 0.6 -s period.

Table 3-6 summarizes the drivetrain properties discussed in this section.

Table 3-6. Drivetrain Properties

\begin{tabular}{|l|c|}
\hline Rated Rotor Speed & $12.1 \mathrm{rpm}$ \\
\hline Rated Generator Speed & $1173.7 \mathrm{rpm}$ \\
\hline Gearbox Ratio & $97: 1$ \\
\hline Electrical Generator Efficiency & $94.4 \%$ \\
\hline Generator Inertia about High-Speed Shaft & $534.116 \mathrm{~kg} \cdot \mathrm{m}^{2}$ \\
\hline Equivalent Drive-Shaft Torsional-Spring Constant & $867,637,000 \mathrm{~N} \cdot \mathrm{m} / \mathrm{rad}$ \\
\hline Equivalent Drive-Shaft Torsional-Damping Constant & $6,215,000 \mathrm{~N} \cdot \mathrm{m} /(\mathrm{rad} / \mathrm{s})$ \\
\hline Fully-Deployed High-Speed Shaft Brake Torque & $28,116.2 \mathrm{~N} \cdot \mathrm{m}$ \\
\hline High-Speed Shaft Brake Time Constant & $0.6 \mathrm{~s}$ \\
\hline
\end{tabular}

\subsubsection{Tower Properties}

I based the distributed tower properties of the NREL 5-MW baseline wind turbine on the base diameter $(6 \mathrm{~m})$ and thickness $(0.027 \mathrm{~m})$, top diameter $(3.87 \mathrm{~m})$ and thickness $(0.019 \mathrm{~m})$, and effective mechanical steel properties of the tower used in the DOWEC study (as given in Table 9 on page 31 of Ref. [51]). The Young's modulus was taken to be $210 \mathrm{GPa}$, the shear modulus was taken to be $80.8 \mathrm{GPa}$, and the effective density of the steel was taken to be $8,500 \mathrm{~kg} / \mathrm{m}^{3}$. The density of $8,500 \mathrm{~kg} / \mathrm{m}^{3}$ was meant to be an increase above steel's typical value of $7,850 \mathrm{~kg} / \mathrm{m}^{3}$ to account for paint, bolts, welds, and flanges that are not accounted for in the tower thickness data. The radius and thickness of the tower were assumed to be linearly tapered from the tower base to 
tower top. Because the REpower 5M machine had a larger tower-top mass than the DOWEC wind turbine, I scaled up the thickness of the tower relative to the values given earlier in this paragraph to strengthen the tower. I chose an increase of 30\% to ensure that the first fore-aft and side-to-side tower frequencies were placed between the one- and three-per-rev frequencies throughout the operational range of the wind turbine in a Campbell diagram. Table 3-7 gives the resulting distributed tower properties.

Table 3-7. Distributed Tower Properties

\begin{tabular}{|c|c|c|c|c|c|c|c|c|c|c|}
\hline $\begin{array}{l}\text { Elevation } \\
(\mathrm{m})\end{array}$ & $\begin{array}{l}\text { HtFract } \\
(-)\end{array}$ & $\begin{array}{r}\text { TMassDen } \\
(\mathrm{kg} / \mathrm{m})\end{array}$ & $\begin{array}{r}\text { TwFAStif } \\
\left(\mathrm{N} \cdot \mathrm{m}^{2}\right)\end{array}$ & $\begin{array}{r}\text { TwSSStif } \\
\left(\mathrm{N} \cdot \mathrm{m}^{2}\right)\end{array}$ & $\begin{array}{r}\text { TwGJStif } \\
\left(\mathrm{N} \cdot \mathrm{m}^{2}\right)\end{array}$ & $\begin{array}{r}\text { TwEAStif } \\
(\mathrm{N})\end{array}$ & $\begin{array}{r}\text { TwFAlner } \\
(\mathrm{kg} \bullet \mathrm{m})\end{array}$ & $\begin{array}{r}\text { TwSSIner } \\
(\mathrm{kg} \bullet \mathrm{m})\end{array}$ & $\begin{array}{r}\text { TwFAcgOf } \\
(\mathrm{m})\end{array}$ & $\begin{array}{r}\text { TwSScgOf } \\
(\mathrm{m})\end{array}$ \\
\hline 0.00 & 0.0 & 5590.87 & $614.34 \mathrm{E}+9$ & $614.34 \mathrm{E}+9$ & $472.75 \mathrm{E}+9$ & 138.13E+9 & 24866.3 & 24866.3 & 0.0 & 0.0 \\
\hline 8.76 & 0.1 & 5232.43 & $534.82 E+9$ & $534.82 E+9$ & $411.56 E+9$ & 129.27E+9 & 21647.5 & 21647.5 & 0.0 & 0.0 \\
\hline 17.52 & 0.2 & 4885.76 & $463.27 E+9$ & $463.27 E+9$ & $356.50 E+9$ & 120.71E+9 & 18751.3 & 18751.3 & 0.0 & 0.0 \\
\hline 26.28 & 0.3 & 4550.87 & $399.13 E+9$ & $399.13 E+9$ & $307.14 \mathrm{E}+9$ & 112.43E+9 & 16155.3 & 16155.3 & 0.0 & 0.0 \\
\hline 35.04 & 0.4 & 4227.75 & $341.88 \mathrm{E}+9$ & $341.88 \mathrm{E}+9$ & $263.09 \mathrm{E}+9$ & 104.45E+9 & 13838.1 & 13838.1 & 0.0 & 0.0 \\
\hline 43.80 & 0.5 & 3916.41 & $291.01 \mathrm{E}+9$ & $291.01 \mathrm{E}+9$ & $223.94 \mathrm{E}+9$ & $96.76 \mathrm{E}+9$ & 11779.0 & 11779.0 & 0.0 & 0.0 \\
\hline 52.56 & 0.6 & 3616.83 & $246.03 E+9$ & $246.03 E+9$ & 189.32E+9 & $89.36 \mathrm{E}+9$ & 9958.2 & 9958.2 & 0.0 & 0.0 \\
\hline 61.32 & 0.7 & 3329.03 & $206.46 E+9$ & $206.46 E+9$ & 158.87E+9 & $82.25 \mathrm{E}+9$ & 8356.6 & 8356.6 & 0.0 & 0.0 \\
\hline 70.08 & 0.8 & 3053.01 & 171.85E+9 & 171.85E+9 & 132.24E+9 & $75.43 \mathrm{E}+9$ & 6955.9 & 6955.9 & 0.0 & 0.0 \\
\hline 78.84 & 0.9 & 2788.75 & 141.78E+9 & 141.78E+9 & 109.10E+9 & $68.90 \mathrm{E}+9$ & 5738.6 & 5738.6 & 0.0 & 0.0 \\
\hline 87.60 & 1.0 & 2536.27 & $115.82 \mathrm{E}+9$ & $115.82 E+9$ & $89.13 E+9$ & $62.66 \mathrm{E}+9$ & 4688.0 & 4688.0 & 0.0 & 0.0 \\
\hline
\end{tabular}

The entries in the first column, "Elevation," are the vertical locations along the tower centerline relative to the tower base. "HtFract" is the fractional height along the tower centerline from the tower base (0.0) to the tower top (1.0). The rest of columns are similar to those described for the distributed blade properties presented in Table 3-2.

The resulting overall (integrated) tower mass is $347,460 \mathrm{~kg}$ and is centered at $38.234 \mathrm{~m}$ along the tower centerline above the ground. This result follows directly from the overall tower height of $87.6 \mathrm{~m}$.

I specified a structural-damping ratio of $1 \%$ critical in all modes of the isolated tower (without the rotor-nacelle assembly mass present), which corresponds to the values used in the DOWEC study (from page 21 of Ref. [51]).

Table 3-8 summarizes the undistributed tower properties discussed in this section.

Table 3-8. Undistributed Tower Properties

\begin{tabular}{|l|c|}
\hline Height above Ground & $87.6 \mathrm{~m}$ \\
\hline Overall (Integrated) Mass & $347,460 \mathrm{~kg}$ \\
\hline CM Location (w.r.t. Ground along Tower Centerline) & $38.234 \mathrm{~m}$ \\
\hline Structural-Damping Ratio (All Modes) & $1 \%$ \\
\hline
\end{tabular}

\subsubsection{Baseline Control System Properties}

For the NREL 5-MW baseline wind turbine, I chose a conventional variable-speed, variable blade-pitch-to-feather configuration. In such wind turbines, the conventional approach for controlling power-production operation relies on the design of two basic control systems: a generator-torque controller and a full-span rotor-collective blade-pitch controller. The two control systems are designed to work independently, for the most part, in the below-rated and above-rated wind-speed range, respectively. The goal of the generator-torque controller is to 
maximize power capture below the rated operation point. The goal of the blade-pitch controller is to regulate generator speed above the rated operation point.

I based the baseline control system for the NREL 5-MW wind turbine on this conventional design approach. I did not establish additional control actions for nonpower-production operations, such as control actions for normal start-up sequences, normal shutdown sequences, and safety and protection functions. Nor did I develop control actions to regulate the nacelleyaw angle because all of the normal power-production simulations I modeled were per the IEC design standards $[33,34]$. The standards designate small nacelle-yaw errors for these simulations (with the exception that the IEC extreme coherent gust with direction change [ECD] load case expects large yaw errors). The nacelle-yaw control system is generally neglected within aeroservo-elastic simulation because its response is slow enough that it does not generally contribute to large extreme loads or fatigue damage.

I describe the development of my baseline control system next, including the controlmeasurement filter (Section 3.1.6.1), the generator-torque controller (Section 3.1.6.2), the bladepitch controller (Section 3.1.6.3), and the blade-pitch actuator (Section 3.1.6.4). Section 3.1.6.5 shows how these systems are put together in the overall integrated control system.

\subsubsection{Baseline Control-Measurement Filter}

As is typical in utility-scale multimegawatt wind turbines, both the generator-torque and bladepitch controllers use the generator speed measurement as the sole feedback input. To mitigate high-frequency excitation of the control systems, I filtered the generator speed measurement for both the torque and pitch controllers using a recursive, single-pole low-pass filter with exponential smoothing [89]. The discrete-time recursion (difference) equation for this filter is

$$
\begin{gathered}
y[n]=(1-\alpha) u[n]+\alpha y[n-1], \\
\text { with } \\
\alpha=e^{-2 \pi T_{s} f_{c}},
\end{gathered}
$$

where $y$ is the filtered generator speed (output measurement), $u$ is the unfiltered generator speed (input), $\alpha$ is the low-pass filter coefficient, $n$ is the discrete-time-step counter, $T_{s}$ is the discrete time step, and $f_{c}$ is the corner frequency.

By defining the filter state,

$$
\begin{gathered}
x[n]=y[n-1], \\
\text { or } \\
x[n+1]=y[n],
\end{gathered}
$$

one can derive a discrete-time state-space representation of this filter: 


$$
\begin{gathered}
x[n+1]=A_{d} x[n]+B_{d} u[n] \\
y[n]=C_{d} x[n]+D_{d} u[n]
\end{gathered}
$$

where $A_{d}=\alpha$ is the discrete-time state matrix, $B_{d}=1-\alpha$ is the discrete-time input matrix, $C_{d}=\alpha$ is the discrete-time output state matrix, and $D_{d}=1-\alpha$ is the discrete-time input transmission matrix.

The state-space representation of Eq. (3-4) is useful for converting the filter into other forms, such as transfer-function form or frequency-response form [91].

I set the corner frequency (the $-3 \mathrm{~dB}$ point in Figure 3-7) of the low-pass filter to be roughly onequarter of the blade's first edgewise natural frequency (see Section 3.1.8) or $0.25 \mathrm{~Hz}$. For a discrete time step of $0.0125 \mathrm{~s}$, the frequency response of the resulting filter is shown in the Bode plot of Figure 3-7.

I chose the recursive, single-pole filter for its simplicity in implementation and effectiveness in the time domain. The drawbacks to this filter are its gentle roll-off in the stop band (-6 $\mathrm{dB} /$ octave) and the magnitude and nonlinearity of its phase lag in the pass band [89]. I considered other linear low-pass filters, such as Butterworth, Chebyshev, Elliptic, and Bessel filters because of their inherent advantages relative to the chosen filter. Like the chosen filter, a Butterworth filter has a frequency response that is flat in the pass band, but the Butterworth filter offers steeper roll-off in the stop band. Chebyshev filters offer even steeper roll-off in the stop band at the expense of equalized-ripple (equiripple) in the pass band (Type 1) or stop band (Type
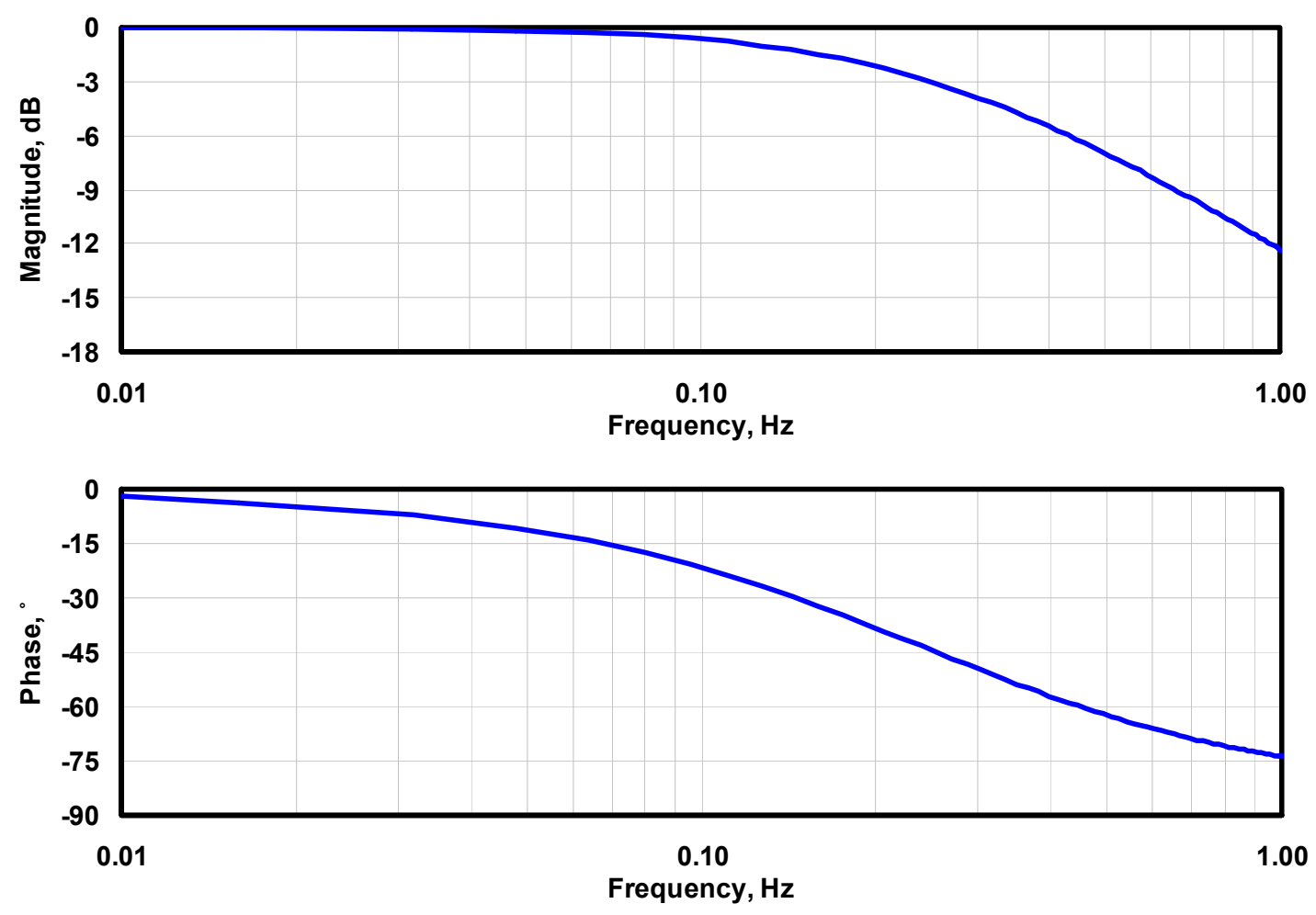

Figure 3-7. Bode plot of generator speed low-pass filter frequency response 
2), respectively. Elliptic filters offer the steepest roll-off of any linear filter, but have equiripple in both the pass and stop bands. Bessel filters offer the flattest group delay (linear phase lag) in the pass band. I designed and tested examples of each of these other low-pass filter types, considering state-space representations of up to fourth order (four states). None were found to give superior performance in the overall system response, however, so they did not warrant the added complexity of implementation.

\subsubsection{Baseline Generator-Torque Controller}

I computed the generator torque as a tabulated function of the filtered generator speed, incorporating five control regions: $1,1 \frac{1}{2}, 2,2 \frac{1}{2}$, and 3. Region 1 is a control region before cut-in wind speed, where the generator torque is zero and no power is extracted from the wind; instead, the wind is used to accelerate the rotor for start-up . Region 2 is a control region for optimizing power capture. Here, the generator torque is proportional to the square of the filtered generator speed to maintain a constant (optimal) tip-speed ratio. In Region 3, the generator power is held constant so that the generator torque is inversely proportional to the filtered generator speed. Region 1 1 2 , a start-up region, is a linear transition between Regions 1 and 2. This region is used to place a lower limit on the generator speed to limit the wind turbine's operational speed range. Region $2 \frac{1}{2}$ is a linear transition between Regions 2 and 3 with a torque slope corresponding to the slope of an induction machine. Region $2 \frac{1}{2}$ is typically needed (as is the case for my 5 -MW turbine) to limit tip speed (and hence noise emissions) at rated power.

I found the peak of the power coefficient as a function of the tip-speed ratio and blade-pitch surface by running FAST with AeroDyn simulations at a number of given rotor speeds and a number of given rotor-collective blade-pitch angles at a fixed wind speed of $8 \mathrm{~m} / \mathrm{s}$. From these simulations, I found that the peak power coefficient of 0.482 occured at a tip-speed ratio of 7.55 and a rotor-collective blade-pitch angle of $0.0^{\circ}$. With the 97:1 gearbox ratio, this resulted in an optimal constant of proportionality of $0.0255764 \mathrm{~N} \cdot \mathrm{m} / \mathrm{rpm}^{2}$ in the Region 2 control law. With the rated generator speed of $1173.7 \mathrm{rpm}$, rated electric power of $5 \mathrm{MW}$, and a generator efficiency of $94.4 \%$, the rated mechanical power is $5.296610 \mathrm{MW}$ and the rated generator torque is $43,093.55 \mathrm{~N} \cdot \mathrm{m}$. I defined Region $1 \frac{1}{2}$ to span the range of generator speeds between $670 \mathrm{rpm}$ and $30 \%$ above this value (or $871 \mathrm{rpm}$ ). The minimum generator speed of $670 \mathrm{rpm}$ corresponds to the minimum rotor speed of $6.9 \mathrm{rpm}$ used by the actual REpower $5 \mathrm{M}$ machine [81]. I took the transitional generator speed between Regions $2 \frac{1}{2}$ and 3 to be $99 \%$ of the rated generator speed, or $1,161.963 \mathrm{rpm}$. The generator-slip percentage in Region $2 \frac{1}{2}$ was taken to be $10 \%$, in accordance with the value used in the DOWEC study (see page 24 of Ref. [51]). Figure 3-8 shows the resulting generator-torque versus generator speed response curve.

Because of the high intrinsic structural damping of the drivetrain, I did not need to incorporate a control loop for damping drivetrain torsional vibration in my baseline generator-torque controller.

I did, however, place a conditional statement on the generator-torque controller so that the torque would be computed as if it were in Region 3-regardless of the generator speed-whenever the previous blade-pitch-angle command was $1^{\circ}$ or greater. This results in improved output power quality (fewer dips below rated) at the expense of short-term overloading of the generator and the gearbox. To avoid this excessive overloading, I saturated the torque to a maximum of $10 \%$ 


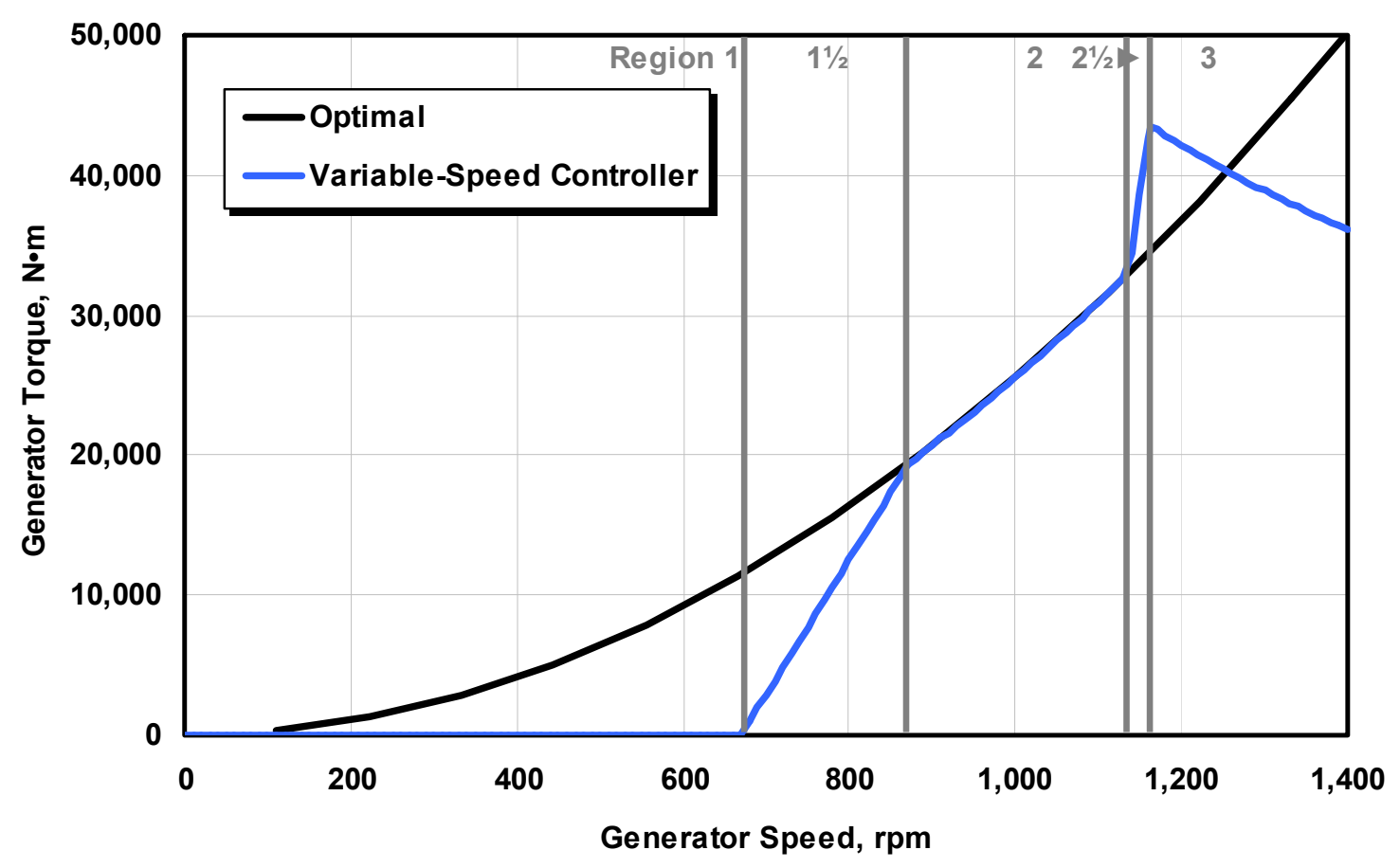

Figure 3-8. Torque-versus-speed response of the variable-speed controller

above rated, or 47,402.91 N•m. I also imposed a torque rate limit of 15,000 N•m/s. In Region 3, the blade-pitch control system takes over.

\subsubsection{Baseline Blade-Pitch Controller}

In Region 3, I computed the full-span rotor-collective blade-pitch-angle commands using gainscheduled proportional-integral (PI) control on the speed error between the filtered generator speed and the rated generator speed (1173.7 rpm).

I designed the blade-pitch control system using a simple single-DOF model of the wind turbine. Because the goal of the blade-pitch control system is to regulate the generator speed, this DOF is the angular rotation of the shaft. To compute the required control gains, it is beneficial to examine the equation of motion of this single-DOF system. From a simple free-body diagram of the drivetrain, the equation of motion is

$$
T_{\text {Aero }}-N_{\text {Gear }} T_{\text {Gen }}=\left(I_{\text {Rotor }}+N_{\text {Gear }}^{2} I_{\text {Gen }}\right) \frac{d}{d t}\left(\Omega_{0}+\Delta \Omega\right)=I_{\text {Drivetrain }} \Delta \dot{\Omega},
$$

where $T_{\text {Aero }}$ is the low-speed shaft aerodynamic torque, $T_{G e n}$ is the high-speed shaft generator torque, $N_{\text {Gear }}$ is the high-speed to low-speed gearbox ratio, $I_{\text {Drivetrain }}$ is the drivetrain inertia cast to the low-speed shaft, $I_{\text {Rotor }}$ is the rotor inertia, $I_{G e n}$ is the generator inertia relative to the highspeed shaft, $\Omega_{0}$ is the rated low-speed shaft rotational speed, $\Delta \Omega$ is the small perturbation of low-speed shaft rotational speed about the rated speed, $\Delta \dot{\Omega}$ is the low-speed shaft rotational acceleration, and $t$ is the simulation time. 
Because the generator-torque controller maintains constant generator power in Region 3, the generator torque in Region 3 is inversely proportional to the generator speed (see Figure 3-8), or

$$
T_{\text {Gen }}\left(N_{\text {Gear }} \Omega\right)=\frac{P_{0}}{N_{\text {Gear }} \Omega}
$$

where $P_{0}$ is the rated mechanical power and $\Omega$ is the low-speed shaft rotational speed.

Similarly, assuming negligible variation of aerodynamic torque with rotor speed, the aerodynamic torque in Region 3 is

$$
T_{\text {Aero }}(\theta)=\frac{P\left(\theta, \Omega_{0}\right)}{\Omega_{0}},
$$

where $P$ is the mechanical power and $\theta$ is the full-span rotor-collective blade-pitch angle.

Using a first-order Taylor series expansion of Eqs. (3-6) and (3-7), one can see that

$$
\begin{gathered}
T_{\text {Gen }} \approx \frac{P_{0}}{N_{\text {Gear }} \Omega_{0}}-\frac{P_{0}}{N_{\text {Gear }} \Omega_{0}^{2}} \Delta \Omega \\
\text { and } \\
T_{\text {Aero }} \approx \frac{P_{0}}{\Omega_{0}}+\frac{1}{\Omega_{0}}\left(\frac{\partial P}{\partial \theta}\right) \Delta \theta,
\end{gathered}
$$

where $\Delta \theta$ is a small perturbation of the blade-pitch angles about their operating point. With proportional-integral-derivative (PID) control, this is related to the rotor-speed perturbations by

$$
\Delta \theta=K_{P} N_{\text {Gear }} \Delta \Omega+K_{I} \int_{0}^{t} N_{\text {Gear }} \Delta \Omega d t+K_{D} N_{\text {Gear }} \Delta \dot{\Omega}
$$

where $K_{P}, K_{I}$, and $K_{D}$ are the blade-pitch controller proportional, integral, and derivative gains, respectively.

By setting $\dot{\varphi}=\Delta \Omega$, combining the above expressions, and simplifying, the equation of motion for the rotor-speed error becomes

$$
\underbrace{\left[I_{\text {Drvivertain }}+\frac{1}{\Omega_{0}}\left(-\frac{\partial P}{\partial \theta}\right) N_{\text {Gear }} K_{D}\right]}_{M_{\varphi}} \ddot{\varphi}+\underbrace{\left[\frac{1}{\Omega_{0}}\left(-\frac{\partial P}{\partial \theta}\right) N_{\text {Gear }} K_{P}-\frac{P_{0}}{\Omega_{0}^{2}}\right]}_{C_{\varphi}} \dot{\varphi}+\underbrace{\left[\frac{1}{\Omega_{0}}\left(-\frac{\partial P}{\partial \theta}\right) N_{\text {Gear }} K_{I}\right]}_{K_{\varphi}} \varphi=0 .
$$

One can see that the idealized PID-controlled rotor-speed error will respond as a second-order system with the natural frequency, $\omega_{\varphi n}$, and damping ratio, $\zeta_{\varphi}$, equal to 


$$
\begin{gathered}
\omega_{\varphi n}=\sqrt{\frac{K_{\varphi}}{M_{\varphi}}} \\
\text { and } \\
\zeta_{\varphi}=\frac{C_{\varphi}}{2 \sqrt{K_{\varphi} M_{\varphi}}}=\frac{C_{\varphi}}{2 M_{\varphi} \omega_{\varphi n}} .
\end{gathered}
$$

In an active pitch-to-feather wind turbine, the sensitivity of aerodynamic power to the rotorcollective blade-pitch angle, $\partial P / \partial \theta$, is negative in Region 3. With positive control gains, then, the derivative term acts to increase the effective inertia of the drivetrain, the proportional term adds damping, and the integral term adds restoring. Also, because the generator torque drops with increasing speed error (to maintain constant power) in Region 3, one can see that the generator-torque controller introduces a negative damping in the speed error response [indicated by the $-P_{0} / \Omega_{0}^{2}$ term in Eq. (3-11)]. This negative damping must be compensated by the proportional term in the blade-pitch controller.

In the design of the blade-pitch controller, Ref. [29] recommends neglecting the derivative gain, ignoring the negative damping from the generator-torque controller, and aiming for the response characteristics given by $\omega_{\varphi n}=0.6 \mathrm{rad} / \mathrm{s}$ and $\zeta_{\varphi}=0.6$ to 0.7 . This specification leads to direct expressions for choosing appropriate PI gains once the sensitivity of aerodynamic power to rotor-collective blade pitch, $\partial P / \partial \theta$, is known:

$$
\begin{gathered}
K_{P}=\frac{2 I_{\text {Drivetrain }} \Omega_{0} \zeta_{\varphi} \omega_{\varphi n}}{N_{\text {Gear }}\left(-\frac{\partial P}{\partial \theta}\right)} \\
\text { and } \\
K_{I}=\frac{I_{\text {Drivertain }} \Omega_{0} \omega_{\varphi n}^{2}}{N_{\text {Gear }}\left(-\frac{\partial P}{\partial \theta}\right)} .
\end{gathered}
$$

The blade-pitch sensitivity, $\partial P / \partial \theta$, is an aerodynamic property of the rotor that depends on the wind speed, rotor speed, and blade-pitch angle. I calculated it for the NREL offshore 5-MW baseline wind turbine by performing a linearization analysis in FAST with AeroDyn at a number of given, steady, and uniform wind speeds; at the rated rotor speed $\left(\Omega_{0}=12.1 \mathrm{rpm}\right)$; and at the corresponding blade-pitch angles that produce the rated mechanical power $\left(P_{0}=5.296610 \mathrm{MW}\right)$. The linearization analysis involves perturbing the rotor-collective blade-pitch angle at each operating point and measuring the resulting variation in aerodynamic power. Within FAST, the partial derivative is computed using the central-difference-perturbation numerical technique. I created a slightly customized copy of FAST with AeroDyn so that the linearization procedure would invoke the frozen-wake assumption, in which the induced wake velocities are held 
constant while the blade-pitch angle is perturbed. This gives a more accurate linearization for heavily loaded rotors (i.e., for operating points in Region 3 closest to rated). Table 3-9 presents the results.

Table 3-9. Sensitivity of Aerodynamic Power to Blade Pitch in Region 3

\begin{tabular}{|l|l|r|r|}
\hline $\begin{array}{l}\text { Wind Speed } \\
(\mathrm{m} / \mathrm{s})\end{array}$ & $\begin{array}{l}\text { Rotor Speed } \\
(\mathrm{rpm})\end{array}$ & $\begin{array}{r}\text { Pitch Angle } \\
\left({ }^{\circ}\right)\end{array}$ & $\begin{array}{r}\partial P / \partial \theta \\
(\text { watt/rad })\end{array}$ \\
\hline $11.4-$ Rated & 12.1 & 0.00 & $-28.24 \mathrm{E}+6$ \\
\hline 12.0 & 12.1 & 3.83 & $-43.73 \mathrm{E}+6$ \\
\hline 13.0 & 12.1 & 6.60 & $-51.66 \mathrm{E}+6$ \\
\hline 14.0 & 12.1 & 8.70 & $-58.44 \mathrm{E}+6$ \\
\hline 15.0 & 12.1 & 10.45 & $-64.44 \mathrm{E}+6$ \\
\hline 16.0 & 12.1 & 12.06 & $-70.46 \mathrm{E}+6$ \\
\hline 17.0 & 12.1 & 13.54 & $-76.53 \mathrm{E}+6$ \\
\hline 18.0 & 12.1 & 14.92 & $-83.94 \mathrm{E}+6$ \\
\hline 19.0 & 12.1 & 16.23 & $-90.67 \mathrm{E}+6$ \\
\hline 20.0 & 12.1 & 17.47 & $-94.71 \mathrm{E}+6$ \\
\hline 21.0 & 12.1 & 18.70 & $-99.04 \mathrm{E}+6$ \\
\hline 22.0 & 12.1 & 19.94 & $-105.90 \mathrm{E}+6$ \\
\hline 23.0 & 12.1 & 21.18 & $-114.30 \mathrm{E}+6$ \\
\hline 24.0 & 12.1 & 22.35 & $-120.20 \mathrm{E}+6$ \\
\hline 25.0 & 12.1 & 23.47 & $-125.30 \mathrm{E}+6$ \\
\hline
\end{tabular}

As Table 3-9 shows, the sensitivity of aerodynamic power to rotor-collective blade pitch varies considerably over Region 3, so constant PI gains are not adequate for effective speed control. The pitch sensitivity, though, varies nearly linearly with blade-pitch angle:

$$
\begin{gathered}
\frac{\partial P}{\partial \theta}=\left[\frac{\frac{\partial P}{\partial \theta}(\theta=0)}{\theta_{K}}\right] \theta+\left[\frac{\partial P}{\partial \theta}(\theta=0)\right] \\
\text { or } \\
\frac{1}{\frac{\partial P}{\partial \theta}}=\frac{1}{\frac{\partial P}{\partial \theta}(\theta=0)\left(1+\frac{\theta}{\theta_{K}}\right)}
\end{gathered}
$$

where $\frac{\partial P}{\partial \theta}(\theta=0)$ is the pitch sensitivity at rated and $\theta_{K}$ is the blade-pitch angle at which the pitch sensitivity has doubled from its value at the rated operating point; that is,

$$
\frac{\partial P}{\partial \theta}\left(\theta=\theta_{K}\right)=2 \frac{\partial P}{\partial \theta}(\theta=0)
$$


On the right-hand side of Eq. (3-16a), the first and second terms in square brackets represent the slope and intercept of the best-fit line, respectively. I computed this regression for the NREL 5MW baseline wind turbine and present the results in Figure 3-9.

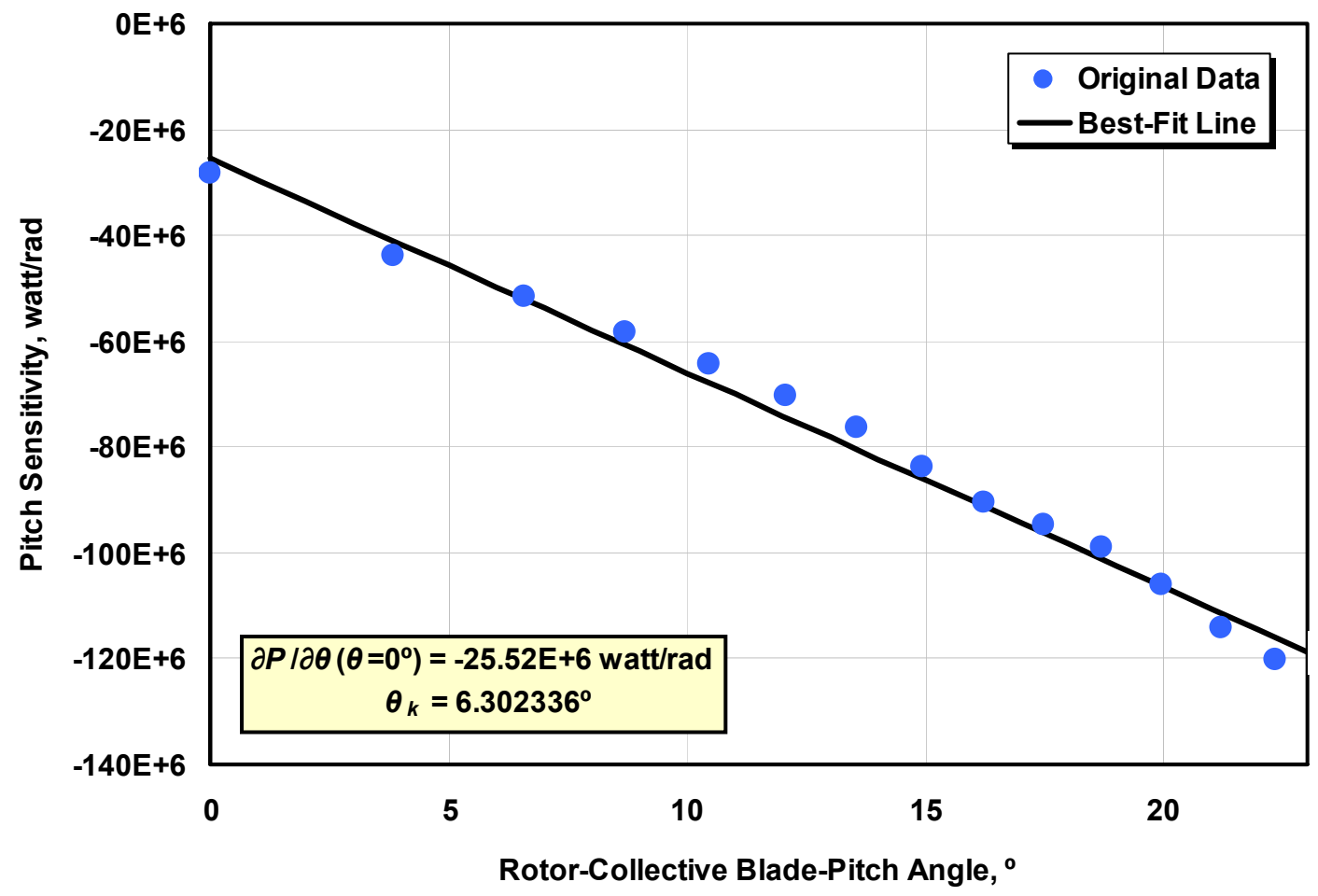

Figure 3-9. Best-fit line of pitch sensitivity in Region 3

The linear relation between pitch sensitivity and blade-pitch angle presents a simple technique for implementing gain scheduling based on blade-pitch angle; that is,

$$
\begin{gathered}
K_{P}(\theta)=\frac{2 I_{\text {Drivetrain }} \Omega_{0} \zeta_{\varphi} \omega_{\varphi n}}{N_{\text {Gear }}\left[-\frac{\partial P}{\partial \theta}(\theta=0)\right]} G K(\theta) \\
\text { and } \\
K_{I}(\theta)=\frac{I_{\text {Drivetrain }} \Omega_{0} \omega_{\varphi n}^{2}}{N_{\text {Gear }}\left[-\frac{\partial P}{\partial \theta}(\theta=0)\right]} G K(\theta),
\end{gathered}
$$

where $G K(\theta)$ is the dimensionless gain-correction factor (from Ref. [29]), which is dependent on the blade-pitch angle: 


$$
G K(\theta)=\frac{1}{1+\frac{\theta}{\theta_{K}}}
$$

In my implementation of the gain-scheduled PI blade-pitch controller, I used the blade-pitch angle from the previous controller time step to calculate the gain-correction factor at the next time step.

Using the properties for the baseline wind turbine and the recommended response characteristics from Ref. [29], the resulting gains are $K_{P}\left(\theta=0^{\circ}\right)=0.01882681 \mathrm{~s}, K_{I}\left(\theta=0^{\circ}\right)=0.008068634$, and $K_{D}=0.0 \mathrm{~s}^{2}$. Figure 3-10 presents the gains at other blade-pitch angles, along with the gaincorrection factor. I used the upper limit of the recommended damping ratio range, $\zeta_{\varphi}=0.7$, to compensate for neglecting negative damping from the generator-torque controller in the determination of $K_{P}$.

Unfortunately, the simple gain-scheduling law derived in this section for the proportional and integral gains cannot retain consistent response characteristics (i.e., constant values of $\omega_{\varphi n}$ and $\zeta_{\varphi}$ ) across all of Region 3 when applied to the derivative gain. I, nevertheless, considered adding a derivative term by selecting and testing a range of gains, but none were found to give better performance in the overall system response. Instead, the baseline control system uses the gains derived previously in this section (without the derivative term).

I set the blade-pitch rate limit to $8 \% \mathrm{~s}$ in absolute value. This is speculated to be the blade-pitch rate limit of conventional 5-MW machines based on General Electric (GE) Wind's long-blade test program. I also set the minimum and maximum blade-pitch settings to $0^{\circ}$ and $90^{\circ}$,

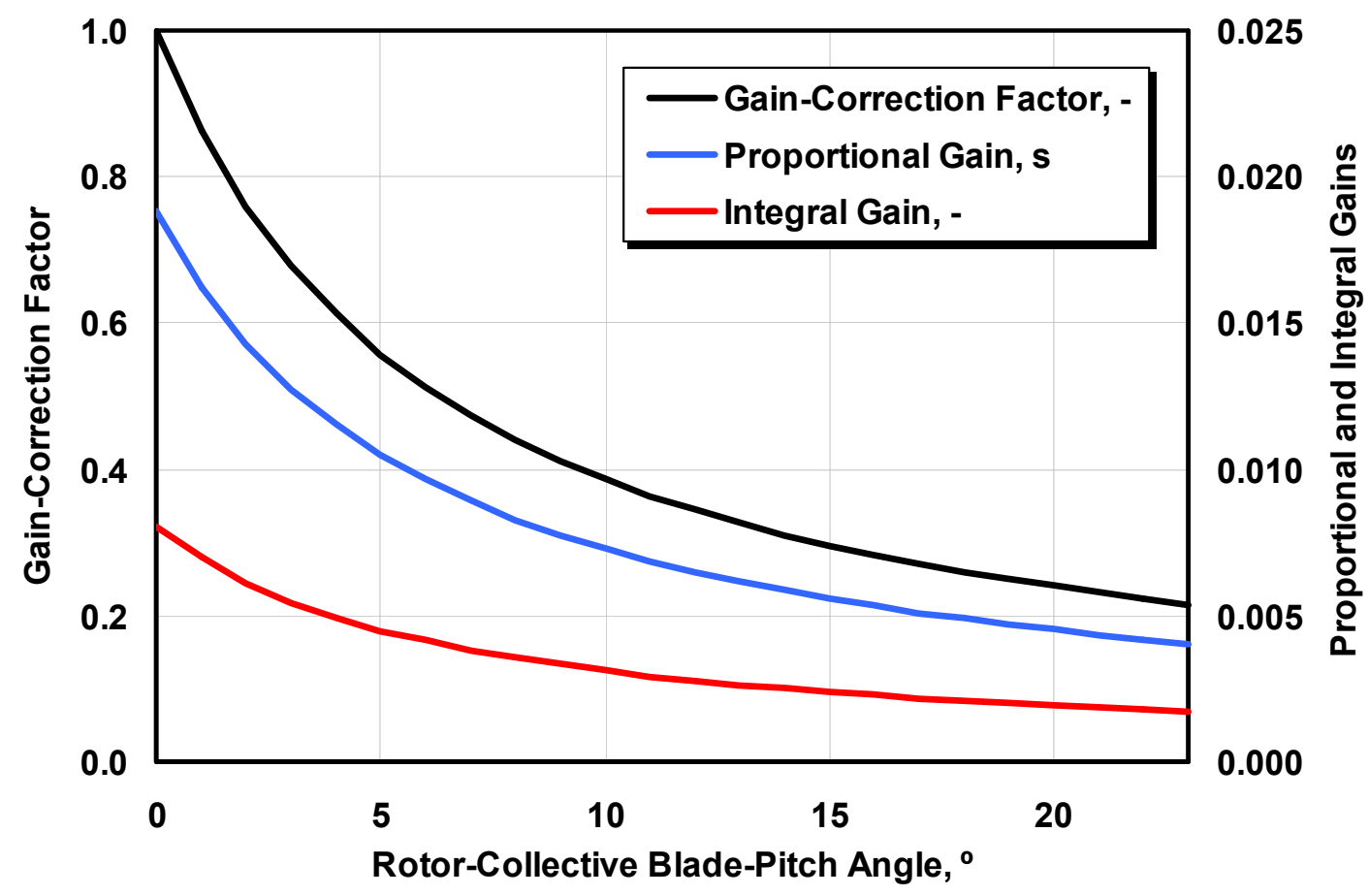

Figure 3-10. Baseline blade-pitch control system gain-scheduling law 
respectively. The lower limit is the set blade pitch for maximizing power in Region 2, as described in Section 3.1.6.2. The upper limit is very close to the fully feathered blade pitch for neutral torque. I saturated the integral term in the PI controller between these limits to ensure a fast response in the transitions between Regions 2 and 3.

\subsubsection{Baseline Blade-Pitch Actuator}

Because of limitations in the FAST code, the FAST model does not include any blade-pitch actuator dynamic effects. Blade-pitch actuator dynamics are, however, needed in ADAMS. To enable successful comparisons between the FAST and ADAMS response predictions I present in subsequent chapters, I found it beneficial to reduce the effect of the blade-pitch actuator response in ADAMS. Consequently, I designed the blade-pitch actuator in the ADAMS model with a very high natural frequency of $30 \mathrm{~Hz}$, which is higher than the highest full-system natural frequency in the FAST model (see Section 3.1.8), and a damping ratio of $2 \%$ critical. This resulted in an equivalent blade-pitch actuator linear-spring constant of 971,350,000 N•m/ $\mathrm{rad}$ and an equivalent blade-pitch actuator linear-damping constant of $206,000 \mathrm{~N} \cdot \mathrm{m} /(\mathrm{rad} / \mathrm{s})$.

\subsubsection{Summary of Baseline Control System Properties}

I implemented the NREL offshore 5-MW wind turbine's baseline control system as an external dynamic link library (DLL) in the style of Garrad Hassan's BLADED wind turbine software package [5]. Appendix C contains the source code for this DLL, and Figure 3-11 presents a

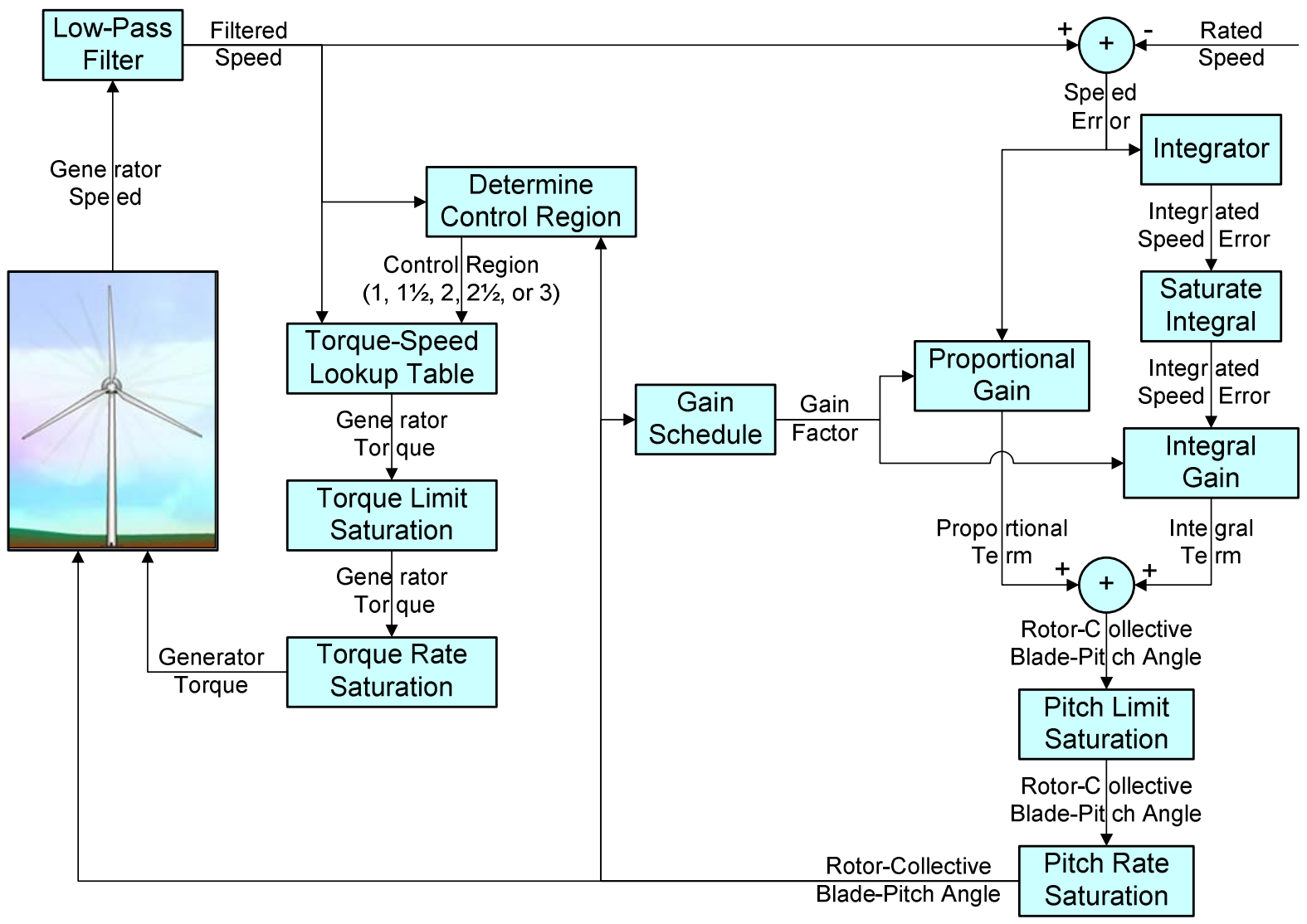

Figure 3-11. Flowchart of the baseline control system 
flowchart of the overall integrated control system calculations. Table 3-10 summarizes the baseline generator-torque and blade-pitch control properties I discussed earlier in this section.

Table 3-10. Baseline Control System Properties

\begin{tabular}{|l|c|}
\hline Corner Frequency of Generator-Speed Low-Pass Filter & $0.25 \mathrm{~Hz}$ \\
\hline Peak Power Coefficient & 0.482 \\
\hline Tip-Speed Ratio at Peak Power Coefficient & 7.55 \\
\hline Rotor-Collective Blade-Pitch Angle at Peak Power Coefficient & $0.0^{\circ}$ \\
\hline Generator-Torque Constant in Region 2 & $0.0255764 \mathrm{~N} \cdot \mathrm{m} / \mathrm{rpm}{ }^{2}$ \\
\hline Rated Mechanical Power & $5.296610 \mathrm{MW}$ \\
\hline Rated Generator Torque & $43,093.55 \mathrm{~N} \cdot \mathrm{m}$ \\
\hline Transitional Generator Speed between Regions 1 and 11/2 & $670 \mathrm{rpm}$ \\
\hline Transitional Generator Speed between Regions 11/2 and 2 & $871 \mathrm{rpm}$ \\
\hline Transitional Generator Speed between Regions 2 $\frac{1}{2}$ and 3 & $1,161.963 \mathrm{rpm}$ \\
\hline Generator Slip Percentage in Region 21/2 & $10^{\%}$ \\
\hline Minimum Blade Pitch for Ensuring Region 3 Torque & $1{ }^{\circ}$ \\
\hline Maximum Generator Torque & $47,402.91 \mathrm{~N} \cdot \mathrm{m}$ \\
\hline Maximum Generator Torque Rate & $15,000 \mathrm{~N} \bullet \mathrm{m} / \mathrm{s}$ \\
\hline Proportional Gain at Minimum Blade-Pitch Setting & $0.01882681 \mathrm{~s}$ \\
\hline Integral Gain at Minimum Blade-Pitch Setting & 0.008068634 \\
\hline Blade-Pitch Angle at which the Rotor Power Has Doubled & $6.302336^{\circ}$ \\
\hline Minimum Blade-Pitch Setting & $0^{\circ}$ \\
\hline Maximum Blade-Pitch Setting & $90^{\circ}$ \\
\hline Maximum Absolute Blade Pitch Rate & $8{ }^{\circ} / \mathrm{s}$ \\
\hline Equivalent Blade-Pitch-Actuator Linear-Spring Constant & $971,350,000 \mathrm{~N} \cdot \mathrm{m} / \mathrm{rad}$ \\
\hline Equivalent Blade-Pitch-Actuator Linear-Damping Constant & $206,000 \mathrm{~N} \bullet \mathrm{m} / \mathrm{rad} / \mathrm{s}$ \\
\hline
\end{tabular}

\subsubsection{FAST with AeroDyn and ADAMS with AeroDyn Models}

Using the turbine properties described previously in this section, I put together models of the NREL offshore 5-MW baseline wind turbine within FAST [39] with AeroDyn [55,67]. The input files for these models are given in Appendix A and Appendix B, for version (v) 6.10a-jmj of FAST and v12.60i-pjm of AeroDyn, respectively. I then generated the higher fidelity ADAMS models through the preprocessor functionality built into the FAST code.

The input files in Appendix A are for the FAST model of the NREL offshore 5-MW baseline wind turbine mounted on the ITI Energy barge. The input files for other versions of the model, such as that for the equivalent land-based version, required only a few minor changes. These include changes to input parameters "PtfmModel" and "PtfmFile," which identify the type and properties of the support platform, and modifications to the prescribed mode shapes in the tower input file, "TwrFile."

Although most of the input-parameter specifications in Appendix A and Appendix B are selfexplanatory, the specifications of the prescribed mode shapes needed by FAST to characterize the flexibility of the blades and tower (see Section 2.1) deserve a special explanation. The required mode shapes depend on the member's boundary conditions. For the blade modes, I used v2.22 of the Modes program [6] to derive the equivalent polynomial representations of the blade mode shapes needed by FAST. The Modes program calculates the mode shapes of rotating blades, assuming that a blade mode shape is unaffected by its coupling with other system modes of motion. This is a common assumption in wind turbine analysis. For the tower modes, 
however, there is a great deal of coupling with the rotor motions, and in floating systems, there is coupling with the platform motions as well. Taking these factors into account, I used the linearization functionality of the full-system ADAMS model to obtain the tower modes for both the floating systems and their equivalent land-based counterparts. In other words, I built an ADAMS model of the coupled wind turbine and support platform system, enabled all system DOFs, and linearized the model. Then I passed a best-fit polynomial through the resulting tower mode shapes to get the equivalent polynomial representations of the tower mode shapes needed by FAST.

Not including platform motions, the FAST model of the land-based version of the NREL 5-MW baseline wind turbine incorporates 16 DOFs as follows:

- Two flapwise and one edgewise bending-mode DOFs for each of the three blades

- One variable-generator speed DOF and one driveshaft torsional DOF

- One nacelle-yaw-actuator DOF

- Two fore-aft and two side-to-side bending-mode DOFs in the tower.

Not including platform motion, the higher fidelity ADAMS model of the land-based version of the wind turbine incorporates 378 DOFs as follows:

- One hundred and two DOFs in each of the three blades, including flapwise and edgewise shear and bending, torsion, and extension DOFs

- One blade-pitch actuator DOF in each of the three blades

- One variable-generator speed DOF and one driveshaft torsional DOF

- One nacelle-yaw actuator DOF

- Sixty-six DOFs in the tower, including fore-aft and side-to-side shear and bending, torsion, and extension DOFs.

The support platform motions in the sea-based versions of the NREL 5-MW baseline wind turbine add six DOFs per model.

I specified a constant time step of $0.0125 \mathrm{~s}$ in FAST's fixed-step-size time-integration scheme and a maximum step size of $0.0125 \mathrm{~s}$ in ADAMS' variable-step-size time integrator. I had AeroDyn perform aerodynamic calculations every other structural time step (i.e., $0.025 \mathrm{~s}$ ) to ensure that there were at least 200-azimuth-step computations per revolution at $12 \mathrm{rpm}$. Data were output at $20 \mathrm{~Hz}$ or every fourth structural time step. I made these time steps as large as possible to ensure numerical stability and suitable output resolution across a range of operating conditions.

\subsubsection{Full-System Natural Frequencies and Steady-State Behavior}

Up to now in this section, I have summarized the specifications of NREL's 5-MW baseline wind turbine. To provide a cursory overview of the overall system behavior of the equivalent landbased turbine, I calculated the full-system natural frequencies and the steady-state response of the system as a function of wind speed. 
I obtained the full-system natural frequencies with both the FAST model and the ADAMS model. In FAST, I calculated the natural frequencies by performing an eigenanalysis on the first-order state matrix created from a linearization analysis. In ADAMS, I obtained the frequencies by invoking a "LINEAR/EIGENSOL" command, which linearizes the complete ADAMS model and computes eigendata. To avoid the rigid-body drivetrain mode, the analyses considered the wind turbine in a stationary condition with the high-speed shaft brake engaged. The blades were pitched to their minimum set point $\left(0^{\circ}\right)$, but aerodynamic damping was ignored. Table 3-11 lists results for the first 13 full-system natural frequencies.

Table 3-11. Full-System Natural Frequencies in Hertz

\begin{tabular}{|l|l|r|r|}
\hline Mode & Description & FAST & ADAMS \\
\hline 1 & 1st Tower Fore-Aft & 0.3240 & 0.3195 \\
\hline 2 & 1st Tower Side-to-Side & 0.3120 & 0.3164 \\
\hline 3 & 1st Drivetrain Torsion & 0.6205 & 0.6094 \\
\hline 4 & 1st Blade Asymmetric Flapwise Yaw & 0.6664 & 0.6296 \\
\hline 5 & 1st Blade Asymmetric Flapwise Pitch & 0.6675 & 0.6686 \\
\hline 6 & 1st Blade Collective Flap & 0.6993 & 0.7019 \\
\hline 7 & 1st Blade Asymmetric Edgewise Pitch & 1.0793 & 1.0740 \\
\hline 8 & 1st Blade Asymmetric Edgewise Yaw & 1.0898 & 1.0877 \\
\hline 9 & 2nd Blade Asymmetric Flapwise Yaw & 1.9337 & 1.6507 \\
\hline 10 & 2nd Blade Asymmetric Flapwise Pitch & 1.9223 & 1.8558 \\
\hline 11 & 2nd Blade Collective Flap & 2.0205 & 1.9601 \\
\hline 12 & 2nd Tower Fore-Aft & 2.9003 & 2.8590 \\
\hline 13 & 2nd Tower Side-to-Side & 2.9361 & 2.9408 \\
\hline
\end{tabular}

The agreement between FAST and ADAMS is quite good. The biggest differences exist in the predictions of the blades' second asymmetric flapwise yaw and pitch modes. By "yaw" and "pitch" I mean that these blade asymmetric modes couple with the nacelle-yaw and nacellepitching motions, respectively. Because of the offsets of the blade section CM from the pitch axis, higher-order modes, and tower-torsion DOFs - which are available in ADAMS, but not in FAST_ADAMS predicts lower natural frequencies in these modes than FAST does.

Bir and I have published [2] a much more exhaustive eigenanalysis for the NREL 5-MW baseline wind turbine. The referenced publication documents the natural frequencies and damping ratios of the land- and sea-based versions of the 5-MW turbine across a range of operating conditions.

I obtained the steady-state response of the land-based 5-MW baseline wind turbine by running a series of FAST with AeroDyn simulations at a number of given, steady, and uniform wind speeds. The simulations lengths were long enough to ensure that all transient behavior had died out; I then recorded the steady-state output values. I ran the simulations using the BEM wake option of AeroDyn and with all available and relevant land-based DOFs enabled. Figure 3-12 shows the results for several output parameters, which are defined as follows:

- "GenSpeed" represents the rotational speed of the generator (high-speed shaft).

- "RotPwr" and "GenPwr" represent the mechanical power within the rotor and the electrical output of the generator, respectively. 

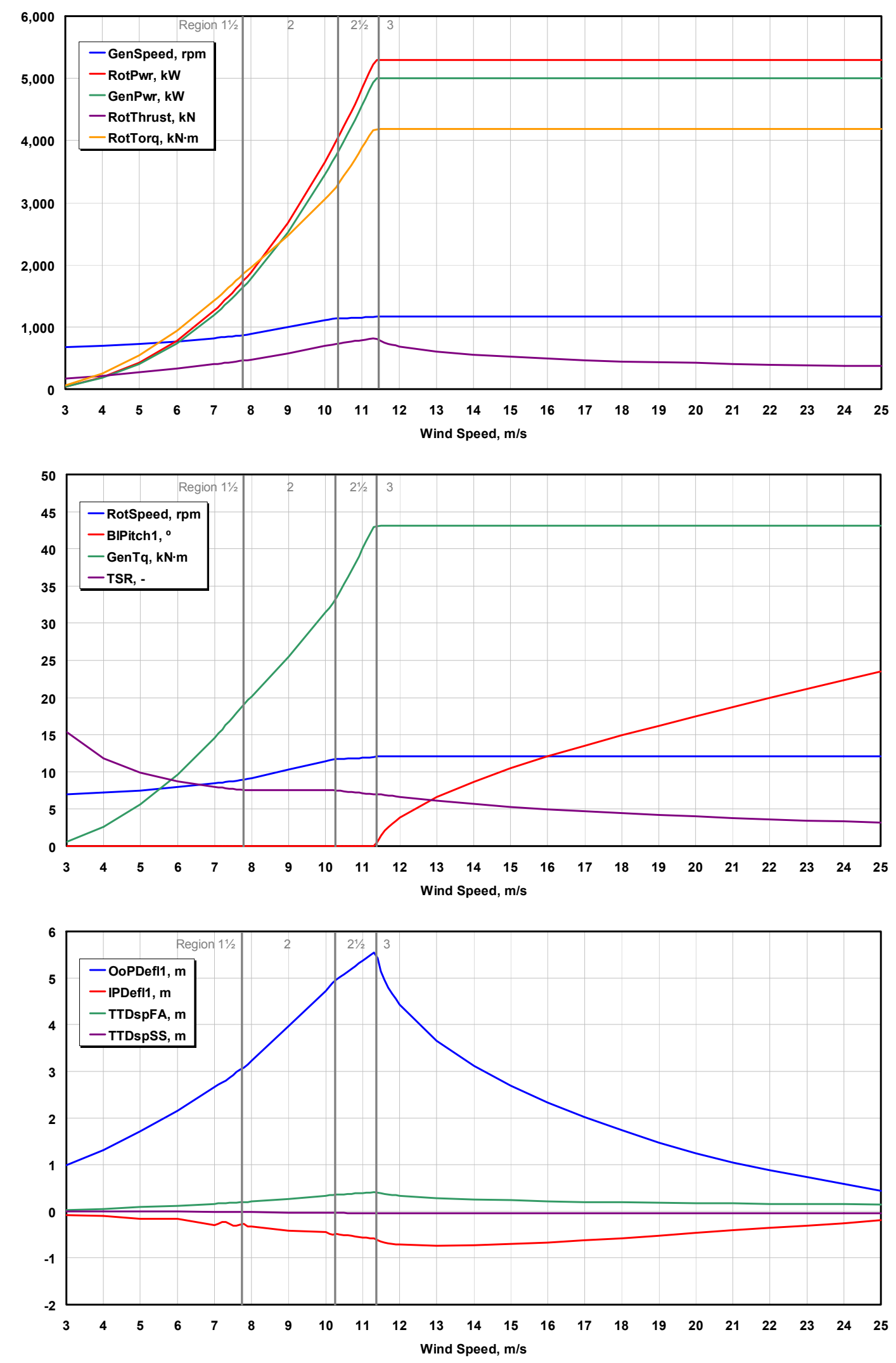

Figure 3-12. Steady-state responses as a function of wind speed 
- "RotThrust" represents the rotor thrust.

- "RotTorq" represents the mechanical torque in the low-speed shaft.

- "RotSpeed" represents the rotational speed of the rotor (low-speed shaft).

- "BlPitch1" represents the pitch angle of Blade 1.

- "GenTq" represents the electrical torque of the generator.

- "TSR" represents the tip-speed ratio.

- "OoPDefl1" and "IPDefl1" represent the out-of-plane and in-plane tip deflections of Blade 1 relative to the undeflected blade-pitch axis.

- "TTDspFA" and "TTDspSS" represent the fore-aft and side-to-side deflection of the tower top relative to the centerline of the undeflected tower.

As planned, the generator and rotor speeds increase linearly with wind speed in Region 2 to maintain constant tip-speed ratio and optimal wind-power conversion efficiency. Similarly, the generator and rotor powers and generator and rotor torques increase dramatically with wind speed in Region 2, increasing cubically and quadratically, respectively. Above rated, the generator and rotor powers are held constant by regulating to a fixed speed with active bladepitch control. The out-of-plane tip deflection of the reference blade (Blade 1) reaches a maximum at the rated operating point before dropping again. This response characteristic is the result of the peak in rotor thrust at rated. This peak is typical of variable generator speed variable blade-pitch-to-feather wind turbines because of the transition that occurs in the control system at rated between the active generator-torque and the active blade-pitch control regions. This peak in response is also visible, though less pronounced, in the in-plane tip deflection of the reference blade and the tower-top fore-aft displacement.

Start-up transient behavior is an artifact of the computational analysis. To mitigate this behavior, I used the steady-state values of the rotor speed and blade-pitch angles found in Figure 3-12 as initial conditions in many of the simulations presented in subsequent chapters.

\subsection{ITI Energy Barge}

For some of the simulation code verification exercises presented in Chapter 4 and for the seabased loads analysis presented in Chapter 5 and Chapter 6, I modeled the NREL 5-MW baseline wind turbine mounted on a floating barge from ITI Energy. I used a preliminary barge concept developed by W. Vijfhuizen under the direction of Professor N. Barltrop of the Department of Naval Architecture and Marine Engineering (NAME) at the Universities of Glasgow and Strathclyde ${ }^{1}$ through a contract with ITI Energy. Not only is the barge intended to support the 5MW wind turbine, but it is also a platform for an OWC wave-power device. To ensure that the simplest possible manufacturing techniques can be used in its fabrication, the barge is square and the wave energy is extracted from a square moon pool located at the center of the barge, which allows the OWC to be installed within the wind turbine's tower. The barge is ballasted with seawater to achieve a reasonable draft, which is not so shallow that it is susceptible to incessant

\footnotetext{
${ }^{1}$ Web site: http://www.na-me.ac.uk/
} 
wave slamming. To prevent it from drifting, the platform is moored by a system of eight catenary lines. Two of these lines emanate from each corner of the bottom of the barge such that they would be $45^{\circ}$ apart at the corner.

I provide some details of the ITI Energy barge and mooring system in Table 3-12 and illustrate the concept with an image generated using ADAMS in Figure 3-13. The concept is documented in much greater detail in Ref. [98]. ${ }^{2}$ Appendix D contains the FAST platform input file, which includes the input parameters related to the support platform, HydroDyn, and the mooring system; the WAMIT input files; and a portion of the WAMIT output files (some of the data are removed to save space). Additionally, some of the WAMIT input and output data are plotted in Chapter 4.

Table 3-12. Summary of ITI Energy Barge Properties

\begin{tabular}{|l|r|}
\hline Size $(\mathrm{W} \times \mathrm{L} \times \mathrm{H})$ & $40 \mathrm{~m} \times 40 \mathrm{~m} \times 10 \mathrm{~m}$ \\
\hline Moon pool $(\mathrm{W} \times \mathrm{L} \times \mathrm{H})$ & $10 \mathrm{~m} \times 10 \mathrm{~m} \times 10 \mathrm{~m}$ \\
\hline Draft, Freeboard & $4 \mathrm{~m}, 6 \mathrm{~m}$ \\
\hline Water Displacement & $6,000 \mathrm{~m}^{3}$ \\
\hline Mass, Including Ballast & $5,452,000 \mathrm{~kg}$ \\
\hline $\mathrm{CM}$ Location below SWL & $0.281768 \mathrm{~m}$ \\
\hline Roll Inertia about CM & $726,900,000 \mathrm{~kg} \cdot \mathrm{m}^{2}$ \\
\hline Pitch Inertia about CM & $726,900,000 \mathrm{~kg} \cdot \mathrm{m}^{2}$ \\
\hline Yaw Inertia about CM & $1,453,900,000 \mathrm{~kg} \cdot \mathrm{m}^{2}$ \\
\hline Anchor (Water) Depth & $150 \mathrm{~m}$ \\
\hline Separation between Opposing Anchors & $773.8 \mathrm{~m}$ \\
\hline Unstretched Line Length & $473.3 \mathrm{~m}$ \\
\hline Neutral Line Length Resting on Seabed & $250 \mathrm{~m}$ \\
\hline Line Diameter & $0.0809 \mathrm{~m}$ \\
\hline Line Mass Density & $130.4 \mathrm{~kg} / \mathrm{m}$ \\
\hline Line Extensional Stiffness & $589,000,000 \mathrm{~N}$ \\
\hline
\end{tabular}

The capabilities of my aero-hydro-servo-elastic simulation tools do not permit me to model an OWC wave-power device or its associated potential for energy extraction. Instead, I modeled the hydrodynamics of the barge by assuming that the moon pool was covered by a fixed plate located just below the free surface. Section 4.1.2 explains this assumption in more detail.

\footnotetext{
${ }^{2}$ Note that some of the properties given in Table 3-12 disagree with the data published in Ref. [98] because I used an updated design. For example, the published freeboard of $4 \mathrm{~m}$ in Ref. [98] was increased to $6 \mathrm{~m}$ after wave tank testing at NAME demonstrated that more freeboard would be beneficial to the system's response. This changed the CM location and inertias slightly. In addition, Ref. [98] used a simple linearized representation of the mooring system. Professor N. Barltrop developed the more detailed mooring system documented in Table 3-12 after Ref. [98] was published.
} 


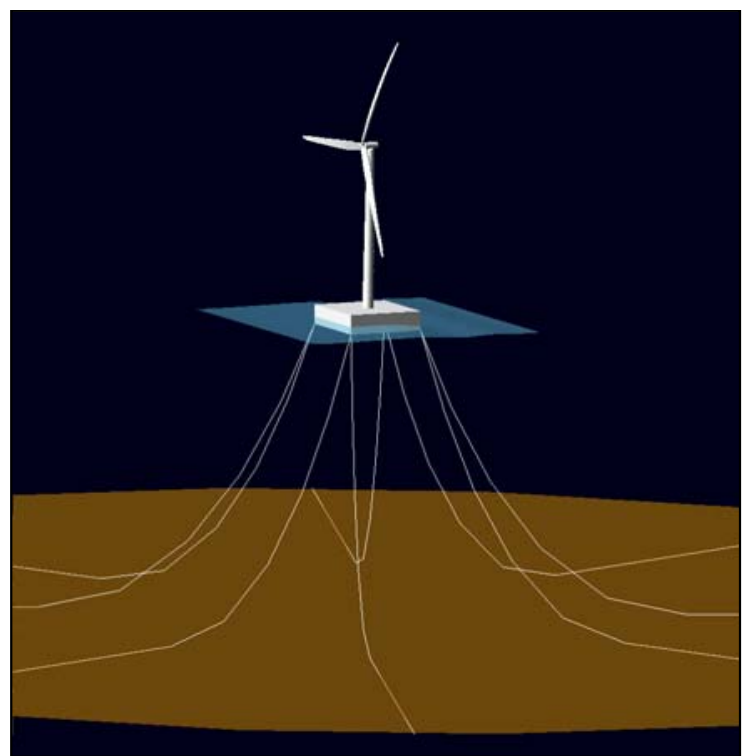

Figure 3-13. Illustration of the 5-MW wind
turbine on the ITI Energy barge

\subsection{MIT / NREL Barge}

Under the direction of Professor P. D. Sclavounos of MIT, E. N. Wayman also developed preliminary concepts of several floating platforms for the NREL offshore 5-MW baseline wind turbine. One of her designs was named the MIT / NREL SDB. I also mounted the 5-MW baseline wind turbine on this floating platform for some of the simulation code verification exercises presented in Chapter 4. (I did not, however, carry out a comprehensive loads analysis for this concept.) The MIT / NREL SDB is a cylindrical barge and has a spread-mooring system with four pairs of taut lines that radiate outward. I list some of the barge data in and provide the FAST platform input file, the WAMIT input files, and a portion of the WAMIT output files in Appendix E. This concept is documented in much greater detail in Refs. [100] and [101].

Table 3-13. Summary of MIT / NREL Barge Properties

\begin{tabular}{|l|r|}
\hline Diameter, Height & $36 \mathrm{~m}, 9.5 \mathrm{~m}$ \\
\hline Draft, Freeboard & $5 \mathrm{~m}, 4.5 \mathrm{~m}$ \\
\hline Water Displacement & $5,089 \mathrm{~m}^{3}$ \\
\hline Mass, Including Ballast & $4,519,150 \mathrm{~kg}$ \\
\hline CM Location below SWL & $3.88238 \mathrm{~m}$ \\
\hline Roll Inertia about CM & $390,147,000 \mathrm{~kg} \cdot \mathrm{m}^{2}$ \\
\hline Pitch Inertia about CM & $390,147,000 \mathrm{~kg} \cdot \mathrm{m}^{2}$ \\
\hline Yaw Inertia about CM & $750,866,000 \mathrm{~kg} \cdot \mathrm{m}^{2}$ \\
\hline Anchor (Water) Depth & $200 \mathrm{~m}$ \\
\hline Separation between Opposing Anchors & $436 \mathrm{~m}$ \\
\hline Unstretched Line Length & $279.3 \mathrm{~m}$ \\
\hline Neutral Line Length Resting on Seabed & $0 \mathrm{~m}$ \\
\hline Line Diameter & $0.127 \mathrm{~m}$ \\
\hline Line Mass Density & $116.0 \mathrm{~kg} / \mathrm{m}$ \\
\hline Line Extensional Stiffness & $1,500,000,000 \mathrm{~N}$ \\
\hline
\end{tabular}




\subsection{Reference-Site Data}

The IEC 61400-3 design standard [34] requires that a loads analysis be based on site-specific external conditions. At the request of ITI Energy, the location of the former Stevenson Weather Station was selected as the reference site for which to obtain environmental (metocean) data for the loads analyses (presented in Chapter 5 and Chapter 6). This site is located at $61^{\circ} 20^{\prime} \mathrm{N}$ latitude, $0^{\circ} 0^{\prime} \mathrm{E}$ longitude on the prime meridian northeast of the Shetland Islands, which are northeast of Scotland. Figure 3-14 illustrates this location with an image courtesy of Google Earth. ${ }^{3}$ This reference site was chosen for its fairly extreme wind and wave conditions, with the implication that if the results of the loads analysis are favorable, the floating wind turbine system under consideration will be applicable at almost any site around the world.

ITI Energy requested that I use data from a Waverider buoy that collected short-term wave statistics at this site from February 1973 to February 1976. Because this data set did not contain windspeed information, however, it was not directly applicable to the loads analysis, which requires joint wind and wave data. Instead, NREL purchased wind and wave data at the reference site through the

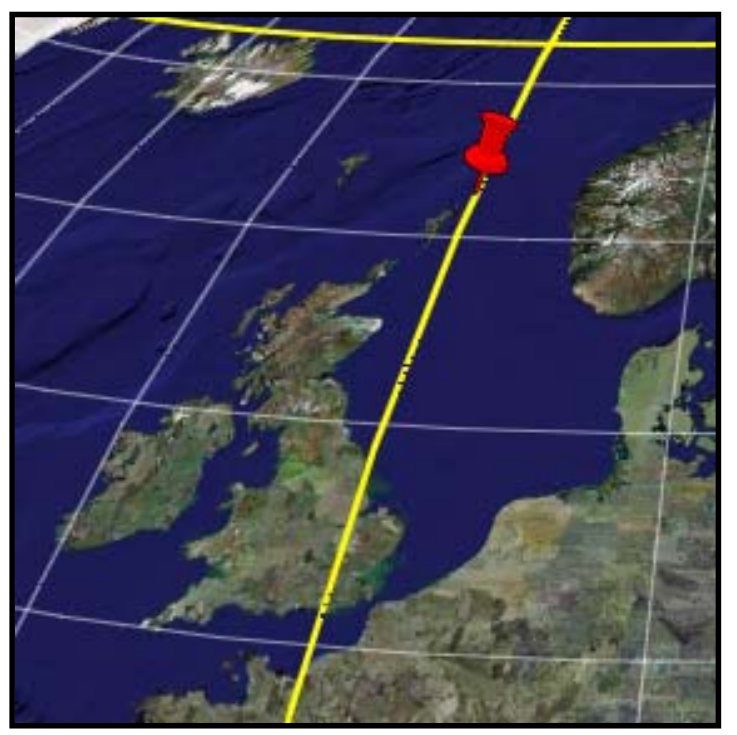

Figure 3-14. Reference-site location online Waveclimate.com service ${ }^{4}$ that is run by the Advisory and Research Group on Geo Observation Systems and Services (ARGOSS) in the Netherlands. ${ }^{5}$ The Waveclimate.com service hosts a worldwide database of wind and wave climate information based on a combination of measurements and a global hindcast model. The measured data come from a composite of radar altimeter, radar scatterometer, and imaging radar (synthetic aperture radar [SAR]) observations, taken from 1985 to present. The Waveclimate.com database has been validated and calibrated with measurements from surface buoys, though not specifically at the chosen reference site. The model is based on the thirdgeneration ocean wind-wave model WaveWatch III [94], which solves the spectral-action, density-balance equation for wave-number-direction spectra. Although I do not show any of the comparisons here, the wave data obtained through the Waveclimate.com service agreed quite well with the wave statistics available from the former Waverider buoy. This gave me confidence in the accuracy of the Waveclimate.com product.

The Waveclimate.com service uses a grid spacing of $1^{\circ}$ latitude by $1^{\circ}$ longitude in the vicinity of the reference site. $\mathrm{We}^{6}$ chose the cell with grid boundaries of $61^{\circ}$ to $62^{\circ} \mathrm{N}$ latitude, $0^{\circ}$ to $1^{\circ} \mathrm{E}$

\footnotetext{
${ }^{3}$ Web site: http://earth.google.com/

${ }^{4}$ Web site: http://www.waveclimate.com/

${ }^{5}$ Web site: http://www.argoss.nl/

${ }^{6}$ My NREL colleague, G. N. Scott, assisted me with processing the data from the Waveclimate.com service. To acknowledge this support, I use "we" in place of "I" where appropriate in this section.
} 
longitude. NREL purchased two sets of data for this cell. The first data set consisted of an estimate of the long-term joint-probability distribution of wind speed, significant wave height, and mean wave period. The second data set was a prediction of the extreme significant wave heights for various return periods.

The joint-probability distribution was provided in terms of 37,992 samples, each based on a 3-h reference (averaging) period, representing a total of about 13 years of data. The samples were grouped in bins with a wind-speed width of 2 knots $(1.029 \mathrm{~m} / \mathrm{s})$, a significant-wave-height width of $1 \mathrm{~m}$, and a mean-wave-period width of $1 \mathrm{~s}$. The reference elevation for the wind-speed data was $10 \mathrm{~m}$ above the MSL. To adjust these data to the turbine's hub height of $90 \mathrm{~m}$, we assumed a vertical power-law shear exponent ${ }^{7}$ of 0.14 . In addition, we scaled all of the wind-speed bins by a factor of $(90 \mathrm{~m} / 10 \mathrm{~m})^{0.14}=1.360$, resulting in an altered bin width of $1.399 \mathrm{~m} / \mathrm{s}$ for the hubheight wind speed, $V_{h u b}$. We also converted the mean wave-period data to peak spectral period, $T_{p}$. By assuming that the wave conditions were represented by the modified Pierson-Moskowitz spectrum [22], all of the wave-period bins were scaled by a factor of 1.408 , resulting in an altered bin width of $1.408 \mathrm{~s}$. The data of significant wave height, $H_{s}$, did not require adjustment.

The resolution of the resulting long-term joint-probability distribution does not entirely conform to the maximum bin widths of $2 \mathrm{~m} / \mathrm{s}, 0.5 \mathrm{~m}$, and $0.5 \mathrm{~s}$ required by the IEC $61400-3$ design standard [34]. I did, however, consider the resolution to be adequate because the loads analysis presented in Chapter 5 and Chapter 6 is preliminary in nature. Similarly, I found it acceptable to base the joint-probability distribution on a 3-h reference period instead of the 1-h period required by the 61400-3 design standard. This is because the marginal long-term probability distributions of significant wave height and peak spectral period do not depend on the averaging period, and because one can assume that the marginal long-term probability distribution of mean wind speed is independent of the averaging period for periods in the range of $10 \mathrm{~min}$ to $3 \mathrm{~h}$ [34].

Using the long-term joint-probability distribution, we characterized the expected value of the significant wave height, $E\left[H_{S} \mid V_{h u b}\right]$, as well as the range of associated peak spectral periods, conditioned on the mean hub-height wind speed from cut-in to cut-out. Figure 3-15 illustrates these data. As shown, the expected value of the significant wave height increases with the mean hub-height wind speed that it is conditioned on-from about $1.6 \mathrm{~m}$ at cut-in, $V_{i n}=3 \mathrm{~m} / \mathrm{s}$, to about $5.9 \mathrm{~m}$ at cut-out, $V_{\text {out }}=25 \mathrm{~m} / \mathrm{s}$. The peak spectral periods have a median that increases and a range that tends to decrease with the expected significant wave heights they are associated with, from about $12.7 \pm 5.6 \mathrm{~s}$ at $1.6 \mathrm{~m}$, to about $15.5 \pm 4.2 \mathrm{~s}$ at $5.9 \mathrm{~m}$.

The Waveclimate.com service's extreme-value analysis yielded predictions of the extreme significant wave heights at the reference site for various return periods. The associated windand wave-period information was not available, however, so we relied on assumptions and estimates to specify them. Although the 61400-3 design standard [34] requires that the extreme individual wave heights be estimated at the reference site, I did not assess them because I did not

${ }^{7}$ The vertical power-law profile is $V(Z)=V\left(Z_{r}\right)\left(\frac{Z}{Z_{r}}\right)^{\alpha}$, where $V(Z)$ is the wind speed at height $Z$ above the ground (or above the mean sea level), $Z_{r}$ is a reference height, and $\alpha$ is the power-law exponent. 


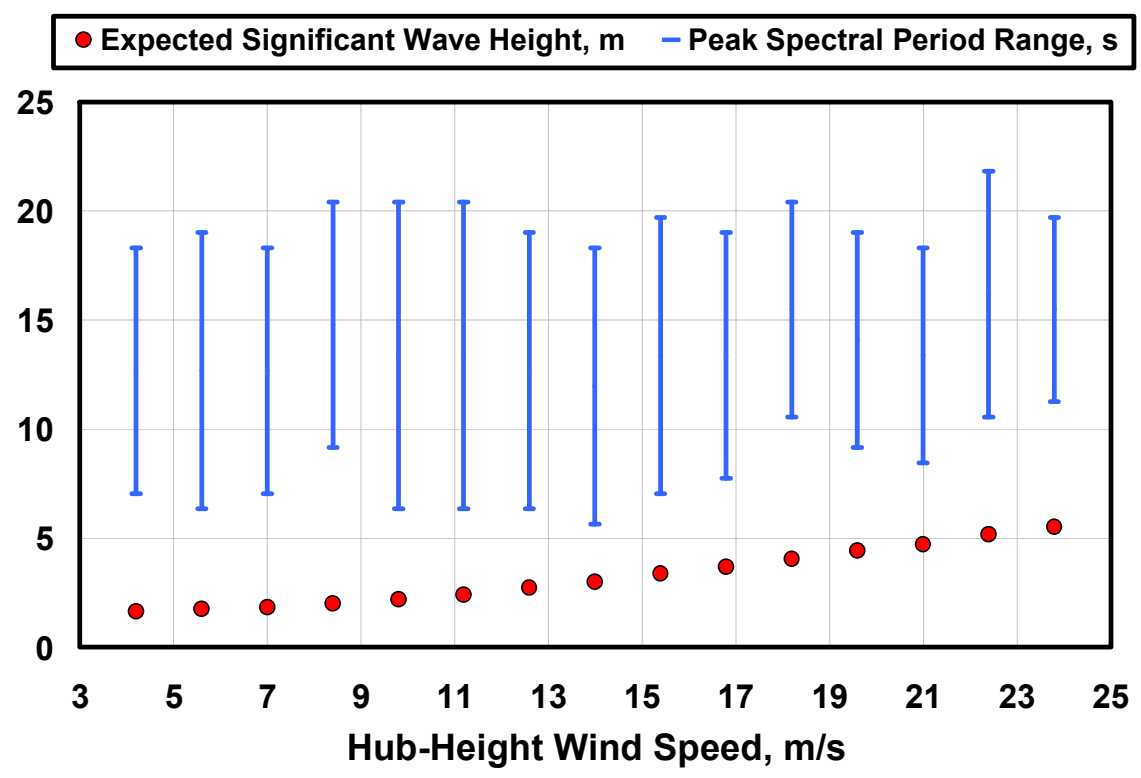

Figure 3-15. Normal sea state conditions at the reference site

use regular, periodic waves in the preliminary loads analysis. Instead, I relied on irregular sea states as described in Chapter 5.

Based on a 3-h reference period, the significant wave height with a recurrence period of 1 year, $H_{s l}$, was predicted by the Waveclimate.com service to be $10.8 \mathrm{~m}$. The service also predicted the significant wave height with a recurrence period of 50 years, $H_{s 50}$, to be $13.8 \mathrm{~m}$. From data available in the joint-probability distribution, we estimated that the range of peak spectral periods associated with the 1-year recurrence of significant wave height would be 15.5 to $19.7 \mathrm{~s}$. Because 50-year recurrence data do not exist in the 13-year record of joint-probability statistics, we had to extrapolate to estimate the range of peak spectral periods associated with the 50-year recurrence of significant wave height. By this extrapolation, we estimated a range of 18.5 to 19.9 s. I assumed that the extreme wind speeds at the reference site conformed to those prescribed by wind turbine class I from the IEC 61400-1 design standard [33]. Based on this assumption and a 10-min averaging period, the reference hub-height wind speed with a recurrence period of 1 year, $V_{l}$, was prescribed to be $40 \mathrm{~m} / \mathrm{s}$ and the reference hub-height wind speed with a recurrence period of 50 years, $V_{50}$, was prescribed to be $50 \mathrm{~m} / \mathrm{s}$.

The water depth at the reference site is roughly $160 \mathrm{~m}$; however, I analyzed the sea-based loads presented in Chapter 5 and Chapter 6 with a depth of $150 \mathrm{~m}$ (as indicated in Table 3-12 for the ITI Energy barge).

I did not quantify several other commonly assessed environmental conditions at the reference site, again because the loads analysis was preliminary. For some of the unquantified conditions, I assumed typical values. I did not assess-nor does the loads analysis account for-the potential loading from sea ice; marine growth; corrosion; wake effects from neighboring wind turbines in a wind farm; earthquakes; variations in water levels from astronomical tides and storm surges; and sea currents generated by wind, tides, storm surges, atmospheric-pressure variations, and near-shore waves (i.e., surf currents). I did not assess the soil conditions at the reference site because my mooring system module assumes that the anchor locations of each 
mooring line are fixed to the inertial frame at the seabed. I assumed standard values of 1.225 $\mathrm{kg} / \mathrm{m}^{3}$ for the air density and $1,025 \mathrm{~kg} / \mathrm{m}^{3}$ for the water density. As dictated by the $61400-3$ design standard [34], I assumed a vertical power-law shear exponent of 0.14 for all normal wind conditions and 0.11 in extreme 1 - and 50-year wind conditions. Similarly, I did not assess the ambient turbulence standard deviation from site data, or from estimations derived from the surface roughness according to the Charnock expression. Instead, I assumed that the wind turbulence at the reference site conformed to the models prescribed by wind turbine turbulencecategory B from the 61400-1 design standard. I also did not assess the correlation of wind and wave direction, opting instead to use the guidance of the 61400-3 design standard (see Chapter 5). I ignored wave directional spreading and used long-crested waves for all sea states. Finally, I did not prescribe a site-specific wave spectrum, but opted instead to use the JONSWAP spectrum defined in Section 2.4.1.1. All these assumptions and omissions will need to be addressed in more detailed follow-on loads-analysis projects. 


\section{Chapter 4 Verification of Simulation Capability}

The aero-servo-elastic capabilities of FAST with AeroDyn and ADAMS with AeroDyn have been well verified and validated in previous studies [7,8,9,12,37,38,63]. But because my hydrodynamics and mooring system modules are novel, they must be verified to ensure that the response predictions from the fully coupled aero-hydro-servo-elastic capability are accurate. In all, I performed seven verification studies to test the accuracy of the new features: three for the hydrodynamics module (Section 4.1), two for the mooring system module (Section 4.2), and two for the complete system (Section 4.3). The last pair of verification exercises compared the results from my time-domain simulation tool with the results from a frequency-domain model. As I discuss in this chapter, the results of all the verification exercises were favorable. This gave me confidence to pursue more thorough investigations into the dynamic behavior of offshore floating wind turbines in Chapter 5 and Chapter 6.

Additionally, though not explicitly documented here, the resulting dynamics from the newly added support platform DOFs in FAST agree well with ADAMS. I furnish some examples of this in Chapter 6.

I used model-to-model comparisons for all these verification exercises. The fully coupled simulation tool will be validated later, once experimental data are made available.

\subsection{Verification of the Hydrodynamics Module}

I performed three verification tests to check HydroDyn's hydrodynamics module. First, as presented in Section 4.1.1, I verified that the PSD of the wave-elevation time series computed by HydroDyn matched the target JONSWAP spectrum prescribed by HydroDyn's wave-spectrum input parameters. Second, I verified that the output from WAMIT, which is used as input to HydroDyn, is similar to that generated by a different radiation / diffraction solver (see Section 4.1.2). Third, I verified that the radiation impulse-response functions computed within my hydrodynamics module were the same as those computed with WAMIT's stand-alone frequencyto-time (F2T) conversion utility [58]. I present these results in Section 4.1.3.

\subsubsection{Wave Elevation versus the Target Wave Spectrum}

Irregular sea states (stochastic waves) are modeled in HydroDyn by the inverse Fourier transform of Eq. (2-9), which represents the superposition of a large number of periodic and parallel wave components. The amplitudes of these wave components, on average, are determined by the prescribed wave spectrum. I say, "on average," because randomness comes in through the realization of the WGN process. That process considers not only a uniformlydistributed random phase, but a normally-distributed amplitude as well (see Section 2.4.1.1). In HydroDyn, Eq. (2-9) is implemented using a computationally efficient FFT routine [92].

I ran a simple test to check that I implemented these mathematical relationships correctly in HydroDyn. I computed four wave-elevation time series, each determined with the PiersonMoskowitz wave spectrum [see Eq. (2-20)] given by a significant wave height, $H_{s}$, of $5.49 \mathrm{~m}$ and a peak spectral period, $T_{p}$, of $14.656 \mathrm{~s}$ or a peak spectral frequency of about $0.429 \mathrm{rad} / \mathrm{s}$. [This 
spectrum is equivalent to a JONSWAP spectrum with the default value (unity) of the peak shape parameter given by Eq. (2-19)]. Each wave-elevation time series was 10,000 s long (i.e., just shy of $3 \mathrm{~h}$ each) and was differentiated through the choice of dissimilar random seeds.

I then computed the PSD of each wave-elevation record, and compared each to the target wave spectrum determined by the given spectral parameters. Figure 4-1 shows the results. To minimize scatter, I grouped the discrete-frequency PSD data of Figure 4-1 in bins of width 0.001 $\mathrm{Hz}$ (about $0.00628 \mathrm{rad} / \mathrm{s}$ ). Because of the normally-distributed amplitudes provided by the WGN process, however, there is still a fair amount of scatter in the PSD of each individual run. But the average of the four PSDs, as indicated by the series labeled "Run Average" in Figure 4-1, is approaching the target spectrum nicely. This outcome would improve by averaging the results of many more simulations.

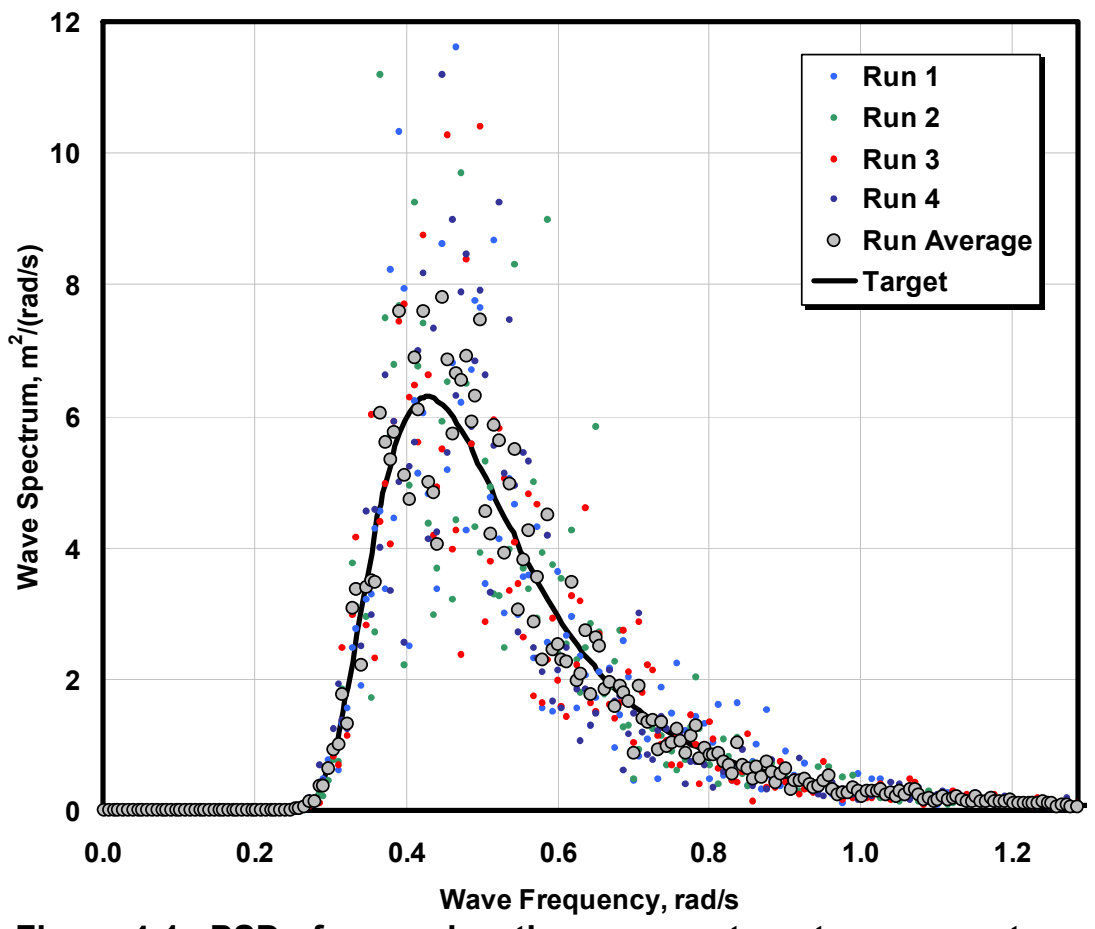

Figure 4-1. PSD of wave elevations versus target wave spectrum

I also calculated the probability density for the aggregate composite of the wave-elevation records computed by, and output from, HydroDyn. As expected, this histogram is Gaussiandistributed with a mean of zero and a standard deviation (for this test case) of $H_{s} / 4=1.37 \mathrm{~m}$. The result is shown with the corresponding probability density function derived from a zerovalued mean and a standard deviation of $1.37 \mathrm{~m}$ in Figure 4-2.

\subsubsection{WAMIT Output / HydroDyn Input}

As I described in Chapter 2, I used WAMIT [58] as a preprocessor for generating the hydrodynamic-added-mass and -damping matrices, $A_{i j}(\omega)$ and $B_{i j}(\omega)$, and wave-excitation force, $X_{i}(\omega, \beta)$, which are inputs to HydroDyn. WAMIT uses the three-dimensional numericalpanel method to solve the linearized hydrodynamic radiation and diffraction problems for the 


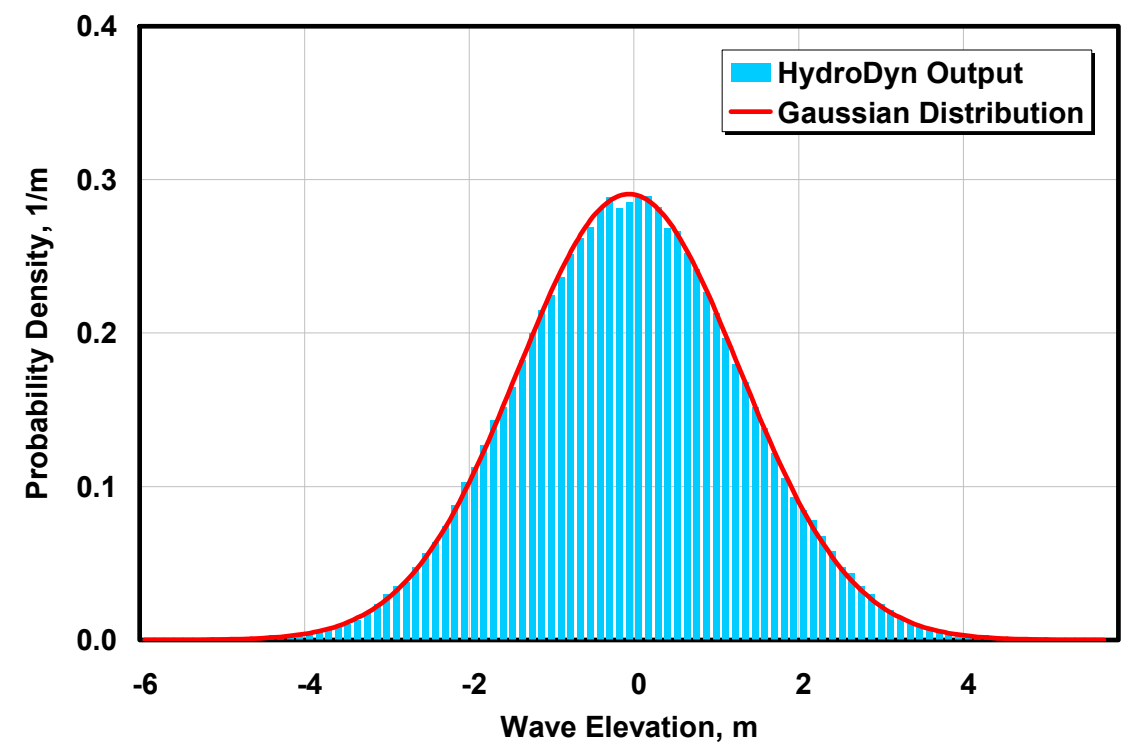

Figure 4-2. Wave-elevation probability density

interaction of surface waves with offshore platforms in the frequency domain. WAMIT ignores the effects of sea current or forward speed on the radiation and diffraction problems, as well as higher-order effects.

Because the hydrodynamic solution my simulation tool generates is only as good as the hydrodynamic inputs, verifying the acceptability of the WAMIT results is beneficial. Consequently, I ran a test to ensure that the WAMIT output I generated is similar to that calculated by a different radiation / diffraction solver. Data used by NAME at the Universities of Glasgow and Strathclyde when devising the ITI Energy barge described in Section 3.2 were available for this comparison. NAME used a custom-made linear hydrodynamic radiation and diffraction solver with capabilities similar to, but independent of, WAMIT.

In WAMIT, I modeled the barge with two geometric planes of symmetry with 2,400 rectangular panels within a quarter of the body. Consistent with linear theory, I needed to mesh only the wetted portion of the body in its undisplaced position. Figure 4-3 shows the panel mesh with both symmetries. To avoid accounting for the OWC in the WAMIT analysis, I covered the moon pool with a fixed plate located $0.01 \mathrm{~m}$ below the free surface. In an attempt by NAME to model the OWC, they considered that the plate was free to move relative to the barge. Figure 4-4 shows the panel mesh for NAME's analysis.

To improve the accuracy of the WAMIT results, I chose to override three default settings, choosing instead to (1) integrate the logarithmic singularity analytically, (2) solve the linear system of equations using a direct solver, and (3) remove the effects of irregular frequencies by automatically projecting the body panels to the free surface. These settings were necessary because some panels are located in a plane near the free surface, the barge has a large waterplane area, and subsequent analysis required high-frequency results. The barge was analyzed in its undisplaced position with infinite water depth in both codes. The hydrodynamic-added-mass and -damping matrices were compared in all six rigid-body modes of motion of the barge (in the matrix subscripts, $1=$ surge, $2=$ sway, $3=$ heave, $4=$ roll, $5=$ pitch, $6=$ yaw), resulting in $6 \times 6$ 


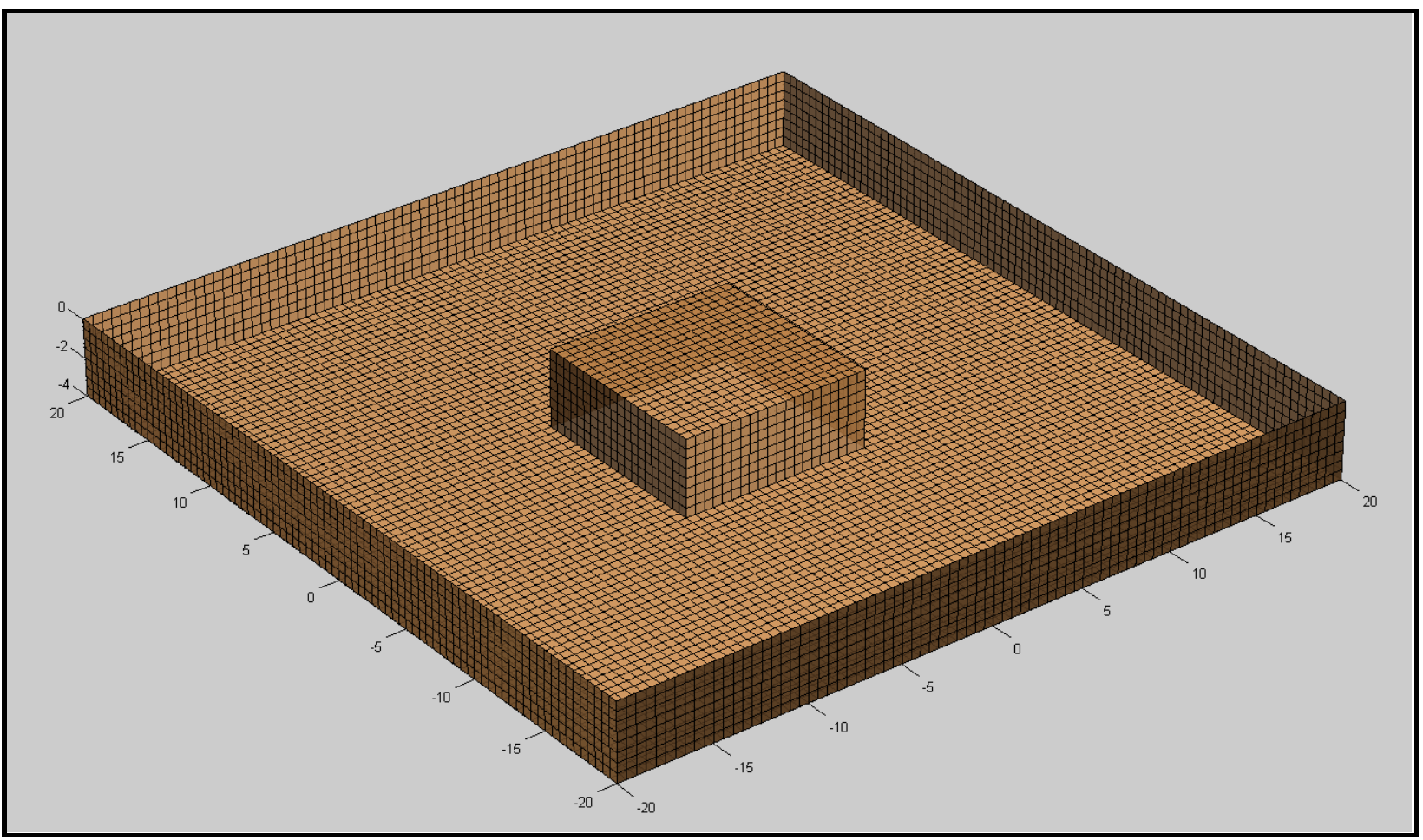

Figure 4-3. Panel mesh of the ITI Energy barge used within WAMIT

matrices at each frequency. Because NAME considerd the plate to be a separate body, their resulting matrices at each frequency were of the size $12 \times 12$. To assist in the comparison, though, NAME reduced these down to $6 \times 6$ matrices via postprocessing. The hydrodynamic wave-excitation force was not considered in this test.

Figure 4-5 shows the results in a side-by-side comparison. All data are dimensional as indicated. Only the upper triangular matrix elements are shown because the hydrodynamicadded-mass and -damping matrices are

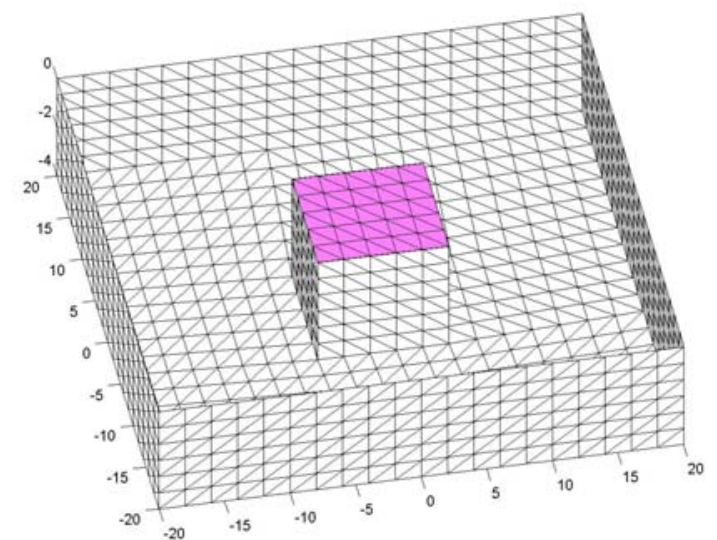

Figure 4-4. Panel mesh of the ITI Energy barge used by NAME symmetric in the absence of sea current or forward speed [22,74]. Also, because of the barge's symmetries, the surge-surge elements of the frequency-dependent added-mass and damping matrices, $A_{11}$ and $B_{11}$, are identical to the swaysway elements, $A_{22}$ and $B_{22}$. Similarly, the roll-roll elements, $A_{44}$ and $B_{44}$, are identical to the pitch-pitch elements, $A_{55}$ and $B_{55}$. Other matrix elements not shown are zero-valued or very close to being zero-valued.

In Figure 4-5, the WAMIT results are given in even increments of frequency. The NAME results are given in even increments of period, so resolution is lost at the higher frequencies. As expected, all matrix elements peak out at some intermediate frequency and level out at higher frequencies. Additionally, the zero- and infinite-frequency limits of all elements of the 

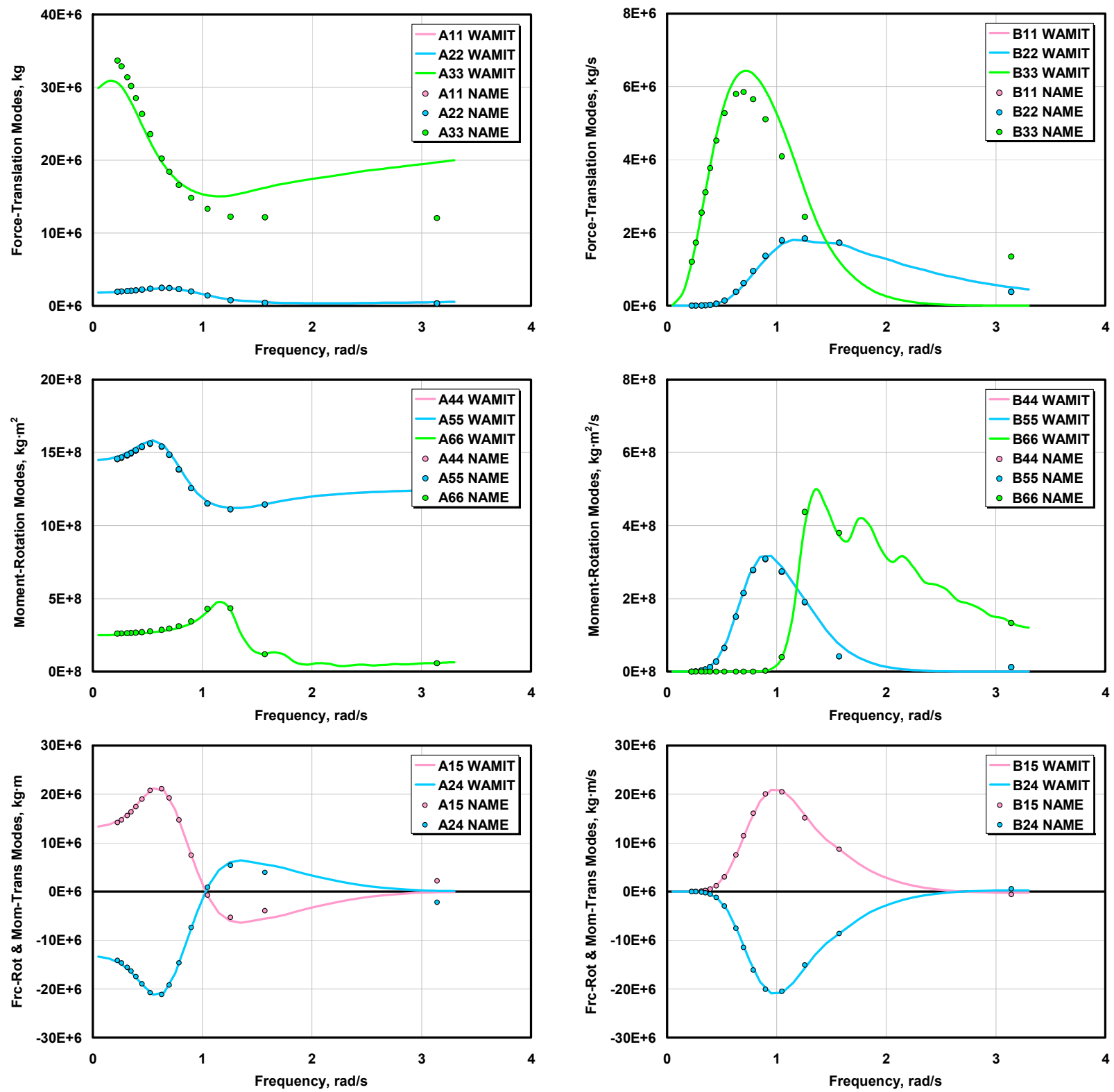

Figure 4-5. Hydrodynamic added mass and damping for the ITI Energy barge

hydrodynamic-damping matrix are zero (not all shown), as required by theory [22,74]. The comparisons between the output of WAMIT and the results of NAME generally agree very well and demonstrate that WAMIT is an acceptable code for generating the hydrodynamic inputs needed by my simulation tool. The biggest discrepancies are in the heave-heave elements of the frequency-dependent added-mass and damping matrices, $A_{33}$ and $B_{33}$. I believe that these differences are artifacts of the dissimilar numerical solutions employed by WAMIT and NAME's radiation / diffraction solver. The differences are not large, however, and I do not believe they are crucial to the accuracy of my hydrodynamics solution. 


\subsubsection{Computation of Radiation Impulse-Response Functions}

The radiation "memory effect" is captured in HydroDyn's hydrodynamics module through the convolution integral of Eq. (2-8). As described in Section 2.4.1.3, the kernel, $K_{i j}(t)$, in this convolution integral is commonly referred to as the impulse-response functions of the radiation problem. Section 2.4.2.1 described how the radiation impulse-response functions can be found from the solution of the frequency-domain radiation problem. In HydroDyn specifically, these functions are found using the cosine transform of the frequency-dependent hydrodynamicdamping matrix, as given in Eq. (2-28b), using a computationally efficient FFT routine [92]. As in the verification of the wave-elevation computation presented in Section 4.1.1, verifying that this cosine transform was implemented correctly is advantageous.

I performed this verification by testing that the radiation impulse-response functions computed within HydroDyn are the same as those computed by WAMIT's stand-alone F2T conversion utility. I implemented the cosine transform within HydroDyn, as opposed to having HydroDyn read in the output of WAMIT's F2T utility, because many of the other computer codes available to solve the frequency-domain hydrodynamics problem, such as the SWIM module [48] of SML, do not contain the F2T conversion functionality. In this test, I used the WAMIT output of the frequency-dependent hydrodynamic-damping matrix for the ITI Energy barge from the previously presented verification test.

Because the comparison between the F2T results and my own is so good (i.e., the results are essentially identical), I present only one set of results in Figure 4-6. As before, all data are dimensional as indicated, and because of the symmetries of the barge, the surge-surge elements are identical to the sway-sway elements, and the roll-roll elements are identical to the pitch-pitch elements. Most of the response decays to zero after about $20 \mathrm{~s}$ (as shown) and has all but vanished at $60 \mathrm{~s}$ (not shown). Consequently, to speed up the calculations of the memory effect in my simulation tool, I generally truncate the numerical convolution after $60 \mathrm{~s}$ of memory.

\subsection{Verification of the Mooring System Module}

I performed two verification tests to check my quasi-static mooring system module. First, as presented in Section 4.2.1, I verified that my mooring system module correctly solves a classic benchmark problem for the static equilibrium of a suspended-cable mechanism. Second, as presented in Section 4.2.2, I verified that the nonlinear force-displacement relationships for a mooring system in surge, as computed by my module, were the same as those calculated by an independent analysis performed by NAME.

\subsubsection{Benchmark Problem}

A classic test problem [95] for checking the accuracy of a mooring system program is that of a horizontally suspended cable with one support free to slide laterally. Figure 4-7 illustrates this problem. For a cable of an unstretched length of $L=200$, a weight per unit length of $\omega=0.1$, an extensional stiffness of $E A=10^{5}$, and a horizontal load (equivalent to the horizontal tension at the fairlead) of $H_{F}=5.77$ applied at the free end (the fairlead), the theoretical static-equilibrium solution is for a horizontal span of $x_{F}=152.2$ and a vertical sag of 58.0. (No units are specified for any parameter in Ref. [95]; instead, consistent units were assumed.) 

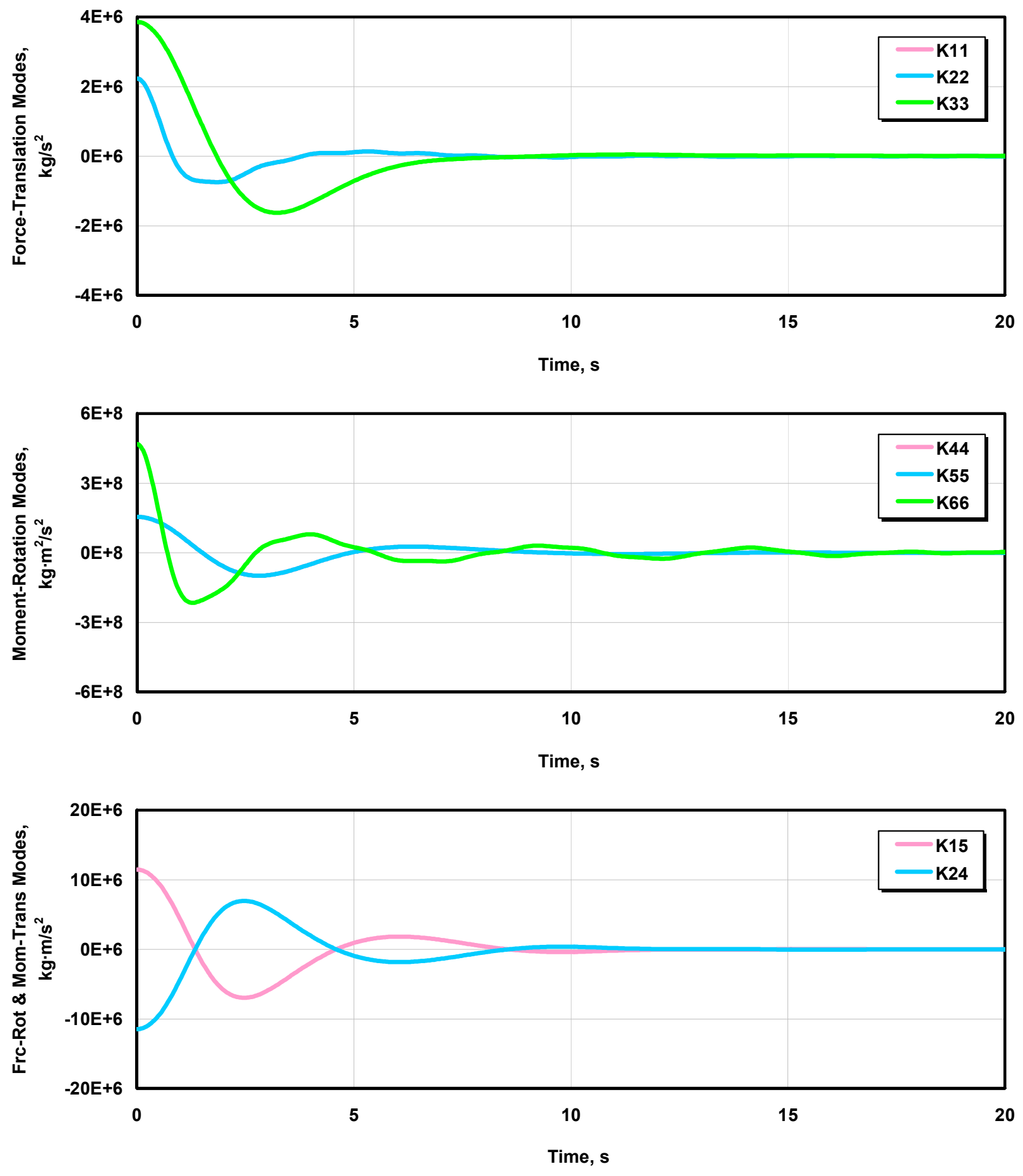

Figure 4-6. Radiation impulse-response functions for the ITI Energy barge

This benchmark problem involves finding a static-equilibrium position of the fairlead. I tested my mooring system module (in the form without seabed interaction) by solving this problem through time integration of the nonlinear equations of motion. The platform, where the fairlead attaches, was given one horizontal-translation DOF and a small, inconsequential mass. A small amount of linear damping was added to the motion to ensure that it eventually settled out. I then ran the time-marching solver until the solution settled out and converged. I had to solve the 


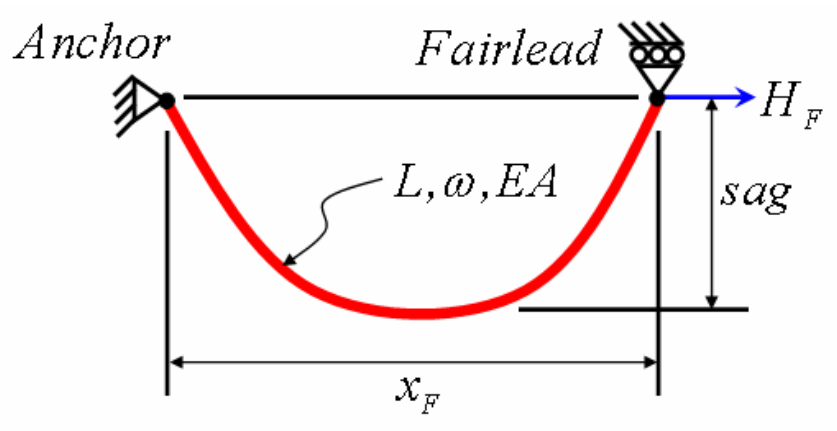

Figure 4-7. Benchmark problem for a suspended cable

static-equilibrium problem in this way because my mooring system module is interfaced to FAST and ADAMS, both of which operate in the time domain. If my mooring system module was implemented correctly, the horizontal span and vertical sag should settle out at the correct solution regardless of the lateral offset chosen as an initial condition for the DOF. Indeed, this is exactly what happens.

Figure 4-8 shows the time-series solution of the horizontal span (displacement) when the fairlead was positioned to the left of the anchor at time zero, at a lateral offset of -100 . FAST and ADAMS gave identical results. The solution is seen to converge to the correct result after about $120 \mathrm{~s}$. Other initial conditions showed similar behavior with the same result.

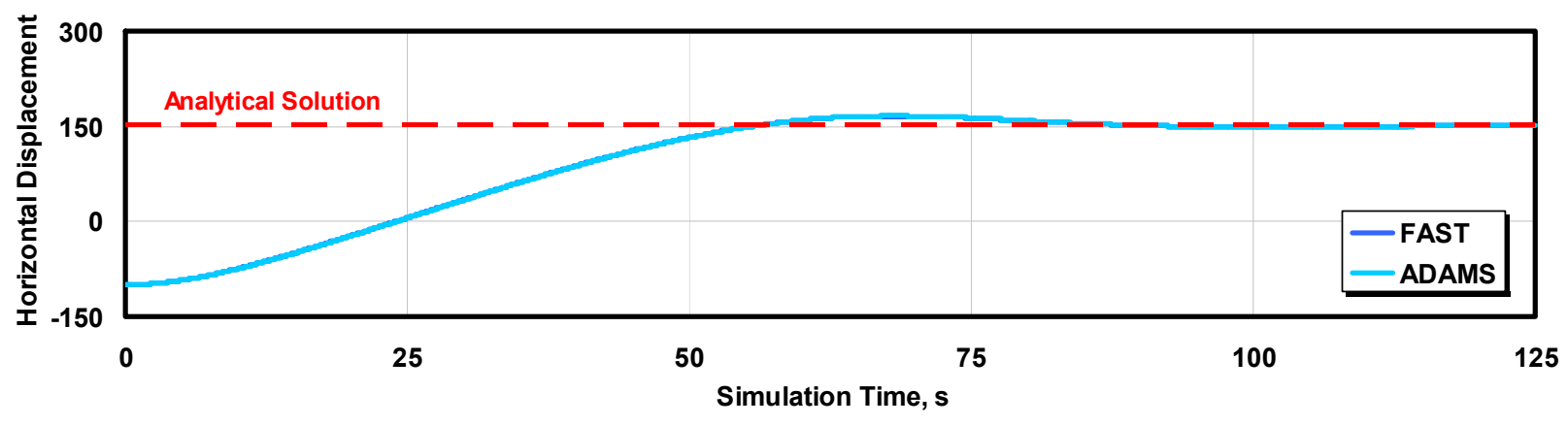

Figure 4-8. Solution of the suspended-cable benchmark problem

\subsubsection{Nonlinear Force-Displacement Relationships}

Nonlinearities are evident in the force-displacement relationships of most mooring systems. Because these nonlinearities may be important in the dynamic response of offshore floating wind turbines, I must check to ensure that my quasi-static mooring system module is computing them correctly.

NAME used a custom-made mooring analysis program to develop the mooring system for the ITI Energy barge described in Section 3.2. NAME's program accounts for homogenous taut or catenary lines with horizontal (but not vertical) elastic stretching. A portion of a line may rest on the seabed in NAME's mooring program, but the program does not account for seabed friction. Even though NAME's program has fewer capabilities than the mooring system module I have developed, comparing my response with NAME's enabled me to verify my analysis module in the form with seabed interaction. 
The layout of the mooring system for the ITI Energy barge, which consists of eight catenary lines, was discussed in Section 3.2. In this verification test, however, I modified the layout to make the mooring lines parallel to the sides of the barge because this is the only way NAME's program could model it. With this modification, each pair of lines is $90^{\circ}$ apart at the corner and opposing lines are parallel to each other. NAME computed the force-displacement relationships for surge motions of the barge for each line independently as well as opposing lines jointly. To reproduce NAME's results, I built a model of the barge and mooring system in ADAMS and translated the barge in surge through a time-marching simulation. This time-dependent motion of the barge does not affect the results of my analysis because the mooring lines are treated quasi-statically in my module.

As in previous verification tests, the results from this exercise compared very well. Because the agreement is so good (i.e., the results are essentially identical), again, only my results are presented, as shown in Figure 4-9. There is a horizontal tension of about $100 \mathrm{kN}$ in each line when the barge is in its neutral position. The force-displacement curve for opposing lines, which represents the net horizontal restraining force on the barge, remains fairly linear between $+20 \mathrm{~m}$ and $-20 \mathrm{~m}$ of surge motion. Beyond a surge displacement of about $40 \mathrm{~m}$, the resistance of the mooring system increases dramatically. At $50 \mathrm{~m}$ of surge displacement, the horizontal tension in each line is greater than $1,000 \mathrm{kN}$.

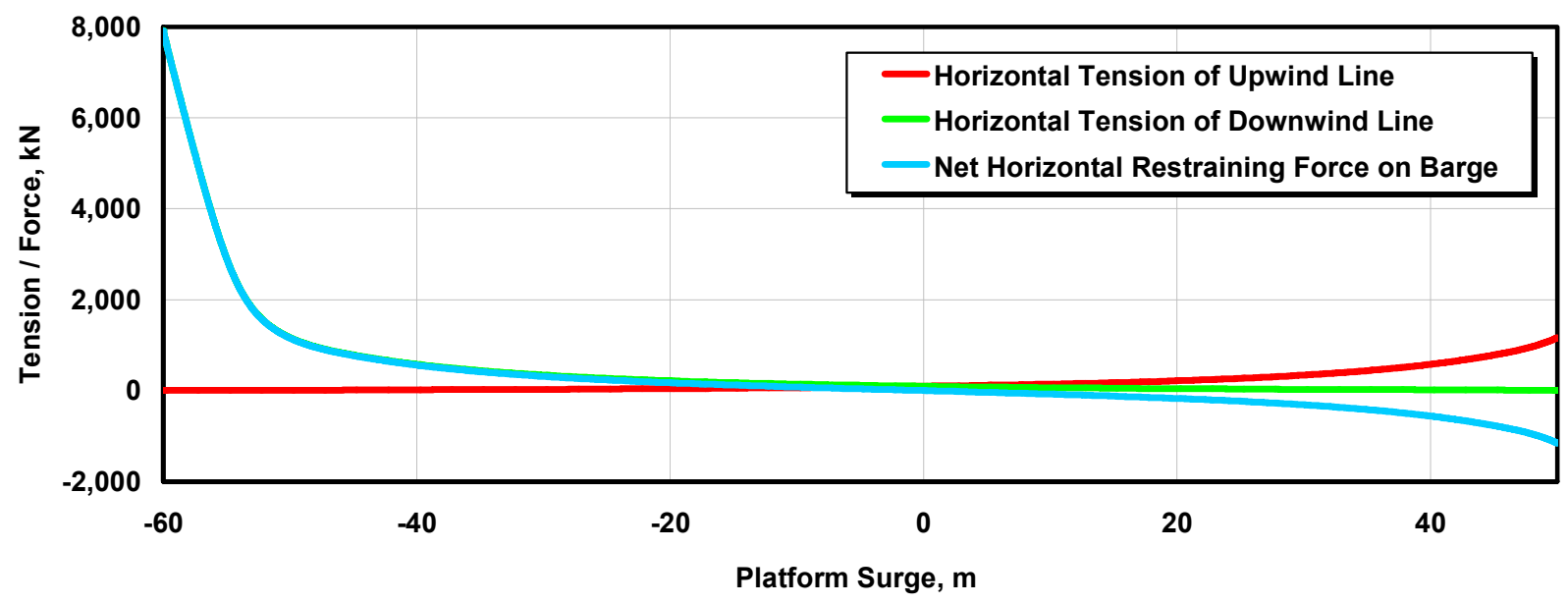

Figure 4-9. Force-displacement relationships for the ITI Energy mooring system

\subsection{Time Domain versus Frequency Domain Verification}

Because my fully coupled aero-hydro-servo-elastic simulation tool is the first of its kind to be developed, finding independent model results to use for verification is difficult. The timedomain models that others have previously developed and used to analyze offshore floating wind turbines were either not rigorous enough to yield sufficient verification data or were unavailable for my use $[23,31,57,75,87,103,105,106]$. Many of the previous studies related to offshore floating wind turbines used frequency-domain models $[13,59,98,100,101]$. I can use the results of a frequency-domain analysis to verify my simulation tool because the hydrodynamic theory in my module was derived from the time-domain representation of the frequency-domain problem (see Section 2.4.2.1). I present two such verifications here. 
Frequency-domain solutions describe the sinusoidal steady-state response of a platform to incident waves that propagate at a single amplitude, frequency, and direction. As discussed in Section 2.4.2.1, the solution to the frequency-domain problem is generally given in terms of RAOs, which are the complex-valued amplitudes of motions for each DOF of the support platform, normalized per unit of wave amplitude. In a time-domain model, the sinusoidal steady-state response of a floating platform can be found by introducing regular, periodic waves as forcing functions, and simulating in time long enough to ensure all transient behavior has died out. As a first verification of my fully coupled model, we ${ }^{1}$ used such time-series simulations to back out the RAOs at discrete incident-wave frequencies, and repeated the process to find the RAOs at each desired frequency. For this verification test, we used Wayman's frequencydomain results for the MIT / NREL SDB (see Ref. [101]).

As I also discussed in Section 2.4.2.1, the response of a floating platform to stochastic sea states in the frequency-domain problem can only be characterized statistically because the frequencydomain representation is not valid for transient analysis. Specifically, the motion of a linearized floating body will have a response that is Gaussian-distributed when it is excited by a sea state with a Gaussian-distributed wave elevation. The standard deviations of the motion response are dictated by the Wiener-Khinchine theorem [85,101]. In a time-domain model, the distributions of the motion response can be ascertained by postprocessing the output of a series of simulations that are long enough to ensure the the results are statistically reliable. (The process can be repeated to find the distributions at each desired sea state.) We used this procedure as a second verification of my fully coupled, time-domain model, again using Wayman's [101] frequencydomain results for the MIT / NREL SDB for comparison.

For these verification tests, we used the NREL offshore 5-MW baseline wind turbine described in Section 3.1 installed on the MIT / NREL barge (SDB) described in Section 3.3. I chose this configuration and Wayman's [101] frequency-domain results because that was the only configuration and the only study documented with enough information for me to build a system model and compare results for all six platform modes of motion.

Wayman used WAMIT to compute the frequency-domain hydrodynamic properties of the MIT / NREL SDB and modeled the spread-mooring system described in Section 3.3 with linear restoring only in the surge and sway DOFs. Wayman used the LINES module [50] of SML to find linear restoring coefficients of $4,000 \mathrm{kN} / \mathrm{m}$. The attributes of the wind turbine were included in Wayman's linearized system model by augmenting the body-mass matrix with the mass properties of the turbine and by augmenting the hydrodynamic-damping and -restoring matrices with damping and restoring contributions from rotor aerodynamics and gyroscopics. Wayman ignored the elasticity of the wind turbine and considered only the six rigid-body modes of the barge [101].

\footnotetext{
${ }^{1}$ My NREL colleague, M. L. Buhl, Jr., assisted me in running my simulation tool and plotting the results presented in Section 4.3. To acknowledge this support, I use "we" in place of "I" and "our" in place of "my" where appropriate.
} 


\subsubsection{Verification with Steady-State Response}

For this comparison, I constructed a FAST with AeroDyn and HydroDyn model of the NREL offshore 5-MW baseline wind turbine installed on the MIT / NREL barge. To ensure reasonable similarity to Wayman's model and to isolate the behavior of the hydrodynamics and mooring system, I modeled the turbine without any control system (i.e., using constant rotor speed and fixed blade pitch) or any modes of motion other than the six rigid-body DOFs of the floating support platform. For environmental conditions, a constant unsheared $11.2-\mathrm{m} / \mathrm{s}$ wind (as Wayman used) and regular periodic waves of unit amplitude (a peak-to-peak height of $2 \mathrm{~m}$ ) were used. Both the wind and waves were codirectional and aligned with the surge coordinate.

When we first attempted to run the time-domain simulations, I modeled the spread-mooring system with my quasi-static mooring system module interfaced to FAST. We soon discovered, however, that the nonlinear restoring of the spread-mooring system prohibited the response from ever reaching a sinusoidal steady-state condition, which eliminated any possibility of backing out the RAOs. To get around this, I decided to remove the interface to my mooring module, and instead, modeled the mooring system as Wayman did with linear restoring coefficients (in surge and sway only). As a consequence, the results presented next are not useful for verifying my time-domain implementation of the mooring system module. They are, though, still useful for verifying the time-domain implementation of my hydrodynamics module.

With the linearized mooring system model, we ran a series of 2,000-s simulations to give them time to reach a periodic steady state. Even after all that time, the platform motion was still not perfectly sinusoidal for the sway, roll, and yaw responses. We ran 10 simulations and varied the discrete frequency of the incident waves from 0.15 to $1.05 \mathrm{rad} / \mathrm{s}$ in even increments. Using the last cycle from each simulation, we computed the amplitudes of the oscillations for the three translational and three rotational platform responses. Because the incident waves were unit amplitude, these response amplitudes are equivalent to the magnitudes of the RAOs. For the rotational responses, we normalized the RAOs by the platform radius $(18 \mathrm{~m})$, as Wayman did [101]. We added our results to the nondimensional RAO plots that Wayman had generated. In these tests, we did not compare the phases of the response.

As shown in Figure 4-10, our time-domain predictions closely mimic those from Wayman's frequency-domain analysis for the platform-surge and -heave modes. This gave me confidence that my time-domain implementation of the platform hydrodynamics was correct. The platformpitch curves seem to have a similar character, but portions differ in both magnitude and frequency. The other three parameters - sway, roll, and yaw-have such small responses that comparison is difficult. Because the oscillations of these modes had not become completely sinusoidal after 2,000 s, we question whether those comparisons are meaningful. Even though there is no excitation of the platform-yaw mode from aerodynamics or hydrodynamics in this configuration, the yaw response is nonzero because the spinning inertia of the rotor, combined with the pitching motion of the platform, induces a gyroscopic yaw moment. 

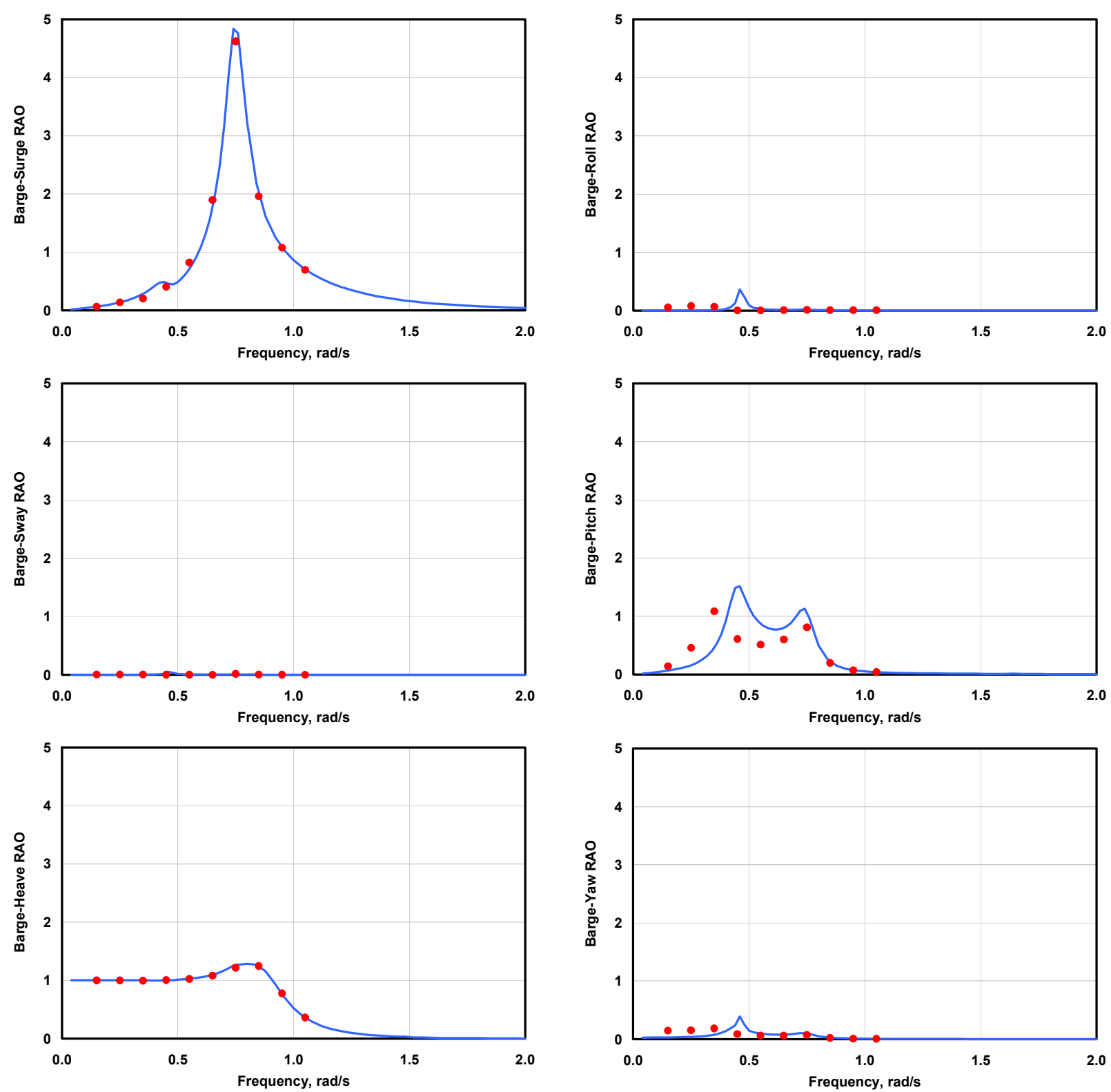

Figure 4-10. RAO comparisons for the MIT / NREL barge

I believe that the differences in the pitch RAO are caused by the variation between my model and Wayman's for the aerodynamic damping in pitch. Wayman showed that the platform damping in pitch is completely dominated by rotor aerodynamics, not by wave radiation (see Appendix A.1 of Ref. [101]). This is not true for the other modes of motion, such as surge and heave. In Wayman's analysis, the aerodynamic damping in barge pitch was constant (it was derived by using FAST with AeroDyn to linearize the rotor aerodynamic thrust about the mean pitch orientation of the platform). In my model, the aerodynamic damping in barge pitch varies as the turbine oscillates against and with the wind. 


\subsubsection{Verification with Stochastic Response}

To verify the stochastic response, I built three FAST with AeroDyn and HydroDyn models of the NREL offshore 5-MW baseline wind turbine installed on the MIT / NREL barge. The first was the same one used in the RAO comparison: it had a rigid turbine, no control system, and a linearized form of the mooring system in surge and sway. For the second model, I replaced the linearized mooring line model with the standard interface between FAST and my quasi-static mooring system module. To see how well these simpler models agreed with higher fidelity simulations, in the third model I replaced the rigid turbine with a fully flexible one and enabled the variable-speed generator-torque and blade-pitch control systems.

The published results [101] of Wayman's frequency-domain study included mean and standard deviations of the Gaussian-distributed responses at a variety of sea states, wind speeds, and water depths. I chose to compare all three of my models with only one of these cases. The case I chose used winds roughly at rated $(11.2 \mathrm{~m} / \mathrm{s})$, a water depth of $200 \mathrm{~m}$, and the same wave conditions considered in my test of the wave-elevation time series (see Section 4.1.1). We used steady unsheared winds in the first two models, but for the third model with an active control system, we used turbulent and sheared winds, with a mean hub-height speed of $11.2 \mathrm{~m} / \mathrm{s}$ and IEC category B turbulence [33]. As before, the wind and waves were codirectional and aligned with the surge coordinate.

For each model, we computed the probability densities for the output of all but the first $30 \mathrm{~s}$ of a series of four 10,000-s simulations (i.e., just shy of $3 \mathrm{~h}$ each), which used different random seeds for the stochastic waves (just as in Section 4.1.1). We constructed an aggregate of the four cases before computing the probability densities. We plotted our resulting histograms against the normal probability density functions derived from the means and standard deviations of Wayman's frequency-domain analysis $[101] .^{2}$

Figure 4-11 presents the comparison between our time-domain results and Wayman's frequencydomain results. Because the differences between the results of my second and third models were much smaller than the changes brought about by the switch to nonlinear mooring lines, the figure shows only the results from the first and third models. As with the RAOs, the surge and heave predictions from my model with the linearized mooring lines agree very well. The spread for the pitch response is narrower for our simulation with the linearized mooring system than it is in Wayman's predictions. This is consistent with what the pitch RAO comparison showed in Figure 4-10 - that is, Wayman's RAO was greater at $0.429 \mathrm{rad} / \mathrm{s}$ than the magnitude predicted by my model.

\footnotetext{
${ }^{2}$ Note that I had to make one correction to Wayman's results published in Ref. [101]. I discovered that when Wayman computed the standard deviations of motion for the rotational modes of the platform, the results were incorrectly dimensionalized. To correct for this mistake, all of the standard deviations of motion presented for the rotational modes in Ref. [101] must be scaled up by a factor of $\sqrt{180 / \pi}$ to reach the values Wayman meant to publish. The results presented here account for this correction.
} 

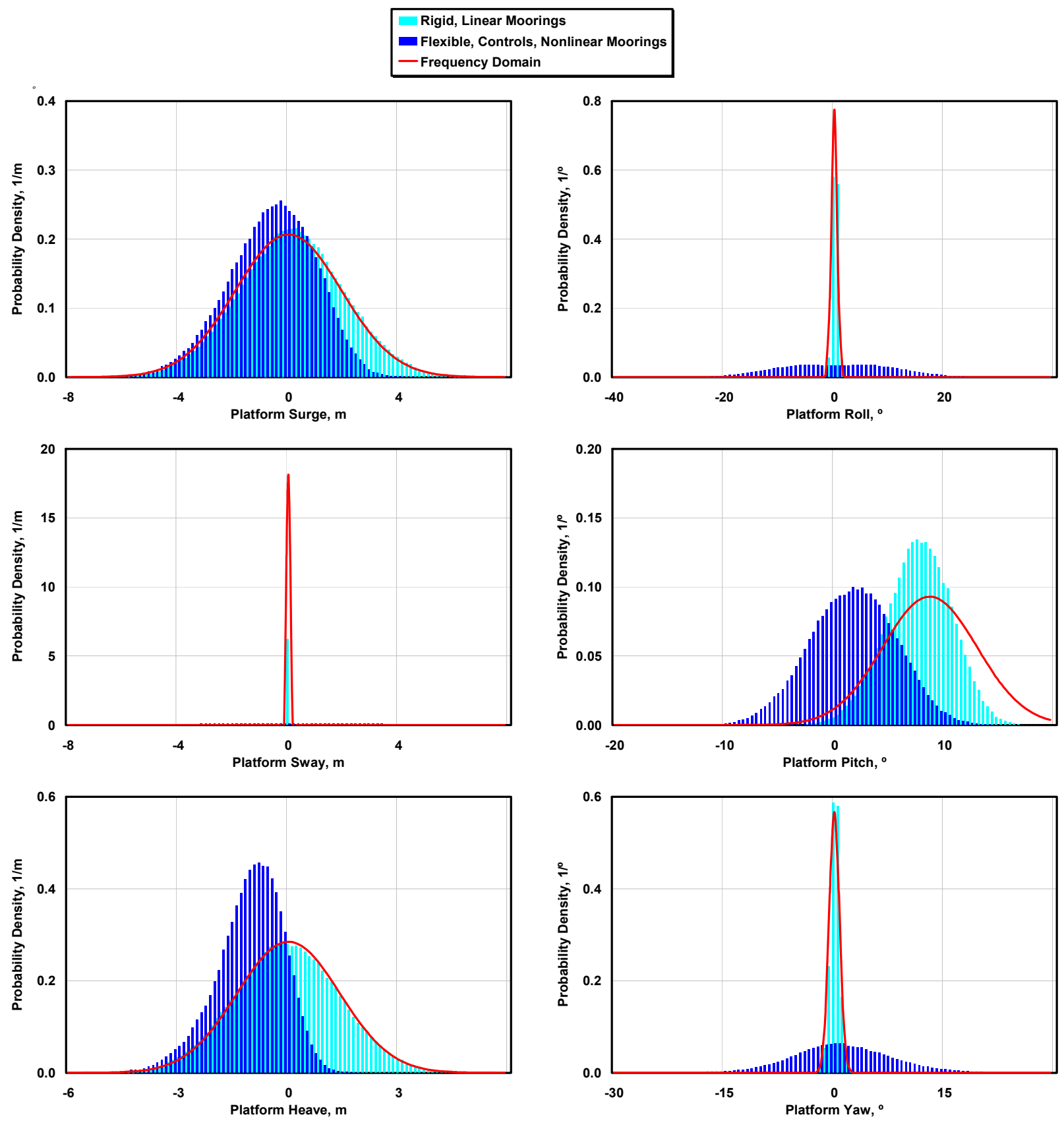

Figure 4-11. Probability density comparisons for the MIT / NREL barge

After introducing the nonlinear mooring system module into the FAST simulations, the mean surge, pitch, and heave responses decreased considerably (see Figure 4-11). This is because once the lines go taut, the stiffness increases dramatically and the mooring system essentially acts as a four-bar linkage. This keeps the platform from rising as high or from traveling as far downwind. The thrust on the rotor tries to pitch the turbine downwind, but the higher tensioned upwind mooring lines prevent the upwind end of the barge from lifting so far out of the water; the platform, in turn, is pushed slightly upwind. Because there is more coupling in the system in the higher fidelity model, the spread of values for the sway, roll, and yaw is also much greater than in the simpler model. 


\section{Chapter 5 Loads-Analysis Overview and Description}

I ran two preliminary sets of loads analyses. The first was for the NREL offshore 5-MW baseline wind turbine installed on land. Its aim was to establish the response of the baseline wind turbine without the effects of hydrodynamic loading or platform motion. The second loads analysis was for the same wind turbine mounted offshore on the floating ITI Energy barge. I used the same wind turbine control system in both analyses. Using the same turbine model (identical from the blade tip to the tower base) and control system in both the on- and offshore load sets has precedent because the design process prescribed in the IEC 61400-3 design standard [34] endorses deriving a sea-based wind turbine design from that of a land-based wind turbine.

Ultimately, for the wind turbine installed on the floating barge, design modifications will have to be made to ensure that favorable performance is achieved and structural integrity is maintained. Indeed, my loads analysis is just the first step in an iterative design process. And by starting with the simplest concept (i.e., an onshore wind turbine mounted atop an offshore barge), one can avoid unnecessary complication in the final design. Even though I ran only one step in the iterative process in this work, comparing the response of the floating system to the response of the turbine installed on land allowed me to quantify the impact brought about by the dynamic couplings between the turbine and floating barge in the presence of combined wind and wave loading. This comparison point outs where modifications must be made to arrive at a suitable design for the floating system. Such design modifications will have to be addressed through additional loads-analysis iterations in subsequent projects.

I used the IEC 61400-1 design standard [33] for land-based turbines and the IEC 61400-3 design standard [34] for sea-based turbines as guides for my preliminary loads analysis. The 61400-3 design standard is still in draft form, and discussion about its design requirement prescriptions continues. Moreover, the 61400-3 design standard explicitly states that "the design requirements specified in this standard are not necessarily sufficient to ensure the engineering integrity of floating offshore wind turbines" [34, p. 7]. For the purposes of my preliminary loads analysis (which is principally a feasibility study), however, I assumed that the stated design requirements were sufficient. I made no attempt to identify other possible platform-specific design conditions.

In Section 5.1, I present an overview and description of the simulations run in the land- and seabased loads analyses. Section 5.2 then discusses how we ${ }^{1}$ processed the loads-analysis data. Chapter 6 presents the results of the loads analyses.

\subsection{Design Load Cases}

Loads analysis involves verifying the structural integrity of a wind turbine by running a series of design load cases (DLCs) to determine the extreme (ultimate) and fatigue loads (i.e., forces and

\footnotetext{
${ }^{1}$ My NREL colleague, M. L. Buhl, Jr., developed the scripts used to run the loads analyses and assisted me in processing the loads-analysis data. To acknowledge this support, I use "we" in place of "I" and "our" in place of "my" where appropriate in Chapter 5.
} 
moments) expected over the lifetime of the machine. The loads are examined within the primary members of the wind turbine, including the blades, drivetrain, nacelle, and tower, and for the floating system, the mooring lines. The required DLCs cover essential design-driving situations such as normal operating conditions, start-up events, shutdown events, and parked or idling states, together with appropriate normal and extreme external conditions and likely fault scenarios.

Each IEC design standard prescribes numerous DLCs. For this preliminary loads analysis, I did not consider it necessary to run all the DLCs prescribed by the design standards; instead, I used a subset, eliminating the fatigue-type DLCs and processing only the anticipated ultimate loads. This omission follows from standard design practice for land-based and fixed-bottom sea-based wind turbines in which the structure is configured to survive ultimate loads before it is checked for fatigue [96]. Because fatigue behavior often governs the design of wind turbines, however, the effect of platform motion on wind turbine fatigue damage will have to be assessed by processing the omitted fatigue-type DLCs in a subsequent project.

As described in Section 3.1.6, the control system for the reference turbine does not include logic for start-up or shutdown sequences, so I eliminated the 3.x, 4.x, and 5.x DLCs defined in the design standards. I do, however, consider shutdowns that follow fault scenarios in DLC 2.x. I also ignored the 8.x cases, which relate to transport, assembly, maintenance, and repair. The four DLCs I omitted may have governed the ultimate loading of some historical wind turbines, but I believe omitting them was reasonable because, from my experience with land-based turbines, they have not dominated the ultimate loads.

The remaining ultimate-type DLCs included the following design situations: power production, DLC 1.x; power production with occurrence of fault, DLC 2.x; parked (idling), DLC 6.x; and parked with fault, DLC 7.x. Table 5-1 summarizes the DLCs I selected. In this table, the DLCs are indicated for each design situation by their associated wind conditions, wave conditions, and operational behavior of the control system, fault scenarios, and other events. For the land-based cases, I disregarded the wave conditions and cantilevered the base of the tower to the ground.

Table 5-1. Summary of Selected Design Load Cases

\begin{tabular}{|c|c|c|c|c|c|c|c|}
\hline \multirow[t]{2}{*}{ DLC } & \multicolumn{2}{|r|}{ Winds } & \multicolumn{3}{|c|}{ Waves } & \multirow[t]{2}{*}{ Controls / Events } & \multirow{2}{*}{$\begin{array}{l}\text { Load } \\
\text { Factor }\end{array}$} \\
\hline & Model & Speed & Model & Height & Direction & & \\
\hline \multicolumn{8}{|c|}{ 1) Power Production } \\
\hline 1.1 & NTM & $V_{\text {in }}<V_{\text {hub }}<V_{\text {out }}$ & NSS & $H_{s}=E\left[H_{s} \mid V_{\text {hub }}\right]$ & $\beta=0^{\circ}$ & Normal operation & $1.25 * 1.2$ \\
\hline 1.3 & ETM & $V_{\text {in }}<V_{\text {hub }}<V_{\text {out }}$ & NSS & $H_{s}=E\left[H_{s} \mid V_{\text {hub }}\right]$ & $\beta=0^{\circ}$ & Normal operation & 1.35 \\
\hline 1.4 & ECD & $V_{\text {hub }}=V_{r}, V_{r} \pm 2 \mathrm{~m} / \mathrm{s}$ & NSS & $H_{s}=E\left[H_{s} \mid V_{\text {hub }}\right]$ & $\beta=0^{\circ}$ & Normal operation; $\pm \Delta$ wind dir'n. & 1.35 \\
\hline 1.5 & EWS & $V_{\text {in }}<V_{\text {hub }}<V_{\text {out }}$ & NSS & $H_{s}=E\left[H_{s} \mid V_{\text {hub }}\right]$ & $\beta=0^{\circ}$ & Normal operation; $\pm \Delta$ ver. \& hor. shr. & 1.35 \\
\hline $1.6 a$ & NTM & $V_{\text {in }}<V_{\text {hub }}<V_{\text {out }}$ & ESS & $H_{s}=1.09^{*} H_{s 50}$ & $\beta=0^{\circ}$ & Normal operation & 1.35 \\
\hline \multicolumn{8}{|c|}{ 2) Power Production Plus Occurrence of Fault } \\
\hline 2.1 & NTM & $V_{\text {hub }}=V_{r}, V_{\text {out }}$ & NSS & $H_{s}=E\left[H_{s} \mid V_{\text {hub }}\right]$ & $\beta=0^{\circ}$ & Pitch runaway $\rightarrow$ Shutdown & 1.35 \\
\hline 2.3 & EOG & $V_{\text {hub }}=V_{r}, V_{r} \pm 2 \mathrm{~m} / \mathrm{s}, V_{\text {out }}$ & NSS & $H_{s}=E\left[H_{s} \mid V_{\text {hub }}\right]$ & $\beta=0^{\circ}$ & Loss of load $\rightarrow$ Shutdown & 1.10 \\
\hline \multicolumn{8}{|c|}{ 6) Parked (Idling) } \\
\hline $6.1 \mathrm{a}$ & EWM & $V_{\text {hub }}=0.95^{*} V_{50}$ & ESS & $H_{s}=1.09^{*} H_{s 50}$ & $\beta=0^{\circ}, \pm 30^{\circ}$ & Yaw $=0^{\circ}, \pm 8^{\circ}$ & 1.35 \\
\hline $6.2 a$ & EWM & $V_{\text {hub }}=0.95^{*} V_{50}$ & ESS & $H_{s}=1.09^{*} H_{s 50}$ & $\beta=0^{\circ}, \pm 30^{\circ}$ & Loss of grid $\rightarrow-180^{\circ}<$ Yaw $<180^{\circ}$ & 1.10 \\
\hline $6.3 a$ & EWM & $V_{\text {hub }}=0.95^{*} V_{1}$ & ESS & $H_{s}=1.09^{*} H_{s 1}$ & $\beta=0^{\circ}, \pm 30^{\circ}$ & Yaw $=0^{\circ}, \pm 20^{\circ}$ & 1.35 \\
\hline \multicolumn{8}{|c|}{ 7) Parked (Idling) and Fault } \\
\hline $7.1 \mathrm{a}$ & EWM & $V_{\text {hub }}=0.95^{*} V_{1}$ & ESS & $H_{s}=1.09^{*} H_{s 1}$ & $\beta=0^{\circ}, \pm 30^{\circ}$ & Seized blade; Yaw $=0^{\circ}, \pm 8^{\circ}$ & 1.10 \\
\hline
\end{tabular}


The wind and wave models are defined in Table 5-2 for readers who are unfamiliar with the IEC terminology.

In general, the 61400-3 sea-based design standard is a superset of the 61400-1 land-based design standard. When the two IEC design standards differed in details, I chose to use the specifications of the 61400-3 design standard for both my land- and sea-based loads analyses. This allowed me to compare the results fairly. For example, the normal wind profile that is used in both the deterministic- and turbulent-wind models should consist of a vertical power-law shear exponent of 0.2 for land-based wind turbines according to the 61400-1 design standard and a value of 0.14 for sea-based turbines according to the 61400-3 design standard. To facilitate the response comparisons, I decided to use 0.14 for both.

Table 5-2. Definition of Wind and Wave Models

\begin{tabular}{|c|c|c|}
\hline Abbr. & Definition & Description \\
\hline$E C D$ & $\begin{array}{l}\text { Extreme Coherent Gust with } \\
\text { Direction Change }\end{array}$ & $\begin{array}{l}\text { This deterministic-wind model consists of an unsheared gust superimposed on a uniform } \\
\text { wind profile with a vertical power-law shear exponent of } 0.14 \text {. The gust rises to } 15 \mathrm{~m} / \mathrm{s} \\
\text { over a } 10 \text {-s period. Occurring concurrently, the wind direction changes inversely } \\
\text { proportional to the given hub-height wind speed. Both positive and negative direction } \\
\text { changes are considered. }\end{array}$ \\
\hline EOG & Extreme Operating Gust & $\begin{array}{l}\text { This deterministic-wind model consists of an unsheared gust superimposed on a uniform } \\
\text { wind profile with a vertical power-law shear exponent of } 0.14 \text {. Over a } 10.5-\mathrm{s} \text { transient, } \\
\text { the gust first dips, rises to a maximum, then dips again before disappearing. Its } \\
\text { magnitude depends on the wind-turbine class (IB in this project) and increases with the } \\
\text { given hub-height wind speed. }\end{array}$ \\
\hline ESS & Extreme Sea State & $\begin{array}{l}\text { This irregular sea state is similar to the NSS but uses a JONSWAP spectrum that is } \\
\text { derived from 1- and } 50 \text {-year return values of the significant wave height and peak } \\
\text { spectral period. Like the NSS, the sea state is modeled as a summation of sinusoidal } \\
\text { wave components whose amplitude is determined by the wave spectrum, each parallel } \\
\text { (long-crested) and described by Airy wave theory. }\end{array}$ \\
\hline ETM & Extreme Turbulence Model & $\begin{array}{l}\text { This model is similar to the NTM but consists of full-field 3-component stochastic winds } \\
\text { with a higher turbulence standard deviation, based on the wind-turbine class (IB in this } \\
\text { project) and increases with the given hub-height wind speed. Like the NTM, the full-field } \\
\text { turbulence is superimposed on a normal wind profile with a vertical power-law shear } \\
\text { exponent of } 0.14 \text {. }\end{array}$ \\
\hline EWM & $\begin{array}{l}\text { Turbulent Extreme Wind } \\
\text { Model }\end{array}$ & $\begin{array}{l}\text { This model consists of full-field 3-component stochastic winds with a turbulence standard } \\
\text { deviation of } 0.11 \text { times the } 10 \text {-min average wind speed at hub height, plus } 0.2 \mathrm{~m} / \mathrm{s} \text { for } 1 \text {-h } \\
\text { long simulations. The full-field turbulence is superimposed on a wind profile with a } \\
\text { vertical power-law shear exponent of } 0.11 \text {. }\end{array}$ \\
\hline EWS & Extreme Wind Shear & $\begin{array}{l}\text { This deterministic-wind model consists of a linear shear superimposed on a uniform wind } \\
\text { profile with a vertical power-law shear exponent of } 0.14 \text {. Over a } 12 \text {-s transient, the shear } \\
\text { rises to a maximum, then decreases again before disappearing. Its magnitude depends } \\
\text { on the wind turbine turbulence category (B in this project) and increases with the given } \\
\text { hub-height wind speed. Positive and negative vertical and horizontal shears are } \\
\text { considered independently. }\end{array}$ \\
\hline NSS & Normal Sea State & $\begin{array}{l}\text { This irregular sea state is modeled as a summation of sinusoidal wave components } \\
\text { whose amplitude is determined by the wave spectrum, each parallel (long-crested) and } \\
\text { described by Airy wave theory. The sea state is derived from the JONSWAP spectrum, } \\
\text { whose formulation is based on the given values of the significant wave height and peak } \\
\text { spectral period. The JONSWAP spectrum reduces down to the Pierson-Moskowitz } \\
\text { spectrum in all but the most extreme sea states. }\end{array}$ \\
\hline NTM & Normal Turbulence Model & $\begin{array}{l}\text { This model consists of full-field 3-component stochastic winds with a turbulence standard } \\
\text { deviation given by the } 90 \% \text { quantile, based on the wind turbine turbulence category (B in } \\
\text { this project) and increases with the given hub-height wind speed. The full-field } \\
\text { turbulence is superimposed on a wind profile with a vertical power-law shear exponent of } \\
0.14 \text {. }\end{array}$ \\
\hline
\end{tabular}


The 61400-3 design standard specifies subsidiary cases for the DLCs involving extreme waves of 1- or 50-year recurrence because it is generally difficult to account for both the irregularity and nonlinearity of the extreme waves simultaneously within simulation. This, in turn, follows from the fact that models for nonlinear irregular wave kinematics are not common in engineering usage. The subsidiary DLCs involve analysis with (a) turbulent winds and stochastic sea states used in conjunction with full-system flexibility and dynamics, or (b and c) steady winds and deterministic nonlinear design waves used in conjunction with a quasi-steady computation with appropriate corrections for dynamic amplification. (The letters "a," "b," and "c" refer to subcases used in the 61400-3 design standard.) I chose the former method as indicated by the "a" in DLCs 1.6a, 6.1a, 6.2a, 6.3a, and 7.1a because it is not possible to model nonlinear waves in my simulation tool, which is based on the linearized radiation and diffraction method (see Chapter 2).

I ran all load-case simulations for both the land- and sea-based turbine configurations using FAST [39] v6.10a-jmj with AeroDyn [55,67] v12.60i-pjm and HydroDyn. I also reran some of the simulations in MSC.ADAMS v2005.2.0 with A2AD [20,54] v12.21a-jmj, AeroDyn v12.60ipjm, and HydroDyn to verify the responses predicted by FAST. (Unless otherwise specified, all results presented in this work were produced by FAST.) All simulations were run with all appropriate and available DOFs enabled. In FAST, these included - for the wind turbine - two flapwise and one edgewise bending-mode DOFs per blade, one drivetrain torsion DOF, one variable generator speed DOF, one nacelle-yaw DOF, and two fore-aft and two side-to-side tower bending-mode DOFs. For the floating system, three translational (surge, sway, and heave) and three rotational (roll, pitch, and yaw) DOFs were enabled for the platform.

In my loads analyses, I made a couple of small modifications to the properties of the NREL offshore 5-MW baseline wind turbine documented in Section 3.1 and to the FAST model given in Appendix A. (These modifications are included in the simulations presented in Chapter 5 and Chapter 6, but not in those presented in Chapter 3, Chapter 4, and Chapter 7.) To account for manufacturing variability, all loads-analysis simulations included a mass imbalance in the rotor, which instigates a once-per-rev excitation of the system when the rotor is spinning. I implemented the rotor-mass imbalance by making one blade $0.5 \%$ heavier and one blade $0.5 \%$ lighter than the mass of the nominal (reference) blade. This is the same way in which a mass imbalance was applied in the DOWEC study (as given on page 19 of Ref. [51]). I did not model an aerodynamic imbalance (such as different blade-pitch angles or twist distributions) because AeroDyn does not currently have that capability. All loads-analysis simulations also incorporated a blade structural-damping ratio of $2.5 \%$ critical, which is a higher amount of damping than the $0.477465 \%$ value mentioned in Section 3.1.1 and used in the DOWEC study (from page 20 of Ref. [51]). In my experience, the higher number is more representative.

For the power-production cases with and without faults, DLCs 1.x and 2.x, I used the quasisteady BEM axial-induction model with the Beddoes-Leishman dynamic-stall model in AeroDyn. I chose the BEM induction model over AeroDyn's GDW induction model because the latter is not suitable when the turbulent-wake state is approached (particularly at low wind speeds) [67]. I did not wish to see a change in response at the wind speed where I would have had to switch between the different models. Similarly, I chose the axial-induction model over AeroDyn's option for a combination of axial- and tangential- (rotational-) induction models because the tangential-induction model is not numerically stable at all rotor speeds under 
consideration (particularly the low rotor speeds during a shutdown event). In addition, I disabled both the BEM induction model and the dynamic-stall model in AeroDyn for DLCs 6.x and 7.1a, relying instead on simple lookup-table aerodynamics with geometric angles of attack. I made this choice because the BEM and dynamic-stall models are not applicable in parked (idling) cases, particularly at the very high post-stall angles of attack.

The generator-torque and blade-pitch control systems are operating properly and the turbine is producing power normally in DLCs 1.x and prior to the fault in DLCs 2.x. In DLCs 6.x and 7.x, the control system is disabled. Instead, the rotor is idling in these DLCs with no generator or brake reaction torques, and all blades are fully feathered to the maximum pitch setting of $90^{\circ}$ (exception: one blade is seized at the minimum set point in DLC 7.1a - see the next paragraph). As described in Section 3.1.6, the control system for the 5-MW baseline wind turbine does not include logic for the active control of nacelle yaw. In all DLCs, then, I secured the nacelle at given yaw angles with a spring and damper to represent compliance in the yaw drive. I describe the given nacelle-yaw angles in the following discussion of wind conditions.

For DLCs 2.x and 7.x, which involve fault conditions, the IEC design standards require choosing faults with the worst consequences. I chose common design-driving faults based on my experience with other land-based wind turbine loads analyses. For DLC 2.1, I simulated a fault in the rotor-collective blade-pitch control system where one blade ignores its command and runs away to the minimum set point of $0^{\circ}$ at the full pitch rate of $8^{\circ} / \mathrm{s}$. I assumed that the turbine's protection system detects this fault in this situation by simulating a shutdown of the turbine. The shutdown is initiated after a 0.2 -s delay (to account for the time it takes the protection system to detect the fault and take action) by feathering the other two blades at full pitch rate to the maximum pitch setting of $90^{\circ}$. For DLC 2.3, I simulated a fault where the load is lost, implying that the generator reaction torque is zero. In this situation, I again assumed that the turbine's protection system detects the fault and shuts down the turbine by feathering all blades after a $0.2-$ $\mathrm{s}$ delay at full pitch rate to the maximum pitch setting. For DLC 7.1a, I simulated the fault condition where one blade is seized at the minimum set point (i.e., flat into the wind) while idling with the other two blades fully feathered.

The hub-height wind speeds, $V_{h u b}$, considered within each DLC are listed in Table 5-1. In the turbulent-wind models (ETM, EWM, and NTM), $V_{h u b}$ represents the average hub-height wind speed over a simulation. In the deterministic-wind models (ECD, EOG, and ECD), $V_{h u b}$ represents the steady wind speed at hub height in the absence of the transient gust. For the cases where a wind-speed range is indicated from cut-in to cut-out, $V_{\text {in }}<V_{\text {hub }}<V_{\text {out }}$, I used a set of simulations with discrete values of $V_{h u b}$ centered within bins of $2 \mathrm{~m} / \mathrm{s}$ width (i.e., discrete values of $4,6, \ldots, 24 \mathrm{~m} / \mathrm{s}$ ). This resolution came from guidance in the IEC design standards. Even though the design standards recommend that DLC 2.1 be analyzed at all wind speeds between cut-in and cut-out, I chose to analyze this load case only at the rated $\left(V_{r}\right)$ and cut-out $\left(V_{\text {out }}\right)$ wind speeds, again based on my experience that they produce the highest loads. The extreme wind conditions were considered with the 1- and 50-year recurrence values of the mean reference hubheight wind speed, $V_{1}$ and $V_{50}$, respectively, as shown in Table 5-1.

We generated the turbulent full-field three-component wind conditions with TurbSim [36] v1.20. We used the Kaimal wind spectrum because TurbSim does not have the capability of generating turbulent-wind inflow with the IEC-recommended Mann model [33]. (The IEC design standards 
also allow for the use of the Kaimal wind spectrum.) We generated the deterministic-wind conditions with a customized copy of IECWind [56] v5.00. We had to customize IECWind so that it would generate wind inflow with a vertical power-law shear exponent of 0.14 because it was originally developed only for the 61400-1 design standard.

All winds were generated with a mean wind direction and a vertical inclination of the mean flow angle of $0^{\circ}$, except in DLC 1.4 where the wind direction departs from $0^{\circ}$ during the gust. In our simulations, a mean wind direction of $0^{\circ}$ implies that the rotor is aligned properly with the wind when the platform and nacelle are not displaced. For the power-production cases with and without faults, I aligned the rotor with the wind at the start of the simulation by securing the nacelle-yaw angle at $0^{\circ}$. For the parked (idling) cases, I also included nonzero-mean nacelle-yaw misalignments as directed by the design standards and indicated by the yaw specifications in Table 5-1. DLC 6.2a considers the full range of nacelle-yaw misalignments, $-180^{\circ}<$ Yaw $<$ $180^{\circ}$, because of an assumed inability of the nacelle-yaw controller to align the rotor with the wind when electrical power is unavailable because the grid is lost. To cover the range of yaw misalignments in this case, I used a set of simulations with discrete nacelle-yaw angles in increments of $20^{\circ}$ (i.e., discrete values of $-160^{\circ},-140^{\circ}, \ldots, 180^{\circ}$ ).

Per the guidance of the 61400-3 design standard (and as shown in Table 5-1), all normal irregular sea states (NSS) were considered with a significant wave height, $H_{s}$, given by the expected value conditioned on the relevant mean hub-height wind speed, $E\left[H_{s} \mid V_{h u b}\right]$, and based on the long-term joint-probability distribution of metocean parameters at the reference site (see Section 3.3). The range of peak spectral periods associated with each expected significant wave height, $T_{p}$, was split uniformly into three bins and was considered in the loads analysis by running three sets of simulations with discrete values of $T_{p}$ centered within those bins. The extreme stochastic sea states (ESS) were considered with the 1- or 50-year recurrence values of the significant wave height, $H_{s 1}$ and $H_{s 50}$, respectively. I used $H_{s 50}$ as a conservative estimate for the severe 50-year significant wave heights conditioned on the relevant mean hub-height wind speeds in DLC 1.6a. I did this because I did not have the opportunity to compute the latter values, which must be determined by extrapolating the appropriate site-specific metocean data such that the combination of the significant wave height and wind speed has a recurrence period of 50 years. This practice again follows the guidance of the 61400-3 design standard. As in the normal wind conditions, I ran three sets of simulations with discrete values of $T_{p}$ in the extreme sea states to represent the range of wave peak spectral periods associated with $H_{s l}$. But, in the simulations with extreme sea states using $H_{s 50}$, I only used one value of $T_{p}$ (the midpoint in the range) because the reference-site data included only a very small range of associated peak spectral periods.

I considered wave propagation to be codirectional with the winds in DLCs 1.x and 2.x, $\beta=0^{\circ}$, except in DLC 1.4 where the wind direction departs from the wave direction during the gust. For the parked (idling) cases, I also included wave misalignments as directed by the 61400-3 design standard and indicated by the $\beta$ specifications in Table 5-1. The design standard requires one to consider wind and wave misalignments of up to $30^{\circ}$ before reducing the severity of the sea state, so in DLCs 6.x and 7.1a I considered three wave heading directions, one aligned with the wind and two misaligned with the wind by $\pm 30^{\circ}$. 
The design standards specify the minimum quantity and length of each simulation in each load case. More than one simulation is required for each pair of turbulent-wind and stochastic-wave conditions to obtain statistically reliable results. The simulations at each pair of turbulent-wind and stochastic-wave conditions were differentiated by choosing varying seeds in their respective pseudo-random number generators. I paired the wind and wave seeds so that when $n$ seeds were required, I ran $n$ total simulations instead of all $n^{2}$ combinations of the two seeds.

For DLCs 1.1 and 1.3, the 61400-3 design standard requires that six 10-min simulations ${ }^{2}$ be run at each wind and wave condition, differentiated with variations in the wind and wave seeds. For DLCs 1.4 and 1.5, the design standard requires six 1-min simulations at each wind and wave condition, differentiated with variations in the wave seed (the deterministic-wind models do not require random seeds to be specified). For DLC 2.1, the design standard requires twelve 10-min simulations at each wind and wave condition, differentiated with variations in the wind and wave seeds. For DLC 2.3, the design standard requires six 1-min simulations at each wind and wave condition, differentiated with variations in the wave seed and the time at which the load is lost relative to the gust. Finally, for cases with extreme sea states-DLCs 1.6a, 6.x, and 7.1a-the design standard requires six 1-h simulations at each wind, wave, and nacelle-yaw condition, differentiated with variations in the wind and wave seeds. In this last group of DLCs, a factor of 1.09 is needed to scale the 1- and 50-year recurrence values of significant wave heights that correspond to a $3-\mathrm{h}$ reference period to the 1-h length of the simulation. Similarly, a factor of 0.95 is needed to scale the 1 - and 50-year recurrence values of the 10-min average wind speeds to the 1-h length of the simulation. These scale factors also come from the 61400-3 design standard.

For the power-production cases with and without faults, I initialized the rotor speed and bladepitch angles based on the given mean hub-height wind speed for each simulation to mitigate the start-up transient behavior, which is an artifact of the computational analysis. I initialized the rotor speed and blade-pitch angles to the values they would trim to in the land-based wind turbine (see Section 3.1.8), based on the action of the control system if the given wind speeds were steady and uniform. Nevertheless, I added $30 \mathrm{~s}$ to the required simulation times before outputting simulation data to eliminate any remaining start-up transient behavior that may have spuriously affected my loads predictions. Thus, I actually ran the 1-min simulations for $90 \mathrm{~s}$, and so on. All of the transient gusts, shears, and direction changes in the deterministic-wind models were initiated $60 \mathrm{~s}$ into the simulation (i.e., $30 \mathrm{~s}$ after the end of the 30 -s start-up transient). The blade-pitch control system faults in DLC 2.1 were also initiated $60 \mathrm{~s}$ into the simulation. The loss of load in DLC 2.3 was initiated at varying times during the 10.5-s gust, depending on the random seed.

Accounting for all of the combinations of wind conditions, wave conditions, and control scenarios, together with the number of required seeds, I ran a total of 2,190 separate sea-based simulations and 452 separate land-based simulations in my loads analysis. To manage the quantity and variety of simulations, we developed and utilized custom-made scripts written in Perl and the Windows batch command language. Using scripts greatly reduced the chance of

\footnotetext{
${ }^{2}$ For all simulations ran for less than $1 \mathrm{~h}$, I generated wave-elevation records based on $1 \mathrm{~h}$ to ensure that I captured an appropriate frequency content.
} 
mistakes (and eliminated a great deal of tedium). The main Perl script used an input file to specify which of the aforementioned DLCs would be run and with what specific parameters. This script was developed to process all the cases sequentially on one computer, or in parallel using the job-queuing utility known as Condor, ${ }^{3}$ which permits one to distribute a set of simulations among all idle computers on a network. We used the sequential method only for debugging. Using Condor on the 45 to 60 available networked processors at NREL / NWTC enabled me to run most of the DLC simulations for each loads analysis overnight. (If I had had to run them all sequentially on a single computer, it would have taken just over three weeks of processing time per loads analysis!)

\subsection{Postprocessing and Partial Safety Factors}

In addition to examining the time-series output from simulations, we processed all of the loadsanalysis data using the postprocessing computer program Crunch [11] v3.00.00, called with another custom-made Windows batch script. We processed each DLC separately in Crunch because the processing requirements varied by DLC and because Crunch cannot process files of different lengths. Because of memory restrictions, we had to run Crunch on a 64-bit server with $16 \mathrm{~GB}$ of random access memory (RAM) to hold the biggest DLC data set in memory all at the same time. We processed the loads data with Crunch in two different ways. First, we had Crunch compute the statistics (i.e.; minimum, mean, and maximum value; standard deviation; skewness; and kurtosis) of each output parameter for each simulation in each DLC. These data enabled me to characterize the dynamic response of the land- and sea-based systems under the influence of the wind conditions, wave conditions, and control scenarios pertinent to each DLC. Second, we had Crunch generate extreme-event tables for each DLC. These tables list the extreme minimum and maximum loads for a group of similar output parameters, along with the associated values of the other parameters that occur when the extreme load is reached. The tables also list the specific simulation that triggered the extreme loads and the times at which they occurred, as well other information that may be relevant to the event, such as instantaneous hub-height wind speed and wave elevation.

As Crunch read in the simulation output for the extreme-event processing, we had it apply partial safety factors (PSFs) to the blade tip-to-tower clearance outputs, to the internal loads in the wind turbine, and for the floating system, to the tensions in the mooring lines. We did not apply the PSFs to other output parameters, including the blade-tip and tower-top deflections; the floating platform displacements; and the control actions such as the generator-torque and power output, and the blade-pitch angles. The PSFs for loads, as specified in the IEC design standards, varied by DLC, and I document them in the last column of Table 5-1. In addition, an extra factor of 1.2 is stated for DLC 1.1. The IEC design standards require that the ultimate loads predicted under normal operation with normal wind-turbulence and stochastic-wave conditions be based on the statistical extrapolation of the load response. To eliminate this extra step, I decided to use a rule of thumb resulting from experience with other land-based loads-analysis exercises. From my and others' experiences, the extrapolation typically increases the predicted ultimate load by $20 \%$. This factor is further justified by the example extrapolation given in Appendix F of the 61400-1 design standard [33]. So I increased the normal 1.25 load PSF for DLC 1.1 by $20 \%$, to a value of

\footnotetext{
${ }^{3}$ Web site: $\underline{\text { http://www.cs.wisc.edu/condor/ }}$
} 
1.5. I did not, however, increase the load PSF for the calculation of the blade tip-to-tower clearance outputs in DLC 1.1 as per the design standard, which says that one should not extrapolate deflections.

According to the IEC 61400-1 design standard [33], the PSFs for loads "take account of possible unfavorable deviations / uncertainties of the load from the characteristic value [and] uncertainties in the loading model." We applied the PSFs to the loads in our extreme-event processing to enable a useful comparison of the loads between the DLCs. In other words, it is necessary to weight each DLC properly when determining the DLC that causes the overall ultimate (maximum) load because loads from abnormal design situations, which are less likely to occur, should be given a lower weighting (and are given a lower load PSF) than normal loads that are more likely to occur. To obtain the global extremes across all DLCs, we combined all of the extreme-event tables from each DLC using a slightly customized copy of the Perl script CombEEv [10] v1.20. We had to customize CombEEv so that it would not only generate the global extreme-event tables, but also the absolute extremes for each output parameter (i.e., the absolute maximum value of the minima and maxima). I used the absolute extremes for each output parameter to compare the land- and sea-based loads results.

The IEC design standards also document PSFs for materials and consequences of failure. I did not apply these, however, because they are the same across all load cases. This means that they will cancel out in the comparison. I also made no attempt to compare the load predictions to the material or buckling strengths of the individual components. 


\section{Chapter 6 Loads-Analysis Results and Discussion}

I ran loads analyses for extreme (ultimate) loads using the simulation capability documented in Chapter 2; the properties of the wind turbine, floating ITI Energy barge, and reference site described in Chapter 3; and the load-case conditions and procedures explained in Chapter 5. I now present the results of this analysis. Because of the sheer volume of results, which includes more than $100 \mathrm{~GB}$ of data, I cannot present them all. Instead, I focus on results that are characteristic of the overall system responses.

My loads analysis helped to identify problems with both the land- and sea-based system configurations. I discovered a side-to-side instability in the tower of the idling land-based wind turbine when $w^{1}$ were processing the loads-analysis data for DLC 6.2a. In the sea-based system, I discovered an instability in the yaw motion of the floating platform that manifested itself in the fault conditions of DLCs 2.1 and 7.1a. Finally, I determined that the floating barge system is susceptible to excessive platform-pitching motion in large and / or steep waves, especially in extreme waves such as those occurring during 1- and 50-year events in DLCs 1.6a, 6.x, and 7.1a. These design problems all led to unreasonable loading of the wind turbine, which dominated the final predictions in ultimate loads.

To gain insight into the dynamic behavior of the onshore and floating systems and to enable a fair comparison between the two systems, I split the results into groups and present each group separately. In Section 6.1, I present the land- and sea-based results for DLCs 1.1, 1.3, 1.4, and 1.5 , which consider the wind turbine in normal operation with a variety of external wind and wave conditions, not including extreme 1- or 50-year events. These results embody the response of the systems unencumbered by the aforementioned design problems. I then present (Section 6.2) the findings from the other load cases, DLCs 1.6a, 2.x, 6.x, and 7.1a, which are concerned with the wind turbine when it is experiencing a fault, when it is idling, and / or when it is being excited by 1- and 50-year wind and wave conditions. My presentation of this latter group of DLCs includes a description of the ensuing design problems and possible mitigation measures.

\subsection{Normal Operation}

I processed the loads-analysis results from the normal operation cases to characterize the dynamic response of the land- and sea-based systems (Section 6.1.1); to identify the designdriving conditions and quantify the resulting ultimate loads (Sections 6.1.2 and 6.1.3, respectively); and to measure the impact of installing the wind turbine on the ITI Energy barge (Section 6.1.4). Section 6.1.5 draws conclusions from this analysis.

\footnotetext{
${ }^{1}$ My NREL colleague, M. L. Buhl, Jr., assisted me in processing the loads-analysis data. Another NREL colleague, Dr. G. S. Bir, assisted me in examining the instabilities. To acknowledge this support, I use "we" in place of "I" and "our" in place of "my" where appropriate in this chapter.
} 


\subsubsection{Characterizing the Dynamic Response}

Figure 6-1 presents the minimum, mean, and maximum values from each simulation in DLC 1.1 for several output parameters. These values are not scaled by the PSFs for loads described in Section 5.2. The statistics from both the land- and sea-based systems are presented side by side. The results for the floating system are further grouped by the peak spectral period of the incident waves in each sea state.

The mean values (indicated by the middle dots) of all parameters are very similar between land and sea, except for the mean value of the platform pitch, which is zero for the land-based wind turbine because its tower is cantilevered to the ground at its base. The mean values also correlate well with the steady-state responses presented in Figure 3-12. As in Figure 3-12, the mean value of the rotor speed in Figure 6-1 increases linearly with mean hub-height wind speed below rated $(11.4 \mathrm{~m} / \mathrm{s})$ to maintain constant tip-speed ratio and optimal wind-power conversion efficiency. Similarly, the mean generator power and rotor torque increase dramatically with wind speed up to rated, increasing cubically and quadratically, respectively. Above rated, the mean generator power is held constant by regulating to a fixed speed with active blade-pitch control and a generator torque that is inversely proportional to the generator speed. The mean values of the out-of-plane tip deflection and root-bending moment of the reference blade (Blade 1) reach a maximum at the rated operating point before dropping again. This response characteristic is the result of a peak in rotor thrust at rated (not shown in Figure 6-1, but seen in Figure 3-12). This peak in response is also visible, though less pronounced, in the mean values of the platform pitch, tower-top fore-aft displacement, and tower-base fore-aft bending moment.

The mean values are similar between land and sea, but Figure 6-1 shows that the excursions of the minimum and maximum values (indicated by the lower and upper horizontal dashes, respectively) in the sea-based results are much larger. The widest spread between the minimum and maximum values in the land-based simulations occurs in the generator power parameter just above rated. This is a result of the large difference in control actions when switching between Regions 2 and 3 while operating in turbulent winds near optimal wind-power conversion efficiency. The excursions of minimum and maximum values for all parameters in the sea-based simulations, however, increase with wind speed. More precisely, they increase with the pitch motion of the floating platform, which increases with wind speed. This is because the barge has a natural tendency to move with the surface waves and because the expected value of the significant wave height increases with wind speed, as shown in Figure 3-15. The pitching of the barge causes large variations in the generator power and rotor speed, which may lead to a loss of energy capture and an increase in aeroacoustic emissions. The pitching of the barge also causes large load excursions - more so for the tower-base loads than for the loads in the blades and drivetrain - because the floating system acts as an inverted pendulum, with the largest effect from inertia loading nearest the pivot point. The magnitudes of the minimum and maximum loads in the floating system are largest with sea states derived from large significant wave heights and from peak spectral periods in the range of 10 to $15 \mathrm{~s}$. The wave-period range of 10 to $15 \mathrm{~s}$ is particularly dominant because the resulting waves are more likely to excite the rigidbody - turbine plus barge - pitch mode. That mode has a natural frequency of about $0.0863 \mathrm{~Hz}$, which equates to a natural period of about $11.6 \mathrm{~s}$. So even though the expected significant wave height is lower at a mean hub-height wind speed of $22 \mathrm{~m} / \mathrm{s}$ than at $24 \mathrm{~m} / \mathrm{s}$, the loads in the 

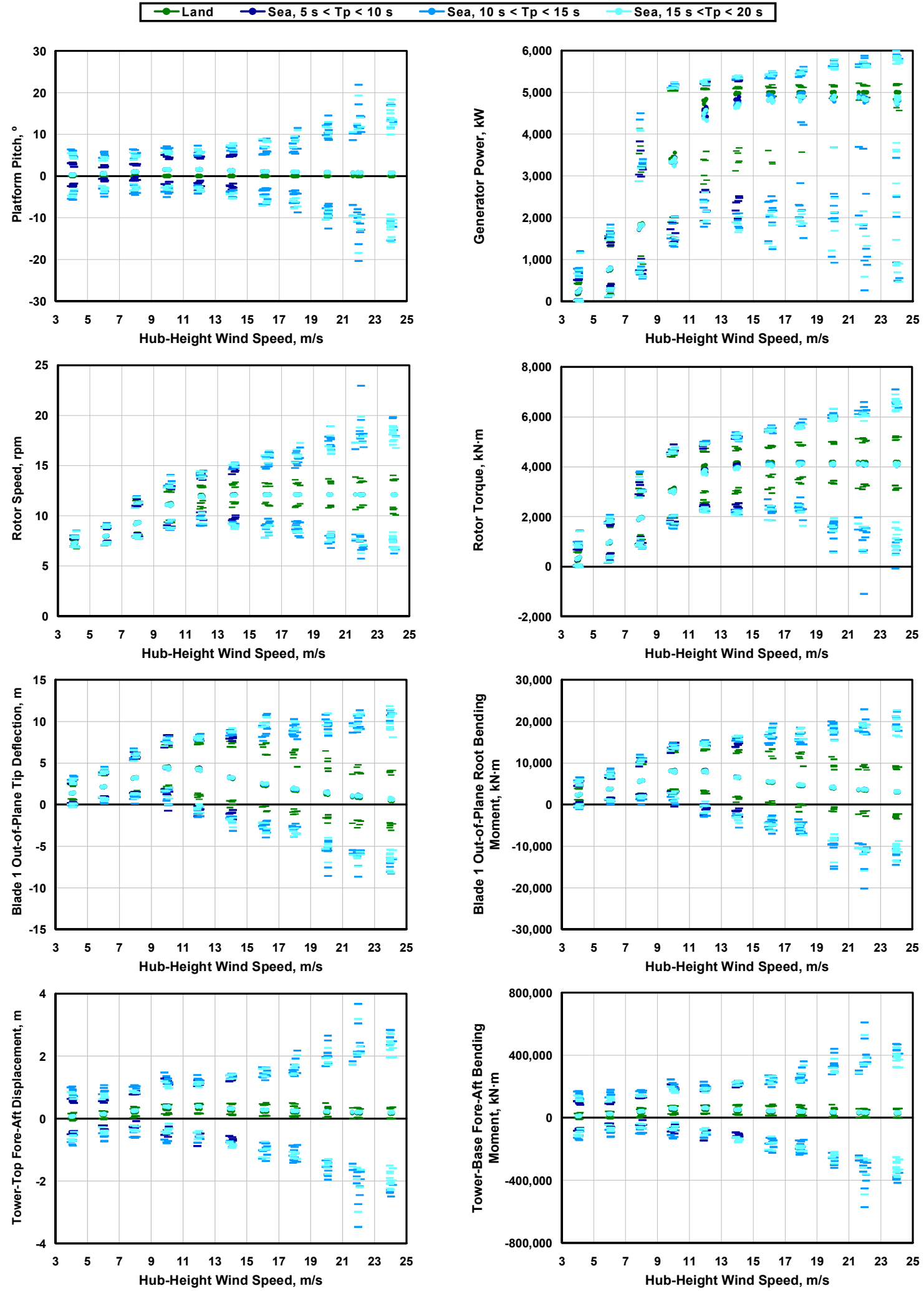

Figure 6-1. Statistics from each simulation in DLC 1.1

floating system are higher at $22 \mathrm{~m} / \mathrm{s}$ than at $24 \mathrm{~m} / \mathrm{s}$, where the wave periods are higher (but outside the critical wave-period range). 
Similar statistical trends exist for both the land- and sea-based system responses in DLC 1.3 (not shown). The only difference is that some of the output parameters, particularly for the landbased wind turbine, have slightly larger load excursions that result from increased turbulence in the wind inflow.

\subsubsection{Identifying Design-Driving Load Cases}

I identified the design-driving load conditions and quantified the resulting ultimate loads by examining the extreme-event tables. We generated 21 tables for the land-based loads and 32 tables for the sea-based loads. Each table contains a distinct group of similar output parameters, such as the internal loads in the blades, drivetrain, nacelle, and tower, and for the floating system, tensions in the mooring lines. The extreme events for the root moments of the reference blade (Blade 1) in the land- and sea-based analyses are presented in Table 6-1 and Table 6-2, respectively. The extreme events for the tower-base moments in the land- and sea-based analyses are presented in Table 6-3 and Table 6-4, respectively. All of the extreme-event tables we generated for both the land- and sea-based loads analyses are contained in Appendix F.

Table 6-1. Extreme Events for the Blade 1 Root Moments - Land

\begin{tabular}{|c|c|c|c|c|c|c|}
\hline Parameter & Type & File & $\begin{array}{r}\text { Root } \mathrm{Mxc1} \\
(\mathrm{kN}-\mathrm{m})\end{array}$ & $\begin{array}{r}\text { RootMyc1 } \\
(\mathrm{kN}-\mathrm{m}) \\
\end{array}$ & $\begin{array}{r}\text { RootMze1 } \\
(\mathrm{kN}-\mathrm{m})\end{array}$ & $\begin{array}{r}\text { Time } \\
\text { (sec) }\end{array}$ \\
\hline RootMxcl & Min & DLC1.3_0063_Land_24.0v0_s03. out & $-7.48 \mathrm{E}+03$ & $-2.13 \mathrm{E}+03$ & $-1.68 \mathrm{E}+02$ & $5.99 \mathrm{E}+02$ \\
\hline RootMxcl & Max & DLCl.1_0061_Land_24.0V0_s01. out & 1. $11 \mathrm{E}+04$ & 1. $15 \mathrm{E}+04$ & $-1.11 \mathrm{E}+02$ & $5.66 \mathrm{E}+02$ \\
\hline RootMycl & Min & DLC1.4_0006_Land_ECD-R+20. out & $1.93 \mathrm{E}+03$ & $-6.81 \mathrm{E}+03$ & $-2.24 \mathrm{E}+02$ & $6.92 \mathrm{E}+01$ \\
\hline RootMycl & $\operatorname{Max}$ & DLCl.4_0002_Land_ECD+R. out & $-7.78 \mathrm{E}+02$ & $2.22 \mathrm{E}+04$ & $1.18 \mathrm{E}+02$ & $7.90 \mathrm{E}+01$ \\
\hline RootMzcl & Min & DLC1. 4 0006 Land ECD $-\mathrm{R}+20$. out & $-1.75 \mathrm{E}+02$ & $-5.18 \mathrm{E}+03$ & $-2.81 \mathrm{E}+02$ & $6.88 \mathrm{E}+01$ \\
\hline RootMzcl & $\operatorname{Max}$ & DLC1.3_0038_Land_16.0v0_S02.out & $-2.00 \mathrm{E}+03$ & 1. $77 \mathrm{E}+04$ & $2.11 \mathrm{E}+02$ & 1. $53 \mathrm{E}+02$ \\
\hline
\end{tabular}

Table 6-2. Extreme Events for the Blade 1 Root Moments - Sea

\begin{tabular}{|c|c|c|c|c|c|c|}
\hline Parameter & Type & File & $\begin{array}{r}\text { Root } \mathrm{MkC1} \\
(\mathrm{kN} \cdot \mathrm{m}) \\
\end{array}$ & $\begin{array}{r}\text { RootMyc1 } \\
(\mathrm{kN}-\mathrm{m}) \\
\end{array}$ & $\begin{array}{r}\text { RootMze1 } \\
(\mathrm{kN} \cdot \mathrm{m}) \\
\end{array}$ & $\begin{array}{r}\text { Time } \\
(s e c)\end{array}$ \\
\hline RootMxcl & Min & DLC1.1_0149_Sea_20.0V0_04.4Hs_14.1Tp_S02.out & $-1.21 \mathrm{E}+04$ & $-1.28 \mathrm{E}+04$ & $-2.26 \mathrm{E}+02$ & $1.96 \mathrm{E}+02$ \\
\hline RootMxcl & $\operatorname{Max}$ & DLC1.1_0190_Sea_24.0V0_05.5Hs_12.7Tp_s04.out & 1. $55 \mathrm{E}+04$ & $1.66 \mathrm{E}+04$ & $-1.43 \mathrm{E}+02$ & $5.92 \mathrm{E}+02$ \\
\hline RootMycl & Min & DLC1.1_0164_Sea_22.0V0_04.7Hs_13.4Tp_s01.out & $7.43 \mathrm{E}+03$ & $-3.03 \mathrm{E}+04$ & $6.87 \mathrm{E}+02$ & $2.60 \mathrm{E}+02$ \\
\hline RootMycl & $\operatorname{Max}$ & DLCl.1_0164_Sea_22.0V0_04.7Hs_13.4Tp_s01.out & 4. $72 \mathrm{E}+03$ & $3.43 \mathrm{E}+04$ & $3.15 \mathrm{E}+02$ & $2.56 \mathrm{E}+02$ \\
\hline RootMzcl & Min & DLC1.4_0030_Sea_ECD+R_02.4Hs_17.6Tp_S04.out & $-2.14 \mathrm{E}+03$ & $-8.11 \mathrm{E}+03$ & $-4.70 \mathrm{E}+02$ & $8.80 \mathrm{E}+01$ \\
\hline RootMzcl & $\operatorname{Max}$ & DLCl.1_0164_Sea_22.0V0_04.7Hs_13.4Tp_S01.out & $7.21 \mathrm{E}+03$ & $-2.99 \mathrm{E}+04$ & $7.21 \mathrm{E}+02$ & $2.60 \mathrm{E}+02$ \\
\hline
\end{tabular}

Table 6-3. Extreme Events for the Tower-Base Moments - Land

\begin{tabular}{|c|c|c|c|c|c|c|}
\hline parameter & Type & File & $\begin{array}{r}\text { TwrBsMxt } \\
(\mathbf{k N}-\mathbf{m})\end{array}$ & $\begin{array}{r}\text { TwrBsMyt } \\
(\mathbf{k N}-\mathrm{m}) \\
\end{array}$ & $\begin{array}{r}\text { TwrBsMzt } \\
(\mathbf{k N}-\mathbf{m})\end{array}$ & $\begin{array}{r}\text { Time } \\
\text { (sec) }\end{array}$ \\
\hline TwrBsMxt & Min & DLC1.3_0057_Land_22.0V0_S03. out & $-2.77 \mathrm{E}+04$ & $2.78 \mathrm{E}+04$ & $-8.14 \mathrm{E}+02$ & 4. $83 \mathrm{E}+02$ \\
\hline TwrBsMxt & $\operatorname{Max}$ & DLCl.3_0064_Land_24.0V0_S04. out & $4.09 \mathrm{E}+04$ & $2.64 \mathrm{E}+04$ & $1.83 \mathrm{E}+03$ & $9.16 \mathrm{E}+01$ \\
\hline TwrBsMyt & Min & DLC1.3_0004_Land_04.0V0_S04. out & $-4.77 \mathrm{E}+03$ & $-3.53 \mathrm{E}+04$ & $1.58 \mathrm{E}+03$ & $3.40 \mathrm{E}+02$ \\
\hline TwrBsMyt & $\operatorname{Max}$ & DLC1.3_0051_Land_20.0V0_S03. out & $9.04 \mathrm{E}+03$ & $1.53 \mathrm{E}+0.5$ & $1.49 \mathrm{E}+03$ & 1. $19 \mathrm{E}+02$ \\
\hline TwrBsMzt & Min & DLC1.3_0062_Land_24.0V0_S02. out & $2.63 \mathrm{E}+03$ & $4.45 \mathrm{E}+04$ & $-1.23 \mathrm{E}+04$ & $5.62 \mathrm{E}+02$ \\
\hline TwrBsMzt & $\operatorname{Max}$ & DLC1.3_0063_Land_24.0v0_S03.out & $4.90 \mathrm{E}+03$ & $3.02 \mathrm{E}+04$ & $1.20 \mathrm{E}+04$ & $5.99 \mathrm{E}+02$ \\
\hline
\end{tabular}

Table 6-4. Extreme Events for the Tower-Base Moments - Sea

\begin{tabular}{|c|c|c|c|c|c|c|}
\hline Parameter & Type & File & $\begin{array}{r}\text { TwrBskxt } \\
(\mathbf{k N}-\mathrm{m})\end{array}$ & $\begin{array}{r}\text { TwrBsMyt } \\
(\mathbf{k N}-\mathrm{m})\end{array}$ & $\begin{array}{r}\text { TwrBsMzt } \\
(\mathbf{k N}-\mathrm{m})\end{array}$ & $\begin{array}{l}\text { Time } \\
\text { (sec) }\end{array}$ \\
\hline TwrBsMxt & Min & DLC1.1_0182_Sea_24.0V0_05.5Hs_15.5Tp_sol.out & $-1.99 \mathrm{E}+05$ & $-1.21 \mathrm{E}+0.5$ & $5.70 \mathrm{E}+02$ & $6.11 \mathrm{E}+02$ \\
\hline TwrBsMxt & $\operatorname{Max}$ & DLC1.1_0182_Sea_24.0V0_05.5Hs_15.5Tp_s01.out & 2. $15 \mathrm{E}+0.5$ & $3.12 \mathrm{E}+0.5$ & $-9.88 \mathrm{E}+03$ & $6.17 \mathrm{E}+02$ \\
\hline TwrBsMyt & Min & DLC1.1_0164_Sea_22.0V0_04.7Hs_13.4Tp_s01.out & $7.71 \mathrm{E}+04$ & $-8.58 \mathrm{E}+0.5$ & $1.25 \mathrm{E}+04$ & $2.62 \mathrm{E}+02$ \\
\hline TwrBsMyt & $\operatorname{Max}$ & DLC1.1_0164_Sea_22.0V0_04.7Hs_13.4Tp_s01.out & $2.75 \mathrm{E}+04$ & $9.14 \mathrm{E}+0.5$ & $1.24 \mathrm{E}+04$ & $2.56 \mathrm{E}+02$ \\
\hline TwrBsMzt & Min & DLC1.4_0026_Sea_ECD+R_02.4Hs_13.4Tp_s03.out & $-6.40 \mathrm{E}+03$ & $-1.85 \mathrm{E}+04$ & $-2.16 \mathrm{E}+04$ & $8.33 \mathrm{E}+01$ \\
\hline TwrBsMzt & $\operatorname{Max}$ & DLC1.3_0164_Sea_22.0V0_04.7Hs_13.4Tp_S0l. out & $9.02 \mathrm{E}+04$ & $-6.21 \mathrm{E}+05$ & $2.15 \mathrm{E}+04$ & $2.63 \mathrm{E}+02$ \\
\hline
\end{tabular}


The extreme-event tables record

- The extreme minimum and maximum loads (the shaded values on the block diagonal) for each parameter (identified in the first column)

- The name of the simulation output file that triggered the extreme load (third column)

- The time at which the extreme load was reached (last column)

- The associated values of the other parameters that occur when the extreme load is reached (off-diagonal values).

The loads data have all been weighted using the PSFs for loads described in Section 5.2. In an actual turbine design, these loads data would be fed into a finite-element analysis (FEA) program to determine the detailed stress distributions within individual turbine components, such as the blades, hub, shaft, and tower. I did not perform this extra step, however, because my project is only a conceptual and feasibility study.

In the parameter names for the blade-root moment tables, "Mxc1," "Myc1," and "Mzc1" refer to the internal moments about the $x$-, $y$-, and $z$-axes of the coordinate system of Blade 1 , which is fixed in the hub so as not to rotate with the pitch control motion of the blade. The $x$-axis of this coordinate system is directed nominally downwind, the $y$-axis is located in the plane of rotation, and the $z$-axis is directed from the hub to the tip of Blade 1. (Reference [39] illustrates this coordinate system and others related to the analyzed loads.) The parameters, then, correspond to the in-plane bending moment, the out-of-plane bending moment, and the pitching (torsion) moment at the root of Blade 1, respectively. In the parameter names for the tower base, "Mxt," "Myt," and "Mzt" refer to the internal moments about the $x$-, $y$-, and $z$-axes of the tower-base coordinate system. The $x$-axis of this coordinate system is directed nominally downwind, the $y$ axis is directed transverse to the nominal wind direction, and the $z$-axis is directed vertically from the tower base to the yaw bearing. The parameters correspond to the roll (side-to-side) bending moment, the pitch (fore-aft) bending moment, and the yaw (torsion) moment at the tower base, respectively. The file names list the DLC, the simulation number, the land or sea basis, the wind and wave conditions, and the random-seed identifier.

For the wind turbine installed on land, Table 6-1 shows that DLCs 1.3 and 1.4 drive most of the extreme root moments in Blade 1 and Table 6-3 shows that DLC 1.3 produces all of the extreme moments in the base of the tower. In contrast, Table 6-2 and Table 6-4 show that DLC 1.1 plays more of a role in triggering the ultimate loads for the wind turbine mounted on the barge. In particular, the sea-based simulation numbered 164 in DLC 1.1 generates (1) the minimum and maximum out-of-plane bending moments in the root of Blade 1, (2) the maximum pitching moment in the root of Blade 1, (3) the minimum and maximum pitch bending moments in the tower base, and (4) the maximum yaw moment in the tower base-all within a 7-s period of time (i.e., from time 256 to $263 \mathrm{~s}$ ).

\subsubsection{Design-Driving Load Events}

To determine the exact sequence of events and the physics behind the dynamic response that led to the extreme load of each output parameter, I examined the time-series output from each of the dominant simulations identified by the extreme-event tables. 
Figure 6-2 presents a portion of the time history for several output parameters from the sea-based simulation numbered 164 in DLC 1.1. Results from independent FAST with AeroDyn and HydroDyn and ADAMS with AeroDyn and HydroDyn runs are shown side by side. As indicated within the associated file name in Table 6-2 and Table 6-4, this particular simulation has a random-seed identifier of 01 , stochastic winds with a mean hub-height wind speed of 22 $\mathrm{m} / \mathrm{s}$, and irregular waves with a significant wave height of $4.7 \mathrm{~m}$ and a peak spectral period of $13.4 \mathrm{~s}$.

The parameter names in Figure 6-2 that have not been previously defined earlier in this chapter are as follows:

- "WindVxi" represents the instantaneous nominally downwind component of the wind speed at the undeflected hub location.

- "WaveElev" represents the instantaneous wave elevation relative to the SWL at the origin of the undisplaced platform.

- "PtfmPitch" represents the instantaneous pitch angle of the platform (barge).

- "GenPwr" represents the instantaneous electrical output of the generator.

- "RotSpeed" represents the instantaneous rotational speed of the rotor (low-speed shaft).

- "RotTorq" represents the instantaneous mechanical torque in the low-speed shaft.

The response of the floating system during the first half of the time histories in Figure 6-2 is characteristic of its response in many other simulations. The incident waves cause the barge to pitch back and forth. The ensuing motion in the supported wind turbine causes all the other parameters to exhibit the same oscillatory behavior. Moreover, the pitching causes a large translation of the wind turbine's nacelle, which results in an oscillating inflow to the rotor. As the platform pitches downwind (positive slope), the rotor's relative wind speed decreases, causing the applied aerodynamic torque to drop. The control system responds by driving the blade-pitch angles to zero (not shown). As the aerodynamic torque drops, there is a mismatch with the generator torque, so the rotor speed decreases as well. (The reverse is true when pitching upwind.) The rotor speed exhibits much more variation than one would see in a landbased wind turbine. (The rotor torque shown in Figure 6-2 equals the difference between the applied aerodynamic torque and the rotor-inertia acceleration or deceleration by d'Alembert's principle [25], which is why the phase of the response may not follow intuition.) 

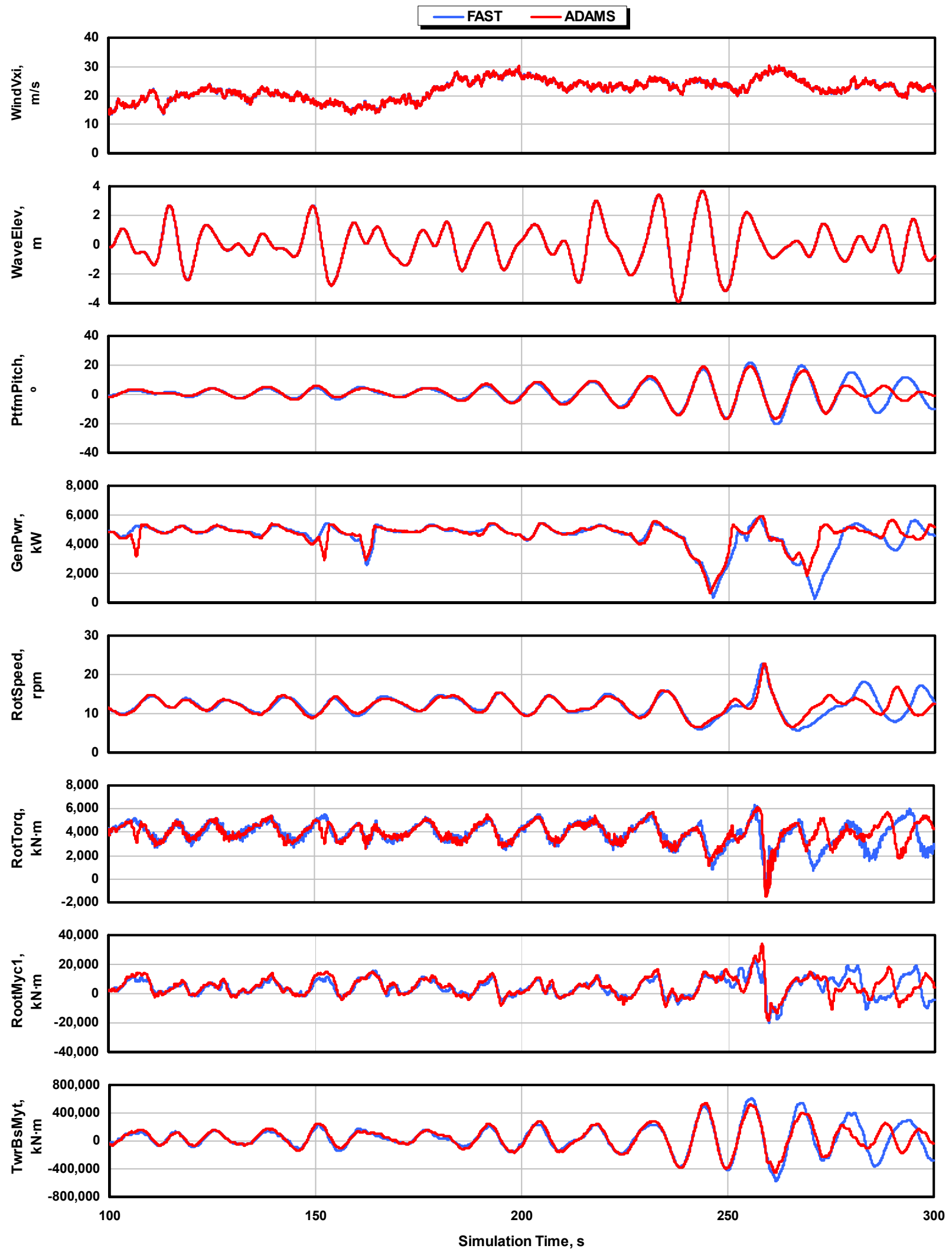

Figure 6-2. Time histories from sea-based simulation number 164 in DLC 1.1 
During the second half of the time history in Figure 6-2, the response of the floating system changes considerably. As shown, a series of large incident waves begins to impinge on the barge. These waves have a height of around $7 \mathrm{~m}$ and propagate near the barge-pitch natural frequency of $0.0863 \mathrm{~Hz}$. Concurrent with these waves are sustained hub-height winds near cutout $(25 \mathrm{~m} / \mathrm{s})$ that are then followed by a gust to $30 \mathrm{~m} / \mathrm{s}$. These wind and wave conditions bring about excessive pitch motion of the barge that leads to large loads in the blades and tower and large excursions in the rotor-speed and generator power output, as well as extreme values in the rotor-thrust, tower-top-displacement, and nacelle-acceleration output parameters (not all shown). In fact, this one series of events drives one-quarter of the extreme values for all of the most relevant output parameters. The loads plotted in Figure 6-2 are not scaled by the PSFs, which is why the extreme values of the blade-root out-of-plane and tower-base pitch bending moments seen in the FAST time histories do not exactly match the values listed in the extreme-event tables shown earlier.

There are differences between the FAST and ADAMS predictions in Figure 6-2, mostly after the series of events that trigger the largest loads. I believe that these differences are caused by the greater structural fidelity of the ADAMS simulator, which includes torsion and mass offsets in the blade model that are not accounted for in FAST. A clear consequence of these differences is that the blade-pitch angles are smaller for ADAMS than for FAST because the control system in FAST must compensate for the lack of blade twist. This difference is visible in the simulation results I present next.

By examining Table 6-1 and the other extreme-event tables presented in Appendix F, I discovered that DLC 1.4 drives the extreme out-of-plane blade-tip deflections and several blade loads in both the land- and sea-based system configurations. Figure 6-3 presents a portion of the time history for several output parameters during the sea-based simulation of this design-driving event. Again, results from independent FAST with AeroDyn and HydroDyn and ADAMS with AeroDyn and HydroDyn runs are shown side by side and the data are not scaled by the PSFs for loads.

Of the parameter names not previously defined above or earlier in this chapter,

- "BlPitch1" represents the instantaneous pitch angle of Blade 1.

- "OoPDefl1" and "IPDefl1" represent the instantaneous out-of-plane and in-plane tip deflections of Blade 1 relative to the undeflected blade-pitch axis.

- "NcIMUTAxs" represents the instantaneous acceleration of the inertial measurement unit, which is located in the nacelle at, and aligned with the centerline of, the main lowspeed shaft bearing.

- "PtfmYaw" represents the instantaneous yaw angle of the platform (barge). 

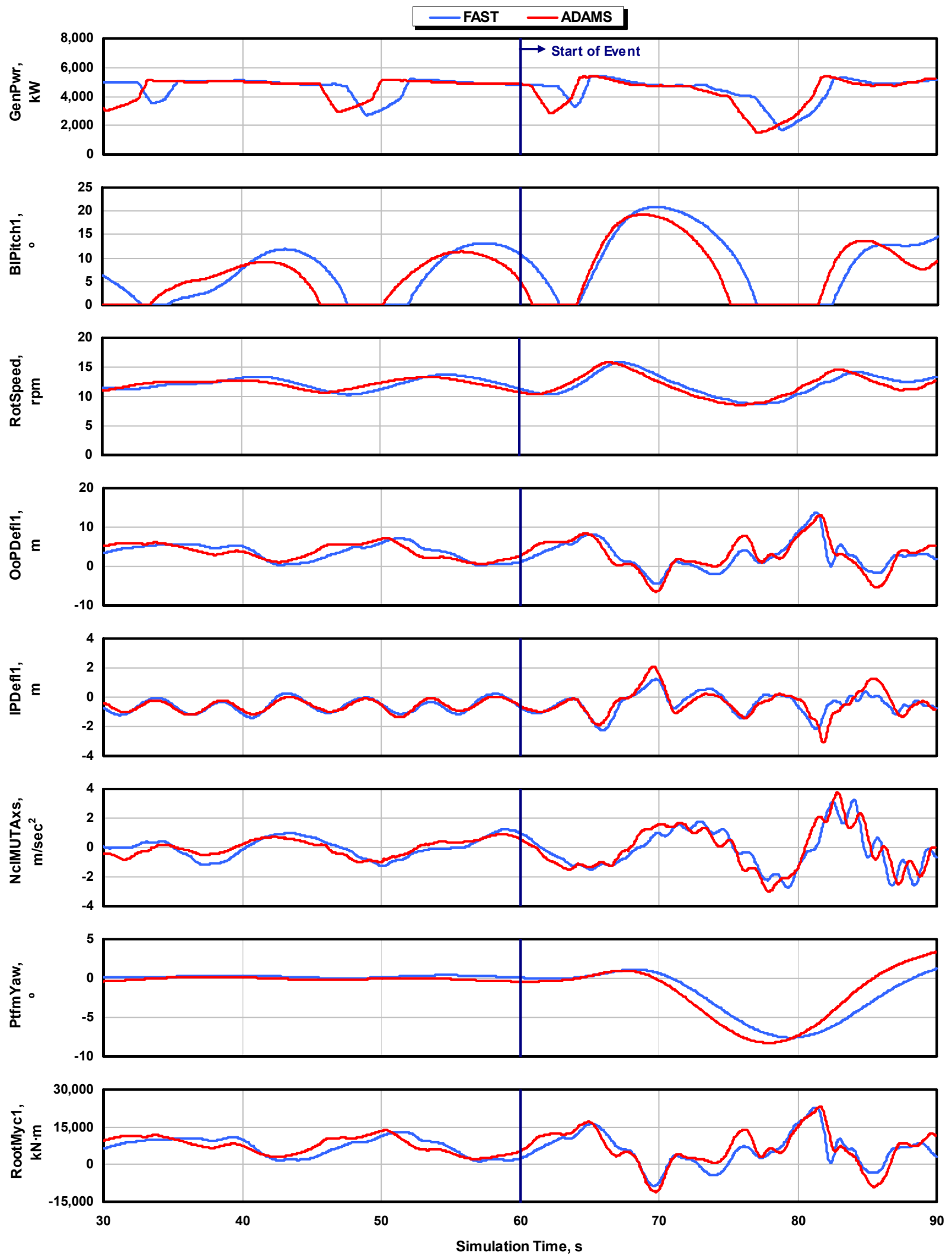

Figure 6-3. Time histories from sea-based simulation number 101 in DLC 1.4 
This particular simulation is numbered 101 and has a random-seed identifier of 04, a steady hubheight wind speed of $13.4 \mathrm{~m} / \mathrm{s}(2 \mathrm{~m} / \mathrm{s}$ above rated) before the ECD event, and irregular waves with a significant wave height of $2.7 \mathrm{~m}$ and a peak spectral period of $12.7 \mathrm{~s}$. Before the ECD event, which starts at $60 \mathrm{~s}$, the barge oscillates in pitch, again because of the impinging surface waves. This is seen in Figure 6-3 through the oscillatory effect on the out-of-plane blade-tip deflection and root-bending moment, the nacelle fore-aft acceleration, the rotor speed, and the blade-pitch angle. The blade-pitch angle varies depending on the action of the control system responding to the oscillating rotor speed, which in turn is a result of the oscillating wind inflow relative to the rotor. In an interesting result, even when the hub-height wind speed is above rated and steady, there are still short periods of below-rated operation where power is lost.

The ECD event starts at $60 \mathrm{~s}$ and takes $10 \mathrm{~s}$ to reach the $15 \mathrm{~m} / \mathrm{s}$ increase in wind speed and the concurrent $54^{\circ}$ change in wind direction. The wind direction shifts to the left when looking downwind. As shown in Figure 6-3, the escalation in wind speed from the gust generates a rise in the rotor speed and an increase in the blade-pitch angle as the control system tries to compensate. But the change in wind direction produces a large nacelle-yaw error that eventually causes the wind speed relative to the rotor to drop. This, in turn, causes the rotor speed and blade-pitch angle to decrease. The maximum out-of-plane tip deflection (of nearly $14 \mathrm{~m}$ !) occurs after this series of events, when the blade is pointing horizontally into the wind just after the blade-pitch angle reaches its $0^{\circ}$ minimum set point. The condition where this occurs is severest for the blade deflections and loads because the blade is flat into the wind during that time. Although the extreme out-of-plane bending moment at the root of Blade 1 is higher in DLC 1.1, this series of events in DLC 1.4 brings about the maximum out-of-plane bending moment in Blade 1 at 50\% span (not shown in Figure 6-3, but included in Appendix F.2). The ECD event also perturbs the yaw angle of the barge. After the event is over, the barge begins to yaw slightly into the wind. There is still about a $50^{\circ}$ nacelle-yaw error at $90 \mathrm{~s}$ into the simulation, but the mooring lines eventually restrain the platform from yawing any farther.

Of all the different hub-height wind speeds I ran simulations for in DLC 1.4, the simulations at a wind speed of $2 \mathrm{~m} / \mathrm{s}$ above rated led to the largest out-of-plane blade-tip deflections in the floating turbine. This wind speed was associated with the highest wave heights and the resulting barge-pitch motion exacerbated an existing problem. In the wind turbine installed on land, the DLC 1.4 simulations run at rated wind speed generated the largest out-of-plane blade-tip deflections and bending moments.

For the land-based wind turbine, DLC 1.3 played a significant role in driving many of the extreme values of the most relevant output parameters not driven by DLC 1.4. Although I do not present any of the time histories, DLC 1.3, with its extreme wind turbulence, was particularly dominant because the resulting wind inflow contained many drastic jumps or drops in wind speed. These wind speed changes generated ultimate loads because the control system could not react fast enough. Jumps in wind speed from below to above rated, or from above to below rated, created particularly large deflections and loads because of the peak in thrust at rated. Large variations in wind speed near cut-out were also problematic.

The wind turbine mounted on the floating barge was more affected by the waves than the wind. Consequently, DLC 1.1 in the sea-based analysis, which has the higher effective PSF for loads, dominated the loads results more than DLC 1.3, which has higher levels of wind turbulence. In 
other words, higher PSF for loads were more important than higher levels of wind turbulence. For example, the maximum magnitude of acceleration in the nacelle at the main shaft bearing was $10.1 \mathrm{~m} / \mathrm{s}^{2}$ (just over $1 \mathrm{~g}$ ) in the floating turbine, as driven by large waves in DLC 1.1 , but only $1.4 \mathrm{~m} / \mathrm{s}^{2}$ in the land-based turbine, as driven by extreme wind turbulence in DLC 1.3.

The only loads in the floating system that appeared to be driven more by wind than waves were the tensions in the mooring lines. The tensions, particularly at the anchors and the fairleads of the upwind mooring lines, were driven by simulations involving sustained winds at or near rated wind speed. This is because sustained winds at rated generate the highest sustained rotor-thrust forces, which push the barge far downwind (i.e., a large surge displacement), and tug on the upwind mooring lines. Even at maximum tension, though, there was enough slack in the mooring lines to keep them from pulling upward on the anchors. This result means that inexpensive anchors could be used.

In relation to DLCs 1.1, 1.3, and 1.4, DLC 1.5, which considers transient wind-shear events, did not play a significant role in driving ultimate loads in either the land- or sea-based wind turbine systems.

\subsubsection{Comparing Land- and Sea-Based Loads}

We took the absolute extreme values of each parameter (i.e., the absolute maximum values of the minima and maxima, from the block diagonals of the extreme-event tables) of the sea-based analysis of DLCs 1.1, 1.3, 1.4, and 1.5 and divided them by the corresponding absolute extremes of the land-based analysis. The resulting dimensionless ratios quantify the impact of installing the NREL 5-MW baseline wind turbine on the floating ITI Energy barge. I present the ratios for many of the parameters in Figure 6-4. A ratio of unity (indicated by the dashed horizontal line) would imply that the absolute extreme is unaffected by the dynamic couplings between the turbine and the floating barge in the presence of combined wind and wave loading. Ratios greater than unity imply an increase in load or response that may have to be addressed by modifying the system design in subsequent analysis iterations.

The chart in the upper-left corner of Figure 6-4 presents the sea-to-land ratios for the absolute extremes of the generator power, generator torque, generator (high-speed shaft) speed, and rotor (low-speed shaft) speed. The sea-to-land ratio of the generator torque is unity because the variable-speed control system, which is identical in the land and sea analyses, places a limit on the torque command to avoid excessive overloading of the generator and gearbox (see Section 3.1.6.2). Nevertheless, greater generator power excursions are seen in the sea-based system because of the increased excursions in generator speed. This may have to be addressed in the floating wind turbine to avoid generator burnout. The sea-to-land ratios of the generator and rotor speed are identical because the high- and low-speed shafts are directly coupled through the gearbox. The rotor-speed excursions in the floating wind turbine are $60 \%$ higher than those seen in the turbine installed on land. These excursions are the result of the oscillatory wind inflow relative to the rotor from the pitching motion of the barge, as discussed earlier. They will most assuredly lead to an increase in aeroacoustic emissions from the rotor, which may or may not be important offshore. 

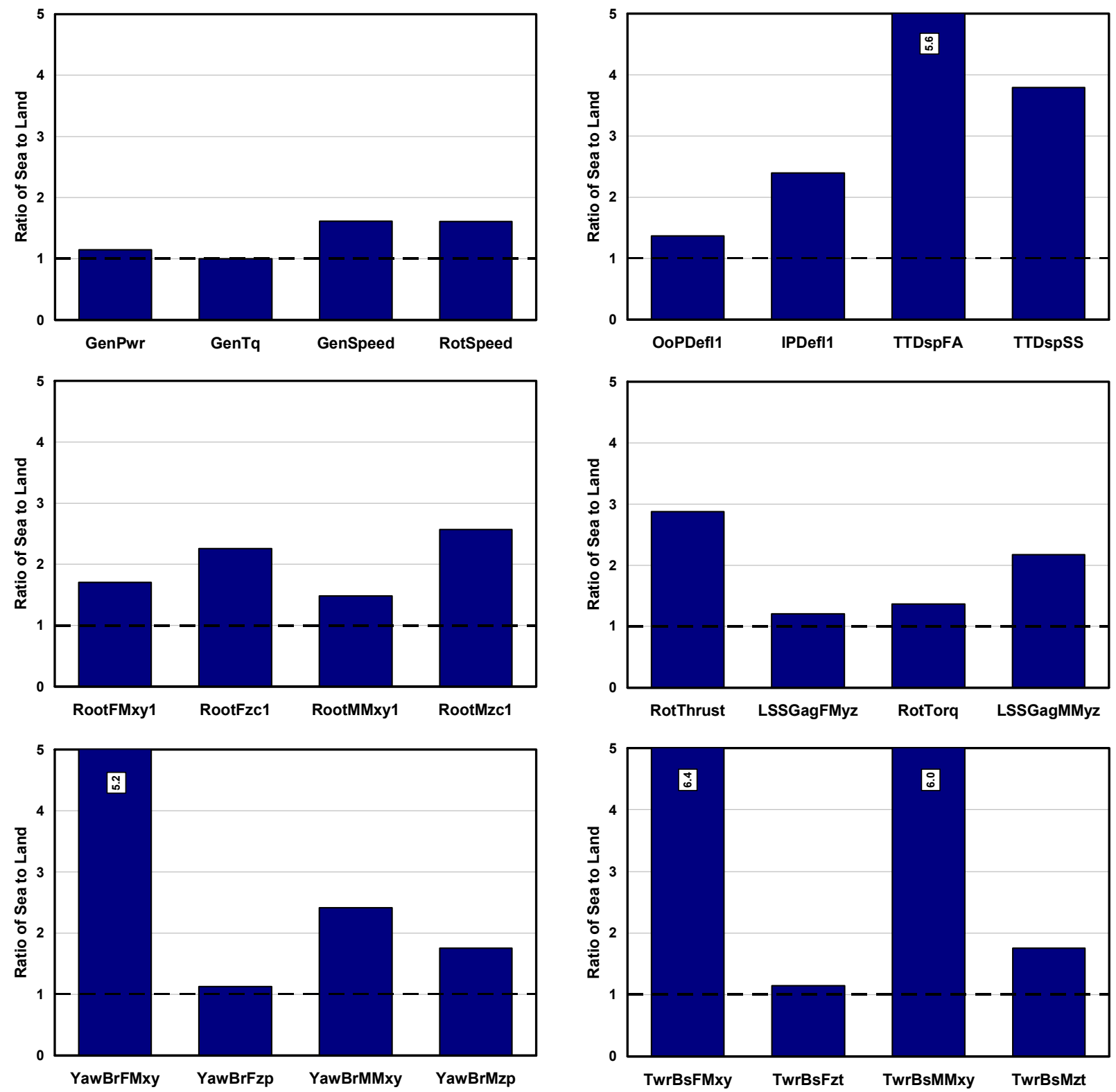

Figure 6-4. Sea-to-land ratios from DLCs 1.1, 1.3, 1.4, and 1.5

The chart in the upper-right corner of Figure 6-4 presents the sea-to-land ratios for the absolute extremes of the out-of-plane and in-plane tip deflections of Blade 1 and the fore-aft and side-toside displacements of the tower top (i.e., yaw bearing). The sea-to-land ratio for the tower-top fore-aft displacement exceeds the upper bound of 5 placed on the ordinate, which is why its value of 5.6 is listed. This ratio is much larger than the sea-to-land ratio of the out-of-plane blade-tip deflection again because of the inverted-pendulum effect discussed earlier. The larger excursions of in-plane blade-tip deflection in the sea-based system are the result of faster rotor rotational accelerations, corresponding with the elevated excursions in rotor speed. The tower side-to-side displacements are larger in the floating wind turbine because larger yaw errors are present between the nominal wind direction and the rotor axis. These, in turn, come from the yaw motion of the barge. That motion is excited by a gyroscopic yaw moment resulting from the 
spinning inertia of the rotor in combination with the pitching motion of the barge. Larger yaw motions are permissible because of the yaw compliance of the mooring system.

The remaining four charts in Figure 6-4, from the middle-left to the lower-right corner, present the sea-to-land ratios for the absolute extremes of the forces and moments in the root of Blade 1, in the low-speed shaft at the main bearing, in the yaw bearing, and in the tower base, respectively. In the parameter names, "FMxy" refers to the magnitude of the internal shear force in the transverse $x$ - and $y$-plane of the various coordinate systems. These were found by taking the vector sum of the shear forces along the $x$ - and $y$-axes. Similarly, "MMxy" refers to the magnitude of the internal bending moment in the transverse $x$ - and $y$-plane of the various coordinate systems, calculated by taking the vector sum of the bending moments about the $x$ - and $y$-axes. We computed the maximum values of the vector sums as opposed to the more conservative calculation of the vector sums of the maximum values. The remaining parameters refer to the axial forces along, and the torsion moments about, the primary axis of the members.

The sea-to-land ratios of the internal shear force and bending moment magnitudes, in general, increase as one follows the load path from the blade tip, through the drivetrain and nacelle, to the tower base. This increase in relative loading between the sea- and land-based systems results again from the inverted-pendulum effect in the floating wind turbine; that is, there is more effect of loading from inertia farther down the load path. The axial forces in the blades are increased in the floating wind turbine relative to the onshore turbine because of the centripetal effect from elevated excursions in rotor speed. The axial forces at the yaw bearing and tower base are larger in the floating system than in the onshore system because of the heave motion of the barge as it follows the up and down elevation of the waves. The sea-to-land ratio of the rotor thrust, "RotThrust," is large because it is computed not as the applied aerodynamic thrust, but as the internal force within the low-speed shaft, which by d'Alembert's principle [25] is the difference between the applied thrust and the fore-aft rotor-inertia acceleration or deceleration. The inertia effect itself is large again because of the barge-pitch motion. To withstand the increased loading for the wind turbine mounted on the floating barge, the tower will certainly have to be strengthened, and the blades and drivetrain may have to be as well.

Of course, all the results presented so far were derived from the environmental conditions at the chosen reference site. As I discussed in Section 3.3, we chose this reference site for its fairly extreme wind and wave conditions, with the implication that if the results of the loads analysis are favorable, the floating wind turbine system under consideration will be applicable at almost any site around the world. The loads-analysis results indicate, however, that without design modifications, there is the potential for loads in the floating wind turbine that are much larger (up to 6.4 times as large at the tower base, as indicated in Figure 6-4) than what would be seen in an equivalent onshore wind turbine. Because of this, it is beneficial to examine whether or not the existing concept, without modification, may be better suited at a site where conditions are less severe.

I studied the effect of the choice in reference site by rerunning the sea-based loads analysis with varying environmental conditions. Instead of choosing different locations to reobtain metocean data, I took a simpler approach and obtained varying environmental conditions by modifying the existing data from the chosen reference site. I chose to adjust only the data of significant wave height because the size of the waves was the key parameter that led to the excessive pitching of 
the barge and subsequent loading of the floating wind turbine. I did not modify the wind-speed data because I wanted to maintain a fair comparison to the results from the land-based wind turbine, which is unaffected by wave conditions. Neither did I modify the wave-period data because the range of wave periods considered are typical of sites around the world and because I did not want to adversely affect the wave steepness. (The wave steepness is related to the wave height and wavelength, the latter of which is dictated by the wave frequency or period.) I adjusted the data of significant wave height by scaling down the magnitude of each data sample provided by the Waveclimate.com service, which corresponds to scaling consistently across all other conditions. I chose wave-height scaling factors of $75 \%, 50 \%, 25 \%$, and $0 \%$. For example, with a wave-height scaling factor of $50 \%$, the expected value of the significant wave height would increase with the mean hub-height wind speed that it is conditioned on, from about $0.8 \mathrm{~m}$ (instead of $1.6 \mathrm{~m}$ ) at cut-in to about $3.0 \mathrm{~m}$ (instead of $5.9 \mathrm{~m}$ ) at cut-out (the original data are plotted in Figure 3-15). A wave-height scaling factor of $0 \%$ represents still water with no incident surface waves (but outgoing waves can still be generated by wave radiation).

I reran the sea-based loads analysis with the reference-site data adjusted by each wave-height scaling factor. Figure 6-5 presents the sea-to-land ratios for the absolute extremes from the rerun loads analysis of DLCs 1.1, 1.3, 1.4, and 1.5. The leftmost ratios of each parameter, labeled " $100 \%$ - Original," correspond to the ratios presented in Figure 6-4, which result from the original reference-site data. The remaining ratios of each parameter correspond with decreasing severity in the wave conditions, from left to right.

For most parameters, the sea-to-land ratios decline rapidly at first, then drop off more slowly with decreasing severity in the wave conditions. So, interestingly, the response is nonlinear even though the hydrodynamic model is primarily based on linear radiation and diffraction theory (see Section 2.4). This implies that other nonlinear features of the model-such as aerodynamic loading, turbine dynamics, and control actions - are affecting the response. Moreover, even in still water, the wind turbine mounted on the barge experiences higher loading than the turbine that is installed on land. In fact, the absolute extreme magnitude of the internal bending moment at the base of the tower is still $60 \%$ higher for the floating wind turbine in still water than for the turbine on land. This implies that the barge pitches because of the wind inflow as well as wave excitation. Nevertheless, the results show that the potential loads in the floating wind turbine will be considerably less at a sheltered site than at a site in the open ocean.

\subsubsection{Drawing Conclusions about Responses in Normal Operation}

To summarize the results presented in this section, the pitching motion of the barge brings about load excursions in the supported wind turbine that exceeds those experienced by the turbine when it is installed on land. The load excursions are worse in the tower than in the blades because of the increased effect of inertia from the barge-pitch motion. To arrive at a technically feasible concept, the design will need to be modified, except possibly at the most sheltered of sites. This is not surprising, however, because I made no attempt to minimize the motions of the floating platform in this analysis, which was the first step of the design-iteration process outlined in the beginning of Chapter 5 .

Two forms of design modifications are possible. First, the turbine, especially the tower, could be strengthened to enable it to withstand the increased loading. However, this approach may not be 
- $100 \%$ - Original $\square 75 \% \square 50 \% \square 25 \% \square 0 \%$ - Still Water
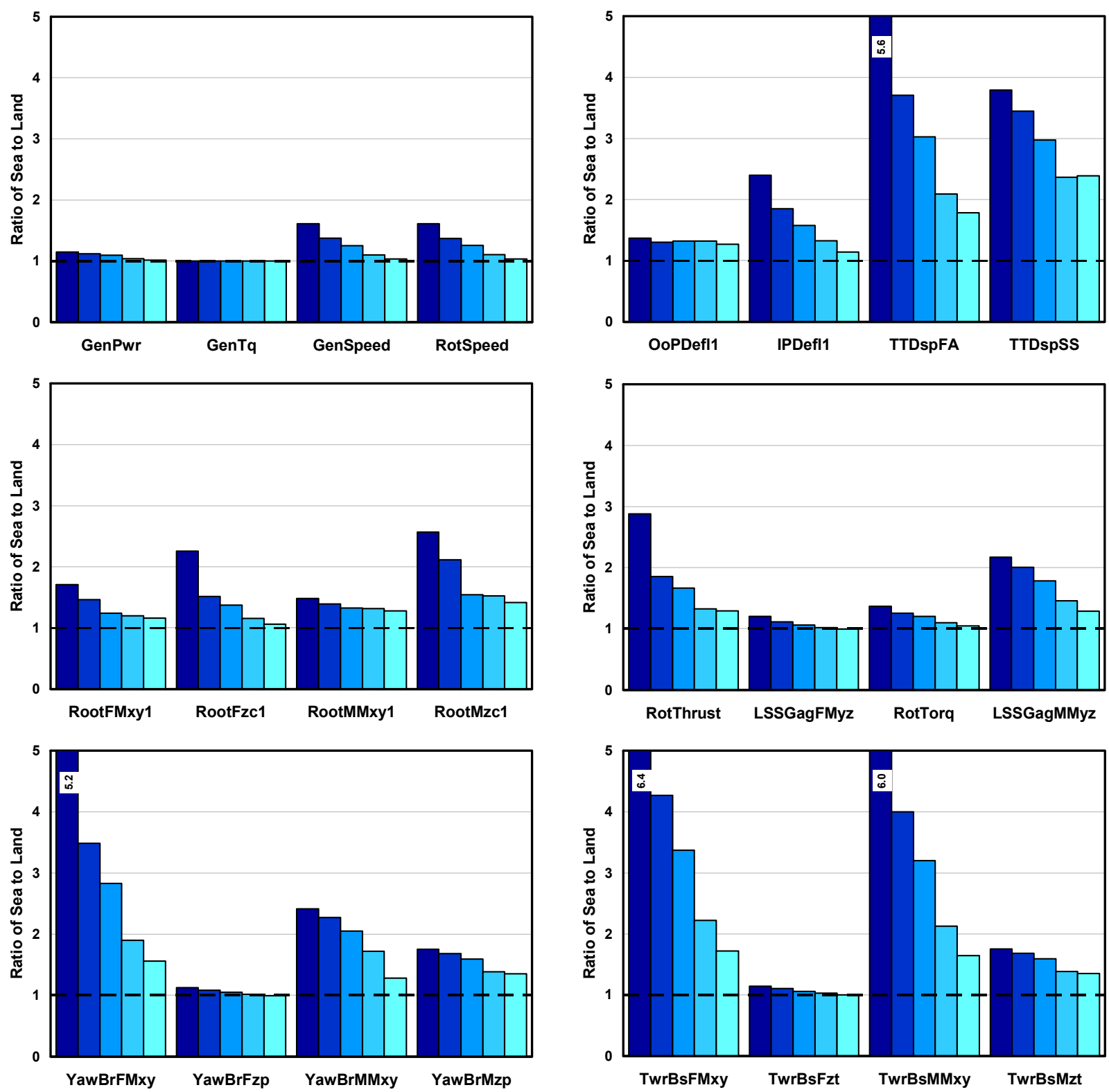

Figure 6-5. Sea-to-land ratios for variations in significant wave height

cost-effective even though the wind turbine in an offshore floating system represents a smaller fraction of the total installed cost than in an onshore system. Second, design alterations may be able to improve the response of the floating system to diminish the increases in loading.

One possible approach to improving the response of the floating system is to add design features that will increase damping to stabilize the barge-pitch motion. In ITI Energy's original concept, not only is the barge designed to support the NREL offshore 5-MW baseline wind turbine, but it is also a platform for an OWC wave-power device. Unfortunately, I could not model the OWC device with my current simulation tool, as described in Sections 3.2 and 4.1.2. But if positioned suitably and controlled properly, the OWC may be able to introduce damping of the barge-pitch motion while extracting wave energy. This concept is currently being researched through 
analytical modeling and wave-tank testing at NAME at the Universities of Glasgow and Strathclyde [98]. Other actuator opportunities include gyro actuators, used commonly for system stabilization in aerospace and spacecraft applications, or hydrodynamic thrusters, used commonly for station-keeping in naval architecture.

To dampen the barge-pitch motion, it may also be possible to develop a wind turbine control system that relies on the conventional wind turbine actuation of blade pitch, generator torque, and nacelle yaw. In Chapter 7, I present the results of my analysis examining the influence of conventional blade-pitch control systems on the pitch damping of the wind turbine-plus floating barge-system.

Beyond active control, a simpler solution for improving the barge-pitch damping may be to introduce passive damping devices into the underlying design. Tuned-mass dampers (TMDs) are frequently employed in skyscrapers to dampen wind-induced vibrations. Similarly, a TMD could be placed at the top of the wind turbine's tower; when tuned at the natural period of the rigid-body - turbine plus barge — pitch mode, such a system could dampen pitching (and rolling) motion dramatically. It may also be possible to dampen the barge rotational motions with the equivalent of passive anti-roll stabilizers installed within or on top of the barge. (Anti-roll stabilizers, which are commonly installed on ships, act like TMDs, but are made of water-filled U-tube tanks [22].) The platform's hydrodynamic radiation damping and viscous drag could also be increased by incorporating damping orifices in the planform of the barge or horizontal damping plates and / or bilge keels positioned below the free surface.

Instead of trying to improve the barge-pitch damping, it may be better to add DOFs in or between the floating platform and the wind turbine to eliminate the direct coupling between the motions of the platform and the turbine. For example, articulated joints could be installed in the

floating platform, as in the Versabuoy offshore system, ${ }^{2}$ or between the wind turbine's tower and nacelle, as in the Wind Eagle turbine [46].

Finally, the easiest solution may be to modify the geometry of the floating platform or the layout of the mooring system, or both, to reduce the barge's natural pitch motion. This would, consequently, minimize the impact on the supported wind turbine. Possibilities include streamlining the shape of the barge to allow surface waves to more easily pass by (i.e., as in a spar-buoy concept), shifting the CM closer to the COB through ballast (i.e., as in a spar-buoy concept), or introducing tauter mooring lines (i.e., as in a TLP concept).

\subsection{Other Load Cases}

As mentioned in this chapter's introduction, I identified problems with both the land- and seabased system configurations when we processed the loads-analysis results for DLCs 1.6a, 2.x, 6.x, and 7.1a. These DLCs are concerned with the wind turbine when it is experiencing a fault, when it is idling, and / or when it is being excited by 1 - and 50 -year wind and wave conditions. I describe these problems in more detail here, but because the loads resulting from the problems were unreasonable, I do not quantify them. I also discuss potential design modifications that may be used to correct the problems.

\footnotetext{
${ }^{2}$ Web site: http://www.vbuoy.com/index.html
} 
Section 6.2.1 describes the tower side-to-side instability of the land-based wind turbine, Section 6.2.2 describes the platform-yaw instability of the sea-based wind turbine, and Section 6.2.3 describes the susceptibility of the barge to excessive motions in extreme waves. All of these results are based on the loads-analysis data. Though not presented here, we have also analyzed the instabilities using linear system models and obtained consistent results (see Ref. [2]).

DLC 2.3 considers an extreme operating wind gust concurrent with a loss of load. I did not include the results for this case in Section 6.1 because of the way I split the results into meaningful groups. Because DLC 2.3 did not cause problems in either the land- or sea-based wind turbine systems - relative to DLCs 1.6a, 2.1, 6.x, and 7.1a-I do not discuss any of the results from DLC 2.3 in this work.

\subsubsection{Tower Side-to-Side Instability of Land-Based Wind Turbine}

The first problem I discovered was a side-to-side instability in the tower of the wind turbine installed on land. This instability was identified when we were analyzing the loads predicted by land-based DLC 6.2a. The instability occurs when the turbine is idling with all blades fully feathered to the maximum pitch setting of $90^{\circ}$, but only when the rotor is positioned at certain azimuth angles and is misaligned with the mean wind direction by $20^{\circ}$ to $40^{\circ}$ on either side of $0^{\circ}$. (DLC 6.2a considers the full range of yaw misalignments because of an assumed inability of the nacelle-yaw controller to align the rotor with the wind when electrical power is unavailable because the grid is lost.) DLC 6.2a required me to analyze this situation with extreme 50-year wind conditions, $V_{50}=50 \mathrm{~m} / \mathrm{s}$. After more study, though, we discovered that the instability is predicted at much lower wind speeds, as low as a mean hub-height wind speed of $25 \mathrm{~m} / \mathrm{s}$. The instability is more severe, however, at the higher wind speeds. The instability leads to excessive limit-cycle oscillations in the tower-top side-to-side displacement and the tower-base side-to-side (roll) bending moment. The oscillations occur at about $0.32 \mathrm{~Hz}$, which corresponds with the natural frequency of the first side-to-side bending mode of the tower (see Table 3-11).

In the sea-based simulations of DLC 6.2a, it is difficult to distinguish an instability from the excessive barge motions generated by the extreme 50-year wave conditions (described as the third problem in Section 6.2.3 below). To eliminate the excessive barge motions, we reanalyzed the floating wind turbine with all of the specifications of DLC 6.2a except the 50-year wave conditions, which we replaced with still water. In this situation, the instability is nonexistent; in a fascinating finding, the barge compliance actually helps to eradicate the side-to-side instability in the land-based tower, at least when no incident surface waves are present. In other words, the tower is prevented from going unstable because of the barge's compliance in still water.

The reason for the instability in the land-based turbine is almost certainly because the amount of structural damping in the first side-to-side bending mode of the tower is exceeded by the amount of energy the rotor absorbs within the critical range of rotor-azimuth and nacelle-yaw-error angles. This probably results from the range of wind-inflow angles of attack on the blades during those conditions. It is difficult to determine what causes what, though, because of the classic chicken-and-egg problem: Which comes first? The oscillation in angles of attack or the instability? It is also difficult to determine if the predicted instability is physical or an artifact of my analysis. As described in Section 5.1, I ran this DLC without AeroDyn's induction or dynamic-stall models enabled because they are not applicable in this idling case, particularly at 
very high post-stall angles of attack. Examining the time histories of the angles of attack might be worthwhile for determining if the simple lookup-table aerodynamic methods that I used are adequate in this situation. The examination might also allow one to determine if other aerodynamic theories are more appropriate and would predict different behavior. But I did not consider this action necessary in this preliminary analysis.

Nevertheless, the information I do know about the tower side-to-side instability of the land-based wind turbine suggests two possible remedies that may be pursued if the problem is real instead of virtual. First, it may be possible to modify the shape of the airfoils in the blades to reduce the amount of energy absorption at the problematic angles of attack. ${ }^{3}$ A second possibility is to apply a fail-safe brake to park the rotor in extreme winds and to keep it away from the critical azimuth positions. The downside to this second solution is that it may cause excessive wear on the brake and become a source of routine maintenance. In the time history presented in Figure 6-6, however, I show that this solution does work. The figure shows the results of two separate FAST simulations. The first simulation considered the onshore wind turbine idling with all blades fully feathered in steady uniform $50-\mathrm{m} / \mathrm{s}$ winds and a nacelle-yaw error of $30^{\circ}$. The second simulation used the same set of conditions, but at $150 \mathrm{~s}$ into the simulation, we applied a high-speed shaft brake. Before the brake is engaged, the responses predicted by the two simulations are identical. After the brake is engaged, the amplitude of the limit-cycle oscillation in the tower-top side-to-side displacement is reduced significantly. (The cycles are very close together and difficult to discern because the frequency of about $0.32 \mathrm{~Hz}$ is small for the range of simulation times considered on the abscissa.)

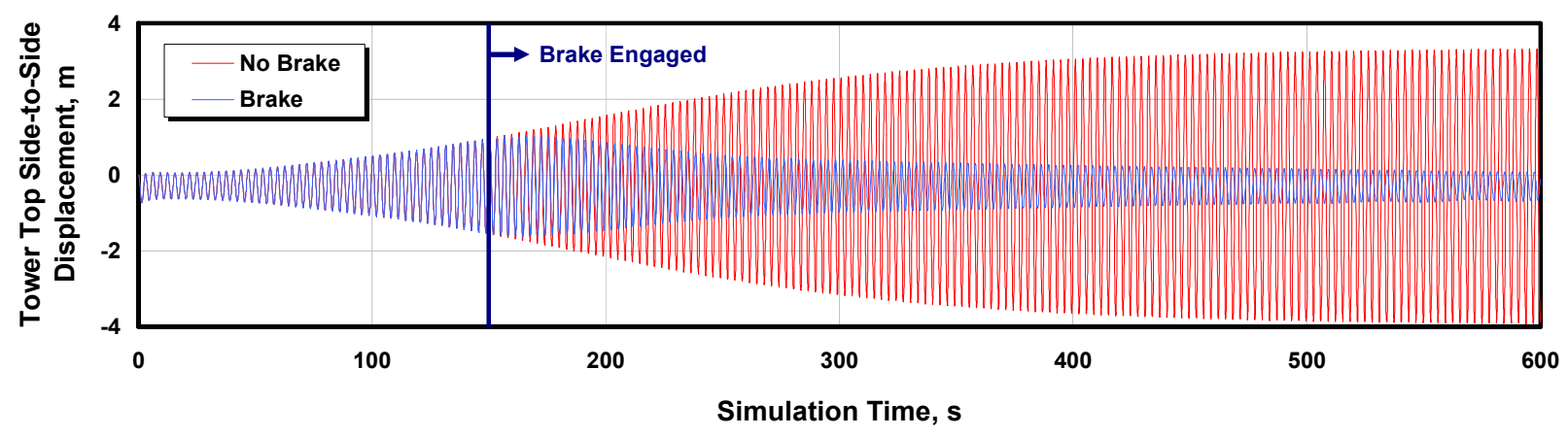

Figure 6-6. Time history of the tower side-to-side instability

\subsubsection{Platform-Yaw Instability of Sea-Based Wind Turbine}

The second problem I discovered was a yaw instability in the barge of the floating wind turbine. The instability occurs when the rotor is idling with a fault, where one blade (the faulted blade) is seized at the minimum pitch set point of $0^{\circ}$ and the other two blades are fully feathered to the maximum pitch setting of $90^{\circ}$. We identified this instability during analysis of the loads predicted by sea-based DLCs 2.1 and 7.1a. In DLC 2.1, which considers normal wind and wave

3 Even though the NREL 5-MW baseline wind turbine model is heavily based on the publicly available specifications of the REpower 5M prototype wind turbine, there is no reason to conclude that the REpower 5M machine has a tower side-to-side instability. The airfoil properties (which influence the instability) of the NREL 5MW baseline wind turbine are likely very different from those of the REpower 5M turbine. 
conditions, the instability occurs after the protection system has detected the blade-pitch fault and shut down the turbine. The instability is more severe in DLC 7.1a, which required me to analyze this fault condition under extreme 1-year wind and wave conditions with misalignments in the mean wind and wave directions of $\pm 8^{\circ}$ and $\pm 30^{\circ}$, respectively. After more study, however, we discovered that the instability is predicted by my simulation tool regardless of the yaw misalignment and also in still water. The instability is caused by a coupling of the barge-yaw motion with the azimuthal motion of the seized blade, and leads to excessive limit-cycle oscillations in the barge-yaw displacement. This, in turn, may cause a knotting of the mooring lines (although my simulation tool cannot model line-to-line interference), excessive loading of the wind turbine from the ensuing dynamics, or both. The oscillations occur at about $0.02 \mathrm{~Hz}$, which corresponds with the natural frequency of the rigid-body — turbine plus barge-yaw mode.

The idling-plus-fault condition does not cause a problem in the land-based wind turbine because it has very little yaw compliance. This condition may cause problems, however, that are more pronounced in floating spar-buoy or TLP concepts than in the floating barge because the former concepts are likely to be more compliant in yaw because smaller moment arms are available to resist yaw moments. In the floating barge, the yaw instability may also be less severe than my simulation tool predicts because my model considers hydrodynamic damping in yaw only from wave radiation (i.e., potential flow), which is negligible at the yaw mode natural frequency (see $B_{66}$ in Figure $4-5$ at about $0.02 \mathrm{~Hz}=0.1257 \mathrm{rad} / \mathrm{s}$ ). In actuality, hydrodynamic viscous damping in yaw may be more dominant. Hydrodynamic viscous damping in yaw comes from vortex shedding off the corners of the barge and skin friction, neither of which are accounted for in my model (Morison's equation considers viscous drag only in surge, sway, roll, and pitch-see Section 2.4.2.2). It may be worthwhile to try to quantify the viscous-drag contributions through wave-tank tests for the vortex-shedding drag and / or ship-resistance formulas for the skinfriction drag to see if they provide enough damping to eliminate the yaw instability. But, as in our investigation of the tower side-to-side instability, I did not consider this action necessary at this time.

As in the tower side-to-side instability, several pathways may be pursued to eliminate the yaw instability of the barge if the problem is real instead of virtual. Possibilities include

- Supplementing the existing yaw damping by installing damping plates below the free surface

- Increasing the yaw stiffness by adding a so-called "crowfoot" at the connection between each mooring line and the barge

- Applying a fail-safe shaft brake to park the rotor when shutdown

- Reducing the pitch angle of the fully feathered blades to generate a low, but persistent, aerodynamic torque that will produce a slow roll of the rotor while idling.

The latter two solutions would prevent the seized blade from coupling with the platform-yaw motion. Again, we tested the brake approach with FAST simulations and present the results in Figure 6-7. The first simulation considered the floating wind turbine idling with one blade flat into the wind (faulted) and with steady uniform $40-\mathrm{m} / \mathrm{s}$ winds and no incident surface waves (still water). The second simulation used the same set of conditions, but at $100 \mathrm{~s}$ into the simulation, we applied a high-speed shaft brake. Before the brake is engaged, the responses 


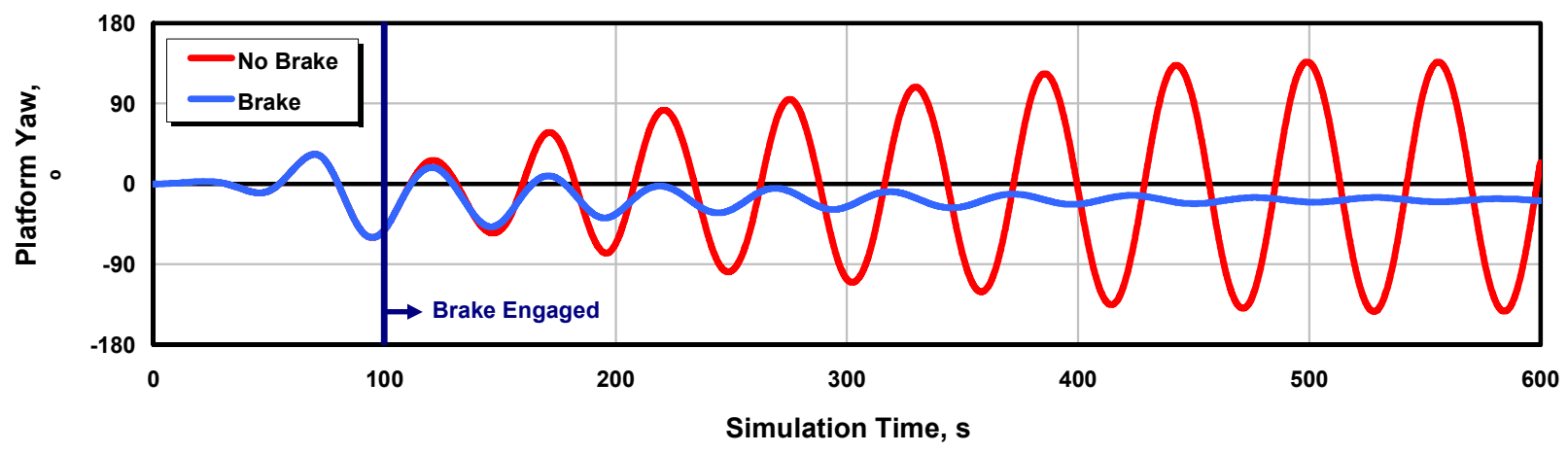

Figure 6-7. Time history of the platform-yaw instability

predicted by the two simulations are identical. After the brake is engaged, the limit-cycle oscillation in the barge-yaw displacement is eliminated.

\subsubsection{Excessive Barge Motions in Extreme Waves}

The final problem I discovered through my loads analysis was that the floating barge is susceptible to excessive motions when the incident waves are large and / or steep, such as during extreme 1- or 50-year wave conditions. This is especially true with the harsh conditions that occur at the chosen reference site. The problems exist whether the wind turbine is idling, as in DLCs 6.x and 7.1a, or producing power, as in DLC 1.6a. The response is worse, however, in the idling turbine because the operating turbine introduces more aerodynamic damping. The response is also worse in the 50-year wave conditions than in the 1-year conditions. The problems in DLCs 1.6a or 6.x are not related to a system instability because the problems disappear in simulations where the extreme wave conditions are exchanged with still water.

The problematic motions usually occur in a series of stages. First, the barge begins to pitch back and forth as it moves with the incident surface waves. The large pitching motion leads to excessive deflections in the blades and tower of the wind turbine. When the blades deflect asymmetrically because of variations in the rotor-aximuth angle, turbulence in the wind, or misalignment of the waves from the barge's planes of symmetry, the barge gets excited in other modes of motion, such as roll and yaw. ${ }^{4}$ The overall result is excessive deflections and loading throughout the entire system, from the blades to the moorings. The problem is so bad that even though the blade tips of the undeflected or undisplaced floating turbine are positioned $30 \mathrm{~m}$ above the SWL (see Section 3.1), the system gets jostled so severely that the blade tips pass below the free surface in many of the DLC simulations involving 1- and 50-year wave conditions.

All of the simulations exhibiting this problem contain waves and responses that most assuredly violate linear hydrodynamic radiation and diffraction theory, and my simulation tool is invalid in

\footnotetext{
${ }^{4}$ This response shows the importance of the fully coupled dynamics solution, demonstrating that the motions of the support platform are coupled with the motions of the wind turbine. The simulations in Section 6.1 demonstrate that the wind turbine excites the floating platform yaw motions through the rotor's gyroscopic effect. The simulations in DLCs 6.x and 7.1a, however, show that asymmetric deflections in the wind turbine also contribute to excitation of the platform motions. (The coupling between the wind turbine and floating platform motions is not the result of rotor gyroscopics because the rotor is idling — not spinning —in these DLCs.)
} 
these situations. It is unclear, however, whether the "real" responses would fare better or worse. This is simply a fundamental limitation with my analysis, if not with most other computational capabilities available in the offshore $O \& G$ industry today. To get around these limitations, wave-tank testing of a scaled model under equivalent conditions is required. This work has already been initiated at NAME at the Universities of Glasgow and Strathclyde [98].

More likely than not, unless the barge is installed only at sheltered sites, modifications to the system design will be required to eliminate the vulnerability of the barge to extreme waves. The design modifications listed in Section 6.1.5 will help to solve this problem, except those modifications that involve active wind turbine control (which are not applicable to an idling turbine), or active control of other actuators (which cannot always be relied on during extreme events). I plan to examine many of these design modifications in subsequent projects. 


\section{Chapter 7 Influence of Conventional Control on Barge-Pitch Damping}

Chapter 6 showed that the pitching motion of the ITI Energy barge brings about load excursions in the supported wind turbine that exceed those the turbine experiences when it is installed on land. One possible approach to improving the response of the floating system is to incorporate design features that will increase damping to stabilize the barge-pitch motion. Damping can be tailored through passive design features and active control. The NREL offshore 5-MW baseline wind turbine I developed and used in the land- and sea-based loads analyses relied on a conventional variable-speed, variable blade-pitch-to-feather control system (see Section 3.1.6). A consequence of conventional pitch-to-feather control of wind turbines, though, is that steadystate rotor thrust is reduced with increasing wind speed above rated (see Figure 3-12). As pointed out by Nielsen, Hanson, and Skaare in Ref. [75, p. 673], "this effect may introduce negative damping in the system that may lead to large resonant motions of [a] floating wind turbine." As the loads-analysis results of Chapter 6 demonstrate, it is important that the damping of the barge-pitch mode be positive and kept as large as possible.

Section 7.2 addresses the influence of conventional wind turbine control methodologies to the pitch damping of the floating wind turbine system analyzed in Chapter 6. In this work, my aim was to modify the baseline control system of the NREL 5-MW turbine to improve the pitch damping of the ITI Energy barge. Moreover, I wanted to make these improvements using conventional wind turbine control techniques to establish a modified baseline with which I could compare more advanced or unconventional control scenarios. Even though I performed this work specifically for the NREL baseline wind turbine and the ITI Energy barge, the analysis process is valid for other concepts in which floating platforms support wind turbines controlled by blade pitch. Section 7.3 qualitatively discusses other potential methods for improving the damping performance using wind turbine control.

First, however, it is important to describe the barge-pitch damping problem in more detail. Section 7.1 presents more details and a quantification of the problem.

\subsection{Overview of the Platform-Pitch-Damping Problem}

The barge-pitch damping problem can be analyzed by considering the rigid-body platform-pitch mode as a single DOF. The equation of motion for this simple model is

$$
\left(I_{\text {Mass }}+A_{\text {Radiation }}\right) \ddot{\xi}+\left(B_{\text {Radiation }}+B_{\text {Viscous }}\right) \dot{\xi}+\left(C_{\text {Hydrostatic }}+C_{\text {Lines }}\right) \xi=L_{H H} T,
$$

where $\xi$ is the platform-pitch angle (i.e., rotational displacement), $\dot{\xi}$ is the platform-pitch rotational velocity, $\ddot{\xi}$ is the platform-pitch rotational acceleration, $I_{\text {Mass }}$ is the pitch inertia associated with wind turbine and barge mass, $A_{\text {Radiation }}$ is the added inertia (added mass) associated with hydrodynamic radiation in pitch, $B_{\text {Radiation }}$ is the damping associated with hydrodynamic radiation in pitch, $B_{V i s c o u s}$ is the linearized damping associated with hydrodynamic viscous drag in pitch, $C_{\text {Hydrostatic }}$ is the hydrostatic restoring in pitch, $C_{\text {Lines }}$ is the linearized 
hydrostatic restoring in pitch from all mooring lines, $T$ is the aerodynamic rotor thrust, and $L_{H H}$ is the hub height (i.e., rotor-thrust moment arm).

Though not directly evident from Eq. (7-1), the aerodynamic rotor thrust also contributes to the platform-pitch damping. To consider its effect, it is convenient to state the equation of motion in terms of the translational motion of the hub instead of the pitching motion of the platform. For small pitch angles, the translational displacement of the hub, $x$, is linearly related to the platformpitch angle by

$$
x=L_{H H} \xi .
$$

Like the blade-pitch sensitivity discussed in Section 3.1.6.3, the aerodynamic rotor thrust depends on the wind speed, rotor speed, and blade-pitch angle. To be clear, its dependence on the wind speed is actually a dependence on the relative wind speed at the hub because the hub can move in this simple model of the platform-pitch mode. If the hub translation varies slowly, the wake of the rotor will respond to changes in hub speed just as it does to changes in wind speed. Considering variations in aerodynamic rotor thrust only with hub speed, a first-order Taylor series expansion gives

$$
T=T_{0}-\frac{\partial T}{\partial V} \dot{x}
$$

where $T_{0}$ is the aerodynamic rotor thrust at a linearization point and $V$ is the rotor-disk-averaged wind speed.

The negative sign appears in Eq. (7-3) because, from Figure 2-1, positive platform-pitch angles correspond to downwind translational displacements of the hub, resulting in a reduction of the relative wind speed. By combining Eqs. (7-1) through (7-3) and simplifying, the equation of motion of the platform-pitch mode stated in terms of the translational motion of the hub becomes

$$
\underbrace{\left(\frac{I_{\text {Mass }}+A_{\text {Radiation }}}{L_{H H}^{2}}\right)}_{M_{x}} \ddot{x}+\underbrace{\left(\frac{B_{\text {Radiation }}+B_{\text {Viscous }}}{L_{H H}^{2}}+\frac{\partial T}{\partial V}\right)}_{C_{x}} \dot{x}+\underbrace{\left(\frac{C_{\text {Hydrostatic }}+C_{\text {Lines }}}{L_{H H}^{2}}\right)}_{K_{x}} x=T_{0} \text {. }
$$

As in the PID-controlled rotor-speed error, one can see that the isolated rigid-body platformpitch DOF will respond as a second-order system with the natural frequency, $\omega_{x n}$, and damping ratio, $\zeta_{x}$, equal to

$$
\begin{gathered}
\omega_{x n}=\sqrt{\frac{K_{x}}{M_{x}}} \\
\text { and } \\
\zeta_{x}=\frac{C_{x}}{2 \sqrt{K_{x} M_{x}}} .
\end{gathered}
$$


Most of the terms in the effective mass, damping, and stiffness coefficients in Eq. (7-4) are easy to quantify. In particular, the terms related to the effective mass and stiffness, including the added inertia (added mass) in pitch and the linearized pitch restoring of the mooring system, are easily computed from a linearization analysis in FAST with HydroDyn. This linearization analysis resulted in a platform-pitch natural frequency for the ITI Energy barge with the NREL 5-MW baseline wind turbine of $\omega_{x n}=0.5420 \mathrm{rad} / \mathrm{s}=0.0863 \mathrm{~Hz}{ }^{1}$

Two terms in Eq. (7-4), the damping associated with hydrodynamic radiation in pitch, $B_{\text {Radiation }}$, and the thrust sensitivity to wind speed, $\partial T / \partial V$, are more difficult to quantify. The former is problematic because the hydrodynamic wave-radiation loads in the true linear hydrodynamicloading expressions are actually described by a convolution integral (see Section 2.4.1.3), which is used to capture the wave-radiation memory effect. This convolution term is not convenient in this analysis or in the design of modern control systems. For use in controls engineering, for instance, Ref. [53] describes a method of converting the convolution term to state-space form by adding "radiation memory states." To get around this complication in this analysis, however, I neglected the memory effect and approximated $B_{\text {Radiation }}$ as the amount of linear radiation damping at the platform-pitch natural frequency, $\omega_{x n}$ (i.e., $B_{\text {Radiation }} \approx 0.86 \mathrm{E}+8 \mathrm{~kg} \cdot \mathrm{m}^{2} / \mathrm{s}$ from Figure 4-5). This choice is consistent with the linear time-domain representation of the frequency-domain problem described in Section 2.4.2.1.

The thrust sensitivity to wind speed, $\partial T / \partial V$, can be computed in multiple ways. One way would be to estimate this sensitivity (at each wind speed) as the slope of the steady-state thrust versus wind-speed response discussed in Section 3.1.8 and presented in Figure 3-12. (Because the aerodynamic rotor thrust depends on wind speed, among other factors, the thrust sensitivity to wind speed depends on wind speed as well.) This way of computing the thrust sensitivity to wind speed characterizes the sensitivity of an ideal closed-loop blade-pitch speed-regulation system. I say "ideal" because a real blade-pitch control system (see Section 3.1.6.3) responds to rotor-speed error (not variations in wind speed) and because the steady-state speed is constant with wind speed throughout Region 3 where the rotor-speed control system functions (again, see Figure 3-12).

A second way of estimating the thrust sensitivity to wind speed would be to perform a linearization analysis in FAST with AeroDyn. FAST with AeroDyn could be used to compute $\partial T / \partial V$ at each of a number of given, steady, and uniform wind speeds and at the associated rotor speeds and blade-pitch angles from the steady-state response. This would be accomplished

\footnotetext{
${ }^{1}$ This frequency falls in the range of typical sea states, which have peak spectral periods in the range of 5 to $20 \mathrm{~s}$ (see Figure 3-15) corresponding to frequencies in the range of 0.05 to $0.2 \mathrm{~Hz}$ (i.e., 0.314 to $1.257 \mathrm{rad} / \mathrm{s}$ ). The barge will tend to oscillate at the excitation frequency of the incident waves, but the motions will be most severe when the wave-excitation frequency is at or near the barge's natural frequency. If the barge were to oscillate at its natural frequency with a pitch amplitude of $A_{\xi}$, the amplitude of the hub translational velocity would be $A_{\xi} L_{H H} \omega_{x n}$ and the amplitude of the hub translational acceleration would be $A_{\xi} L_{H H} \omega_{x n}^{2}$. For $A_{\xi}=5^{\circ}$, this translates into hub velocity and acceleration amplitudes of about $4.26 \mathrm{~m} / \mathrm{s}$ and $2.31 \mathrm{~m} / \mathrm{s}^{2}=0.24 \mathrm{~g}$ 's, respectively; for $A_{\xi}=10^{\circ}$, this translates into hub velocity and acceleration amplitudes of about $8.51 \mathrm{~m} / \mathrm{s}$ and $4.61 \mathrm{~m} / \mathrm{s}^{2}=0.47 \mathrm{~g}$ 's, respectively. At these amplitudes, the wind turbine control system will continuously switch between below- and above-rated control regions, except at the very lowest and highest mean hub-height wind speeds.
} 
by perturbing the wind speed at each operating point and measuring the variation in the resulting aerodynamic thrust (using the same process described in Section 3.1.6.3 for computing the aerodynamic power sensitivity to blade-pitch angle). This way of computing the thrust sensitivity to wind speed characterizes the sensitivity of an open-loop system because the bladepitch angle is not varied with the perturbations in wind speed.

I calculated $\partial T / \partial V$ using both methods and show the results in Table 7-1. I found the slope of the steady-state thrust versus wind-speed response in the ideal closed-loop method from the central-difference approximation of the derivative using the two wind speeds on either side of each given wind speed. This is why I did not estimate the slope at the cut-in and cut-out wind speeds. The magnitude of this slope is largest near rated, where the slope changes sign. In the open-loop method, the thrust sensitivity increases with wind speed below rated and remains flat and positive above rated.

Using these thrust sensitivities to wind speed and other properties of the ITI Energy barge with the NREL 5MW baseline wind turbine, I estimated the barge-pitch damping ratios according to Eq. (7-6). Figure 7-1 presents these ratios. The barge-pitch damping ratio is largest in magnitude and changes sign at the rated wind speed of $11.4 \mathrm{~m} / \mathrm{s}$ for the ideal closed-loop method, just like the thrust sensitivity to wind speed. Just above rated, the damping ratio is less than $-10 \%$. Near the cut-out wind speed of $25 \mathrm{~m} / \mathrm{s}$, the positive-valued hydrodynamic-radiation and viscous damping exceed the magnitude of the negative-valued aerodynamic damping, so the barge-pitch damping ratio becomes slightly positive again. In the open-loop method, the barge-

Table 7-1. Pitch-to-Feather Sensitivity of Aerodynamic Thrust to Wind Speed

\begin{tabular}{|l|l|l|r|r|}
\hline $\begin{array}{l}\text { Wind Speed } \\
(\mathrm{m} / \mathrm{s})\end{array}$ & $\begin{array}{l}\text { Rotor Speed } \\
(\mathrm{rpm})\end{array}$ & $\begin{array}{l}\text { Pitch Angle } \\
\left({ }^{\circ}\right)\end{array}$ & $\begin{array}{r}\text { Open-Loop } \partial T / \partial V \\
(\mathrm{~N} /(\mathrm{m} / \mathrm{s}))\end{array}$ & $\begin{array}{r}\text { Ideal Closed-Loop } \partial T / \partial V \\
(\mathrm{~N} /(\mathrm{m} / \mathrm{s}))\end{array}$ \\
\hline $3.0-$ Cut-In & 6.97 & 0.00 & $39.43 \mathrm{E}+3$ & $48.61 \mathrm{E}+3$ \\
\hline 4.0 & 7.18 & 0.00 & $32.81 \mathrm{E}+3$ & $57.17 \mathrm{E}+3$ \\
\hline 5.0 & 7.51 & 0.00 & $36.17 \mathrm{E}+3$ & $64.83 \mathrm{E}+3$ \\
\hline 6.0 & 7.94 & 0.00 & $39.86 \mathrm{E}+3$ & $73.89 \mathrm{E}+3$ \\
\hline 7.0 & 8.47 & 0.00 & $43.63 \mathrm{E}+3$ & $90.33 \mathrm{E}+3$ \\
\hline 8.0 & 9.16 & 0.00 & $46.49 \mathrm{E}+3$ & $106.74 \mathrm{E}+3$ \\
\hline 9.0 & 10.30 & 0.00 & $52.26 \mathrm{E}+3$ & $105.69 \mathrm{E}+3$ \\
\hline 10.0 & 11.43 & 0.00 & $57.97 \mathrm{E}+3$ & $-0.769 \mathrm{E}+3$ \\
\hline 11.0 & 11.89 & 0.00 & $57.64 \mathrm{E}+3$ & $-91.09 \mathrm{E}+3$ \\
\hline 12.0 & 12.10 & 3.82 & $74.80 \mathrm{E}+3$ & $-66.00 \mathrm{E}+3$ \\
\hline 13.0 & 12.10 & 6.60 & $79.98 \mathrm{E}+3$ & $-43.99 \mathrm{E}+3$ \\
\hline 14.0 & 12.10 & 8.67 & $82.50 \mathrm{E}+3$ & $-33.37 \mathrm{E}+3$ \\
\hline 15.0 & 12.10 & 10.45 & $83.82 \mathrm{E}+3$ & $-26.39 \mathrm{E}+3$ \\
\hline 16.0 & 12.10 & 12.05 & $84.49 \mathrm{E}+3$ & $-21.42 \mathrm{E}+3$ \\
\hline 17.0 & 12.10 & 13.54 & $84.90 \mathrm{E}+3$ & $-17.68 \mathrm{E}+3$ \\
\hline 18.0 & 12.10 & 14.92 & $85.85 \mathrm{E}+3$ & $-14.79 \mathrm{E}+3$ \\
\hline 19.0 & 12.10 & 16.23 & $86.43 \mathrm{E}+3$ & $-12.79 \mathrm{E}+3$ \\
\hline 20.0 & 12.10 & 17.47 & $85.41 \mathrm{E}+3$ & $-11.77 \mathrm{E}+3$ \\
\hline 21.0 & 12.10 & 18.70 & $84.33 \mathrm{E}+3$ & $-10.79 \mathrm{E}+3$ \\
\hline 22.0 & 12.10 & 19.94 & $84.29 \mathrm{E}+3$ & $-9.26 \mathrm{E}+3$ \\
\hline 23.0 & 12.10 & 21.18 & $85.34 \mathrm{E}+3$ & $-7.92 \mathrm{E}+3$ \\
\hline 24.0 & 12.10 & 22.35 & $85.22 \mathrm{E}+3$ & \\
\hline $25.0-$ Cut-Out & 12.10 & 23.47 & $84.39 \mathrm{E}+3$ & \\
\hline
\end{tabular}




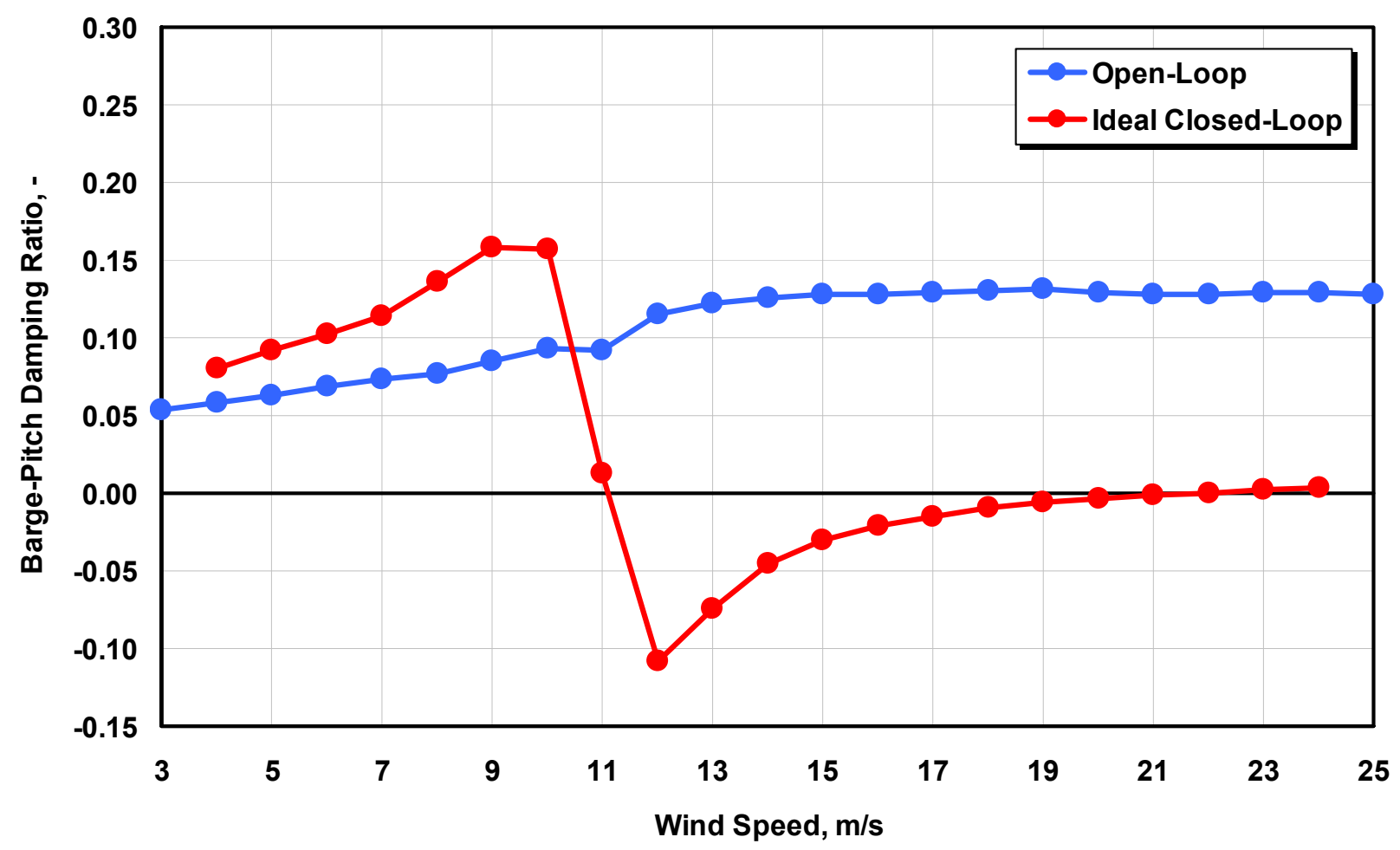

Figure 7-1. Pitch-to-feather barge-pitch damping ratios

pitch damping ratio remains positive across all wind speeds. The ratio increases with wind speed below rated and remains flat above rated. With real blade-pitch-control speed regulation above rated, the actual damping ratio is difficult to quantify with this simple model, but will fall somewhere between the bounds imposed by the open- and ideal closed-loop results.

\subsection{Influence of Conventional Wind Turbine Control Methodologies}

To improve the barge-pitch damping, I modified the baseline control system with a number of conventional wind turbine control methodologies. These included (1) adding a second bladepitch control loop through feedback of tower-top acceleration, (2) changing from variable bladepitch-to-feather to variable blade-pitch-to-stall speed-control regulation, and (3) detuning the gains in the variable blade-pitch-to-feather rotor-speed controller. I developed and tested (through simulation) each approach independently. The rationale behind each approach and the main findings are presented in Sections 7.2.1, 7.2.2, and 7.2.3, respectively.

\subsubsection{Feedback of Tower-Top Acceleration}

The conventional approach to improving the tower fore-aft damping in land-based wind turbines is to append the conventional blade-pitch controller for rotor-speed regulation with an additional blade-pitch control loop, which uses a tower-top acceleration measurement [3]. Naturally, the same technique could be applied to modify the platform-pitch damping of an offshore floating wind turbine. The intent of the new control loop would be to augment the aerodynamic rotor thrust with adjustments to the blade-pitch angle based on the tower-top acceleration measurement. To see the effect of blade-pitch angle on the platform-pitch damping, consider 
variations in the aerodynamic thrust with full-span rotor-collective blade-pitch angles, $\theta$, in addition to hub speed, as was accounted for in Eq. (7-3):

$$
T=T_{0}-\frac{\partial T}{\partial V} \dot{x}+\frac{\partial T}{\partial \theta} \Delta \theta
$$

In Eq. (7-7), $\Delta \theta$ is a small perturbation of the blade-pitch angles about their operating point. If the blade-pitch rate in the tower-feedback (TFB) control loop is proportional to a tower-top acceleration measurement through a gain $K_{P x}$, then:

$$
\begin{gathered}
\Delta \dot{\theta}=K_{P x} \ddot{x} \\
\text { or } \\
\Delta \theta=K_{P x} \int_{0}^{t} \ddot{x} d t=K_{P x} \dot{x} .
\end{gathered}
$$

By combining Eqs. (7-8b) and (7-7) with the equations presented in Section 7.1 and simplifying, the addition of a TFB control loop modifies the effective damping coefficient from Eq. (7-4) and becomes

$$
C_{x}=\frac{B_{\text {Radiation }}+B_{\text {Viscous }}}{L_{H H}^{2}}+\frac{\partial T}{\partial V} \underbrace{-K_{P x} \frac{\partial T}{\partial \theta}}_{\text {New Term }} .
$$

The effective mass and stiffness coefficients from Eq. (7-4) are left unchanged by the addition of the TFB control loop.

In an active blade-pitch-to-feather wind turbine, the thrust sensitivity to rotor-collective blade pitch, $\partial T / \partial \theta$, is negative-valued from cut-in to cut-out so damping is increased with a positive control gain. Once the thrust sensitivity to rotor-collective blade pitch is known and a control gain is chosen, the effective increase in platform-pitch damping ratio, $\Delta \zeta_{x}$, according to the given model is

$$
\Delta \zeta_{x}=-\frac{K_{P x}}{2 \sqrt{K_{x} M_{x}}}\left(\frac{\partial T}{\partial \theta}\right)
$$

Alternatively, a proper control gain can be chosen specifically from any desired increase in platform-pitch damping according to the given model. Just like the thrust sensitivity to wind speed, though, the thrust sensitivity to rotor-collective blade-pitch angle depends on the wind speed, rotor speed, and blade-pitch angle. Consequently, one cannot obtain a constant increase in damping ratio across control regions without gain-scheduling. The gain-scheduling law for the TFB control system will not, however, be as simple as the law used in the blade-pitch rotorspeed-regulation controller because the thrust senstitivy to blade pitch is not linearly related to 
the blade-pitch angle. I calculated the thrust sensitivity to blade pitch from a linearization analysis in FAST with AeroDyn, and show the results in Table 7-2.

In the middle of Region $3(18 \mathrm{~m} / \mathrm{s})$, a modest 0.05 increase in effective damping ratio requires a control gain of $K_{P x}=0.007556 \mathrm{rad} /(\mathrm{m} / \mathrm{s})$ and a large 0.5 increase in effective damping ratio requires a control gain of $K_{P x}=0.07556 \mathrm{rad} /(\mathrm{m} / \mathrm{s})$. Naturally, the larger the control gain, the larger the blade-pitch-rate requirement. Conversely, to limit the blade-pitch rate, one has to minimize the effective increase in damping ratio. From footnote 1 on page 125, barge-pitch amplitudes in the range of $A_{\xi}=5$ to $10^{\circ}$ can result in hub-acceleration amplitudes ranging from 2.31 to $4.61 \mathrm{~m} / \mathrm{s}^{2}$. In the middle of Region 3 according to the given model, damping these motions using the TFB control loop developed previously will require blade-pitch-rate amplitudes in the range of $\Delta \dot{\theta}=1.0$ to $2.0^{\circ}$ /s for the modest 0.05 increase in effective damping ratio and $\Delta \dot{\theta}=10.0$ to $20.0^{\circ} \mathrm{s}$ for the large 0.5 increase in effective damping ratio. Consequently, only moderately large increases in effective damping ratio are achievable with the given blade-pitch-rate limit of $8 \%$ s (from Section 3.1.6.4).

I incorporated the TFB control loop into my FAST with AeroDyn and HydroDyn simulations using an extension of the baseline control system DLL. As implemented, the TFB blade-pitch angle commands were found by measuring the tower-top fore-aft acceleration, integrating to find the tower-top fore-aft velocity, and then multiplying by the control gain. This blade-pitch-angle command was then added to the blade-pitch-angle command from the rotor-speed controller,

Table 7-2. Pitch-to-Feather Sensitivity of Aerodynamic Thrust to Blade Pitch

\begin{tabular}{|l|l|l|r|}
\hline $\begin{array}{l}\text { Wind Speed } \\
(\mathrm{m} / \mathrm{s})\end{array}$ & $\begin{array}{l}\text { Rotor Speed } \\
(\mathrm{rpm})\end{array}$ & $\begin{array}{l}\text { Pitch Angle } \\
\left({ }^{\circ}\right)\end{array}$ & $\begin{array}{r}\partial T / 2 \theta \\
(\mathrm{N} / \mathrm{rad})\end{array}$ \\
\hline $3.0-$ Cut-In & 6.97 & 0.00 & $-1.556 \mathrm{E}+6$ \\
\hline 4.0 & 7.18 & 0.00 & $-1.646 \mathrm{E}+6$ \\
\hline 5.0 & 7.51 & 0.00 & $-1.783 \mathrm{E}+6$ \\
\hline 6.0 & 7.94 & 0.00 & $-1.982 \mathrm{E}+6$ \\
\hline 7.0 & 8.47 & 0.00 & $-2.238 \mathrm{E}+6$ \\
\hline 8.0 & 9.16 & 0.00 & $-2.533 \mathrm{E}+6$ \\
\hline 9.0 & 10.30 & 0.00 & $-3.201 \mathrm{E}+6$ \\
\hline 10.0 & 11.43 & 0.00 & $-3.939 \mathrm{E}+6$ \\
\hline 11.0 & 11.89 & 0.00 & $-3.988 \mathrm{E}+6$ \\
\hline 12.0 & 12.10 & 3.82 & $-4.603 \mathrm{E}+6$ \\
\hline 13.0 & 12.10 & 6.60 & $-4.664 \mathrm{E}+6$ \\
\hline 14.0 & 12.10 & 8.67 & $-4.702 \mathrm{E}+6$ \\
\hline 15.0 & 12.10 & 10.45 & $-4.733 \mathrm{E}+6$ \\
\hline 16.0 & 12.10 & 12.05 & $-4.765 \mathrm{E}+6$ \\
\hline 17.0 & 12.10 & 13.54 & $-4.806 \mathrm{E}+6$ \\
\hline 18.0 & 12.10 & 14.92 & $-4.905 \mathrm{E}+6$ \\
\hline 19.0 & 12.10 & 16.23 & $-4.983 \mathrm{E}+6$ \\
\hline 20.0 & 12.10 & 17.47 & $-4.944 \mathrm{E}+6$ \\
\hline 21.0 & 12.10 & 18.70 & $-4.906 \mathrm{E}+6$ \\
\hline 22.0 & 12.10 & 19.94 & $-4.979 \mathrm{E}+6$ \\
\hline 23.0 & 12.10 & 21.18 & $-5.125 \mathrm{E}+6$ \\
\hline 24.0 & 12.10 & 22.35 & $-5.182 \mathrm{E}+6$ \\
\hline $25.0-$ Cut-Out & 12.10 & 23.47 & $-5.200 \mathrm{E}+6$ \\
\hline
\end{tabular}


which had already been saturated for the angle and rate limits. Said another way, I did not saturate the TFB control system commands.

For the NREL 5-MW wind turbine mounted atop the ITI Energy barge, I tested the system response at a variety of wind and wave conditions with both the modest and high TFB gains. Figure 7-2 shows a response with the high $K_{P x}=0.07556 \mathrm{rad} /(\mathrm{m} / \mathrm{s})$ TFB gain for a simulation with stochastic winds with a mean hub-height wind speed of $18 \mathrm{~m} / \mathrm{s}$ and irregular waves with a significant wave height of $3.673 \mathrm{~m}$ and a peak spectral period of $13.376 \mathrm{~s}$. (These waves have the expected value of the significant wave height and the median value of the peak spectral period conditioned on the mean hub-height wind speed at the chosen reference site; see Figure 3-15). The system response with the unmodified (baseline) control system is shown for comparison in Figure 7-2. I ran the simulations with all appropriate and available DOFs enabled, as applied in the loads analysis and described in Section 5.1, but without considering the rotormass imbalance or the increased blade structural-damping ratio. In Figure 7-2, the ordinates "GenPwr," "GenSpeed," "B1Pitch1," and "PtfmPitch" correspond to the instantaneous electrical output of the generator, generator (high-speed shaft) rotational speed, pitch angle of Blade 1, and platform-pitch angle, respectively.

It may seem surprising at first that the results do not show a large improvement in the damping of the barge-pitch motion ("PtfmPitch"). The exacerbated excursions in generator speed and electrical output are more prominent. These results can be explained by thinking about the problem in more detail than is provided in the simple model. The relative wind speed is highest when the system is pitching into the wind (i.e., from maximum to minimum barge-pitch angles).
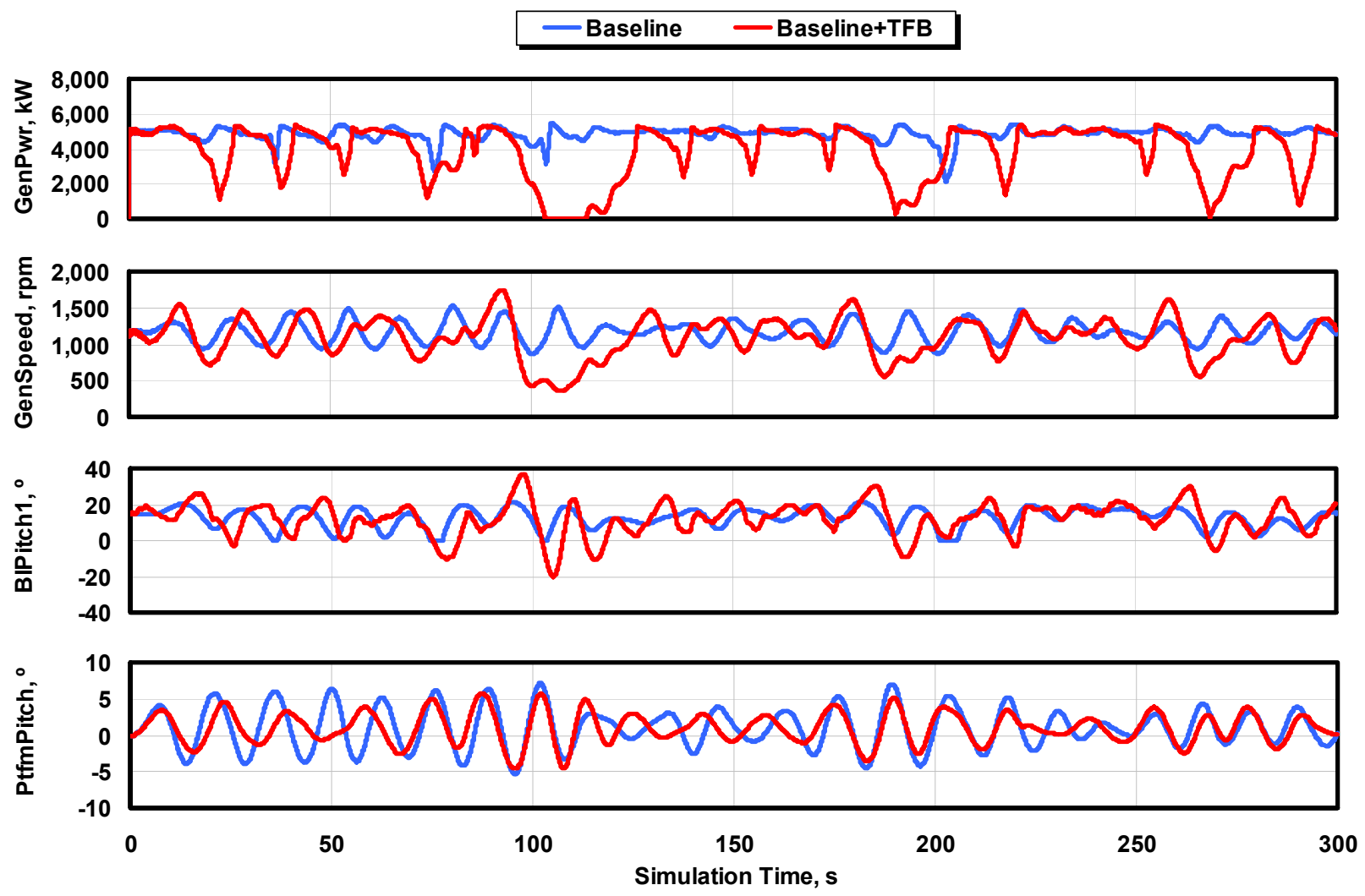

Figure 7-2. System responses with and without a tower-feedback control loop 
This causes the rotor-speed control system to pitch the blades to feather (more positive) to shed power and regulate speed while the TFB damping control system pitches the blades to stall (more negative) to increase thrust and introduce damping. The reverse is true when the turbine is pitching with the wind. In this case, the relative wind speed is lowest when the system pitches downwind, causing the rotor-speed control system to pitch the blades to stall while the TFB damping control system commands pitching to feather. Said another way, the two blade-pitch control systems are at odds and fight with each other in this situation. This can be seen by the blade-pitch angle responses of Figure 7-2, where in many instances, the pitch-angle commands in the baseline control system move in the opposite direction to the pitch-angle commands in the combined baseline and TFB control system.

Similar results (not shown) are obtained with the more modest $K_{P x}=0.007556 \mathrm{rad} /(\mathrm{m} / \mathrm{s}$ ) TFB gain. Here, the generator speed and power excursions are not as badly exacerbated, but there is also less improvement in the damping of the barge-pitch motion.

The simple model I describe in this section is routinely applied in the design of TFB damping control algorithms for land-based wind turbines. But, because the amplitude of the overall tower motion is less in land-based turbines, the problem with generator speed and power excursions is less of an issue. Instead, the control system designer for land-based wind turbines must make a basic trade-off between improved tower damping and increased generator speed and power excursions. For the floating system considered in this work, however, the severity of the towertop motions induced by the barge's movement with surface waves renders the conventional TFB damping control system ineffective.

\subsubsection{Active Pitch-to-Stall Speed-Control Regulation}

As described in the introduction to this chapter, the problem to be addressed is that the reduction in steady-state rotor thrust with increasing wind speed in Region 3, which occurs as a result of variable blade-pitch-to-feather speed-control regulation, may introduce negative damping in the platform-pitch mode. This implies that variable blade-pitch-to-stall speed-control regulation may be more effective at damping the barge-pitch motions because drag (and hence thrust) increase as power is shed (to regulate speed) in increasing relative winds in wind turbines controlled by an active pitch-to-stall system. Although variable blade-pitch-to-stall speedcontrol regulation has been shown to work effectively in simulation, it has not been widely pursued in the wind industry because of the "uncertainty that remains in the theoretical understanding of stalled rotor aerodynamics" [3, p. 234]. In spite of this uncertainty, I tested the effects of active pitch-to-stall speed-control regulation for the floating wind barge concept.

Before pursuing the design of the pitch-to-stall controller, I decided to smooth the airfoil-data coefficients (as presented in Figure 3-1 through Figure 3-6) near stall to eliminate the existing fluctuations that could have led to numerical problems in the BEM aerodynamic-induction solution algorithm. I modified the airfoils by manually manipulating the lift coefficients. 
Once the airfoil data were corrected, I redeveloped the full-span rotor-collective blade-pitch controller according to the same procedure I used to arrive at the blade-pitch-to-feather speedcontrol gains (see Section 3.1.6.3). Like I did for the pitch-to-feather controller design, I calculated the sensitivity of aerodynamic power to blade pitch, $\partial P / \partial \theta$, by performing a linearization analysis in FAST with AeroDyn. Table 7-3 shows the results for operation in Region 3.

The blade-pitch angles that produce the rated mechanical power (5.296610 MW) are negativeand double-valued over the wind-speed range of Region 3, first decreasing, then increasing, with increasing wind speed. By being double-valued, it is impossible to use the same gain-scheduling law I implemented in the active pitch-to-feather controller. But because, the variation in bladepitch sensitivity across Region 3 is less pronounced, gain scheduling is less of a requirement. Instead, I chose constant gains using the value of $\partial P / \partial \theta$ in the middle of Region $3(18 \mathrm{~m} / \mathrm{s})$ to develop the PID gains. Using the recommended response characteristics of $\omega_{\varphi n}=0.6 \mathrm{rad} / \mathrm{s}$ and $\zeta_{\varphi}=0.7$ [29] resulted in gains of $K_{P}=-0.00731238 \mathrm{~s}, K_{I}=-0.00313388$, and $K_{D}=0.0 \mathrm{~s}^{2}$. The gains are negative-valued because $\partial P / \partial \theta$ is positive-valued. The gains are also smaller in magnitude than the pitch-to-feather gains at rated because the blade-pitch sensitivity — or control authority - is higher in pitch-to-stall operation.

I incorporated the blade-pitch-to-stall speed-regulation controller into my FAST with AeroDyn and HydroDyn simulations by a simple modification of the baseline control system DLL. I modified the gains to the values derived in this section and I fixed the maximum and minimum pitch settings at $0^{\circ}$ and $-90^{\circ}$, respectively. To eliminate the influence of the gain-scheduling law, I set $\theta_{K}$ to an arbitrarily large value. Like I did for the baseline system in Figure 3-12, I obtained the steady-state response of the land-based NREL 5-MW wind turbine by running a series of FAST with AeroDyn simulations at a number of given, steady, and uniform wind speeds. The results for the same output parameters plotted in Figure 3-12 are shown in Figure 7-3.

Table 7-3. Sensitivity of Aerodynamic Power to Blade Pitch (to Stall)

\begin{tabular}{|l|l|r|r|}
\hline $\begin{array}{l}\text { Wind Speed } \\
(\mathrm{m} / \mathrm{s})\end{array}$ & $\begin{array}{l}\text { Rotor Speed } \\
(\mathrm{rpm})\end{array}$ & $\begin{array}{r}\text { Pitch Angle } \\
\left({ }^{\circ}\right)\end{array}$ & $\begin{array}{r}\partial P / \partial \theta \\
(\text { watt/rad })\end{array}$ \\
\hline $11.4-$ Rated & 12.1 & 0.00 & $-28.24 \mathrm{E}+6$ \\
\hline 12.0 & 12.1 & -6.76 & $27.72 \mathrm{E}+6$ \\
\hline 13.0 & 12.1 & -7.50 & $46.94 \mathrm{E}+6$ \\
\hline 14.0 & 12.1 & -7.79 & $47.14 \mathrm{E}+6$ \\
\hline 15.0 & 12.1 & -8.05 & $49.82 \mathrm{E}+6$ \\
\hline 16.0 & 12.1 & -8.22 & $54.43 \mathrm{E}+6$ \\
\hline 17.0 & 12.1 & -8.26 & $59.92 \mathrm{E}+6$ \\
\hline 18.0 & 12.1 & -8.20 & $65.70 \mathrm{E}+6$ \\
\hline 19.0 & 12.1 & -8.05 & $71.22 \mathrm{E}+6$ \\
\hline 20.0 & 12.1 & -7.84 & $75.64 \mathrm{E}+6$ \\
\hline 21.0 & 12.1 & -7.60 & $81.00 \mathrm{E}+6$ \\
\hline 22.0 & 12.1 & -7.32 & $84.96 \mathrm{E}+6$ \\
\hline 23.0 & 12.1 & -7.03 & $88.59 \mathrm{E}+6$ \\
\hline 24.0 & 12.1 & -6.74 & $89.94 \mathrm{E}+6$ \\
\hline 25.0 & 12.1 & -6.46 & $91.10 \mathrm{E}+6$ \\
\hline
\end{tabular}



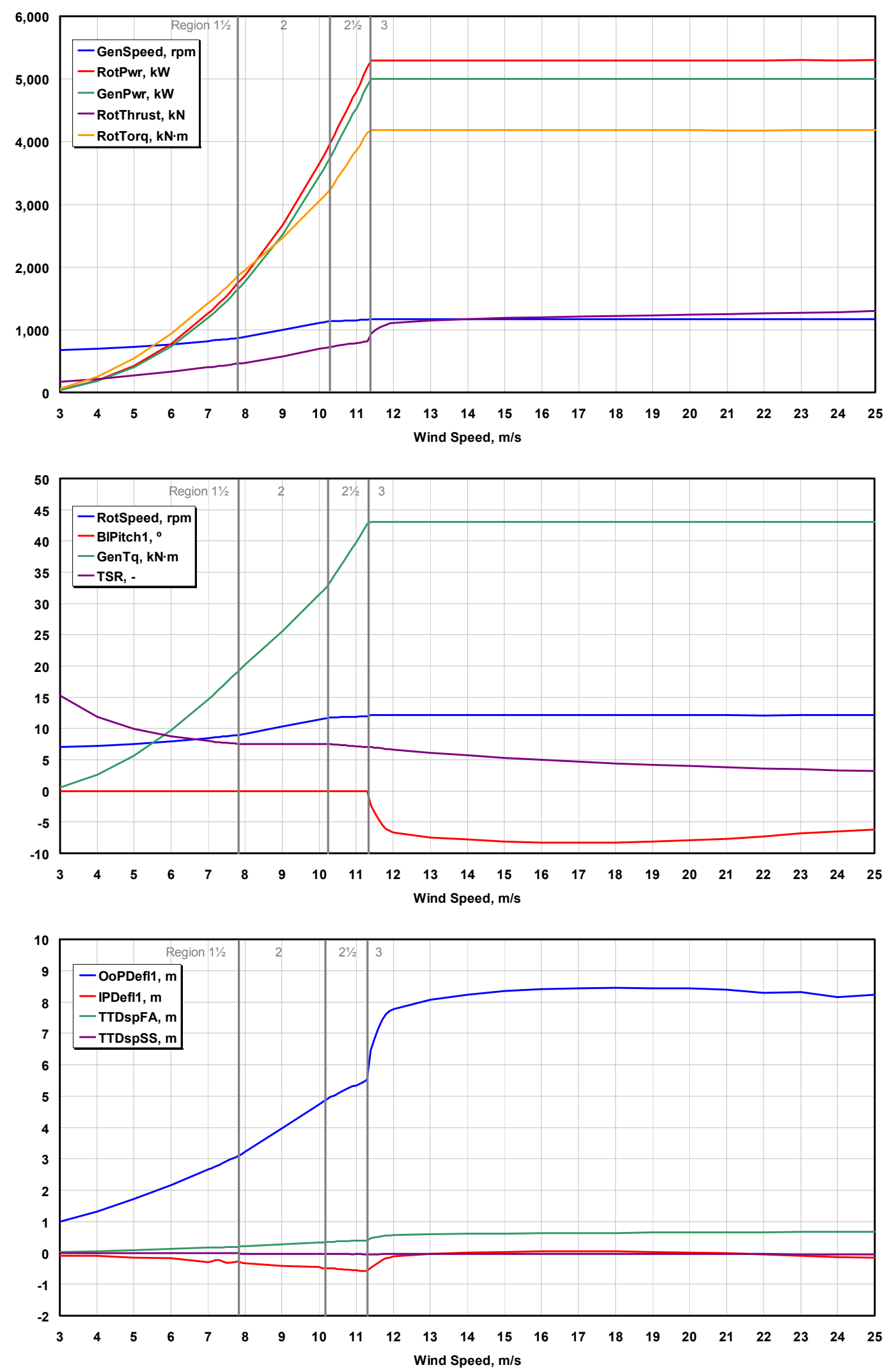

Figure 7-3. Steady-state pitch-to-stall responses as a function of wind speed 
As shown in Figure 7-3, the intended increase in rotor thrust ("RotThrust") with wind speed across all control regions is noticeable, although the increase is small from just above rated to cut-out. As a result of the thrust increase, and in contrast to the pitch-to-feather response, the out-of-plane blade-tip deflection ("OoPDefl1") and the tower-top fore-aft displacement ("TTDspFA") both increase dramatically upon entering Region 3. I left the baseline generatortorque controller unchanged so the steady-state system response is identical to the response of the pitch-to-feather system below rated wind speed.

As in Section 7.2.1, I tested the system response of the NREL 5-MW wind turbine mounted atop the ITI Energy barge with this new control system at a variety of wind and wave conditions. Figure 7-4 compares the active pitch-to-feather and active pitch-to-stall system responses for the simulation with the same wind and wave conditions applied to the simulations presented in Figure 7-2 (i.e., an 18-m/s stochastic wind, a 3.673-m significant wave height, and a 13.376-s peak spectral period). Again, I ran the simulations with all appropriate and available DOFs enabled, but without considering the rotor-mass imbalance or the increased blade structuraldamping ratio.

As shown in Figure 7-4, active blade-pitch-to-stall control regulates generator speed - and hence electrical power-very well. It performs even better than the baseline active blade-pitch-tofeather controller. What it does not do, however, is dampen the barge-pitch motions as intended. In fact, the barge-pitch motions are exaggerated in comparison to the response using the baseline active pitch-to-feather controller. This seemingly contradictory result can be understood by examining the barge-pitch damping ratios resulting from the active pitch-to-stall control. As for
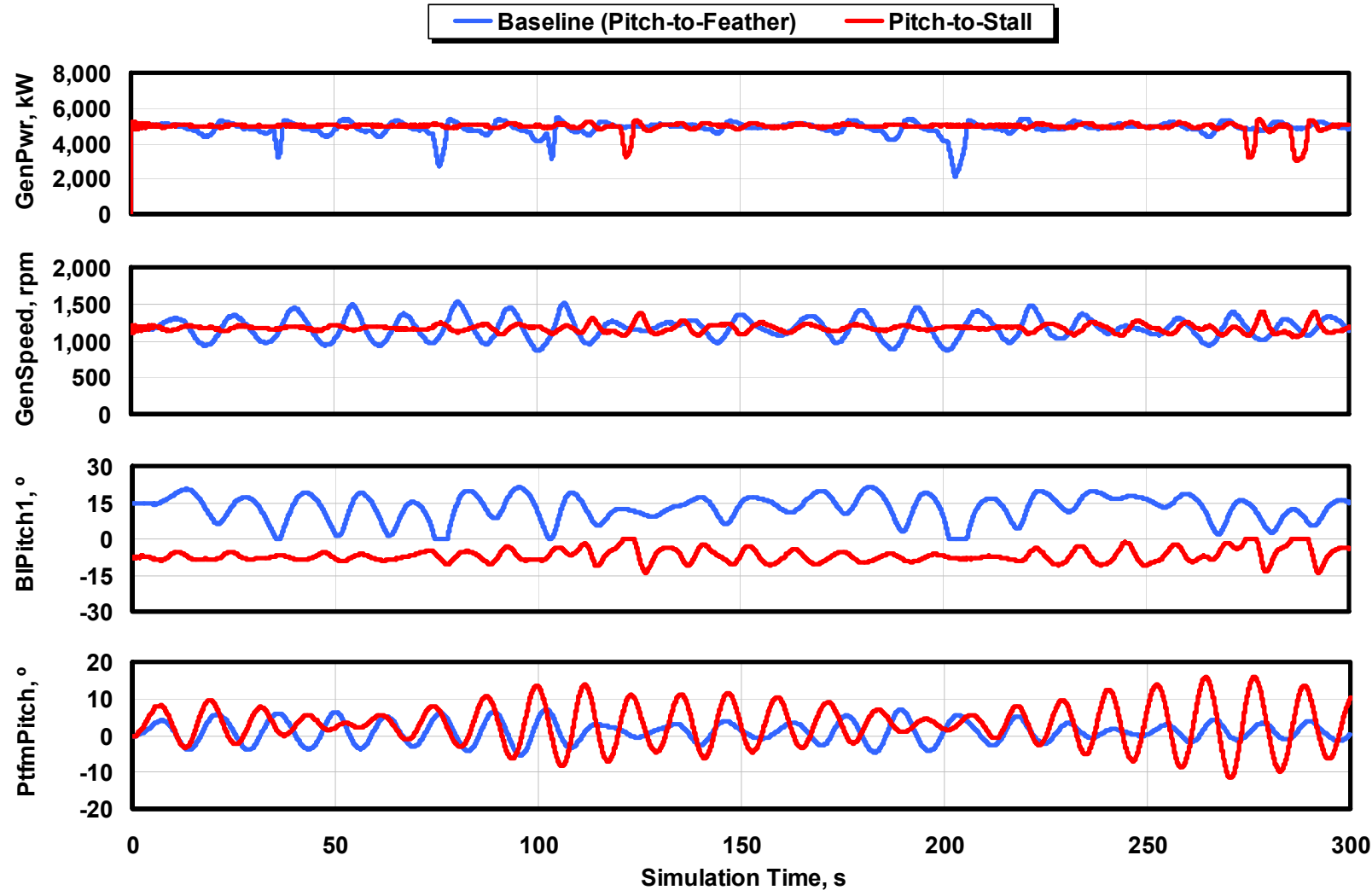

Figure 7-4. Comparison of pitch-to-feather and pitch-to-stall system responses 
the pitch-to-feather system, the thrust sensitivity to wind speed ( $\partial T / \partial V$ ) can be found using the open-loop and ideal closed-loop methods, and both methods can be used to estimate the bargepitch damping ratios. The results of these calculations are presented in Table 7-4 and Figure 7-5.

In Figure 7-5, the barge-pitch damping ratios associated with the pitch-to-stall system are plotted along with the original values presented in Figure 7-1 for the pitch-to-feather system. Because the two systems are identical below rated wind speed, so are the barge-pitch damping ratios. Above rated wind speed, however, the pitch-to-stall ratios diverge from the pitch-to-feather ratios. Unlike the pitch-to-feather values, the barge-pitch damping ratio remains positive-valued across control regions for the ideal closed-loop pitch-to-stall method because the thrust sensitivity to wind speed remains positive-valued (see the last column of Table 7-4). In the pitch-to-stall system slightly above rated, the open- and ideal closed-loop bounds imposed by the barge-pitch damping ratios converge toward each other and remain at or near $2.5 \%$ across the remainder of Region 3.

As I mentioned at the end of Section 7.1, with real blade-pitch-control speed regulation above rated wind speed, the actual damping ratio will lie somewhere between the bounds imposed by the open- and ideal closed-loop results. This implies that the real blade-pitch-to-stall controller, regardless of its gains, will give the system a slightly stable barge-pitch damping ratio near 2.5\% across Region 3, starting just above rated. Moreover, because the barge-pitch motions for the pitch-to-stall system are larger than those for the pitch-to-feather system in the time histories presented in Figure 7-4, I can conclude that the real blade-pitch-to-feather speed controller

Table 7-4. Pitch-to-Stall Sensitivity of Aerodynamic Thrust to Wind Speed

\begin{tabular}{|l|l|l|r|r|}
\hline $\begin{array}{l}\text { Wind Speed } \\
(\mathrm{m} / \mathrm{s})\end{array}$ & $\begin{array}{l}\text { Rotor Speed } \\
(\mathrm{rpm})\end{array}$ & $\begin{array}{l}\text { Pitch Angle } \\
\left({ }^{\circ}\right)\end{array}$ & $\begin{array}{r}\text { Open-Loop } \partial T / \partial V \\
(\mathrm{~N} /(\mathrm{m} / \mathrm{s}))\end{array}$ & $\begin{array}{r}\text { Ideal Closed-Loop } \partial T / \partial V \\
(\mathrm{~N} /(\mathrm{m} / \mathrm{s}))\end{array}$ \\
\hline $3.0-$ Cut-In & 6.97 & 0.00 & $29.43 \mathrm{E}+3$ & $48.61 \mathrm{E}+3$ \\
\hline 4.0 & 7.18 & 0.00 & $32.81 \mathrm{E}+3$ & $57.17 \mathrm{E}+3$ \\
\hline 5.0 & 7.51 & 0.00 & $36.17 \mathrm{E}+3$ & $64.83 \mathrm{E}+3$ \\
\hline 6.0 & 7.94 & 0.00 & $39.86 \mathrm{E}+3$ & $73.89 \mathrm{E}+3$ \\
\hline 7.0 & 8.47 & 0.00 & $43.63 \mathrm{E}+3$ & $90.33 \mathrm{E}+3$ \\
\hline 8.0 & 9.16 & 0.00 & $46.49 \mathrm{E}+3$ & $106.74 \mathrm{E}+3$ \\
\hline 9.0 & 10.30 & 0.00 & $52.26 \mathrm{E}+3$ & $105.69 \mathrm{E}+3$ \\
\hline 10.0 & 11.43 & 0.00 & $57.97 \mathrm{E}+3$ & $207.25 \mathrm{E}+3$ \\
\hline 11.0 & 11.89 & 0.00 & $57.64 \mathrm{E}+3$ & $179.29 \mathrm{E}+3$ \\
\hline 12.0 & 12.10 & -6.76 & $15.69 \mathrm{E}+3$ & $31.74 \mathrm{E}+3$ \\
\hline 13.0 & 12.10 & -7.50 & $9.09 \mathrm{E}+3$ & $18.75 \mathrm{E}+3$ \\
\hline 14.0 & 12.10 & -7.79 & $9.79 \mathrm{E}+3$ & $16.07 \mathrm{E}+3$ \\
\hline 15.0 & 12.10 & -8.05 & $9.96 \mathrm{E}+3$ & $13.58 \mathrm{E}+3$ \\
\hline 16.0 & 12.10 & -8.22 & $8.82 \mathrm{E}+3$ & $10.97 \mathrm{E}+3$ \\
\hline 17.0 & 12.10 & -8.26 & $7.39 \mathrm{E}+3$ & $9.11 \mathrm{E}+3$ \\
\hline 18.0 & 12.10 & -8.20 & $5.81 \mathrm{E}+3$ & $8.26 \mathrm{E}+3$ \\
\hline 19.0 & 12.10 & -8.05 & $4.49 \mathrm{E}+3$ & $8.08 \mathrm{E}+3$ \\
\hline 20.0 & 12.10 & -7.84 & $3.77 \mathrm{E}+3$ & $7.87 \mathrm{E}+3$ \\
\hline 21.0 & 12.10 & -7.60 & $2.78 \mathrm{E}+3$ & $11.15 \mathrm{E}+3$ \\
\hline 22.0 & 12.10 & -7.32 & $2.89 \mathrm{E}+3$ & $12.79 \mathrm{E}+3$ \\
\hline 23.0 & 12.10 & -7.03 & $3.42 \mathrm{E}+3$ & $14.10 \mathrm{E}+3$ \\
\hline 24.0 & 12.10 & -6.74 & $4.89 \mathrm{E}+3$ & \\
\hline $25.0-$ Cut-Out & 12.10 & -6.46 & $6.81 \mathrm{E}+3$ & \\
\hline & & & & \\
\hline
\end{tabular}




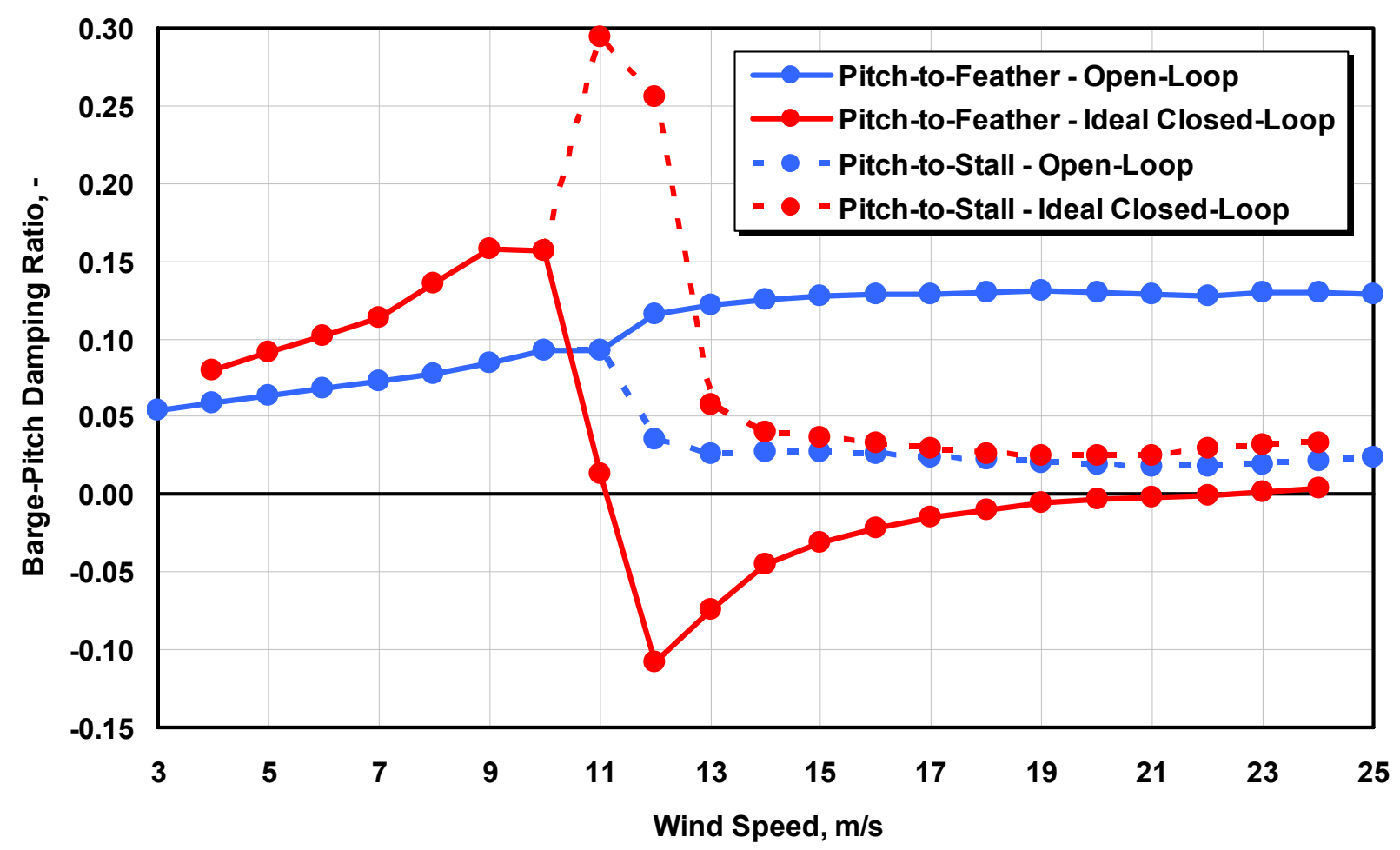

Figure 7-5. Pitch-to-feather and -stall barge-pitch damping ratios

actually has an effective damping ratio higher than $2.5 \%$ (at least for the conditions considered). In other words, the real pitch-to-feather barge-pitch damping ratio is actually much greater than what is predicted by the ideal closed-loop results. It is still, however, beneficial to increase the damping as much as possible.

One possibility for increasing the barge-pitch damping through active pitch-to-stall control is to tailor the airfoil-data coefficients so that rotor thrust increases more with wind speed in Region 3 than what resulted with the existing airfoils. Experimental data from NREL's Phase VI Unsteady Aerodynamics Experiment (UAE) [27], which tested a passive stall-regulated wind turbine in the National Full-Scale Aerodynamics Complex at the National Aeronautics and Space Administration (NASA) Ames Research Center, showed a nearly steady increase in rotor thrust with wind speed from cut-in to cut-out. This would translate into a nearly constant steady-state thrust sensitivity to wind speed, $\partial T / \partial V$, across all wind speeds for the UAE wind turbine. If the NREL 5-MW wind turbine were modified to behave comparably (e.g., to make its thrust sensitivity to wind speed in Region 3 similar to that seen in Region 2), the ideal closed-loop barge-pitch damping ratio would increase to about $15 \%$ in Region 3 . This is slightly higher damping than the open-loop damping ratio obtained with active pitch-to-feather control. To get the necessary augmentation in rotor thrust, however, the existing airfoils will need to be modified and the rotor will need to be redesigned. Both tasks are beyond the scope of this work. I also suspect that it would be quite difficult to obtain damping ratios much above $15 \%$ through rotorthrust augmentation and active pitch-to-stall speed regulation, even though a higher amount of damping is desirable. 
One might also think that combining the controller developed in this section with the TFB control loop developed in Section 7.2.1 would be another possibility for improving the bargepitch damping through active pitch-to-stall speed-regulation control. But unfortunately, a pitchto-stall control system cannot be combined with a classic TFB control loop for two reasons. First, the thrust sensitivity to rotor-collective blade pitch changes sign midway through Region 3 , implying that the TFB control gain would also have to change sign midway through Region 3 . Otherwise, the TFB control loop would actually act to reduce the effective platform-pitch damping in certain operating regions. Second, the magnitude of the thrust sensitivity to blade pitch is much smaller with pitch-to-stall control than with pitch-to-feather control. This implies that one could not achieve any significant increase in platform-pitch damping without very large control gains and resulting blade-pitch-rate requirements. Both inadequacies are observable from the pitch-to-stall thrust sensitivity, which I calculated from a FAST with AeroDyn linearization analysis and present in Table 7-5.

Table 7-5. Pitch-to-Stall Sensitivity of Aerodynamic Thrust to Blade Pitch

\begin{tabular}{|l|l|l|r|}
\hline $\begin{array}{l}\text { Wind Speed } \\
(\mathrm{m} / \mathrm{s})\end{array}$ & $\begin{array}{l}\text { Rotor Speed } \\
(\mathrm{rpm})\end{array}$ & $\begin{array}{l}\text { Pitch Angle } \\
\left({ }^{\circ}\right)\end{array}$ & $\begin{array}{r}\partial T / 2 \theta \\
(\mathrm{N} / \mathrm{rad})\end{array}$ \\
\hline $3.0-$ Cut-In & 6.97 & 0.00 & $-1.556 \mathrm{E}+6$ \\
\hline 4.0 & 7.18 & 0.00 & $-1.646 \mathrm{E}+6$ \\
\hline 5.0 & 7.51 & 0.00 & $-1.783 \mathrm{E}+6$ \\
\hline 6.0 & 7.94 & 0.00 & $-1.982 \mathrm{E}+6$ \\
\hline 7.0 & 8.47 & 0.00 & $-2.238 \mathrm{E}+6$ \\
\hline 8.0 & 9.16 & 0.00 & $-2.533 \mathrm{E}+6$ \\
\hline 9.0 & 10.30 & 0.00 & $-3.201 \mathrm{E}+6$ \\
\hline 10.0 & 11.43 & 0.00 & $-3.939 \mathrm{E}+6$ \\
\hline 11.0 & 11.89 & 0.00 & $-3.988 \mathrm{E}+6$ \\
\hline 12.0 & 12.10 & -6.76 & $-1.216 \mathrm{E}+6$ \\
\hline 13.0 & 12.10 & -7.50 & $-0.388 \mathrm{E}+6$ \\
\hline 14.0 & 12.10 & -7.79 & $-0.279 \mathrm{E}+6$ \\
\hline 15.0 & 12.10 & -8.05 & $-0.109 \mathrm{E}+6$ \\
\hline 16.0 & 12.10 & -8.22 & $0.123 \mathrm{E}+6$ \\
\hline 17.0 & 12.10 & -8.26 & $0.331 \mathrm{E}+6$ \\
\hline 18.0 & 12.10 & -8.20 & $0.536 \mathrm{E}+6$ \\
\hline 19.0 & 12.10 & -8.05 & $0.725 \mathrm{E}+6$ \\
\hline 20.0 & 12.10 & -7.84 & $0.887 \mathrm{E}+6$ \\
\hline 21.0 & 12.10 & -7.60 & $1.069 \mathrm{E}+6$ \\
\hline 22.0 & 12.10 & -7.32 & $1.182 \mathrm{E}+6$ \\
\hline 23.0 & 12.10 & -7.03 & $1.254 \mathrm{E}+6$ \\
\hline 24.0 & 12.10 & -6.74 & $1.251 \mathrm{E}+6$ \\
\hline $25.0-$ Cut-Out & 12.10 & -6.46 & $1.214 \mathrm{E}+6$ \\
\hline & & &
\end{tabular}

\subsubsection{Detuning the Gains in the Pitch-to-Feather Controller}

Neither the addition of the TFB control loop presented in Section 7.2.1 nor the modification to pitch-to-stall rotor-speed regulation presented in Section 7.2.2 gave satisfactory improvements in the barge-pitch response. This section describes one more approach I took to improve the platform-pitch damping of the ITI Energy wind barge concept through conventional wind turbine control methods. 
This control strategy was the simplest modification I made to the baseline control system developed in Section 3.1.6.3, involving only a reduction of gains in the active blade-pitch-tofeather controller. The basic premise behind this control strategy is the understanding that reducing the gains in the rotor-speed controller will cause the floating wind turbine system to behave less like the results for the ideal closed-loop pitch-to-feather method, and more like the results for the open-loop control method. Because of knowledge about barge-pitch damping ratios acquired from Figure 7-1 or Figure 7-5, this end result is important.

To maintain a reasonable relationship between the proportional and integral gains in the rotorspeed control system, I reduced the gains by choosing a smaller controller-response natural frequency $\left(\omega_{\varphi n}\right)$. I preserved the recommended controller damping ratio $\left(\zeta_{\varphi}=0.6\right.$ to 0.7$)$. The recommended value found in Ref. [29], and the value selected for the baseline control system, of $\omega_{\varphi n}=0.6 \mathrm{rad} / \mathrm{s}$ is slightly above the barge-pitch natural frequency of $\omega_{x n}=0.5420 \mathrm{rad} / \mathrm{s}$ (see Section 7.1). This relationship between frequencies has the potential to introduce negative damping of the barge-pitch mode. Larsen and Hanson [57] found that the smallest controllerresponse natural frequency must be less than the smallest critical support-structure natural frequency to ensure that the support structure motions of an offshore floating wind turbine with active pitch-to-feather control remain positively damped.

Reducing $\omega_{\varphi n}$ by one-third will ensure that the controller-response natural frequency is lower than the the barge-pitch natural frequency and also lower than wave-excitation frequency of all but the most severe sea states. Using the properties for the NREL 5-MW wind turbine, along with $\omega_{\varphi n}=0.4 \mathrm{rad} / \mathrm{s}$ and $\zeta_{\varphi}=0.7$, I derived the resulting reduced (detuned) gains of $K_{P}\left(\theta=0^{\circ}\right)=$ $0.01255121 \mathrm{~s}, K_{I}\left(\theta=0^{\circ}\right)=0.003586059$, and $K_{D}=0.0 \mathrm{~s}^{2}$. Figure 7-6 shows the gains at other blade-pitch angles, along with the gain-correction factor (which is the same as it was in the

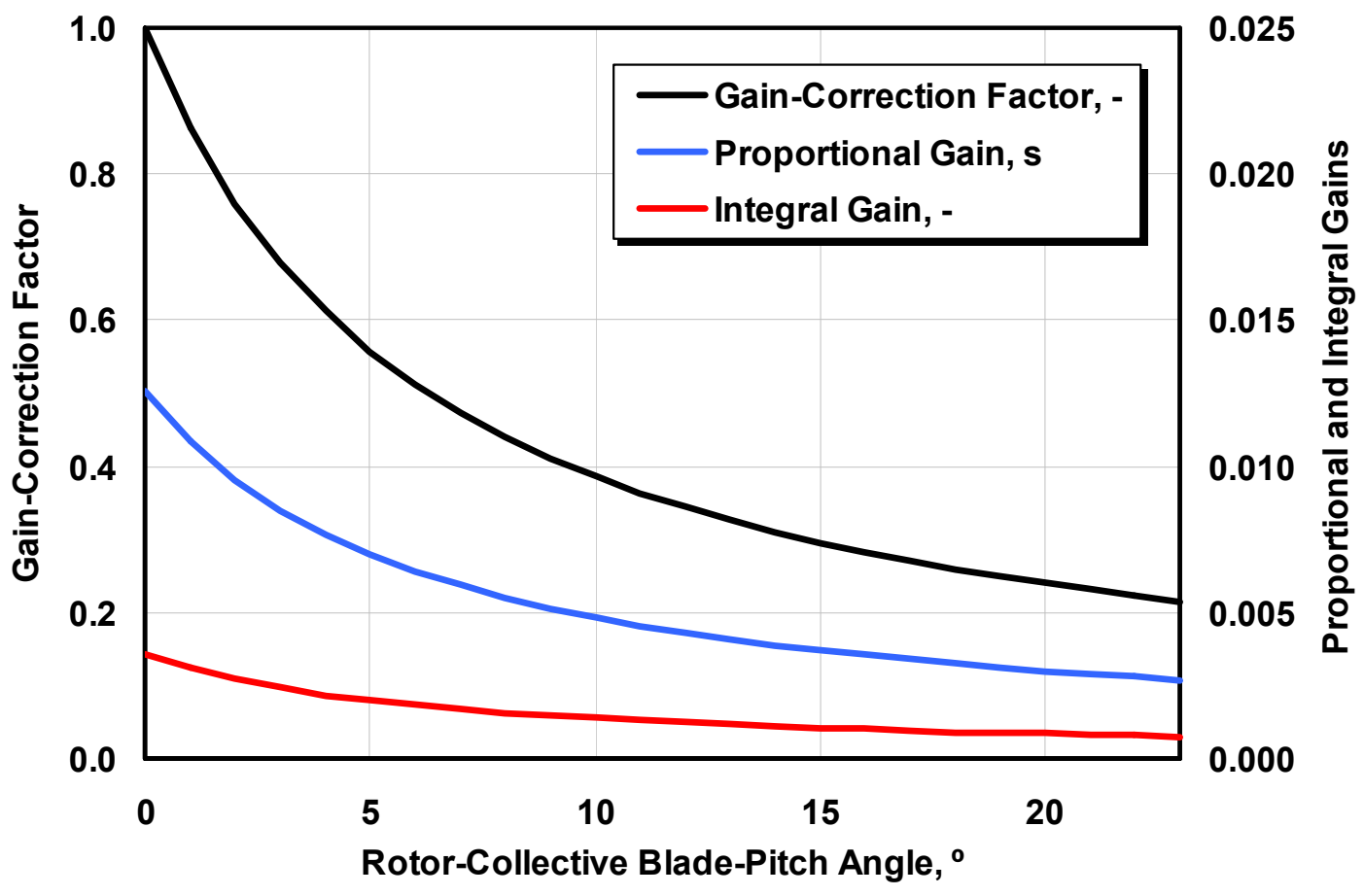

Figure 7-6. Detuned blade-pitch control system gain-scheduling law 
baseline control system). As in Section 3.1.6.3, I used the upper limit of the recommended damping ratio range $\left(\zeta_{\varphi}=0.7\right)$ to compensate for neglecting negative damping from the generator-torque controller in the determination of $K_{P}$.

As in Sections 7.2.1 and 7.2.2, I tested the system response of the NREL 5-MW wind turbine mounted atop the ITI Energy barge with this new control system. Figure 7-7 compares the system responses with the detuned and original (baseline) blade-pitch-to-feather gains for a simulation with the same wind and wave conditions used in the simulations presented in Figure 7-2 and Figure 7-4 (i.e., an 18-m/s stochastic wind, a 3.673-m significant wave height, and a 13.376-s peak spectral period). As before, I ran the simulations with all appropriate and available DOFs enabled, but without considering the rotor-mass imbalance or the increased blade structural-damping ratio.

As shown in Figure 7-7, the detuned blade-pitch control system is marginally effective at reducing the barge-pitch motions. Furthermore, it attains this positive performance without negatively affecting the generator speed and power excursions. As a matter of fact, the generator speed and power excursions have actually been diminished. And all of this has been accomplished with a reduction in blade-pitch duty cycle!

There is an upper bound, though, to the amount of improvement in the barge-pitch damping that

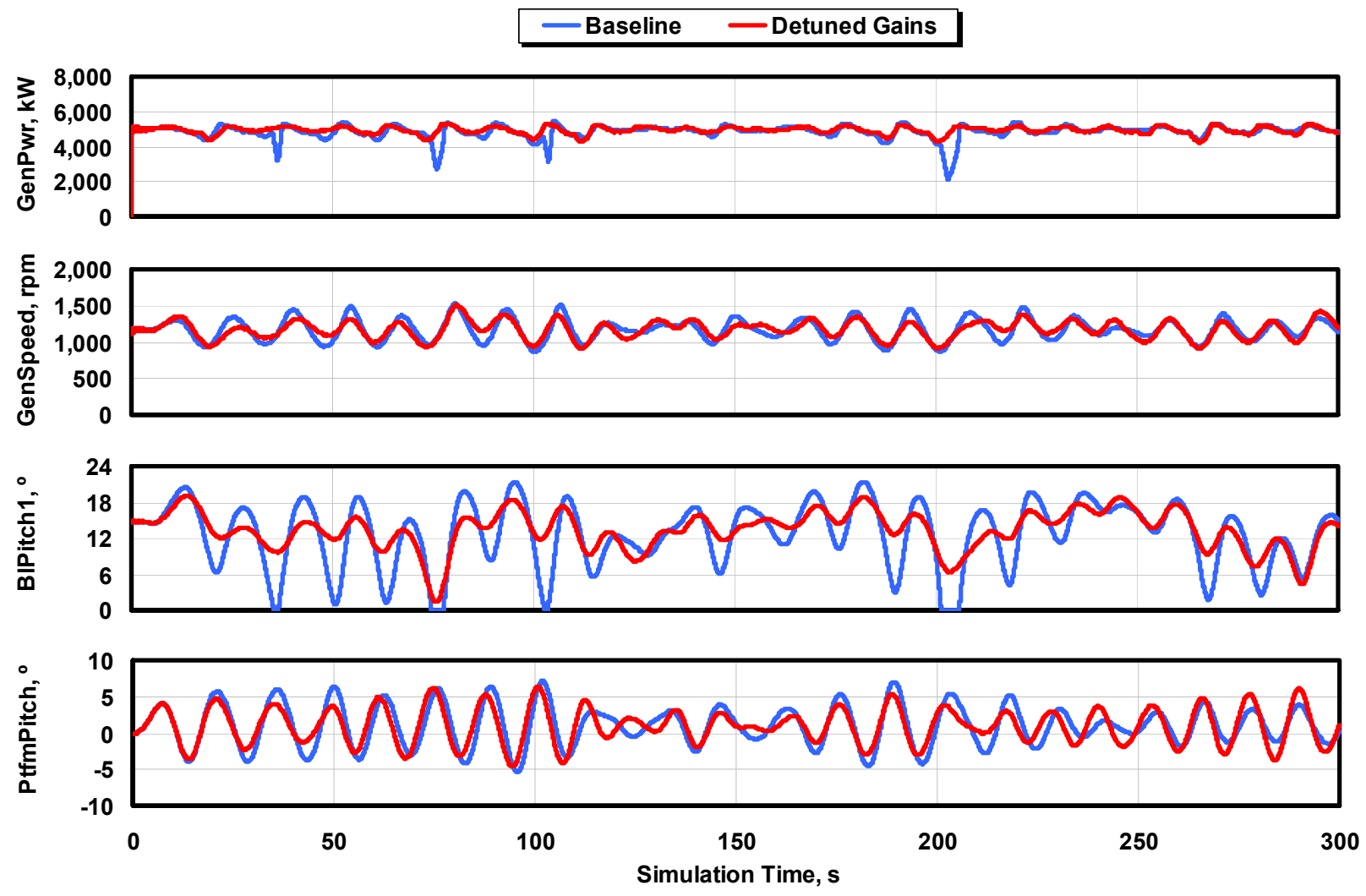

Figure 7-7. System responses with and without detuned blade-pitch control gains 
is attainable with a basic detuning of the blade-pitch control system gains. That upper bound is simply the amount of damping shown in Figure 7-1 or Figure 7-5 for the open-loop pitch-tofeather control system, or roughly $13 \%$ in Region 3.

In addition, one cannot expect that further and further reductions in the blade-pitch controller gains will continue to produce improved damping of the barge-pitch motions without eventually bringing about exacerbated excursions in the system response. This is because the rotor-speed error is unstable in the open-loop (uncontrolled) scenario in Region 3. [The rotor-speed error response is negatively damped in Eq. (3-11) if all blade-pitch control gains are zero]. To confirm this behavior, I reran simulations with detuned blade-pitch control gains derived from varying values of $\omega_{\varphi n}$, from 0.1 to $0.5 \mathrm{rad} / \mathrm{s}$ in steps of $0.1 \mathrm{rad} / \mathrm{s}$. As expected, with $\omega_{\varphi n}=0.1 \mathrm{or} 0.2 \mathrm{rad} / \mathrm{s}$ the system responses (not shown) exhibited much higher excursions in barge-pitch, generator speed, and electrical power output. With $\omega_{\varphi n}=0.5 \mathrm{rad} / \mathrm{s}$ and $\omega_{\varphi n}=0.3 \mathrm{rad} / \mathrm{s}$, I obtained responses (not shown) very similar to the system responses obtained for gains derived with $\omega_{\varphi n}=$ $0.4 \mathrm{rad} / \mathrm{s}$. (The barge-pitch damping from the simulation with $\omega_{\varphi n}=0.4 \mathrm{rad} / \mathrm{s}$ was slightly better than the damping with $\omega_{\varphi n}=0.3$ or $0.5 \mathrm{rad} / \mathrm{s}$.)

To determine the overall effect of the detuned blade-pitch control system, I reran the sea-based loads analysis with the control system gains derived from $\omega_{\varphi n}=0.4 \mathrm{rad} / \mathrm{s}$. Figure 7-8 presents the sea-to-land ratios for the absolute extremes from the rerun loads analysis of DLCs 1.1, 1.3, 1.4, and 1.5. I ran this loads analysis with the same model parameters and load-case prescriptions as in Section 6.1, with the only difference being the blade-pitch control system gains. $^{2}$

The parameter names in Figure 7-8 refer to the same simulation outputs plotted in Figure 6-4 and Figure 6-5. Moreover, the ratios of each parameter plotted on the left and labeled as "Baseline" in Figure 7-8 are the same ratios presented in Figure 6-4; they also correspond to the leftmost ratios presented in Figure 6-5 (labeled as "100\% - Original"). The ratios of each parameter plotted on the right and labeled as "Detuned Gains" in Figure 7-8 were computed using the landbased loads derived with the baseline control system. In other words, the land-based loads were not recomputed using the detuned control system.

For most parameters shown in Figure 7-8, the sea-to-land ratios in the sea-based system with the detuned gains are less than the sea-to-land ratios with the baseline gains. ${ }^{3}$ This demonstrates that detuning the gains in the blade-pitch controller of the sea-based system had a beneficial effect on the system response. For example, the sea-to-land ratios for the generator- and rotor-speed excursions of the system with the detuned gains (the upper-right chart in Figure 7-8) have dropped by more than $10 \%$ relative to the system with the baseline gains. The speed excursions in the system with the detuned gains are now only $40 \%$ higher than those seen in the turbine

\footnotetext{
${ }^{2}$ Note that the design problems identified in DLCs 2.1a, 6.x, and 7.1a (see Section 6.2) did not depend on the actions of the control system. They would, then, be unaffected by the reductions in the active blade-pitch-to-feather controller gains. These design problems will have to be resolved independently of control system improvements.

${ }^{3}$ For the parameters where this is not the case, the extreme loads are not dictated by barge-pitching motion. For example, the axial forces at the yaw bearing ("YawBrFzp") and the tower base ("TwrBsFzt") are dictated by heave motion, which has not been effected by the detuned gains.
} 


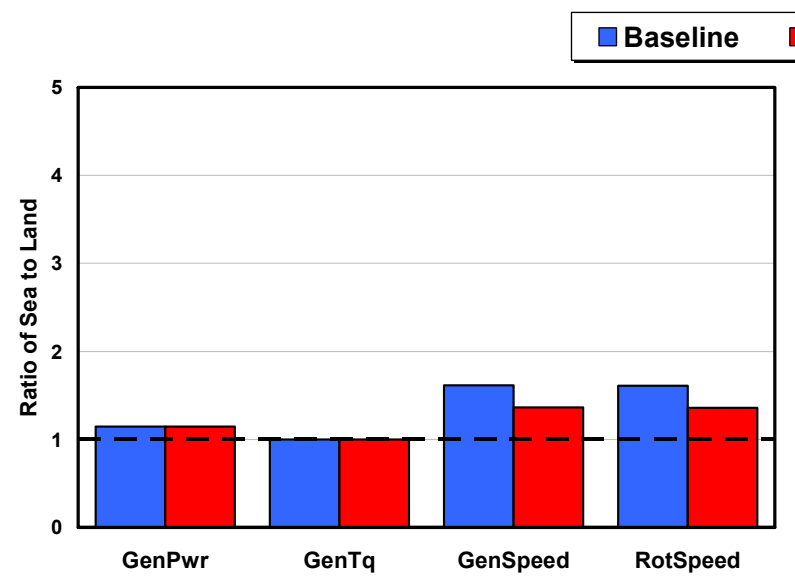

$\square$ Detuned Gains
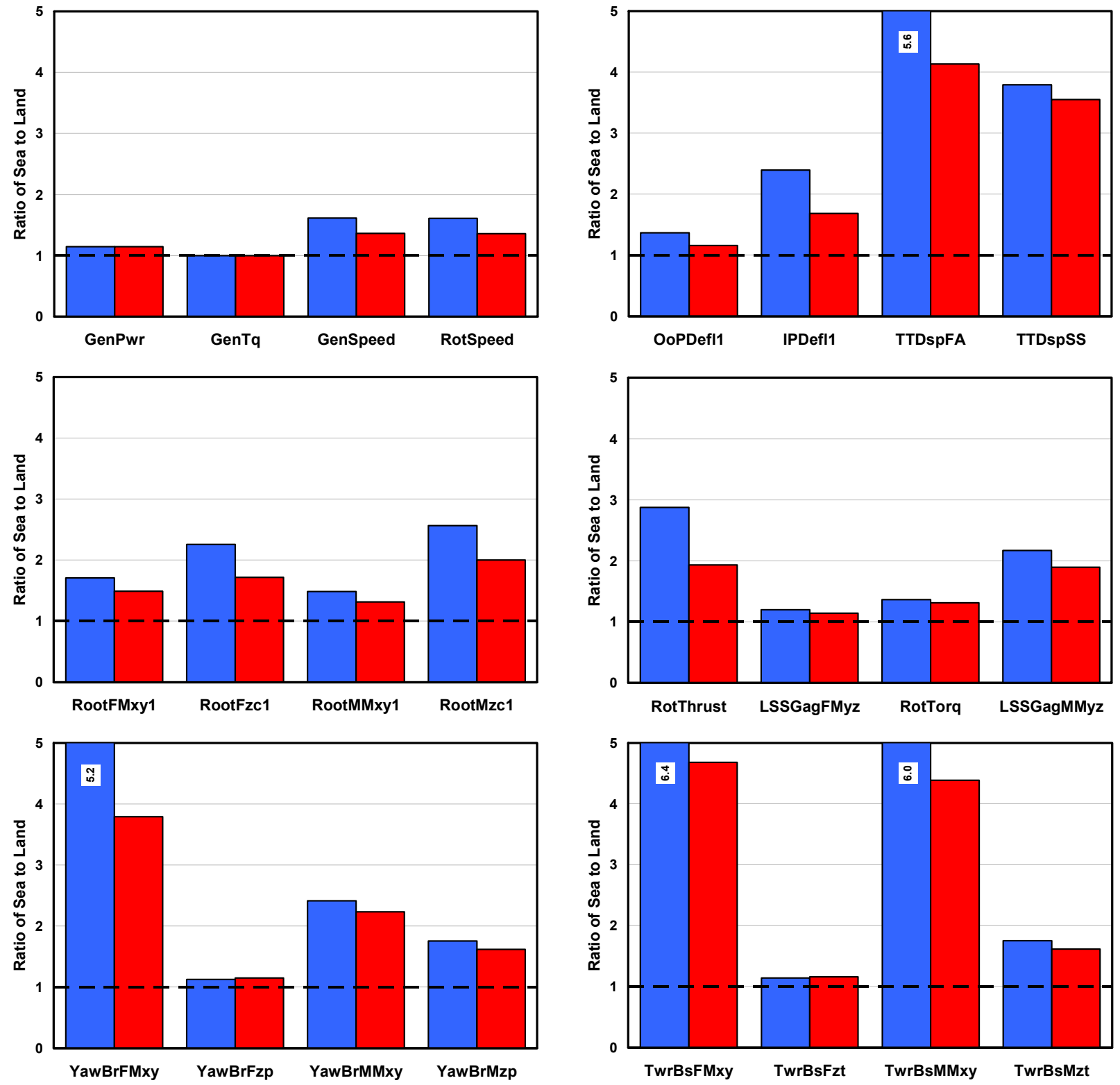

Figure 7-8. Sea-to-land ratios with and without detuned blade-pitch control gains

installed on land. (In the system with the baseline gains, the sea-based excursions were $60 \%$ higher).

In general, the detuned gains have more effect on the sea-to-land ratios of the deflections and loads as one follows the load path from the blade tip, through the drivetrain and nacelle, to the tower base. At the root of Blade 1 (the middle-left chart in Figure 7-8), for example, the sea-toland ratios of the internal shear force ("RootFMxyl") and bending moment ("RootMMxyl") magnitudes have dropped by more than $10 \%$. But at the base of the tower (the lower-right chart in Figure 7-8), the sea-to-land ratios have dropped by more than $25 \%$. This further demonstrates the effect of the inverted pendulum discussed in Section 6.1. Detuning the gains in the blade- 
pitch controller reduced the barge-pitch motions, which have more effect on loading from inertia farther down the load path.

The excursions in the internal shear force ("TwrBsFMxy") and bending moment ("TwrBsMMxy") magnitudes at the tower base, however, are still more than $400 \%$ higher than the excursions seen in the turbine installed on land. This demonstrates that detuning the gains in the baseline blade-pitch controller still did not entirely resolve the barge-pitch-motion problem. To arrive at a technically feasible concept, modifications to the system design will still be required (except possibly at the most sheltered of sites, as discussed in Section 6.1).

\subsection{Other Ways to Improve the Pitch Damping with Turbine Control}

As demonstrated in Section 7.2, conventional wind turbine control methodologies are limited in what they can do to improve the platform-pitch motions while limiting rotor-speed excursions of the ITI Energy wind barge concept. A number of other unconventional methods are also worth considering. Because a quantitative consideration of each method is beyond the scope of this work, I leave these considerations for future work. But this section highlights some of the possibilities.

I. Edwards of ITI Energy proposed one idea for an unconventional wind turbine control system. Edwards suggested that part of the problem with the barge-pitch damping in Region 3 might be that the generator is already operating at full (rated) power, so that there is no "head room" for absorbing more power as the barge pitches into the wind as a result of wave excitation. This implies that it might be better to regulate to some "below-rated" power level across all (even high) wind speeds to leave room for absorbing more power. This would, perhaps, permit the wind turbine rotor to capture not only wind power, but some of the wave power as well. Assessing this control strategy would require a study that examines the trade-off between improving the damping of the barge and reducing the capacity factor of the wind turbine.

Another unconventional wind turbine control strategy, proposed by Dr. R. Thresher of NREL / NWTC, would be to regulate the rotor speed of the turbine using nacelle-yaw actuation, instead of blade-pitch actuation. This strategy could eliminate the problems from the drop in steadystate rotor thrust with increasing wind speed above rated resulting from blade-pitch-to-feather control. One would, however, have to determine whether the gyroscopic moments induced by the required yaw rates would have undesirable consequences.

A simple, but unconventionial, modification to the control strategy would be to change Region 3 from a constant generator power to a constant generator-torque control region. With this change, the generator-torque controller would not introduce negative damping in the rotor-speed response (which must be compensated by the blade-pitch controller), and so, might reduce the rotor-speed excursions. Larson and Hanson [57] demonstrated the effectiveness of this modification for one offshore floating wind turbine concept. This change, however, would not improve the barge-pitch damping.

Conventional wind turbine control methodologies rely on the independent development and concatenation of multiple single-input, single-output (SISO) PID-based control loops using the conventional turbine actuators of blade pitch, generator torque, and nacelle yaw (and, as 
required, shaft brakes and other actuators). Naturally, modern control theories, such as disturbance-accommodating control (DAC) [35], offer the potential to bring about improved performance. Previous controls studies by Stol [90], Hand [26], and Wright [104] have demonstrated the applicability of combining a state estimator, a wind-disturbance estimator, and full-state feedback using DAC to develop multiple-input, multiple-output (MIMO) state-spacebased control systems for mitigating dynamic loads and stabilizing flexible modes of land-based wind turbines without compromising energy capture. But these studies have not yet been extended to offshore floating wind turbines.

Through MIMO state-space-based control, it may be possible to enhance rotor-speed regulation and platform-pitch damping through unified control of the generator torque and blade-pitch angles. For example, because rotor-speed regulation requires a blade-pitch command that is opposite of the one required to add damping to the barge-pitch motion (see Section 7.2.1), it might be possible to develop a combined generator-torque and blade-pitch controller to address both objectives simultaneously. The generator-torque commands may be able to mitigate the rotor-speed excursions while the blade-pitch commands attempt to augment aerodynamic rotor thrust to dampen the platform-pitch motion. When used in conjunction with off-axis flow through nacelle-yaw actuation, it may also be possible to introduce platform-roll damping through the blade-pitch commands (and thrust augmentation).

Rotor-collective blade-pitch control can be used to adapt rotor thrust, which induces a moment on the floating platform through the hub-height moment arm, but independent blade-pitch control may also be useful. For example, independent blade-pitch control can be used to introduce pitching moments within the rotor itself through asymmetric aerodynamic loading of the rotor. If developed properly, it may be possible to use such a moment to counteract the hydrodynamic pitching loads on the platform brought about by surface waves.

Section 6.1.5 discusses other design alterations, beyond wind turbine control, that may be applied to improve the response of the floating wind barge system. 


\section{Chapter 8 Conclusions and Recommendations}

The vast deepwater wind resource represents a potential to use offshore floating wind turbines to power much of the world with renewable energy. Before I began this project, many floating wind turbine concepts had been proposed, but few had or could have been evaluated because available modeling capabilities were limited.

The limitations of previous time- and frequency-domain studies on offshore floating wind turbines motivated my development of simulation capability for modeling the fully coupled aerohydro-servo-elastic response of such systems. As presented in Chapter 2, I developed this capability by combining the computational methodologies of the onshore wind turbine and offshore O\&G industries. The aero-servo-elastic onshore wind turbine simulation capability of FAST with AeroDyn and MSC.ADAMS with A2AD and AeroDyn were interfaced with the external hydrodynamic wave-body interaction programs SWIM and WAMIT. To establish these interfaces, I developed modules for treating time-domain hydrodynamics (HydroDyn) and quasistatic mooring system responses. I developed the HydroDyn hydrodynamics module to account for linear hydrostatic restoring; nonlinear viscous drag from incident-wave kinematics, sea currents, and platform motion; the added-mass and damping contributions from linear wave radiation, including free-surface memory effects; and the incident-wave excitation from linear diffraction in regular or irregular seas. I developed my quasi-static mooring line module to account for the elastic stretching of an array of homogenous taut or slack catenary lines with seabed interaction. The simulation capability was developed with enough sophistication to address the primary limitations of the previous frequency- and time-domain studies. In addition, the simulation program has the features required to perform integrated loads analyses. To make it useful for examining the technical feasibility of a variety of offshore floating wind turbine concepts, I also made my simulation capability universal enough to analyze a variety of turbine, support platform, and mooring system configurations.

To support this and other concept studies aimed at assessing offshore wind technology, I developed the specifications of a representative utility-scale multimegawatt turbine now known as the NREL offshore 5-MW baseline wind turbine. This wind turbine is a conventional threebladed upwind variable-speed variable blade-pitch-to-feather-controlled turbine. In Chapter 3, I discussed the development of this wind turbine and gave an overview of the properties of two floating barges - the ITI Energy barge and MIT / NREL barge. Also in Chapter 3, I presented the metocean data at a reference site in the northern North Sea. I used the wind turbine, barges, and metocean data in my model-verification, loads analysis, and controls-development efforts.

Through model-to-model comparisons, I tested my newly developed simulation capability, as presented in Chapter 4, to ensure its correctness. I verified that the PSD and probability density of the wave-elevation record computed by HydroDyn matched the prescribed target spectrum and Gaussian distribution, respectively. I demonstrated that WAMIT produces acceptable input for HydroDyn, and from this hydrodynamic input, I showed that HydroDyn correctly generates the radiation impulse-response functions. I also showed that my quasi-static mooring system module correctly solves a classic benchmark problem for the static equilibrium of a suspended cable structure. In addition, I demonstrated that my mooring system module predicts nonlinear force-displacement relationships consistent with an independent analysis. Finally, the results 
from my fully coupled time-domain analysis were shown to agree with results generated from a frequency-domain approach. The results of all the verification exercises were favorable and gave me confidence to pursue more thorough investigations into the dynamic behavior of offshore floating wind turbines.

I then used my simulation capability to perform a preliminary, but integrated, loads analysis for the NREL 5-MW baseline wind turbine mounted both on land and offshore on the floating ITI Energy barge, which has slack catenary moorings. I introduced the loads analyses in Chapter 5 and discussed the results in Chapter 6. I based the analyses on the ultimate load cases and procedures dictated by the on- and offshore IEC wind turbine design standards and the severe environmental conditions at the chosen reference site. By comparing the responses of the landand sea-based systems, I was able to quantify the impact brought about by the dynamic couplings between the turbine and floating barge in the presence of combined wind and wave loading.

I characterized the dynamic responses by showing that the mean values of the loads and deflections in the floating turbine were very similar to those that existed on land. The excursions of the loads and deflections, however, exceeded those found on land. I showed that the increased load excursions in the floating system were produced by the barge's pitching motion, and so were higher in the tower than in the blades because of the increased effect of inertia. I discussed how the barge concept was susceptible to excessive pitching during extreme wave conditions, but showed how the load excursions were reduced with decreasing severity in the waves. Relative to the fixed land-based support, I found that the added compliance in the barge led to an instability of the floating system in yaw when the wind turbine was idling with a faulted blade. I discussed how the compliance of the floating barge did, however, mitigate a tower side-to-side instability discovered in the land-based turbine.

In Chapter 7, I presented the influence of conventional wind turbine blade-pitch control actions on the pitch damping of the NREL 5-MW baseline wind turbine mounted atop the ITI Energy barge. I was concerned that the drop in steady-state wind turbine rotor thrust with wind speed above rated would lead to negative damping of the barge-pitch mode and contribute to the large system-pitch motions. I demonstrated that neither the addition of a control loop through feedback of tower-top acceleration nor the modification to pitch-to-stall rotor-speed regulation gave satisfactory improvements in the barge-pitch response. The latter modification helped me conclude, however, that the actual barge-pitch damping was considerably higher than that implied by the steady-state rotor thrust response, but that it was still beneficial to increase the damping as much as possible. I also showed in Chapter 7 that detuning the gains in the baseline blade-pitch-to-feather controller helped, but still did not entirely resolve the barge-pitch-motion problem.

In summary of my accomplishments, I have satisfied my project objectives by (1) developing a comprehensive simulation capability for modeling the coupled dynamic response of offshore floating wind turbines, (2) verifying the simulation capability through model-to-model comparisons, and (3) applying the simulation capability to the integrated loads analysis for one of the promising system concepts. At the end of all this work, I have not produced a floating wind turbine concept free of problems (although doing so was not one of my objectives). To arrive at a technically and economically feasible concept, modifications to the system designs I presented in this work are still required. 
My recommendations for future research were scattered throughout this work where appropriate. In summary, though, future opportunities include enhancing and validating the simulation capability, modifying the turbine and barge system designs, performing additional iterations in the design loads analysis, applying advanced control solutions, and extending the work to other promising floating platform concepts.

Though not specific to the modeling of offshore wind turbines, it would be advantageous to add a torsion DOF to the modal representation of the tower in FAST. In addition, extending the modal representation of the blades to include mass and elastic offsets, torsion DOFs, and coupled mode-shape properties would be useful.

Additional enhancements to improve the simulation of floating offshore wind turbines are also possible. For example, one could add capabilities that would allow for modeling and testing various mechanisms for stabilizing the barge-pitch motion, such as TMDs, OWCs, or other active and passive control devices. For the detailed analysis of some designs (see Section 2.2), it would be beneficial to introduce second-order effects into my HydroDyn hydrodynamics module, including the effects of intermittent wetting and mean-drift, slow-drift, and sumfrequency excitation. It would also be advantageous to add the potential loading from VIV and from sea ice, and to replace my quasi-static mooring system module with a fully coupled module that can handle the line dynamics. Finally, the models should be validated against experimental data derived from wave-tank tests and sub- and full-scale prototypes installed offshore.

This work can also be extended to enable the simulation tools to model the coupled dynamic response of fixed-bottom offshore wind turbines. For monopile support structures in shallow water, nonlinear wave-kinematics models and Morison's equation for the wave-induced loading must be introduced. For tripod and space-frame designs in intermediate depths, more sophisticated structural-dynamic and hydrodynamic models, including member-to-member interactions, will be required. Having a single code capable of modeling a large range of support structures and water depths would allow one to perform conceptual studies that attempt to find the optimal transition depth between fixed-bottom and floating platform support structures.

Independent of code enhancements, the simulation capability as it exists now can also be applied in many important research projects. For instance, the loads-analysis process I used in this work is also applicable to other floating platform concepts, including TLPs and spar buoys. The process could also be applied for varying wind turbine concepts with unconventional features, such as light-weight rotors, ratings higher than $5 \mathrm{MW}$, two instead of three blades, or downwind instead of upwind rotors. (Reference [14] discusses how these unconventional features might be advantageous in floating systems). Such loads analyses should be performed to determine which concept_ or hybrid thereof-has the best overall technical advantage.

For the particular system concept analyzed in this work, Sections 6.1.5, 6.2, and 7.3 suggested design modifications and active and passive control features that could potentially reduce the barge motions, improve the turbine response, and eliminate the instabilities. These recommended future projects included

- Incorporating actively controlled OWCs into the barge

- Incorporating passively controlled TMDs or anti-roll stabilizers into the barge or turbine 
- Introducing unconventional and / or advanced control strategies into the wind turbine

- Decoupling the turbine and barge motions by adding new articulations

- Modifying the geometry of the turbine, barge, and / or mooring system.

After improvements to the system design are made, it would be constructive to rerun the loads analysis to reassess the concept's technical feasibility. It would also be beneficial to expand the set of load cases considered. It would, for example, be useful to add the load cases that would allow one to quantify the impact of the platform motions on the fatigue life of the supported wind turbine.

Once suitable design modifications have made the concept more technically feasible, it will be important to assess the economics of the system, including the influences of manufacturing, installation, and decommissioning. System-wide optimization will improve the economics.

If the technical challenges can be solved in an economically feasible way, the possibility of using offshore floating wind turbines to power much of the world with an indigenous, nonpolluting, and inexhaustible energy source can become real. 


\section{References}

[1] Agarwal, P. and Manuel, L., "Simulation of Offshore Wind Turbine Response for Extreme Limit States," Proceedings of OMAE2007 $26^{\text {th }}$ International Conference on Offshore Mechanics and Arctic Engineering, 10-15 June 2007, San Diego, CA [CDROM], Houston, TX: The American Society of Mechanical Engineers (ASME International) Ocean, Offshore and Arctic Engineering (OOAE) Division, June 2007, OMAE2007-29326.

[2] Bir, G. and Jonkman, J., "Aeroelastic Instabilities of Large Offshore and Onshore Wind Turbines," Journal of Physics: Conference Series, The Second Conference on The Science of Making Torque From Wind, Copenhagen, Denmark, 28-31 August 2007, [online journal], Vol. 75, 2007, 012069, URL: http://www.iop.org/EJ/article/17426596/75/1/012069/jpconf7_75 012069.pdf?request-id=PNODaQdu3BGLGoay2wi7Kg, [cited 28 August 2007].

[3] Bossanyi, E. A., "Wind Turbine Control for Load Reduction," Wind Energy, Vol. 6, No. 3, June 2003, pp. 229-244.

[4] Bossanyi, E. A., GH Bladed Theory Manual, Issue No. 12, 282/BR/009, Bristol, UK: Garrad Hassan and Partners Limited, December 2003.

[5] Bossanyi, E. A., GH Bladed Version 3.6 User Manual, 282/BR/010, Bristol, UK: Garrad Hassan and Partners Limited, December 2003.

[6] Buhl, M., "Modes: A Simple Mode-Shape Generator for Both Towers and Rotating Blades," NWTC Design Codes [online database], URL: http://wind.nrel.gov/designcodes/preprocessors/modes/ [cited 22 July 2005].

[7] Buhl, M. L., Jr., Wright, A. D., and Tangler, J. L, "Wind Turbine Design Codes: A Preliminary Comparison of the Aerodynamics," NREL/CP-500-23975, Golden, CO: National Renewable Energy Laboratory, December 1997.

[8] Buhl, M. L., Jr., Wright, A. D., and Pierce, K. G., "Wind Turbine Design Codes: A Comparison of the Structural Response," A Collection of the 2000 ASME Wind Energy Symposium Technical Papers Presented at the $38^{\text {th }}$ AIAA Aerospace Sciences Meeting and Exhibit, 10-13 January 2000, Reno Nevada, USA, New York: American Institute of Aeronautics and Astronautics, Inc. (AIAA) and American Society of Mechanical Engineers (ASME), January 2000, pp. 12-22, NREL/CP-500-27470, Golden, CO: National Renewable Energy Laboratory.

[9] Buhl, M. L., Jr., Wright, A. D., and Pierce, K. G., "FAST_AD Code Verification: A Comparison to ADAMS," A Collection of the 2001 ASME Wind Energy Symposium Technical Papers Presented at the $39^{\text {th }}$ AIAA Aerospace Sciences Meeting and Exhibit, 11-14 January 2001, Reno Nevada, USA, New York: American Institute of Aeronautics and Astronautics, Inc. (AIAA) and American Society of Mechanical Engineers (ASME), 
January 2001, pp. 368-377, NREL/CP-500-28848, Golden, CO: National Renewable Energy Laboratory.

[10] Buhl, M. L., Jr., CombEEv User's Guide, NREL/EL-500-31664, Golden, CO: National Renewable Energy Laboratory, October 2003.

[11] Buhl, M. L., Jr., Crunch User's Guide, NREL/EL-500-30122, Golden, CO: National Renewable Energy Laboratory, October 2003.

[12] Buhl, M. L., Jr. and Manjock, A., "A Comparison of Wind Turbine Aeroelastic Codes Used for Certification," 44 ${ }^{\text {th }}$ AIAA Aerospace Sciences Meeting and Exhibit, 9-12 January 2006, Reno, NV, AIAA Meeting Papers on Disc [CD-ROM], Reston, VA: American Institute of Aeronautics and Astronautics, January 2006, AIAA-2006-786, NREL/CP-500-39113, Golden, CO: National Renewable Energy Laboratory.

[13] Bulder, B. H., et al, Study to Feasibility of and Boundary Conditions for Floating Offshore Wind Turbines, Novem 2002-CMC-R43, ECN, MARIN, Lagerway the Windmaster, TNO, TUD, MSC, December 2002.

[14] Butterfield, S., Musial, W., Jonkman, J., Sclavounos, P., and Wayman, L., "Engineering Challenges for Floating Offshore Wind Turbines," Copenhagen Offshore Wind 2005 Conference and Expedition Proceedings, 26-28 October 2005, Copenhagen, Denmark [CD-ROM], Copenhagen, Denmark: Danish Wind Energy Association, October 2005, NREL/CP-500-38776, Golden, CO: National Renewable Energy Laboratory.

[15] Cheng, P. W., A Reliability Based Design Methodology for Extreme Responses of Offshore Wind Turbines, Ph.D. Dissertation, Wind Energy Research Institute, Delft University of Technology, Delft, The Netherlands, 2002.

[16] Cummins, W. E., "The Impulse Response Function and Ship Motions," Schiffstechnik, Vol. 9, October 1962, pp. 101-109.

[17] Demirbilek, Z. and Vincent, C. L., "Part II: Coastal Hydrodynamics; Chapter II-1: Water Wave Mechanics," Coastal Engineering Manual [online publication], URL: http://users.coastal.ufl.edu/ sheppard/eoc6430/Coastal_Engineering_Manual.htm, [cited 22 September 2005].

[18] de Vries, E., "Multibrid: 'A New Offshore Wind Turbine Contender'," Renewable Energy World [online journal], Vol. 7, No. 5, September-October 2004, URL: http://www.renewable-energy-

world.com/articles/article display.cfm?ARTICLE ID=272695\&p=121, $\quad$ [cited 1 November 2004].

[19] Eecen, P. J., Wind Waves: Forces Due to Waves on Offshore Wind Turbines, ECN-C--03097, Petten, The Netherlands: Energy Research Centre of The Netherlands, September 2003. 
[20] Elliott, A. S., "Analyzing Rotor Dynamics with a General-Purpose Code," Mechanical Engineering, Vol. 112, No. 12, December 1990, pp. 21-25.

[21] Emmerhoff, O. J., The Slow-Drift Motions of Offshore Structures, Ph.D. Dissertation, Department of Ocean Engineering, Massachusetts Institute of Technology, Cambridge, MA, USA, 1994.

[22] Faltinsen, O. M., Sea Loads on Ships and Offshore Structures, Cambridge University Press, Cambridge, UK, 1990.

[23] Fulton, G. R., Malcolm, D. J., and Moroz, E., "Design of a Semi-Submersible Platform for a 5MW Wind Turbine," 44 $4^{\text {th }}$ AIAA Aerospace Sciences Meeting and Exhibit, 9-12 January 2006, Reno, NV, AIAA Meeting Papers on Disc [CD-ROM], Reston, VA: American Institute of Aeronautics and Astronautics, January 2006, AIAA-2006-997.

[24] Goezinne, F., "Terms of reference DOWEC," DOWEC Dutch Offshore Wind Energy Converter 1997-2003 Public Reports [CD-ROM], DOWEC 10041_000, 176-FG-R0300, September 2001.

[25] Greenwood, D. T., Principles of Dynamics, $2^{\text {nd }}$ ed., Prentice-Hall, Inc., Englewood Cliffs, NJ, USA, 1998.

[26] Hand, M. M., Mitigation of Wind Turbine/Vortex Interaction Using Disturbance Accommodating Control, Ph.D. Dissertation, Department of Mechanical Engineering, University of Colorado, Boulder, CO, USA, December 2003, NREL/TP-500-35172, Golden, CO: National Renewable Energy Laboratory.

[27] Hand, M. M., Simms, D. A., Fingersh, L. J., Jager, D. W., Cotrell, J. R., Schreck, S., and Larwood, S. M., Unsteady Aerodynamics Experiment Phase VI: Wind Tunnel Test Configurations and Available Data Campaigns, NREL/TP-500-29955, Golden, CO: National Renewable Energy Laboratory, December 2001.

[28] Hansen, C., "AirfoilPrep: An Excel workbook for generating airfoil tables for AeroDyn and WT_Perf," NWTC Design Codes [online database], URL: http://wind.nrel.gov/designcodes/preprocessors/airfoilprep/ [cited 1 November 2004].

[29] Hansen, M. H., Hansen, A., Larsen, T. J., Фye, S., Sørensen, and Fuglsang, P., Control Design for a Pitch-Regulated, Variable-Speed Wind Turbine, Risø-R-1500(EN), Roskilde, Denmark: Risø National Laboratory, January 2005.

[30] Henderson, A. R., Morgan, C. S., Smith, B., Sørensen, H. C., Barthelmie, R. J., and Boesmans, B., "Offshore Wind Energy in Europe - A Review of the State-of-the-Art," Wind Energy, Vol. 6, No. 1, February 2003, pp. 35-52.

[31] Henderson, A. R. and Patel, M. H., "On the Modelling of a Floating Offshore Wind Turbine," Wind Energy, Vol. 6, No. 1, February 2003, pp. 53-86. 
[32] Horn, R. A. and Johnson, C. R., Matrix Analysis, Cambridge University Press, Cambridge, UK, 1985, pp. 427-455.

[33] IEC 61400-1 Ed. 3, Wind Turbines - Part 1: Design Requirements, International Electrotechnical Commission (IEC), 2005.

[34] IEC 61400-3, Wind Turbines - Part 3: Design Requirements for Offshore Wind Turbines, International Electrotechnical Commission (IEC), 2006 (to be published).

[35] Johnson, C. D., "Theory of Disturbance Accommodating Controllers," Advances in Control and Dynamic Systems, Vol. 12, 1976.

[36] Jonkman, B. J. and Buhl, M. L., Jr., TurbSim User's Guide, NREL/EL-500-41136, Golden, CO: National Renewable Energy Laboratory, March 2007.

[37] Jonkman, J. M., Modeling of the UAE Wind Turbine for Refinement of FAST_AD, M.S. Dissertation, Department of Mechanical Engineering, Colorado State University, Fort Collins, CO, USA, November 2001, NREL/TP-500-34755, Golden, CO: National Renewable Energy Laboratory.

[38] Jonkman, J. M. and Buhl, M. L., Jr., "New Developments for the NWTC's FAST Aeroelastic HAWT Simulator," A Collection of the 2004 ASME Wind Energy Symposium Technical Papers Presented at the $42^{\text {nd }}$ AIAA Aerospace Sciences Meeting and Exhibit, 5-8 January 2004, Reno Nevada, USA, New York: American Institute of Aeronautics and Astronautics, Inc. (AIAA) and American Society of Mechanical Engineers (ASME), January 2004, pp. 181-191, NREL/CP-500-35077, Golden, CO: National Renewable Energy Laboratory.

[39] Jonkman, J. M. and Buhl, M. L., Jr., FAST User's Guide, NREL/EL-500-29798, Golden, CO: National Renewable Energy Laboratory, October 2004.

[40] Jonkman, J. M. and Sclavounos, P. D., "Development of Fully Coupled Aeroelastic and Hydrodynamic Models for Offshore Wind Turbines," $44^{\text {th }}$ AIAA Aerospace Sciences Meeting and Exhibit, 9-12 January 2006, Reno, NV, AIAA Meeting Papers on Disc [CDROM], Reston, VA: American Institute of Aeronautics and Astronautics, January 2006, AIAA-2006-995, NREL/CP-500-39066, Golden, CO: National Renewable Energy Laboratory.

[41] Jonkman, J. M. and Buhl, M. L., Jr., "Development and Verification of a Fully Coupled Simulator for Offshore Wind Turbines," $45^{\text {th }}$ AIAA Aerospace Sciences Meeting and Exhibit, 8-11 January 2007, Reno, NV, AIAA Meeting Papers on Disc [CD-ROM], Reston, VA: American Institute of Aeronautics and Astronautics, January 2007, AIAA2007-212, NREL/CP-500-40979, Golden, CO: National Renewable Energy Laboratory.

[42] Jonkman, J., Butterfield, S., Musial, W., and Scott, G., Definition of a 5-MW Reference Wind Turbine for Offshore System Development, NREL/TP-500-38060, Golden, CO: National Renewable Energy Laboratory, February 2007 (to be published). 
[43] Jonkman, J. M. and Buhl, M. L., Jr., "Loads Analysis of a Floating Offshore Wind Turbine Using Fully Coupled Simulation," WINDPOWER 2007 Conference and Exhibition, Los Angeles, California, 3-6 June 2007 [CD-ROM], Washington, D.C.: American Wind Energy Association, June 2007, NREL/CP-500-41714, Golden, CO: National Renewable Energy Laboratory.

[44] Jonkman, J. M., "Improving the Pitch Damping of a Floating Wind Turbine Using Conventional Control," 46 $6^{\text {th }}$ AIAA Aerospace Sciences Meeting and Exhibit, 7-10 January 2008, Reno, NV, AIAA Meeting Papers on Disc [CD-ROM], Reston, VA: American Institute of Aeronautics and Astronautics, January 2008, (to be published).

[45] Kane, T. R. and Levinson, D. A., Dynamics: Theory and Applications, McGraw-Hill Inc., New York, 1985.

[46] Kelley, N. D., Wright, A. D., and Osgood, R. M., "A Progress Report on the Characterization and Modeling of a Very Flexible Wind Turbine Design," A Collection of the 1999 ASME Wind Energy Symposium Technical Papers Presented at the $37^{\text {th }}$ AIAA Aerospace Sciences Meeting and Exhibit, 11-14 January 1999, Reno Nevada, USA, New York: American Institute of Aeronautics and Astronautics, Inc. (AIAA) and American Society of Mechanical Engineers (ASME), January 1999, pp. 243-252, NREL/CP-50025513, Golden, CO: National Renewable Energy Laboratory.

[47] Kim, S. and Sclavounos, P. D., "Fully Coupled Response Simulations of Theme Offshore Structures in Water Depths of Up to 10,000 Feet," Proceedings of the Eleventh International Offshore and Polar Engineering Conference, 17-22 June 2001, Stavanger, Norway, Vol. III, Mountain View, CA: The International Society of Offshore and Polar Engineers (ISOPE), June 2001, pp. 457-466.

[48] Kim, S., SWIM 2001: Frequency-Domain Analysis of Offshore Platforms, User Manual, Cambridge, MA: Massachusetts Institute of Technology, 2004.

[49] Kim, S., MOTION 2001: Time-Domain Response Analysis of Offshore Platforms, User Manual, Cambridge, MA: Massachusetts Institute of Technology, 2004.

[50] Kim, S., LINES 2001: Nonlinear Static \& Dynamic Analysis of Mooring Line/Riser/Tether Arrays, User Manual, Cambridge, MA: Massachusetts Institute of Technology, 2004.

[51] Kooijman, H. J. T., Lindenburg, C., Winkelaar, D., and van der Hooft, E. L., "DOWEC 6 MW Pre-Design: Aero-elastic modeling of the DOWEC 6 MW pre-design in PHATAS," DOWEC Dutch Offshore Wind Energy Converter 1997-2003 Public Reports [CD-ROM], DOWEC 10046_009, ECN-CX--01-135, Petten, the Netherlands: Energy Research Center of the Netherlands, September 2003.

[52] Kühn, M., Dynamics and Design Optimization of Offshore Wind Energy Conversion Systems, Ph.D. Dissertation, Wind Energy Research Institute, Delft University of Technology, Delft, The Netherlands, 2001. 
[53] Kristiansen, E., Hjulstad, Å., and Egeland, O., "State-Space Representation of Radiation Forces in Time-Domain Vessel Motions," Ocean Engineering, Vol. 32, 2005, pp. 21952216.

[54] Laino, D. J. and Hansen, A. C., User's Guide to the Computer Software Routines AeroDyn Interface for $A D A M S^{\circledR}$, Salt Lake City, UT: Windward Engineering LLC, Prepared for the National Renewable Energy Laboratory under Subcontract No. TCX-929209-01, September 2001.

[55] Laino, D. J. and Hansen, A. C., User's Guide to the Wind Turbine Dynamics Aerodynamics Computer Software AeroDyn, Salt Lake City, UT: Windward Engineering LLC, Prepared for the National Renewable Energy Laboratory under Subcontract No. TCX-9-29209-01, December 2002.

[56] Laino, D. J., "IECWind: A Program to Create IEC Wind Data Files," NWTC Design Codes [online database], URL: http://wind.nrel.gov/designcodes/preprocessors/iecwind/ [cited 22 July 2005].

[57] Larsen, T. J. and Hanson, T. D., "A Method to Avoid Negative Damped Low Frequent Tower Vibrations for a Floating, Pitch Controlled Wind Turbine," Journal of Physics: Conference Series, The Second Conference on The Science of Making Torque From Wind, Copenhagen, Denmark, 28-31 August 2007, [online journal], Vol. 75, 2007, 012073, URL: http://www.iop.org/EJ/article/1742-6596/75/1/012073/jpconf7 75 012073.pdf?request-id=SpEuhRBu3BG0xV3r2wi7Kg, [cited 28 August 2007].

[58] Lee, C. H. and Newman, J. N., WAMIT ${ }^{\circledR}$ User Manual, Versions 6.3, 6.3PC, 6.3S, 6.3SPC, WAMIT, Inc., Chestnut Hill, MA, USA, 2006.

[59] Lee, K. H., Responses of Floating Wind Turbines to Wind and Wave Excitation, M.S. Dissertation, Department of Ocean Engineering, Massachusetts Institute of Technology, Cambridge, MA, USA, January 2005.

[60] Lindenburg, C., "Aeroelastic Modelling of the LMH64-5 Blade," DOWEC Dutch Offshore Wind Energy Converter 1997-2003 Public Reports [CD-ROM], DOWEC 10083_001, DOWEC-02-KL-083/0, Petten, the Netherlands: Energy Research Center of the Netherlands, December 2002.

[61] Lindenburg, C., PHATAS Release "NOV-2003" and "APR-2005” USER'S MANUAL: Program for Horizontal Axis wind Turbine Analysis and Simulation, ECN-I--05-005, Petten, the Netherlands: Energy Research Center of the Netherlands, May 2005.

[62] LM Glasfiber Group, Wind Turbine Blades, Product Overview, Standard Products Max. Rated Power $\quad<=5000 \mathrm{~kW}$ [online publication], URL: http://www.lmglasfiber.dk/UK/Products/Wings/ProductOverView/50000kw.htm [cited 4 January 2005]. 
[63] Majock, A., Evaluation Report: Design Codes FAST and ADAMS ${ }^{\circledR}$ for Load Calculations of Onshore Wind Turbines, Report No. 72042, Hamburg, Germany: Germanischer Lloyd WindEnergie GmbH, May 26, 2005.

[64] Malcolm, D. J. and Hansen, A. C., WindPACT Turbine Rotor Design Study, NREL/SR500-32495, Golden, CO: National Renewable Energy Laboratory, August 2002.

[65] Massel, S. R., Ocean Surface Waves: Their Physics and Prediction, Advanced Series on Ocean Engineering, Vol. 11, Singapore - New Jersey - London - Hong Kong: World Scientific, 1996.

[66] McCabe, A. P., Bradshaw, A., and Widden, M. B., "A Time-Domain Model of a Floating Body Using Transforms," $6^{\text {th }}$ European Wave and Tidal Conference, 30 August - 2 September 2005, Glasgow, Scotland, August 2005.

[67] Moriarty, P. J. and Hansen, A. C., AeroDyn Theory Manual, NREL/EL-500-36881, Golden, CO: National Renewable Energy Laboratory, December 2005.

[68] Multibrid Technology, Technical Data Multibrid M5000 [online publication], URL: http://www.multibrid.com/download/Datenblatt_M5000_eng.pdf [cited 1 November 2004].

[69] Multibrid Technology, The Concept in Detail [online publication], URL: http://www.multibrid.com/english/concept.htm [cited 4 January 2005].

[70] Musial, W., Butterfield, S., and Boone, A., "Feasibility of Floating Platform Systems for Wind Turbines," A Collection of the 2004 ASME Wind Energy Symposium Technical Papers Presented at the 42 ${ }^{\text {nd }}$ AIAA Aerospace Sciences Meeting and Exhibit, 5-7 January 2004, Reno Nevada, USA, New York: American Institute of Aeronautics and Astronautics, Inc. (AIAA) and American Society of Mechanical Engineers (ASME), January 2004, pp. 476-486, NREL/CP-500-36504, Golden, CO: National Renewable Energy Laboratory.

[71] Musial, W. and Butterfield, S., "Future for Offshore Wind Energy in the United States," EnergyOcean Proceedings, June 2004, Palm Beach Florida, USA, NREL/CP-500-36313, Golden, CO: National Renewable Energy Laboratory.

[72] Musial, W., Butterfield, S., and Ram, B., "Energy from Offshore Wind," 2006 Offshore Technology Conference, 1-4 May 2006, Houston, TX [CD-ROM], Richardson, TX: Offshore Technology Conference, May 2006, OTC 18355, NREL/CP-500-39450, Golden, CO: National Renewable Energy Laboratory.

[73] National Renewable Energy Laboratory, About the Program: WindPACT [online publication], URL: http://www.nrel.gov/wind/windpact/ [cited 4 January 2005].

[74] Newman, J. N., Marine Hydrodynamics, The MIT Press, Cambridge, MA, USA, 1997. 
[75] Nielsen, F. G., Hanson, T. D., and Skaare, B., "Integrated Dynamic Analysis of Floating Offshore Wind Turbines," Proceedings of OMAE2006 $25^{\text {th }}$ International Conference on Offshore Mechanics and Arctic Engineering, 4-9 June 2006, Hamburg, Germany [CDROM], Houston, TX: The American Society of Mechanical Engineers (ASME International) Ocean, Offshore and Arctic Engineering (OOAE) Division, June 2006, OMAE2006-92291.

[76] Ogilvie, T. F. "Recent Progress toward the Understanding and Prediction of Ship Motions," Fifth Symposium on Naval Hydrodynamics, September, 1964, pp. 3-128.

[77] Passon, P. and Kühn, M., "State-of-the-art and Development Needs of Simulation Codes for Offshore Wind Turbines," Copenhagen Offshore Wind 2005 Conference and Expedition Proceedings, 26-28 October 2005, Copenhagen, Denmark [CD-ROM], Copenhagen, Denmark: Danish Wind Energy Association, October 2005.

[78] Passon, P., Kühn, M., Butterfield, S., Jonkman, J., Camp, T., and Larsen, T. J., "OC3Benchmark Exercise of Aero-Elastic Offshore Wind Turbine Codes," Journal of Physics: Conference Series, The Second Conference on The Science of Making Torque From Wind, Copenhagen, Denmark, 28-31 August 2007, [online journal], Vol. 75, 2007, 012071, URL: http://www.iop.org/EJ/article/1742-6596/75/1/012071/jpconf7 75 012071.pdf?request-id=8kI1 Ig5u3BGgUobT2wi7Kg, [cited 28 August 2007].

[79] Peyrot, A. H. and Goulois, A. M., "Analysis of Cable Structures," Computers \& Structures, Vol. 10, 1979, pp. 805-813.

[80] Press, W. H., Teukolsky, S. A., Vetterling, W. T., and Flannery, B. P., Numerical Recipes in Fortran: The Art of Scientific Computing, $2^{\text {nd }}$ ed., Cambridge University Press, Cambridge, UK, 1992, pp. 490-498.

[81] REpower Systems, REpower $5 M$ [online publication], URL: http://www.repower.de/typo3/fileadmin/download/produkte/5m_uk.pdf [cited 4 January 2005].

[82] REpower Systems, REpower Systems AG - Renewable Energy for the Future [online publication], URL: http://www.repower.de/ [cited 4 January 2005].

[83] Rubinstein, R. Y., Simulation and the Monte Carlo Method, John Wiley \& Sons, Inc., New York, 1981, pp. 86-91.

[84] Saigal, R. K., Dolan, D., Der Kiureghian, A., Camp, T., and Smith, C. E., "Comparison of Design Guidelines for Offshore Wind Energy Systems," 2007 Offshore Technology Conference, April 30 - May 3, 2007, Houston, TX [CD-ROM], Richardson, TX: Offshore Technology Conference, May 2007, OTC 18984.

[85] Sclavounos, P. D., "13.022 Surface Waves and Their Interaction with Floating Bodies, Lecture Notes," Massachusetts Institute of Technology (MIT) OpenCourseWare [online publication], URL: http://ocw.mit.edu/OcwWeb/Ocean-Engineering/13-022Surface- 
Waves-and-their-Interaction-With-Floating-BodiesSpring2002/CourseHome/index.htm, [cited 27 September 2005].

[86] Sclavounos, P. D., "Nonlinear Particle Kinematics of Ocean Waves," Journal of Fluid Mechanics, Vol. 540, 2005, pp. 133-142.

[87] Skaare, B., Hanson, T. D., and Nielsen, F. G., "Importance of Control Strategies on Fatigue Life of Floating Wind Turbines," Proceedings of OMAE2007 $26^{\text {th }}$ International Conference on Offshore Mechanics and Arctic Engineering, 10-15 June 2007, San Diego, CA [CD-ROM], Houston, TX: The American Society of Mechanical Engineers (ASME International) Ocean, Offshore and Arctic Engineering (OOAE) Division, June 2007, OMAE2007-29277.

[88] Smith, K., WindPACT Turbine Design Scaling Studies; Technical Area 2: Turbine, Rotor, and Blade Logistics, NREL/SR-500-29439, Golden, CO: National Renewable Energy Laboratory, June 2001.

[89] Smith, S. W., The Scientist and Engineer's Guide to Digital Signal Processing, San Diego, CA: California Technical Publishing, 2006.

[90] Stol, K. A., Dynamics Modeling and Periodic Control of Horizontal-Axis Wind Turbines, Ph.D. Dissertation, Department of Aerospace Engineering Sciences, University of Colorado, Boulder, CO, USA, 2001.

[91] Strum, R. D. and Kirk, D. E., Contemporary Linear Systems Using MATLAB ${ }^{\circledR}$, Brooks/Cole, Pacific Grove, California, USA, 2000, pp. 221-297.

[92] Swarztrauber, P. N., "FFTPACK," NCAR's Mathematical and Statistical Libraries [online database], URL: http://www.cisl.ucar.edu/softlib/FFTPACK.html [cited 12 July 2007], Boulder, CO: The National Center for Atmospheric Research (NCAR).

[93] Tarp-Johansen, N. J., RECOFF Home Page [online publication], URL: http://www.risoe.dk/vea/recoff/, [cited 1 November 2004].

[94] Tolman, H. L., User manual and system documentation of WAVEWATCH-III version 2.22, Environmental Modeling Center Marine Modeling and Analysis Branch (MMAB) Technical Note 222, Washington DC: National Ocean and Atmospheric Administration (NOAA), National Weather Service (NWS), and National Centers for Environmental Prediction (NCEP), September 2002.

[95] Underwood, P. G., "Dynamic Relaxation," Computational Methods for Transient Analysis, Vol. 1, 1983, pp. 245-265.

[96] van der Tempel, J., Design of Support Structures for Offshore Wind Turbines, Ph.D. Dissertation, Offshore Engineering Group, Delft University of Technology, Delft, The Netherlands, 2006. 
[97] Veldkamp, H. F. and van der Tempel, J., "Influence of Wave Modeling on the Prediction of Fatigue for Offshore Wind Turbines," Wind Energy, Vol. 8, No. 1, January/March 2005, pp. 49-65.

[98] Vijfhuizen, W. J. M. J, Design of a Wind and Wave Power Barge, M.S. Dissertation, Department of Naval Architecture and Mechanical Engineering, Universities of Glasgow and Strathclyde, Glasgow, Scotland, September 2006.

[99] Watson, G., et al, "A Framework for Offshore Wind Energy Development in the United States," Massachusetts Technology Collaborative (MTC) [online publication], URL: http://www.mtpc.org/offshore/final_09_20.pdf, [cited 17 November 2005].

[100] Wayman, E. N., Sclavounos, P. D., Butterfield, S., Jonkman, J., and Musial, W., "Coupled Dynamic Modeling of Floating Wind Turbine Systems," 2006 Offshore Technology Conference, 1-4 May 2006, Houston, TX [CD-ROM], Richardson, TX: Offshore Technology Conference, May 2006, OTC 18287, NREL/CP-500-39481, Golden, CO: National Renewable Energy Laboratory.

[101] Wayman, E., Coupled Dynamics and Economic Analysis of Floating Wind Turbine Systems, M.S. Dissertation, Department of Mechanical Engineering, Massachusetts Institute of Technology, Cambridge, MA, USA, June 2006.

[102] Wehausen, J. V., "Initial-Value Problem for the Motion in an Undulating Sea of a Body with Fixed Equilibrium Position," Journal of Engineering Mathematics, Vol. 1, No. 1, January 1967, pp. 1-17.

[103] Withee, J. E., Fully Coupled Dynamic Analysis of a Floating Wind Turbine System, Ph.D. Dissertation, Department of Ocean Engineering, Massachusetts Institute of Technology, Cambridge, MA, USA, 2004.

[104] Wright, A. D., Modern Control Design for Flexible Wind Turbines, Ph.D. Dissertation, Department of Aerospace Engineering Sciences, University of Colorado, Boulder, CO, USA, July 2004, NREL/TP-500-35816, Golden, CO: National Renewable Energy Laboratory.

[105] Zambrano, T., MacCready, T., Kiceniuk, T., Jr., Roddier, D. G., and Cermelli, C. A., "Dynamic Modeling of Deepwater Offshore Wind Turbine Structures in Gulf of Mexico Storm Conditions," Proceedings of OMAE2006 $25^{\text {th }}$ International Conference on Offshore Mechanics and Arctic Engineering, 4-9 June 2006, Hamburg, Germany [CDROM], Houston, TX: The American Society of Mechanical Engineers (ASME International) Ocean, Offshore and Arctic Engineering (OOAE) Division, June 2006, OMAE2006-92029.

[106] Zambrano, T., MacCready, T., Roddier, D. G., and Cermelli, C. A., "Design and Installation of a Tension Moored Wind Turbine," Proceedings of OMAE2007 $26^{\text {th }}$ International Conference on Offshore Mechanics and Arctic Engineering, 10-15 June 2007, San Diego, CA [CD-ROM], Houston, TX: The American Society of Mechanical 
Engineers (ASME International) Ocean, Offshore and Arctic Engineering (OOAE) Division, June 2007, OMAE2007-29587. 


\section{Appendix A FAST Input Files for the 5-MW Wind Turbine}

\section{A.1 Primary Input File}

\begin{tabular}{|c|c|c|}
\hline \multicolumn{3}{|c|}{$\begin{array}{l}\text { NREL 5.0 MW Baseline Wind Turbine for Use in Offshore Analysis. } \\
\text { Properties from Dutch Offshore Wind Energy Converter (DOWEC) 6MW Pre-Design (10046_009.pdf) and REpower 5M 5MW (5m_uk.pdf); C }\end{array}$} \\
\hline False & Echo & - Echo input data to "echo.out" (flag) \\
\hline 3 & ADAMSPrep & - ADAMS preprocessor mode $\{1$ : Run FAST, 2: use FAST as a preprocessor to create an ADAMS model, $3:$ do \\
\hline 1 & AnalMode & - Analysis mode $\{1$ : Run a time-marching simulation, 2: create a periodic linearized model\} (switch) \\
\hline 3 & NumBl & - Number of blades (-) \\
\hline 630.0 & TMax & - Total run time (s) \\
\hline 0.0125 & DT & - Integration time step (s) \\
\hline & & TURBINE CONTROL \\
\hline 0 & YCMode & - Yaw control mode $\{0$ : none, 1: user-defined from routine UserYawCont, 2: user-defined from Simulink\} \\
\hline 9999.9 & TYCOn & - Time to enable active yaw control (s) [unused when YCMode=0] \\
\hline 1 & PCMode & - Pitch control mode $\{0$ : none, 1: user-defined from routine PitchCntrl, 2: user-defined from Simulink \\
\hline 0.0 & TPCOn & - Time to enable active pitch control (s) [unused when PCMode=0] \\
\hline 2 & VSContrl & - Variable-speed control mode \{0: none, 1: simple VS, 2: user-defined from routine UserVSCont, 3 : use \\
\hline 9999.9 & VS_RtGnSp & - Rated generator speed for simple variable-speed generator control (HSS side) (rpm) [used only when \\
\hline 9999.9 & VS_RtTq & - Rated generator torque/constant generator torque in Region 3 for simple variable-speed generator co \\
\hline 9999.9 & VS_Rgn2K & - Generator torque constant in Region 2 for simple variable-speed generator control (HSS side) (N-m/r \\
\hline 9999.9 & VS_SlPc & - Rated generator slip percentage in Region $21 / 2$ for simple variable-speed generator control (\%) [us \\
\hline 2 & GenModel & - Generator model \{1: simple, 2: Thevenin, 3: user-defined from routine UserGen\} (switch) [used only \\
\hline True & GenTiStr & - Method to start the generator $\{\mathrm{T}$ : timed using TimGenOn, F: generator speed using SpdGenOn\} ( $f l a g)$ \\
\hline & GenTiStp & - Method to stop the generator $\{\mathrm{T}$ : timed using TimGenOf, $\mathrm{F}:$ when generator power $=0\}(\mathrm{flag})$ \\
\hline 9999.9 & SpdGenOn & - Generator speed to turn on the generator for a startup (HSS speed) (rpm) [used only when GenTiStr=F \\
\hline 0.0 & TimGenOn & - Time to turn on the generator for a startup (s) [used only when GenTiStr=True] \\
\hline 9999.9 & TimGenOf & - Time to turn off the generator ( $s$ ) [used only when GenTiStp=True] \\
\hline 1 & HSSBrMode & - HSS brake model $\{1$ : simple, 2 : user-defined from routine UserHSSBr\} (switch) \\
\hline 9999.9 & THSSBrDp & - Time to initiate deployment of the HSS brake (s) \\
\hline 9999.9 & TiDynBrk & - Time to initiate deployment of the dynamic generator brake [CURRENTLY IGNORED] (s) \\
\hline 9999.9 & $\operatorname{TTpBrDp}(1)$ & - Time to initiate deployment of tip brake 1 (s) \\
\hline 9999.9 & $\operatorname{TTpBrDp}(2)$ & - Time to initiate deployment of tip brake 2 (s) \\
\hline 9999.9 & $\operatorname{TTpBrDp}(3)$ & - Time to initiate deployment of tip brake $3(\mathrm{~s})$ [unused for 2 blades] \\
\hline 9999.9 & TBDepISp(1) & - Deployment-initiation speed for the tip brake on blade 1 (rpm) \\
\hline 9999.9 & TBDepISp(2) & - Deployment-initiation speed for the tip brake on blade 2 (rpm) \\
\hline 9999.9 & TBDepISp(3) & - Deployment-initiation speed for the tip brake on blade 3 (rpm) [unused for 2 blades] \\
\hline 9999.9 & TYawManS & - Time to start override yaw maneuver and end standard yaw control (s) \\
\hline 0.3 & YawManRat & - Yaw rate (in absolute value) at which override yaw maneuver heads toward final yaw angle (deg/s) \\
\hline 0.0 & NacYawF & - Final yaw angle for override yaw maneuvers (degrees) \\
\hline 9999.9 & TPitMans(1) & - Time to start override pitch maneuver for blade 1 and end standard pitch control (s) \\
\hline 9999.9 & TPitManS(2) & - Time to start override pitch maneuver for blade 2 and end standard pitch control (s) \\
\hline 9999.9 & TPitManS(3) & - Time to start override pitch maneuver for blade 3 and end standard pitch control (s) [unused for 2 \\
\hline 8.0 & PitManRat(1) & )- Pitch rate (in absolute value) at which override pitch maneuver for blade 1 heads toward final pitc \\
\hline 8.0 & PitManRat(2) & )- Pitch rate (in absolute value) at which override pitch maneuver for blade 2 heads toward final pitc \\
\hline 8.0 & PitManRat(3) & )- Pitch rate (in absolute value) at which override pitch maneuver for blade 3 heads toward final pitc \\
\hline 0.0 & BlPitch(1) & - Blade 1 initial pitch (degrees) \\
\hline 0.0 & BlPitch(2) & - Blade 2 initial pitch (degrees) \\
\hline 0.0 & BlPitch(3) & - Blade 3 initial pitch (degrees) [unused for 2 blades] \\
\hline 0.0 & BlPitchF(1) & - Blade 1 final pitch for override pitch maneuvers (degrees) \\
\hline 0.0 & BlPitchF(2) & - Blade 2 final pitch for override pitch maneuvers (degrees) \\
\hline 0.0 & BlPitchF(3) & - Blade 3 final pitch for override pitch maneuvers (degrees) [unused for 2 blades] \\
\hline & & NMENTAL CONDITIONS \\
\hline 9.8 & vity & - Gravitational acceleration (m/s^2) \\
\hline & & FEATURE FLAGS $\cdots$ \\
\hline True & FlapDOF1 & - First flapwise blade mode DOF (flag) \\
\hline True & FlapDOF2 & - Second flapwise blade mode DOF (flag) \\
\hline True & EdgeDOF & - First edgewise blade mode DOF (flag) \\
\hline False & TeetDOF & - Rotor-teeter DOF (flag) [unused for 3 blades] \\
\hline True & DrTrDOF & - Drivetrain rotational-flexibility DOF (flag) \\
\hline True & GenDOF & - Generator DOF (flag) \\
\hline True & YawDOF & - Yaw DOF (flag) \\
\hline True & TWFADOF1 & - First fore-aft tower bending-mode DOF (flag) \\
\hline True & TWFADOF 2 & - Second fore-aft tower bending-mode DOF (flag) \\
\hline True & TWSSDOF1 & - First side-to-side tower bending-mode DOF (flag) \\
\hline True & TWSSDOF 2 & - Second side-to-side tower bending-mode DOF (flag) \\
\hline True & CompAero & - Compute aerodynamic forces (flag) \\
\hline False & CompNoise & - Compute aerodynamic noise (flag) \\
\hline & & INITIAL CONDITIONS \\
\hline 0.0 & OoPDefl & - Initial out-of-plane blade-tip displacement (meters) \\
\hline 0.0 & IPDefl & - Initial in-plane blade-tip deflection (meters) \\
\hline 0.0 & TeetDefl & - Initial or fixed teeter angle (degrees) [unused for 3 blades] \\
\hline 0.0 & Azimuth & - Initial azimuth angle for blade 1 (degrees) \\
\hline 12.1 & RotSpeed & - Initial or fixed rotor speed (rpm) \\
\hline 0.0 & NacYaw & - Initial or fixed nacelle-yaw angle (degrees) \\
\hline 0.0 & TTDspFA & - Initial fore-aft tower-top displacement (meters) \\
\hline 0.0 & TTDspSS & - Initial side-to-side tower-top displacement (meters) \\
\hline
\end{tabular}




\begin{tabular}{|c|c|c|}
\hline & TipRad & - The distance from the rotor apex to the blade tip (meters) \\
\hline 1.5 & HubRad & - The distance from the rotor apex to the blade root (meters) \\
\hline 1 & PSpnElN & - Number of the innermost blade element which is still part of the pitchable portion of the blade for \\
\hline 0.0 & UndSling & - Undersling length [distance from teeter pin to the rotor apex] (meters) [unused for 3 blades] \\
\hline 0.0 & HubCM & - Distance from rotor apex to hub mass [positive downwind] (meters) \\
\hline-5.01910 & OverHang & - Distance from yaw axis to rotor apex [3 blades] or teeter pin [2 blades] (meters) \\
\hline 1.9 & NacCMxn & - Downwind distance from the tower-top to the nacelle CM (meters) \\
\hline 0.0 & NacCMyn & - Lateral distance from the tower-top to the nacelle CM (meters) \\
\hline 1.75 & NacCMzn & - Vertical distance from the tower-top to the nacelle CM (meters) \\
\hline 87.6 & TowerHt & - Height of tower above ground level [onshore] or MSL [offshore] (meters) \\
\hline 1.96256 & Twr2Shft & - Vertical distance from the tower-top to the rotor shaft (meters) \\
\hline 0.0 & TwrRBHt & - Tower rigid base height (meters) \\
\hline-5.0 & ShftTilt & - Rotor shaft tilt angle (degrees) \\
\hline 0.0 & Delta3 & - Delta-3 angle for teetering rotors (degrees) [unused for 3 blades] \\
\hline-2.5 & PreCone(1) & - Blade 1 cone angle (degrees) \\
\hline-2.5 & PreCone (2) & - Blade 2 cone angle (degrees) \\
\hline-2.5 & PreCone (3) & - Blade 3 cone angle (degrees) [unused for 2 blades] \\
\hline 0.0 & AzimB1Up & - Azimuth value to use for I/O when blade 1 points up (degrees) \\
\hline & & MASS AND INERTIA \\
\hline 0.0 & YawBrMass & - Yaw bearing mass $(\mathrm{kg})$ \\
\hline $240.00 \mathrm{E} 3$ & NacMass & - Nacelle mass (kg) \\
\hline $56.78 \mathrm{E} 3$ & HubMass & - Hub mass (kg) \\
\hline 0.0 & TipMass (1) & - Tip-brake mass, blade 1 (kg) \\
\hline 0.0 & TipMass (2) & - Tip-brake mass, blade $2(\mathrm{~kg})$ \\
\hline 0.0 & TipMass (3) & - Tip-brake mass, blade 3 (kg) [unused for 2 blades] \\
\hline $2607.89 \mathrm{E} 3$ & NacYIner & - Nacelle inertia about yaw axis ( $\mathrm{kg} \mathrm{m}^{\wedge} 2$ ) \\
\hline 534.116 & GenIner & - Generator inertia about HSS ( $\mathrm{kg} \mathrm{m} \mathrm{m}^{\wedge}$ ) \\
\hline $115.926 \mathrm{E} 3$ & HubIner & - Hub inertia about rotor axis [3 blades] or teeter axis [2 blades] $\left(\mathrm{kg} \mathrm{m}^{\wedge} 2\right)$ \\
\hline & $----1--$ & DRIVETRAIN - \\
\hline 100.0 & GBoxEff & - Gearbox efficiency (\%) \\
\hline 94.4 & GenEff & - Generator efficiency [ignored by the Thevenin and user-defined generator models] (\%) \\
\hline 97.0 & GBRatio & - Gearbox ratio (-) \\
\hline False & GBRevers & - Gearbox reversal $\{\mathrm{T}$ : if rotor and generator rotate in opposite directions $\}$ (flag) \\
\hline $28.1162 \mathrm{E} 3$ & HSSBrTqF & - Fully deployed HSS-brake torque (N-m) \\
\hline 0.6 & HSSBrDT & - Time for HSS-brake to reach full deployment once initiated (sec) [used only when HSSBrMode=1] \\
\hline & DynBrkFi & - File containing a mech-gen-torque vs HSS-speed curve for a dynamic brake [CURRENTLY IGNORED] (quote \\
\hline 867.637E6 & DTTorSpr & - Drivetrain torsional spring (N-m/rad) \\
\hline $6.215 \mathrm{E} 6$ & DTTorDmp & - Drivetrain torsional damper ( $\mathrm{N}-\mathrm{m} /(\mathrm{rad} / \mathrm{s}))$ \\
\hline & & SIMPLE INDUCTION GENERATOR - \\
\hline 9999.9 & SIG_SIPC & - Rated generator slip percentage (\%) [used only when VSContrl=0 and GenModel=1] \\
\hline 9999.9 & SIG_SySp & - Synchronous (zero-torque) generator speed $(\mathrm{rpm})$ [used only when VSContrl=0 and GenModel=1] \\
\hline 9999.9 & SIG_RtTq & - Rated torque $(\mathrm{N}-\mathrm{m})$ [used only when VSContrl=0 and GenModel $=1]$ \\
\hline 9999.9 & SIG_PORt & - Pull-out ratio (Tpullout/Trated) (-) [used only when VSContrl=0 and GenModel=1] \\
\hline$-\ldots-1-10$ & 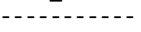 & THEVENIN-EQUIVALENT INDUCTION GENERATOR - \\
\hline 9999.9 & TEC_Freq & - Line frequency [50 or 60] (Hz) [used only when VSContrl=0 and GenModel=2] \\
\hline 9998 & TEC_NPol & - Number of poles [even integer $>0$ ] $(-)$ [used only when VSContrl=0 and GenModel=2] \\
\hline 9999.9 & TEC_SRes & - Stator resistance (ohms) [used only when vSContrl=0 and GenModel=2] \\
\hline 9999.9 & TEC_RRes & - Rotor resistance (ohms) [used only when VSContrl=0 and GenModel=2] \\
\hline 9999.9 & TEC_VLL & - Line-to-line RMS voltage (volts) [used only when VSContrl=0 and GenModel=2] \\
\hline 9999.9 & TEC_SLR & - Stator leakage reactance (ohms) [used only when VSContrl=0 and GenModel=2] \\
\hline 9999.9 & TEC_RLR & - Rotor leakage reactance (ohms) [used only when VSContrl=0 and GenModel=2] \\
\hline 9999.9 & TEC_MR & - Magnetizing reactance (ohms) [used only when VSContrl=0 and GenModel=2] \\
\hline & & PLATFORM - \\
\hline 3 & PtfmModel & - Platform model $\{0$ : none, 1: onshore, 2: fixed bottom offshore, 3: floating offshore $\}$ (switch) \\
\hline "NRELOffshr & Bsline5MW_P. & latform_ITIBarge4.dat" PtfmFile - Name of file containing platform properties (quoted string) [u \\
\hline & & TOWER \\
\hline 20 & TwrNodes & - Number of tower nodes used for analysis (-) \\
\hline "NRELOffshrE & Bsline5MW_TC & ower_ITIBarge4.dat" TwrFile - Name of file containing tower properties (quoted string) \\
\hline & & NACĒELE-YAW - \\
\hline $9028.32 \mathrm{E} 6$ & YawSpr & - Nacelle-yaw spring constant $(\mathrm{N}-\mathrm{m} / \mathrm{rad})$ \\
\hline 19.16E6 & YawDamp & - Nacelle-yaw damping constant (N-m/(rad/s)) \\
\hline 0.0 & YawNeut & - Neutral yaw position--yaw spring force is zero at this yaw (degrees) \\
\hline 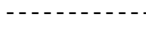 & & (-1, \\
\hline False & $\begin{array}{l}\text { Furling } \\
\text { FurlFile }\end{array}$ & $\begin{array}{l}\text { - Read in additional model properties for furling turbine ( } f l a g \text { ) } \\
\text { - Name of file containing furling properties (quoted string) [unused when Furling=False] }\end{array}$ \\
\hline & & ROTOR-TEETER - \\
\hline 0 & TeetMod & - Rotor-teeter spring/damper model $\{0$ : none, 1: standard, 2: user-defined from routine UserTeet $\}$ (swi \\
\hline 0.0 & TeetDmpP & - Rotor-teeter damper position (degrees) [used only for 2 blades and when TeetMod=1] \\
\hline 0.0 & TeetDmp & - Rotor-teeter damping constant $(\mathrm{N}-\mathrm{m} /(\mathrm{rad} / \mathrm{s}))$ [used only for 2 blades and when TeetMod=1] \\
\hline 0.0 & TeetCDmp & - Rotor-teeter rate-independent Coulomb-damping moment $(\mathrm{N}-\mathrm{m})$ [used only for 2 blades and when TeetMod \\
\hline 0.0 & TeetSStP & - Rotor-teeter soft-stop position (degrees) [used only for 2 blades and when TeetMod=1] \\
\hline 0.0 & TeetHStP & - Rotor-teeter hard-stop position (degrees) [used only for 2 blades and when TeetMod=1] \\
\hline 0.0 & TeetSSSp & - Rotor-teeter soft-stop linear-spring constant $(\mathrm{N}-\mathrm{m} / \mathrm{rad})$ [used only for 2 blades and when TeetMod=1] \\
\hline 0.0 & TeetHSSp & - Rotor-teeter hard-stop linear-spring constant $(\mathrm{N}-\mathrm{m} / \mathrm{rad})$ [used only for 2 blades and when TeetMod=1] \\
\hline & & - \\
\hline 0.0 & TBDrConN & - Tip-brake drag constant during normal operation, Cd*Area $\left(m^{\wedge} 2\right)$ \\
\hline 0.0 & TBDrConD & - Tip-brake drag constant during fully-deployed operation, Cd*Area $\left(\mathrm{m}^{\wedge} 2\right)$ \\
\hline 0.0 & TpBrDT & - Time for tip-brake to reach full deployment once released (sec) \\
\hline Fiot & $\mathrm{Bcl}$ ine $5 \mathrm{MW} \mathrm{R}$ & B]dFile \\
\hline $\begin{array}{l}\text { "NRELOffshrE } \\
\text { "NRELOffshrE }\end{array}$ & Bsline5MW_B. & BldFile(1) - Name of file containing properties for blade 1 (quoted string) \\
\hline "NRELOffshrt & Bsline5MW_B. & $\begin{array}{l}\text { - Name of tile contalning properties for blade } 2 \text { (quoted string) } \\
\text { Bldfile(3) - Name of file containing properties for blade } 3 \text { (quoted string) }\end{array}$ \\
\hline & & \\
\hline
\end{tabular}




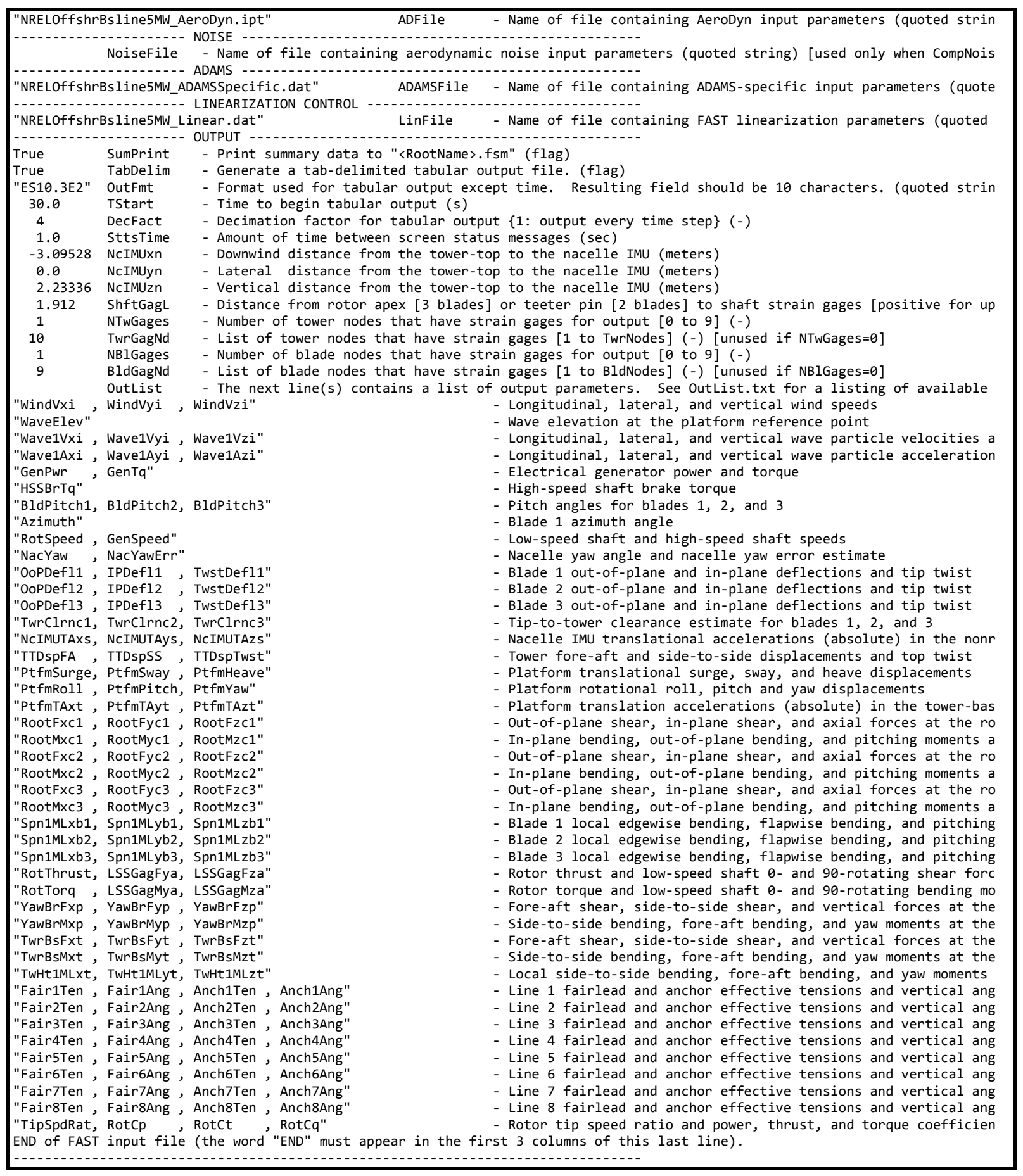

\section{A.2 Blade Input File - NRELOffshrBsline5MW_Blade.dat}

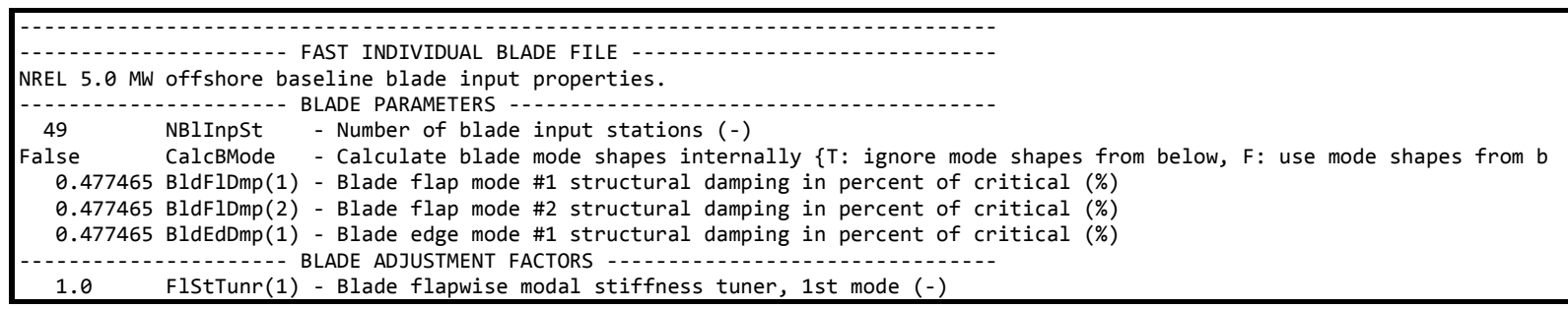




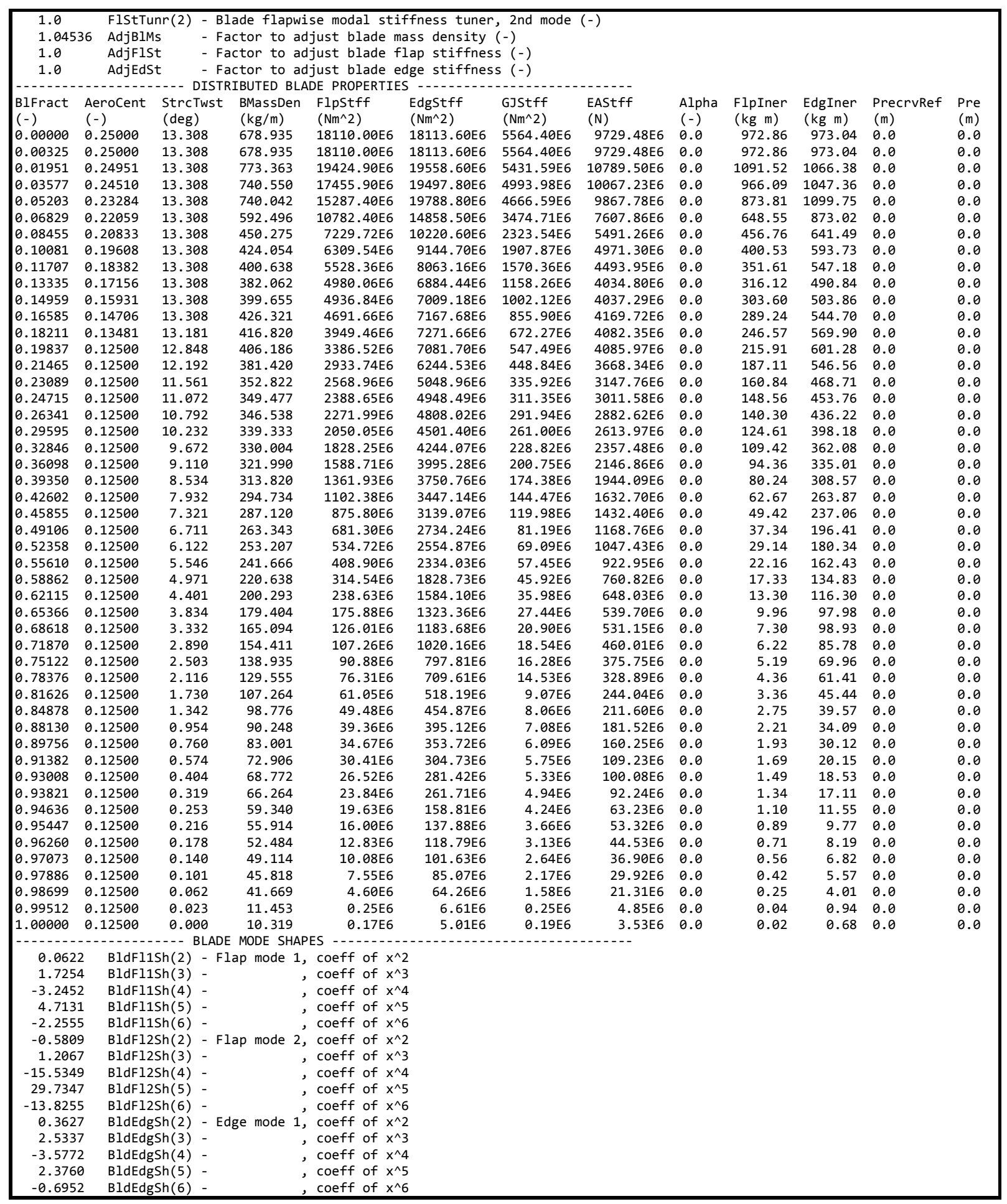

\section{A.3 Tower Input File - NRELOffshrBsline5MW_Tower_ITIBarge4.dat}

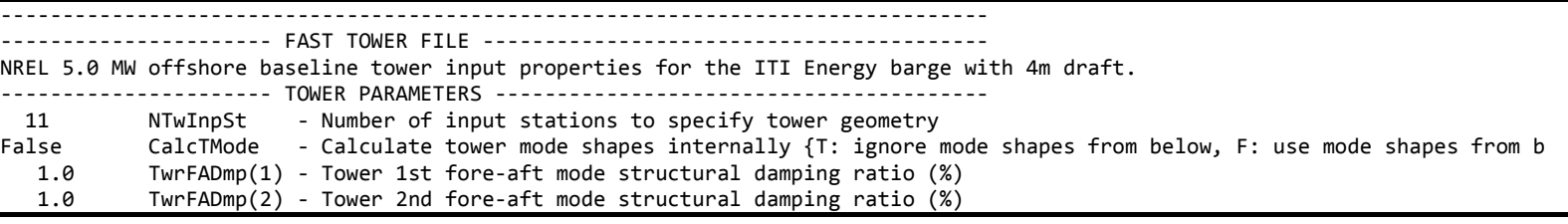




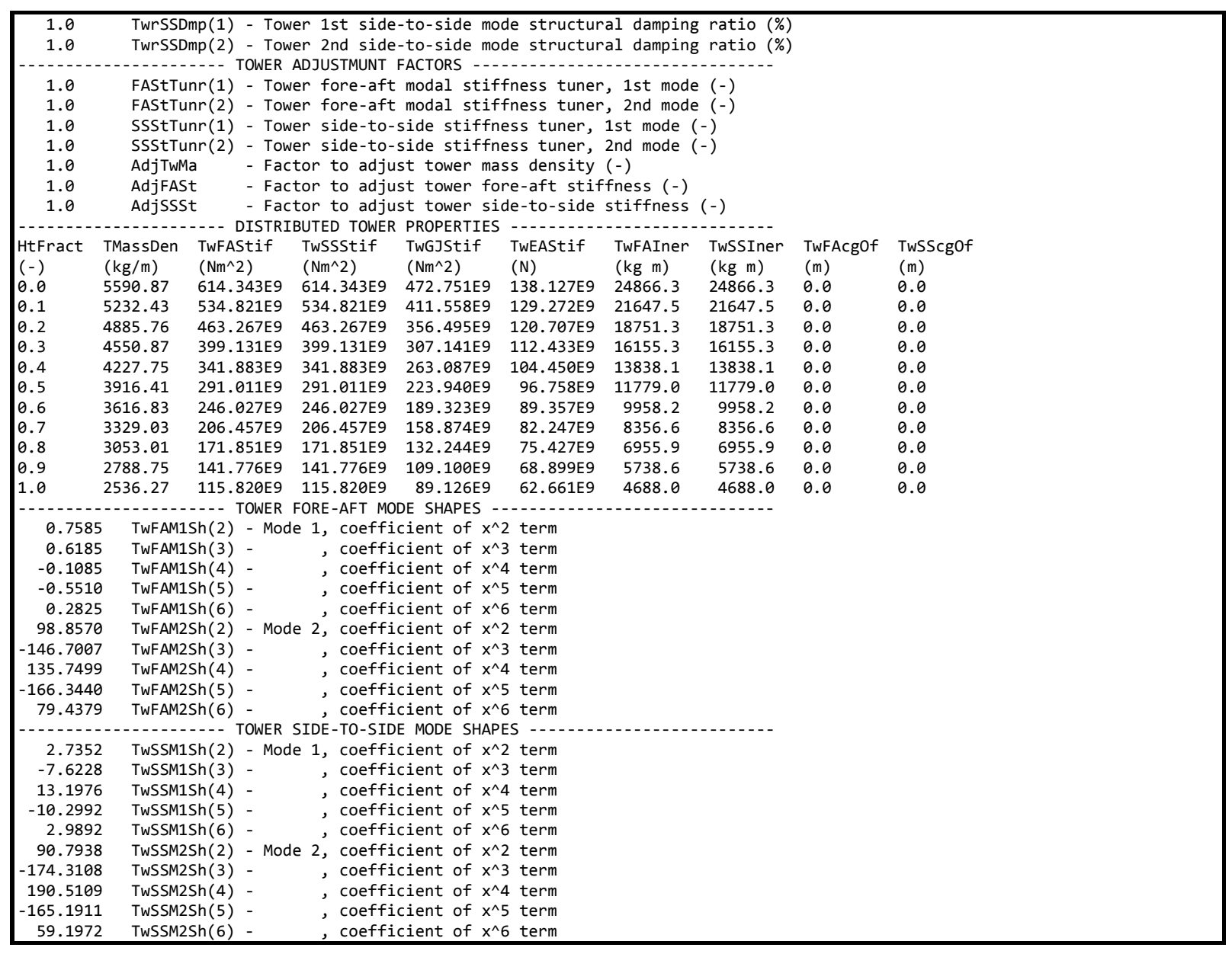

\section{A.4 ADAMS Input File - NRELOffshrBsline5MW_ADAMSSpecific.dat}

\begin{tabular}{|c|c|c|c|c|}
\hline & $\begin{array}{l}\text { Offshore baseline ADAMS-Specific input properties. } \\
\text { SaveGrphcs - Save GRAPHICS output (flag) } \\
\text { MakeLINacf - Make an ADAMS/LINEAR control / command file (flag) } \\
\text { CRatioTGJ - Ratio of damping to stiffness for the tower torsion deflection (-) } \\
\text { CRatioTEA - Ratio of damping to stiffness for the tower extensional deflection (-) } \\
\text { CRatioBGJ } \\
\text { CRatioBEA }\end{array}$ & $\begin{array}{l}\text { NREL } 5.0 \text { Mh } \\
\text { True } \\
\text { False } \\
0.01 \\
0.01 \\
0.01 \\
0.01 \\
971.350 \mathrm{E} 6 \\
0.206 \mathrm{E} \\
-1 .-1 \\
20 \\
3.000 \\
1.935 \\
7.0 \\
1.75 \\
1.75 \\
1.0 \\
2.39 \\
1.195 \\
4.78 \\
0.75 \\
0.2 \\
0.4 \\
0.875 \\
0.18 \\
0.0\end{array}$ & $\begin{array}{l}\text { SaveGrphcs } \\
\text { MakeLINacf } \\
\text { CRatioTGJ } \\
\text { CRatioTEA } \\
\text { CRatioBGJ } \\
\text { CRatioBEA } \\
\text { BPActrSpr } \\
\text { BPActrDmp } \\
\text { NSides } \\
\text { TwrBaseRad } \\
\text { TwrTopRad } \\
\text { NacLength } \\
\text { NacRadBot } \\
\text { NacRadTop } \\
\text { GBoxLength } \\
\text { GenLength } \\
\text { HSSLength } \\
\text { LSSLength } \\
\text { GenRad } \\
\text { HSSRad } \\
\text { LSSRad } \\
\text { HubCylRad } \\
\text { ThkOvrChrd } \\
\text { BoomRad }\end{array}$ & $\begin{array}{l}\text { FAST } 2 \text { ADAMS PREPROCESSOR, ADAMS-SPECIFIC DATA FILE } \\
\text { Seline ADAMS-specific input properties. } \\
\text { FEATURE FLAGS - } \\
\text { - Save GRAPHICS output (flag) } \\
\text { - Make an ADAMS/LINEAR control / command file (flag) } \\
\text { DAMPING PARAMETERS - Ratio of damping to stiffness for the tower torsion deflection (-) } \\
\text { - Ratio of damping to stiffness for the tower extensional deflection (-) } \\
\text { - Ratio of damping to stiffness for the blade torsion deflections (-) } \\
\text { - Ratio of damping to stiffness for the blade extensional deflections (-) } \\
\text { BLADE PITCH ACTUATOR PARAMETERS - } \\
\text { - Blade pitch actuator spring Stiffness constant (N-m/rad) } \\
\text { - Blade pitch actuator damping constant (N-m/(rad/s)) } \\
\text { - Number of sides used in GRAPHICS CYLINDER and FRUSTUM statements (-) } \\
\text { - Tower base radius used for linearly tapered tower GRAPHICS CYLINDERS (m) } \\
\text { - Tower top radius used for linearly tapered tower GRAPHICS CYLINDERS (m) } \\
\text { - Length of nacelle used for the nacelle GRAPHICS (m) } \\
\text { - Bottom (opposite rotor) radius of nacelle FRUSTUM used for the nacelle GRAPHICS (m) } \\
\text { - Top (rotor end) } \\
\text { - Length, width, and height of the gearbox BOX for gearbox GRAPHICS (m) } \\
\text { - Length of the generator CYLINDER used for generator GRAPHICS (m) } \\
\text { - Length of the high-speed shaft CYLINDER used for HSS GRAPHICS (m) } \\
\text { - Length of the low-speed shaft CYLINDER used for LSS GRAPHICS (m) } \\
\text { - Radius of the generator CYLINDER used for generator GRAPHICS (m) } \\
\text { - Radius of the high-speed shaft CYLINDER used for HSS GRAPHICS (m) } \\
\text { - Radius of the low - speed shaft CYLINDER used for LSS GRAPHICS (m) } \\
\text { - Radius of hub CYLINDER used for hub GRAPHICS (m) } \\
\text { - Ratio of blade thickness to blade chord used for blade element BOX GRAPHICS (-) } \\
\text { - Radius of the tail boom CYLINDER used for tail boom GRAPHICS (m) }\end{array}$ \\
\hline
\end{tabular}




\section{A.5 Linearization Input File - NRELOffshrBsline5MW_Linear.dat}

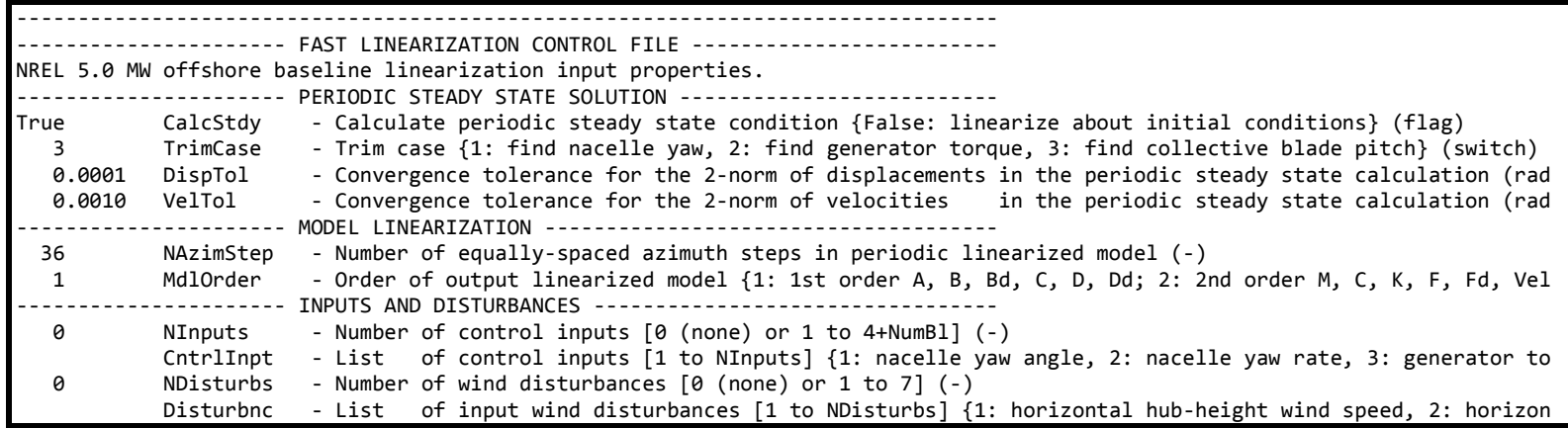




\section{Appendix B AeroDyn Input Files for the 5-MW Wind Turbine}

\section{B.1 Primary Input File - NRELOffshrBsline5MW_AeroDyn.ipt}

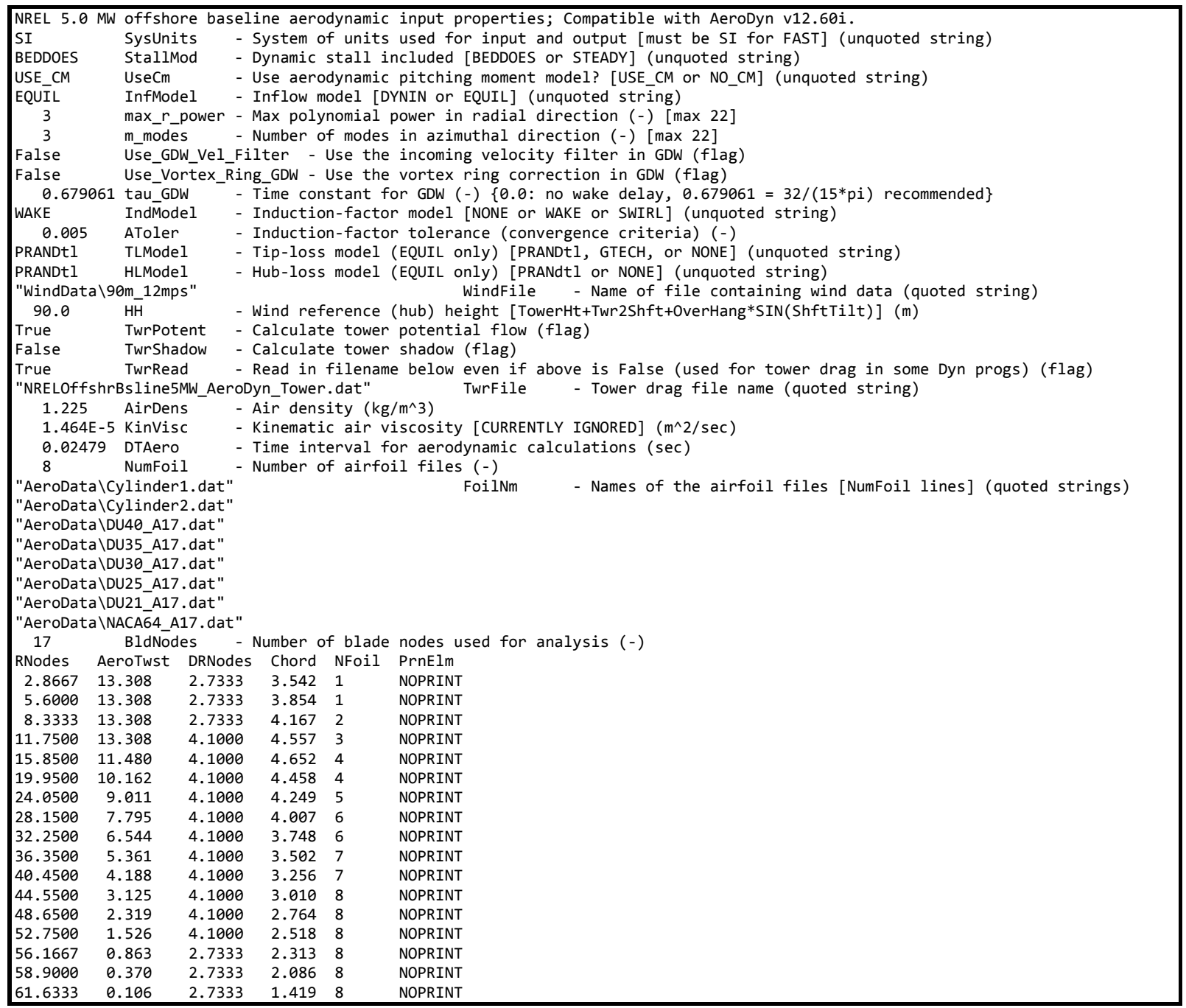

\section{B.2 Tower Input File - NRELOffshrBsline5MW_AeroDyn_Tower.dat}

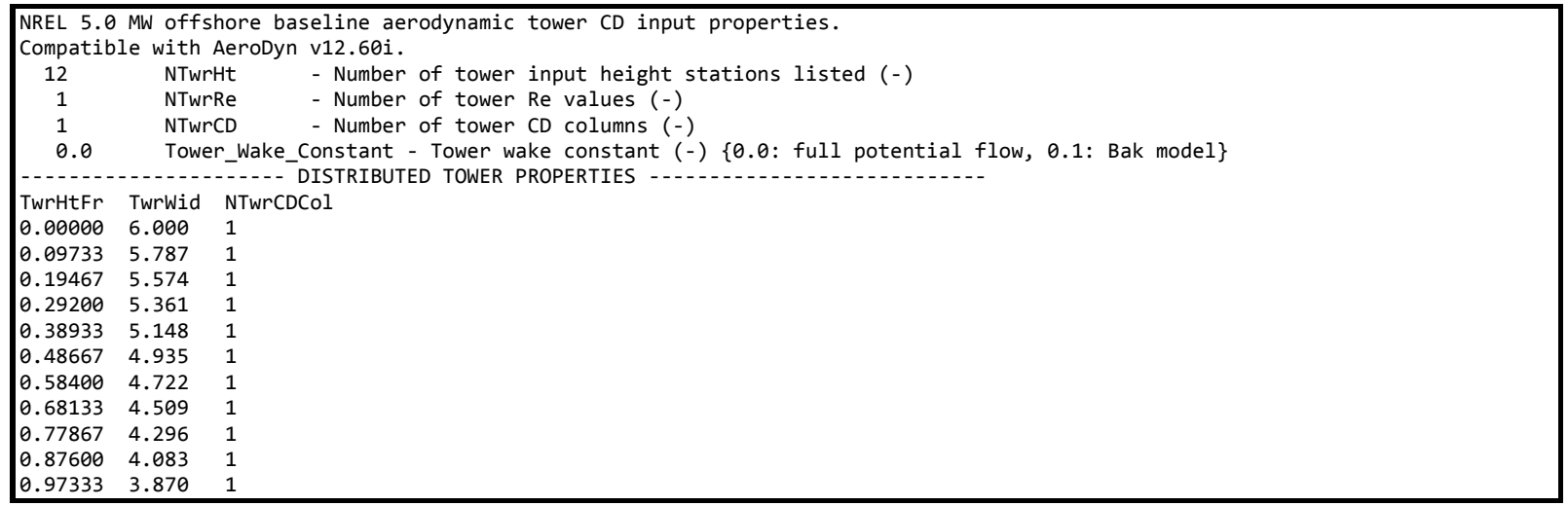




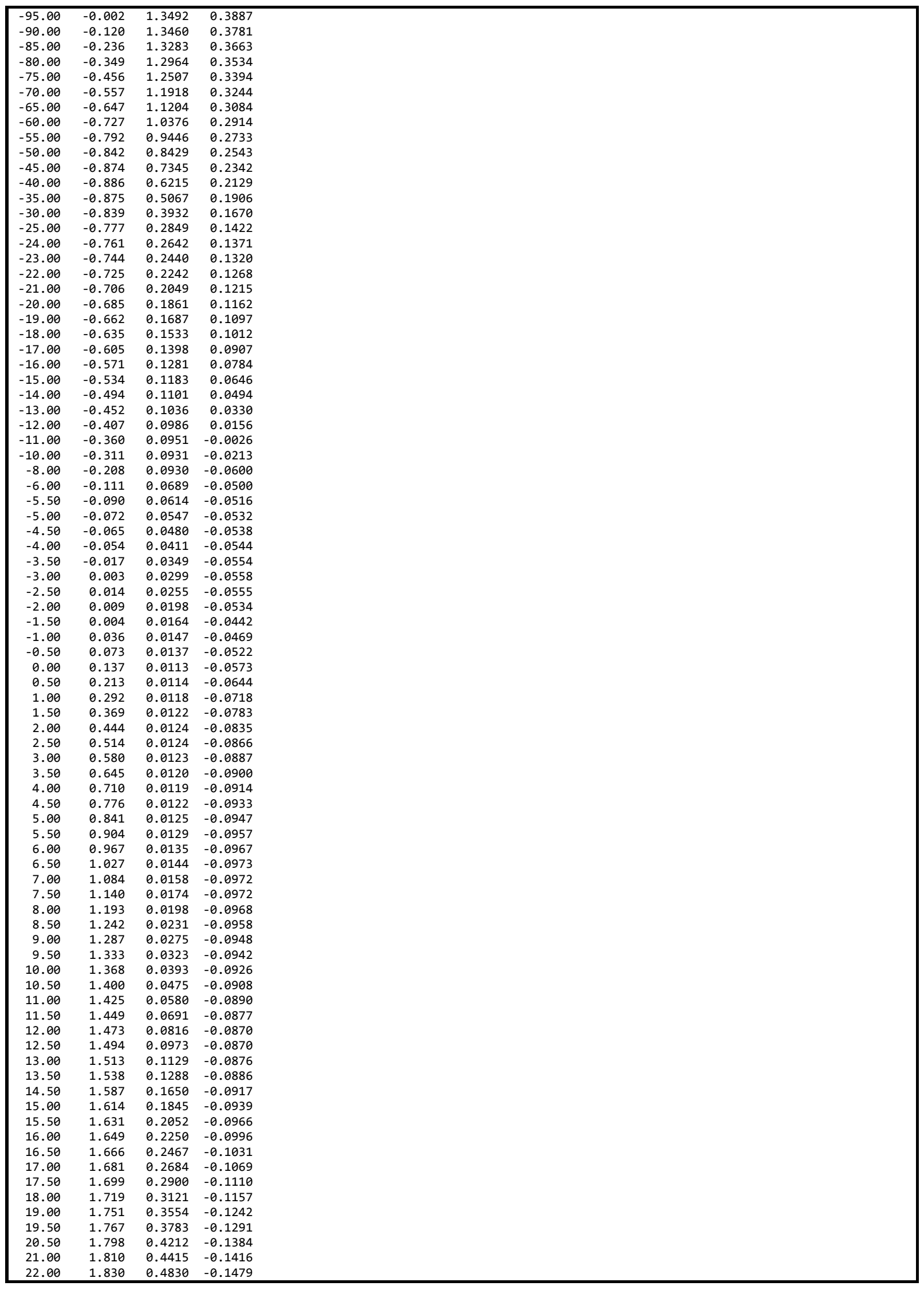




\begin{tabular}{|rrrr|}
\hline 23.00 & 1.847 & 0.5257 & -0.1542 \\
24.00 & 1.861 & 0.5694 & -0.1603 \\
25.00 & 1.872 & 0.6141 & -0.1664 \\
26.00 & 1.881 & 0.6593 & -0.1724 \\
28.00 & 1.894 & 0.7513 & -0.1841 \\
30.00 & 1.904 & 0.8441 & -0.1954 \\
32.00 & 1.915 & 0.9364 & -0.2063 \\
35.00 & 1.929 & 1.0722 & -0.2220 \\
40.00 & 1.903 & 1.2873 & -0.2468 \\
45.00 & 1.820 & 1.4796 & -0.2701 \\
50.00 & 1.690 & 1.6401 & -0.2921 \\
55.00 & 1.522 & 1.7609 & -0.3127 \\
60.00 & 1.323 & 1.8360 & -0.3321 \\
65.00 & 1.106 & 1.8614 & -0.3502 \\
70.00 & 0.880 & 1.8347 & -0.3672 \\
75.00 & 0.658 & 1.7567 & -0.3830 \\
80.00 & 0.449 & 1.6334 & -0.3977 \\
85.00 & 0.267 & 1.4847 & -0.4112 \\
90.00 & 0.124 & 1.3879 & -0.4234 \\
95.00 & 0.002 & 1.3912 & -0.4343 \\
100.00 & -0.118 & 1.3795 & -0.4437 \\
105.00 & -0.235 & 1.3528 & -0.4514 \\
110.00 & -0.348 & 1.3114 & -0.4573 \\
115.00 & -0.453 & 1.2557 & -0.4610 \\
120.00 & -0.549 & 1.1864 & -0.4623 \\
125.00 & -0.633 & 1.1041 & -0.4606 \\
130.00 & -0.702 & 1.0102 & -0.4554 \\
135.00 & -0.754 & 0.9060 & -0.4462 \\
140.00 & -0.787 & 0.7935 & -0.4323 \\
145.00 & -0.797 & 0.6750 & -0.4127 \\
150.00 & -0.782 & 0.5532 & -0.3863 \\
155.00 & -0.739 & 0.4318 & -0.3521 \\
160.00 & -0.664 & 0.3147 & -0.3085 \\
170.00 & -0.410 & 0.1144 & -0.1858 \\
175.00 & -0.226 & 0.0702 & -0.1022 \\
180.00 & 0.000 & 0.0602 & 0.0000 \\
\hline
\end{tabular}

\section{B.6 Airfoil-Data Input File - DU35_A17.dat}

\begin{tabular}{|c|c|c|c|}
\hline \\
\hline \multicolumn{4}{|c|}{$\begin{array}{l}\text { DU35 airfoil with an aspect ratio of } 17 \text {. Original }-180 \text { to } 180 \mathrm{deg} \mathrm{Cl}, \mathrm{Cd} \text {, and } \mathrm{Cm} \text { versus AOA data taken from Appendix A of DOW } \\
\mathrm{Cl} \text { and } \mathrm{Cd} \text { values corrected for rotational stall delay and } \mathrm{Cd} \text { values corrected using the Viterna method for } \theta \text { to } 90 \mathrm{deg} \text { AOA by }\end{array}$} \\
\hline 1 & Numbe & of airfc & il tables in this file \\
\hline 0.0 & Table & ID parame & \\
\hline 11.50 & Stall & angle $(\mathrm{d} \epsilon$ & \\
\hline 0.0 & No lo & ger used & enter zero \\
\hline 0.0 & No lo & ger used & enter zero \\
\hline 0.0 & No 10 & ger used & enter zero \\
\hline-1.8330 & Zero & in angle & f attack (deg) \\
\hline 7.1838 & Cn sl & pe for ze & ro lift (dimensionless) \\
\hline 1.6717 & Cn ex & rapolated & to value at positive stall angle of attack \\
\hline-0.3075 & Cn at & stall va & ue for negative angle of attack \\
\hline 0.00 & Angle & of attacl & for minimum CD (deg) \\
\hline 0.0094 & Minim & Im CD valu & \\
\hline-180.00 & 0.000 & 0.0407 & 0.0000 \\
\hline-175.00 & 0.223 & 0.0507 & 0.0937 \\
\hline-170.00 & 0.405 & 0.1055 & 0.1702 \\
\hline-160.00 & 0.658 & 0.2982 & 0.2819 \\
\hline-155.00 & 0.733 & 0.4121 & 0.3213 \\
\hline-150.00 & 0.778 & 0.5308 & 0.3520 \\
\hline-145.00 & 0.795 & 0.6503 & 0.3754 \\
\hline-140.00 & 0.787 & 0.7672 & 0.3926 \\
\hline-135.00 & 0.757 & 0.8785 & 0.4046 \\
\hline-130.00 & 0.708 & 0.9819 & 0.4121 \\
\hline-125.00 & 0.641 & 1.0756 & 0.4160 \\
\hline-120.00 & 0.560 & 1.1580 & 0.4167 \\
\hline-115.00 & 0.467 & 1.2280 & 0.4146 \\
\hline-110.00 & 0.365 & 1.2847 & 0.4104 \\
\hline-105.00 & 0.255 & 1.3274 & 0.4041 \\
\hline-100.00 & 0.139 & 1.3557 & 0.3961 \\
\hline-95.00 & 0.021 & 1.3692 & 0.3867 \\
\hline-90.00 & -0.098 & 1.3680 & 0.3759 \\
\hline-85.00 & -0.216 & 1.3521 & 0.3639 \\
\hline-80.00 & -0.331 & 1.3218 & 0.3508 \\
\hline-75.00 & -0.441 & 1.2773 & 0.3367 \\
\hline-70.00 & -0.544 & 1.2193 & 0.3216 \\
\hline-65.00 & -0.638 & 1.1486 & 0.3054 \\
\hline-60.00 & -0.720 & 1.0660 & 0.2884 \\
\hline-55.00 & -0.788 & 0.9728 & 0.2703 \\
\hline-50.00 & -0.840 & 0.8705 & 0.2512 \\
\hline-45.00 & -0.875 & 0.7611 & 0.2311 \\
\hline-40.00 & -0.889 & 0.6466 & 0.2099 \\
\hline-35.00 & -0.880 & 0.5299 & 0.1876 \\
\hline
\end{tabular}




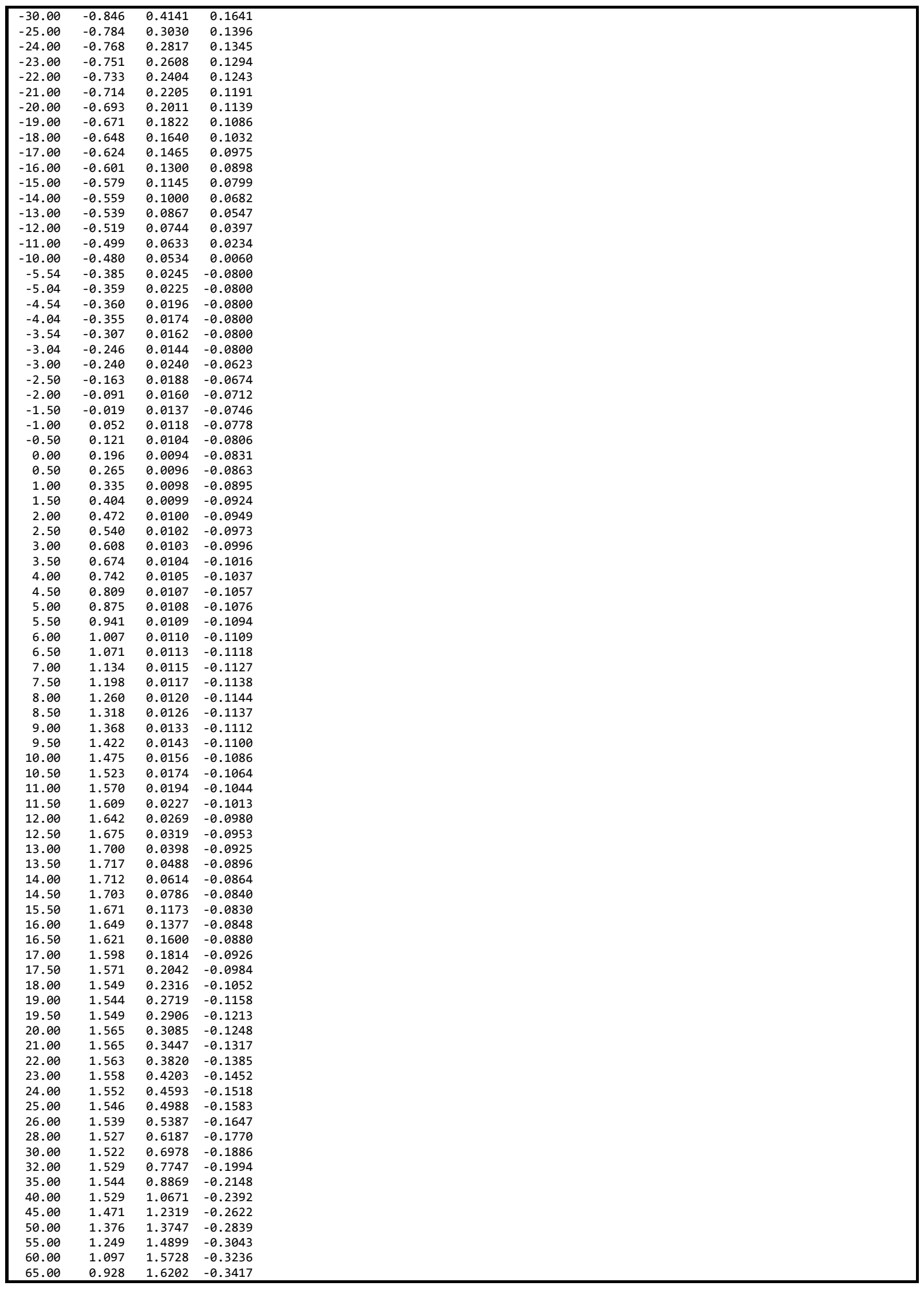




\begin{tabular}{|rrrr|}
\hline 70.00 & 0.750 & 1.6302 & -0.3586 \\
75.00 & 0.570 & 1.6031 & -0.3745 \\
80.00 & 0.396 & 1.5423 & -0.3892 \\
85.00 & 0.237 & 1.4598 & -0.4028 \\
90.00 & 0.101 & 1.4041 & -0.4151 \\
95.00 & -0.022 & 1.4053 & -0.4261 \\
100.00 & -0.143 & 1.3914 & -0.4357 \\
105.00 & -0.261 & 1.3625 & -0.4437 \\
11.00 & -0.374 & 1.3188 & -0.4498 \\
115.00 & -0.480 & 1.2608 & -0.4538 \\
120.00 & -0.575 & 1.1891 & -0.4553 \\
125.00 & -0.659 & 1.1046 & -0.4540 \\
130.00 & -0.727 & 1.0086 & -0.4492 \\
135.00 & -0.778 & 0.9025 & -0.4405 \\
140.00 & -0.809 & 0.7883 & -0.4270 \\
145.00 & -0.818 & 0.6684 & -0.4078 \\
150.00 & -0.800 & 0.5457 & -0.3821 \\
155.00 & -0.754 & 0.4236 & -0.3484 \\
160.00 & -0.677 & 0.3066 & -0.3054 \\
170.00 & -0.417 & 0.1085 & -0.1842 \\
175.00 & -0.229 & 0.0510 & -0.1013 \\
180.00 & 0.000 & 0.0407 & 0.0000 \\
\hline
\end{tabular}

\section{B.7 Airfoil-Data Input File - DU30_A17.dat}

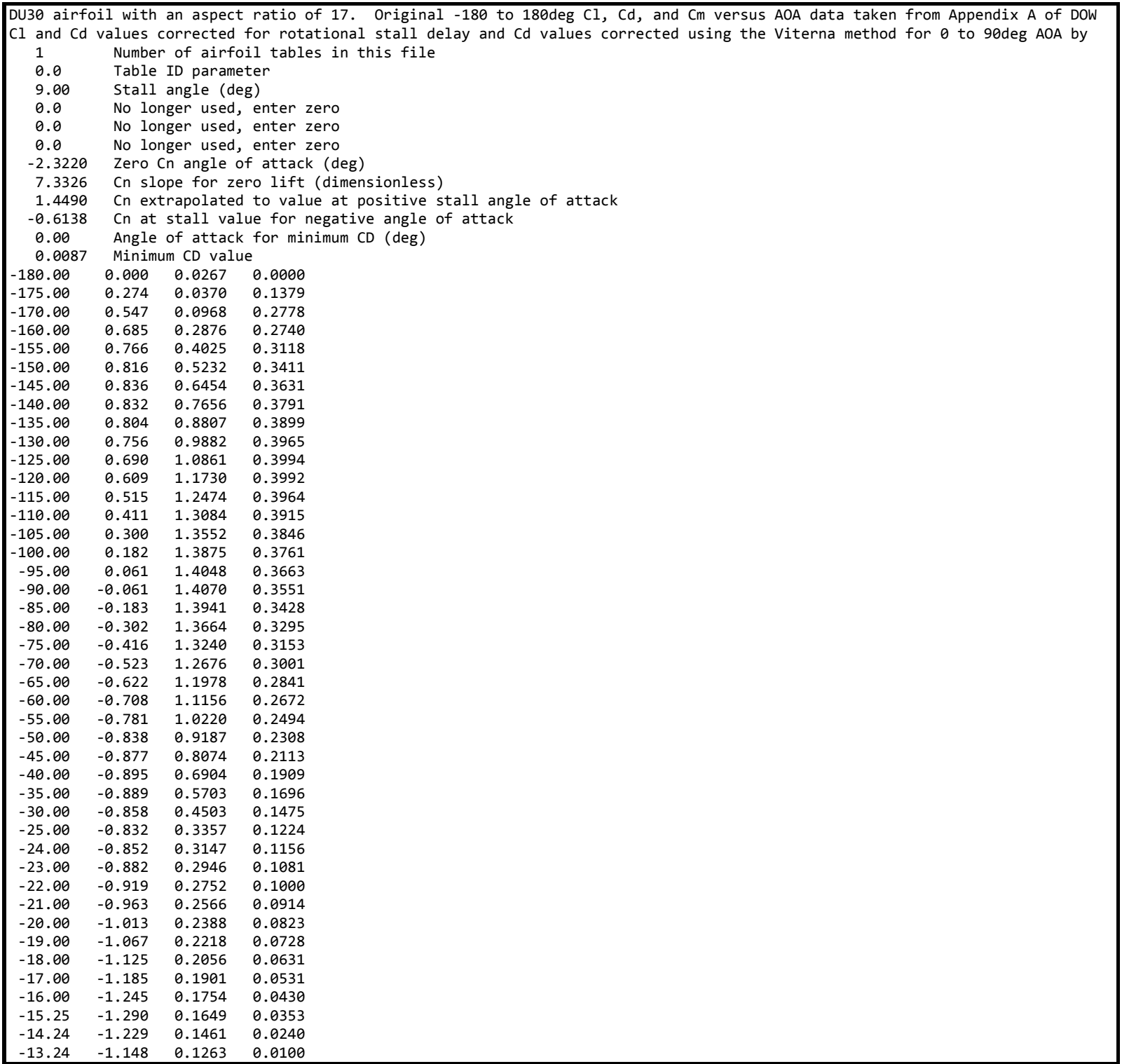




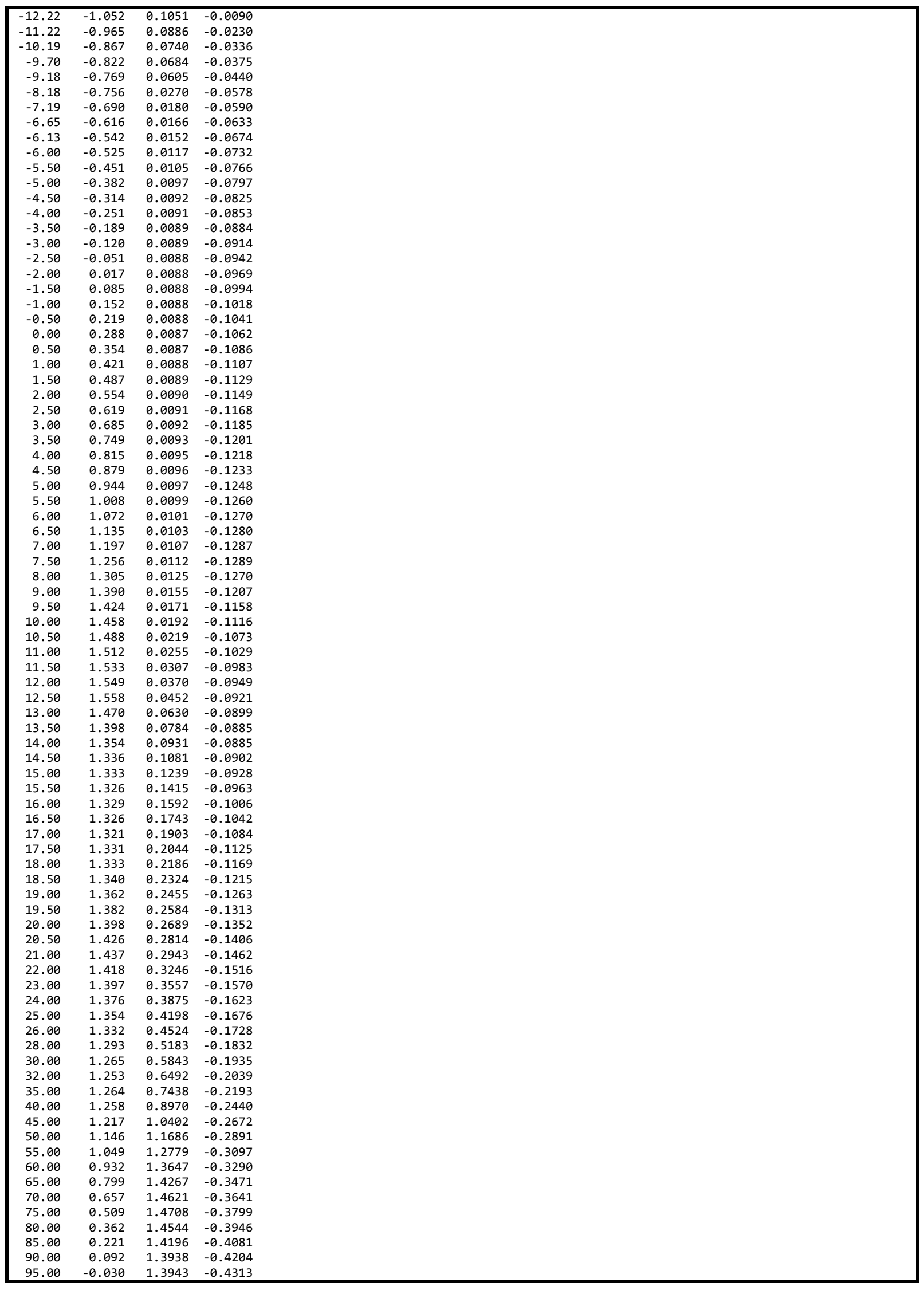




\begin{tabular}{|llll|}
\hline 100.00 & -0.150 & 1.3798 & -0.4408 \\
105.00 & -0.267 & 1.3504 & -0.4486 \\
110.00 & -0.379 & 1.3063 & -0.4546 \\
115.00 & -0.483 & 1.2481 & -0.4584 \\
120.00 & -0.578 & 1.1763 & -0.4597 \\
125.00 & -0.660 & 1.0919 & -0.4582 \\
130.00 & -0.727 & 0.9962 & -0.4532 \\
135.00 & -0.777 & 0.8906 & -0.4441 \\
140.00 & -0.807 & 0.7771 & -0.4303 \\
145.00 & -0.815 & 0.6581 & -0.4109 \\
150.00 & -0.797 & 0.5364 & -0.3848 \\
155.00 & -0.750 & 0.4157 & -0.3508 \\
160.00 & -0.673 & 0.3000 & -0.3074 \\
170.00 & -0.547 & 0.1051 & -0.2786 \\
175.00 & -0.274 & 0.0388 & -0.1380 \\
180.00 & 0.000 & 0.0267 & 0.0000
\end{tabular}

\section{B.8 Airfoil-Data Input File - DU25_A17.dat}

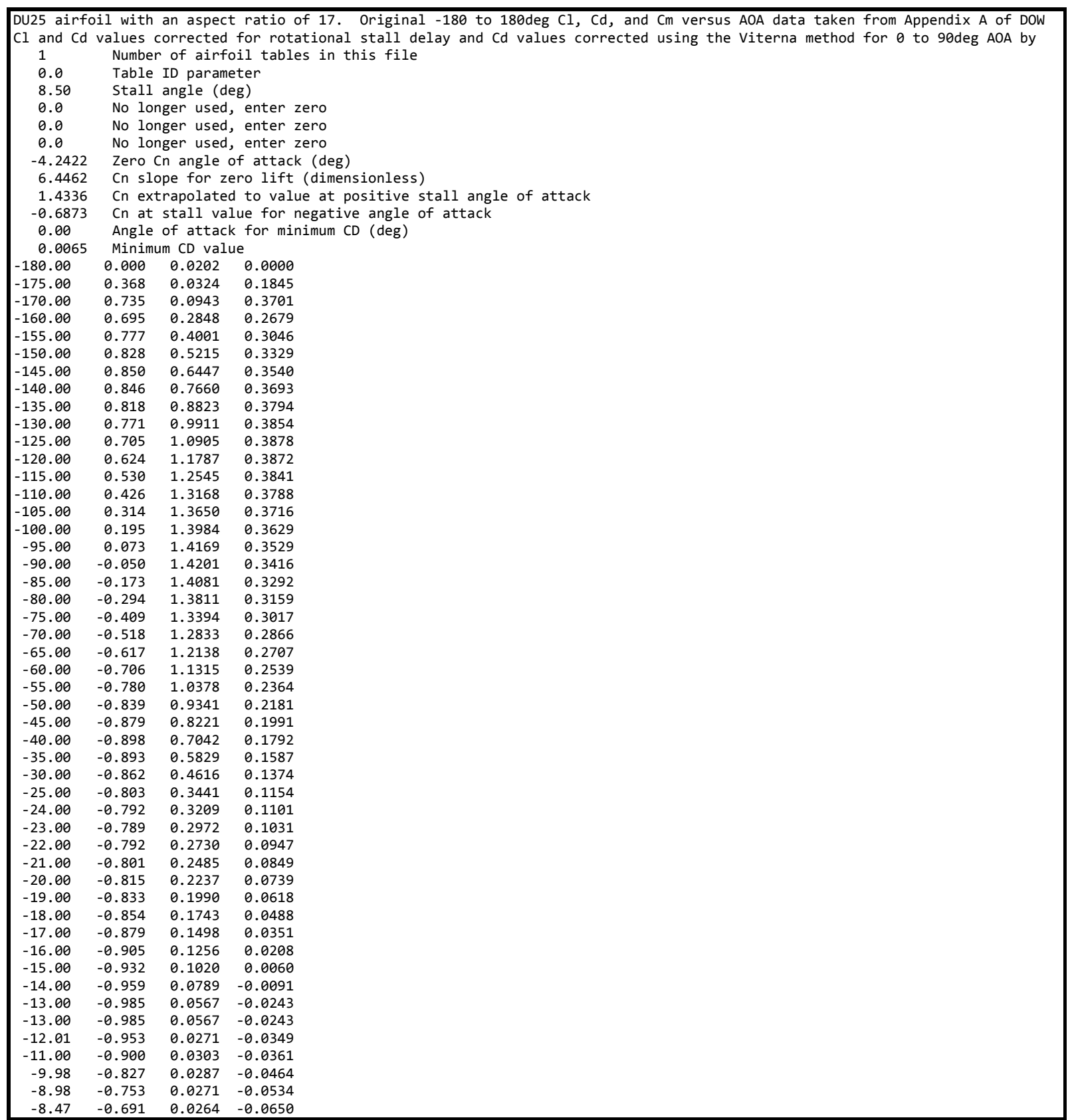




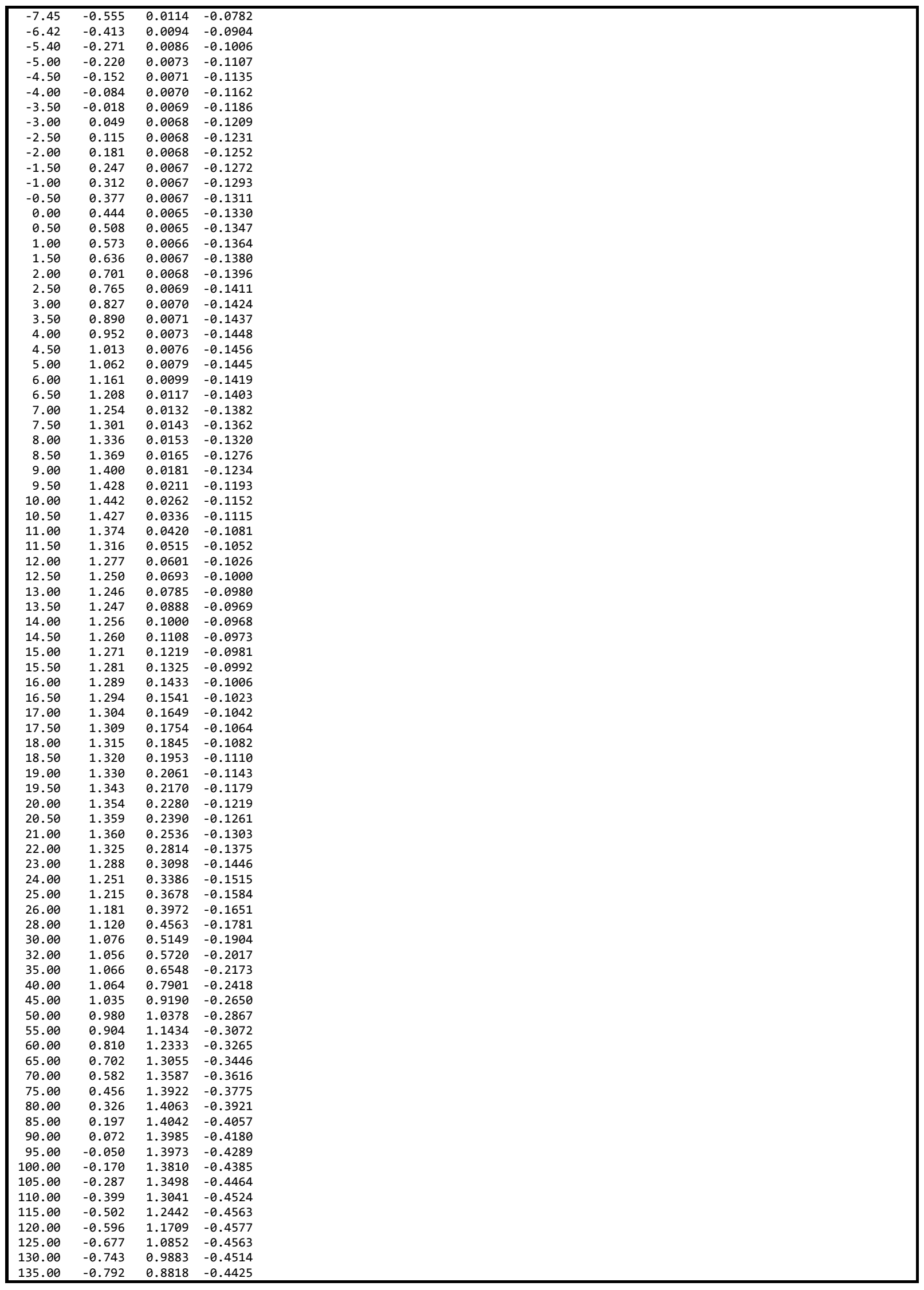




\begin{tabular}{|rrrr|}
\hline 140.00 & -0.821 & 0.7676 & -0.4288 \\
145.00 & -0.826 & 0.6481 & -0.4095 \\
150.00 & -0.806 & 0.5264 & -0.3836 \\
155.00 & -0.758 & 0.4060 & -0.3497 \\
160.00 & -0.679 & 0.2912 & -0.3065 \\
170.00 & -0.735 & 0.0995 & -0.3706 \\
175.00 & -0.368 & 0.0356 & -0.1846 \\
180.00 & 0.000 & 0.0202 & 0.0000 \\
\hline
\end{tabular}

\section{B.9 Airfoil-Data Input File - DU21_A17.dat}

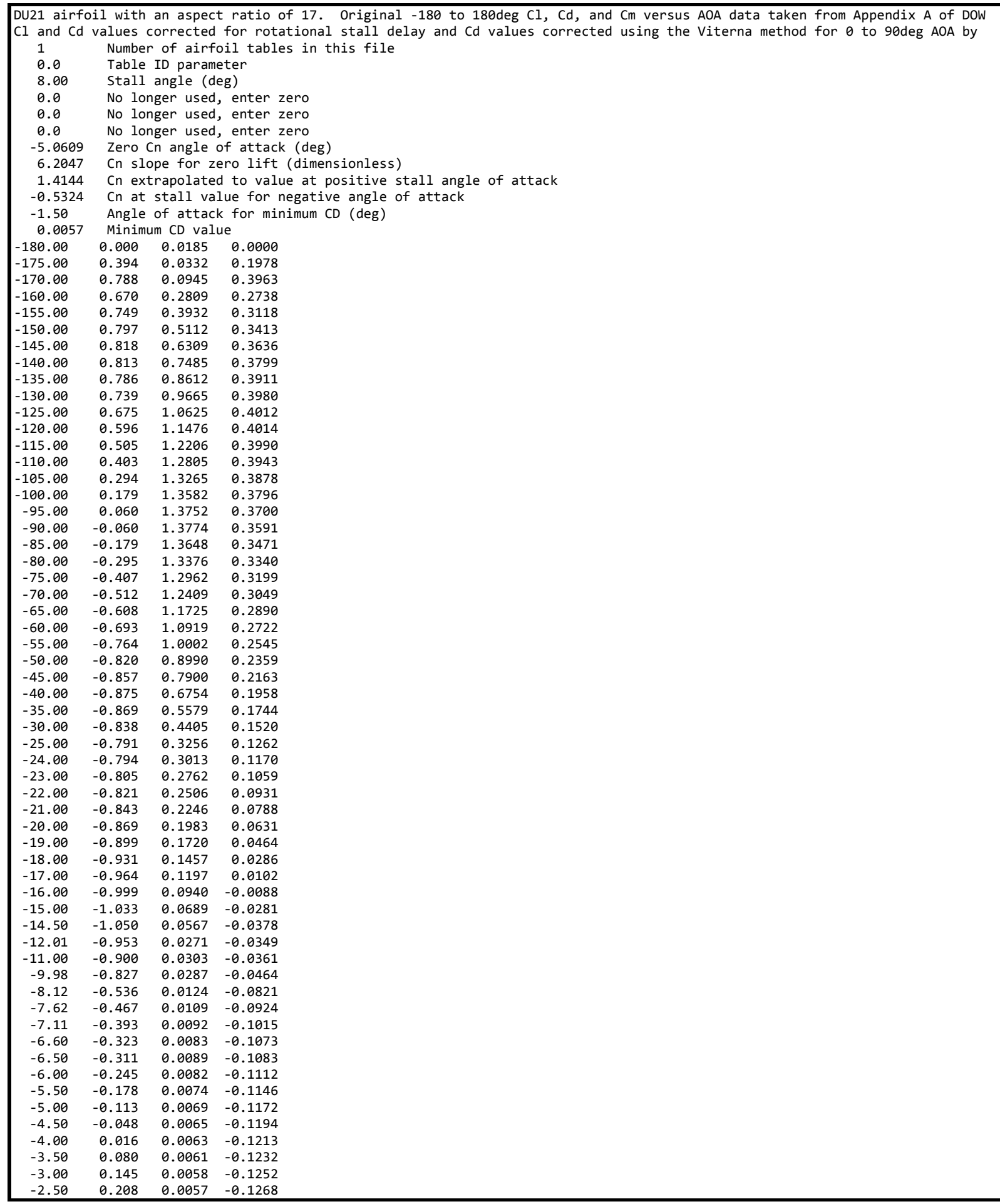




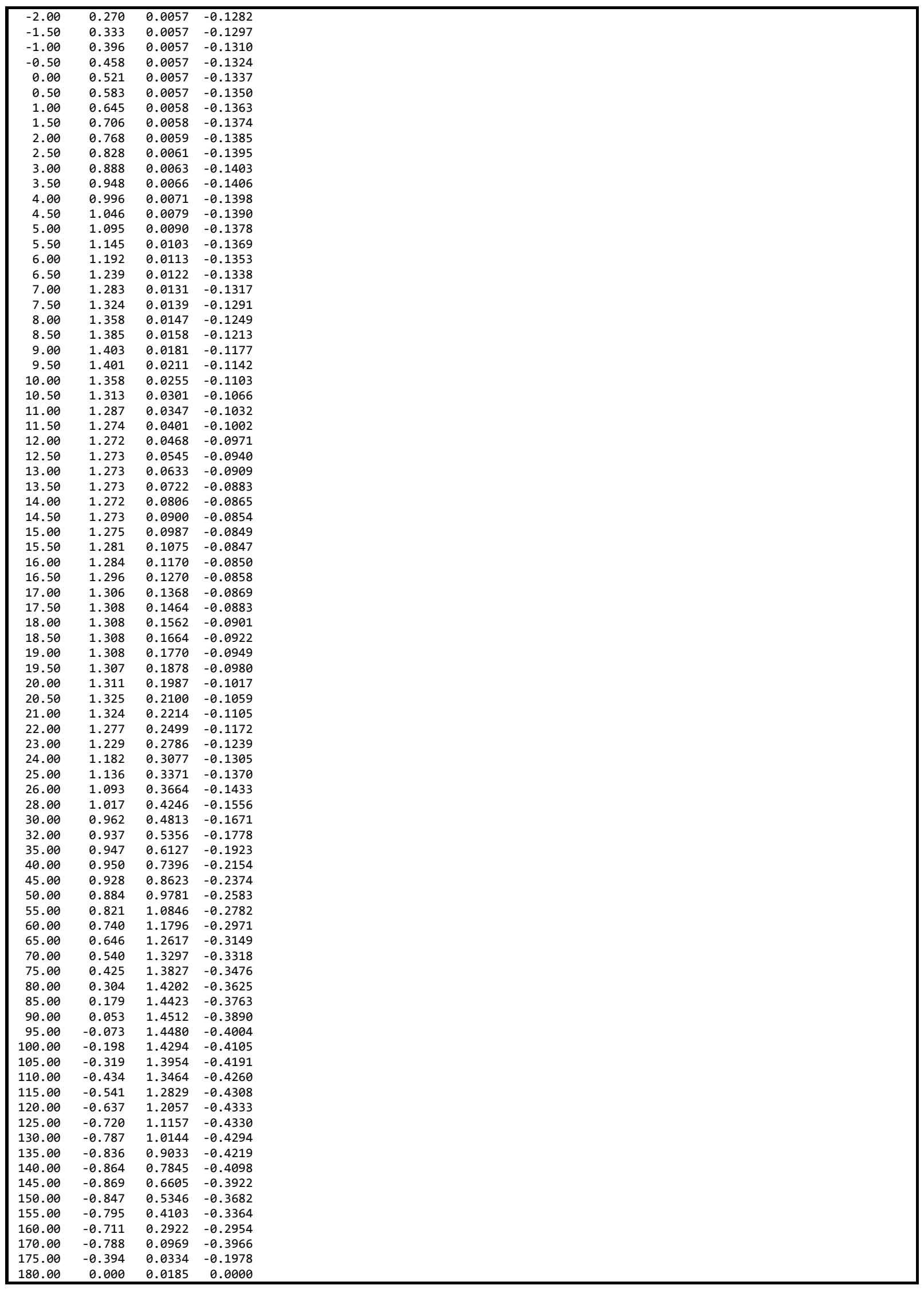




\section{B.10 Airfoil-Data Input File - NACA64_A17.dat}

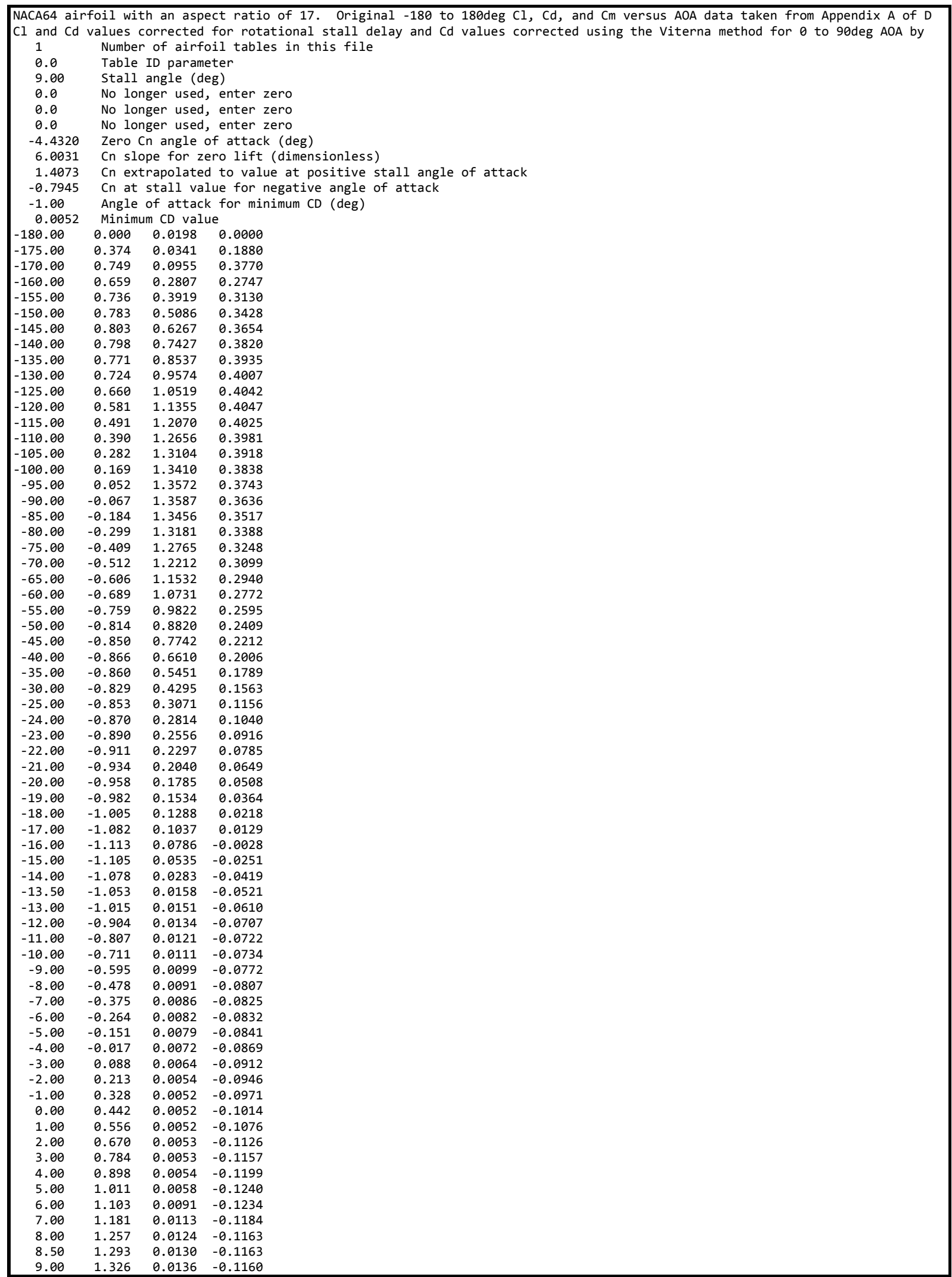




\begin{tabular}{|c|c|c|c|}
\hline 9.50 & 1.356 & 0.0143 & -0.1154 \\
\hline 10.00 & 1.382 & 0.0150 & -0.1149 \\
\hline 10.50 & 1.400 & 0.0267 & -0.1145 \\
\hline 11.00 & 1.415 & 0.0383 & -0.1143 \\
\hline 11.50 & 1.425 & 0.0498 & -0.1147 \\
\hline 12.00 & 1.434 & 0.0613 & -0.1158 \\
\hline 12.50 & 1.443 & 0.0727 & -0.1165 \\
\hline 13.00 & 1.451 & 0.0841 & -0.1153 \\
\hline 13.50 & 1.453 & 0.0954 & -0.1131 \\
\hline 14.00 & 1.448 & 0.1065 & -0.1112 \\
\hline 14.50 & 1.444 & 0.1176 & -0.1101 \\
\hline 15.00 & 1.445 & 0.1287 & -0.1103 \\
\hline 15.50 & 1.447 & 0.1398 & -0.1109 \\
\hline 16.00 & 1.448 & 0.1509 & -0.1114 \\
\hline 16.50 & 1.444 & 0.1619 & -0.1111 \\
\hline 17.00 & 1.438 & 0.1728 & -0.1097 \\
\hline 17.50 & 1.439 & 0.1837 & -0.1079 \\
\hline 18.00 & 1.448 & 0.1947 & -0.1080 \\
\hline 18.50 & 1.452 & 0.2057 & -0.1090 \\
\hline 19.00 & 1.448 & 0.2165 & -0.1086 \\
\hline 19.50 & 1.438 & 0.2272 & -0.1077 \\
\hline 20.00 & 1.428 & 0.2379 & -0.1099 \\
\hline 21.00 & 1.401 & 0.2590 & -0.1169 \\
\hline 22.00 & 1.359 & 0.2799 & -0.1190 \\
\hline 23.00 & 1.300 & 0.3004 & -0.1235 \\
\hline 24.00 & 1.220 & 0.3204 & -0.1393 \\
\hline 25.00 & 1.168 & 0.3377 & -0.1440 \\
\hline 26.00 & 1.116 & 0.3554 & -0.1486 \\
\hline 28.00 & 1.015 & 0.3916 & -0.1577 \\
\hline 30.00 & 0.926 & 0.4294 & -0.1668 \\
\hline 32.00 & 0.855 & 0.4690 & -0.1759 \\
\hline 35.00 & 0.800 & 0.5324 & -0.1897 \\
\hline 40.00 & 0.804 & 0.6452 & -0.2126 \\
\hline 45.00 & 0.793 & 0.7573 & -0.2344 \\
\hline 50.00 & 0.763 & 0.8664 & -0.2553 \\
\hline 55.00 & 0.717 & 0.9708 & -0.2751 \\
\hline 60.00 & 0.656 & 1.0693 & -0.2939 \\
\hline 65.00 & 0.582 & 1.1606 & -0.3117 \\
\hline 70.00 & 0.495 & 1.2438 & -0.3285 \\
\hline 75.00 & 0.398 & 1.3178 & -0.3444 \\
\hline 80.00 & 0.291 & 1.3809 & -0.3593 \\
\hline 85.00 & 0.176 & 1.4304 & -0.3731 \\
\hline 90.00 & 0.053 & 1.4565 & -0.3858 \\
\hline 95.00 & -0.074 & 1.4533 & -0.3973 \\
\hline 100.00 & -0.199 & 1.4345 & -0.4075 \\
\hline 105.00 & -0.321 & 1.4004 & -0.4162 \\
\hline 110.00 & -0.436 & 1.3512 & -0.4231 \\
\hline 115.00 & -0.543 & 1.2874 & -0.4280 \\
\hline 120.00 & -0.640 & 1.2099 & -0.4306 \\
\hline 125.00 & -0.723 & 1.1196 & -0.4304 \\
\hline 130.00 & -0.790 & 1.0179 & -0.4270 \\
\hline 135.00 & -0.840 & 0.9064 & -0.4196 \\
\hline 140.00 & -0.868 & 0.7871 & -0.4077 \\
\hline 145.00 & -0.872 & 0.6627 & -0.3903 \\
\hline 150.00 & -0.850 & 0.5363 & -0.3665 \\
\hline 155.00 & -0.798 & 0.4116 & -0.3349 \\
\hline 160.00 & -0.714 & 0.2931 & -0.2942 \\
\hline 170.00 & -0.749 & 0.0971 & -0.3771 \\
\hline 175.00 & -0.374 & 0.0334 & -0.1879 \\
\hline 180.00 & 0.000 & 0.0198 & 0.0000 \\
\hline
\end{tabular}




\title{
Appendix C Source Code for the Baseline Turbine Control System DLL
}

\author{
SUBROUTINE DISCON ( avRSWAP, avIFAIL, ACCINFILE, aVCOUTNAME, avCMSG) \\ !DEC\$ ATTRIBUTES DLLEXPORT, ALIAS: 'DISCON' : : DISCON \\ ! This Bladed-style DLL controller is used to implement a variable-speed \\ ! generator-torque controller and PI collective blade pitch controller for \\ ! the NREL Offshore 5MW baseline wind turbine. This routine was written by \\ ! J. Jonkman of NREL/NWTC for use in the IEA Annex XXIII OC3 studies.
}

IMPLICIT

NONE

! Passed Variables:

\begin{tabular}{|c|c|c|c|}
\hline $\operatorname{REAL}(4)$, & INTENT ( INOUT) & $:$ : avrSWAP & ! The swap array, used to pass data to, and $r$ \\
\hline $\operatorname{INTEGER}(4)$, & INTENT( OUT $)$ & : : aviFAIL & ! A flag used to indicate the success of this \\
\hline $\operatorname{INTEGER}(1)$, & INTENT (IN ) & $:: \operatorname{accINFILE}(*)$ & ! The address of the first record of an array \\
\hline $\operatorname{INTEGER}(1)$, & INTENT( OUT) & $:: \operatorname{avcMSG}(*)$ & ! The address of the first record of an array \\
\hline $\operatorname{INTEGER}(1)$, & INTENT (IN ) & $::$ avcOUTNAME $(*)$ & ! The address of the first record of an array \\
\hline
\end{tabular}

! Local Variables:

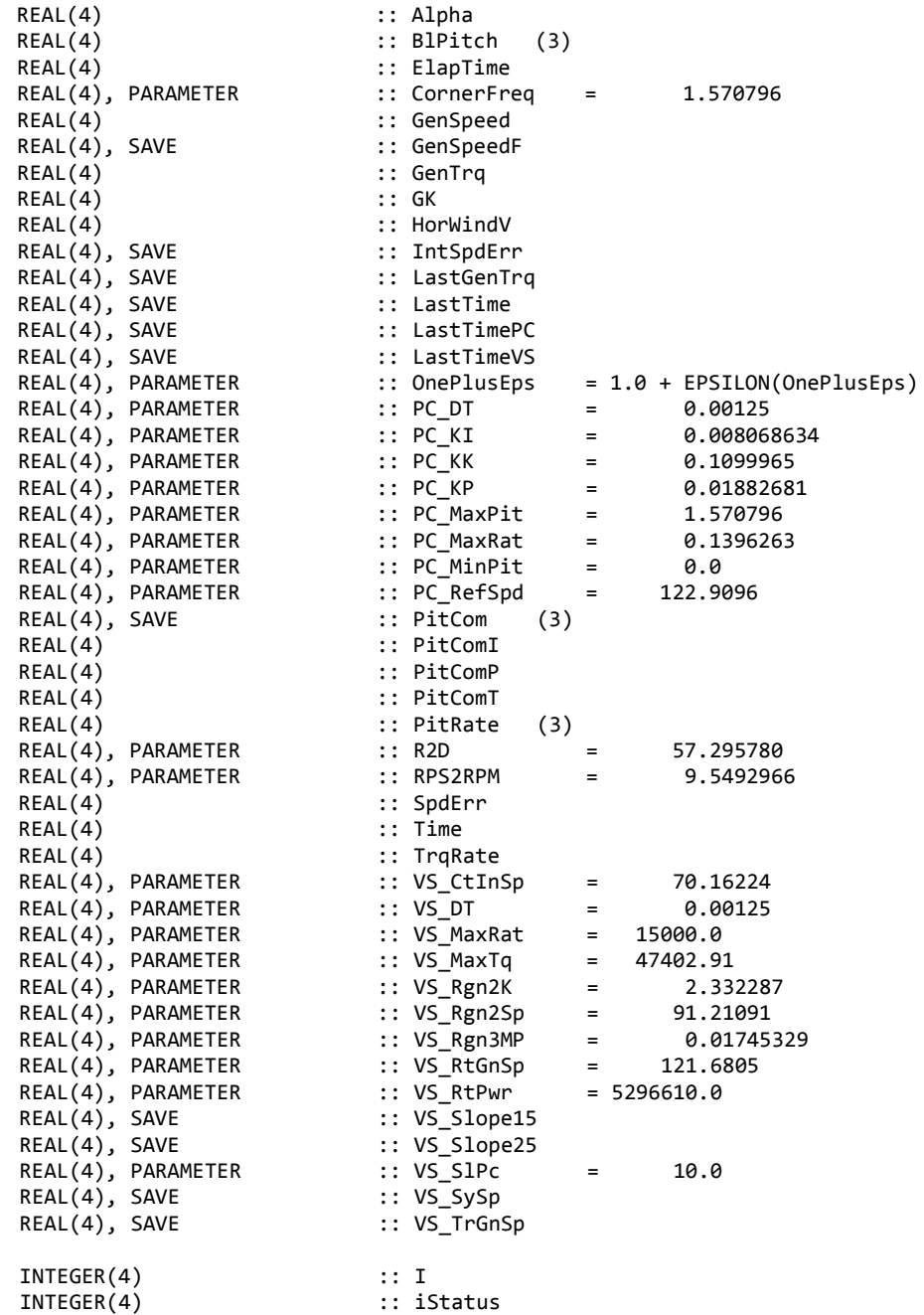

! Current coefficient in the recursive, singl Current values of the blade pitch angles, $r$ ! Elapsed time since the last call to the con ! Corner frequency (-3dB point) in the recurs ! Current HSS (generator) speed, rad/s.

( Filtered HSS (generator) speed, $\mathrm{rad} / \mathrm{s}$.

! Electrical generator torque, $\mathrm{N}-\mathrm{m}$.

! Current value of the gain correction factor

! Horizontal hub-heigh wind speed, $\mathrm{m} / \mathrm{s}$.

! Current integral of speed error w.r.t. time

! Commanded electrical generator torque the 1

! Last time this DLL was called, sec.

! Last time the pitch controller was called,

! Last time the torque controller was called,

! The number slighty greater than unity in si

! Communication interval for pitch controlle

! Integral gain for pitch controller at rated

! Pitch angle were the the derivative of the

! Proportional gain for pitch controller at $r$

! Maximum pitch setting in pitch controller,

! Maximum pitch rate (in absolute value) in

! Minimum pitch setting in pitch controller,

! Desired (reference) HSS speed for pitch con

! Commanded pitch of each blade the last time

! Integral term of command pitch, rad.

! Proportional term of command pitch, rad.

! Total command pitch based on the sum of the

! Pitch rates of each blade based on the curr

! Factor to convert radians to degrees.

! Factor to convert radians per second to rev

! Current speed error, rad/s.

! Current simulation time, sec.

! Torque rate based on the current and last $t$

Transitional generator speed (HSS side) bet

! Communication interval for torque controlle

! Maximum torque rate (in absolute value) in

! Maximum generator torque in Region 3 (HSS $\mathrm{s}$

! Generator torque constant in Region 2 (HSS

Transitional generator speed (HSS side) bet

! Minimum pitch angle at which the torque is

! Rated generator speed (HSS side), rad/s. --

! Rated generator generator power in Region 3

! Torque/speed slope of region $11 / 2$ cut-in $t$

! Torque/speed slope of region $21 / 2$ inductio

! Rated generator slip percentage in Region 2

! Synchronous speed of region 2 1/2 induction

! Transitional generator speed (HSS side) bet

! Generic index.

! A status flag set by the simulation as foll 


\begin{tabular}{|c|c|c|c|}
\hline $\operatorname{INTEGER}(4)$ & $:: \mathrm{K}$ & & ! Loops through blades. \\
\hline $\operatorname{INTEGER(4)}$ & $::$ NumBl & & ! Number of blades, (-). \\
\hline INTEGER(4), PARAMETER & $::$ UnDb & $=85$ & ! I/0 unit for the debugging information \\
\hline $\operatorname{INTEGER(1)}$ & :: iInFile & $(256)$ & ! CHARACTER string cInFile stored as a 1 -byt \\
\hline $\operatorname{INTEGER(1)}$ & :: iMessage & $(256)$ & ! CHARACTER string cMessage stored as a 1 -byt \\
\hline $\operatorname{INTEGER}(1)$, SAVE & $::$ iOutName & $(1024)$ & ! CHARACTER string cOutName stored as a 1-byt \\
\hline LOGICAL (1), PARAMETER & $::$ PC_DbgOut & $=. \mathrm{FALSE}$. & ! Flag to indicate whether to output debuggin \\
\hline CHARACTER( 256$)$ & :: cInFile & & ! CHARACTER string giving the name of the pa \\
\hline CHARACTER( 256$)$ & $::$ cMessage & & ! CHARACTER string giving a message that will \\
\hline CHARACTER(1024), SAVE & $::$ cOutName & & ! CHARACTER string giving the simulation run \\
\hline 1), PARAMETER & : : Tab & $=\operatorname{CHAR}(9)$ & ! The tab character. \\
\hline CHARACTER( 25$)$, PARAMETER & : : FmtDat & $\left(\mathrm{F} 8.3,99\left({ }^{\prime} " / / \mathrm{Tab} / / \mathrm{I}^{\prime}, \mathrm{E}\right.\right.$ & ! The format of the debugging data \\
\hline
\end{tabular}

! Set EQUIVALENCE relationships between INTEGER(1) byte arrays and CHARACTER strings:

EQUIVALENCE (iInFile, cInFile )

EQUIVALENCE (iMessage, cMessage)

EQUIVALENCE (iOutName, cOutName)

! Load variables from calling program (See Appendix A of Bladed User's Guide):

$\begin{array}{lll}\text { istatus } & =\operatorname{NINT}(\operatorname{avrSWAP}(1)) \\ \text { NumBl } & =\operatorname{NINT}(\operatorname{avrSWAP}(61)) \\ & & \\ \text { BlPitch }(1) & = & \operatorname{avrSWAP}(4) \\ \text { BlPitch }(2) & = & \operatorname{avrSWAP}(33) \\ \text { BlPitch (3) } & = & \operatorname{avrSWAP}(34) \\ \text { GenSpeed } & = & \operatorname{avrSWAP}(20) \\ \text { HorWindV } & = & \operatorname{avrSWAP}(27) \\ \text { Time } & = & \operatorname{avrSWAP}(2)\end{array}$

! Initialize avifAIL to 0 :

aviFAIL $\quad=0$

! Read any External Controller Parameters specified in the User Interface

! and initialize variables:

IF ( iStatus $=0$ ) THEN ! .TRUE. if were on the first call to the DLL

! Convert byte arrays to CHARACTER strings, for convenience:

DO I $=1, \operatorname{MIN}(256, \operatorname{NINT}(\operatorname{avrSWAP}(50)))$

infile $(\mathrm{I})=\operatorname{accINFILE}(\mathrm{I}) \quad$ ! Sets cInfile by EQUIVALENCE

ENDDO

DO I $=1, \operatorname{MIN}(1024, \operatorname{NINT}(\operatorname{avrSWAP}(51)))$

iOutName $(\mathrm{I})=\operatorname{avcOUTNAME}(\mathrm{I})$ ! Sets coutName by EQUIVALENCE ENDDO

! Inform users that we are using this user-defined routine:

aviFAIL $=1$

cMessage $=$ 'Running with torque and pitch control of the NREL offshore $1 / /$ \&

'5MW baseline wind turbine from DISCON.dll as written by $\mathrm{J} .1 / / \&$

'Jonkman of NREL/NWTC for use in the IEA Annex XXIII OC3 ' // \&

'studies.

! Determine some torque control parameters not specified directly:

VS_SySp $=$ VS_RtGnSp $/\left(1.0+0.01 * V S \_S I P C\right)$

VS_Slope15 $=$ ( VS_Rgn2K*VS_Rgn2Sp*VS_Rgn2Sp $) /($ VS_Rgn2Sp - VS_CtInSp $)$

VS Slope25 = (VSRtPwr/VS RtGnSp )/( VS RtGnSp - VS SySp)

IF ( VS Rgn2K $==\overline{0} .0$ ) THEN ! . TRUE. if the Region 2 torque is flat, and thus, the denominator in the ELSE condition is VS_TrGnSp = VS_SySp

ELSE

! . TRUE. if the Region 2 torque is quadratic with speed
SQRT(VS Slope25*(VS Slope25 - 4.0*VS Rgn2K*VS SySp ) ) $) /(2.0 *$ VS Rgn2K $)$

ENDIF

! Check validity of input parameters: 


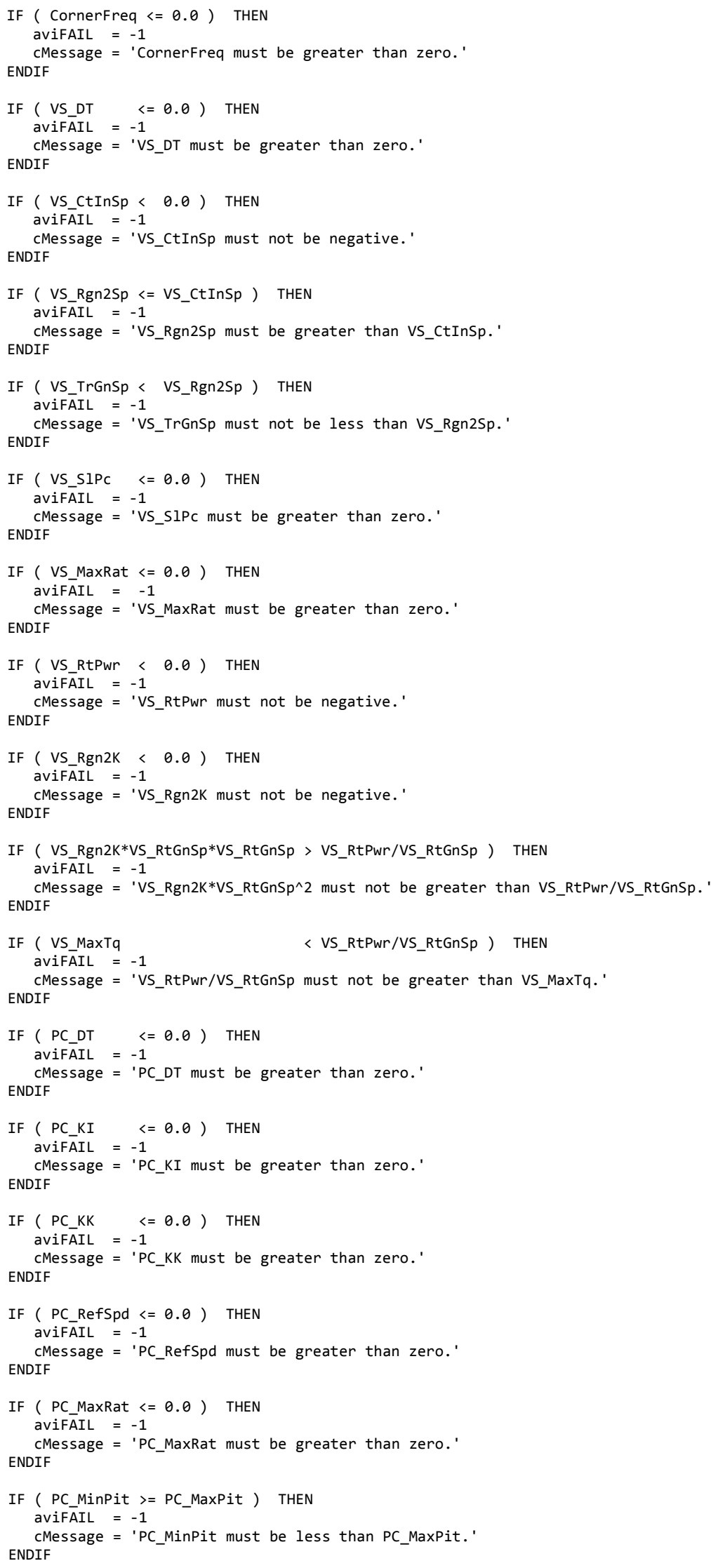


! If we're debugging the pitch controller, open the debug file and write the

! header:

IF ( PC_DbgOut ) THEN

OPEN ( UnDb, FILE=TRIM( cOutName $) / /^{\prime}$. dbg' , STATUS='REPLACE' )

WRITE (UnDb, ' $\left.(/ / / / /)^{\prime}\right)$

WRITE (UnDb,' (A)') 'Time '//Tab//'ElapTime'//Tab//'HorWindV'//Tab//'GenSpeed'//Tab//'GenSpeedF'//Tab//'RelSpdErr'//Tab 'SpdErr '//Tab//'IntSpdErr'//Tab//'GK '//Tab//'PitComP'//Tab//'PitComI'//Tab//'PitComT'//Tab// 'PitRate1'//Tab//'PitCom1'

WRITE (UnDb, '(A)') '(sec)'//Tab//'(sec) '//Tab//'(m/sec) '//Tab//'(rpm) '//Tab//'(rpm) '//Tab//'(\%) '//Tab '(rad/s)'//Tab//'(rad) '//Tab//'(-)'//Tab//'(deg) '//Tab//'(deg) '//Tab//'(deg) '//Tab//

ENDIF

! Initialize the SAVEd variables:

! NOTE: LastGenTrq, though SAVEd, is initialized in the torque controller

below for simplicity, not here.

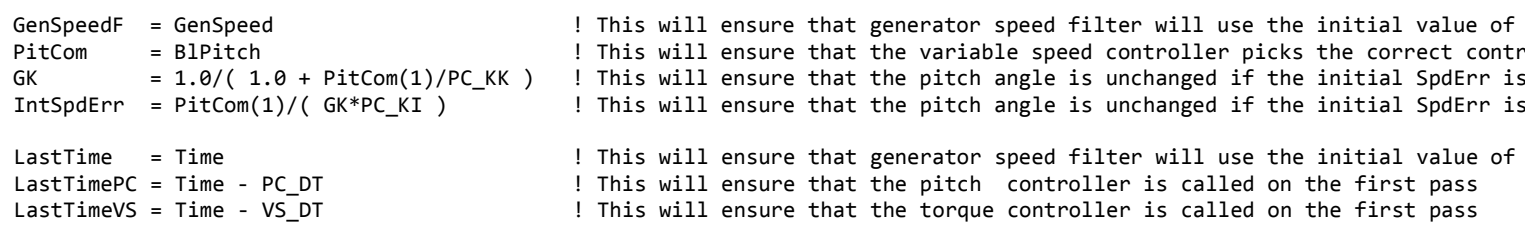

ENDIF

! Main control calculations:

IF $(($ iStatus $>=0)$.AND. ( avifAIL $>=0)$ ) THEN ! Only compute control calculations if no error has occured and we are

! Abort if the user has not requested a pitch angle actuator (See Appendix A

of Bladed User's Guide):

IF $(\operatorname{NINT}(\operatorname{avrSWAP}(10)) /=0)$ THEN ! .TRUE. if a pitch angle actuator hasn't been requested

aviFAIL $=-1$

cMessage $=$ 'Pitch angle actuator not requested.'

ENDIF

! Set unused outputs to zero (See Appendix A of Bladed User's Guide):

$\operatorname{avrSWAP}(36)=0.0$ ! Shaft brake status: $\theta=0 \mathrm{ff}$

$\operatorname{avrSWAP}(41)=0.0$ ! Demanded yaw actuator torque

$\operatorname{avrSWAP}(46)=0.0$ ! Demanded pitch rate (Collective pitch)

$\operatorname{avrSWAP}(48)=0.0$ ! Demanded nacelle yaw rate

$\operatorname{avrSWAP}(65)=0.0$ ! Number of variables returned for logging

$\operatorname{avrSWAP}(72)=0.0$ ! Generator startup resistance

$\operatorname{avrSWAP}(79)=0.0$ ! Request for loads: $\theta=$ none

$\operatorname{avrSWAP}(80)=0.0$ ! Variable slip current status

$\operatorname{avrSWAP}(81)=0.0$ ! Variable slip current demand

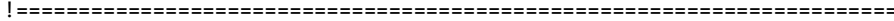

! Filter the HSS (generator) speed measurement:

! NOTE: This is a very simple recursive, single-pole, low-pass filter with

! exponential smoothing.

! Update the coefficient in the recursive formula based on the elapsed time

! since the last call to the controller:

Alpha $=\operatorname{EXP}($ ( LastTime - Time $) *$ CornerFreq $)$

! Apply the filter:

GenSpeedF $=(1.0-$ Alpha $) *$ GenSpeed + Alpha*GenSpeedF 
! Variable-speed torque control:

! Compute the elapsed time since the last call to the controller:

ElapTime $=$ Time - LastTimeVS

! Only perform the control calculations if the elapsed time is greater than

! or equal to the communication interval of the torque controller:

! NOTE: Time is scaled by OnePlusEps to ensure that the contoller is called

! at every time step when VS_DT $=$ DT, even in the presence of numerical precision errors.

IF ( ( Time*OnePlusEps - LastTimeVS ) >= VS_DT ) THEN

! Compute the generator torque, which depends on which region we are in:

IF ( ( GenSpeedF >=VS_RtGnSp ) .OR. ( PitCom(1) >=VS_Rgn3MP ) ) THEN ! We are in region 3 - power is constant GenTrq = VS RtPwr/GenSpeed

ELSEIF ( GenSpeedF <= VS_CtInSp ) THEN GenTrq $=0.0$

ELSEIF ( GenSpeedF < VS_Rgn2Sp ) THEN GenTrq = VS Slope15*(GenSpeedF - VS CtInSp )

ELSEIF ( GenSpeedF < VS_TrGnSp ) THEN GenTrq = VS_Rgn2K*GenSpeedF*GenSpeedF ELSE GenTrq = VS_Slope25*( GenSpeedF - VS_SySp )

! We are in region 1 - torque is zero

! We are in region $11 / 2$ - linear ramp in to

! We are in region 2 - optimal torque is pro

! We are in region $21 / 2$ - simple induction ENDIF

! Saturate the commanded torque using the maximum torque limit:

GenTrq = MIN(GenTrq , VS_MaxTq ) ! Saturate the command using the maximum torque limit

! Saturate the commanded torque using the torque rate limit:

$\begin{array}{ll}\text { IF }(\text { iStatus }==0) \text { LastGenTrq }=\text { GenTrq } & \text { ! Initialize the value of LastGenTrq on the first pass only } \\ \text { TrqRate }=(\text { GenTrq }- \text { LastGenTrq }) / \text { ElapTime } & \text { ! Torque rate (unsaturated) } \\ \text { TrqRate }=\text { MIN }(\text { MAX }(\text { TrqRate, -VS_MaxRat ), VS_MaxRat ) } & \text { ! Saturate the torque rate using its maximum absolute value } \\ \text { GenTrq = LastGenTrq + TrqRate*ElapTime } & \text { ! Saturate the command using the torque rate limit }\end{array}$

! Reset the values of LastTimeVS and LastGenTrq to the current values:

LastTimeVS $=$ Time

LastGenTrq $=$ GenTrq

ENDIF

! Set the generator contactor status, avrSWAP(35), to main (high speed)

! variable-speed generator, the torque override to yes, and command the

! generator torque (See Appendix A of Bladed User's Guide):

$\operatorname{avrSWAP}(35)=1.0 \quad$ ! Generator contactor status: 1=main (high speed) variable-speed generator

$\operatorname{avrSWAP}(56)=0.0 \quad$ ! Torque override: $\theta=$ yes

$\operatorname{avrSWAP}(47)=$ LastGenTrq $!$ Demanded generator torque

! Pitch control:

! Compute the elapsed time since the last call to the controller:

ElapTime $=$ Time - LastTimePC

! Only perform the control calculations if the elapsed time is greater than

! or equal to the communication interval of the pitch controller:

! NOTE: Time is scaled by OnePlusEps to ensure that the contoller is called

! at every time step when PC_DT $=$ DT, even in the presence of

! numerical precision errors.

IF ( ( Time*OnePlusEps - LastTimePC ) >= PC_DT ) THEN

! Compute the gain scheduling correction factor based on the previously

! commanded pitch angle for blade 1: 


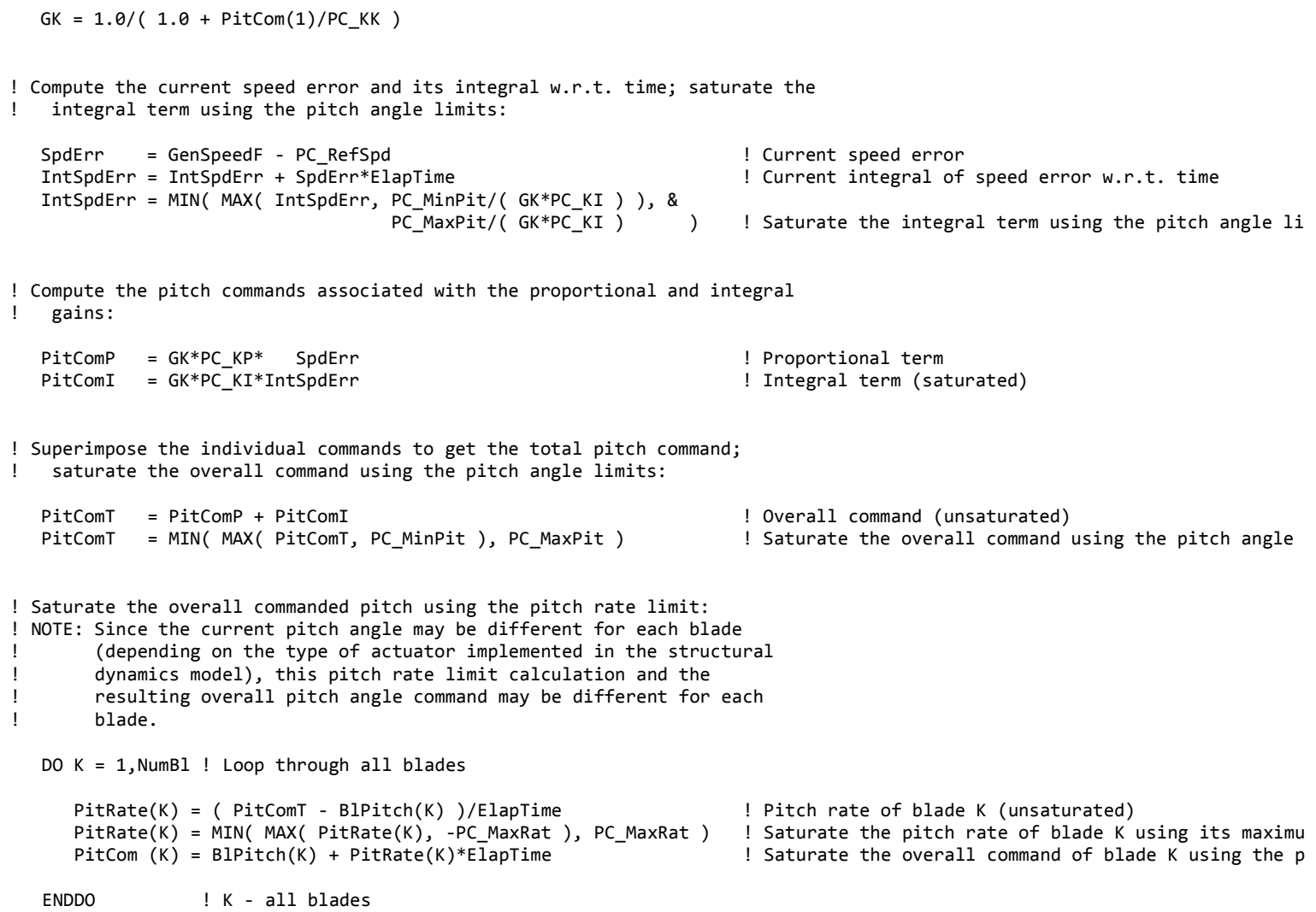


RETUR

END SUBROUTINE DISCON

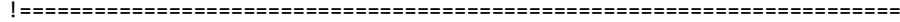




\section{Appendix D Input Files for the ITI Energy Barge}

\section{D.1 FAST Platform / HydroDyn Input File}

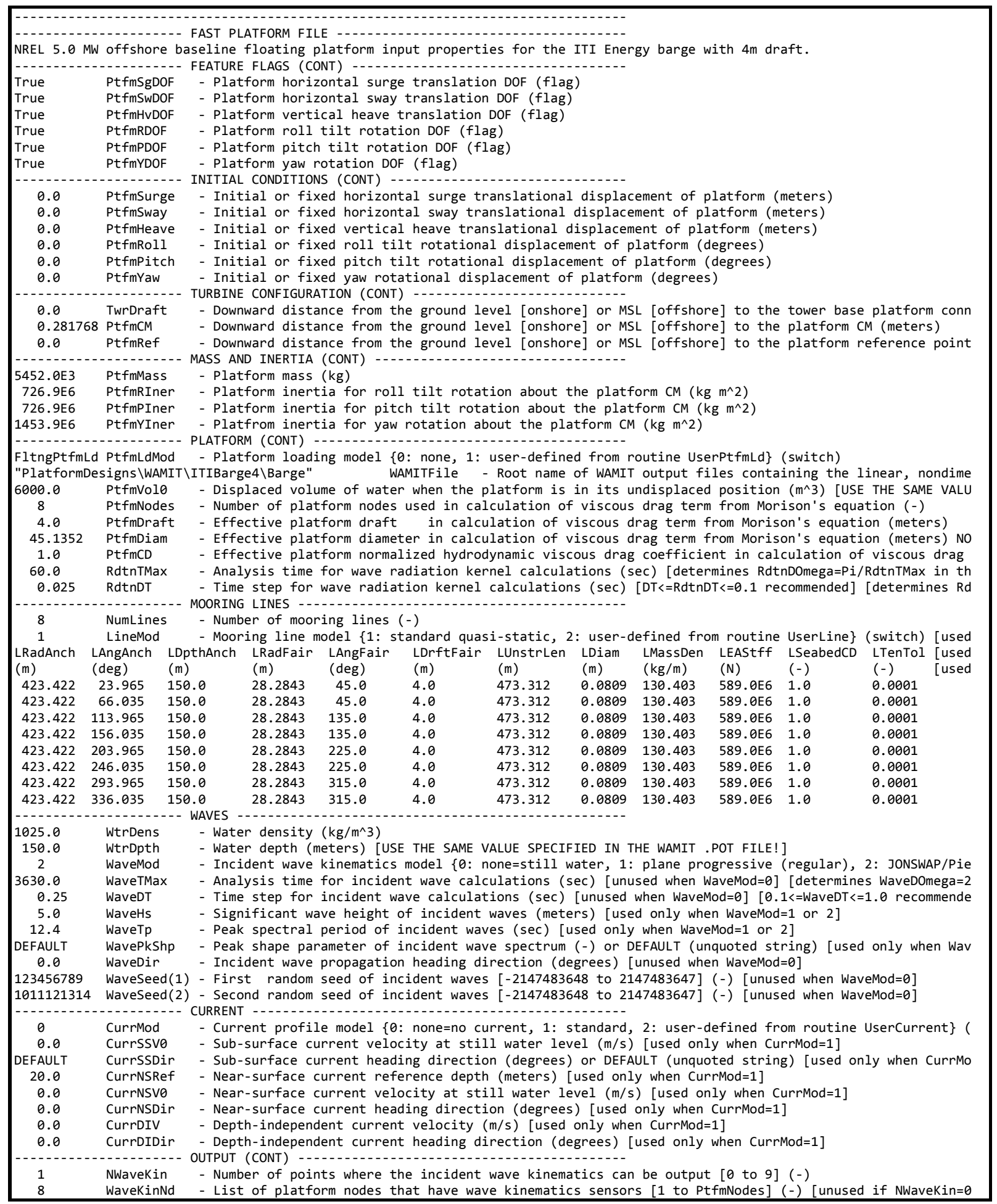




\section{D.2 WAMIT Input File - CONFIG.WAM}

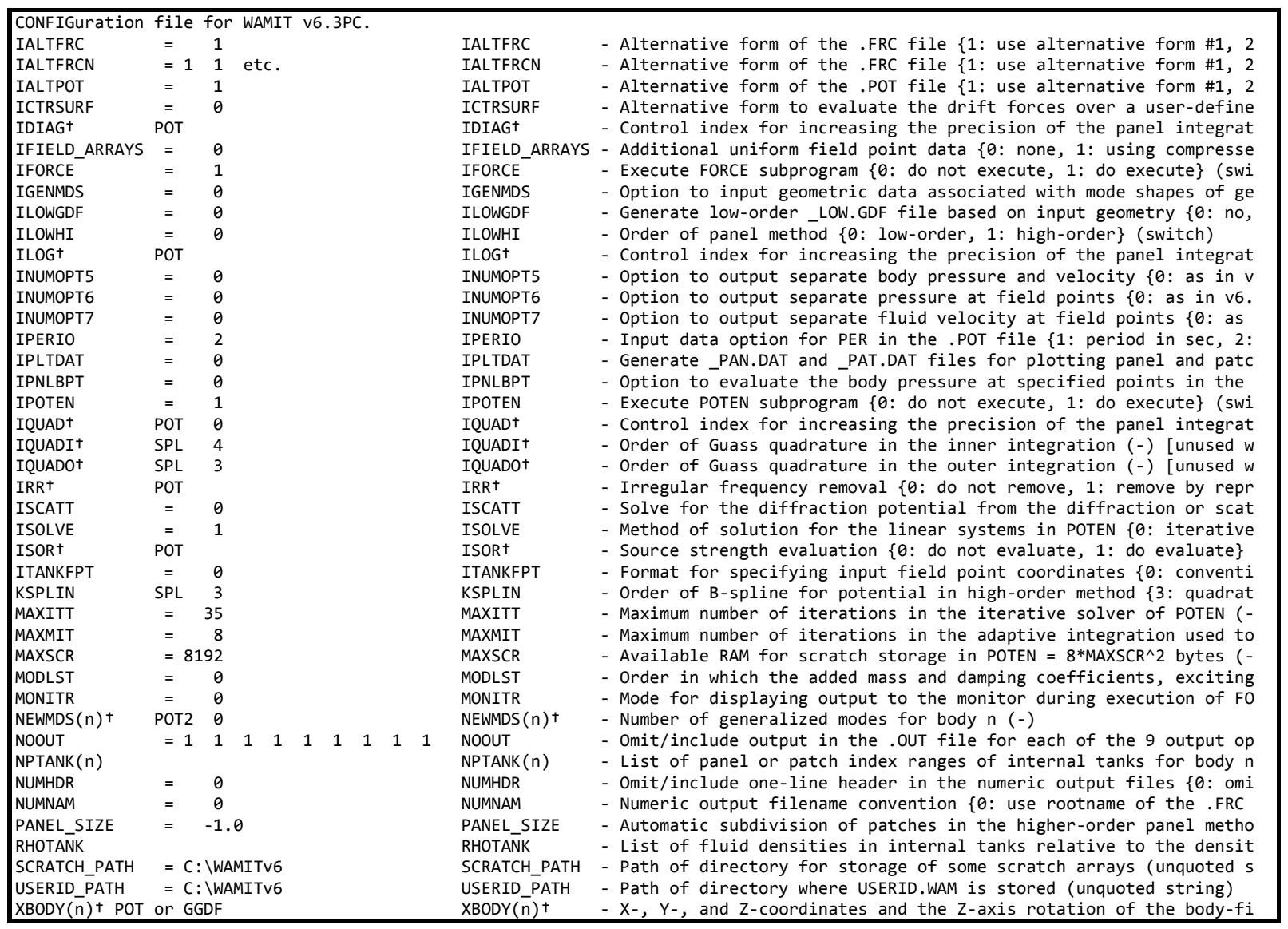

\section{D.3 WAMIT Input File - Barge.POT}

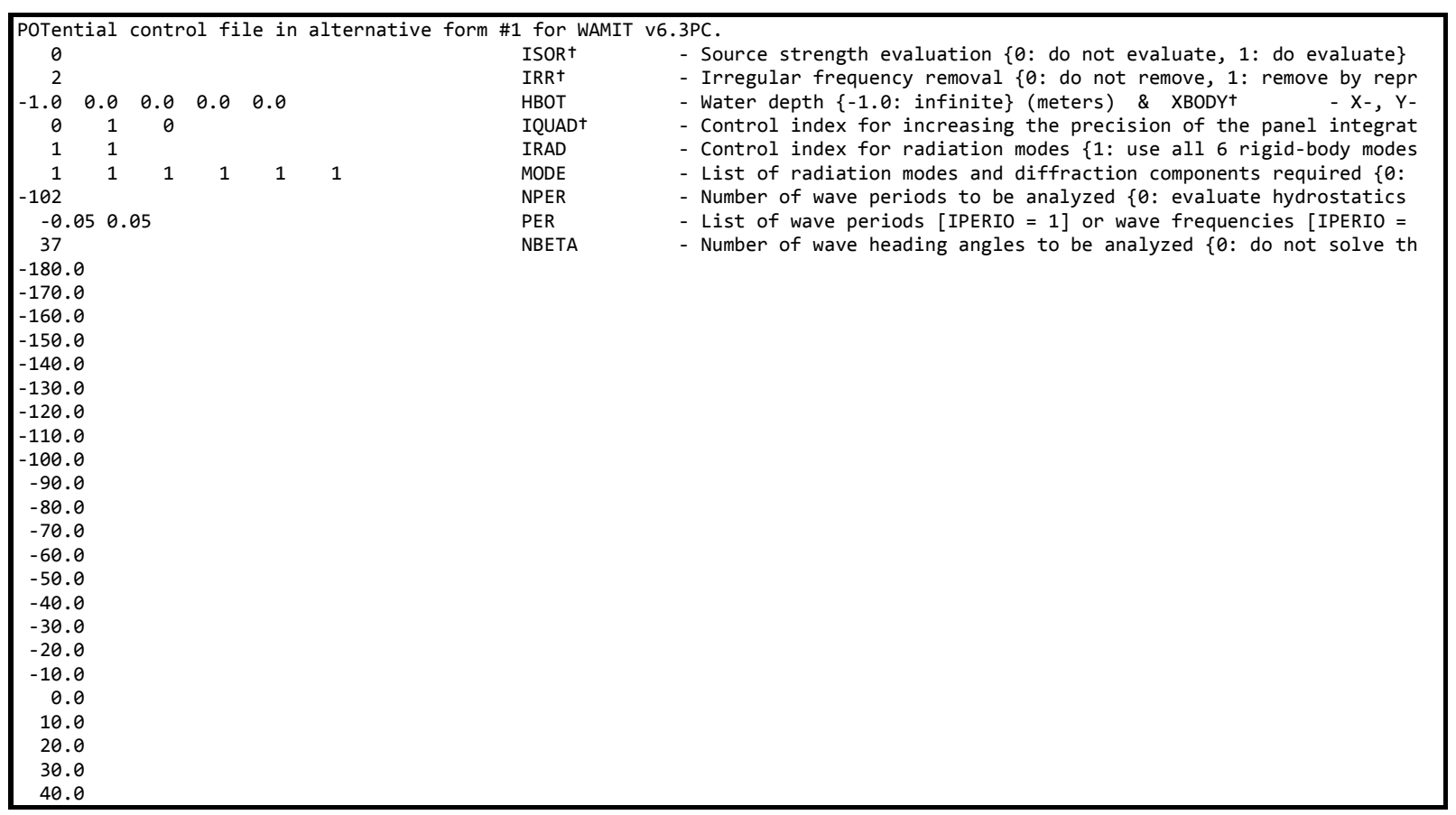




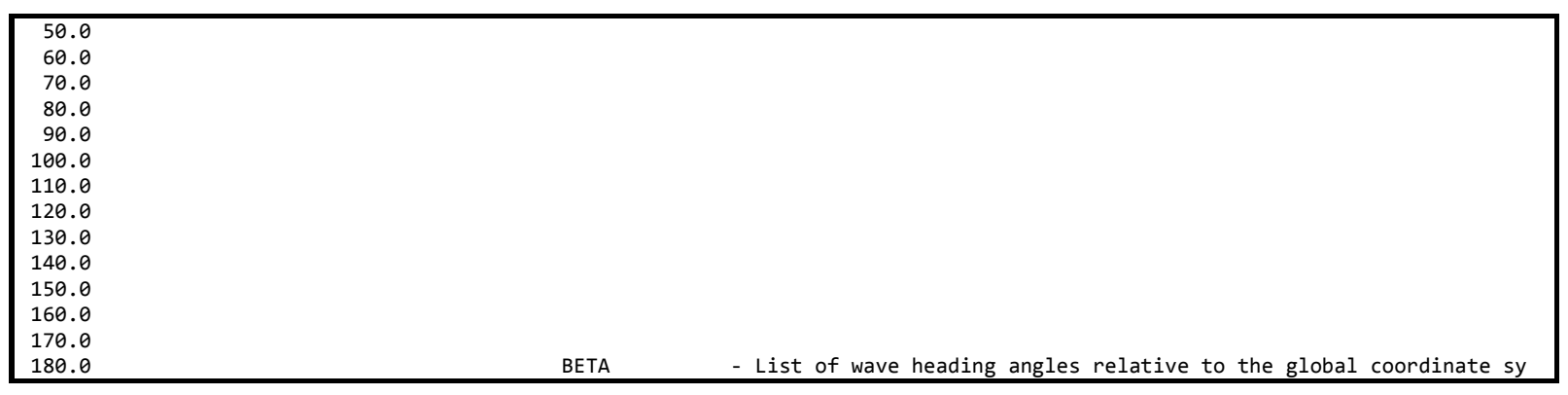

\section{D.4 WAMIT Input File - Barge.FRC}

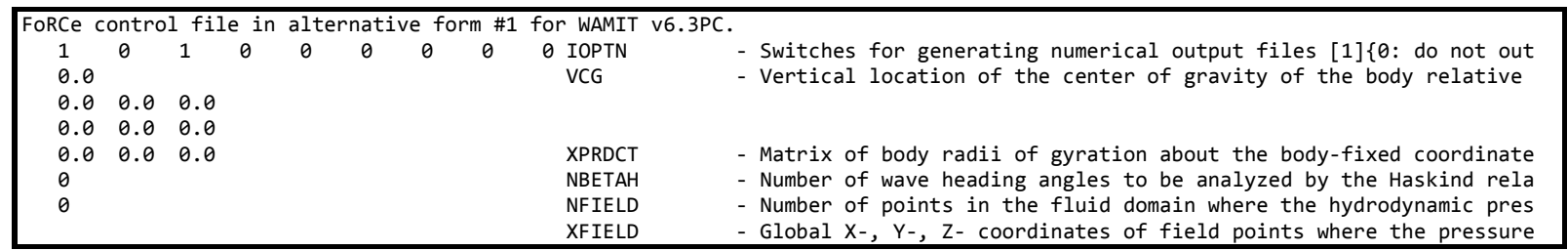

\section{D.5 WAMIT Input File - Barge.GDF}

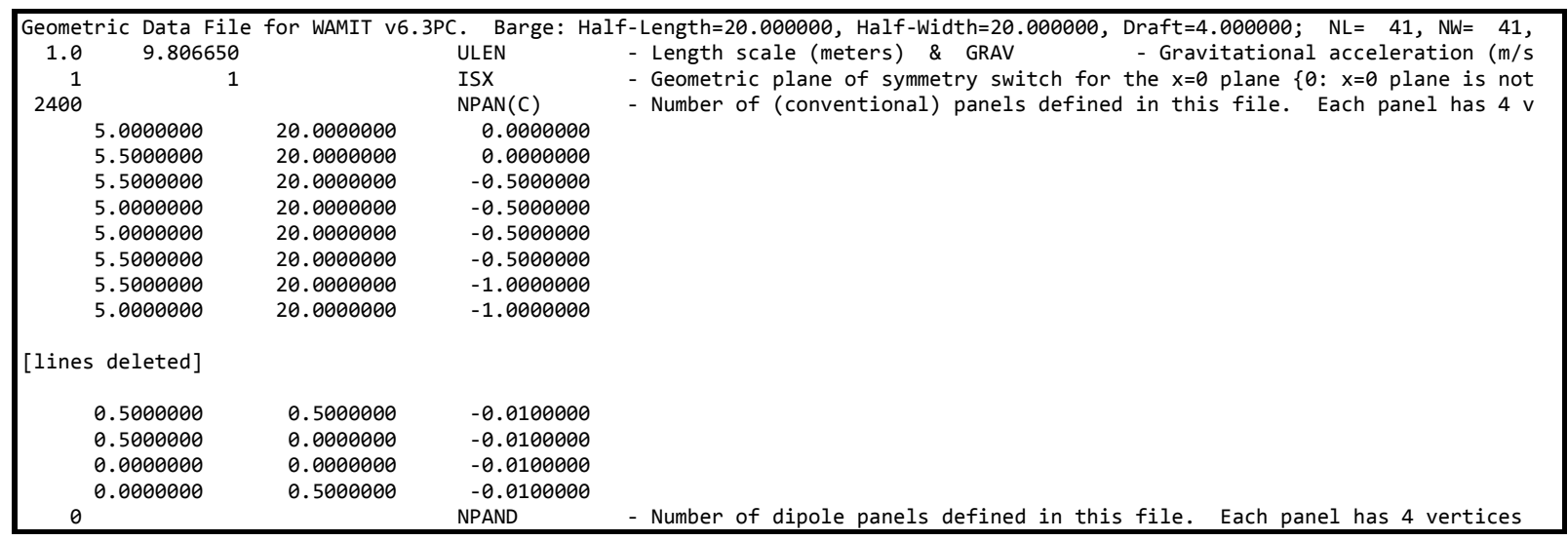

\section{D.6 WAMIT Output File - Barge.hst}

\begin{tabular}{|lll|}
\hline 1 & 1 & $0.000000 \mathrm{E}+00$ \\
1 & 2 & $0.000000 \mathrm{E}+00$ \\
1 & 3 & $0.000000 \mathrm{E}+00$ \\
1 & 4 & $0.000000 \mathrm{E}+00$ \\
1 & 5 & $0.000000 \mathrm{E}+00$ \\
1 & 6 & $0.000000 \mathrm{E}+00$ \\
2 & 1 & $0.000000 \mathrm{E}+00$ \\
2 & 2 & $0.000000 \mathrm{E}+00$ \\
2 & 3 & $0.000000 \mathrm{E}+00$ \\
2 & 4 & $0.000000 \mathrm{E}+00$ \\
2 & 5 & $0.000000 \mathrm{E}+00$ \\
2 & 6 & $0.000000 \mathrm{E}+00$ \\
3 & 1 & $0.000000 \mathrm{E}+00$ \\
3 & 2 & $0.000000 \mathrm{E}+00$ \\
3 & 3 & $1.600000 \mathrm{E}+03$ \\
3 & 4 & $0.000000 \mathrm{E}+00$ \\
3 & 5 & $0.000000 \mathrm{E}+00$ \\
3 & 6 & $0.000000 \mathrm{E}+00$ \\
4 & 1 & $0.000000 \mathrm{E}+00$ \\
4 & 2 & $0.000000 \mathrm{E}+00$ \\
4 & 3 & $0.000000 \mathrm{E}+00$ \\
4 & 4 & $2.013000 \mathrm{E}+05$ \\
4 & 5 & $0.000000 \mathrm{E}+00$ \\
4 & 6 & $0.000000 \mathrm{E}+00$ \\
5 & 1 & $0.000000 \mathrm{E}+00$ \\
\hline
\end{tabular}




\begin{tabular}{|lll|}
\hline 5 & 2 & $0.000000 \mathrm{E}+00$ \\
5 & 3 & $0.000000 \mathrm{E}+00$ \\
5 & 4 & $0.000000 \mathrm{E}+00$ \\
5 & 5 & $2.013000 \mathrm{E}+05$ \\
5 & 6 & $0.000000 \mathrm{E}+00$ \\
6 & 1 & $0.000000 \mathrm{E}+00$ \\
6 & 2 & $0.000000 \mathrm{E}+00$ \\
6 & 3 & $0.000000 \mathrm{E}+00$ \\
6 & 4 & $0.000000 \mathrm{E}+00$ \\
6 & 5 & $0.000000 \mathrm{E}+00$ \\
6 & 6 & $0.000000 \mathrm{E}+00$
\end{tabular}

\section{D.7 WAMIT Output File - Barge.1}

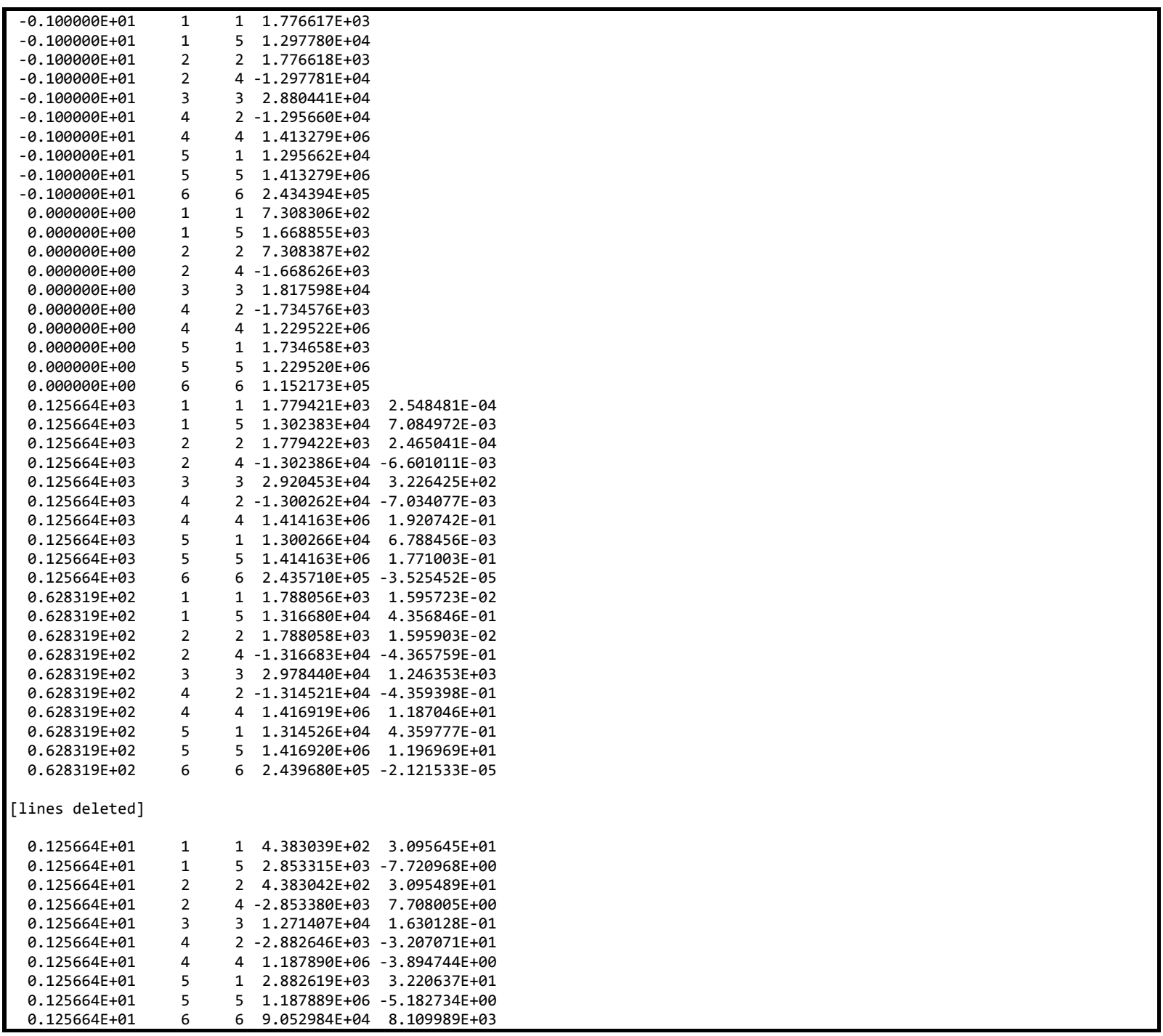

\section{D.8 WAMIT Output File - Barge.3}

$\begin{array}{llllrrrr}0.125664 \mathrm{E}+03 & -0.180000 \mathrm{E}+03 & 1 & 1.983066 \mathrm{E}+00 & -9.000000 \mathrm{E}+01 & -6.338097 \mathrm{E}-08 & -1.983066 \mathrm{E}+00 \\ 0.125664 \mathrm{E}+03 & -0.180000 \mathrm{E}+03 & 2 & 4.825996 \mathrm{E}-08 & -9.000000 \mathrm{E}+01 & -1.542444 \mathrm{E}-15 & -4.825996 \mathrm{E}-08 \\ 0.125664 \mathrm{E}+03 & -0.180000 \mathrm{E}+03 & 3 & 1.591020 \mathrm{E}+03 & 2.962686 \mathrm{E}-03 & 1.591020 \mathrm{E}+03 & 8.226946 \mathrm{E}-02 \\ 0.125664 \mathrm{E}+03 & -0.180000 \mathrm{E}+03 & 4 & 1.325606 \mathrm{E}-06 & 9.000000 \mathrm{E}+01 & 4.236804 \mathrm{E}-14 & 1.325606 \mathrm{E}-06 \\ 0.125664 \mathrm{E}+03 & -0.180000 \mathrm{E}+03 & 5 & 5.447115 \mathrm{E}+01 & -9.000000 \mathrm{E}+01 & -1.740960 \mathrm{E}-06 & -5.447115 \mathrm{E}+01 \\ 0.125664 \mathrm{E}+03 & -0.180000 \mathrm{E}+03 & 6 & 5.515950 \mathrm{E}-15 & 9.000000 \mathrm{E}+01 & 5.515950 \mathrm{E}-15 & 4.165130 \mathrm{E}-24 \\ 0.125664 \mathrm{E}+03 & -0.170000 \mathrm{E}+03 & 1 & 1.952940 \mathrm{E}+00 & -9.000000 \mathrm{E}+01 & -6.241811 \mathrm{E}-08 & -1.952940 \mathrm{E}+00 \\ 0.125664 \mathrm{E}+03 & -0.170000 \mathrm{E}+03 & 2 & 3.443574 \mathrm{E}-01 & -9.000000 \mathrm{E}+01 & -1.100605 \mathrm{E}-08 & -3.443574 \mathrm{E}-01 \\ 0.125664 \mathrm{E}+03 & -0.170000 \mathrm{E}+03 & 3 & 1.591020 \mathrm{E}+03 & 2.962686 \mathrm{E}-03 & 1.591020 \mathrm{E}+03 & 8.226946 \mathrm{E}-02 \\ 0.125664 \mathrm{E}+03 & -0.170000 \mathrm{E}+03 & 4 & 9.458789 \mathrm{E}+00 & 9.000000 \mathrm{E}+01 & 3.023152 \mathrm{E}-07 & 9.458789 \mathrm{E}+00\end{array}$




\begin{tabular}{|c|c|c|c|c|c|c|}
\hline $0.125664 \mathrm{E}+03$ & $-0.170000 \mathrm{E}+03$ & 5 & $5.364359 \mathrm{E}+01$ & $-9.000000 \mathrm{E}+01$ & $-1.714512 \mathrm{E}-06$ & $-5.364359 E+01$ \\
\hline $125664 \mathrm{E}+03$ & $-0.170000 E+03$ & 6 & $4.453025 \mathrm{E}-08$ & $3.766301 \mathrm{E}-08$ & $4.453025 \mathrm{E}-08$ & $2.927167 \mathrm{E}-17$ \\
\hline $125664 \mathrm{E}+03$ & $-0.160000 E+03$ & 1 & $1.863475 \mathrm{E}+00$ & $-9.000000 \mathrm{E}+01$ & $-5.955869 E-08$ & $-1.863475 E+00$ \\
\hline $0.125664 \mathrm{E}+03$ & $-0.160000 E+03$ & 2 & $6.782514 \mathrm{E}-01$ & $-9.000000 \mathrm{E}+01$ & $-2.167768 \mathrm{E}-08$ & $-6.782514 \mathrm{E}-01$ \\
\hline $0.125664 \mathrm{E}+03$ & $-0.160000 E+03$ & 3 & $1.591020 \mathrm{E}+03$ & $2.962687 \mathrm{E}-03$ & $1.591020 \mathrm{E}+03$ & $8.226947 \mathrm{E}-02$ \\
\hline $0.125664 E+03$ & $-0.160000 E+03$ & 4 & $1.863024 \mathrm{E}+01$ & $9.000000 \mathrm{E}+01$ & $5.954440 \mathrm{E}-07$ & $1.863024 \mathrm{E}+01$ \\
\hline $0.125664 E+03$ & $-0.160000 E+03$ & 5 & $5.118615 E+01$ & $-9.000000 \mathrm{E}+01$ & $-1.635966 \mathrm{E}-06$ & $-5.118615 E+01$ \\
\hline $0.125664 \mathrm{E}+03$ & $-0.160000 E+03$ & 6 & $6.214594 \mathrm{E}-08$ & $5.072097 \mathrm{E}-08$ & $6.214594 \mathrm{E}-08$ & $5.501456 \mathrm{E}-17$ \\
\hline $0.125664 \mathrm{E}+03$ & $-0.150000 E+03$ & 1 & $1.717387 \mathrm{E}+00$ & $-9.000000 \mathrm{E}+01$ & $-5.488958 E-08$ & $-1.717387 E+00$ \\
\hline $0.125664 \mathrm{E}+03$ & $-0.150000 \mathrm{E}+03$ & 2 & $9.915367 \mathrm{E}-01$ & $-9.000000 \mathrm{E}+01$ & $-3.169064 \mathrm{E}-08$ & $-9.915367 E-01$ \\
\hline+03 & $-0.150000 E+03$ & 3 & $1.591020 \mathrm{E}+03$ & $2.962686 \mathrm{E}-03$ & $91020 E+03$ & $6 E-02$ \\
\hline+03 & -0.150 & 4 & $1 \mathrm{E}+01$ & 9.00 & $8.704816 E-07$ & $2.723551 \mathrm{E}+01$ \\
\hline $0.125664 \mathrm{E}+03$ & $-0.150000 E+03$ & 5 & 4. $717344 \mathrm{E}+01$ & $-9.000000 \mathrm{E}+01$ & $-1.507716 \mathrm{E}-06$ & $-4.717344 \mathrm{E}+01$ \\
\hline $0.125664 \mathrm{E}+03$ & $-0.150000 E+03$ & 6 & $6.074003 E-08$ & $6.990674 \mathrm{E}-08$ & $6.074003 E-08$ & $7.410908 \mathrm{E}-17$ \\
\hline $0.125664 \mathrm{E}+03$ & $-0.140000 \mathrm{E}+03$ & 1 & $1.519122 \mathrm{E}+00$ & $-9.000000 \mathrm{E}+01$ & $-4.855279 E-08$ & -1.51 \\
\hline $0.125664 \mathrm{E}+03$ & $-0.140000 E+03$ & 2 & 1. $274695 \mathrm{E}+00$ & $-9.000000 E+01$ & $-4.074073 E-08$ & $-1.274695 \mathrm{E}+00$ \\
\hline $0.125664 E+03$ & $-0.140000 E+03$ & 3 & $1.591020 \mathrm{E}+03$ & $2.962686 \mathrm{E}-03$ & $1.591020 E+03$ & $8.226946 \mathrm{E}-02$ \\
\hline $0.125664 \mathrm{E}+03$ & $-0.140000 \mathrm{E}+03$ & 4 & $3.501334 \mathrm{E}+01$ & $9.000000 \mathrm{E}+01$ & $1.119071 \mathrm{E}-06$ & $3.501334 \mathrm{E}+01$ \\
\hline $0.125664 \mathrm{E}+03$ & $-0.140000 \mathrm{E}+03$ & 5 & $4.172728 \mathrm{E}+01$ & $-9.000000 \mathrm{E}+01$ & $-1.333655 E-06$ & $-4.172728 \mathrm{E}+01$ \\
\hline $0.125664 E+03$ & $-0.140000 E+03$ & 6 & $3.637167 \mathrm{E}-08$ & $1.327815 \mathrm{E}-07$ & $3.637167 E-08$ & $8.429042 \mathrm{E}-17$ \\
\hline $5664 \mathrm{E}+03$ & $-0.130000 E+03$ & 1 & $1.274693 \mathrm{E}+00$ & $-9.000000 \mathrm{E}+01$ & $-4.074061 E-08$ & $-1.274693 \mathrm{E}+00$ \\
\hline $0.125664 \mathrm{E}+03$ & $-0.130000 \mathrm{E}+03$ & 2 & $122 \mathrm{E}+00$ & $-9.000000 \mathrm{E}+01$ & $-4.855287 E-08$ & $-1.519122 \mathrm{E}+00$ \\
\hline $4 \mathrm{E}+03$ & -0.1300 & 3 & $20 E+03$ & $6 \mathrm{E}-03$ & $1.591020 \mathrm{E}+03$ & $47 E-02$ \\
\hline$E+03$ & -0.1 & 4 & $5 E+01$ & $\theta E+01$ & -06 & $E+01$ \\
\hline+03 & -0.1 & 5 & $E+01$ & $\theta 0 E+01$ & $=-06$ & $3 E+01$ \\
\hline $0.125664 \mathrm{E}+03$ & -0.130 & 6 & $4 E-09$ & $7 E-07$ & $64 E-09$ & $979 E-17$ \\
\hline $0.125664 \mathrm{E}+03$ & -0.120 & 1 & $9 E-01$ & -9.006 & $2 E-08$ & $39 E-01$ \\
\hline $0.125664 \mathrm{E}+03$ & -0.120 & 2 & $1 E+00$ & -9.000 & $E-08$ & $1 E+00$ \\
\hline $0.125664 E+03$ & $-0.120000 E+03$ & 3 & $20 E+03$ & $86 E-03$ & 1.59 & $E-02$ \\
\hline $0.125664 E+03$ & $-0.120000 E+03$ & 4 & $4.717342 \mathrm{E}+01$ & $9.000000 \mathrm{E}+01$ & $1.507717 \mathrm{E}-06$ & $4.717342 E+01$ \\
\hline $0.125664 \mathrm{E}+03$ & $-0.120000 E+03$ & 5 & $2.723554 \mathrm{E}+01$ & $-9.000000 \mathrm{E}+01$ & $-8.704814 E-07$ & $-2.723554 \mathrm{E}+01$ \\
\hline $0.125664 \mathrm{E}+03$ & $-0.120000 \mathrm{E}+03$ & 6 & $1.997914 \mathrm{E}-08$ & $1.800000 \mathrm{E}+02$ & $-1.997914 \mathrm{E}-08$ & $7.410738 \mathrm{E}-17$ \\
\hline $0.125664 \mathrm{E}+03$ & $-0.110000 \mathrm{E}+03$ & 1 & $6.782510 \mathrm{E}-01$ & $-9.000000 \mathrm{E}+01$ & $-2.167763 E-08$ & $-6.782510 E-01$ \\
\hline $0.125664 \mathrm{E}+03$ & $-0.110000 E+03$ & 2 & $177 E+00$ & $\theta 0 E+01$ & -5.955889 & $477 E+00$ \\
\hline$E+03$ & $-0.11 e$ & 3 & $E+03$ & -03 & $=03$ & 8. \\
\hline 0.125 & -0.1 & 4 & $E+01$ & $\theta \mathrm{E}+01$ & -06 & 5. \\
\hline+03 & -0.1 & 5 & $E+01$ & $\mathrm{E}+01$ & $=-07$ & -1 \\
\hline$=+03$ & -0.1 & 6 & $E-08$ & $E+02$ & -1 & \\
\hline$=+03$ & -0.1 & 1 & $E-01$ & -9.00 & -1.1 & $8 \mathrm{E}-01$ \\
\hline $4 \mathrm{E}+03$ & -0.100 & 2 & $45 E+00$ & -9.006 & $31 E-08$ & $45 E+00$ \\
\hline $0.125664 \mathrm{E}+03$ & -0.100 & 3 & $1.591021 \mathrm{E}+03$ & $5 E-03$ & 1. $591021 \mathrm{E}+03$ & $8.226947 \mathrm{E}-02$ \\
\hline $0.125664 \mathrm{E}+03$ & $-0.100000 \mathrm{E}+03$ & 4 & $5.364361 \mathrm{E}+01$ & $\partial \theta \mathrm{E}+01$ & $1.714512 \mathrm{E}-06$ & $5.364361 E+01$ \\
\hline $0.125664 E+03$ & $-0.100000 E+03$ & 5 & $9.458813 \mathrm{E}+00$ & $-9.000000 \mathrm{E}+01$ & $-3.023147 E-07$ & $-9.458813 E+00$ \\
\hline $0.125664 E+03$ & $-0.100000 E+03$ & 6 & $1.732498 \mathrm{E}-08$ & $1.800000 \mathrm{E}+02$ & $-1.732498 \mathrm{E}-08$ & $2.927118 \mathrm{E}-17$ \\
\hline $0.125664 \mathrm{E}+03$ & $-0.900000 \mathrm{E}+02$ & 1 & $2.412994 \mathrm{E}-08$ & $9.000000 \mathrm{E}+01$ & $7.712198 \mathrm{E}-16$ & $2.412994 \mathrm{E}-08$ \\
\hline $0.125664 \mathrm{E}+03$ & $-0.900000 \mathrm{E}+02$ & 2 & $1.983073 \mathrm{E}+00$ & $-9.000000 \mathrm{E}+01$ & $-6.338124 E-08$ & $-1.983073 E+00$ \\
\hline $0.125664 E+03$ & $-0.900000 \mathrm{E}+02$ & 3 & $21 E+03$ & $6 E-03$ & $221 E+03$ & 8.22 \\
\hline $0.125664 \mathrm{E}+03$ & $-0.900000 \mathrm{E}+02$ & 4 & $5.447108 \mathrm{E}+01$ & $\theta 0 E+01$ & $1.740961 \mathrm{E}-06$ & $5.447108 \mathrm{E}+01$ \\
\hline$E+03$ & -0.90 & 5 & $=-07$ & 9.00 & -14 & 6. \\
\hline $0.125664 \mathrm{E}+03$ & -0.900 & 6 & $8 E-15$ & $\partial E+01$ & -15 & -2 \\
\hline+03 & $-0 . \varepsilon-1-1$ & 1 & -01 & 01 & $=-08$ & \\
\hline+03 & -0.8 & 2 & $E+00$ & -9.00 & -6 & -1. \\
\hline $0.125664 \mathrm{E}+03$ & -0.800 & 3 & $1 \mathrm{E}+03$ & 2.96 & +03 & $E-02$ \\
\hline 0.1 & -0.80 & 4 & +01 & 9.6 & -06 & -01 \\
\hline 0.1256 & $-0.800000 \mathrm{E}+02$ & 5 & $9.458814 \mathrm{E}+00$ & $9.000000 \mathrm{E}+01$ & $7 E-07$ & $E+00$ \\
\hline $0.125664 \mathrm{E}+03$ & $-0.800000 \mathrm{E}+02$ & 6 & $1.799070 \mathrm{E}-08$ & -9.322 & $1.799070 \mathrm{E}-08$ & $-2.927141 E-17$ \\
\hline $0.125664 E+03$ & $-0.700000 E+02$ & 1 & $6.782510 \mathrm{E}-01$ & $9.000000 \mathrm{E}+01$ & $2.167763 E-08$ & $6.782510 \mathrm{E}-01$ \\
\hline $0.125664 \mathrm{E}+03$ & $-0.700000 E+02$ & 2 & $1.863477 \mathrm{E}+00$ & $-9.000000 \mathrm{E}+01$ & $-5.955889 E-08$ & $-1.863477 E+00$ \\
\hline $0.125664 \mathrm{E}+03$ & $-0.700000 E+02$ & 3 & $1.591020 \mathrm{E}+03$ & $2.962686 \mathrm{E}-03$ & $1.591020 \mathrm{E}+03$ & $8.226948 \mathrm{E}-02$ \\
\hline $0.125664 \mathrm{E}+03$ & $-0.700000 E+02$ & 4 & $5.118610 \mathrm{E}+01$ & $9.000000 \mathrm{E}+01$ & $1.635968 \mathrm{E}-06$ & $5.118610 E+01$ \\
\hline $0.125664 \mathrm{E}+03$ & $-0.700000 E+02$ & 5 & $1.863021 \mathrm{E}+01$ & $9.000000 \mathrm{E}+01$ & $5.954436 \mathrm{E}-07$ & $1.863021 \mathrm{E}+01$ \\
\hline $0.125664 \mathrm{E}+03$ & $-0.700000 \mathrm{E}+02$ & 6 & $1.993885 \mathrm{E}-08$ & $-1.580865 E-07$ & $1.993885 \mathrm{E}-08$ & $-5.501388 \mathrm{E}-17$ \\
\hline $0.125664 \mathrm{E}+03$ & -0.6000 & 1 & $9 E-01$ & $9.000000 \mathrm{E}+01$ & $E-08$ & -01 \\
\hline $0.125664 \mathrm{E}+03$ & -0.600 & 2 & $L E+00$ & -9 & $E-08$ & -1 \\
\hline$=03$ & -0.6 & 3 & $E+03$ & 2.96 & $1.591020 \mathrm{E}+03$ & $7 E-02$ \\
\hline$E+03$ & -0.6006 & 4 & $42 E+01$ & +01 & -06 & $E+01$ \\
\hline $0.125664 \mathrm{E}+03$ & $-0.600000 E+02$ & 5 & $2.723554 \mathrm{E}+01$ & $9.000000 \mathrm{E}+01$ & -07 & $54 \mathrm{E}+01$ \\
\hline $0.125664 \mathrm{E}+03$ & -0.600 & 6 & 1.9979 & -2.12 & -08 & -7.4 \\
\hline+03 & $-0.500000 E+02$ & 1 & 1. $274693 \mathrm{E}+00$ & 9.000 & -08 & $E+00$ \\
\hline $0.125664 \mathrm{E}+03$ & $-0.500000 E+02$ & 2 & $1.519122 \mathrm{E}+00$ & $-9.000000 \mathrm{E}+01$ & $-4.855287 E-08$ & $-1.519122 \mathrm{E}+00$ \\
\hline $0.125664 \mathrm{E}+03$ & $-0.500000 E+02$ & 3 & $1.591020 \mathrm{E}+03$ & $2.962686 \mathrm{E}-03$ & $1.591020 \mathrm{E}+03$ & $8.226947 \mathrm{E}-02$ \\
\hline $0.125664 \mathrm{E}+03$ & $-0.500000 \mathrm{E}+02$ & 4 & 4. $172725 \mathrm{E}+01$ & $9.000000 \mathrm{E}+01$ & $1.333656 \mathrm{E}-06$ & $4.172725 E+01$ \\
\hline $0.125664 E+03$ & $-0.500000 E+02$ & 5 & $3.501334 \mathrm{E}+01$ & $9.000000 \mathrm{E}+01$ & $1.119070 \mathrm{E}-06$ & $3.501334 E+01$ \\
\hline $0.125664 \mathrm{E}+03$ & $-0.500000 E+02$ & 6 & $4.064337 \mathrm{E}-09$ & $-1.800000 \mathrm{E}+02$ & $-4.064337 E-09$ & $-8.428993 E-17$ \\
\hline $0.125664 \mathrm{E}+03$ & $-0.400000 \mathrm{E}+02$ & 1 & $1.519122 \mathrm{E}+00$ & $9.000000 \mathrm{E}+01$ & $4.855279 E-08$ & $1.519122 \mathrm{E}+00$ \\
\hline $0.125664 \mathrm{E}+03$ & $-0.400000 \mathrm{E}+02$ & 2 & $695 \mathrm{E}+00$ & $-9.000000 \mathrm{E}+01$ & $-4.074073 E-08$ & $-1.274695 \mathrm{E}+00$ \\
\hline $0.125664 \mathrm{E}+03$ & $-0.400000 \mathrm{E}+02$ & 3 & $1.591020 \mathrm{E}+03$ & $2.962686 \mathrm{E}-03$ & $1.591020 \mathrm{E}+03$ & $946 \mathrm{E}-02$ \\
\hline $4 \mathrm{E}+03$ & $-0.400000 \mathrm{E}+02$ & 4 & $3.501334 \mathrm{E}+01$ & $9.000000 \mathrm{E}+01$ & $1.119071 \mathrm{E}-06$ & $3.501334 \mathrm{E}+01$ \\
\hline $0.125664 \mathrm{E}+03$ & $-0.400000 E+02$ & 5 & 4. $172728 \mathrm{E}+01$ & $9.000000 \mathrm{E}+01$ & $1.333655 \mathrm{E}-06$ & $4.172728 \mathrm{E}+01$ \\
\hline $0.125664 E+03$ & $-0.400000 E+02$ & 6 & $3.637167 \mathrm{E}-08$ & $-1.800000 \mathrm{E}+02$ & $-3.637167 E-08$ & $-8.429042 \mathrm{E}-17$ \\
\hline $0.125664 \mathrm{E}+03$ & $-0.300000 E+02$ & 1 & $1.717387 \mathrm{E}+00$ & $9.000000 \mathrm{E}+01$ & $5.488958 \mathrm{E}-08$ & $1.717387 \mathrm{E}+00$ \\
\hline $0.125664 \mathrm{E}+03$ & $-0.300000 \mathrm{E}+02$ & 2 & $9.915367 \mathrm{E}-01$ & $-9.000000 \mathrm{E}+01$ & $-3.169064 \mathrm{E}-08$ & $-9.915367 E-01$ \\
\hline $0.125664 E+03$ & $-0.300000 E+02$ & 3 & $1.591020 \mathrm{E}+03$ & $2.962686 \mathrm{E}-03$ & $1.591020 \mathrm{E}+03$ & $8.226946 \mathrm{E}-02$ \\
\hline & & & & & & \\
\hline
\end{tabular}
$\begin{array}{lllllll}0.125664 \mathrm{E}+03 & -0.300000 \mathrm{E}+02 & 4 & 2.723551 \mathrm{E}+01 & 9.000000 \mathrm{E}+01 & 8.704816 \mathrm{E}-07 & 2.723551 \mathrm{E}+01\end{array}$ 


\begin{tabular}{|c|c|c|c|c|c|c|}
\hline $0.125664 \mathrm{E}+03$ & $-0.300000 E+02$ & 5 & $4.717344 \mathrm{E}+01$ & $9.000000 \mathrm{E}+01$ & $1.507716 \mathrm{E}-06$ & $4.717344 \mathrm{E}+01$ \\
\hline $0.125664 \mathrm{E}+03$ & $-0.300000 E+02$ & 6 & $6.074003 E-08$ & $-1.800000 E+02$ & $-6.074003 E-08$ & $-7.410908 \mathrm{E}-17$ \\
\hline $0.125664 \mathrm{E}+03$ & $-0.200000 \mathrm{E}+02$ & 1 & $1.863475 \mathrm{E}+00$ & $9.000000 \mathrm{E}+01$ & $5.955869 E-08$ & $1.863475 \mathrm{E}+00$ \\
\hline $0.125664 \mathrm{E}+03$ & $-0.200000 \mathrm{E}+02$ & 2 & $6.782514 \mathrm{E}-01$ & $-9.000000 E+01$ & $-2.167768 \mathrm{E}-08$ & $-6.782514 E-01$ \\
\hline $0.125664 \mathrm{E}+03$ & $-0.200000 \mathrm{E}+02$ & 3 & $1.591020 E+03$ & $2.962687 \mathrm{E}-03$ & $1.591020 \mathrm{E}+03$ & $8.226947 \mathrm{E}-02$ \\
\hline $0.125664 E+03$ & $-0.200000 E+02$ & 4 & $1.863024 \mathrm{E}+01$ & $9.000000 E+01$ & $5.954440 \mathrm{E}-07$ & $1.863024 \mathrm{E}+01$ \\
\hline $0.125664 E+03$ & $-0.200000 E+02$ & 5 & $5.118615 E+01$ & $9.000000 E+01$ & $1.635966 \mathrm{E}-06$ & $5.118615 E+01$ \\
\hline $0.125664 E+03$ & $-0.200000 E+02$ & 6 & $6.214594 \mathrm{E}-08$ & $-1.800000 E+02$ & $-6.214594 E-08$ & $-5.501456 \mathrm{E}-17$ \\
\hline $0.125664 \mathrm{E}+03$ & $-0.100000 E+02$ & 1 & $1.952940 E+00$ & $9.000000 \mathrm{E}+01$ & $6.241811 E-08$ & $1.952940 \mathrm{E}+00$ \\
\hline $0.125664 \mathrm{E}+03$ & $-0.100000 E+02$ & 2 & $3.443574 \mathrm{E}-01$ & $-9.000000 E+01$ & $-1.100605 E-08$ & $-3.443574 E-01$ \\
\hline $0.125664 \mathrm{E}+03$ & $-0.100000 \mathrm{E}+02$ & 3 & $1.591020 E+03$ & $2.962686 \mathrm{E}-03$ & $1.591020 \mathrm{E}+03$ & $8.226946 \mathrm{E}-02$ \\
\hline $0.125664 \mathrm{E}+03$ & $-0.100000 \mathrm{E}+02$ & 4 & $9.458788 \mathrm{E}+00$ & $9.000000 \mathrm{E}+01$ & $3.023151 \mathrm{E}-07$ & $9.458788 \mathrm{E}+00$ \\
\hline $0.125664 \mathrm{E}+03$ & $-0.100000 \mathrm{E}+02$ & 5 & $5.364359 E+01$ & $9.000000 E+01$ & $1.714512 \mathrm{E}-06$ & $5.364359 E+01$ \\
\hline $0.125664 \mathrm{E}+03$ & $-0.100000 \mathrm{E}+02$ & 6 & $4.114693 E-08$ & $-1.800000 E+02$ & $-4.114693 E-08$ & $-2.927184 \mathrm{E}-17$ \\
\hline $0.125664 \mathrm{E}+03$ & $0.000000 \mathrm{E}+00$ & 1 & $1.983066 \mathrm{E}+00$ & $9.000000 \mathrm{E}+01$ & $6.338097 E-08$ & $1.983066 \mathrm{E}+00$ \\
\hline $0.125664 \mathrm{E}+03$ & $0.000000 E+00$ & 2 & $0.000000 E+00$ & $9.000000 \mathrm{E}+01$ & $0.000000 E+00$ & $0.000000 E+00$ \\
\hline $0.125664 \mathrm{E}+03$ & $0.000000 E+00$ & 3 & $1.591020 E+03$ & $2.962686 \mathrm{E}-03$ & $1.591020 \mathrm{E}+03$ & $8.226946 \mathrm{E}-02$ \\
\hline $0.125664 \mathrm{E}+03$ & $0.000000 \mathrm{E}+00$ & 4 & $0.000000 \mathrm{E}+00$ & $9.000000 \mathrm{E}+01$ & $0.000000 E+00$ & $0.000000 \mathrm{E}+00$ \\
\hline $0.125664 E+03$ & $0.000000 E+00$ & 5 & $5.447115 E+01$ & $9.000000 \mathrm{E}+01$ & $1.740960 \mathrm{E}-06$ & $5.447115 E+01$ \\
\hline $0.125664 E+03$ & $0.000000 E+00$ & 6 & $0.000000 E+00$ & $9.000000 E+01$ & $0.000000 E+00$ & $0.000000 E+00$ \\
\hline $0.125664 E+03$ & $0.100000 E+02$ & 1 & $1.952940 E+00$ & $9.000000 E+01$ & $6.241811 \mathrm{E}-08$ & $1.952940 E+00$ \\
\hline $0.125664 E+03$ & $0.100000 E+02$ & 2 & $3.443574 \mathrm{E}-01$ & $9.000000 E+01$ & $1.100605 \mathrm{E}-08$ & $3.443574 \mathrm{E}-01$ \\
\hline $0.125664 \mathrm{E}+03$ & $0.100000 E+02$ & 3 & $1.591020 E+03$ & $2.962686 \mathrm{E}-03$ & $1.591020 E+03$ & $8.226946 \mathrm{E}-02$ \\
\hline $0.125664 \mathrm{E}+03$ & $0.100000 E+02$ & 4 & $9.458788 \mathrm{E}+00$ & $-9.000000 \mathrm{E}+01$ & $-3.023151 E-07$ & $-9.458788 \mathrm{E}+00$ \\
\hline $0.125664 \mathrm{E}+03$ & $0.100000 E+02$ & 5 & $5.364359 E+01$ & $9.000000 \mathrm{E}+01$ & $1.714512 \mathrm{E}-06$ & $5.364359 E+01$ \\
\hline $0.125664 \mathrm{E}+03$ & $0.100000 \mathrm{E}+02$ & 6 & $4.114693 \mathrm{E}-08$ & $4.076011 \mathrm{E}-08$ & $4.114693 \mathrm{E}-08$ & $2.927184 \mathrm{E}-17$ \\
\hline $0.125664 E+03$ & $0.200000 E+02$ & 1 & $1.863475 E+00$ & $9.000000 E+01$ & $5.955869 E-08$ & $1.863475 \mathrm{E}+00$ \\
\hline $0.125664 \mathrm{E}+03$ & $0.200000 E+02$ & 2 & $6.782514 \mathrm{E}-01$ & $9.000000 \mathrm{E}+01$ & $2.167768 \mathrm{E}-08$ & $6.782514 \mathrm{E}-01$ \\
\hline $0.125664 \mathrm{E}+03$ & $0.200000 E+02$ & 3 & $1.591020 E+03$ & $2.962687 \mathrm{E}-03$ & 1. $591020 E+03$ & $8.226947 \mathrm{E}-02$ \\
\hline $0.125664 \mathrm{E}+03$ & $0.200000 E+02$ & 4 & $1.863024 \mathrm{E}+01$ & $-9.000000 E+01$ & $-5.954440 E-07$ & $-1.863024 \mathrm{E}+01$ \\
\hline $0.125664 \mathrm{E}+03$ & $0.200000 E+02$ & 5 & $5.118615 E+01$ & $9.000000 \mathrm{E}+01$ & $1.635966 \mathrm{E}-06$ & $5.118615 \mathrm{E}+01$ \\
\hline $0.125664 \mathrm{E}+03$ & $0.200000 E+02$ & 6 & $6.214594 \mathrm{E}-08$ & $5.072097 \mathrm{E}-08$ & $6.214594 \mathrm{E}-08$ & $5.501456 \mathrm{E}-17$ \\
\hline $0.125664 \mathrm{E}+03$ & $0.300000 E+02$ & 1 & $1.717387 E+00$ & $9.000000 \mathrm{E}+01$ & $5.488958 \mathrm{E}-08$ & $1.717387 \mathrm{E}+00$ \\
\hline $0.125664 \mathrm{E}+03$ & $0.300000 E+02$ & 2 & $9.915367 E-01$ & $9.000000 \mathrm{E}+01$ & $3.169064 \mathrm{E}-08$ & $9.915367 \mathrm{E}-01$ \\
\hline $0.125664 E+03$ & $0.300000 E+02$ & 3 & $1.591020 E+03$ & $2.962686 \mathrm{E}-03$ & $1.591020 E+03$ & $8.226946 \mathrm{E}-02$ \\
\hline $0.125664 \mathrm{E}+03$ & $0.300000 E+02$ & 4 & $2.723551 \mathrm{E}+01$ & $-9.000000 E+01$ & $-8.704816 \mathrm{E}-07$ & $-2.723551 E+01$ \\
\hline $0.125664 \mathrm{E}+03$ & $0.300000 E+02$ & 5 & $4.717344 \mathrm{E}+01$ & $9.000000 \mathrm{E}+01$ & $1.507716 \mathrm{E}-06$ & $4.717344 \mathrm{E}+01$ \\
\hline $0.125664 \mathrm{E}+03$ & $0.300000 E+02$ & 6 & $6.074003 E-08$ & $6.990674 \mathrm{E}-08$ & $6.074003 E-08$ & $7.410908 \mathrm{E}-17$ \\
\hline $0.125664 \mathrm{E}+03$ & $0.400000 E+02$ & 1 & $1.519122 \mathrm{E}+00$ & $9.000000 \mathrm{E}+01$ & $4.855279 E-08$ & 1. $519122 \mathrm{E}+00$ \\
\hline $0.125664 E+03$ & $0.400000 E+02$ & 2 & 1. $274695 \mathrm{E}+00$ & $9.000000 E+01$ & $4.074073 E-08$ & 1. $274695 \mathrm{E}+00$ \\
\hline $0.125664 \mathrm{E}+03$ & $0.400000 E+02$ & 3 & $1.591020 E+03$ & $2.962686 \mathrm{E}-03$ & 1. $591020 E+03$ & $8.226946 \mathrm{E}-02$ \\
\hline $0.125664 \mathrm{E}+03$ & $0.400000 E+02$ & 4 & $3.501334 E+01$ & $-9.000000 E+01$ & $-1.119071 E-06$ & $-3.501334 \mathrm{E}+01$ \\
\hline $0.125664 \mathrm{E}+03$ & $0.400000 \mathrm{E}+02$ & 5 & $4.172728 \mathrm{E}+01$ & $9.000000 \mathrm{E}+01$ & $1.333655 \mathrm{E}-06$ & $4.172728 \mathrm{E}+01$ \\
\hline $0.125664 \mathrm{E}+03$ & $0.400000 E+02$ & 6 & $3.637167 E-08$ & $1.327815 \mathrm{E}-07$ & $3.637167 \mathrm{E}-08$ & $8.429042 \mathrm{E}-17$ \\
\hline $0.125664 \mathrm{E}+03$ & $0.500000 E+02$ & 1 & 1. $274693 E+00$ & $9.000000 \mathrm{E}+01$ & $4.074062 E-08$ & 1. $274693 \mathrm{E}+00$ \\
\hline $0.125664 \mathrm{E}+03$ & $0.500000 E+02$ & 2 & $1.519122 \mathrm{E}+00$ & $9.000000 \mathrm{E}+01$ & $4.855287 E-08$ & $1.519122 \mathrm{E}+00$ \\
\hline $0.125664 E+03$ & $0.500000 E+02$ & 3 & $1.591020 E+03$ & $2.962686 \mathrm{E}-03$ & $1.591020 E+03$ & $8.226947 E-02$ \\
\hline $0.125664 E+03$ & $0.500000 E+02$ & 4 & $4.172725 E+01$ & $-9.000000 E+01$ & $-1.333656 E-06$ & $-4.172725 E+01$ \\
\hline $0.125664 \mathrm{E}+03$ & $0.500000 E+02$ & 5 & $3.501334 \mathrm{E}+01$ & $9.000000 \mathrm{E}+01$ & 1.119070E-06 & $3.501334 \mathrm{E}+01$ \\
\hline $0.125664 \mathrm{E}+03$ & $0.500000 E+02$ & 6 & 4.064337E-09 & $1.188252 \mathrm{E}-06$ & 4.064337E-09 & $8.428993 E-17$ \\
\hline $0.125664 \mathrm{E}+03$ & $0.600000 E+02$ & 1 & $9.915339 \mathrm{E}-01$ & $9.000000 \mathrm{E}+01$ & $3.169052 \mathrm{E}-08$ & $9.915339 \mathrm{E}-01$ \\
\hline $0.125664 \mathrm{E}+03$ & $0.600000 E+02$ & 2 & 1. $717391 \mathrm{E}+00$ & $9.000000 \mathrm{E}+01$ & $5.488981 \mathrm{E}-08$ & $1.717391 \mathrm{E}+00$ \\
\hline $0.125664 \mathrm{E}+03$ & $0.600000 E+02$ & 3 & $1.591020 E+03$ & $2.962686 \mathrm{E}-03$ & $1.591020 E+03$ & $8.226947 E-02$ \\
\hline $0.125664 E+03$ & $0.600000 E+02$ & 4 & $4.717342 E+01$ & $-9.000000 E+01$ & $-1.507717 E-06$ & $-4.717342 E+01$ \\
\hline $0.125664 \mathrm{E}+03$ & $0.600000 E+02$ & 5 & $2.723554 \mathrm{E}+01$ & $9.000000 \mathrm{E}+01$ & $8.704814 \mathrm{E}-07$ & $2.723554 \mathrm{E}+01$ \\
\hline $0.125664 \mathrm{E}+03$ & $0.600000 E+02$ & 6 & $1.997914 \mathrm{E}-08$ & $1.800000 \mathrm{E}+02$ & $-1.997914 \mathrm{E}-08$ & $7.410738 \mathrm{E}-17$ \\
\hline $0.125664 \mathrm{E}+03$ & $0.700000 E+02$ & 1 & $6.782510 E-01$ & $9.000000 \mathrm{E}+01$ & $2.167763 E-08$ & $6.782510 E-01$ \\
\hline $0.125664 \mathrm{E}+03$ & $0.700000 E+02$ & 2 & $1.863477 \mathrm{E}+00$ & $9.000000 \mathrm{E}+01$ & $5.955889 E-08$ & $1.863477 \mathrm{E}+00$ \\
\hline $0.125664 \mathrm{E}+03$ & $0.700000 E+02$ & 3 & 1. $591020 E+03$ & $2.962686 \mathrm{E}-03$ & $1.591020 E+03$ & $8.226948 \mathrm{E}-02$ \\
\hline $0.125664 E+03$ & $0.700000 E+02$ & 4 & $5.118610 E+01$ & $-9.000000 E+01$ & $-1.635968 E-06$ & $-5.118610 E+01$ \\
\hline $0.125664 E+03$ & $0.700000 E+02$ & 5 & $1.863021 \mathrm{E}+01$ & $9.000000 E+01$ & $5.954436 \mathrm{E}-07$ & $1.863021 E+01$ \\
\hline $0.125664 E+03$ & $0.700000 E+02$ & 6 & $1.993885 \mathrm{E}-08$ & $1.800000 \mathrm{E}+02$ & $-1.993885 E-08$ & $5.501388 \mathrm{E}-17$ \\
\hline $0.125664 \mathrm{E}+03$ & $0.800000 E+02$ & 1 & $3.443569 \mathrm{E}-01$ & $9.000000 \mathrm{E}+01$ & $1.100603 \mathrm{E}-08$ & $3.443569 E-01$ \\
\hline $0.125664 \mathrm{E}+03$ & $0.800000 \mathrm{E}+02$ & 2 & $1.952945 \mathrm{E}+00$ & $9.000000 \mathrm{E}+01$ & $6.241831 E-08$ & $1.952945 \mathrm{E}+00$ \\
\hline $0.125664 \mathrm{E}+03$ & $0.800000 E+02$ & 3 & $1.591021 \mathrm{E}+03$ & $2.962685 E-03$ & 1. $591021 \mathrm{E}+03$ & $8.226947 \mathrm{E}-02$ \\
\hline $0.125664 \mathrm{E}+03$ & $0.800000 E+02$ & 4 & $5.364361 \mathrm{E}+01$ & $-9.000000 \mathrm{E}+01$ & $-1.714512 \mathrm{E}-06$ & $-5.364361 E+01$ \\
\hline $0.125664 \mathrm{E}+03$ & $0.800000 E+02$ & 5 & $9.458814 \mathrm{E}+00$ & $9.000000 \mathrm{E}+01$ & $3.023147 E-07$ & $9.458814 \mathrm{E}+00$ \\
\hline $0.125664 \mathrm{E}+03$ & $0.800000 E+02$ & 6 & $1.799070 \mathrm{E}-08$ & $1.800000 \mathrm{E}+02$ & $-1.799070 E-08$ & $2.927141 \mathrm{E}-17$ \\
\hline $0.125664 \mathrm{E}+03$ & $0.900000 E+02$ & 1 & $2.412994 \mathrm{E}-08$ & $9.000000 \mathrm{E}+01$ & $7.712198 \mathrm{E}-16$ & $2.412994 \mathrm{E}-08$ \\
\hline $0.125664 \mathrm{E}+03$ & $0.900000 E+02$ & 2 & $1.983073 E+00$ & $9.000000 \mathrm{E}+01$ & $6.338124 \mathrm{E}-08$ & $1.983073 \mathrm{E}+00$ \\
\hline $0.125664 \mathrm{E}+03$ & $0.900000 E+02$ & 3 & $1.591021 \mathrm{E}+03$ & $2.962686 \mathrm{E}-03$ & 1. $591021 \mathrm{E}+03$ & $8.226948 \mathrm{E}-02$ \\
\hline $0.125664 \mathrm{E}+03$ & $0.900000 E+02$ & 4 & $5.447108 \mathrm{E}+01$ & $-9.000000 E+01$ & $-1.740961 E-06$ & $-5.447108 \mathrm{E}+01$ \\
\hline $0.125664 \mathrm{E}+03$ & $0.900000 E+02$ & 5 & $6.628035 E-07$ & $9.000000 \mathrm{E}+01$ & $2.118402 \mathrm{E}-14$ & $6.628035 E-07$ \\
\hline $0.125664 E+03$ & $0.900000 \mathrm{E}+02$ & 6 & 1. $249348 \mathrm{E}-15$ & $9.000000 \mathrm{E}+01$ & $-1.249348 \mathrm{E}-15$ & $2.082531 E-24$ \\
\hline $0.125664 E+03$ & $0.100000 E+03$ & 1 & $3.443568 \mathrm{E}-01$ & $-9.000000 E+01$ & $-1.100603 E-08$ & $-3.443568 E-01$ \\
\hline $0.125664 \mathrm{E}+03$ & $0.100000 E+03$ & 2 & $1.952945 E+00$ & $9.000000 \mathrm{E}+01$ & $6.241831 \mathrm{E}-08$ & $1.952945 E+00$ \\
\hline $0.125664 \mathrm{E}+03$ & $0.100000 E+03$ & 3 & $1.591021 \mathrm{E}+03$ & $2.962685 E-03$ & $1.591021 \mathrm{E}+03$ & $8.226947 E-02$ \\
\hline $0.125664 \mathrm{E}+03$ & $0.100000 E+03$ & 4 & $5.364361 \mathrm{E}+01$ & $-9.000000 \mathrm{E}+01$ & $-1.714512 \mathrm{E}-06$ & $-5.364361 E+01$ \\
\hline $0.125664 \mathrm{E}+03$ & $0.100000 E+03$ & 5 & $9.458813 E+00$ & $-9.000000 E+01$ & $-3.023147 E-07$ & $-9.458813 E+00$ \\
\hline $0.125664 \mathrm{E}+03$ & $0.100000 E+03$ & 6 & $1.732498 \mathrm{E}-08$ & $-9.680328 E-08$ & $1.732498 \mathrm{E}-08$ & $-2.927118 \mathrm{E}-17$ \\
\hline $0.125664 \mathrm{E}+03$ & $0.110000 E+03$ & 1 & $6.782510 \mathrm{E}-01$ & $-9.000000 E+01$ & $-2.167763 E-08$ & $-6.782510 E-01$ \\
\hline $0.125664 \mathrm{E}+03$ & $0.110000 E+03$ & 2 & $1.863477 \mathrm{E}+00$ & $9.000000 \mathrm{E}+01$ & $5.955889 \mathrm{E}-08$ & $1.863477 \mathrm{E}+00$ \\
\hline $0.125664 \mathrm{E}+03$ & $0.110000 E+03$ & 3 & $1.591020 E+03$ & $2.962686 \mathrm{E}-03$ & $1.591020 \mathrm{E}+03$ & $8.226948 \mathrm{E}-02$ \\
\hline $0.125664 \mathrm{E}+03$ & $0.110000 E+03$ & 4 & $5.118610 E+01$ & $-9.000000 E+01$ & $-1.635968 E-06$ & $-5.118610 E+01$ \\
\hline
\end{tabular}




\begin{tabular}{|c|c|c|c|c|c|c|}
\hline $0.125664 \mathrm{E}+03$ & $0.110000 E+03$ & 5 & $1.863021 \mathrm{E}+01$ & $-9.000000 \mathrm{E}+01$ & $-5.954436 E-07$ & $-1.863021 E+01$ \\
\hline $0.125664 E+03$ & $0.110000 E+03$ & 6 & $1.964059 \mathrm{E}-08$ & $-1.604866 E-07$ & $1.964059 \mathrm{E}-08$ & $-5.501368 E-17$ \\
\hline $125664 \mathrm{E}+03$ & $0.120000 \mathrm{E}+03$ & 1 & $9.915339 \mathrm{E}-01$ & $-9.000000 E+01$ & $-3.169052 E-08$ & $-9.915339 E-01$ \\
\hline $0.125664 \mathrm{E}+03$ & $0.120000 \mathrm{E}+03$ & 2 & $1.717391 \mathrm{E}+00$ & $9.000000 \mathrm{E}+01$ & $5.488981 \mathrm{E}-08$ & $1.717391 \mathrm{E}+00$ \\
\hline $0.125664 \mathrm{E}+03$ & $0.120000 \mathrm{E}+03$ & 3 & $1.591020 \mathrm{E}+03$ & $2.962686 \mathrm{E}-03$ & $1.591020 \mathrm{E}+03$ & $8.226947 E-02$ \\
\hline $0.125664 \mathrm{E}+03$ & $0.120000 E+03$ & 4 & $4.717342 \mathrm{E}+01$ & $-9.000000 \mathrm{E}+01$ & $-1.507717 E-06$ & $-4.717342 E+01$ \\
\hline $0.125664 \mathrm{E}+03$ & $0.120000 \mathrm{E}+03$ & 5 & $2.723554 \mathrm{E}+01$ & $-9.000000 \mathrm{E}+01$ & $-8.704814 E-07$ & $-2.723554 E+01$ \\
\hline $0.125664 E+03$ & $0.120000 E+03$ & 6 & $1.997914 \mathrm{E}-08$ & $-2.125237 E-07$ & $1.997914 \mathrm{E}-08$ & $-7.410738 \mathrm{E}-17$ \\
\hline $0.125664 \mathrm{E}+03$ & $0.130000 \mathrm{E}+03$ & 1 & $1.274693 \mathrm{E}+00$ & $-9.000000 E+01$ & $-4.074061 E-08$ & $-1.274693 E+00$ \\
\hline $0.125664 \mathrm{E}+03$ & $0.130000 \mathrm{E}+03$ & 2 & $1.519122 \mathrm{E}+00$ & $9.000000 \mathrm{E}+01$ & $4.855287 E-08$ & $1.519122 \mathrm{E}+00$ \\
\hline $0.125664 \mathrm{E}+03$ & $0.130000 E+03$ & 3 & $1.591020 \mathrm{E}+03$ & $2.962686 \mathrm{E}-03$ & $1.591020 E+03$ & $8.226947 \mathrm{E}-02$ \\
\hline $0.125664 \mathrm{E}+03$ & $0.130000 \mathrm{E}+03$ & 4 & $4.172725 \mathrm{E}+01$ & $-9.000000 \mathrm{E}+01$ & $-1.333656 \mathrm{E}-06$ & $-4.172725 \mathrm{E}+01$ \\
\hline $0.125664 \mathrm{E}+03$ & $0.130000 \mathrm{E}+03$ & 5 & $3.501333 \mathrm{E}+01$ & $-9.000000 \mathrm{E}+01$ & $-1.119070 \mathrm{E}-06$ & $-3.501333 \mathrm{E}+01$ \\
\hline+03 & $0.130000 E+03$ & 6 & $664 \mathrm{E}-09$ & $-1.800000 \mathrm{E}+02$ & $-9.002664 \mathrm{E}-09$ & $-8.428979 E-17$ \\
\hline $0.125664 \mathrm{E}+03$ & 0.140 & 1 & $122 \mathrm{E}+00$ & $-9.000000 \mathrm{E}+01$ & E-08 & $-1.519122 \mathrm{E}+00$ \\
\hline $0.125664 \mathrm{E}+03$ & $0.140000 \mathrm{E}+03$ & 2 & $1695 E+00$ & $E+01$ & .08 & $E+00$ \\
\hline 0.125 & 0.14 & 3 & & & +03 & \\
\hline 0.12 & 0.1 & 4 & +01 & -9.0 & $E-06$ & -3.5 \\
\hline $0.125664 \mathrm{E}+03$ & $0.140000 \mathrm{E}+03$ & 5 & $4.172728 \mathrm{E}+01$ & -9.00 & $-1.333655 E-06$ & $-4.172728 \mathrm{E}+01$ \\
\hline 0.1256 & $0.140000 E+03$ & 6 & $3.637167 \mathrm{E}-08$ & -1.8000 & -3.637 & $-8.429042 E-17$ \\
\hline $0.125664 \mathrm{E}+03$ & $0.150000 E+03$ & 1 & $387 E+00$ & -9.00 & -5.488958 & $-1.717387 E+00$ \\
\hline $0.125664 \mathrm{E}+03$ & $0.150000 \mathrm{E}+03$ & 2 & $9.915367 \mathrm{E}-01$ & $9.000000 \mathrm{E}+01$ & $3.169064 \mathrm{E}-08$ & $9.915367 \mathrm{E}-01$ \\
\hline $0.125664 \mathrm{E}+03$ & $0.150000 \mathrm{E}+03$ & 3 & $1.591020 \mathrm{E}+03$ & $2.962686 \mathrm{E}-03$ & $1.591020 \mathrm{E}+03$ & $8.226946 \mathrm{E}-02$ \\
\hline $0.125664 \mathrm{E}+03$ & $0.150000 \mathrm{E}+03$ & 4 & $2.723551 \mathrm{E}+01$ & $000 \mathrm{E}+01$ & $-8.704816 E-07$ & $-2.723551 \mathrm{E}+01$ \\
\hline $0.125664 \mathrm{E}+03$ & 0.1506 & 5 & $4.717344 \mathrm{E}+01$ & $000 E+01$ & $-1.507716 \mathrm{E}-06$ & $-4.717344 \mathrm{E}+01$ \\
\hline 0.125 & $E+03$ & 6 & $33 E-08$ & $E+02$ & -6.6 & $-7.410908 \mathrm{E}-17$ \\
\hline 0.125 & $\mathrm{E}+03$ & 1 & $3475 E+00$ & $\mathrm{E}+01$ & $99-08$ & $3475 E+00$ \\
\hline $\mathrm{E}+03$ & $\mathrm{E}+03$ & 2 & $4 \mathrm{E}-01$ & $E+01$ & $3 E-08$ & $4 \mathrm{E}-01$ \\
\hline+03 & $E+03$ & 3 & $E+03$ & -03 & +03 & $E-02$ \\
\hline 0.12 & 0.16 & 4 & 01 & $E+01$ & $E-07$ & $4 \mathrm{E}+01$ \\
\hline 0.125 & 0.160 & 5 & $5 \mathrm{E}+01$ & -9.6 & -1.6 & $5 E+01$ \\
\hline 0.1256 & 0.160 & 6 & $4 E-08$ & -1.8 & -6.2 & $6 \mathrm{E}-17$ \\
\hline $0.125664 \mathrm{E}+03$ & 0.170 & 1 & $1.952940 \mathrm{E}+00$ & $-9.000000 \mathrm{E}+01$ & $1 E-08$ & $\theta E+00$ \\
\hline $0.125664 \mathrm{E}+03$ & $0.170000 E+03$ & 2 & $3574 E-01$ & 9.00000 & 1.100605 & $3.443574 \mathrm{E}-01$ \\
\hline $0.125664 \mathrm{E}+03$ & $0.170000 \mathrm{E}+03$ & 3 & $1.591020 \mathrm{E}+03$ & $2.962686 \mathrm{E}-03$ & $1.591020 E+03$ & $8.226946 \mathrm{E}-02$ \\
\hline $0.125664 \mathrm{E}+03$ & $0.170000 \mathrm{E}+03$ & 4 & $9.458789 \mathrm{E}+00$ & $-9.000000 \mathrm{E}+01$ & $52 E-07$ & $-9.458789 E+00$ \\
\hline $0.125664 \mathrm{E}+03$ & $0.170000 \mathrm{E}+03$ & 5 & $59 E+01$ & $-9.000000 \mathrm{E}+01$ & $2 E-06$ & $9 \mathrm{E}+01$ \\
\hline $0.125664 \mathrm{E}+03$ & 0.170 & 6 & $4.453025 \mathrm{E}-08$ & $-1.800000 \mathrm{E}+02$ & -4.453 & $-2.927167 E-17$ \\
\hline 0.125 & 0.180 & 1 & $56 \mathrm{E}+00$ & -9.0 & -6.3 & -1.9 \\
\hline 0.125 & +03 & 2 & $6 \mathrm{E}-08$ & & & 08 \\
\hline 0.125 & +03 & 3 & $\partial E+03$ & & 03 & \\
\hline 03 & -03 & 4 & & -9 & -4 & -1 \\
\hline & 0.1 & 5 & 01 & -9.0 & -1.7 & $5 E+01$ \\
\hline 0.125 & 0.180 & 6 & $E-15$ & -9.0 & $\mathrm{E}-15$ & $30 E-24$ \\
\hline 0.628 & -0.186 & 1 & $8 \mathrm{E}+00$ & -9.0 & E-05 & $58 \mathrm{E}+00$ \\
\hline $0.628319 \mathrm{E}+02$ & -0.180 & 2 & $74 E-07$ & $E+01$ & $E-13$ & $374 E-07$ \\
\hline $0.628319 E+02$ & -0.180 & 3 & $453 E+03$ & $E-02$ & $52 E+03$ & $050 E+00$ \\
\hline $0.628319 \mathrm{E}+02$ & $-0.180000 \mathrm{E}+03$ & 4 & $5.262239 \mathrm{E}-06$ & $8.999989 \mathrm{E}+01$ & $1.048258 \mathrm{E}-11$ & $5.262239 \mathrm{E}-06$ \\
\hline $0.628319 \mathrm{E}+02$ & $-0.180000 \mathrm{E}+03$ & 5 & $2.162391 \mathrm{E}+02$ & $-9.000011 \mathrm{E}+01$ & $-4.307330 \mathrm{E}-04$ & $-2.162391 \mathrm{E}+02$ \\
\hline $0.628319 \mathrm{E}+02$ & $-0.180000 \mathrm{E}+03$ & 6 & $1.158811 \mathrm{E}-12$ & $9.000000 \mathrm{E}+01$ & $1.158811 \mathrm{E}-12$ & $2.892725 \mathrm{E}-23$ \\
\hline $0.628319 E+02$ & $-0.170000 \mathrm{E}+03$ & 1 & $7.795320 \mathrm{E}+00$ & $-9.000011 E+01$ & $-1.552790 \mathrm{E}-05$ & $-7.795320 E+00$ \\
\hline $0.628319 \mathrm{E}+02$ & -0.17000 & 2 & 1. $374557 \mathrm{E}+00$ & -9.000 & $0 E-06$ & $-1.374557 \mathrm{E}+00$ \\
\hline $0.628319 \mathrm{E}+02$ & -0.170 & 3 & $453 E+03$ & 4.6 & $2 \mathrm{E}+03$ & $\theta \mathrm{E}+\theta \theta$ \\
\hline 0.628 & $-0.17 t$ & 4 & $856 \mathrm{E}+01$ & 8.9 & 7. & $6 \mathrm{E}+01$ \\
\hline $0.628319 \mathrm{E}+02$ & -0.170 & 5 & $2.129541 \mathrm{E}+02$ & -9.0 & $1 E-04$ & $E+02$ \\
\hline+02 & -0. & 6 & 7. & & 06 & 2 . \\
\hline & -0 . & 1 & 7. & -9. & -1 & -7 \\
\hline $9 \mathrm{E}+02$ & $-0.16 e$ & 2 & $E+00$ & -9.0 & -5.3 & $2 \mathrm{E}+00$ \\
\hline 0.6 & -0.16 & 3 & 1.5 & 4.6 & 1.5 & 1.2 \\
\hline $0.628319 E+02$ & -0.160 & 4 & $3 E+01$ & +01 & $5 E-04$ & $3 E+01$ \\
\hline $0.628319 E+02$ & $-0.160000 \mathrm{E}+03$ & 5 & $2.031979 \mathrm{E}+02$ & $-9.000011 E+01$ & $-4.047586 E-04$ & $-2.031979 E+02$ \\
\hline $0.628319 \mathrm{E}+02$ & $-0.160000 \mathrm{E}+03$ & 6 & $1.178375 \mathrm{E}-05$ & $2.250318 \mathrm{E}-09$ & $1.178375 \mathrm{E}-05$ & $4.628120 \mathrm{E}-16$ \\
\hline $0.628319 \mathrm{E}+02$ & $-0.150000 \mathrm{E}+03$ & 1 & $6.855124 \mathrm{E}+00$ & $-9.000011 \mathrm{E}+01$ & $-1.365508 \mathrm{E}-05$ & $-6.855124 \mathrm{E}+00$ \\
\hline $0.628319 E+02$ & $-0.150000 E+03$ & 2 & $3.957861 \mathrm{E}+00$ & $-9.000011 E+01$ & $-7.883886 E-06$ & $-3.957861 \mathrm{E}+00$ \\
\hline $0.628319 \mathrm{E}+02$ & $-0.150000 E+03$ & 3 & $1.563449 \mathrm{E}+03$ & 4.6580 & $1.563449 E+03$ & $1.271050 \mathrm{E}+00$ \\
\hline $0.628319 \mathrm{E}+02$ & $-0.150000 \mathrm{E}+03$ & 4 & $1.081174 \mathrm{E}+02$ & $8.999989 \mathrm{E}+01$ & $2.153707 E-04$ & $1.081174 \mathrm{E}+02$ \\
\hline $0.628319 E+02$ & $-0.150000 E+03$ & 5 & $1.872679 \mathrm{E}+02$ & $-9.000011 \mathrm{E}+01$ & $-3.730287 E-04$ & $-1.872679 \mathrm{E}+02$ \\
\hline $0.628319 \mathrm{E}+02$ & $-0.150000 \mathrm{E}+03$ & 6 & $1.025948 \mathrm{E}-05$ & $99 \mathrm{E}-09$ & $1.025948 \mathrm{E}-05$ & 4. $990625 E-16$ \\
\hline $19 \mathrm{E}+02$ & -0.14 & 1 & +00 & -9.0000 & $-1.207868 E-05$ & -6.0 \\
\hline $9 \mathrm{E}+02$ & $-0.140000 \mathrm{E}+03$ & 2 & $5.088108 \mathrm{E}+00$ & $-9.000011 \mathrm{E}+01$ & $-1.013529 E-05$ & $-5.088108 \mathrm{E}+00$ \\
\hline $0.628319 E+02$ & $-0.140000 \mathrm{E}+03$ & 3 & $51 E+03$ & 02 & $150 E+03$ & $49 E+00$ \\
\hline $0.628319 \mathrm{E}+02$ & $-0.140000 \mathrm{E}+03$ & 4 & 1. $389937 \mathrm{E}+02$ & $8.999989 \mathrm{E}+01$ & $2.768744 \mathrm{E}-04$ & 1. $389937 \mathrm{E}+02$ \\
\hline $0.628319 \mathrm{E}+02$ & -0.140 & 5 & $1.656466 \mathrm{E}+02$ & $-9.000011 \mathrm{E}+01$ & $-3.299649 E-04$ & $-1.656466 \mathrm{E}+02$ \\
\hline $0.628319 E+02$ & $-0.140000 \mathrm{E}+03$ & 6 & $4.580391 \mathrm{E}-06$ & $8.884777 \mathrm{E}-09$ & $4.580391 \mathrm{E}-06$ & $7.102749 E-16$ \\
\hline $0.628319 \mathrm{E}+02$ & $-0.130000 E+03$ & 1 & $5.088099 \mathrm{E}+00$ & $-9.000011 E+01$ & $-1.013527 E-05$ & $-5.088099 E+00$ \\
\hline $0.628319 \mathrm{E}+02$ & $-0.130000 \mathrm{E}+03$ & 2 & $6.063747 \mathrm{E}+00$ & $-9.000011 \mathrm{E}+01$ & $-1.207870 \mathrm{E}-05$ & $-6.063747 E+00$ \\
\hline $0.628319 E+02$ & $-0.130000 E+03$ & 3 & $1.563450 \mathrm{E}+03$ & $4.658020 E-02$ & $1.563450 E+03$ & 1. $271050 \mathrm{E}+00$ \\
\hline $0.628319 E+02$ & $-0.130000 \mathrm{E}+03$ & 4 & $1.656468 \mathrm{E}+02$ & 8.999989 & $3.299647 E-04$ & $1.656468 \mathrm{E}+02$ \\
\hline $0.628319 \mathrm{E}+02$ & $-0.130000 \mathrm{E}+03$ & 5 & 1. $389937 \mathrm{E}+02$ & $-9.000011 \mathrm{E}+01$ & $-2.768741 E-04$ & $-1.389937 E+02$ \\
\hline $0.628319 E+02$ & $-0.130000 E+03$ & 6 & $3.366579 E-06$ & 1.800000 & $-3.366579 E-06$ & $7.103030 \mathrm{E}-16$ \\
\hline $0.628319 E+02$ & $-0.120000 E+03$ & 1 & $3.957853 \mathrm{E}+00$ & $-9.000011 \mathrm{E}+01$ & $-7.883853 E-06$ & $-3.957853 E+00$ \\
\hline $0.628319 \mathrm{E}+02$ & $-0.120000 \mathrm{E}+03$ & 2 & $6.855131 \mathrm{E}+00$ & $\mathrm{E}+01$ & $-1.365513 E-05$ & -6.855131 \\
\hline & $000 E+03$ & 3 & $148 E+03$ & $4.658024 \mathrm{E}-02$ & $1.563448 \mathrm{E}+03$ & 1. $271050 \mathrm{E}+00$ \\
\hline $028319 E+6$ & & & & & & \\
\hline
\end{tabular}
$\begin{array}{lllllll}0.628319 \mathrm{E}+02 & -0.120000 \mathrm{E}+03 & 4 & 1.872677 \mathrm{E}+02 & 8.999989 \mathrm{E}+01 & 3.730291 \mathrm{E}-04 & 1.872677 \mathrm{E}+02\end{array}$ 


\begin{tabular}{|c|c|c|c|c|c|c|}
\hline $0.628319 E+02$ & $-0.120000 E+03$ & 5 & $1.081174 \mathrm{E}+02$ & $-9.000011 E+01$ & $-2.153705 E-04$ & $-1.081174 \mathrm{E}+02$ \\
\hline $0.628319 E+02$ & $-0.120000 E+03$ & 6 & $9.737923 \mathrm{E}-06$ & $1.800000 \mathrm{E}+02$ & $-9.737923 E-06$ & $5.062944 \mathrm{E}-16$ \\
\hline $0.628319 \mathrm{E}+02$ & $-0.110000 \mathrm{E}+03$ & 1 & $2.707341 \mathrm{E}+00$ & $-9.000011 \mathrm{E}+01$ & $-5.392900 E-06$ & $-2.707341 \mathrm{E}+00$ \\
\hline $0.628319 E+02$ & $-0.110000 E+03$ & 2 & $7.438230 \mathrm{E}+00$ & $-9.000011 \mathrm{E}+01$ & $-1.481663 E-05$ & $-7.438230 E+00$ \\
\hline $0.628319 E+02$ & $-0.110000 \mathrm{E}+03$ & 3 & $1.563451 \mathrm{E}+03$ & $4.658015 \mathrm{E}-02$ & $1.563451 \mathrm{E}+03$ & $1.271050 \mathrm{E}+00$ \\
\hline $0.628319 E+02$ & $-0.110000 \mathrm{E}+03$ & 4 & $2.031977 \mathrm{E}+02$ & $8.999989 \mathrm{E}+01$ & $4.047584 \mathrm{E}-04$ & $2.031977 \mathrm{E}+02$ \\
\hline $0.628319 E+02$ & $-0.110000 \mathrm{E}+03$ & 5 & $7.395638 \mathrm{E}+01$ & $-9.000011 \mathrm{E}+01$ & $-1.473225 E-04$ & $-7.395638 \mathrm{E}+01$ \\
\hline $0.628319 E+02$ & $-0.110000 \mathrm{E}+03$ & 6 & 1.101663E-05 & $1.800000 \mathrm{E}+02$ & $-1.101663 E-05$ & $4.711616 \mathrm{E}-16$ \\
\hline $0.628319 E+02$ & $-0.100000 \mathrm{E}+03$ & 1 & $1.374556 \mathrm{E}+00$ & $-9.000011 \mathrm{E}+01$ & $-2.738055 E-06$ & $-1.374556 \mathrm{E}+00$ \\
\hline $0.628319 E+02$ & $-0.100000 \mathrm{E}+03$ & 2 & $7.795338 \mathrm{E}+00$ & $-9.000011 \mathrm{E}+01$ & $-1.552795 E-05$ & $-7.795338 E+00$ \\
\hline $0.628319 E+02$ & $-0.100000 \mathrm{E}+03$ & 3 & $1.563453 \mathrm{E}+03$ & $4.658010 \mathrm{E}-02$ & $1.563453 \mathrm{E}+03$ & $1.271050 \mathrm{E}+00$ \\
\hline $0.628319 E+02$ & $-0.100000 E+03$ & 4 & $2.129536 \mathrm{E}+02$ & $8.999989 E+01$ & $4.241898 E-04$ & $2.129536 E+02$ \\
\hline $0.628319 \mathrm{E}+02$ & $-0.100000 \mathrm{E}+03$ & 5 & $3.754859 \mathrm{E}+01$ & $-9.000011 \mathrm{E}+01$ & $-7.479772 \mathrm{E}-05$ & $-3.754859 E+01$ \\
\hline $0.628319 \mathrm{E}+02$ & $-0.100000 \mathrm{E}+03$ & 6 & $7.200288 \mathrm{E}-06$ & $1.800000 \mathrm{E}+02$ & $-7.200288 E-06$ & $2.515480 \mathrm{E}-16$ \\
\hline $0.628319 E+02$ & $-0.900000 \mathrm{E}+02$ & 1 & $9.631858 E-08$ & $9.000000 \mathrm{E}+01$ & 1.918623E-13 & $9.631858 E-08$ \\
\hline $0.628319 E+02$ & $-0.900000 \mathrm{E}+02$ & 2 & $7.915587 \mathrm{E}+00$ & $-9.000011 E+01$ & $-1.576749 E-05$ & $-7.915587 \mathrm{E}+00$ \\
\hline $0.628319 E+02$ & $-0.900000 \mathrm{E}+02$ & 3 & 1. $563453 \mathrm{E}+03$ & $4.658009 E-02$ & $1.563453 \mathrm{E}+03$ & $1.271050 \mathrm{E}+00$ \\
\hline $0.628319 E+02$ & $-0.900000 \mathrm{E}+02$ & 4 & $2.162391 \mathrm{E}+02$ & 8.999989E+01 & $4.307332 E-04$ & $2.162391 \mathrm{E}+02$ \\
\hline $0.628319 E+02$ & $-0.900000 \mathrm{E}+02$ & 5 & $2.631120 \mathrm{E}-06$ & $8.999989 \mathrm{E}+01$ & $5.241285 \mathrm{E}-12$ & $2.631120 \mathrm{E}-06$ \\
\hline $0.628319 E+02$ & $-0.900000 \mathrm{E}+02$ & 6 & $5.470291 \mathrm{E}-13$ & $-9.000000 \mathrm{E}+01$ & $5.470291 \mathrm{E}-13$ & $-1.498696 \mathrm{E}-23$ \\
\hline $0.628319 E+02$ & $-0.800000 \mathrm{E}+02$ & 1 & $1.374556 \mathrm{E}+00$ & $8.999989 E+01$ & $2.738056 \mathrm{E}-06$ & $1.374556 \mathrm{E}+00$ \\
\hline $0.628319 E+02$ & $-0.800000 \mathrm{E}+02$ & 2 & $7.795338 \mathrm{E}+00$ & $-9.000011 \mathrm{E}+01$ & $-1.552795 E-05$ & $-7.795338 E+00$ \\
\hline $0.628319 E+02$ & $-0.800000 \mathrm{E}+02$ & 3 & $1.563453 \mathrm{E}+03$ & $4.658010 \mathrm{E}-02$ & $1.563453 \mathrm{E}+03$ & $1.271050 \mathrm{E}+00$ \\
\hline $0.628319 E+02$ & $-0.800000 \mathrm{E}+02$ & 4 & $2.129536 \mathrm{E}+02$ & $8.999989 E+01$ & $4.241898 E-04$ & $2.129536 \mathrm{E}+02$ \\
\hline $0.628319 E+02$ & $-0.800000 \mathrm{E}+02$ & 5 & $3.754860 \mathrm{E}+01$ & $8.999989 E+01$ & 7.479774E-05 & $3.754860 \mathrm{E}+01$ \\
\hline $0.628319 E+02$ & $-0.800000 \mathrm{E}+02$ & 6 & 7.190672E-06 & $-1.999489 E-09$ & $7.190672 E-06$ & $-2.509377 E-16$ \\
\hline $0.628319 E+02$ & $-0.700000 \mathrm{E}+02$ & 1 & $2.707341 \mathrm{E}+00$ & 8.999989E+01 & $5.392901 \mathrm{E}-06$ & $2.707341 \mathrm{E}+00$ \\
\hline $0.628319 E+02$ & $-0.700000 \mathrm{E}+02$ & 2 & $7.438230 E+00$ & $-9.000011 E+01$ & $-1.481663 \mathrm{E}-05$ & $-7.438230 E+00$ \\
\hline $0.628319 E+02$ & $-0.700000 \mathrm{E}+02$ & 3 & $1.563451 \mathrm{E}+03$ & $4.658015 E-02$ & $1.563451 \mathrm{E}+03$ & $1.271050 \mathrm{E}+00$ \\
\hline $0.628319 E+02$ & $-0.700000 \mathrm{E}+02$ & 4 & $2.031977 E+02$ & $8.999989 \mathrm{E}+01$ & $4.047584 \mathrm{E}-04$ & $2.031977 \mathrm{E}+02$ \\
\hline $0.628319 E+02$ & $-0.700000 \mathrm{E}+02$ & 5 & $7.395638 \mathrm{E}+01$ & $8.999989 E+01$ & $1.473225 \mathrm{E}-04$ & $7.395638 \mathrm{E}+01$ \\
\hline $0.628319 E+02$ & $-0.700000 \mathrm{E}+02$ & 6 & $1.094496 \mathrm{E}-05$ & $-2.450852 E-09$ & $1.094496 \mathrm{E}-05$ & $-4.681757 E-16$ \\
\hline $0.628319 E+02$ & $-0.600000 \mathrm{E}+02$ & 1 & $3.957853 \mathrm{E}+00$ & $8.999989 \mathrm{E}+01$ & $7.883853 \mathrm{E}-06$ & $3.957853 \mathrm{E}+00$ \\
\hline $0.628319 E+02$ & $-0.600000 \mathrm{E}+02$ & 2 & $6.855131 \mathrm{E}+00$ & $-9.000011 \mathrm{E}+01$ & $-1.365513 E-05$ & $-6.855131 E+00$ \\
\hline $0.628319 E+02$ & $-0.600000 \mathrm{E}+02$ & 3 & $1.563448 \mathrm{E}+03$ & $4.658024 \mathrm{E}-02$ & $1.563448 \mathrm{E}+03$ & 1. $271050 \mathrm{E}+00$ \\
\hline $0.628319 E+02$ & $-0.600000 \mathrm{E}+02$ & 4 & $1.872677 \mathrm{E}+02$ & 8.999989E+01 & $3.730291 E-04$ & $1.872677 \mathrm{E}+02$ \\
\hline $0.628319 E+02$ & $-0.600000 \mathrm{E}+02$ & 5 & $1.081174 \mathrm{E}+02$ & 8.999989E+01 & $2.153705 E-04$ & 1. $081174 \mathrm{E}+02$ \\
\hline $0.628319 E+02$ & $-0.600000 \mathrm{E}+02$ & 6 & $9.737923 E-06$ & $-2.978924 \mathrm{E}-09$ & $9.737923 E-06$ & $-5.062944 E-16$ \\
\hline $0.628319 E+02$ & $-0.500000 \mathrm{E}+02$ & 1 & $5.088100 \mathrm{E}+00$ & $8.999989 E+01$ & 1.013527E-05 & $5.088100 \mathrm{E}+00$ \\
\hline $0.628319 E+02$ & $-0.500000 \mathrm{E}+02$ & 2 & $6.063747 \mathrm{E}+00$ & $-9.000011 \mathrm{E}+01$ & $-1.207870 E-05$ & $-6.063747 E+00$ \\
\hline $0.628319 E+02$ & $-0.500000 E+02$ & 3 & $1.563450 \mathrm{E}+03$ & $4.658020 \mathrm{E}-02$ & $1.563450 \mathrm{E}+03$ & 1. $271050 \mathrm{E}+00$ \\
\hline $0.628319 E+02$ & $-0.500000 \mathrm{E}+02$ & 4 & $1.656468 \mathrm{E}+02$ & $8.999989 E+01$ & 3.299647E-04 & $1.656468 \mathrm{E}+02$ \\
\hline $0.628319 E+02$ & $-0.500000 \mathrm{E}+02$ & 5 & 1. 389937E+02 & 8. $999989 E+01$ & $2.768741 \mathrm{E}-04$ & 1. $389937 \mathrm{E}+02$ \\
\hline $0.628319 E+02$ & $-0.500000 \mathrm{E}+02$ & 6 & $3.283122 \mathrm{E}-06$ & $-1.234711 \mathrm{E}-08$ & $3.283122 \mathrm{E}-06$ & $-7.075052 \mathrm{E}-16$ \\
\hline $0.628319 \mathrm{E}+02$ & $-0.400000 \mathrm{E}+02$ & 1 & $6.063741 \mathrm{E}+00$ & $8.999989 \mathrm{E}+01$ & $1.207868 \mathrm{E}-05$ & $6.063741 \mathrm{E}+00$ \\
\hline $0.628319 E+02$ & $-0.400000 \mathrm{E}+02$ & 2 & $5.088108 \mathrm{E}+00$ & $-9.000011 \mathrm{E}+01$ & $-1.013529 E-05$ & $-5.088108 \mathrm{E}+00$ \\
\hline $0.628319 \mathrm{E}+02$ & $-0.400000 \mathrm{E}+02$ & 3 & $1.563451 \mathrm{E}+03$ & $4.658015 \mathrm{E}-02$ & $1.563450 \mathrm{E}+03$ & 1. $271049 \mathrm{E}+00$ \\
\hline $0.628319 E+02$ & $-0.400000 \mathrm{E}+02$ & 4 & 1. 389937E+02 & $8.999989 E+01$ & $2.768744 E-04$ & 1. 389937E+02 \\
\hline $0.628319 E+02$ & $-0.400000 \mathrm{E}+02$ & 5 & $1.656466 \mathrm{E}+02$ & $8.999989 E+01$ & $3.299649 E-04$ & $1.656466 \mathrm{E}+02$ \\
\hline $0.628319 E+02$ & $-0.400000 \mathrm{E}+02$ & 6 & $4.580391 \mathrm{E}-06$ & $-1.800000 \mathrm{E}+02$ & $-4.580391 E-06$ & $-7.102749 E-16$ \\
\hline $0.628319 E+02$ & $-0.300000 \mathrm{E}+02$ & 1 & $6.855124 \mathrm{E}+00$ & 8.999989E+01 & $1.365508 \mathrm{E}-05$ & $6.855124 \mathrm{E}+00$ \\
\hline $0.628319 E+02$ & $-0.300000 \mathrm{E}+02$ & 2 & $3.957861 \mathrm{E}+00$ & $-9.000011 \mathrm{E}+01$ & $-7.883886 E-06$ & $-3.957861 E+00$ \\
\hline $0.628319 E+02$ & $-0.300000 \mathrm{E}+02$ & 3 & $1.563449 \mathrm{E}+03$ & $4.658022 E-02$ & $1.563449 E+03$ & $1.271050 \mathrm{E}+00$ \\
\hline $0.628319 E+02$ & $-0.300000 \mathrm{E}+02$ & 4 & 1.081174E+02 & $8.999989 \mathrm{E}+01$ & $2.153707 E-04$ & 1.081174E+02 \\
\hline $0.628319 \mathrm{E}+02$ & $-0.300000 E+02$ & 5 & $1.872679 \mathrm{E}+02$ & $8.999989 E+01$ & $3.730287 E-04$ & $1.872679 \mathrm{E}+02$ \\
\hline $0.628319 E+02$ & $-0.300000 \mathrm{E}+02$ & 6 & $1.025948 \mathrm{E}-05$ & $-1.800000 \mathrm{E}+02$ & $-1.025948 \mathrm{E}-05$ & $-4.990625 E-16$ \\
\hline $0.628319 E+02$ & $-0.200000 \mathrm{E}+02$ & 1 & $7.438222 E+00$ & $8.999989 E+01$ & $1.481658 \mathrm{E}-05$ & $7.438222 \mathrm{E}+00$ \\
\hline $0.628319 E+02$ & $-0.200000 \mathrm{E}+02$ & 2 & $2.707342 \mathrm{E}+00$ & $-9.000011 \mathrm{E}+01$ & $-5.392911 \mathrm{E}-06$ & $-2.707342 \mathrm{E}+00$ \\
\hline $0.628319 \mathrm{E}+02$ & $-0.200000 \mathrm{E}+02$ & 3 & $1.563451 \mathrm{E}+03$ & 4.658017E-02 & $1.563451 \mathrm{E}+03$ & $1.271050 \mathrm{E}+00$ \\
\hline $0.628319 E+02$ & $-0.200000 \mathrm{E}+02$ & 4 & 7.395633E+01 & $8.999989 E+01$ & $1.473225 \mathrm{E}-04$ & 7. $395633 E+01$ \\
\hline $0.628319 E+02$ & $-0.200000 \mathrm{E}+02$ & 5 & 2.031979E+02 & $8.999989 E+01$ & $4.047586 E-04$ & $2.031979 E+02$ \\
\hline $0.628319 E+02$ & $-0.200000 \mathrm{E}+02$ & 6 & $1.178375 \mathrm{E}-05$ & $-1.800000 \mathrm{E}+02$ & $-1.178375 E-05$ & $-4.628120 \mathrm{E}-16$ \\
\hline $0.628319 E+02$ & $-0.100000 \mathrm{E}+02$ & 1 & $7.795320 E+00$ & 8.999989E+01 & 1.552790E-05 & $7.795320 E+00$ \\
\hline $0.628319 E+02$ & $-0.100000 \mathrm{E}+02$ & 2 & $1.374557 \mathrm{E}+00$ & $-9.000011 \mathrm{E}+01$ & $-2.738060 \mathrm{E}-06$ & $-1.374557 \mathrm{E}+00$ \\
\hline $0.628319 E+02$ & $-0.100000 \mathrm{E}+02$ & 3 & $1.563453 E+03$ & $4.658012 E-02$ & $1.563452 \mathrm{E}+03$ & $1.271050 \mathrm{E}+00$ \\
\hline $0.628319 E+02$ & $-0.100000 \mathrm{E}+02$ & 4 & $3.754856 E+01$ & 8.999989E+01 & 7.479774E-05 & $3.754856 E+01$ \\
\hline $0.628319 E+02$ & $-0.100000 \mathrm{E}+02$ & 5 & $2.129541 \mathrm{E}+02$ & $8.999989 E+01$ & 4.241891E-04 & $2.129541 \mathrm{E}+02$ \\
\hline $0.628319 E+02$ & $-0.100000 \mathrm{E}+02$ & 6 & $7.794393 E-06$ & $-1.800000 \mathrm{E}+02$ & $-7.794393 E-06$ & $-2.503381 E-16$ \\
\hline $0.628319 E+02$ & $0.000000 \mathrm{E}+00$ & 1 & $7.915568 \mathrm{E}+00$ & 8. $999989 E+01$ & $1.576742 \mathrm{E}-05$ & $7.915568 \mathrm{E}+00$ \\
\hline $0.628319 E+02$ & $0.000000 \mathrm{E}+00$ & 2 & $0.000000 E+00$ & $9.000000 \mathrm{E}+01$ & $0.000000 \mathrm{E}+00$ & $0.000000 \mathrm{E}+00$ \\
\hline $0.628319 E+02$ & $0.000000 \mathrm{E}+00$ & 3 & $1.563453 E+03$ & $4.658011 \mathrm{E}-02$ & $1.563452 \mathrm{E}+03$ & 1. $271050 \mathrm{E}+00$ \\
\hline $0.628319 E+02$ & $0.000000 \mathrm{E}+00$ & 4 & $0.000000 E+00$ & $9.000000 \mathrm{E}+01$ & $0.000000 \mathrm{E}+00$ & $0.000000 \mathrm{E}+00$ \\
\hline $0.628319 E+02$ & $0.000000 \mathrm{E}+00$ & 5 & $2.162391 \mathrm{E}+02$ & $8.999989 \mathrm{E}+01$ & $4.307330 \mathrm{E}-04$ & $2.162391 \mathrm{E}+02$ \\
\hline $0.628319 E+02$ & $0.000000 \mathrm{E}+00$ & 6 & $0.000000 E+00$ & $9.000000 \mathrm{E}+01$ & $0.000000 \mathrm{E}+00$ & $0.000000 \mathrm{E}+00$ \\
\hline $0.628319 E+02$ & $0.100000 \mathrm{E}+02$ & 1 & $7.795320 E+00$ & $8.999989 E+01$ & $1.552790 \mathrm{E}-05$ & $7.795320 \mathrm{E}+00$ \\
\hline $0.628319 \mathrm{E}+02$ & $0.100000 \mathrm{E}+02$ & 2 & $1.374557 \mathrm{E}+00$ & $8.999989 E+01$ & $2.738060 E-06$ & $1.374557 \mathrm{E}+00$ \\
\hline $0.628319 E+02$ & $0.100000 \mathrm{E}+02$ & 3 & $1.563453 \mathrm{E}+03$ & $4.658012 \mathrm{E}-02$ & $1.563452 \mathrm{E}+03$ & $1.271050 \mathrm{E}+00$ \\
\hline $0.628319 E+02$ & $0.100000 \mathrm{E}+02$ & 4 & $3.754856 E+01$ & $-9.000011 \mathrm{E}+01$ & $-7.479774 E-05$ & $-3.754856 E+01$ \\
\hline $0.628319 E+02$ & $0.100000 \mathrm{E}+02$ & 5 & $2.129541 \mathrm{E}+02$ & $8.999989 E+01$ & 4.241891E-04 & $2.129541 \mathrm{E}+02$ \\
\hline $0.628319 E+02$ & $0.100000 \mathrm{E}+02$ & 6 & $7.794393 E-06$ & $1.840210 \mathrm{E}-09$ & 7.794393E-06 & $2.503381 \mathrm{E}-16$ \\
\hline $0.628319 E+02$ & $0.200000 \mathrm{E}+02$ & 1 & $7.438222 \mathrm{E}+00$ & 8.999989E+01 & $1.481658 \mathrm{E}-05$ & $7.438222 \mathrm{E}+00$ \\
\hline $0.628319 E+02$ & $0.200000 \mathrm{E}+02$ & 2 & $2.707342 \mathrm{E}+00$ & 8.999989E+01 & $5.392911 \mathrm{E}-06$ & $2.707342 \mathrm{E}+00$ \\
\hline $0.628319 E+02$ & $0.200000 \mathrm{E}+02$ & 3 & $1.563451 \mathrm{E}+03$ & $4.658017 \mathrm{E}-02$ & $1.563451 \mathrm{E}+03$ & 1. $271050 \mathrm{E}+00$ \\
\hline $0.628319 E+02$ & $0.200000 \mathrm{E}+02$ & 4 & $7.395633 E+01$ & $-9.000011 E+01$ & $-1.473225 E-04$ & $-7.395633 E+01$ \\
\hline
\end{tabular}




\begin{tabular}{|c|c|c|c|c|c|c|}
\hline $0.628319 \mathrm{E}+02$ & $0.200000 \mathrm{E}+02$ & 5 & $2.031979 E+02$ & $8.999989 \mathrm{E}+01$ & $4.047586 \mathrm{E}-04$ & $2.031979 E+02$ \\
\hline $0.628319 \mathrm{E}+02$ & $0.200000 \mathrm{E}+02$ & 6 & $1.178375 \mathrm{E}-05$ & $2.250318 \mathrm{E}-09$ & $1.178375 \mathrm{E}-05$ & $4.628120 \mathrm{E}-16$ \\
\hline $0.628319 E+02$ & $0.300000 \mathrm{E}+02$ & 1 & $6.855124 \mathrm{E}+00$ & $8.999989 E+01$ & 1. $365508 \mathrm{E}-05$ & $6.855124 \mathrm{E}+00$ \\
\hline $0.628319 E+02$ & $0.300000 \mathrm{E}+02$ & 2 & $3.957861 \mathrm{E}+00$ & $8.999989 E+01$ & $7.883886 \mathrm{E}-06$ & $3.957861 \mathrm{E}+00$ \\
\hline $0.628319 E+02$ & $0.300000 \mathrm{E}+02$ & 3 & $1.563449 \mathrm{E}+03$ & $4.658022 \mathrm{E}-02$ & 1. $563449 \mathrm{E}+03$ & 1. $271050 \mathrm{E}+00$ \\
\hline $0.628319 E+02$ & $0.300000 \mathrm{E}+02$ & 4 & 1. $081174 \mathrm{E}+02$ & $-9.000011 E+01$ & $-2.153707 E-04$ & $-1.081174 \mathrm{E}+02$ \\
\hline $0.628319 E+02$ & $0.300000 \mathrm{E}+02$ & 5 & $1.872679 \mathrm{E}+02$ & $8.999989 E+01$ & $3.730287 E-04$ & $1.872679 \mathrm{E}+02$ \\
\hline $0.628319 E+02$ & $0.300000 \mathrm{E}+02$ & 6 & $1.025948 \mathrm{E}-05$ & $2.787099 \mathrm{E}-09$ & $1.025948 \mathrm{E}-05$ & $4.990625 \mathrm{E}-16$ \\
\hline $0.628319 E+02$ & $0.400000 E+02$ & 1 & $6.063741 \mathrm{E}+00$ & $8.999989 E+01$ & 1. $207868 \mathrm{E}-05$ & $6.063741 \mathrm{E}+00$ \\
\hline $0.628319 E+02$ & $0.400000 E+02$ & 2 & $5.088108 \mathrm{E}+00$ & $8.999989 E+01$ & 1. $013529 E-05$ & $5.088108 \mathrm{E}+00$ \\
\hline $0.628319 E+02$ & $0.400000 E+02$ & 3 & $1.563451 \mathrm{E}+03$ & $4.658015 E-02$ & $1.563450 \mathrm{E}+03$ & 1. $271049 \mathrm{E}+00$ \\
\hline $0.628319 E+02$ & $0.400000 \mathrm{E}+02$ & 4 & 1. 389937E+02 & $-9.000011 \mathrm{E}+01$ & $-2.768744 E-04$ & $-1.389937 \mathrm{E}+02$ \\
\hline $0.628319 E+02$ & $0.400000 \mathrm{E}+02$ & 5 & $1.656466 \mathrm{E}+02$ & $8.999989 E+01$ & $3.299649 E-04$ & $1.656466 \mathrm{E}+02$ \\
\hline $0.628319 E+02$ & $0.400000 \mathrm{E}+02$ & 6 & $4.580391 E-06$ & $8.884777 E-09$ & $4.580391 E-06$ & $7.102749 \mathrm{E}-16$ \\
\hline $0.628319 E+02$ & $0.500000 \mathrm{E}+02$ & 1 & $5.088100 \mathrm{E}+00$ & $8.999989 E+01$ & 1.013527E-05 & $5.088100 \mathrm{E}+00$ \\
\hline $0.628319 \mathrm{E}+02$ & $0.500000 \mathrm{E}+02$ & 2 & $6.063747 \mathrm{E}+00$ & $8.999989 E+01$ & $1.207870 \mathrm{E}-05$ & $6.063747 \mathrm{E}+00$ \\
\hline $0.628319 E+02$ & $0.500000 \mathrm{E}+02$ & 3 & $1.563450 \mathrm{E}+03$ & $4.658020 \mathrm{E}-02$ & 1. $563450 \mathrm{E}+03$ & 1. $271050 \mathrm{E}+00$ \\
\hline $0.628319 E+02$ & $0.500000 \mathrm{E}+02$ & 4 & $1.656468 \mathrm{E}+02$ & $-9.000011 \mathrm{E}+01$ & $-3.299647 E-04$ & $-1.656468 \mathrm{E}+02$ \\
\hline $0.628319 E+02$ & $0.500000 E+02$ & 5 & 1. $389937 E+02$ & 8.999989E+01 & $2.768741 E-04$ & 1. $389937 \mathrm{E}+02$ \\
\hline $0.628319 E+02$ & $0.500000 \mathrm{E}+02$ & 6 & $3.283122 \mathrm{E}-06$ & $1.800000 \mathrm{E}+02$ & $-3.283122 E-06$ & $7.075052 \mathrm{E}-16$ \\
\hline $0.628319 E+02$ & $0.600000 \mathrm{E}+02$ & 1 & $3.957853 E+00$ & $8.999989 E+01$ & $7.883853 E-06$ & $3.957853 \mathrm{E}+00$ \\
\hline $0.628319 E+02$ & $0.600000 \mathrm{E}+02$ & 2 & $6.855131 \mathrm{E}+00$ & $8.999989 E+01$ & 1. $365513 \mathrm{E}-05$ & $6.855131 E+00$ \\
\hline $0.628319 E+02$ & $0.600000 \mathrm{E}+02$ & 3 & $1.563448 \mathrm{E}+03$ & $4.658024 \mathrm{E}-02$ & $1.563448 \mathrm{E}+03$ & 1. $271050 \mathrm{E}+00$ \\
\hline $0.628319 E+02$ & $0.600000 \mathrm{E}+02$ & 4 & $1.872677 \mathrm{E}+02$ & $-9.000011 \mathrm{E}+01$ & $-3.730291 E-04$ & $-1.872677 E+02$ \\
\hline $0.628319 E+02$ & $0.600000 \mathrm{E}+02$ & 5 & $1.081174 \mathrm{E}+02$ & $8.999989 E+01$ & $2.153705 E-04$ & $1.081174 \mathrm{E}+02$ \\
\hline $0.628319 E+02$ & $0.600000 \mathrm{E}+02$ & 6 & $9.737923 \mathrm{E}-06$ & $1.800000 \mathrm{E}+02$ & $-9.737923 E-06$ & $5.062944 \mathrm{E}-16$ \\
\hline $0.628319 E+02$ & $0.700000 \mathrm{E}+02$ & 1 & $2.707341 \mathrm{E}+00$ & $8.999989 E+01$ & $5.392901 E-06$ & $2.707341 \mathrm{E}+00$ \\
\hline $0.628319 E+02$ & $0.700000 \mathrm{E}+02$ & 2 & $7.438230 E+00$ & $8.999989 E+01$ & 1. $481663 \mathrm{E}-05$ & $7.438230 E+00$ \\
\hline $0.628319 E+02$ & $0.700000 \mathrm{E}+02$ & 3 & $1.563451 \mathrm{E}+03$ & $4.658015 E-02$ & $1.563451 \mathrm{E}+03$ & 1. $271050 \mathrm{E}+00$ \\
\hline $0.628319 E+02$ & $0.700000 \mathrm{E}+02$ & 4 & $2.031977 E+02$ & $-9.000011 E+01$ & $-4.047584 E-04$ & $-2.031977 E+02$ \\
\hline $0.628319 E+02$ & $0.700000 \mathrm{E}+02$ & 5 & $7.395638 \mathrm{E}+01$ & $8.999989 E+01$ & $1.473225 \mathrm{E}-04$ & $7.395638 \mathrm{E}+01$ \\
\hline $0.628319 E+02$ & $0.700000 \mathrm{E}+02$ & 6 & $1.094496 \mathrm{E}-05$ & $1.800000 \mathrm{E}+02$ & $-1.094496 E-05$ & 4. $681757 \mathrm{E}-16$ \\
\hline $0.628319 E+02$ & $0.800000 \mathrm{E}+02$ & 1 & $1.374556 \mathrm{E}+00$ & $8.999989 E+01$ & $2.738056 \mathrm{E}-06$ & $1.374556 \mathrm{E}+00$ \\
\hline $0.628319 E+02$ & $0.800000 \mathrm{E}+02$ & 2 & $7.795338 \mathrm{E}+00$ & $8.999989 E+01$ & $1.552795 \mathrm{E}-05$ & $7.795338 \mathrm{E}+00$ \\
\hline $0.628319 E+02$ & $0.800000 \mathrm{E}+02$ & 3 & $1.563453 \mathrm{E}+03$ & $4.658010 E-02$ & $1.563453 \mathrm{E}+03$ & 1. $271050 \mathrm{E}+00$ \\
\hline $0.628319 E+02$ & $0.800000 \mathrm{E}+02$ & 4 & $2.129536 \mathrm{E}+02$ & $-9.000011 E+01$ & $-4.241898 E-04$ & $-2.129536 \mathrm{E}+02$ \\
\hline $0.628319 E+02$ & $0.800000 \mathrm{E}+02$ & 5 & $3.754860 \mathrm{E}+01$ & 8.999989E+01 & 7.479774E-05 & $3.754860 \mathrm{E}+01$ \\
\hline $0.628319 E+02$ & $0.800000 \mathrm{E}+02$ & 6 & $7.190672 E-06$ & $1.800000 \mathrm{E}+02$ & $-7.190672 E-06$ & $2.509377 \mathrm{E}-16$ \\
\hline $0.628319 E+02$ & $0.900000 \mathrm{E}+02$ & 1 & $9.631858 \mathrm{E}-08$ & $9.000000 \mathrm{E}+01$ & $1.918623 \mathrm{E}-13$ & $9.631858 \mathrm{E}-08$ \\
\hline $0.628319 E+02$ & $0.900000 E+02$ & 2 & $7.915587 E+00$ & $8.999989 E+01$ & 1.576749E-05 & $7.915587 E+00$ \\
\hline $0.628319 E+02$ & $0.900000 \mathrm{E}+02$ & 3 & $1.563453 \mathrm{E}+03$ & 4.658009E-02 & 1. $563453 \mathrm{E}+03$ & 1. $271050 \mathrm{E}+00$ \\
\hline $0.628319 E+02$ & $0.900000 \mathrm{E}+02$ & 4 & $2.162391 \mathrm{E}+02$ & $-9.000011 E+01$ & $-4.307332 E-04$ & $-2.162391 E+02$ \\
\hline $0.628319 E+02$ & $0.900000 \mathrm{E}+02$ & 5 & $2.631120 \mathrm{E}-06$ & $8.999989 E+01$ & $5.241285 E-12$ & $2.631120 \mathrm{E}-06$ \\
\hline $0.628319 E+02$ & $0.900000 E+02$ & 6 & $5.470291 \mathrm{E}-13$ & $9.000000 \mathrm{E}+01$ & $-5.470291 E-13$ & $1.498696 \mathrm{E}-23$ \\
\hline $0.628319 E+02$ & $0.100000 \mathrm{E}+03$ & 1 & $1.374556 \mathrm{E}+00$ & $-9.000011 E+01$ & $-2.738055 E-06$ & $-1.374556 \mathrm{E}+00$ \\
\hline $0.628319 E+02$ & $0.100000 \mathrm{E}+03$ & 2 & $7.795338 \mathrm{E}+00$ & 8.999989E+01 & $1.552795 E-05$ & $7.795338 \mathrm{E}+00$ \\
\hline $0.628319 E+02$ & $0.100000 E+03$ & 3 & $1.563453 \mathrm{E}+03$ & $4.658010 \mathrm{E}-02$ & 1. $563453 \mathrm{E}+03$ & 1. $271050 \mathrm{E}+00$ \\
\hline $0.628319 E+02$ & $0.100000 \mathrm{E}+03$ & 4 & $2.129536 \mathrm{E}+02$ & $-9.000011 E+01$ & $-4.241898 E-04$ & $-2.129536 \mathrm{E}+02$ \\
\hline $0.628319 E+02$ & $0.100000 \mathrm{E}+03$ & 5 & $3.754859 \mathrm{E}+01$ & $-9.000011 E+01$ & $-7.479772 \mathrm{E}-05$ & $-3.754859 E+01$ \\
\hline $0.628319 E+02$ & $0.100000 E+03$ & 6 & $7.200288 \mathrm{E}-06$ & $-2.001675 E-09$ & $7.200288 E-06$ & $-2.515480 E-16$ \\
\hline $0.628319 E+02$ & $0.110000 E+03$ & 1 & $2.707341 \mathrm{E}+00$ & $-9.000011 \mathrm{E}+01$ & $-5.392900 E-06$ & $-2.707341 E+00$ \\
\hline $0.628319 E+02$ & $0.110000 \mathrm{E}+03$ & 2 & $7.438230 \mathrm{E}+00$ & 8.999989E+01 & $1.481663 \mathrm{E}-05$ & $7.438230 \mathrm{E}+00$ \\
\hline $0.628319 E+02$ & $0.110000 E+03$ & 3 & $1.563451 \mathrm{E}+03$ & 4.658015E-02 & $1.563451 \mathrm{E}+03$ & 1. $271050 \mathrm{E}+00$ \\
\hline $0.628319 E+02$ & $0.110000 \mathrm{E}+03$ & 4 & $2.031977 E+02$ & $-9.000011 E+01$ & $-4.047584 E-04$ & $-2.031977 E+02$ \\
\hline $0.628319 E+02$ & $0.110000 \mathrm{E}+03$ & 5 & $7.395638 \mathrm{E}+01$ & $-9.000011 E+01$ & $-1.473225 E-04$ & $-7.395638 E+01$ \\
\hline $0.628319 E+02$ & $0.110000 \mathrm{E}+03$ & 6 & 1. $101663 \mathrm{E}-05$ & $-2.450438 E-09$ & $1.101663 E-05$ & $-4.711616 E-16$ \\
\hline $0.628319 E+02$ & $0.120000 \mathrm{E}+03$ & 1 & $3.957853 \mathrm{E}+00$ & $-9.000011 E+01$ & $-7.883853 E-06$ & $-3.957853 E+00$ \\
\hline $0.628319 E+02$ & $0.120000 \mathrm{E}+03$ & 2 & $6.855131 \mathrm{E}+00$ & $8.999989 \mathrm{E}+01$ & $1.365513 \mathrm{E}-05$ & $6.855131 \mathrm{E}+00$ \\
\hline $0.628319 E+02$ & $0.120000 \mathrm{E}+03$ & 3 & $1.563448 \mathrm{E}+03$ & $4.658024 \mathrm{E}-02$ & 1. $563448 \mathrm{E}+03$ & 1. $271050 \mathrm{E}+00$ \\
\hline $0.628319 E+02$ & $0.120000 E+03$ & 4 & $1.872677 \mathrm{E}+02$ & $-9.000011 \mathrm{E}+01$ & $-3.730291 E-04$ & $-1.872677 E+02$ \\
\hline $0.628319 E+02$ & $0.120000 E+03$ & 5 & 1. $081174 \mathrm{E}+02$ & $-9.000011 \mathrm{E}+01$ & $-2.153705 E-04$ & $-1.081174 \mathrm{E}+02$ \\
\hline $0.628319 E+02$ & $0.120000 \mathrm{E}+03$ & 6 & $9.737923 E-06$ & $-2.978924 \mathrm{E}-09$ & $9.737923 \mathrm{E}-06$ & $-5.062944 E-16$ \\
\hline $0.628319 E+02$ & $0.130000 \mathrm{E}+03$ & 1 & $5.088099 E+00$ & $-9.000011 E+01$ & $-1.013527 E-05$ & $-5.088099 E+00$ \\
\hline $0.628319 E+02$ & $0.130000 E+03$ & 2 & $6.063747 \mathrm{E}+00$ & 8.999989E+01 & 1. $207870 \mathrm{E}-05$ & $6.063747 \mathrm{E}+00$ \\
\hline $0.628319 E+02$ & $0.130000 E+03$ & 3 & $1.563450 \mathrm{E}+03$ & $4.658020 E-02$ & $1.563450 \mathrm{E}+03$ & $1.271050 \mathrm{E}+00$ \\
\hline $0.628319 E+02$ & $0.130000 \mathrm{E}+03$ & 4 & $1.656468 \mathrm{E}+02$ & $-9.000011 \mathrm{E}+01$ & $-3.299647 E-04$ & $-1.656468 \mathrm{E}+02$ \\
\hline $0.628319 E+02$ & $0.130000 \mathrm{E}+03$ & 5 & 1. $389937 \mathrm{E}+02$ & $-9.000011 E+01$ & $-2.768741 E-04$ & $-1.389937 E+02$ \\
\hline $0.628319 E+02$ & $0.130000 \mathrm{E}+03$ & 6 & $3.366579 E-06$ & $-1.208864 \mathrm{E}-08$ & $3.366579 E-06$ & $-7.103030 \mathrm{E}-16$ \\
\hline $0.628319 E+02$ & $0.140000 \mathrm{E}+03$ & 1 & $6.063741 \mathrm{E}+00$ & $-9.000011 \mathrm{E}+01$ & $-1.207868 \mathrm{E}-05$ & $-6.063741 E+00$ \\
\hline $0.628319 E+02$ & $0.140000 E+03$ & 2 & $5.088108 \mathrm{E}+00$ & 8.999989E+01 & 1. $013529 \mathrm{E}-05$ & $5.088108 \mathrm{E}+00$ \\
\hline $0.628319 E+02$ & $0.140000 \mathrm{E}+03$ & 3 & $1.563451 \mathrm{E}+03$ & $4.658015 \mathrm{E}-02$ & $1.563450 \mathrm{E}+03$ & 1. $271049 \mathrm{E}+00$ \\
\hline $0.628319 E+02$ & $0.140000 \mathrm{E}+03$ & 4 & 1. $389937 \mathrm{E}+02$ & $-9.000011 E+01$ & $-2.768744 E-04$ & $-1.389937 E+02$ \\
\hline $0.628319 E+02$ & $0.140000 \mathrm{E}+03$ & 5 & 1. $656466 \mathrm{E}+02$ & $-9.000011 E+01$ & $-3.299649 E-04$ & $-1.656466 \mathrm{E}+02$ \\
\hline $0.628319 E+02$ & $0.140000 E+03$ & 6 & $4.580391 E-06$ & $-1.800000 \mathrm{E}+02$ & $-4.580391 E-06$ & $-7.102749 E-16$ \\
\hline $0.628319 E+02$ & $0.150000 \mathrm{E}+03$ & 1 & $6.855124 \mathrm{E}+00$ & $-9.000011 \mathrm{E}+01$ & $-1.365508 \mathrm{E}-05$ & $-6.855124 \mathrm{E}+00$ \\
\hline $0.628319 E+02$ & $0.150000 \mathrm{E}+03$ & 2 & $3.957861 \mathrm{E}+00$ & $8.999989 E+01$ & $7.883886 \mathrm{E}-06$ & $3.957861 \mathrm{E}+00$ \\
\hline $0.628319 E+02$ & $0.150000 E+03$ & 3 & $1.563449 \mathrm{E}+03$ & $4.658022 \mathrm{E}-02$ & 1. $563449 \mathrm{E}+03$ & 1. $271050 \mathrm{E}+00$ \\
\hline $0.628319 E+02$ & $0.150000 \mathrm{E}+03$ & 4 & 1. $081174 \mathrm{E}+02$ & $-9.000011 \mathrm{E}+01$ & $-2.153707 E-04$ & $-1.081174 \mathrm{E}+02$ \\
\hline $0.628319 E+02$ & $0.150000 \mathrm{E}+03$ & 5 & $1.872679 \mathrm{E}+02$ & $-9.000011 \mathrm{E}+01$ & $-3.730287 E-04$ & $-1.872679 E+02$ \\
\hline $0.628319 E+02$ & $0.150000 E+03$ & 6 & $1.025948 \mathrm{E}-05$ & $-1.800000 \mathrm{E}+02$ & $-1.025948 E-05$ & $-4.990625 E-16$ \\
\hline $0.628319 E+02$ & $0.160000 \mathrm{E}+03$ & 1 & $7.438222 \mathrm{E}+00$ & $-9.000011 E+01$ & $-1.481658 \mathrm{E}-05$ & $-7.438222 E+00$ \\
\hline $0.628319 E+02$ & $0.160000 \mathrm{E}+03$ & 2 & $2.707342 \mathrm{E}+00$ & $8.999989 E+01$ & $5.392911 E-06$ & $2.707342 \mathrm{E}+00$ \\
\hline $0.628319 E+02$ & $0.160000 \mathrm{E}+03$ & 3 & $1.563451 \mathrm{E}+03$ & $4.658017 \mathrm{E}-02$ & $1.563451 E+03$ & 1. $271050 \mathrm{E}+00$ \\
\hline $0.628319 E+02$ & $0.160000 \mathrm{E}+03$ & 4 & $7.395633 E+01$ & $-9.000011 E+01$ & $-1.473225 E-04$ & $-7.395633 E+01$ \\
\hline
\end{tabular}




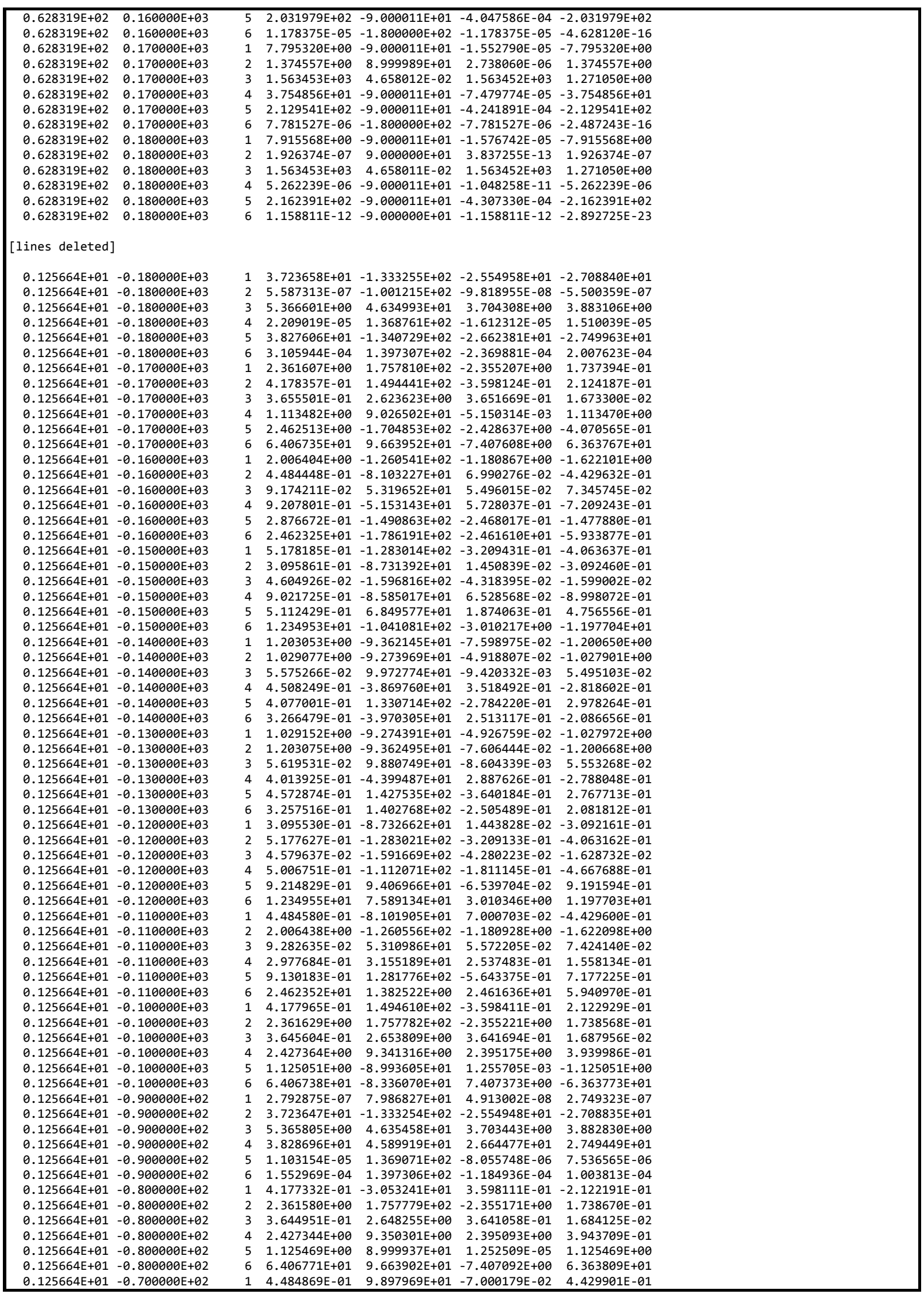


$0.125664 \mathrm{E}+01-0.700000 \mathrm{E}+02$ $0.125664 \mathrm{E}+01-0.700000 \mathrm{E}+02$ $0.125664 \mathrm{E}+01-0.700000 \mathrm{E}+02$ $0.125664 \mathrm{E}+01-0.700000 \mathrm{E}+02$ $0.125664 E+01-0.700000 E+02$ $0.125664 E+01-0.600000 E+02$ $0.125664 \mathrm{E}+01-0.600000 \mathrm{E}+02$ $0.125664 E+01-0.600000 E+02$ $0.125664 \mathrm{E}+01-0.600000 \mathrm{E}+02$ $0.125664 \mathrm{E}+01-0.600000 \mathrm{E}+02$ $0.125664 \mathrm{E}+01-0.600000 \mathrm{E}+02$ $0.125664 \mathrm{E}+01-0.500000 \mathrm{E}+02$ $0.125664 \mathrm{E}+01-0.500000 \mathrm{E}+02$ $0.125664 \mathrm{E}+01-0.500000 \mathrm{E}+02$ $0.125664 \mathrm{E}+01-0.500000 \mathrm{E}+02$ $0.125664 \mathrm{E}+01-0.500000 \mathrm{E}+02$ $0.125664 E+01-0.500000 E+02$ $0.125664 \mathrm{E}+01-0.400000 \mathrm{E}+02$ $0.125664 \mathrm{E}+01-0.400000 \mathrm{E}+02$ $0.125664 E+01-0.400000 E+02$ $0.125664 \mathrm{E}+01-0.400000 \mathrm{E}+02$ $0.125664 \mathrm{E}+01-0.400000 \mathrm{E}+02$ $0.125664 \mathrm{E}+01-0.400000 \mathrm{E}+02$ $0.125664 \mathrm{E}+01-0.300000 \mathrm{E}+02$ $0.125664 \mathrm{E}+01-0.300000 \mathrm{E}+02$ $0.125664 \mathrm{E}+01-0.300000 \mathrm{E}+02$ $0.125664 \mathrm{E}+01-0.300000 \mathrm{E}+02$ $0.125664 \mathrm{E}+01-0.300000 \mathrm{E}+02$ $0.125664 E+01-0.300000 E+02$ $0.125664 E+01-0.200000 E+02$ $0.125664 \mathrm{E}+01-0.200000 \mathrm{E}+02$ $0.125664 \mathrm{E}+01-0.200000 \mathrm{E}+02$ $0.125664 \mathrm{E}+01-0.200000 \mathrm{E}+02$ $0.125664 \mathrm{E}+01-0.200000 \mathrm{E}+02$ $0.125664 \mathrm{E}+01-0.200000 \mathrm{E}+02$ $0.125664 \mathrm{E}+01-0.100000 \mathrm{E}+02$ $0.125664 \mathrm{E}+01-0.100000 \mathrm{E}+02$ $0.125664 E+01-0.100000 E+02$ $0.125664 \mathrm{E}+01-0.100000 \mathrm{E}+02$ $0.125664 \mathrm{E}+01-0.100000 \mathrm{E}+02$ $0.125664 \mathrm{E}+01-0.100000 \mathrm{E}+02$ $0.125664 \mathrm{E}+01 \quad 0.000000 \mathrm{E}+00$ $0.125664 \mathrm{E}+01 \quad 0.000000 \mathrm{E}+00$ $0.125664 \mathrm{E}+01 \quad 0.000000 \mathrm{E}+00$ $0.125664 \mathrm{E}+01 \quad 0.000000 \mathrm{E}+00$ $0.125664 \mathrm{E}+01 \quad 0.000000 \mathrm{E}+00$ $0.125664 \mathrm{E}+01 \quad 0.000000 \mathrm{E}+00$ $0.125664 \mathrm{E}+01 \quad 0.100000 \mathrm{E}+02$ $0.125664 \mathrm{E}+01 \quad 0.100000 \mathrm{E}+02$ $0.125664 \mathrm{E}+01 \quad 0.100000 \mathrm{E}+02$ $0.125664 \mathrm{E}+01 \quad 0.100000 \mathrm{E}+02$ $0.125664 \mathrm{E}+01 \quad 0.100000 \mathrm{E}+02$ $0.125664 \mathrm{E}+01 \quad 0.100000 \mathrm{E}+02$ $0.125664 \mathrm{E}+01 \quad 0.200000 \mathrm{E}+02$ $0.125664 \mathrm{E}+01 \quad 0.200000 \mathrm{E}+02$ $0.125664 \mathrm{E}+01 \quad 0.200000 \mathrm{E}+02$ $0.125664 \mathrm{E}+01 \quad 0.200000 \mathrm{E}+02$ $0.125664 \mathrm{E}+01 \quad 0.200000 \mathrm{E}+02$ $0.125664 \mathrm{E}+01 \quad 0.200000 \mathrm{E}+02$ $0.125664 \mathrm{E}+01 \quad 0.300000 \mathrm{E}+02$ $0.125664 \mathrm{E}+01 \quad 0.300000 \mathrm{E}+02$ $0.125664 \mathrm{E}+01 \quad 0.300000 \mathrm{E}+02$ $0.125664 \mathrm{E}+01 \quad 0.300000 \mathrm{E}+02$ $0.125664 \mathrm{E}+01 \quad 0.300000 \mathrm{E}+02$ $0.125664 \mathrm{E}+01 \quad 0.300000 \mathrm{E}+02$ $0.125664 \mathrm{E}+01 \quad 0.400000 \mathrm{E}+02$ $0.125664 \mathrm{E}+01 \quad 0.400000 \mathrm{E}+02$ $0.125664 \mathrm{E}+01 \quad 0.400000 \mathrm{E}+02$ $0.125664 \mathrm{E}+01 \quad 0.400000 \mathrm{E}+02$ $0.125664 \mathrm{E}+01 \quad 0.400000 \mathrm{E}+02$ $0.125664 \mathrm{E}+01 \quad 0.400000 \mathrm{E}+02$ $0.125664 \mathrm{E}+01 \quad 0.500000 \mathrm{E}+02$ $0.125664 \mathrm{E}+01 \quad 0.500000 \mathrm{E}+02$ $0.125664 \mathrm{E}+01 \quad 0.500000 \mathrm{E}+02$ $0.125664 \mathrm{E}+01 \quad 0.500000 \mathrm{E}+02$ $0.125664 \mathrm{E}+01 \quad 0.500000 \mathrm{E}+02$ $0.125664 \mathrm{E}+01 \quad 0.500000 \mathrm{E}+02$ $0.125664 \mathrm{E}+01 \quad 0.600000 \mathrm{E}+02$ $0.125664 \mathrm{E}+01 \quad 0.600000 \mathrm{E}+02$ $0.125664 \mathrm{E}+01 \quad 0.600000 \mathrm{E}+02$ $0.125664 \mathrm{E}+01 \quad 0.600000 \mathrm{E}+02$ $0.125664 \mathrm{E}+01 \quad 0.600000 \mathrm{E}+02$ $0.125664 \mathrm{E}+01 \quad 0.600000 \mathrm{E}+02$ $0.125664 \mathrm{E}+01 \quad 0.700000 \mathrm{E}+02$
$2 \quad 2.006445 \mathrm{E}+00 \quad-1.260550 \mathrm{E}+02 \quad-1.180915 \mathrm{E}+00 \quad-1.622116 \mathrm{E}+00$

$\begin{array}{lllll}9.283326 \mathrm{E}-02 & 5.308544 \mathrm{E}+01 & 5.575783 \mathrm{E}-02 & 7.422317 \mathrm{E}-02\end{array}$

$4 \quad 2.975250 \mathrm{E}-01 \quad 3.139752 \mathrm{E}+01 \quad 2.539594 \mathrm{E}-01 \quad 1.550024 \mathrm{E}-01$

$5 \quad 9.139579 \mathrm{E}-01-5.182080 \mathrm{E}+01 \quad 5.649385 \mathrm{E}-01-7.184452 \mathrm{E}-01$

$6 \quad 2.462373 \mathrm{E}+01-1.786176 \mathrm{E}+02-2.461657 \mathrm{E}+01-5.940315 \mathrm{E}-01$

$1 \quad 3.095530 \mathrm{E}-01 \quad 9.267338 \mathrm{E}+01 \quad-1.443828 \mathrm{E}-02 \quad 3.092161 \mathrm{E}-01$

$2 \quad 5.177627 \mathrm{E}-01-1.283021 \mathrm{E}+02-3.209133 \mathrm{E}-01-4.063162 \mathrm{E}-01$

3 4.579637E-02 -1.591669E+02 -4.280223E-02 -1.628732E-02

$4 \quad 5.006751 \mathrm{E}-01-1.112071 \mathrm{E}+02-1.811145 \mathrm{E}-01-4.667688 \mathrm{E}-01$

$5 \quad 9.214829 \mathrm{E}-01-8.593034 \mathrm{E}+01 \quad 6.539704 \mathrm{E}-02 \quad-9.191594 \mathrm{E}-01$

$6 \quad 1.234955 \mathrm{E}+01-1.041087 \mathrm{E}+02-3.010346 \mathrm{E}+00-1.197703 \mathrm{E}+01$

$1 \quad 1.029144 \mathrm{E}+00 \quad 8.725648 \mathrm{E}+01 \quad 4.926003 \mathrm{E}-02 \quad 1.027964 \mathrm{E}+00$

$2 \quad 1.203070 \mathrm{E}+00-9.362509 \mathrm{E}+01-7.606713 \mathrm{E}-02-1.200663 \mathrm{E}+00$

$3 \quad 5.628183 \mathrm{E}-02 \quad 9.891718 \mathrm{E}+01 \quad-8.724050 \mathrm{E}-03 \quad 5.560157 \mathrm{E}-02$

$4 \quad 4.014029 \mathrm{E}-01-4.381311 \mathrm{E}+01 \quad 2.896531 \mathrm{E}-01-2.778945 \mathrm{E}-01$

$5 \quad 4.588585 \mathrm{E}-01-3.740073 \mathrm{E}+01 \quad 3.645203 \mathrm{E}-01-2.787042 \mathrm{E}-01$

$6 \quad 3.258539 \mathrm{E}-01-3.974574 \mathrm{E}+01 \quad 2.505456 \mathrm{E}-01-2.083450 \mathrm{E}-01$

$1 \quad 1.203053 \mathrm{E}+00 \quad 8.637856 \mathrm{E}+01 \quad 7.598975 \mathrm{E}-02 \quad 1.200650 \mathrm{E}+00$

$2 \quad 1.029077 \mathrm{E}+00-9.273969 \mathrm{E}+01-4.918807 \mathrm{E}-02-1.027901 \mathrm{E}+00$

$3 \quad 5.575266 \mathrm{E}-02 \quad 9.972774 \mathrm{E}+01 \quad-9.420332 \mathrm{E}-03 \quad 5.495103 \mathrm{E}-02$

$4 \quad 4.508249 \mathrm{E}-01-3.869760 \mathrm{E}+01 \quad 3.518492 \mathrm{E}-01-2.818602 \mathrm{E}-01$

$5 \quad 4.077001 \mathrm{E}-01-4.692863 \mathrm{E}+01 \quad 2.784220 \mathrm{E}-01-2.978264 \mathrm{E}-01$

$6 \quad 3.266479 \mathrm{E}-01 \quad 1.402970 \mathrm{E}+02-2.513117 \mathrm{E}-01 \quad 2.086656 \mathrm{E}-01$

$1 \quad 5.178185 \mathrm{E}-01 \quad 5.169857 \mathrm{E}+01 \quad 3.209431 \mathrm{E}-01 \quad 4.063637 \mathrm{E}-\theta 1$

$2 \quad 3.095861 \mathrm{E}-01-8.731392 \mathrm{E}+01 \quad 1.450839 \mathrm{E}-02-3.092460 \mathrm{E}-01$

$3 \quad 4.604926 \mathrm{E}-02-1.596816 \mathrm{E}+02-4.318395 \mathrm{E}-02-1.599002 \mathrm{E}-02$

$4 \quad 9.021725 \mathrm{E}-01-8.585017 \mathrm{E}+01 \quad 6.528568 \mathrm{E}-02-8.998072 \mathrm{E}-01$

$5 \quad 5.112429 \mathrm{E}-01-1.115042 \mathrm{E}+02-1.874063 \mathrm{E}-01-4.756556 \mathrm{E}-01$

$6 \quad 1.234953 \mathrm{E}+01 \quad 7.589193 \mathrm{E}+01 \quad 3.010217 \mathrm{E}+00 \quad 1.197704 \mathrm{E}+01$

$1 \quad 2.006404 \mathrm{E}+00 \quad 5.394589 \mathrm{E}+01 \quad 1.180867 \mathrm{E}+00 \quad 1.622101 \mathrm{E}+00$

$2 \quad 4.484448 \mathrm{E}-01-8.103227 \mathrm{E}+01 \quad 6.990276 \mathrm{E}-02-4.429632 \mathrm{E}-01$

$3 \quad 9.174211 \mathrm{E}-02 \quad 5.319652 \mathrm{E}+01 \quad 5.496015 \mathrm{E}-02 \quad 7.345745 \mathrm{E}-02$

$4 \quad 9.207801 \mathrm{E}-01-5.153143 \mathrm{E}+01 \quad 5.728037 \mathrm{E}-01 \quad-7.209243 \mathrm{E}-01$

$5 \quad 2.876672 \mathrm{E}-01 \quad 3.091370 \mathrm{E}+01 \quad 2.468017 \mathrm{E}-01 \quad 1.477880 \mathrm{E}-01$

$\begin{array}{lllll}6.462325 \mathrm{E}+01 & 1.380886 \mathrm{E}+00 & 2.461610 \mathrm{E}+01 & 5.933877 \mathrm{E}-01\end{array}$

$12.361786 \mathrm{E}+00-4.219011 \mathrm{E}+00 \quad 2.355386 \mathrm{E}+00-1.737545 \mathrm{E}-01$

$2 \quad 4.178132 \mathrm{E}-01 \quad 1.494453 \mathrm{E}+02 \quad-3.597974 \mathrm{E}-01 \quad 2.123997 \mathrm{E}-01$

$3 \quad 3.654533 \mathrm{E}-01 \quad 2.633396 \mathrm{E}+00 \quad 3.650674 \mathrm{E}-01 \quad 1.679085 \mathrm{E}-02$

$4 \quad 1.113104 \mathrm{E}+00 \quad 9.029066 \mathrm{E}+01 \quad-5.646759 \mathrm{E}-03 \quad 1.113090 \mathrm{E}+00$

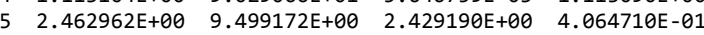

$\begin{array}{llllll}6 & 6.406702 \mathrm{E}+01 & -8.336032 \mathrm{E}+01 & 7.407757 \mathrm{E}+00 & -6.363732 \mathrm{E}+01\end{array}$

$\begin{array}{lllll}1 & 3.723658 \mathrm{E}+01 & 4.667451 \mathrm{E}+01 & 2.554958 \mathrm{E}+01 & 2.708840 \mathrm{E}+01\end{array}$

$2 \quad 0.000000 \mathrm{E}+00 \quad 9.000000 \mathrm{E}+01 \quad 0.000000 \mathrm{E}+00 \quad 0.000000 \mathrm{E}+00$

$3 \quad 5.366601 \mathrm{E}+00 \quad 4.634993 \mathrm{E}+01 \quad 3.704308 \mathrm{E}+00 \quad 3.883106 \mathrm{E}+00$

$40.000000 \mathrm{E}+00 \quad 9.000000 \mathrm{E}+01 \quad 0.000000 \mathrm{E}+00 \quad 0.000000 \mathrm{E}+00$

$\begin{array}{lllll}5 & 3.827606 \mathrm{E}+01 & 4.592707 \mathrm{E}+01 & 2.662381 \mathrm{E}+01 & 2.749963 \mathrm{E}+01\end{array}$

$\begin{array}{lllll}6 & 0.000000 \mathrm{E}+00 & 9.000000 \mathrm{E}+01 & 0.000000 \mathrm{E}+00 & 0.000000 \mathrm{E}+00\end{array}$

$12.361786 \mathrm{E}+00-4.219011 \mathrm{E}+00 \quad 2.355386 \mathrm{E}+00-1.737545 \mathrm{E}-01$

$2 \quad 4.178132 \mathrm{E}-01-3.055469 \mathrm{E}+01 \quad 3.597974 \mathrm{E}-01-2.123997 \mathrm{E}-01$

$3 \quad 3.654533 \mathrm{E}-01 \quad 2.633396 \mathrm{E}+00 \quad 3.650674 \mathrm{E}-01 \quad 1.679085 \mathrm{E}-02$

$4 \quad 1.113104 \mathrm{E}+00 \quad-8.970934 \mathrm{E}+01 \quad 5.646759 \mathrm{E}-03-1.113090 \mathrm{E}+00$

$5 \quad 2.462962 \mathrm{E}+00 \quad 9.499172 \mathrm{E}+00 \quad 2.429190 \mathrm{E}+00 \quad 4.064710 \mathrm{E}-01$

$6 \quad 6.406702 E+01 \quad 9.663969 E+01-7.407757 E+00 \quad 6.363732 E+01$

$\begin{array}{lllll}1 & 2.006404 \mathrm{E}+00 & 5.394589 \mathrm{E}+01 & 1.180867 \mathrm{E}+00 & 1.622101 \mathrm{E}+00\end{array}$

$2 \quad 4.484448 \mathrm{E}-01 \quad 9.896774 \mathrm{E}+01 \quad-6.990276 \mathrm{E}-02 \quad 4.429632 \mathrm{E}-01$

$3 \quad 9.174211 \mathrm{E}-02 \quad 5.319652 \mathrm{E}+01 \quad 5.496015 \mathrm{E}-02 \quad 7.345745 \mathrm{E}-02$

$4 \quad 9.207801 \mathrm{E}-01 \quad 1.284686 \mathrm{E}+02 \quad-5.728037 \mathrm{E}-01 \quad 7.209243 \mathrm{E}-01$

$5 \quad 2.876672 \mathrm{E}-01 \quad 3.091370 \mathrm{E}+01 \quad 2.468017 \mathrm{E}-01 \quad 1.477880 \mathrm{E}-01$

$6 \quad 2.462325 \mathrm{E}+01-1.786191 \mathrm{E}+02-2.461610 \mathrm{E}+01-5.933877 \mathrm{E}-01$

$1 \quad 5.178185 \mathrm{E}-01 \quad 5.169857 \mathrm{E}+01 \quad 3.209431 \mathrm{E}-01 \quad 4.063637 \mathrm{E}-01$

$2 \quad 3.095861 \mathrm{E}-01 \quad 9.268608 \mathrm{E}+01 \quad-1.450839 \mathrm{E}-02 \quad 3.092460 \mathrm{E}-01$

$3 \quad 4.604926 \mathrm{E}-02-1.596816 \mathrm{E}+02-4.318395 \mathrm{E}-02-1.599002 \mathrm{E}-02$

$4 \quad 9.021725 \mathrm{E}-01 \quad 9.414983 \mathrm{E}+01 \quad-6.528568 \mathrm{E}-02 \quad 8.998072 \mathrm{E}-01$

$5 \quad 5.112429 \mathrm{E}-01-1.115042 \mathrm{E}+02-1.874063 \mathrm{E}-01-4.756556 \mathrm{E}-01$

$6 \quad 1.234953 \mathrm{E}+01-1.041081 \mathrm{E}+02-3.010217 \mathrm{E}+00-1.197704 \mathrm{E}+01$

$\begin{array}{lllll}1.203053 \mathrm{E}+00 & 8.637856 \mathrm{E}+01 & 7.598975 \mathrm{E}-02 & 1.200650 \mathrm{E}+00\end{array}$

$2 \quad 1.029077 \mathrm{E}+00 \quad 8.726032 \mathrm{E}+01 \quad 4.918807 \mathrm{E}-02 \quad 1.027901 \mathrm{E}+00$

$3 \quad 5.575266 \mathrm{E}-02 \quad 9.972774 \mathrm{E}+01 \quad-9.420332 \mathrm{E}-03 \quad 5.495103 \mathrm{E}-02$

$4 \quad 4.508249 \mathrm{E}-01 \quad 1.413024 \mathrm{E}+02-3.518492 \mathrm{E}-01 \quad 2.818602 \mathrm{E}-01$

$5 \quad 4.077001 \mathrm{E}-01 \quad-4.692863 \mathrm{E}+01 \quad 2.784220 \mathrm{E}-01 \quad-2.978264 \mathrm{E}-01$

3.266479E-01 -3.970305E+01 2.513117E-01 -2.086656E-01

$1 \quad 1.029144 \mathrm{E}+00 \quad 8.725648 \mathrm{E}+01 \quad 4.926003 \mathrm{E}-02 \quad 1.027964 \mathrm{E}+00$

$2 \quad 1.203070 \mathrm{E}+00 \quad 8.637492 \mathrm{E}+01 \quad 7.606713 \mathrm{E}-02 \quad 1.200663 \mathrm{E}+00$

$3 \quad 5.628183 \mathrm{E}-02 \quad 9.891718 \mathrm{E}+01 \quad-8.724050 \mathrm{E}-03 \quad 5.560157 \mathrm{E}-02$

$4 \quad 4.014029 \mathrm{E}-01 \quad 1.361869 \mathrm{E}+02 \quad-2.896531 \mathrm{E}-01 \quad 2.778945 \mathrm{E}-01$

$5 \quad 4.588585 \mathrm{E}-01-3.740073 \mathrm{E}+01 \quad 3.645203 \mathrm{E}-01-2.787042 \mathrm{E}-01$

$6 \quad 3.258539 \mathrm{E}-01 \quad 1.402543 \mathrm{E}+02 \quad-2.505456 \mathrm{E}-01 \quad 2.083450 \mathrm{E}-01$

$1 \quad 3.095530 \mathrm{E}-01 \quad 9.267338 \mathrm{E}+01 \quad-1.443828 \mathrm{E}-02 \quad 3.092161 \mathrm{E}-01$

$2 \quad 5.177627 \mathrm{E}-01 \quad 5.169791 \mathrm{E}+01 \quad 3.209133 \mathrm{E}-01 \quad 4.063162 \mathrm{E}-01$

$3 \quad 4.579637 \mathrm{E}-02-1.591669 \mathrm{E}+02-4.280223 \mathrm{E}-02-1.628732 \mathrm{E}-02$

$4 \quad 5.006751 \mathrm{E}-01 \quad 6.879288 \mathrm{E}+01 \quad 1.811145 \mathrm{E}-01 \quad 4.667688 \mathrm{E}-01$

$5 \quad 9.214829 \mathrm{E}-01-8.593034 \mathrm{E}+01 \quad 6.539704 \mathrm{E}-02-9.191594 \mathrm{E}-01$

$6 \quad 1.234955 \mathrm{E}+01 \quad 7.589134 \mathrm{E}+01 \quad 3.010346 \mathrm{E}+00 \quad 1.197703 \mathrm{E}+01$

$1 \quad 4.484869 \mathrm{E}-01 \quad 9.897969 \mathrm{E}+01 \quad-7.000179 \mathrm{E}-02 \quad 4.429901 \mathrm{E}-01$ 


\begin{tabular}{|c|c|c|c|c|c|c|}
\hline $0.125664 \mathrm{E}+01$ & $0.700000 \mathrm{E}+02$ & 2 & $2.006445 E+00$ & $5.394504 \mathrm{E}+01$ & $1.180915 \mathrm{E}+00$ & $1.622116 \mathrm{E}+00$ \\
\hline $0.125664 \mathrm{E}+01$ & $0.700000 \mathrm{E}+02$ & 3 & $9.283326 \mathrm{E}-02$ & $5.308544 \mathrm{E}+01$ & $5.575783 \mathrm{E}-02$ & 7.422317E-02 \\
\hline $0.125664 \mathrm{E}+01$ & $0.700000 \mathrm{E}+02$ & 4 & $2.975250 \mathrm{E}-01$ & $-1.486025 E+02$ & $-2.539594 E-01$ & $-1.550024 \mathrm{E}-01$ \\
\hline $0.125664 \mathrm{E}+01$ & $0.700000 \mathrm{E}+02$ & 5 & $9.139579 \mathrm{E}-01$ & $-5.182080 E+01$ & $5.649385 \mathrm{E}-01$ & $-7.184452 E-01$ \\
\hline $0.125664 \mathrm{E}+01$ & $0.700000 \mathrm{E}+02$ & 6 & $2.462373 E+01$ & $1.382357 \mathrm{E}+00$ & $2.461657 \mathrm{E}+01$ & $5.940315 \mathrm{E}-01$ \\
\hline $0.125664 \mathrm{E}+01$ & $0.800000 \mathrm{E}+02$ & 1 & $4.177332 \mathrm{E}-01$ & $-3.053241 \mathrm{E}+01$ & $3.598111 \mathrm{E}-01$ & $-2.122191 E-01$ \\
\hline $0.125664 \mathrm{E}+01$ & $0.800000 \mathrm{E}+02$ & 2 & $2.361580 E+00$ & $-4.222116 \mathrm{E}+00$ & $2.355171 \mathrm{E}+00$ & $-1.738670 \mathrm{E}-01$ \\
\hline $0.125664 \mathrm{E}+01$ & $0.800000 \mathrm{E}+02$ & 3 & $3.644951 \mathrm{E}-01$ & $2.648255 E+00$ & $3.641058 \mathrm{E}-01$ & $1.684125 \mathrm{E}-02$ \\
\hline $0.125664 \mathrm{E}+01$ & $0.800000 E+02$ & 4 & $2.427344 \mathrm{E}+00$ & $-1.706497 E+02$ & $-2.395093 E+00$ & $-3.943709 E-01$ \\
\hline $0.125664 \mathrm{E}+01$ & $0.800000 \mathrm{E}+02$ & 5 & $1.125469 \mathrm{E}+00$ & $8.999937 E+01$ & $1.252509 \mathrm{E}-05$ & $1.125469 \mathrm{E}+00$ \\
\hline $0.125664 \mathrm{E}+01$ & $0.800000 \mathrm{E}+02$ & 6 & $6.406771 \mathrm{E}+01$ & $-8.336099 E+01$ & $7.407092 \mathrm{E}+00$ & $-6.363809 E+01$ \\
\hline $0.125664 \mathrm{E}+01$ & $0.900000 \mathrm{E}+02$ & 1 & $2.792875 \mathrm{E}-07$ & $7.986827 E+01$ & $4.913002 \mathrm{E}-08$ & $2.749323 E-07$ \\
\hline $0.125664 \mathrm{E}+01$ & $0.900000 \mathrm{E}+02$ & 2 & $3.723647 E+01$ & $4.667456 E+01$ & $2.554948 \mathrm{E}+01$ & $2.708835 E+01$ \\
\hline $0.125664 \mathrm{E}+01$ & $0.900000 \mathrm{E}+02$ & 3 & $5.365805 E+00$ & $4.635458 \mathrm{E}+01$ & $3.703443 E+00$ & $3.882830 E+00$ \\
\hline $0.125664 \mathrm{E}+01$ & $0.900000 E+02$ & 4 & $3.828696 \mathrm{E}+01$ & $-1.341008 \mathrm{E}+02$ & $-2.664477 E+01$ & $-2.749449 E+01$ \\
\hline $0.125664 \mathrm{E}+01$ & $0.900000 E+02$ & 5 & 1. $103154 \mathrm{E}-05$ & $1.369071 \mathrm{E}+02$ & $-8.055748 E-06$ & $7.536565 E-06$ \\
\hline $0.125664 \mathrm{E}+01$ & $0.900000 \mathrm{E}+02$ & 6 & $1.552969 \mathrm{E}-04$ & $-4.026945 E+01$ & $1.184936 \mathrm{E}-04$ & $-1.003813 E-04$ \\
\hline $0.125664 \mathrm{E}+01$ & $0.100000 E+03$ & 1 & 4.177965E-01 & $1.494610 \mathrm{E}+02$ & $-3.598411 E-01$ & $2.122929 E-01$ \\
\hline $0.125664 \mathrm{E}+01$ & $0.100000 \mathrm{E}+03$ & 2 & $2.361629 E+00$ & $-4.221781 E+00$ & $2.355221 \mathrm{E}+00$ & $-1.738568 \mathrm{E}-01$ \\
\hline $0.125664 \mathrm{E}+01$ & $0.100000 \mathrm{E}+03$ & 3 & $3.645604 \mathrm{E}-01$ & $2.653809 E+00$ & $3.641694 \mathrm{E}-01$ & $1.687956 \mathrm{E}-02$ \\
\hline $0.125664 \mathrm{E}+01$ & $0.100000 \mathrm{E}+03$ & 4 & $2.427364 \mathrm{E}+00$ & $-1.706587 E+02$ & $-2.395175 E+00$ & $-3.939986 \mathrm{E}-01$ \\
\hline $0.125664 \mathrm{E}+01$ & $0.100000 \mathrm{E}+03$ & 5 & $1.125051 \mathrm{E}+00$ & $-8.993605 E+01$ & $1.255705 \mathrm{E}-03$ & $-1.125051 E+00$ \\
\hline $0.125664 \mathrm{E}+01$ & $0.100000 \mathrm{E}+03$ & 6 & $6.406738 \mathrm{E}+01$ & $9.663930 \mathrm{E}+01$ & $-7.407373 E+00$ & $6.363773 E+01$ \\
\hline $0.125664 \mathrm{E}+01$ & $0.110000 \mathrm{E}+03$ & 1 & $4.484580 E-01$ & $-8.101905 E+01$ & $7.000703 E-02$ & $-4.429600 \mathrm{E}-01$ \\
\hline $0.125664 \mathrm{E}+01$ & $0.110000 \mathrm{E}+03$ & 2 & $2.006438 \mathrm{E}+00$ & $5.394444 \mathrm{E}+01$ & $1.180928 \mathrm{E}+00$ & $1.622098 \mathrm{E}+00$ \\
\hline $0.125664 \mathrm{E}+01$ & $0.110000 \mathrm{E}+03$ & 3 & $9.282635 \mathrm{E}-02$ & $5.310986 E+01$ & $5.572205 \mathrm{E}-02$ & $7.424140 \mathrm{E}-02$ \\
\hline $0.125664 \mathrm{E}+01$ & $0.110000 \mathrm{E}+03$ & 4 & $2.977684 \mathrm{E}-01$ & $-1.484481 E+02$ & $-2.537483 E-01$ & $-1.558134 \mathrm{E}-01$ \\
\hline $0.125664 \mathrm{E}+01$ & $0.110000 \mathrm{E}+03$ & 5 & $9.130183 E-01$ & $1.281776 \mathrm{E}+02$ & $-5.643375 E-01$ & $7.177225 E-01$ \\
\hline $0.125664 \mathrm{E}+01$ & $0.110000 \mathrm{E}+03$ & 6 & $2.462352 \mathrm{E}+01$ & $-1.786175 E+02$ & $-2.461636 \mathrm{E}+01$ & $-5.940970 \mathrm{E}-01$ \\
\hline $0.125664 \mathrm{E}+01$ & $0.120000 \mathrm{E}+03$ & 1 & $3.095530 E-01$ & $-8.732662 E+01$ & $1.443828 \mathrm{E}-02$ & $-3.092161 E-01$ \\
\hline $0.125664 \mathrm{E}+01$ & $0.120000 \mathrm{E}+03$ & 2 & $5.177627 \mathrm{E}-01$ & $5.169791 \mathrm{E}+01$ & $3.209133 \mathrm{E}-01$ & $4.063162 \mathrm{E}-01$ \\
\hline $0.125664 \mathrm{E}+01$ & $0.120000 \mathrm{E}+03$ & 3 & 4.579637E-02 & $-1.591669 \mathrm{E}+02$ & $-4.280223 E-02$ & $-1.628732 E-02$ \\
\hline $0.125664 \mathrm{E}+01$ & $0.120000 \mathrm{E}+03$ & 4 & $5.006751 E-01$ & $6.879288 \mathrm{E}+01$ & $1.811145 E-01$ & $4.667688 \mathrm{E}-01$ \\
\hline $0.125664 \mathrm{E}+01$ & $0.120000 E+03$ & 5 & $9.214829 E-01$ & $9.406966 \mathrm{E}+01$ & $-6.539704 E-02$ & $9.191594 \mathrm{E}-01$ \\
\hline $0.125664 \mathrm{E}+01$ & $0.120000 \mathrm{E}+03$ & 6 & 1. $234955 E+01$ & $-1.041087 E+02$ & $-3.010346 E+00$ & $-1.197703 E+01$ \\
\hline $0.125664 \mathrm{E}+01$ & $0.130000 \mathrm{E}+03$ & 1 & $1.029152 \mathrm{E}+00$ & $-9.274391 E+01$ & $-4.926759 E-02$ & $-1.027972 E+00$ \\
\hline $0.125664 \mathrm{E}+01$ & $0.130000 E+03$ & 2 & 1. $203075 E+00$ & $8.637505 E+01$ & $7.606444 \mathrm{E}-02$ & 1. $200668 \mathrm{E}+00$ \\
\hline $0.125664 \mathrm{E}+01$ & $0.130000 E+03$ & 3 & $5.619531 \mathrm{E}-02$ & $9.880749 \mathrm{E}+01$ & $-8.604339 E-03$ & $5.553268 \mathrm{E}-02$ \\
\hline $0.125664 \mathrm{E}+01$ & $0.130000 \mathrm{E}+03$ & 4 & $4.013925 \mathrm{E}-01$ & 1. $360051 \mathrm{E}+02$ & $-2.887626 E-01$ & $2.788048 \mathrm{E}-01$ \\
\hline $0.125664 \mathrm{E}+01$ & $0.130000 E+03$ & 5 & $4.572874 \mathrm{E}-01$ & $1.427535 \mathrm{E}+02$ & $-3.640184 E-01$ & $2.767713 E-01$ \\
\hline $0.125664 \mathrm{E}+01$ & $0.130000 \mathrm{E}+03$ & 6 & $3.257516 \mathrm{E}-01$ & $-3.972321 E+01$ & $2.505489 E-01$ & $-2.081812 E-01$ \\
\hline $0.125664 \mathrm{E}+01$ & $0.140000 E+03$ & 1 & 1. $203053 E+00$ & $-9.362145 E+01$ & $-7.598975 E-02$ & $-1.200650 \mathrm{E}+00$ \\
\hline $0.125664 \mathrm{E}+01$ & $0.140000 E+03$ & 2 & $1.029077 \mathrm{E}+00$ & $8.726032 \mathrm{E}+01$ & 4.918807E-02 & $1.027901 \mathrm{E}+00$ \\
\hline $0.125664 \mathrm{E}+01$ & $0.140000 E+03$ & 3 & $5.575266 \mathrm{E}-02$ & $9.972774 \mathrm{E}+01$ & $-9.420332 E-03$ & $5.495103 E-02$ \\
\hline $0.125664 \mathrm{E}+01$ & $0.140000 \mathrm{E}+03$ & 4 & $4.508249 \mathrm{E}-01$ & 1. $413024 \mathrm{E}+02$ & $-3.518492 \mathrm{E}-01$ & $2.818602 \mathrm{E}-01$ \\
\hline $0.125664 \mathrm{E}+01$ & $0.140000 E+03$ & 5 & $4.077001 \mathrm{E}-01$ & 1. $330714 \mathrm{E}+02$ & $-2.784220 E-01$ & $2.978264 \mathrm{E}-01$ \\
\hline $0.125664 \mathrm{E}+01$ & $0.140000 E+03$ & 6 & $3.266479 \mathrm{E}-01$ & $1.402970 E+02$ & $-2.513117 E-01$ & $2.086656 \mathrm{E}-01$ \\
\hline $0.125664 \mathrm{E}+01$ & $0.150000 \mathrm{E}+03$ & 1 & $5.178185 E-01$ & $-1.283014 \mathrm{E}+02$ & $-3.209431 E-01$ & $-4.063637 E-01$ \\
\hline $0.125664 \mathrm{E}+01$ & $0.150000 \mathrm{E}+03$ & 2 & $3.095861 \mathrm{E}-01$ & $9.268608 \mathrm{E}+01$ & $-1.450839 E-02$ & $3.092460 \mathrm{E}-01$ \\
\hline $0.125664 \mathrm{E}+01$ & $0.150000 \mathrm{E}+03$ & 3 & $4.604926 \mathrm{E}-02$ & $-1.596816 \mathrm{E}+02$ & $-4.318395 E-02$ & $-1.599002 E-02$ \\
\hline $0.125664 \mathrm{E}+01$ & $0.150000 \mathrm{E}+03$ & 4 & $9.021725 \mathrm{E}-01$ & $9.414983 \mathrm{E}+01$ & $-6.528568 \mathrm{E}-02$ & $8.998072 E-01$ \\
\hline $0.125664 \mathrm{E}+01$ & $0.150000 \mathrm{E}+03$ & 5 & $5.112429 \mathrm{E}-01$ & $6.849577 E+01$ & $1.874063 \mathrm{E}-01$ & $4.756556 \mathrm{E}-01$ \\
\hline $0.125664 \mathrm{E}+01$ & $0.150000 \mathrm{E}+03$ & 6 & 1. $234953 \mathrm{E}+01$ & $7.589193 \mathrm{E}+01$ & $3.010217 \mathrm{E}+00$ & $1.197704 \mathrm{E}+01$ \\
\hline $0.125664 \mathrm{E}+01$ & $0.160000 \mathrm{E}+03$ & 1 & $2.006404 \mathrm{E}+00$ & $-1.260541 \mathrm{E}+02$ & $-1.180867 E+00$ & $-1.622101 \mathrm{E}+00$ \\
\hline $0.125664 \mathrm{E}+01$ & $0.160000 \mathrm{E}+03$ & 2 & $4.484448 \mathrm{E}-01$ & $9.896774 \mathrm{E}+01$ & $-6.990276 E-02$ & $4.429632 \mathrm{E}-01$ \\
\hline $0.125664 \mathrm{E}+01$ & $0.160000 \mathrm{E}+03$ & 3 & $9.174211 \mathrm{E}-02$ & $5.319652 E+01$ & $5.496015 E-02$ & $7.345745 E-02$ \\
\hline $0.125664 \mathrm{E}+01$ & $0.160000 \mathrm{E}+03$ & 4 & $9.207801 \mathrm{E}-01$ & $1.284686 \mathrm{E}+02$ & $-5.728037 E-01$ & $7.209243 E-01$ \\
\hline $0.125664 \mathrm{E}+01$ & $0.160000 \mathrm{E}+03$ & 5 & $2.876672 E-01$ & $-1.490863 E+02$ & $-2.468017 E-01$ & $-1.477880 E-01$ \\
\hline $0.125664 \mathrm{E}+01$ & $0.160000 \mathrm{E}+03$ & 6 & $2.462325 E+01$ & $1.380886 \mathrm{E}+00$ & $2.461610 \mathrm{E}+01$ & $5.933877 \mathrm{E}-01$ \\
\hline $0.125664 \mathrm{E}+01$ & $0.170000 \mathrm{E}+03$ & 1 & $2.361607 E+00$ & $1.757810 E+02$ & $-2.355207 E+00$ & $1.737394 \mathrm{E}-01$ \\
\hline $0.125664 \mathrm{E}+01$ & $0.170000 \mathrm{E}+03$ & 2 & $4.178357 E-01$ & $-3.055589 E+01$ & $3.598124 \mathrm{E}-01$ & $-2.124187 E-01$ \\
\hline $0.125664 \mathrm{E}+01$ & $0.170000 \mathrm{E}+03$ & 3 & $3.655501 \mathrm{E}-01$ & $2.623623 E+00$ & $3.651669 \mathrm{E}-01$ & $1.673300 \mathrm{E}-02$ \\
\hline $0.125664 \mathrm{E}+01$ & $0.170000 \mathrm{E}+03$ & 4 & $1.113482 \mathrm{E}+00$ & $-8.973499 E+01$ & $5.150314 \mathrm{E}-03$ & $-1.113470 E+00$ \\
\hline $0.125664 \mathrm{E}+01$ & $0.170000 \mathrm{E}+03$ & 5 & $2.462513 E+00$ & $-1.704853 E+02$ & $-2.428637 E+00$ & $-4.070565 E-01$ \\
\hline $0.125664 \mathrm{E}+01$ & $0.170000 \mathrm{E}+03$ & 6 & $6.406735 \mathrm{E}+01$ & $-8.336049 E+01$ & $7.407608 \mathrm{E}+00$ & $-6.363767 E+01$ \\
\hline $0.125664 \mathrm{E}+01$ & $0.180000 \mathrm{E}+03$ & 1 & $3.723658 \mathrm{E}+01$ & $-1.333255 E+02$ & $-2.554958 \mathrm{E}+01$ & $-2.708840 \mathrm{E}+01$ \\
\hline $0.125664 \mathrm{E}+01$ & $0.180000 \mathrm{E}+03$ & 2 & $5.587313 E-07$ & $7.987847 \mathrm{E}+01$ & $9.818955 E-08$ & $5.500359 E-07$ \\
\hline $0.125664 \mathrm{E}+01$ & $0.180000 \mathrm{E}+03$ & 3 & $5.366601 \mathrm{E}+00$ & $4.634993 E+01$ & $3.704308 \mathrm{E}+00$ & $3.883106 \mathrm{E}+00$ \\
\hline $0.125664 \mathrm{E}+01$ & $0.180000 \mathrm{E}+03$ & 4 & $2.209019 E-05$ & $-4.312394 \mathrm{E}+01$ & $1.612312 \mathrm{E}-05$ & $-1.510039 E-05$ \\
\hline $0.125664 \mathrm{E}+01$ & $0.180000 E+03$ & 5 & $3.827606 \mathrm{E}+01$ & $-1.340729 \mathrm{E}+02$ & $-2.662381 E+01$ & $-2.749963 \mathrm{E}+01$ \\
\hline $0.125664 \mathrm{E}+01$ & $0.180000 \mathrm{E}+03$ & 6 & $3.105944 \mathrm{E}-04$ & $-4.026929 \mathrm{E}+01$ & $2.369881 \mathrm{E}-04$ & $-2.007623 E-04$ \\
\hline
\end{tabular}




\section{Appendix E Input Files for the MIT / NREL Barge}

\section{E.1 FAST Platform / HydroDyn Input File}

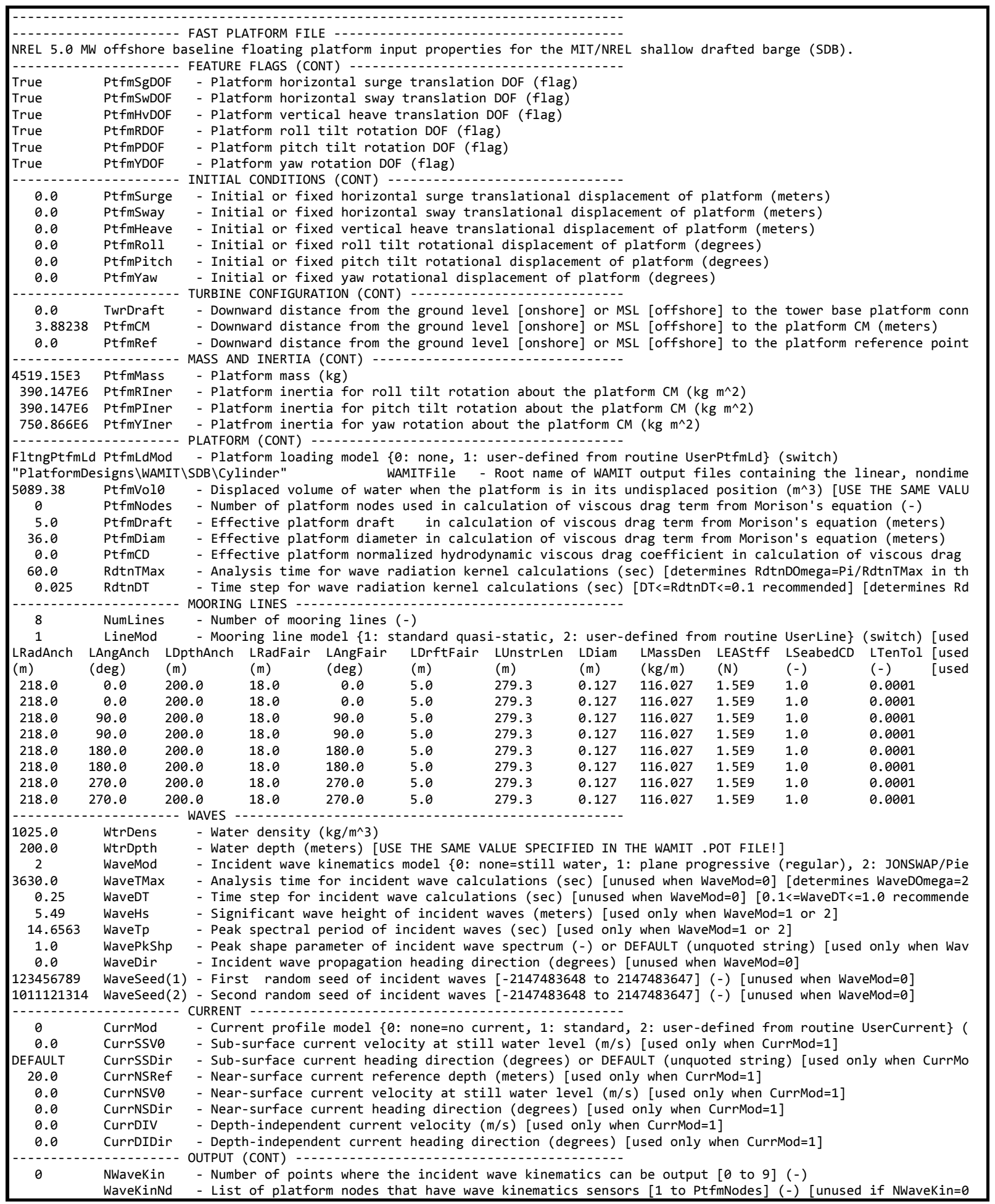




\section{E.2 WAMIT Input File - CONFIG.WAM}

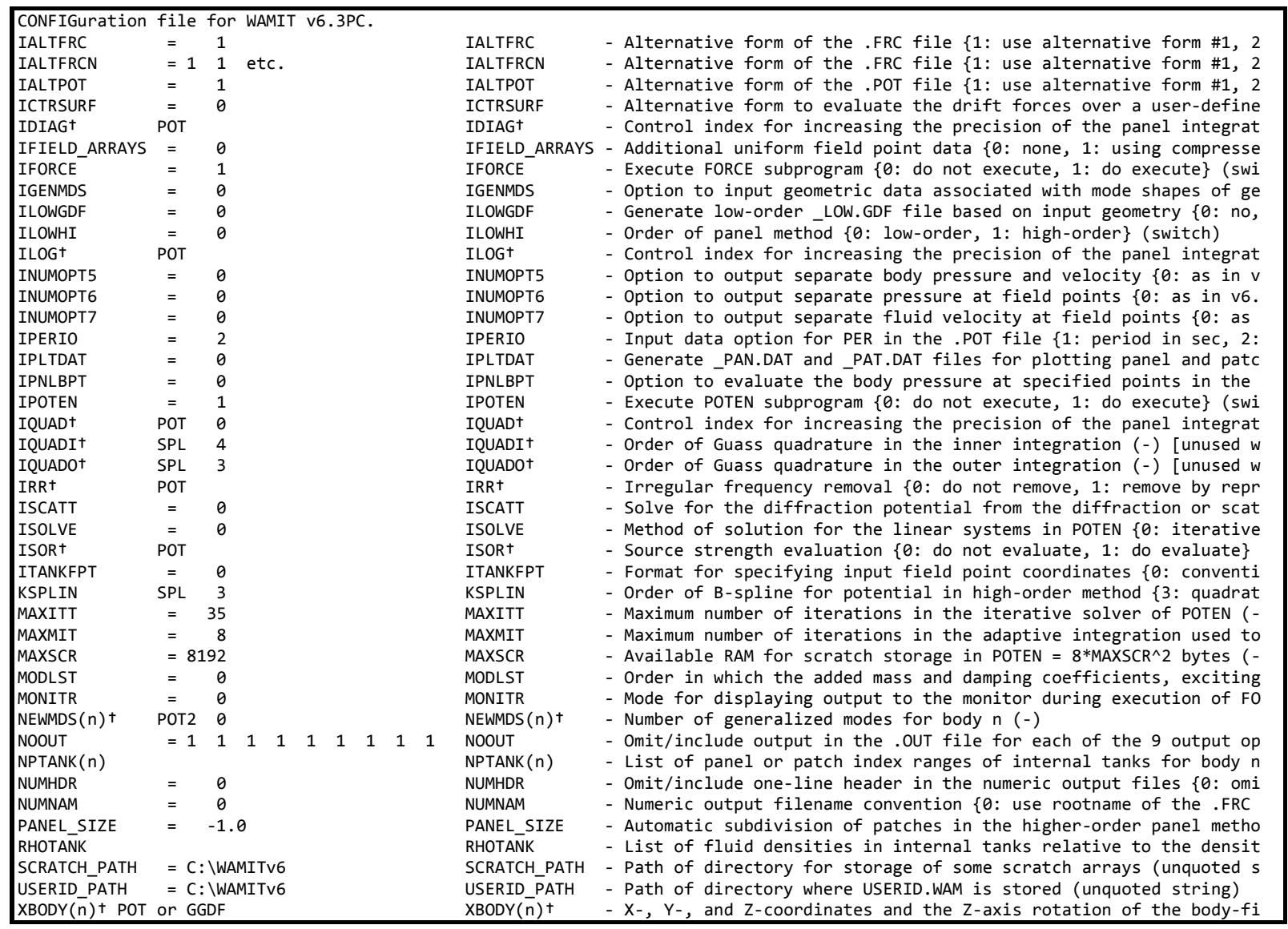

\section{E.3 WAMIT Input File - Cylinder.POT}

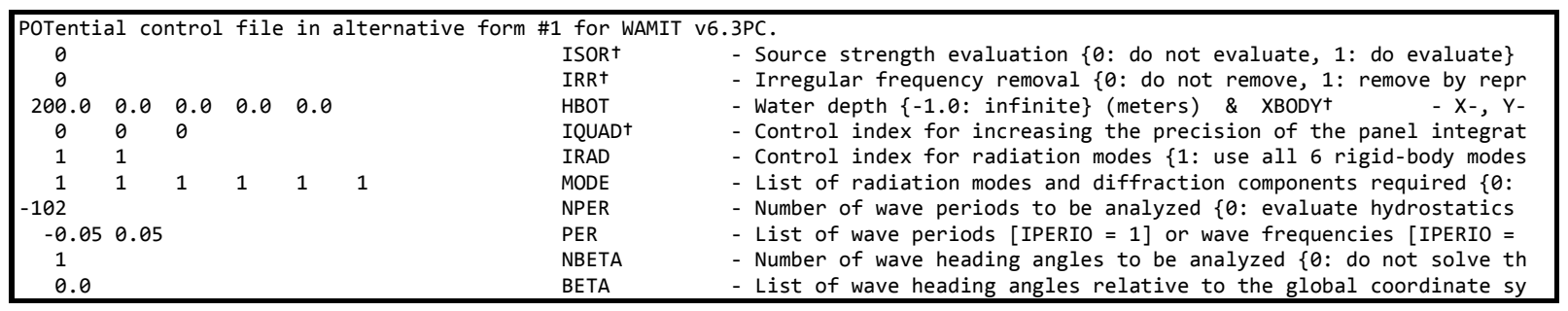

\section{E.4 WAMIT Input File - Cylinder.FRC}

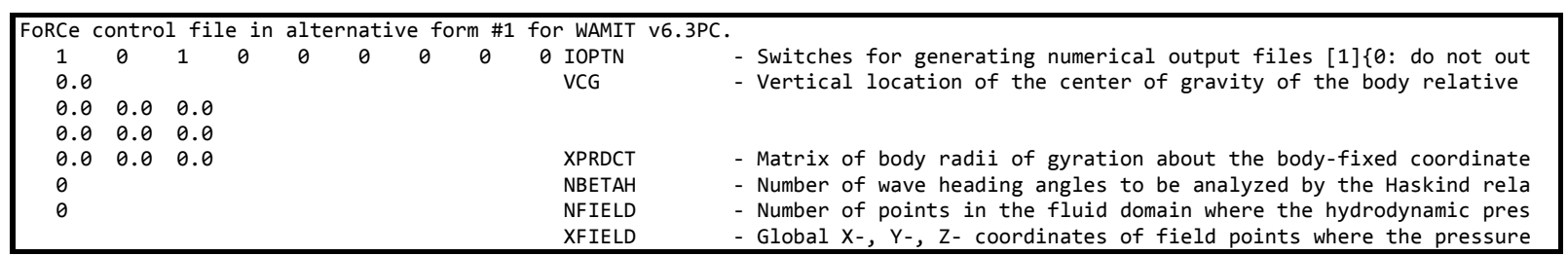




\section{E.5 WAMIT Input File - Cylinder.GDF}

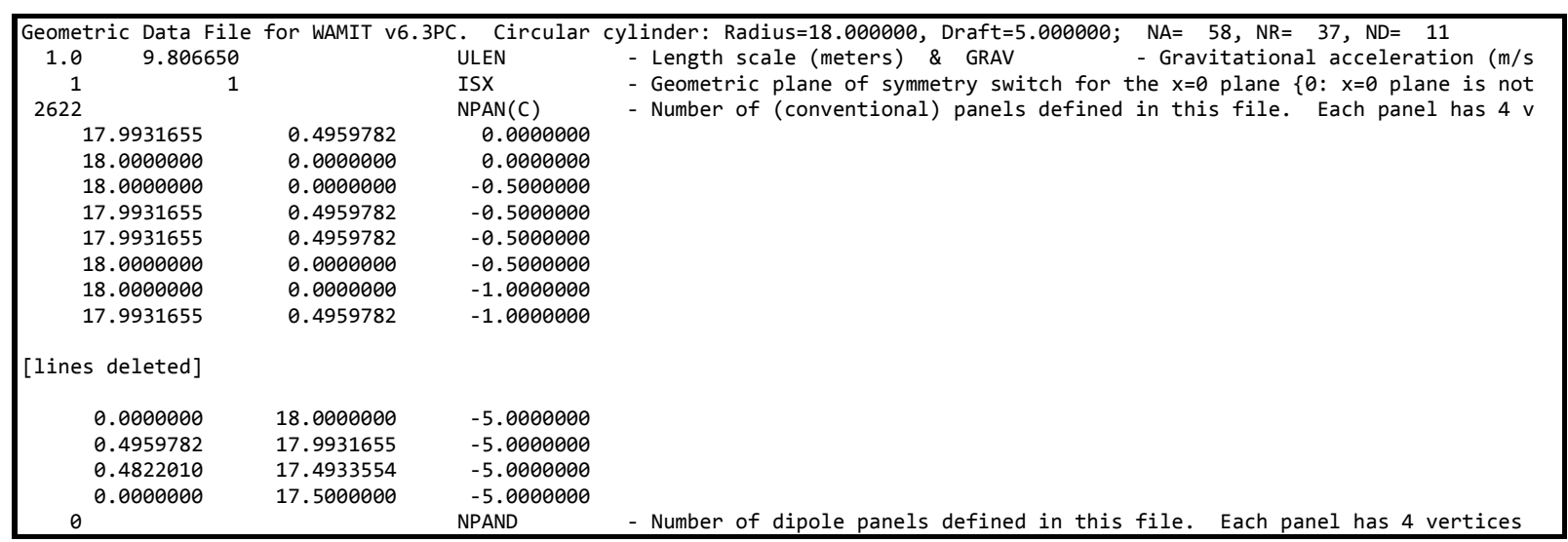

\section{E.6 WAMIT Output File - Cylinder.hst}

\begin{tabular}{|c|c|c|}
\hline 1 & 1 & $0.000000 \mathrm{E}+00$ \\
\hline 1 & 2 & $0.000000 \mathrm{E}+00$ \\
\hline 1 & 3 & $0.000000 E+00$ \\
\hline 1 & 4 & $0.000000 \mathrm{E}+00$ \\
\hline 1 & 5 & $0.000000 E+00$ \\
\hline 1 & 6 & $0.000000 \mathrm{E}+00$ \\
\hline 2 & 1 & $0.000000 \mathrm{E}+00$ \\
\hline 2 & 2 & $0.000000 E+00$ \\
\hline 2 & 3 & $0.000000 \mathrm{E}+00$ \\
\hline 2 & 4 & $0.000000 E+00$ \\
\hline 2 & 5 & $0.000000 \mathrm{E}+00$ \\
\hline 2 & 6 & $0.000000 E+00$ \\
\hline 3 & 1 & $0.000000 \mathrm{E}+00$ \\
\hline 3 & 2 & $0.000000 \mathrm{E}+00$ \\
\hline 3 & 3 & 1. $017745 \mathrm{E}+03$ \\
\hline 3 & 4 & $0.000000 \mathrm{E}+00$ \\
\hline 3 & 5 & $0.000000 \mathrm{E}+00$ \\
\hline 3 & 6 & $0.000000 \mathrm{E}+00$ \\
\hline 4 & 1 & $0.000000 \mathrm{E}+00$ \\
\hline 4 & 2 & $0.000000 \mathrm{E}+00$ \\
\hline 4 & 3 & $0.000000 \mathrm{E}+00$ \\
\hline 4 & 4 & $6.968945 E+04$ \\
\hline 4 & 5 & $0.000000 \mathrm{E}+00$ \\
\hline 4 & 6 & $0.000000 E+00$ \\
\hline 5 & 1 & $0.000000 \mathrm{E}+00$ \\
\hline 5 & 2 & $0.000000 \mathrm{E}+00$ \\
\hline 5 & 3 & $0.000000 \mathrm{E}+00$ \\
\hline 5 & 4 & $0.000000 \mathrm{E}+00$ \\
\hline 5 & 5 & $6.968947 E+04$ \\
\hline 5 & 6 & $0.000000 \mathrm{E}+00$ \\
\hline 6 & 1 & $0.000000 E+00$ \\
\hline 6 & 2 & $0.000000 \mathrm{E}+00$ \\
\hline 6 & 3 & $0.000000 \mathrm{E}+00$ \\
\hline 6 & 4 & $0.000000 E+00$ \\
\hline 6 & 5 & $0.000000 \mathrm{E}+00$ \\
\hline 6 & 6 & $0.000000 E+00$ \\
\hline
\end{tabular}

\section{E.7 WAMIT Output File - Cylinder.1}

\begin{tabular}{|lllr|}
\hline$-0.100000 \mathrm{E}+01$ & 1 & 1 & $1.489954 \mathrm{E}+03$ \\
$-0.100000 \mathrm{E}+01$ & 1 & 5 & $6.071913 \mathrm{E}+03$ \\
$-0.100000 \mathrm{E}+01$ & 2 & 2 & $1.489951 \mathrm{E}+03$ \\
$-0.100000 \mathrm{E}+01$ & 2 & 4 & $-6.071907 \mathrm{E}+03$ \\
$-0.100000 \mathrm{E}+01$ & 3 & 3 & $1.365988 \mathrm{E}+04$ \\
$-0.100000 \mathrm{E}+01$ & 4 & 2 & $-6.064123 \mathrm{E}+03$ \\
$-0.100000 \mathrm{E}+01$ & 4 & 4 & $4.238899 \mathrm{E}+05$ \\
$-0.100000 \mathrm{E}+01$ & 5 & 1 & $6.064096 \mathrm{E}+03$ \\
$-0.100000 \mathrm{E}+01$ & 5 & 5 & $4.238900 \mathrm{E}+05$ \\
$-0.100000 \mathrm{E}+01$ & 6 & 6 & $6.802706 \mathrm{E}-08$ \\
$0.000000 \mathrm{E}+00$ & 1 & 1 & $5.318895 \mathrm{E}+02$ \\
$0.000000 \mathrm{E}+00$ & 1 & 5 & $1.837424 \mathrm{E}+03$ \\
$0.000000 \mathrm{E}+00$ & 2 & 2 & $5.318895 \mathrm{E}+02$ \\
\hline
\end{tabular}




\begin{tabular}{|c|c|c|c|c|}
\hline $0.000000 \mathrm{E}+00$ & 2 & 4 & $-1.837423 E+03$ & \\
\hline $0.000000 \mathrm{E}+00$ & 3 & 3 & $9.698843 \mathrm{E}+03$ & \\
\hline $0.000000 \mathrm{E}+00$ & 4 & 2 & $-1.848423 E+03$ & \\
\hline $0.000000 \mathrm{E}+00$ & 4 & 4 & $3.975065 \mathrm{E}+05$ & \\
\hline $0.000000 \mathrm{E}+00$ & 5 & 1 & $1.848424 \mathrm{E}+03$ & \\
\hline $0.000000 \mathrm{E}+00$ & 5 & 5 & $3.975065 \mathrm{E}+05$ & \\
\hline $0.000000 \mathrm{E}+00$ & 6 & 6 & $6.618992 \mathrm{E}-08$ & \\
\hline $0.125664 \mathrm{E}+03$ & 1 & 1 & $1.492564 \mathrm{E}+03$ & $3.627036 \mathrm{E}-02$ \\
\hline $0.125664 \mathrm{E}+03$ & 1 & 5 & $6.089232 \mathrm{E}+03$ & $4.171622 \mathrm{E}-01$ \\
\hline $0.125664 \mathrm{E}+03$ & 2 & 2 & $1.492562 \mathrm{E}+03$ & $3.618027 \mathrm{E}-02$ \\
\hline $0.125664 \mathrm{E}+03$ & 2 & 4 & $-6.089223 E+03$ & $-4.171455 E-01$ \\
\hline $0.125664 \mathrm{E}+03$ & 3 & 3 & $1.504551 \mathrm{E}+04$ & $1.326158 \mathrm{E}+03$ \\
\hline $0.125664 \mathrm{E}+03$ & 4 & 2 & $-6.081390 \mathrm{E}+03$ & $-4.176246 \mathrm{E}-01$ \\
\hline $0.125664 \mathrm{E}+03$ & 4 & 4 & $4.240218 \mathrm{E}+05$ & $4.771546 \mathrm{E}+00$ \\
\hline $0.125664 \mathrm{E}+03$ & 5 & 1 & $6.081377 \mathrm{E}+03$ & $4.159448 \mathrm{E}-01$ \\
\hline $0.125664 \mathrm{E}+03$ & 5 & 5 & $4.240217 \mathrm{E}+05$ & $4.774776 \mathrm{E}+00$ \\
\hline $0.125664 \mathrm{E}+03$ & 6 & 6 & $6.802735 E-08$ & $-2.658206 \mathrm{E}-16$ \\
\hline $0.628319 E+02$ & 1 & 1 & $1.500499 \mathrm{E}+03$ & $1.688199 \mathrm{E}-01$ \\
\hline $0.628319 \mathrm{E}+02$ & 1 & 5 & $6.141928 \mathrm{E}+03$ & $1.929863 \mathrm{E}+00$ \\
\hline $0.628319 E+02$ & 2 & 2 & $1.500498 \mathrm{E}+03$ & $1.688323 E-01$ \\
\hline $0.628319 E+02$ & 2 & 4 & $-6.141922 E+03$ & $-1.929951 \mathrm{E}+00$ \\
\hline $0.628319 \mathrm{E}+02$ & 3 & 3 & $1.461348 \mathrm{E}+04$ & $1.427834 \mathrm{E}+03$ \\
\hline $0.628319 E+02$ & 4 & 2 & $-6.133953 E+03$ & $-1.927234 \mathrm{E}+00$ \\
\hline $0.628319 E+02$ & 4 & 4 & $4.244209 E+05$ & $2.202481 E+01$ \\
\hline $0.628319 \mathrm{E}+02$ & 5 & 1 & $6.133940 E+03$ & $1.927675 \mathrm{E}+00$ \\
\hline $0.628319 \mathrm{E}+02$ & 5 & 5 & $4.244209 E+05$ & 2. 202487E+01 \\
\hline $0.628319 E+02$ & 6 & 6 & $6.802819 E-08$ & $-9.891171 \mathrm{E}-16$ \\
\hline \multicolumn{5}{|l|}{ [lines deleted] } \\
\hline $0.125664 \mathrm{E}+01$ & 1 & 1 & $4.398125 \mathrm{E}+02$ & $1.828869 \mathrm{E}+01$ \\
\hline $0.125664 \mathrm{E}+01$ & 1 & 5 & $1.690897 \mathrm{E}+03$ & $-7.049192 \mathrm{E}+00$ \\
\hline $0.125664 \mathrm{E}+01$ & 2 & 2 & $4.398125 \mathrm{E}+02$ & $1.828869 \mathrm{E}+01$ \\
\hline $0.125664 \mathrm{E}+01$ & 2 & 4 & $-1.690901 \mathrm{E}+03$ & $7.049183 E+00$ \\
\hline $0.125664 \mathrm{E}+01$ & 3 & 3 & $9.633591 \mathrm{E}+03$ & $2.642366 \mathrm{E}-02$ \\
\hline $0.125664 \mathrm{E}+01$ & 4 & 2 & $-1.706354 E+03$ & $9.886565 E+00$ \\
\hline $0.125664 \mathrm{E}+01$ & 4 & 4 & $3.966836 \mathrm{E}+05$ & $3.810670 \mathrm{E}+00$ \\
\hline $0.125664 \mathrm{E}+01$ & 5 & 1 & $1.706357 \mathrm{E}+03$ & $-9.886564 \mathrm{E}+00$ \\
\hline $0.125664 \mathrm{E}+01$ & 5 & 5 & $3.966838 E+05$ & $3.810673 E+00$ \\
\hline $0.125664 \mathrm{E}+01$ & 6 & 6 & $6.784203 E-08$ & $9.183608 \mathrm{E}-10$ \\
\hline
\end{tabular}

\section{E.8 WAMIT Output File - Cylinder.3}

\begin{tabular}{|c|c|c|c|c|c|c|}
\hline $0.125664 \mathrm{E}+03$ & $0.000000 \mathrm{E}+00$ & 1 & $7.489913 \mathrm{E}+00$ & $8.999969 E+01$ & $4.113390 \mathrm{E}-05$ & $7.489913 \mathrm{E}+00$ \\
\hline $0.125664 \mathrm{E}+03$ & $0.000000 \mathrm{E}+00$ & 2 & $0.000000 \mathrm{E}+00$ & $9.000000 \mathrm{E}+01$ & $0.000000 \mathrm{E}+00$ & $0.000000 \mathrm{E}+00$ \\
\hline $0.125664 \mathrm{E}+03$ & $0.000000 \mathrm{E}+00$ & 3 & $1.012577 \mathrm{E}+03$ & 1.912910E-02 & 1.012577E+03 & $3.380647 E-01$ \\
\hline $0.125664 \mathrm{E}+03$ & $0.000000 \mathrm{E}+00$ & 4 & $0.000000 \mathrm{E}+00$ & $9.000000 \mathrm{E}+01$ & $0.000000 \mathrm{E}+00$ & $0.000000 \mathrm{E}+00$ \\
\hline $0.125664 \mathrm{E}+03$ & $0.000000 \mathrm{E}+00$ & 5 & $8.600130 \mathrm{E}+01$ & $8.999969 \mathrm{E}+01$ & $4.723112 E-04$ & $8.600130 \mathrm{E}+01$ \\
\hline $0.125664 \mathrm{E}+03$ & $0.000000 \mathrm{E}+00$ & 6 & $0.000000 \mathrm{E}+00$ & $9.000000 \mathrm{E}+01$ & $0.000000 \mathrm{E}+00$ & $0.000000 \mathrm{E}+00$ \\
\hline $0.628319 \mathrm{E}+02$ & $0.000000 \mathrm{E}+00$ & 1 & $1.534577 \mathrm{E}+01$ & $8.999855 E+01$ & $3.881812 \mathrm{E}-04$ & $1.534577 \mathrm{E}+01$ \\
\hline $0.628319 \mathrm{E}+02$ & $0.000000 \mathrm{E}+00$ & 2 & $0.000000 \mathrm{E}+00$ & $9.000000 \mathrm{E}+01$ & $0.000000 \mathrm{E}+00$ & $0.000000 \mathrm{E}+00$ \\
\hline $0.628319 \mathrm{E}+02$ & $0.000000 \mathrm{E}+00$ & 3 & $9.976350 \mathrm{E}+02$ & $8.361685 E-02$ & $9.976340 \mathrm{E}+02$ & $1.455937 \mathrm{E}+00$ \\
\hline $0.628319 \mathrm{E}+02$ & $0.000000 \mathrm{E}+00$ & 4 & $0.000000 \mathrm{E}+00$ & $9.000000 \mathrm{E}+01$ & $0.000000 \mathrm{E}+00$ & $0.000000 \mathrm{E}+00$ \\
\hline $0.628319 \mathrm{E}+02$ & $0.000000 \mathrm{E}+00$ & 5 & $1.752071 \mathrm{E}+02$ & $8.999855 E+01$ & $4.431981 \mathrm{E}-03$ & $1.752071 \mathrm{E}+02$ \\
\hline $0.628319 \mathrm{E}+02$ & $0.000000 \mathrm{E}+00$ & 6 & $0.000000 \mathrm{E}+00$ & $9.000000 \mathrm{E}+01$ & $0.000000 \mathrm{E}+00$ & $0.000000 \mathrm{E}+00$ \\
\hline
\end{tabular}

[lines deleted]

$0.125664 \mathrm{E}+01 \quad 0.000000 \mathrm{E}+00$ $0.125664 \mathrm{E}+01 \quad 0.000000 \mathrm{E}+00$ $0.125664 \mathrm{E}+01 \quad 0.000000 \mathrm{E}+00$ $0.125664 \mathrm{E}+01 \quad 0.000000 \mathrm{E}+00$ $0.125664 \mathrm{E}+01 \quad 0.000000 \mathrm{E}+00$ $0.125664 \mathrm{E}+01 \quad 0.000000 \mathrm{E}+00$

$\begin{array}{lrrrr}1 & 5.488204 \mathrm{E}+00 & 6.842252 \mathrm{E}+01 & 2.018336 \mathrm{E}+00 & 5.103597 \mathrm{E}+00 \\ 2 & 0.000000 \mathrm{E}+00 & 9.000000 \mathrm{E}+01 & 0.000000 \mathrm{E}+00 & 0.000000 \mathrm{E}+00 \\ 3 & 5.635286 \mathrm{E}-02 & -1.303021 \mathrm{E}+02 & -3.645004 \mathrm{E}-02 & -4.297720 \mathrm{E}-02 \\ 4 & 0.000000 \mathrm{E}+00 & 9.000000 \mathrm{E}+01 & 0.000000 \mathrm{E}+00 & 0.000000 \mathrm{E}+00 \\ 5 & 2.966837 \mathrm{E}+00 & -1.115774 \mathrm{E}+02 & -1.091078 \mathrm{E}+00 & -2.758925 \mathrm{E}+00 \\ 6 & 0.000000 \mathrm{E}+00 & 9.000000 \mathrm{E}+01 & 0.000000 \mathrm{E}+00 & 0.000000 \mathrm{E}+00\end{array}$ 


\section{Appendix F Extreme-Event Tables for Normal Operation}

\section{F.1 Land-Based Wind Turbine Loads}

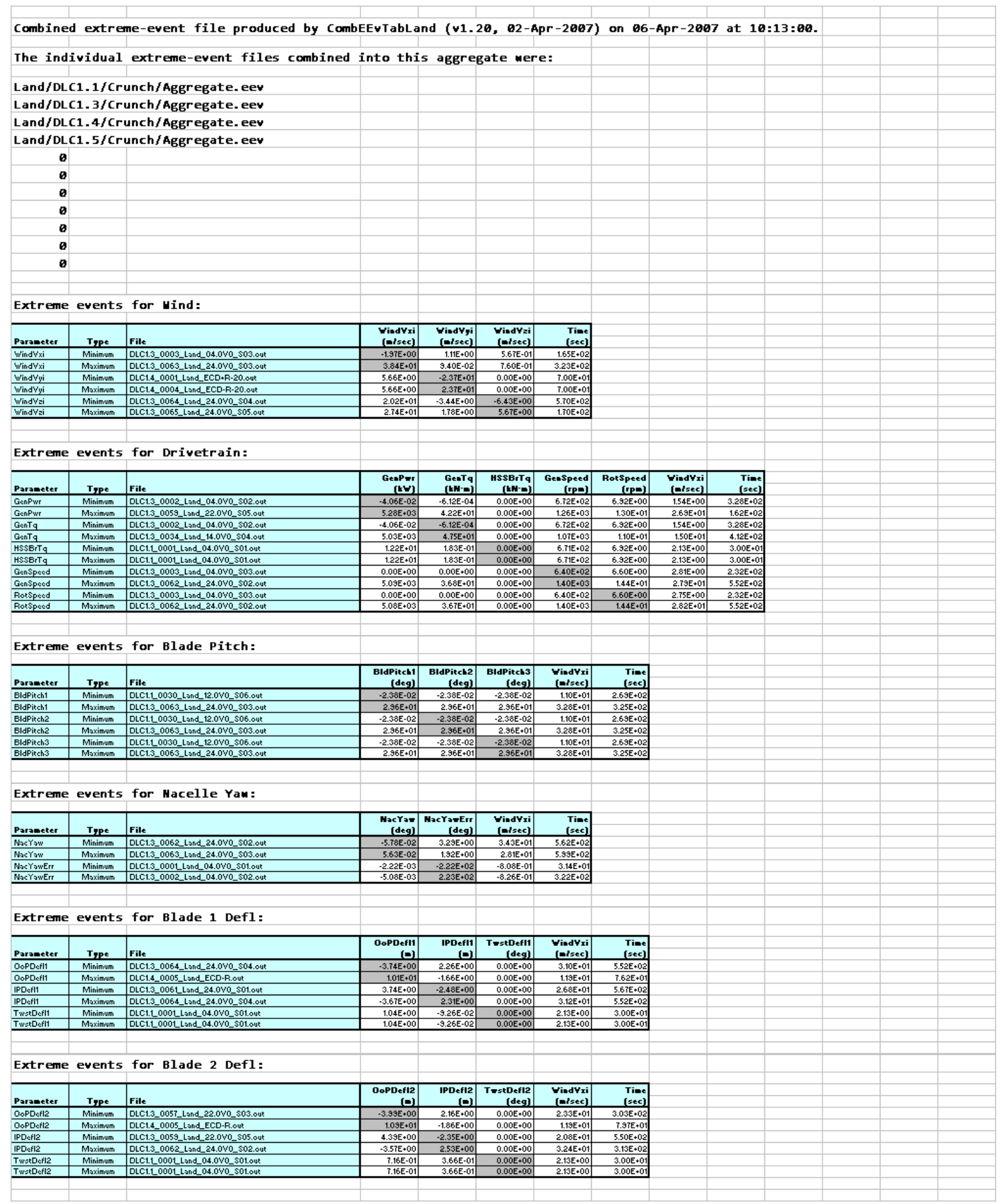




\begin{tabular}{|c|c|c|c|c|c|c|c|}
\hline Extreme & events & for Blade 3 Defl: & & & & & \\
\hline Parameter & Type & File & $\begin{array}{r}\text { OoPDef13 } \\
(0) \\
\end{array}$ & $\begin{array}{r}\text { IPDefl3 } \\
(\square) \\
\end{array}$ & \begin{tabular}{|r|} 
TestDefi3 \\
(deg) \\
\end{tabular} & 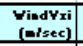 & $\begin{array}{c}\text { Time } \\
\text { (sec) }\end{array}$ \\
\hline $0 \circ P_{0 \times f l 3}$ & Minimum & DLC1.3_0064_Lond_24.0V0_S04.out & $-4.50 \mathrm{E}+00$ & $2.65 \mathrm{E}+00$ & $0.00 \mathrm{E}+00$ & $2.98 \mathrm{E}+01$ & $5.50 \mathrm{E}+02$ \\
\hline $0 \circ P D \& f 13$ & Moximum & DLC1.4_0005_Lond_ECD-R.out & $1.09 \mathrm{E}+01$ & $-1.78 \mathrm{E}+00$ & $0.00 \mathrm{E}+00$ & 1.19E+01 & $7.80 \mathrm{E}+01$ \\
\hline IPDefl3 & Minimum & DLC1.3_0063_Lond_24.0v0_S03.out & $3.99 E+00$ & $-2.41 E+00$ & $0.00 \mathrm{E}+00$ & $2.24 \mathrm{E}+01$ & $2.25 E+02$ \\
\hline PPDefl3 & Moximum & DLC1.3_0064_Lond_24.0V0_S04.out & $-4.48 \mathrm{E}+00$ & $2.70 E+00$ & $0.00 \mathrm{E}+00$ & $3.03 E+01$ & $5.50 \mathrm{E}+02$ \\
\hline TwetDefl3 & Minimum & DLC1._000LLIond_04.0V0_S01.out & $1.111+00$ & $-4.70 \mathrm{E}-01$ & $0.00 E+00$ & $2.13 E+00$ & $3.00 \mathrm{E}+01$ \\
\hline$T_{w a t D e f l 3}$ & Moximum & DLC1.L_000LLLond_04.0V0_S01.0ut & $1.11 \mathrm{E}+00$ & $-4.70 \mathrm{E}-01$ & $0.00 E+00$ & $2.13 \mathrm{E}+00$ & $3.00 \mathrm{E}+0$ \\
\hline
\end{tabular}

Extreme events for Blade Clrnc:

\begin{tabular}{|c|c|c|c|c|c|c|c|c|}
\hline Parameter & Type & File & $\begin{array}{r}\text { BidCIract } \\
(0 \\
\end{array}$ & \begin{tabular}{r|} 
BldClrece \\
$(\Xi)$ \\
\end{tabular} & $\begin{array}{r}\text { BidClrac } 3 \\
(0) \\
\end{array}$ & $\begin{array}{r}\text { Azineth } \\
\text { (deg] }\end{array}$ & $\begin{array}{l}\begin{array}{l}\text { YiddVri } \\
\text { (Elsec) }\end{array} \\
\end{array}$ & $\begin{array}{l}\text { Time } \\
\text { (sec) }\end{array}$ \\
\hline BldCline1 & Minimum & DLC1.4_0005_Lond_ECD-R.out & $-2.93 E+00$ & $6.03 \mathrm{E}+00$ & $3.78 \mathrm{E}+00$ & $7.68 \mathrm{E}+01$ & $1.19 \mathrm{E}+01$ & $7.62 E+01$ \\
\hline EldClrnc1 & Maximum & DLC1.3_0064_Lond_24.0V0_S04.out & $1.56 \mathrm{E}+01$ & $9.84 \mathrm{E}+00$ & $1.14 E+01$ & $1.91 E+02$ & $3.10 E+01$ & $5.52 E+02$ \\
\hline BldClinc2 & Minimum & DLC1.4_0005_Lond_ECD-R.out & $3.43 \mathrm{E}+00$ & $-4.10 E+00$ & $5.66 \mathrm{E}+00$ & $3.22 E+02$ & $1.19 E+01$ & $7.97 E+01$ \\
\hline BldClinc2 & Moximum & DLC1.3_0057_Lond_22.0V0_S03.out & $9.35 E+00$ & $1.59 \mathrm{E}+01$ & $9.27 \mathrm{E}+00$ & $1.12 \mathrm{E}+02$ & $2.33 \mathrm{E}+01$ & $3.03 E+02$ \\
\hline BldClrnc3 & Minimum & DLC1.4_0005_Lond_- & $6.03 \mathrm{E}+00$ & $3.44 E+00$ & $-4.01 \mathrm{E}+00$ & $1.98 \mathrm{E}+0 \mathrm{E}$ & $1.9 \mathrm{E}+01$ & \\
\hline BldClrnc3 & Moximum & DLC1.3_0064_Land_24.0V0_S04.out & $9.56 \mathrm{E}+00$ & $1.16 E+01$ & $1.66 E+01$ & $3.30 \mathrm{E}+02$ & $2.98 \mathrm{E}+01$ & $5.50 \mathrm{E}+02$ \\
\hline
\end{tabular}

Extreme events for Hacelle Acc:

\begin{tabular}{|c|c|c|c|c|c|c|c|c|}
\hline Parameter & Type & File & 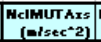 & \begin{tabular}{|r|} 
HelimUT Ays \\
$\left(\operatorname{cosec}^{-2} 2\right)$
\end{tabular} & 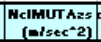 & 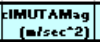 & $\begin{array}{l}\text { YindVri } \\
\text { (ulsec) }\end{array}$ & $\begin{array}{l}\text { Time } \\
\text { (sec) }\end{array}$ \\
\hline NAIMUTAAx & Minimum & DLC1.3_001LLIond_06.0V0_\$05.out & $-1.13 E+00$ & $9.30 \mathrm{E}-02$ & $-2.00 \mathrm{E}-01$ & $1.15 \mathrm{E}+00$ & $\frac{2.22 E+00}{4}$ & $1.52 \mathrm{E}+02$ \\
\hline NelMUTA $A \times s$ & Moximum & DLC1.3_0064_Lond_24.0V0_S04.out & $1.35 E+00$ & $-4.68 \mathrm{E}-02$ & $2.55 \mathrm{E}-01$ & $1.37 \mathrm{E}+00$ & $2.49 E+01$ & $4.90 \mathrm{E}+02$ \\
\hline NeIMUTAys & Minimum & DLC1.3_0065_Lond_24.0V0_S05.out & $3.52 E-01$ & $-8.72 E-01$ & $3.90 \mathrm{E}-02$ & $9.52 \mathrm{E}-01$ & $3.21 E+01$ & $3.02 E+02$ \\
\hline NclMUTAys & Moximum & DLC1.3_0057_Lond_22.0V0_S03.out & $3.42 E-02$ & $8.711-01$ & $3.91 \mathrm{E}-02$ & $8.72 \mathrm{E}-01$ & $3.18 \mathrm{E}+01$ & $4.82 E+02$ \\
\hline NelMUTA: & Minimum & DLC1.3_0065_Lond_24.0V0_SO5.out & $-4.87 \mathrm{TE}-01$ & $6.49 \mathrm{E}-02$ & $-3.13 E-01$ & $5.81 \mathrm{E}-01$ & $3.05 E+01$ & $3.80 \mathrm{E}+01$ \\
\hline NelMUTAzs & Moximum & DLC1.3_0066_Lond_24.0V0_S06.out & $5.32 E-01$ & \begin{tabular}{ll|l}
$-3.41 E-02$ \\
\end{tabular} & $3.28 E-01$ & $6.72 E-01$ & $2.08 E+01$ & $1.62 \mathrm{E}+02$ \\
\hline NclMUTAMag & Minimum & DLC1.L0066_Land_24.0V0_S06.out & $7.26 E-04$ & $-1.66 \mathrm{E}-0.3$ & $4.07 \mathrm{E}-03$ & $-9.00 E-03$ & $2.51 \mathrm{2}+01$ & $4.80 \mathrm{E}+02$ \\
\hline NclMUTAMsa & Moximum & DLC1.3_0064_Lond_24.0V0_S04.out & $1.35 \mathrm{E}+00$ & $-4.68 E-02$ & $2.55 E-01$ & $1.37 \mathrm{~T}+00$ & $2.49 \mathrm{EE}+01$ & $4.90 E+02$ \\
\hline
\end{tabular}

\begin{tabular}{|c|c|c|c|c|c|c|c|}
\hline Extrem & events & for TurTop Disp: & & & & & \\
\hline Paraneter & Type & File & \begin{tabular}{|l|}
$T D$ spFA \\
$(0)$
\end{tabular} & TTDspss & \begin{tabular}{|r|}
$T$ Dsp Test \\
(deg)
\end{tabular} & 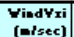 & $\begin{array}{c}\text { Tine } \\
\text { fsect }\end{array}$ \\
\hline$\pi \mathrm{DapFA}$ & Minimum & DLC1.3_0004_Lond_04.0V0_S04.out & $-1.55 E-01$ & $2.01 \mathrm{E}-02$ & $0.00 E+00$ & $2.94 \mathrm{E}+00$ & $3.40 E+02$ \\
\hline TTDspFA & Moximum & DLC1.3_005LLond_20.0V0_S03.out & $6.61 \mathrm{E}-01$ & $-6.29 E-02$ & $0.00 E+00$ & $1.43 \mathrm{E}+01$ & $1.19 \mathrm{E}+02$ \\
\hline TIDspSS & Minimum & DLC1.3_0065_Lond_24.0V0_S05.out & $2.50 \mathrm{E}-01$ & $-2.06 \mathrm{E}-01$ & $0.00 E+00$ & $2.37 E+01$ & $2.47 E+02$ \\
\hline TIDapSS & Moximum & DLC1.3_0063_Lond_24.0V0_\$03.out & $7.47 \mathrm{EE}-03$ & $1.00 \mathrm{E}-01$ & $0.00 E+00$ & $2.98 \mathrm{E}+01$ & $2.80 \mathrm{E}+02$ \\
\hline TDEpTwot & Minimum & DLC1.L000LLond_04.0V0_S01.out & $\frac{3.65 E-02}{365-602}$ & $\begin{array}{l}-6.85 E-03 \\
-655-03\end{array}$ & $0.00 E+00$ & $\frac{2.13 E+00}{2133+00}$ & $3.00 \mathrm{E}+01$ \\
\hline TIDEpTwat & Moximum & DLLC1.L000LLIond_04.0V0_S01.out & $3.65 \mathrm{EE}-02$ & -6.85E-03 & & $2.13 E+000$ & $3.00 E+01$ \\
\hline
\end{tabular}

Extreme events for Blade 1 Root:

\begin{tabular}{|c|c|c|c|c|c|c|c|c|c|c|c|c|}
\hline Parameter & Type & File & $\begin{array}{r}\text { Rootf } x+1 \\
(\mathrm{kH})\end{array}$ & \begin{tabular}{r|} 
Rootf,yci \\
$(\mathbf{k H})$ \\
\end{tabular} & $\begin{array}{r}\text { Rootf zct } \\
(\mathrm{kH})\end{array}$ & 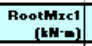 & \begin{tabular}{r|} 
Rootmyc1 \\
$($ tw- 1$)$ \\
\end{tabular} & 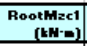 & 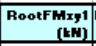 & \begin{tabular}{|r|} 
Rootminxy) \\
$(\mathrm{kH}-\mathrm{C})$ \\
\end{tabular} & $\begin{array}{c}\text { YiedVri } \\
\text { (ntsec] }\end{array}$ & $\begin{array}{c}\text { Tinec } \\
(s \in c) \\
\end{array}$ \\
\hline Root $\mathrm{Fxc1}$ & Minumum & DLC1.4_0006_Lond_ECD-R+20.out & $-1.70 \mathrm{E}+02$ & $-7.76 \mathrm{E}+01$ & $6.85 \mathrm{E}+02$ & $\begin{array}{ll}.41 E+03 \\
\end{array}$ & $-6.68 \mathrm{E}+03$ & $\frac{.2 .43 E+02}{-20}$ & $1.87 \mathrm{E}+02$ & & $1.71 \mathrm{~T}+01$ & \\
\hline RootFxc1 & Moximum & DLC1.3_0033_Lond_14.0V0_S03.out & $6.12 E+02$ & $-2.75 E+02$ & $9.14 E+02$ & $7.09 \mathrm{E}+03$ & $2.11 \mathrm{E}+04$ & $-4.06 \mathrm{E}+01$ & $6.71 \mathrm{71}+02$ & $2.22 E+04$ & $1.05 E+01$ & $6.05 E+02$ \\
\hline Rootfyc1 & Minimum & DLC1.1_0063_Lond_24.0V0_S03.out & $2.73 \mathrm{E}+02$ & $-4.80 E+02$ & $8.40 \mathrm{E}+02$ & $1.05 E+04$ & $6.05 E+03$ & $-8.38 \mathrm{E}+01$ & $5.54 \mathrm{E}+02$ & $1.21 E+04$ & $2.97 E+01$ & $4.32 \mathrm{E}+02$ \\
\hline Rootfyc1 & Moximum & DLC1.L0063_Lond_24.0V0_SO3.out & $5.25 E+01$ & $2.87 \mathrm{~F}+02$ & $1.00 \mathrm{E}+03$ & $-7.42 E+03$ & $-9.49 E+02$ & $-1.62 \mathrm{E}+02$ & $2.92 E+02$ & $7.48 \mathrm{E}+03$ & $2.70 \mathrm{E}+01$ & $5.99 \mathrm{9}+02$ \\
\hline Rootfac1 & Minimum & DLC1.3_0003_Lond_04.0V0_S03.out & $3.02 E+01$ & $2.72 E+00$ & $1.89 \mathrm{E}+01$ & $-6.60 \mathrm{E}+01$ & $1.33 \mathrm{E}+03$ & $-2.27 E+01$ & $3.03 E+01$ & $1.33 \mathrm{E}+03$ & $2.12 E+00$ & $2.26 \mathrm{E}+02$ \\
\hline Rootfact & Maximum & DLC1.1_0062_Lond_24.0V0_S02.out & $9.49 E+01$ & $-1.31 E+02$ & $1.495 \cdot 03$ & $1.74 \mathrm{E}+03$ & $-9.56 \mathrm{E}+02$ & $-1.49 \mathrm{E}+02$ & $1.61 \mathrm{E}+02$ & $1.99 \mathrm{E}+03$ & $3.02 E+01$ & $5.53 \mathrm{E}+02$ \\
\hline RootMxc1 & Minimum & DLC1.3_0063_Lond_24.0V0_S03.out & $1.39 \mathrm{E}+01$ & $2.82 \mathrm{E}+02$ & $9.12 E+02$ & $-7.48 E+03$ & $-2.13 \mathrm{E}+03$ & $-1.68 \mathrm{E}+02$ & $2.83 \mathrm{E}+02$ & $7.78 \mathrm{E}+03$ & $2.77 \mathrm{E}+01$ & $5.99 \mathrm{E}+02$ \\
\hline RootMxc1 & Moximum & DLC1.L006_Lond_24.0V0_\$01.out & $3.96 \mathrm{E}+02$ & $-4.66 \mathrm{E}+02$ & $9.54 E+02$ & 1.11E +04 & $1.15 E+04$ & $-1.111 \mathrm{E}+02$ & $6.11 \mathrm{11}+02$ & $1.60 E+04$ & $2.62 \mathrm{E}+01$ & $5.66 \mathrm{E}+02$ \\
\hline RootMyc1 & Minimum & DLC1.4_0006_Lond_ECD-R+20.out & $-1.67 \mathrm{E}+02$ & $-1.06 \mathrm{E}+02$ & $6.911+02$ & $1.93 \mathrm{E}+03$ & $-6.81 E+03$ & $-2.24 E+02$ & $1.98 \mathrm{E}+02$ & $7.07 E+03$ & $1.70 \mathrm{E}+01$ & $6.92 E+01$ \\
\hline RootMyc1 & Moximum & DLC1.4_0002_Lond_ECD+R.out & $5.69 \mathrm{EE}+02$ & $1.42 E+02$ & $7.15 E+02$ & $-7.78 E+02$ & $2.22 E+04$ & $1.18 E+02$ & $5.87 \mathrm{TE}+02$ & $2.23 E+04$ & $1.19 \mathrm{E}+01$ & $7.90 E+01$ \\
\hline RootMrec & Minimum & DLC1.4_0006_Lond_ECD-P R+20.out & $-1.36 \mathrm{E}+02$ & $8.54 \mathrm{E}+00$ & $6.93 \mathrm{E}+02$ & \begin{tabular}{l|l|}
$-1.75 \mathrm{E}+02$ \\
\end{tabular} & $\frac{-5.18 \mathrm{E}+03}{-12}$ & $-2.81 \mathrm{E}+02$ & $1.36 \mathrm{E}+02$ & $5.18 \mathrm{E}+03$ & $1.72 E+01$ & $6.88 \mathrm{E}+01$ \\
\hline RootMsec & Moximum & DLC1.3_0038_Lond_16.0V0_S02.out & $5.08 \mathrm{E}+02$ & $1.26 \mathrm{E}+02$ & $6.50 \mathrm{E}+02$ & $-2.00 \mathrm{E}+03$ & $1.77 \mathrm{E}+04$ & $2.111+02$ & $5.24 E+02$ & $1.795+04$ & $1.32 E+11$ & $1.53 E+02$ \\
\hline Root $\mathrm{M} M \mathrm{Mx} 1$ & Minimum & DLC1.3_0062_Lond_24.0V0_S02.0ut & $-1.23 \mathrm{E}+00$ & $-6.16 \mathrm{E}-01$ & $1.00 \mathrm{E}+03$ & $-1.86 \mathrm{E}+02$ & $-2.66 \mathrm{E}+03$ & $\begin{array}{ccc}-6.75 E+01 \\
-150\end{array}$ & $1.34 E+00$ & 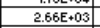 & $2.02 E+01$ & $6.08 \mathrm{E}+02$ \\
\hline RootFMxy1 & Moximum & DLC1.3_0030_Lond_12.0V0_S06.out & $5.67 \mathrm{E}+02$ & $-3.95 E+02$ & $8.61 \mathrm{E}+02$ & $9.70 \mathrm{E}+03$ & $1.87 \mathrm{E}+04$ & $-7.90 E+01$ & $6.91 E+02$ & $2.11 E+04$ & $1.11 \mathrm{E}+01$ & $1.06 \mathrm{E}+02$ \\
\hline RootMMxy1 & Minimum & DLC1.1_0060_Lond_22.0V0_S06. out & $7.40 \mathrm{E} \div 01$ & $-3.12 \mathrm{E}+01$ & $1.10 \mathrm{E}+03$ & $-5.40 \mathrm{E}+00$ & $-4.13 E+00$ & $-9.99 \mathrm{E}+01$ & $8.03 E+01$ & $6.79 E+00$ & $2.05 E+01$ & $5.92 \mathrm{E}+02$ \\
\hline RootMMxy1 & Moximum & DLC1.4_0005_Lond_ECD-R.out & $5.53 \mathrm{E}+02$ & $-3.22 E+02$ & $5.66 \mathrm{E}+02$ & $8.53 \mathrm{E}+03$ & $2.17 \mathrm{FE}+04$ & $-2.03 \mathrm{E}+02$ & $6.40 \mathrm{E}+02$ & $2.33 E+04$ & $1.19 \mathrm{E}+01$ & $7.63 \mathrm{E}+01$ \\
\hline
\end{tabular}

Extreme events for Blade 2 Root:

\begin{tabular}{|c|c|c|c|c|c|c|c|c|c|c|c|c|}
\hline Parameter & Type & File & \begin{tabular}{r|} 
RootFrce \\
$(\mathrm{tw})$ \\
\end{tabular} & \begin{tabular}{r|r|} 
Rootf, $c 2$ \\
$(\mathrm{k} W)$ \\
\end{tabular} & \begin{tabular}{r|} 
RootFzce \\
$(\mathrm{tw})$ \\
\end{tabular} & 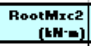 & 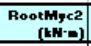 & 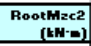 & \begin{tabular}{|r|} 
Rootf Mxx 2 \\
$(\mathbf{t H})$ \\
\end{tabular} & 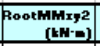 & $\begin{array}{c}\text { GindVri } \\
\text { (n) }\end{array}$ & $\begin{array}{c}\text { Tince } \\
(s \in c) \\
\end{array}$ \\
\hline$\overline{R o o t F \times c 2}$ & Minimum & DLC1.4_0003_Lond_ECD+R+20.out & $-1.80 E+02$ & $1.14 \mathrm{E}+02$ & $1.05 E+03$ & $-2.31 E+03$ & $-6.43 E+03$ & $-2.51 E+02$ & $2.13 \mathrm{E}+02$ & $6.84 \mathrm{E}+03$ & $1.68 \mathrm{E}+01$ & $6.97 \mathrm{9t}+01$ \\
\hline Rootf $\times \leq 2$ & Moximum & DLC1.4_0005_Lond_ECD-R.out & \begin{tabular}{ll|l}
$6.13 E+02$ \\
\end{tabular} & $-3.55 E+02$ & $7.18 \mathrm{E}+02$ & $9.62 E+03$ & $2.36 \mathrm{E}+04$ & $-2.38 \mathrm{E}+02$ & $7.08 \mathrm{E}+02$ & $2.55 E+04$ & $1.19 \mathrm{E}+01$ & $7.98 \mathrm{E}+0.01$ \\
\hline Rootfyc2 & Minimum & DLC1.L0066_Lond_24.0V0_S06.out & $2.59 \mathrm{E}+02$ & $-5.02 E+02$ & $9.14 E+02$ & $1.08 E+04$ & $4.91 E+03$ & $-1.03 E+02$ & $5.64 \mathrm{E}+02$ & 1.18E+04 & $2.76 E+01$ & $5.94 \mathrm{E}+02$ \\
\hline Rootfyc2 & Moximum & DLC1.L0054_Lond_20.0V0_S06.out & $3.91 E+01$ & $2.75 E+02$ & $1.03 E+03$ & $-6.29 E+03$ & $-3.24 E+02$ & $-1.32 E+02$ & $2.78 E+02$ & $6.30 \mathrm{E}+03$ & $1.78 E+01$ & $5.33 \mathrm{E}+02$ \\
\hline Rootface & Minimum & DLC1.3_0003_Lond_04.0V0_\$03.out & $4.63 \mathrm{E}+01$ & $-7.93 \mathrm{E}+00$ & $1.75 E+01$ & $2.00 \mathrm{E}+02$ & $2.09 E+03$ & $-3.69 \mathrm{gE}+01$ & $4.70 \mathrm{E}+01$ & $2.10 \mathrm{E}+03$ & $2.75 E+00$ & $2.32 \mathrm{E}+02$ \\
\hline Rootface & Moximum & DLC1.L0062_Lond_24.0V0_S02.out & $1.86 \mathrm{E}+02$ & $-9.65 E+01$ & $1.49 E+03$ & $1.05 E+03$ & $2.70 E+03$ & $-1.28 E+02$ & $2.10 \mathrm{E}+02$ & $2.90 \mathrm{E}+03$ & $2.79 E+01$ & $5.51 E+02$ \\
\hline RootMxc2 & Minimum & DLC1.3_0065_Lond_24.0\%0_S05.out & $4.92 \mathrm{E}+01$ & $2.49 E+02$ & $9.03 E+02$ & $-7.06 E+03$ & $-1.08 \mathrm{E}+03$ & $-1.53 \mathrm{E}+02$ & $2.55 E+02$ & $7.12 E+03$ & $2.44 E+01$ & $2.79 E+02$ \\
\hline RootMx 2 & Moximum & DLC1.L0063_Lond_24.0V0_S03.out & $4.27 \mathrm{E}+02$ & $-4.79 E+02$ & $8.65 \mathrm{E}+02$ & $1.10 \mathrm{E}+04$ & $1.16 \mathrm{E}+04$ & $-6.83 \mathrm{E}+01$ & $6.42 E+02$ & $1.60 \mathrm{E}+04$ & $2.72 E+01$ & $5.99 \mathrm{9}+02$ \\
\hline RootMy<2 & Minimum & DLC1.3_0062_Lond_24.0V0_S02.out & $-9.40 E+01$ & $-5.96 \mathrm{E}+00$ & $1.10 E+03$ & $-9.24 E+02$ & $-7.16 E+03$ & $-4.98 E+01$ & $9.42 E+01$ & $7.21 E+03$ & $3.34 E+01$ & $3.12 E+02$ \\
\hline RootMy<2 & Moximum & DLC1.4_0005_Lond_ECD-R.out & $\begin{array}{ll}6.08 \mathrm{E}+02 \\
\end{array}$ & $-3.53 E+02$ & $6.99 \mathrm{E}+02$ & $9.58 \mathrm{E}+03$ & $2.36 \mathrm{E}+04$ & $-2.52 E+02$ & $7.03 E+02$ & $2.55 \mathrm{E}+04$ & $1.195+01$ & $7.97 \mathrm{E}+01$ \\
\hline RootMec2 & Minimum & DLC1.4_0003_Lond_ECD+R+20.out & $-1.43 E+02$ & $4.40 E+01$ & $1.08 \mathrm{E}+03$ & $-6.67 \mathrm{TE}+02$ & $-5.04 E+03$ & $-2.80 E+02$ & $1.50 \mathrm{E}+02$ & $5.08 \mathrm{E}+03$ & $1.69 \mathrm{E}+01$ & $6.95 E+01$ \\
\hline RootMec2 & Moximum & DLC1.3_0037_Lond_16.0V0_S01.out & $4.97 E+02$ & $1.90 \mathrm{E}+02$ & $8.70 \mathrm{E}+02$ & $-3.47 \mathrm{~F}+03$ & $1.76 \mathrm{E}+04$ & $1.95 E+02$ & $5.32 \mathrm{E}+02$ & $1.79 E+04$ & $1.26 \mathrm{E}+01$ & $3.75 E+01$ \\
\hline RootFMxy2 & Minimum & DLC1.3_0063_Lond_24.0V0_S03.out & $\begin{array}{ll}3.19 E-02 \\
\end{array}$ & $-1.56 \mathrm{E}-01$ & $1.04 \mathrm{E}+03$ & $-8.37 E+02$ & $-3.25 E+03$ & $-8.70 \mathrm{E}+01$ & $5.19 \mathrm{E}-02$ & $3.36 \mathrm{E}+03$ & $2.57 \mathrm{E}+01$ & $5.31 E+02$ \\
\hline RootFMxy2 & Moximum & DLC1.4_0005_Lond_ECD-R.out & $6.13 E+02$ & $-3.55 E+02$ & $\begin{array}{ll}7.18 \mathrm{E}+02 \\
\end{array}$ & $9.62 E+03$ & $2.36 E+04$ & $-2.38 E+02$ & $7.08 \mathrm{E}+02$ & $2.55 E+04$ & $1.19 \mathrm{E}+01$ & $7.98 \mathrm{E}+01$ \\
\hline RootMMxy2 & Minimum & DLC1._0052_Land_20.0V0_S04.out & $5.46 \mathrm{E}+01$ & $-1.45 E+01$ & $1.20 \mathrm{E}+03$ & $8.50 \mathrm{E}+00$ & $1.64 \mathrm{E}+01$ & $-1.13 E+02$ & $5.65 E+01$ & $1.14 E+01$ & $1.99 E+01$ & $4.92 E+02$ \\
\hline RootMMxy2 & Moximum & DLC1.4_0005_Lond_ECD-R.out & $6.13 E+02$ & $-3.55 E+02$ & $7.18 \mathrm{E}+02$ & $9.62 E+03$ & $2.36 E+04$ & $-2.38 \mathrm{E}+02$ & $7.08 \mathrm{E}+02$ & $2.55 E+04$ & $1.19 \mathrm{E}+01$ & $7.98 E+01$ \\
\hline & & & & & & & & & & & & \\
\hline
\end{tabular}


Extreme events for Blade 3 Root:

\begin{tabular}{|c|c|c|c|c|c|c|c|c|c|c|c|c|}
\hline Parameter & Type & File & $\begin{array}{r}\text { Rootf } \text { res }^{\text {(tw) }} \\
\end{array}$ & \begin{tabular}{|r|} 
Rootfyc3 \\
$(\mathrm{kW})$ \\
\end{tabular} & \begin{tabular}{r|} 
Rootfzcc \\
$(\mathrm{tW})$ \\
\end{tabular} & $\begin{array}{r}\text { RootMrc3 } \\
(\mathbf{k H}-0)\end{array}$ & \begin{tabular}{|r|} 
Rootmyc3 \\
$(\mathbb{k} H-0)$
\end{tabular} & $\begin{array}{r}\text { RootMzc3 } \\
(\text { LW-D }) \\
\end{array}$ & \begin{tabular}{|r|} 
RootFMxy3 \\
$(\mathbf{k W})$ \\
\end{tabular} & \begin{tabular}{|r|} 
RootmMxy3 \\
(tWH-D)
\end{tabular} & $\begin{array}{c}\begin{array}{c}\text { YiedVri } \\
\text { (elsecs) }\end{array} \\
\end{array}$ & $\begin{array}{c}\text { Timec } \\
(s \in c) \\
\end{array}$ \\
\hline RootF $x<3$ & Minimum & DLC1.4_0002_Lond_ECD+R.out & $-1.84 \mathrm{E}+02$ & $1.05 E+02$ & $1.07 E+03$ & $-1.89 \mathrm{E}+03$ & $-6.50 \mathrm{E}+03$ & $-2.34 \mathrm{E}+02$ & $2.11 \mathrm{E}+02$ & $6.76 \mathrm{E}+03$ & $1.26 \mathrm{E}+01$ & $6.88 \mathrm{E}+01$ \\
\hline Root $\times x<3$ & Moximum & DLC1.3_0035_Land_14.0V0_SO5.out & $6.22 E+02$ & $-2.90 \mathrm{E}+02$ & $6.49 E+02$ & $6.87 \mathrm{TE}+03$ & $2.17 E+04$ & $-3.34 \mathrm{E}+01$ & $6.87 \mathrm{TE}+02$ & $2.27 \mathrm{E}+04$ & $1.39 E+01$ & $1.28 E+02$ \\
\hline Rootfyc3 & Minimum & DLC1.L0063_Land_24.0V0_S03.out & $4.59 E+02$ & $-5.03 E+02$ & $8.90 \mathrm{E}+02$ & $1.15 E+04$ & $1.26 \mathrm{E}+04$ & $-5.21 E+01$ & $6.81 \mathrm{E}+02$ & $1.71 E+04$ & $2.62 \mathrm{E}+01$ & $5.98 \mathrm{E}+02$ \\
\hline RootFy,3 & Moximum & DLC1.L0054_Lond_20.0V0_SO6.out & $5.85 E+01$ & $2.72 E+02$ & $9.69 \mathrm{E}+02$ & $-6.28 \mathrm{E}+03$ & $4.46 \mathrm{E}+02$ & $-1.16 \mathrm{E}+02$ & $2.78 \mathrm{E}+02$ & $6.26 \mathrm{E}+03$ & $1.89 \mathrm{E}+01$ & $5.31 \mathrm{E}+02$ \\
\hline Rootfacs & Minimum & DLC1.3_0003_Lond_04.0V0_S03.out & $4.10 \mathrm{E}+01$ & $-1.22 \mathrm{E}+01$ & $1.91 \mathrm{1}+01$ & $2.80 \mathrm{E}+02$ & $1.80 \mathrm{E}+03$ & $-9.34 \mathrm{~S}+00$ & $4.28 \mathrm{E}+01$ & $1.82 \mathrm{E}+03$ & $2.34 \mathrm{E}+00$ & $2.29 \mathrm{E}+02$ \\
\hline RootFac3 & Moximum & DLC1.L0059_Lond_22.0V__S05.out & $2.42 E+02$ & $-3.20 E+01$ & $1.43 E+03$ & $1.95 E+02$ & $5.16 \mathrm{E}+03$ & $-8.38 E+01$ & $2.45 E+02$ & $5.17 \mathrm{E}+03$ & $2.66 \mathrm{E}+01$ & $1.63 E+02$ \\
\hline RootMxe3 & Minimum & DLC1.3_0063_Lond_24.0V0_S03.out & $1.01 E+02$ & $2.62 E+02$ & $8.23 E+02$ & $-7.04 E+03$ & $8.13 E+02$ & $-1.40 E+02$ & $2.81 E+02$ & $7.09 E+03$ & $3.21 E+01$ & $4.31 E+02$ \\
\hline RootMxc3 & Moximum & DLC1.L0063_Lond_24.0V0_S03.out & $4.54 E+02$ & $-4.98 \mathrm{E}+02$ & $8.79 E+02$ & $1.15 E+04$ & $1.25 E+04$ & $-7.02 E+01$ & $6.73 E+02$ & $1.70 \mathrm{E}+04$ & $2.61 E+01$ & $5.98 \mathrm{E}+02$ \\
\hline RootMyes & Minimum & $\begin{array}{l}\text { DLC1.3_0064_Lond_24.0V0_S04.out } \\
\end{array}$ & $-1.22 E+02$ & $7.51 E+01$ & $1.20 \mathrm{E}+03$ & $-3.41 \mathrm{E}+03$ & 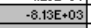 & $-9.61 \mathrm{E}+01$ & $1.44 \mathrm{E}+02$ & $8.82 E+03$ & $2.98 E+01$ & $5.49 E+02$ \\
\hline RootMyes & Moximum & $\begin{array}{l}\text { DLC1.4_0005_lond_ECD-R.out } \\
\end{array}$ & $6.00 \mathrm{E}+02$ & $-3.34 \mathrm{E}+02$ & $6.47 \mathrm{E}+02$ & $8.94 \mathrm{E}+03$ & $2.34 E+04$ & $-2.01 \mathrm{E}+02$ & $6.86 \mathrm{E}+02$ & $2.51 \mathrm{E}+04$ & $1.19 E+01$ & $7.80 \mathrm{E}+01$ \\
\hline RootMres & Minimum & DLC1.4_0003_Lind_ECD $+R+20$. out & $-1.43 E+02$ & $6.17 \mathrm{E}+01$ & $1.22 E+03$ & $\begin{array}{ll}-0.046 \mathrm{E}+03 \\
-1.03\end{array}$ & $\begin{aligned}-5.527 \mathrm{E}+03 \\
-53\end{aligned}$ & 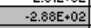 & $1.56 \mathrm{E}+02$ & $5.39 \mathrm{E}+03$ & $1.79 E+01$ & $6.80 \mathrm{E}+01$ \\
\hline RootMzc3 & Moximum & DLC1.3_0048_Lond_18.0V0_SO6.out & $5.53 \mathrm{E}+02$ & $1.58 \mathrm{E}+02$ & $9.08 \mathrm{E}+02$ & $-2.61 \mathrm{E}+03$ & $1.88 \mathrm{E}+04$ & $2.22 E+02$ & $5.75 E+02$ & $1.90 \mathrm{E}+04$ & $1.55 E+01$ & $1.00 E+02$ \\
\hline RootFMxy 3 & Minimum & DLC1.3_0064_Lond_24.0V0_S04.Out & $7.05 E-01$ & $1.84 \mathrm{E}-01$ & $1.14 \mathrm{E}+03$ & $-6.98 \mathrm{E}+02$ & $-2.15 E+03$ & $-1.16 \mathrm{E}+02$ & $1.49 E+00$ & $2.25 E+03$ & $2.03 E+01$ & $6.14 E+02$ \\
\hline RootFMxy3 & Moximum & DLC1.4_0005_Lond_ECD-R.out & $6.01 \mathrm{E}+02$ & $-3.37 E+02$ & $6.67 \mathrm{E}+02$ & $8.91 E+03$ & $2.33 E+04$ & $-1.85 E+02$ & $6.88 E+02$ & $2.49 E+04$ & $1.19 \mathrm{EE}+01$ & $7.81 E+0$ \\
\hline RootMMxy3 3 & Minimum & DLC1._0065_Lond_24.0V0_S05.out & $9.58 \mathrm{E}+01$ & $-6.25 \mathrm{E}+01$ & $1.12 E+03$ & $-3.71 E+00$ & $-7.07 \mathrm{EE}+00$ & $-9.76 \mathrm{E}+01$ & $1.14 \mathrm{E}+02$ & $-2.64 E+00$ & $2.79 E+01$ & $3.31 E+0$ \\
\hline RootMmxy3 & Moximum & DLC1.4_0005_Lond_ECD-R.out & $6.00 \mathrm{E}+02$ & $-3.34 \mathrm{E}+02$ & $6.47 \mathrm{EE}+02$ & $8.94 E+03$ & $2.34 \mathrm{E}+04$ & $-2.01 E+02$ & $\frac{1.1420 \mathrm{C}}{6.86 \mathrm{E}+02}$ & $2.51 E+04$ & e.T. & $7.80 \mathrm{E}$ \\
\hline
\end{tabular}

Extreme events for Blade 1 50\%:

\begin{tabular}{|c|c|c|c|c|c|c|c|c|}
\hline Parameter & Type & File & 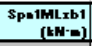 & 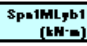 & \begin{tabular}{|r|}
$\begin{array}{r}\text { Spn1mLzb1 } \\
\text { (twH-G) }\end{array}$ \\
\end{tabular} & 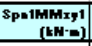 & $\begin{array}{l}\text { GindVri } \\
\text { (ntsec) }\end{array}$ & $\begin{array}{l}\text { Timec } \\
(s \in c) \\
\end{array}$ \\
\hline SprilMLxb1 & Minimum & DLC1.L0065_Lond_24.0V0_S05.out & $-1.50 E+03$ & $5.92 \mathrm{E}+02$ & $-4.02 E+01$ & $1.61 \mathrm{E}+03$ & $2.50 \mathrm{E}+01$ & $3.85 \mathrm{E}+02$ \\
\hline SprimLxb1 & Moximum & DLC1.3_0066_Lond_24.0V0_S06.out & $1.95 E+03$ & $-1.82 \mathrm{E}+03$ & $-7.35 E+01$ & $2.66 \mathrm{E}+03$ & $2.59 \mathrm{E}+01$ & $2.64 \mathrm{E}+\mathrm{C}$ \\
\hline SprilMLyb1 & Minimum & DLC1.3_0064_Lond_24.0V0_S04.out & $5.66 \mathrm{E}+02$ & $-2.95 E+03$ & $-1.03 E+02$ & $3.00 \mathrm{E}+03$ & $3.10 E+01$ & $5.52 \mathrm{E}+$ \\
\hline SprimLyb1 & Moximum & DLC1.4_0005_Lond_ECD-R.out & $1.111 \mathrm{E}+03$ & $6.54 \mathrm{E}+03$ & $-7.93 E+01$ & $6.64 \mathrm{E}+03$ & $1.19 E+01$ & $7.62 \mathrm{E}$ \\
\hline SprimLab1 & Minimum & DLC1.4_0006_Land_ECD-R+20.out & $4.13 E+02$ & $-1.06 \mathrm{E}+03$ & $-1.64 E+02$ & $\begin{array}{ll}1.14 \mathrm{E}+03 \\
\end{array}$ & $1.73 E+01$ & \\
\hline MLLb1 & Maximum & DLC1.3_0038_Lond_16.00 & $-1.01 E+03$ & $4.33 \mathrm{E}+03$ & $6.68 \mathrm{E}+01$ & $4.44 E+03$ & $1.32 \mathrm{E}+01$ & 1.53 \\
\hline$M M x_{y}$ & Minimum & DLC1.L0059_Lo & $-1.03 \mathrm{E}+00$ & $-4.24 \mathrm{E}+00$ & $-9.44 \mathrm{E}+01$ & $3.19 \mathrm{E}-01$ & $2.49 E+01$ & \\
\hline $1 \mathrm{MMMxy} 1$ & Moximum & \begin{tabular}{|l|} 
DLC1.4_0005_Lond_ECD-R.out \\
\end{tabular} & $1.11 \mathrm{E}+03$ & $6.54 \mathrm{E}+03$ & $-7.93 \mathrm{E}+01$ & $6.64 \mathrm{E}+03$ & $1.19 E+01$ & $7.62 \mathrm{E}$ \\
\hline
\end{tabular}

Extreme events for Blade 2 50\%:

\begin{tabular}{|c|c|c|c|c|c|c|c|c|}
\hline Parameter & Type & File & 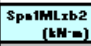 & 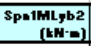 & 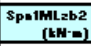 & 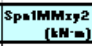 & $\begin{array}{c}\begin{array}{c}\text { ViedVri } \\
\text { (elsecs) }\end{array} \\
\end{array}$ & $\begin{array}{l}\text { Time } \\
(\text { sec) }\end{array}$ \\
\hline SprimLxb2 & Minimum & \begin{tabular}{|l} 
DLC1.L0062_Lond_24.0V0_\$02.out \\
\end{tabular} & 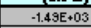 & $\begin{array}{ll}1.17 \mathrm{E}+03 \\
\end{array}$ & $-3.62 \mathrm{E}+01$ & $1.90 \mathrm{E}+03$ & $2.31 \mathrm{E}+01$ & $2.35 \mathrm{E}+02$ \\
\hline SpnilMLxb2 & Maximum & DLC1.3_0062_Lond_24.0V0_S02.out & $1.44 E+03$ & $-1.28 E+03$ & $-8.77 E+01$ & $1.93 \mathrm{E}+03$ & $2.63 \mathrm{E}+01$ & $5.55 E+02$ \\
\hline SprimLyb2 & Minimum & DLC1.3_0062_Lond_24.0V0_S02.out & $5.79 \mathrm{E}+02$ & $-3.06 E+03$ & $-7.94 E+01$ & $3.12 E+03$ & $3.30 \mathrm{E}+01$ & $3.13 E+02$ \\
\hline SprimLyb2 & Moximum & DLC1.4_0005_Land_ECD-R.out & $1.34 E+03$ & $7.05 E+03$ & $-1.11 \mathrm{E}+02$ & $7.18 E+03$ & $1.19 \mathrm{E}+01$ & $7.97 E+01$ \\
\hline SpniMLsb2 & Minimum & DLC1.4_0006_Lond_ECD-R+20.out & $4.17 \mathrm{E}+02$ & $-6.58 E+02$ & $-1.62 E+02$ & $7.79 E+02$ & $1.84 \mathrm{E}+01$ & $6.74 \mathrm{E}+01$ \\
\hline SprimLisb2 & Moximum & DLC1.3_0027_Land_12.0Y0_S03.out & $-7.94 \mathrm{E}+02$ & $4.23 \mathrm{E}+03$ & $6.28 E+01$ & $4.30 \mathrm{E}+0.3$ & $1.30 \mathrm{E}+01$ & $5.28 \mathrm{E}+02$ \\
\hline SprilMMxy2 & Minimum & DLC1.L0066_L & $1.28 \mathrm{E}+01$ & $-1.995+00$ & $-7.54 E+01$ & $-6.33 \mathrm{E}-01$ & $2.47 E+01$ & $2.96 E+0$ \\
\hline SprilMMxy2 & Maximum & \begin{tabular}{|l} 
DLC1.4_0005_Land_ECD-R.out \\
\end{tabular} & $1.34 \mathrm{E}+03$ & $7.05 E+03$ & $-1.111+02$ & $7.18 E+03$ & $1.19 \mathrm{E}+01$ & 7 \\
\hline
\end{tabular}

Extreme events for Blade 3 56\%:

\begin{tabular}{|c|c|c|c|c|c|c|c|c|}
\hline Parameter & Type & File & 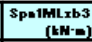 & 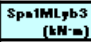 & 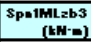 & 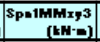 & $\begin{array}{l}\text { YindVxi } \\
\text { (Dlsec) }\end{array}$ & $\begin{array}{c}\text { Tinct } \\
(s \in c) \\
\end{array}$ \\
\hline SpnilMLxb33 & Minimum & DLC1.L0066_Lond_24.0V0_S06.out & $-1.45 E+03$ & $-6.00 \mathrm{E}+02$ & $-8.73 E+01$ & $1.58 \mathrm{E}+03$ & $2.69 \mathrm{E}+01$ & $4.81 E+02$ \\
\hline SprilMLxb3 & Moximum & DLC1.3_0062_Lond_24.0V0_S02.out & $1.72 E+03$ & $-2.36 \mathrm{E}+03$ & $-8.89 \mathrm{E}+01$ & $2.91 \mathrm{E}+03$ & $3.11 E+01$ & $5.53 \mathrm{E}+02$ \\
\hline SprilMLlyb3 & Minimum & DLC1.3_0064_Lond_24.0V0_S04.Out & $5.39 E+02$ & $-3.51 E+03$ & $-9.94 E+01$ & $3.55 \mathrm{E}+03$ & $2.98 \mathrm{E}+01$ & $5.50 \mathrm{E}+02$ \\
\hline SpnilMLyb3 & Maximum & DLC1.4_0005_Lond_ECD-R.out & $1.20 \mathrm{E}+03$ & $7.01 E+03$ & $-9.31 E+01$ & $7.11 \mathrm{E}+03$ & $1.19 E+01$ & $7.80 \mathrm{E}+01$ \\
\hline SpnilMLab3 & Minimum & DLC1.4_0003_Lond_ECD+R+20.out & $3.93 \mathrm{E}+02$ & $-9.97 \mathrm{9} 5+02$ & $-1.65 E+02$ & $1.07 \mathrm{E}+03$ & $1.79 \mathrm{E}+01$ & $6.80 \mathrm{E}+01$ \\
\hline SpnilMLab3 & Maximum & DLC1.3_003LLand_14.0V0_SO1.out & $-7.82 E+02$ & $4.61 \mathrm{E}+03$ & $6.18 E+01$ & $4.68 \mathrm{E}+03$ & $1.15 E+01$ & $5.43 E+02$ \\
\hline SponiMMxy3 & Minimum & DLC1.3_0060_Land_22.0V0_S06.out & $2.42 E+00$ & $1.45 E+00$ & $-8.35 \mathrm{E}+01$ & $2.47 \mathrm{E}+00$ & $2.67 E+01$ & $5.83 \mathrm{E}+01$ \\
\hline SpniMMxy3 & Moximum & |DLC1.4_0005_Lond_ECD-R.out & $1.20 E+03$ & $7.01 E+03$ & $-9.31 E+01$ & $7.111+03$ & $1.19 \mathrm{E}+01$ & $7.80 \mathrm{E}+01$ \\
\hline
\end{tabular}

Extreme events for LSS Main Br:

\begin{tabular}{|c|c|c|c|c|c|c|c|c|c|c|c|c|}
\hline & Type & File & $\begin{array}{r}\text { RotThrust } \\
\text { (twH) }\end{array}$ & \begin{tabular}{|l} 
LSSGagF; \\
(tw)
\end{tabular} & $\begin{array}{r}\text { LSSGagF } 22 \\
\text { (tw) }\end{array}$ & $\begin{array}{r}\text { RotTorg } \\
\text { (tw-D) }\end{array}$ & \begin{tabular}{|r|} 
LSSGagm,ga \\
$(\mathrm{EH}-\mathrm{G})$
\end{tabular} & \begin{tabular}{|r|} 
LSSGagMza \\
$(t \mathrm{H}-\boldsymbol{O})$
\end{tabular} & $\begin{array}{r}s s G a g F M z z \\
(\mathrm{tw})\end{array}$ & $\begin{array}{r}\text { SSGagmMgz } \\
\text { (tH-D) }\end{array}$ & $\begin{array}{r}\text { YindVxi } \\
\text { (edsece) }\end{array}$ & $\begin{array}{c}\text { Time } \\
\text { (sec) }\end{array}$ \\
\hline RotThruat & Minimum & DLC1.4_0002_Land_ECD+R.out & $-1.09 \mathrm{E}+01$ & $1.08 \mathrm{E}+03$ & $-9.95 E+02$ & $4.52 \mathrm{E}+03$ & $-3.88 \mathrm{E}+02$ & $6.39 \mathrm{gE}+03$ & $1.47 \mathrm{E}+03$ & $6.40 \mathrm{E}+03$ & $1.28 \mathrm{E}+01$ & $\mathrm{BE}+01$ \\
\hline RotThrugt & Moximum & DLC1.3_005_Lond_20.0\%0_\$03.out & $1.53 \mathrm{E}+03$ & $1.39 \mathrm{E}+03$ & $-3.42 E+02$ & $5.90 \mathrm{E}+03$ & $-2.90 \mathrm{E}+03$ & $1.54 \mathrm{E}+02$ & $1.43 E+03$ & $2.90 E+03$ & $1.31 E+01$ & $1.19 \mathrm{E}+02$ \\
\hline LSSGogFyo & Minimum & DLC1.L0064_Lond_24.0V0_S04.out & $5.54 E+02$ & $-1.76 \mathrm{E}+03$ & $2.92 E+01$ & $6.54 \mathrm{E}+03$ & $8.59 \mathrm{E}+03$ & $\begin{array}{ll}-3.45 E+02 \\
\end{array}$ & $1.76 \mathrm{E}+03$ & $8.60 \mathrm{E}+03$ & $2.111+01$ & $5.32 \mathrm{E}+02$ \\
\hline LSSGogFys & Moximum & DLC1.L0058_Lond_22.0Y0_S04.out & $5.01 E+02$ & $1.77 \mathrm{TE}+03$ & $-4.84 \mathrm{E}+01$ & $5.92 E+03$ & $-7.02 E+03$ & $-2.40 E+03$ & $1.77 \mathrm{TE}+03$ & $7.41 E+03$ & $2.51 E+01$ & $5.39 \mathrm{E}+02$ \\
\hline LSSGogfas & Minimum & DLC1._0063_Lond_24.0V0_S03.out & $5.34 \mathrm{E}+02$ & $2.23 \mathrm{E}+00$ & $-1.75 E+03$ & $6.34 \mathrm{E}+03$ & $-3.23 E+03$ & $9.03 \mathrm{E}+02$ & $1.75 E+03$ & $3.36 \mathrm{E}+03$ & $2.69 \mathrm{E}+01$ & $4.66 \mathrm{E}+02$ \\
\hline LSSGogfas & Moximum & $\begin{array}{l}\text { DLC1.L0065_Lond_24.0V0_S05.out } \\
\end{array}$ & $4.40 \mathrm{E}+02$ & $1.03 E+02$ & $1.75 \mathrm{E}+03$ & $5.93 \mathrm{E}+03$ & $\begin{array}{cl}-1.19 \mathrm{E}+03 \\
\end{array}$ & $\begin{array}{ll}-6.46 \mathrm{E}+03 \\
\end{array}$ & $1.76 \mathrm{E}+03$ & $6.57 \mathrm{E}+03$ & $2.67 \mathrm{E}+01$ & $1.711 \mathrm{0}+02$ \\
\hline RotTorg & Minimum & $\begin{array}{l}\text { DLC1.3_0003_Lond_04.0\%0_\$03.out } \\
\end{array}$ & $1.04 \mathrm{E}+02$ & $-9.74 \mathrm{E}+01$ & $1.46 \mathrm{E}+03$ & $-7.36 \mathrm{E}+011$ & $\begin{array}{ll}2.58 \mathrm{E}+03 \\
\end{array}$ & $-4.495 E+02$ & $1.46 \mathrm{E}+03$ & $4.61 \mathrm{E}+03$ & $1.22 E+00$ & $1.95 E+02$ \\
\hline RotTorg & Moximum & DLC1.L0062_Land_24.0V0_s02.out & $8.75 E+02$ & $1.59 \mathrm{E}+03$ & $4.80 \mathrm{E}+01$ & $7.84 \mathrm{E}+03$ & $1.06 \mathrm{E}+03$ & $2.61 \mathrm{E}+03$ & $1.59 \mathrm{E}+03$ & $2.8115+03$ & $1.84 \mathrm{E}+01$ & $4.89 \mathrm{E}+01$ \\
\hline LSSGogMMo & Minimum & DLC1.4_0006_Lond_ECD-R+20.out & $1.43 \mathrm{E}+02$ & $-7.48 E+02$ & $-1.23 \mathrm{E}+03$ & $4.84 \mathrm{E}+03$ & $-1.47 E+04$ & $2.11 \mathrm{E}+03$ & $1.44 E+03$ & $1.48 \mathrm{E}+04$ & $1.70 \mathrm{E}+01$ & $6.92 E+01$ \\
\hline ogmys & Moximum & DLC1.3_003LLond_14.0V0_S01.out & $8.64 \mathrm{E}+02$ & $-6.33 \mathrm{E}+02$ & $1.33 \mathrm{E}+03$ & $5.61 \mathrm{E}+03$ & $1.36 \mathrm{E}+04$ & $-1.34 \mathrm{E}+0$ & $1.48 \mathrm{E}+03$ & $1.37 \mathrm{E}+04$ & $1.41 \mathrm{E}+01$ & $4.25 E+02$ \\
\hline & Minimum & DLC1.3. & $2.89 \mathrm{E}+02$ & $-1.29 E+03$ & $1.76 E+02$ & $4.87 \mathrm{E}+03$ & $-3.11 E+03$ & $-1.29 E+04$ & 1.3 & 04 & $2.97 E+01$ & $5.51 E+02$ \\
\hline LSSGogMzo & Moximum & DLC1.4_0002_ & $E+02$ & $-1.30 \mathrm{E}+03$ & $5.75 E+02$ & $5.56 \mathrm{E}+03$ & $2.28 \mathrm{E}+03$ & $1.41 E+04$ & 03 & +04 & +01 & $8.18 E+01$ \\
\hline LSSGogFMyz & Minimum & DLC1.3_0062_Lond_24.0V0_S02.out & $4.08 \mathrm{E}+02$ & $9.96 E+02$ & $.5 .66 \mathrm{E}+02$ & $5.40 \mathrm{E}+03$ & $8.25 E+03$ & $3.60 \mathrm{E}+03$ & $1.15 \mathrm{E}+03$ & +03 & $3.10 \mathrm{E}+01$ & \\
\hline LSSGogFMyz & Moximum & DLC1.L0063_Land_24.0V0_S03.out & $7.06 E+02$ & $5.79 E+02$ & $1.74 \mathrm{E}+03$ & $6.38 \mathrm{E}+03$ & $-1.46 \mathrm{E}+03$ & $-9.92 \mathrm{E}+03$ & $1.83 \mathrm{E}+03$ & $1.00 E+04$ & $2.62 E+01$ & $5.98 \mathrm{E}+02$ \\
\hline LSSGogMMYz & Minimum & DLC1.L0056_Lond_22.0V0_S02.out & $5.19 \mathrm{E}+02$ & $-8.66 \mathrm{E}+02$ & $-1.38 E+03$ & $6.14 \mathrm{E}+03$ & $-1.10 \mathrm{E}+00$ & $-3.40 E+00$ & $1.63 E+03$ & +01 & $2.86 \mathrm{E}+01$ & \\
\hline SSGogMM & Moximum & DLC1.4_0002_Lind_ECD+R.out & $1.10 E+03$ & $1.45 E+0$ & $-1.37 \mathrm{E}+$ & $5.64 \mathrm{E}+03$ & $-1.44 E+04$ & $E+03$ & $1.37 \mathrm{FE}$ & $1.57 \mathrm{EE}+04$ & $1.19 \mathrm{E}+01$ & \\
\hline
\end{tabular}

Extreme events for Yam Bearing:

\begin{tabular}{|c|c|c|c|c|c|c|c|c|c|c|c|c|}
\hline Parameter & Type & File & 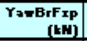 & $\begin{array}{r}Y_{2=B r F, p p} \\
(\text { tw) }\end{array}$ & $\begin{array}{r}\text { YanBrFzp } \\
(\text { LH }\end{array}$ & 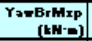 & 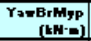 & 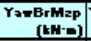 & 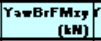 & 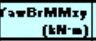 & 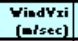 & $\begin{array}{c}\text { Timect } \\
(s \in c)\end{array}$ \\
\hline 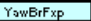 & Minimum & DLC1.3_0003_Lond_04.0V0_\$03.out & $-3.01 E+02$ & $5.76 \mathrm{E}+01$ & $-4.65 E+03$ & $-1.94 \mathrm{E}+02$ & $-1.33 E+03$ & $-9.54 \mathrm{5}+02$ & $3.07 \mathrm{~V}+02$ & $1.34 \mathrm{E}+03$ & $3.42 E+00$ & $2.56 \mathrm{E}+02$ \\
\hline 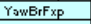 & Moximum & DLC1.3_005LLand_20.0V0_\$03.out & $1.64 E+03$ & $3.73 \mathrm{E}+00$ & $-4.76 \mathrm{E}+03$ & $6.17 \mathrm{EE}+03$ & $3.41 E+03$ & $2.21 E+03$ & $1.64 E+03$ & $7.05 E+03$ & $1.32 E+01$ & $1.19 \mathrm{E}+02$ \\
\hline YowErfyp & Minimum & DLC1.3_0065_Lond_24.0V0_S05.out & $5.58 \mathrm{E}+02$ & $-3.69 \mathrm{E}+02$ & $-4.70 \mathrm{E}+03$ & $6.80 \mathrm{E}+03$ & $4.50 \mathrm{E}+03$ & $1.26 \mathrm{E}+03$ & $6.70 \mathrm{E}+02$ & $8.15 \mathrm{E}+03$ & $2.37 E+01$ & $2.47 \mathrm{TE}+02$ \\
\hline YowErfyp & Moximum & DLC1.3_0057_Lond_22.0v0_\$03.out & $3.57 \mathrm{E}+02$ & $3.50 \mathrm{E}+02$ & $-4.6 \mathrm{gE}+03$ & $4.42 \mathrm{E}+03$ & $-2.81 E+03$ & $-6.35 E+02$ & $4.99 \mathrm{E}+02$ & $5.24 E+03$ & $3.06 \mathrm{E}+01$ & $4.83 \mathrm{E}+02$ \\
\hline YowErFsp & Minimum & DLC1.L0063_Lond_24.0V0_\$03.out & $4.61 \mathrm{E}+02$ & $-1.35 E+02$ & $-5.41 E+03$ & $7.48 \mathrm{E}+03$ & $5.53 \mathrm{E}+02$ & $9.41 E+03$ & $4.80 \mathrm{E}+02$ & $7.50 \mathrm{E}+03$ & $2.62 E+01$ & $5.98 \mathrm{E}+02$ \\
\hline YowErFsp & Moximum & DLC1.3_0062_Lond_24.0V0_\$02.out & $2.53 E+02$ & $-2.90 \mathrm{E}+02$ & $-4.36 \mathrm{E}+03$ & $5.14 E+03$ & $9.85 \mathrm{E}+03$ & $-6.37 E+03$ & $3.86 \mathrm{E}+02$ & $1.111 E+04$ & $3.16 E+01$ & $5.64 E+02$ \\
\hline$Y_{9 w E r M \times p}$ & Minimum & DLC1.3_0003_Lond_04.0V0_S03.out & $-2.16 \mathrm{E}+02$ & $9.32 \mathrm{E}+01$ & $-4.64 E+03$ & $-3.24 E+02$ & $-2.14 E+03$ & $-1.66 \mathrm{E}+03$ & $2.36 \mathrm{E}+02$ & $2.16 \mathrm{E}+03$ & $3.67 \mathrm{E}+00$ & $2.50 \mathrm{E}+02$ \\
\hline 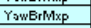 & Moximum & DLC1._0065_Lond_24.0V0_S05.out & $4.47 \mathrm{E}+02$ & $-2.91 E+02$ & $-5.20 \mathrm{E}+03$ & $8.42 E+03$ & $6.70 \mathrm{E}+03$ & $1.48 \mathrm{E}+03$ & $5.33 \mathrm{E}+02$ & $1.08 \mathrm{E}+04$ & $2.20 \mathrm{E}+01$ & $6.08 \mathrm{E}+02$ \\
\hline YawErMyp & Minimum & DLC1.4_0002_Lond_ECD+R.out & $9.53 \mathrm{E}+02$ & $-5.56 \mathrm{E}+01$ & $-4.63 E+03$ & $4.29 \mathrm{gE}+03$ & $-1.18 E+04$ & $-2.93 E+03$ & $9.54 E+02$ & $1.26 \mathrm{E}+04$ & $1.19 \mathrm{E}+01$ & $7.84 \mathrm{E}+01$ \\
\hline$Y_{a w E r M y p}$ & Moximum & DLC1.3_0064_Land_24.0V0_S04.out & $9.99 E+01$ & $-2.22 \mathrm{E}+02$ & $-4.47 \mathrm{E}+03$ & $5.66 \mathrm{E}+03$ & $1.51 E+04$ & $-3.91 E+03$ & $2.44 \mathrm{E}+02$ & $1.62 \mathrm{E}+04$ & $2.91 E+01$ & $5.51 E+02$ \\
\hline YawErMzp & Minimum & DLC1.3_0062_Lond_24.0V0_S02.out & $4.76 \mathrm{E}+02$ & $3.57 \mathrm{E}+01$ & $-4.56 \mathrm{E}+03$ & $4.41 E+03$ & $-8.64 E+02$ & $-1.23 E+04$ & \begin{tabular}{ll|l}
$4.77 E+02$ \\
\end{tabular} & $4.49 \mathrm{EE}+03$ & $3.43 E+01$ & $5.62 E+02$ \\
\hline YowBrMzp & Moximum & DLC1.3_0063_Lond_24.0V0_S03.out & $3.46 E+02$ & $1.53 E+01$ & $-4.81 E+03$ & $6.30 \mathrm{E}+03$ & $5.39 E+03$ & $1.20 \mathrm{E}+04$ & $3.46 \mathrm{E}+02$ & $8.29 E+03$ & $2.81 E+01$ & $5.99 \mathrm{gE}+02$ \\
\hline$Y_{3 w E r F M x y}$ & Minimum & DLC1.L0003_Lond_04.0V0_\$03.out & $4.63 \mathrm{E}-02$ & $3.47 \mathrm{E}-01$ & $-5.16 E+03$ & $7.66 \mathrm{E}+01$ & $-2.07 E+03$ & $-1.97 \mathrm{TE}+02$ & $1.66 \mathrm{E}-01$ & $2.07 E+03$ & $1.97 \mathrm{E}+00$ & $1.61 \mathrm{0}+02$ \\
\hline$Y_{5 w E r f M x y}$ & Moximum & DLC1.3_005LLand_20.0v0_\$03.out & $1.64 E+03$ & $3.73 E+00$ & $-4.76 \mathrm{E}+03$ & $6.17 \mathrm{E}+03$ & $3.41 E+03$ & $2.21 E+03$ & $1.64 E+03$ & $7.05 E+03$ & $1.32 \mathrm{E}+01$ & $1.19 E+02$ \\
\hline YowErMMxy & Minimum & DLC1.3_0005_Land_04.0Y0_S05.out & $2.49 \mathrm{EE}+02$ & $3.07 \mathrm{E}+01$ & $-4.63 \mathrm{E}+03$ & $2.08 \mathrm{E}+01$ & $-1.53 E+01$ & $-2.50 \mathrm{E}+03$ & $2.51 E+02$ & $1.32 E+01$ & $2.46 E+00$ & $6.24 E+02$ \\
\hline$Y_{5 w B_{r} M M x y}$ & Moximum & DLC1.3_0064_Land_24.0V0_S04.out & $9.99 E+01$ & $-2.22 E+02$ & $-4.47 \mathrm{EE}+03$ & $5.66 \mathrm{E}+03$ & $1.51 E+04$ & $-3.91 E+03$ & $2.44 \mathrm{E}+02$ & $1.62 E \div 04$ & $2.91 E+01$ & $5.511 \div 02$ \\
\hline & & & & & & & & & & & & \\
\hline
\end{tabular}


Extreme events for Tower Base:

\begin{tabular}{|c|c|c|c|c|c|c|c|c|c|c|c|c|}
\hline Para & Iype & File & $\begin{array}{r}\begin{array}{r}\text { TerBsFrt } \\
(\mathrm{tw})\end{array} \\
\end{array}$ & $\begin{array}{r}\text { TerBsF, } \\
(\mathbf{k w}) \\
\end{array}$ & 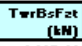 & 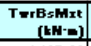 & $\begin{array}{r}\text { TerBsmgt } \\
(\mathbf{H}-\mathbf{E})\end{array}$ & \begin{tabular}{|r|}
$T=r B s H z t$ \\
$(\mathbf{k H}-0)$
\end{tabular} & \begin{tabular}{|r|} 
TerBsFMxy \\
$(\mathrm{LW})$ \\
\end{tabular} & 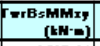 & $\begin{array}{r}\text { GindVri } \\
(-1, j e c)\end{array}$ & $\begin{array}{c}\text { Timec } \\
(s \in c) \\
\end{array}$ \\
\hline$T_{w r B}=F x t$ & Minimum & DLC1.3_0004_Lond_04.0V0_\$04.out & $-4.13 E+02$ & $5.711+01$ & $-9.26 \mathrm{E}+03$ & $-4.42 E+03$ & $-3.49 E+04$ & $1.30 \mathrm{E}+03$ & $4.15 E+02$ & $3.52 E+04$ & $2.93 \mathrm{E}+00$ & $3.39 \mathrm{E}+02$ \\
\hline TwrB $F \times x t$ & Moximum & DLC1.3_005LLond_20.0V0_S03.out & $1.81 \mathrm{E}+03$ & $-4.31 E+01$ & $-9.38 \mathrm{E}+03$ & $9.04 E+03$ & $1.53 \mathrm{E}+05$ & $1.49 \mathrm{E}+03$ & $1.81 E+03$ & $1.53 \mathrm{E}+05$ & 1.43E+01 & $1.19 E+02$ \\
\hline TwrBsfyt & Minimum & DLC1.3_0064_Lond_24.0V0_\$04.out & $2.92 \mathrm{E}+02$ & $-4.65 E+02$ & $-9.23 \mathrm{E}+03$ & $4.09 \mathrm{E}+04$ & $2.64 E+04$ & $1.83 \mathrm{E}+03$ & $5.48 \mathrm{E}+02$ & $4.88 \mathrm{E}+04$ & $2.76 \mathrm{E}+01$ & $9.16 E+01$ \\
\hline$T_{\text {wrEeFyt }}$ & Moximum & \begin{tabular}{|l} 
DLC1.3_0057_Land_22.0V0_S03.out \\
\end{tabular} & $3.79 E+02$ & $4.42 E+02$ & $-9.31 E+03$ & $-2.77 E+04$ & $2.78 \mathrm{E}+04$ & $-8.14 E+02$ & $5.85 \mathrm{E}+02$ & \begin{tabular}{ll|l}
$.93 E+04$ \\
\end{tabular} & $3.111+0+1$ & $4.83 \mathrm{E}+02$ \\
\hline$T_{\text {TwEofat }}$ & Minimum & \begin{tabular}{|l} 
DLC1.L0063_Lond_24.0V0_SO3.out \\
\end{tabular} & $4.06 E+02$ & $-1.69 \mathrm{E}+02$ & $-1.05 E+04$ & $2.15 E+04$ & $3.82 E+04$ & $9.41 E+03$ & $4.40 E+02$ & $\begin{array}{ll}4.38 E+04 \\
\end{array}$ & $2.62 E+01$ & $5.98 \mathrm{E}+02$ \\
\hline TwrEs:Fat & Moximum & DLC1.3_0062_Lond_24.0V0_\$02.out & $6.28 \mathrm{E}+01$ & $-2.96 \mathrm{E}+02$ & $-8.96 \mathrm{E}+03$ & $3.15 E+04$ & $2.26 \mathrm{E}+04$ & $-6.36 \mathrm{E}+03$ & $3.02 E+02$ & $3.88 E+04$ & $3.16 \mathrm{E}+01$ & $5.64 E+02$ \\
\hline TwrEs:Mxt & Minimum & DLC1.3_0057_Lond_22.0V0_S03.out & $3.79 E+02$ & $4.42 E+02$ & $-9.31 E+03$ & $-2.77 E+04$ & $2.78 E+04$ & $-8.14 E+02$ & $5.85 E+02$ & $3.93 E+04$ & $3.11 E+01$ & $4.83 \mathrm{E}+02$ \\
\hline TwrEs:Mxt & Moximum & DLC1.3_0064_Lond_24.0V0_\$04.out & $2.92 E+02$ & $-4.65 E+02$ & $-9.23 E+03$ & $4.09 \mathrm{EE}+04$ & $2.64 E+04$ & $1.83 \mathrm{E}+03$ & $5.48 \mathrm{E}+02$ & $4.88 \mathrm{E}+04$ & $2.76 \mathrm{E}+01$ & $9.16 \mathrm{E}+0$ \\
\hline TwrBeMyt & Minimum & DLC1.3_0004_Lond_04.0V0_\$04.out & $-4.11 E+02$ & $6.37 \mathrm{E}+01$ & $-9.26 \mathrm{E}+03$ & $-4.77 \mathrm{E}+03$ & $-3.53 E+04$ & $1.58 \mathrm{E}+03$ & $4.17 E+02$ & $3.56 \mathrm{E}+04$ & $2.71 E+00$ & $3.40 E+02$ \\
\hline TwrBs:Myt & Moximum & DLC1.3_005LLLond_20.0V0_S03.out & $1.81 \mathrm{E}+03$ & $-4.31 E+01$ & $-9.38 \mathrm{E}+03$ & $9.04 E+03$ & $1.53 \mathrm{E}+05$ & $1.49 E+03$ & $1.81 E+03$ & $1.53 \mathrm{E}+05$ & $1.43 \mathrm{E}+01$ & $1.195+02$ \\
\hline TwrEgmat & Minimum & DLC1.3_0062_Lond_24.0V0_\$02.out & $5.34 E+02$ & $1.71 E+01$ & $-9.16 E+03$ & $2.63 \mathrm{E}+03$ & $45 E+04$ & $-1.23 E+04$ & $5.34 E+02$ & $\begin{array}{ll}4.46 E+04 \\
\end{array}$ & $3.43 E+01$ & $5.62 \mathrm{E}+02$ \\
\hline TwrBeMzet & Moximum & DLC1.3_0063_Lond_24.0V0_\$03.out & $2.32 E+02$ & $3.47 E+00$ & $-9.41 E+03$ & $4.90 \mathrm{E}+03$ & $3.02 \mathrm{E}+04$ & $1.20 E+04$ & $2.32 E+02$ & $3.06 \mathrm{E}+04$ & $2.81 \mathrm{E}+01$ & $5.99 E+02$ \\
\hline$T_{w F E x F x y}$ & Minimum & DLC1.3_0016_Land_08.0V0_S04.out & $5.93 \mathrm{E}-01$ & $-3.63 \mathrm{E}-01$ & $-9.26 \mathrm{E}+03$ & $1.08 \mathrm{E}+03$ & $3.46 \mathrm{E}+03$ & $-1.82 \mathrm{E}+03$ & $-1.12 E-01$ & $3.63 E+03$ & $3.32 E+00$ & $4.54 E+01$ \\
\hline TwEB:FMxy & Maximum & DLC1.3_005LLond_20.0V0_\$03.out & $1.61 \mathrm{E}+03$ & $-4.31 E+01$ & $-9.38 \mathrm{E}+03$ & $9.04 \mathrm{E}+03$ & $1.53 \mathrm{E}+05$ & $1.49 \mathrm{E}+03$ & $1.811+03$ & $1.53 E+05$ & $1.43 E+01$ & $1.19 \mathrm{E}+02$ \\
\hline TwrEs:MMxy & Minimum & DLC1.3_0009_Lond_06.0V0_s03.out & $3.95 E+01$ & $5.86 \mathrm{E}+00$ & $-9.26 \mathrm{E}+03$ & $1.84 \mathrm{E}+01$ & $4.21 E+00$ & $6.04 E+02$ & $4.00 \mathrm{E}+01$ & $1.53 E+01$ & $3.82 \mathrm{E}+00$ & $7.85 E+01$ \\
\hline$T_{w r B g} \mathrm{TMxy}$ & Maximum & DLC1.3_005LLInd_20.0Y0_S03.out & $1.81 \mathrm{E}+03$ & $-4.31 E+01$ & $-9.38 \mathrm{E}+03$ & $9.04 \mathrm{E}+03$ & $1.53 \mathrm{E}+05$ & $1.49 \mathrm{E}+03$ & $1.81 \mathrm{E}+03$ & $1.53 E+05$ & $1.43 E+01$ & $1.19 \mathrm{EE}+02$ \\
\hline
\end{tabular}

Extreme events for Tomer 56\%:

\begin{tabular}{|c|c|c|c|c|c|c|c|c|}
\hline Parameter & Type & File & $\begin{array}{r}\text { T=He1HLxt } \\
\text { (tWHe) }\end{array}$ & 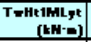 & $\begin{array}{r}\text { T=He1HLzt } \\
\text { (tH-2) }\end{array}$ & 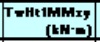 & $\begin{array}{l}\text { YindVri } \\
\text { (ntsec) }\end{array}$ & \\
\hline TwHelMLxt & Minimum & DLC1.3_0057_Lond_22.0V0_S03.out & $-1.13 E+04$ & $1.29 E+04$ & $-6.34 \mathrm{E}+02$ & $1.72 \mathrm{E}+04$ & $3.06 E+01$ & \\
\hline TwhtimLxt & Moximum & DLC1.3_0065_Land_24.0V0_S05.out & $2.42 E+04$ & $3.03 E+04$ & $1.27 E+03$ & $3.88 \mathrm{E}+04$ & $2.37 E+01$ & \\
\hline TwHeIMLye & Minimum & DLC1.3_000 & $-2.21 E+03$ & $-1.83 E+04$ & . & $1.85 E+04$ & $2.94 \mathrm{E}+00$ & \\
\hline TwHelMLLe & Moximum & DLC1.3_005LLIond_20.0V0_S03.out & $5.87 \mathrm{TE}+03$ & $7.89 \mathrm{E}+04$ & $2.22 \mathrm{E}+03$ & $7.92 E+04$ & $1.32 E+01$ & SE \\
\hline TwHelMLzt & Minimum & DLC1.3_0062_Land_24.0V0_S02.out & $3.35 E+03$ & $2.22 E+04$ & $-1.23 E+04$ & $2.25 E+04$ & $3.43 E+01$ & 5. \\
\hline TwHelMLat & Moximum & DLC1.3_0063_Land_24.0V0_S03.out & $5.24 \mathrm{E}+03$ & $2.00 \mathrm{E}+04$ & $1.20 \mathrm{E}+04$ & $2.06 \mathrm{E}+04$ & $2.81 E+01$ & 30 \\
\hline TwHt1MMxy & Minimum & DLC1.L000_Land_04.0V0_\$01.out & & $\begin{array}{l}-2.07 E+00 \\
305\end{array}$ & -4.31E+02 & $4.20 E+00$ & $3.01 E+00$ & \\
\hline$T_{\mathrm{w} H \mathrm{H} \text { 1MMxy }}$ & Moximum & DLC1.3_005LLIand_20.0V0_S03.out & $37 E+03$ & $7.88 \mathrm{E}+04$ & $3.34 \mathrm{~s}+03$ & $7.94 E+04$ & $1.40 \mathrm{E}+01$ & 1.19E \\
\hline
\end{tabular}

Extreme events for Rotor Perf:

\begin{tabular}{|c|c|c|c|c|c|c|c|c|}
\hline Paraneter & Type & File & $\begin{array}{r}\text { RotCp }_{(-1} \\
\end{array}$ & $\begin{array}{r}\text { Rotce } \\
\text { (-) }\end{array}$ & $\begin{array}{r}\text { RotCq } \\
(-1) \\
\end{array}$ & \begin{tabular}{|r|} 
TipSpdRat \\
$(-1)$ \\
\end{tabular} & $\begin{array}{l}\text { YiadVri } \\
\text { (alsec) }\end{array}$ & $\begin{array}{l}\operatorname{Tin}_{\text {Tisec }} \\
\text { (sec) }\end{array}$ \\
\hline RotCp & Minimum & DLC13_0002_Lond_04.0V0_S02.out & $-6.09 \mathrm{E}+10$ & $2.74 E+05$ & $9.03 E+02$ & $-3.47 \mathrm{E}+04$ & $-9.26 \mathrm{E}-03$ & \\
\hline Rotcp & Maximum & DLC1.3_0002_Lond_04.0V0_S02.out & $4.87 \mathrm{EE}+11$ & $6.92 \mathrm{E}+08$ & $1.98 \mathrm{E}+06$ & $2.46 E+05$ & $1.86 \mathrm{E}-04$ & $3.58 \mathrm{E}+02$ \\
\hline RotCt & Minimum & DLC1.3_0005_Lond_04.0V0_S05.out & $1.64 E+08$ & $-5.15 E+05$ & $-3.54 \mathrm{E}+03$ & $-2.63 \mathrm{E}+03$ & $-1.08 \mathrm{E}-02$ & $4.90 \mathrm{E}+02$ \\
\hline RotCl & Moximum & DLC1.3_0002_Lond_04.0V0_S02.out & $4.87 \mathrm{TE}+11$ & $6.92 \mathrm{E}+08$ & $1.98 \mathrm{E}+06$ & $2.46 \mathrm{E}+05$ & $1.86 \mathrm{E}-04$ & $3.58 \mathrm{E}+02$ \\
\hline Rotca & Minimum & DLC1.3_0003_Lond_04.0V0_S03.out & $5.40 E+09$ & $7.04 E+07$ & $-6.39 \mathrm{E}+04$ & $-8.44 E+04$ & $5.90 \mathrm{E}-03$ & $1.61 E+02$ \\
\hline Rorcq & Moximum & DLC1.3_0002_Land_04.0v0_S02.0ut & $4.87 \mathrm{EE}+11$ & $6.92 \mathrm{E}+08$ & $1.98 \mathrm{E}+06$ & $2.46 \mathrm{E}+05$ & $1.86 E-04$ & $3.58 \mathrm{E}+02$ \\
\hline
\end{tabular}

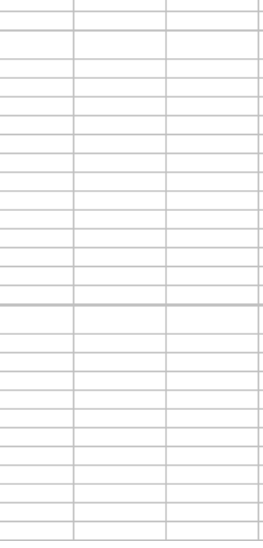

\section{F.2 Sea-Based Wind Turbine Loads}

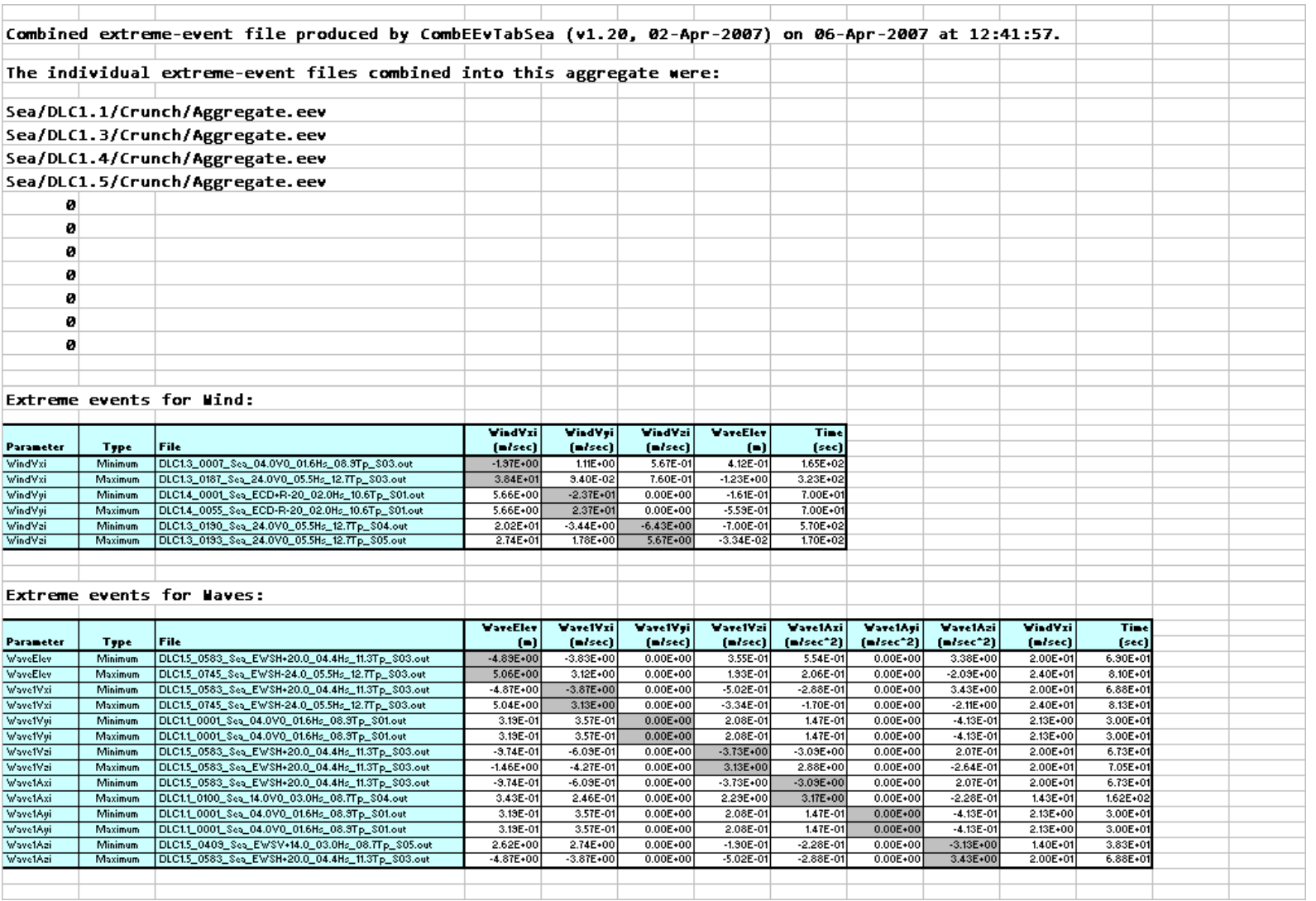


Extreme events for Drivetrain:

\begin{tabular}{|c|c|c|c|c|c|c|c|c|c|c|}
\hline Parameter & Type & File & $\begin{array}{r}\text { GenP=r } \\
\text { (t: })\end{array}$ & 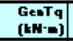 & $\begin{array}{r}\text { HSSBrT } \\
\text { (tkH-W) }\end{array}$ & \begin{tabular}{|r|} 
Gesspeed \\
(tpa)
\end{tabular} & $\begin{array}{r}\text { Rotspetd } \\
\text { (rpD) }\end{array}$ & $\begin{array}{r}\text { YareEler } \\
\text { (0) }\end{array}$ & $\begin{array}{l}\begin{array}{c}\text { YindVri } \\
(\boldsymbol{E} / \mathrm{sec})\end{array} \\
\end{array}$ & $\begin{array}{l}\text { Time } \\
(\mathrm{sec})\end{array}$ \\
\hline$\overline{\text { GenPwr }}$ & Minimum & DLC1.3_0190_Sc2_24.0V0_05.5HE_12.TाP_S04.out & $-3.32 E+00$ & $-4.62 \mathrm{E}-02$ & $0.00 \mathrm{E}+00$ & $7.21 \mathrm{E}+02$ & $7.42 \mathrm{E}+00$ & $-2.47 \mathrm{E}+00$ & $2.16 \mathrm{E}+01$ & $6.05 \mathrm{E}+02$ \\
\hline GenPwr & Moximum & DLC1.3_018L_SQ__24.0V0_05.5Hz_12.TTP_SO1.0ut & $6.05 E+03$ & $3.84 \mathrm{E}+01$ & $0.00 E+00$ & $1.59 \mathrm{E}+03$ & $1.64 \mathrm{E}+01$ & $-1.67 \mathrm{E}+00$ & $1.94 \mathrm{E}+01$ & $1.65 \mathrm{E}+02$ \\
\hline GenTq & Minimum & DLC1.3_0190_SC2_24.0V0_05.5H__12.TTP_S04.OUt & $-3.32 E+00$ & $-4.62 E-02$ & $0.00 \mathrm{E}+00$ & $7.21 \mathrm{E}+02$ & $7.42 E+00$ & $-2.47 E+00$ & $2.16 \mathrm{E}+01$ & $6.05 E+02$ \\
\hline GenTq & Moximum & DLC1.LO058_Se2_10.0V0_02.2Hs_09.2T P_SO2.Out & $5.00 \mathrm{E}+03$ & $4.75 E+01$ & $0.00 \mathrm{E}+00$ & $1.07 \mathrm{E}+03$ & $1.10 \mathrm{E}+01$ & $5.47 \mathrm{E}-01$ & $1.34 \mathrm{E}+01$ & $5.71 E+02$ \\
\hline HSSBrTq & Minimum & DLC1._000L_SeQ_04.0V0_01.6HE_08.STP_SO1.0ut & $5.54 \mathrm{E}+00$ & $8.37 \mathrm{E}-02$ & $0.00 \mathrm{E}+00$ & $6.70 \mathrm{E}+02$ & $6.91 E+00$ & $3.19 \mathrm{E}-01$ & $2.13 \mathrm{E}+00$ & $3.00 \mathrm{E}+01$ \\
\hline HSSBrTq & Moximum & DLC1._000L_Se__04.0V0_01.6HE_08.9TP_S01.out & $5.54 E+00$ & $8.37 \mathrm{E}-02$ & $0.00 E+00$ & $6.70 \mathrm{E}+02$ & $6.91 E+00$ & $3.19 \mathrm{E}-01$ & $2.13 \mathrm{E}+00$ & $3.00 \mathrm{E}+01$ \\
\hline GenSpeed & Minimum & 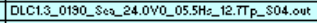 & $2.55 E+03$ & $4.74 \mathrm{E}+01$ & $0.00 \mathrm{E}+00$ & $5.44 \mathrm{E}+02$ & $5.64 \mathrm{E}+00$ & $7.42 E-01$ & $2.17 \mathrm{E}+01$ & $6.00 \mathrm{E}+02$ \\
\hline Genspeed & Maximum & DLC1.3_0164_SeQ_22.0V0_04.7H__13.4T P_S01.out & $5.35 E+03$ & $2.40 \mathrm{E}+01$ & $0.00 E+00$ & $2.26 \mathrm{E}+03$ & $2.31 E+01$ & $-1.23 E-02$ & $2.93 \mathrm{E}+01$ & $2.58 \mathrm{E}+02$ \\
\hline Rotspeed & Minimum & DLC1.3_0190_SC__24.0V0_05.5H__12.TTP_S04.OUt & $2.56 \mathrm{E}+03$ & $4.74 \mathrm{E}+01$ & $0.00 E+00$ & $5.46 \mathrm{E}+02$ & $5.64 E+00$ & $7.32 E-01$ & $2.22 \mathrm{E}+01$ & $6.01 E+02$ \\
\hline Rotspeed & Moximum & DLC1.3_0164_Sc2_22.0V0_04.7HE_13.4T P_S01.0ut & $5.39 E+03$ & $2.44 \mathrm{E}+01$ & $0.00 E+00$ & $2.23 \mathrm{E}+03$ & $2.32 E+01$ & $7.38 \mathrm{E}-02$ & $2.91 E+01$ & $2.58 \mathrm{E}+02$ \\
\hline
\end{tabular}

Extreme events for Blade Pitch:

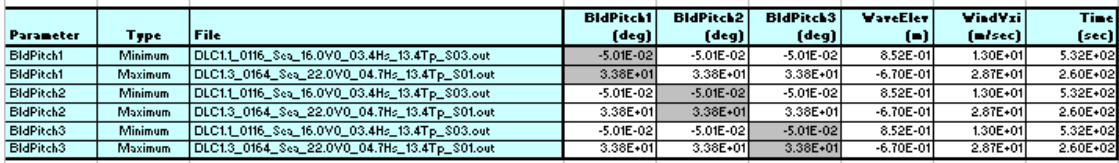

Extreme events for Hacelle Yaw:

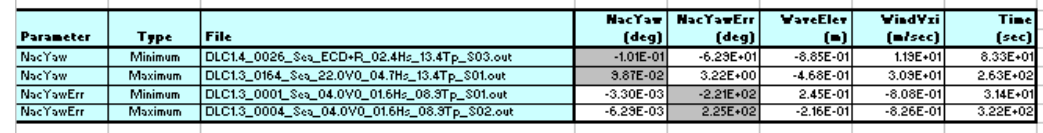

Extreme events for Blade 1 Defl:

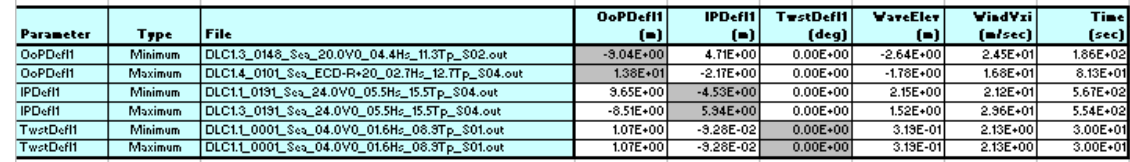

Extreme events for Blade 2 Defl:

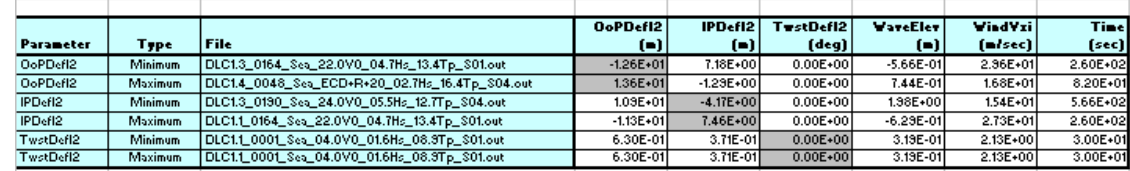

Extreme events for Blade 3 Defl:

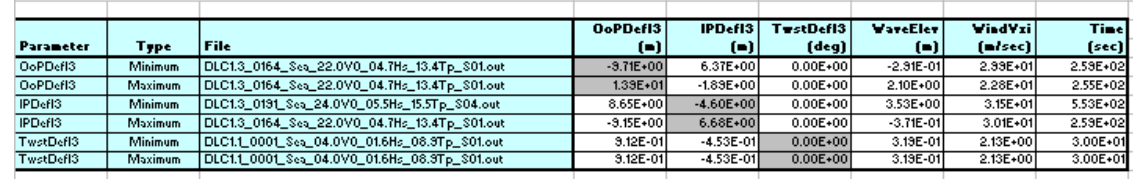

Extreme events for Blade Clrnc:

\begin{tabular}{|c|c|c|c|c|c|c|c|c|c|}
\hline Parameter & Type & File & $\begin{array}{r}\text { BldCirnci } \\
(\mathbf{( 0 )}\end{array}$ & $\begin{array}{r}\text { BidCirece } \\
(\bullet)\end{array}$ & $\begin{array}{r}\text { BIdCirace } \\
(0) \\
\end{array}$ & $\begin{array}{r}\text { Azinet) } \\
\text { (deg) }\end{array}$ & $\begin{array}{r}\text { FareEler } \\
\text { (0) }\end{array}$ & $\begin{array}{l}\begin{array}{l}\text { VindVxi } \\
(=/ \text { sec })\end{array} \\
\end{array}$ & $\begin{array}{l}\text { Tinec } \\
(\mathrm{sec})\end{array}$ \\
\hline EldClrne1 & linimum & $002.7 \mathrm{H} s_{-} 12 . \pi_{\mathrm{P}}$ _S04.out & & $3.84 \mathrm{E}+00$ & & $6.69 \mathrm{E}+01$ & & $1.68 \mathrm{E}+01$ & \\
\hline & ximum & & & & & & & & \\
\hline Bi & Minimum & 4.0 & & 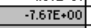 & & & & & \\
\hline EldClrt & Moximum & 3 & & & & & & & \\
\hline EIdCCC & Minimum & DLC1.3_0164_SC2_22.0 & & & & & & & \\
\hline EIddirns 3 & Moximum & |DLC1.3_0164_Se__22.0V0_04.7H__13.4TP_S01.0ut & & & & $3.55 \mathrm{E}$ & -2.911 & $2.99 E+01$ & $2.59 \mathrm{E}$ \\
\hline
\end{tabular}

Extreme events for Hacelle Acc:

\begin{tabular}{|c|c|c|c|c|c|c|c|c|c|}
\hline & Type & File & \begin{tabular}{|r|} 
MclimUTAx \\
$\left(-\sec ^{2} 2\right)$ \\
\end{tabular} & 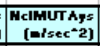 & $\begin{array}{r}\begin{array}{c}\text { NelmUTAzs } \\
\left(=/ \sec ^{-2}\right)\end{array} \\
\end{array}$ & 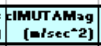 & $\begin{array}{r}\text { VoreEler } \\
\text { (a) }\end{array}$ & $\begin{array}{c}\text { YiedYri } \\
(0 / \text { sec })\end{array}$ & $\begin{array}{l}\text { Time } \\
(\mathrm{sec})\end{array}$ \\
\hline MUTAxs & Minimum & DLC1.3_0164_Sea_22.0V0_04.7Hs_13.4T P_S01.out & $-8.69 \mathrm{E}+00$ & $1.07 \mathrm{E}+00$ & $-2.11 \mathrm{E}+00$ & $9.01 E+00$ & $2.13 \mathrm{E}+00$ & $2.22 \mathrm{E}+01$ & $2.55 \mathrm{E}+02$ \\
\hline MuT & Maximum & C1.L0164_Seq_22.0V0_04.7Hz_13.4T P_S01.out & & & $1.51 E+00$ & & & & \\
\hline NeIMUT & Minimum & $05.5 \mathrm{H} \mathrm{S}_{-} 12 . \mathrm{TT}_{\mathrm{P}-\mathrm{S}} \mathrm{S} 05 . \mathrm{out}$ & & & & & & & $2.31 \mathrm{E}+02$ \\
\hline & Moximum & $05.5 \mathrm{H}$ __15.5TP_S04.out & & & $3.96 \mathrm{E}-01$ & $5.59 \mathrm{E}+00$ & & & $5.69 \mathrm{E}+02$ \\
\hline WUTAE & Minimum & DLC1.3_0164_SeQ_22. & & & & & & $2.26 \mathrm{E}+01$ & $2.53 \mathrm{E}+02$ \\
\hline IMUTAES & Moximum & 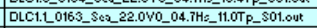 & 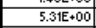 & $\frac{-1.5}{-1.5}$ & 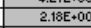 & 5.7 & $\frac{-1.0}{-4.0}$ & $2.05 E+01$ & $2.38 \mathrm{E}+02$ \\
\hline IMIT & Minimum & DLC1.3_0125_ & & & & & & $1.97 E+01$ & $1.68 \mathrm{E}+02$ \\
\hline CIMUTAMsgag & Maximum & DLC1.L0164_Se2_22.0V0_04.7HE_13.4TP_SO1.out & $9.85 \mathrm{E}+00$ & $-1.37 \mathrm{TE}+00$ & $1.51 E+00$ & $1.01 E+01$ & $-2.81 \mathrm{E}+00$ & $2.34 \mathrm{E}+01$ & $2.50 \mathrm{E}+02$ \\
\hline
\end{tabular}

Extreme events for TurTop Disp:

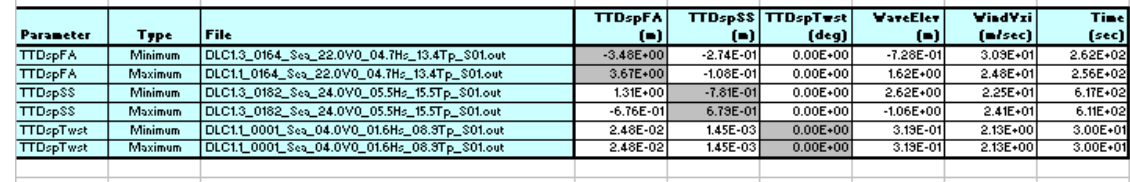

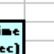

(2)


Extreme events for Platform Dsp:

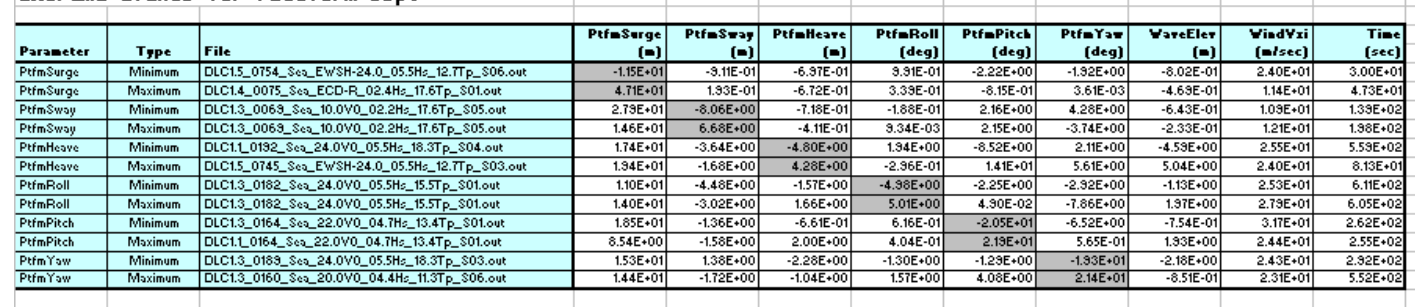

Extreme events for Platform Acc:

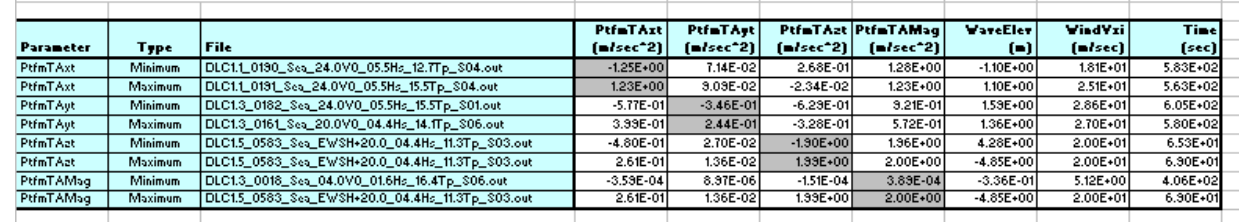

Extreme events for Blade 1 Root:

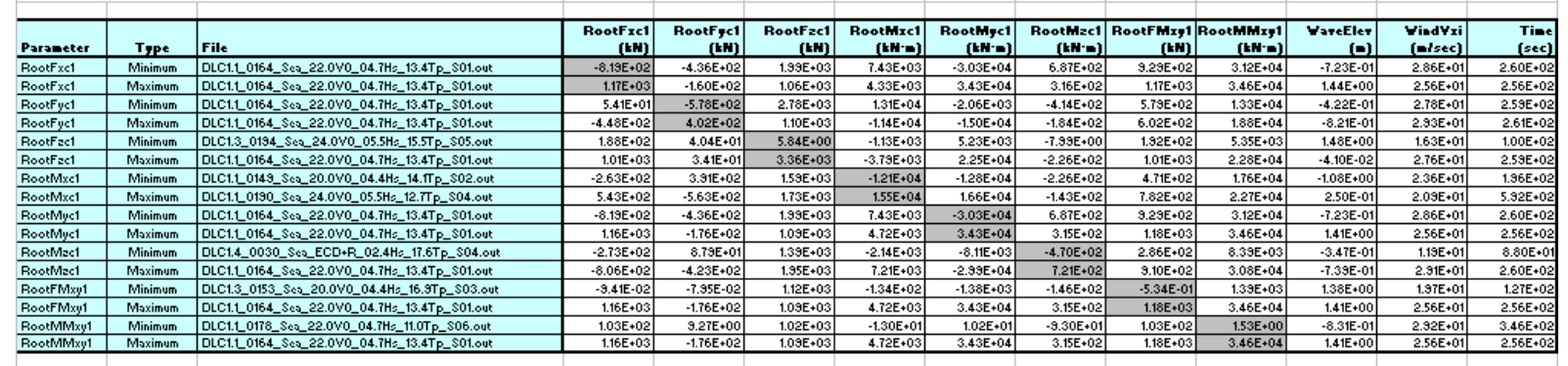

Extreme events for Blade 2 Root:

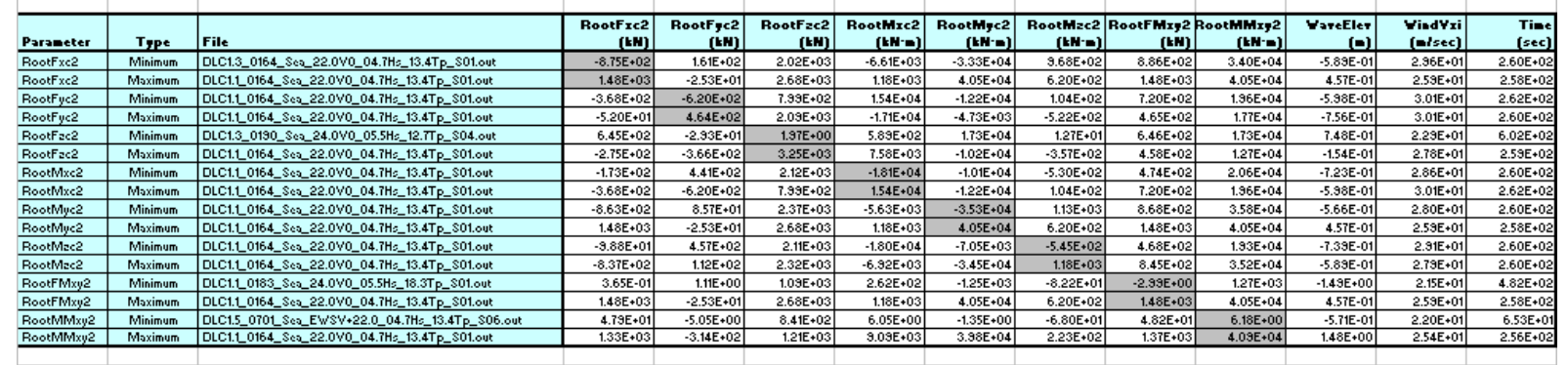

Extreme events for Blade 3 Root:

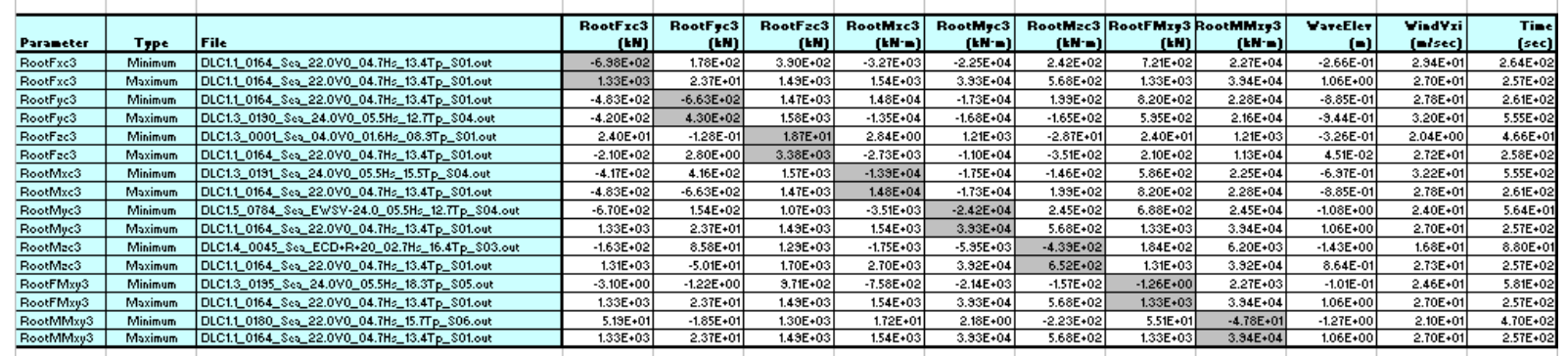

Extreme events for Blade 1 50\%:
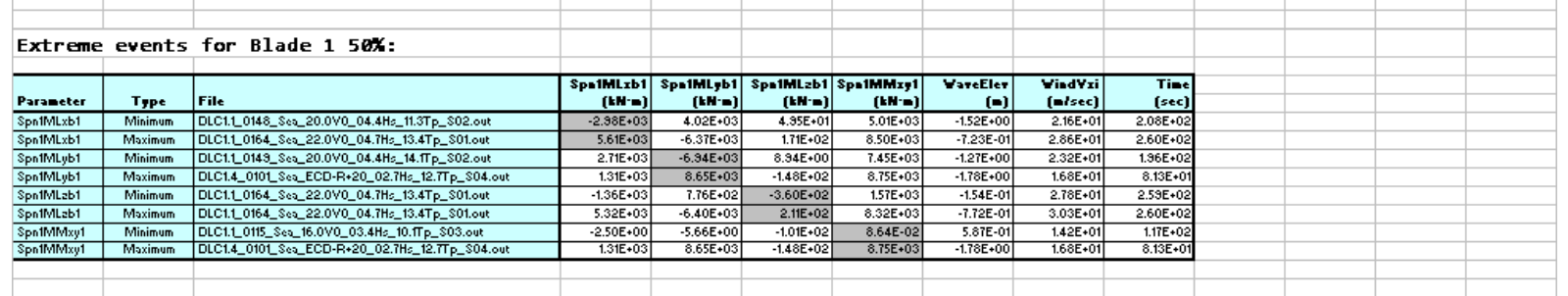
Extreme events for Blade 2 50\%:

\begin{tabular}{|c|c|c|c|c|c|c|c|c|c|}
\hline Parameter & Type & File & 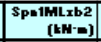 & \begin{tabular}{|r}
$\begin{array}{r}\text { Spa1mL,yb2 } \\
\text { (kH-D) }\end{array}$ \\
\end{tabular} & 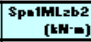 & 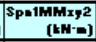 & $\begin{array}{r}\text { YareEler } \\
\text { (o) }\end{array}$ & $\begin{array}{l}\text { YiedVxi } \\
(=/ s e c)\end{array}$ & $\left.\begin{array}{c}T_{i=c} \\
{[\mathrm{sec}}\end{array}\right)$ \\
\hline $\begin{array}{l}\text { SprimLxb2 } \\
\end{array}$ & Minimum & DLC1.L0164_Se__22.0V0_04.7H__13.4T P_S01.0ut & $-3.57 E+03$ & $5.74 \mathrm{E}+03$ & $1.26 \mathrm{E}+02$ & $6.75 E+03$ & $5.79 \mathrm{E}-01$ & $2.61 \mathrm{E}+01$ & $2.57 E+0.0$ \\
\hline SprimLxb2 & Moximum & DLC1.L0164_Sc__22.0Y0_04.7HE_13.4T P_S01.0ut & $5.45 E+03$ & $-4.111 E+03$ & $1.31 E+02$ & $6.85 E+03$ & $-8.30 \mathrm{E}-01$ & $2.89 \mathrm{E}+01$ & $2.61 \mathrm{E}+0$. \\
\hline SpriMLyb2 & Minimum & DLC1.L0164_Se__22.0V0_04.7H__13.4T P_S01.0ut & $4.75 E+03$ & $-9.68 \mathrm{E}+03$ & $3.94 \mathrm{E}+02$ & $1.08 \mathrm{E}+04$ & $-5.89 \mathrm{E}-01$ & $2.79 \mathrm{E}+01$ & $2.60 \mathrm{E}+0$ \\
\hline SprimLity2 & Maximum & DLC1._0164_Se__22.0Y0_04.7H__13.4TP_SO1.0ut & $-2.33 \mathrm{E}+02$ & $8.71 E+03$ & $4.09 \mathrm{E}+01$ & $8.71 E+03$ & $1.41 E+00$ & $2.56 \mathrm{E}+01$ & $2.56 \mathrm{E}+0$. \\
\hline SpriMLzb2 & Minimum & 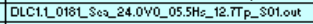 & $-3.20 E+02$ & $-3.63 \mathrm{E}+03$ & $-3.14 E+02$ & $3.65 E+03$ & $-3.80 \mathrm{E}-01$ & $2.61 \mathrm{E}+01$ & $5.18 \mathrm{E}+0 \mathrm{~K}$ \\
\hline SprimLab2 & Moximum & 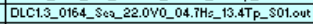 & $5.21 \mathrm{E}+03$ & $-8.55 E+03$ & $4.37 E+02$ & $1.00 E+04$ & $-5.42 E-01$ & $2.94 \mathrm{E}+01$ & $2.59 \mathrm{E}+0$ \\
\hline SprimMxy2 & Minimum & DLC1.3_0133_Seq_18.0V0_03.7Ho_10.TP_SOS03.out & $-1.20 \mathrm{E}-02$ & $8.17 \mathrm{E}-02$ & $-7.54 \mathrm{E}+011$ & $-2.916 \cdot 00$ & $8.65 \mathrm{E}-01$ & $2.05 \mathrm{E}+01$ & $1.01 \mathrm{E}+0$ \\
\hline SprimMxy2 & Moximum & DLC1.LO164_Se__22.0Y0_04.7Hs_13.4T P_SO1.0ut & \begin{tabular}{|c|c|}
$5.38 \mathrm{E}+03$ \\
\end{tabular} & $-9.62 E+03$ & $3.88 E+02$ & $1.10 \mathrm{E}+04$ & $-5.42 E-01$ & $2.78 E+01$ & $\frac{.059 \mathrm{E}+0}{2.52}$ \\
\hline
\end{tabular}

Extreme events for Blade 3 50x:

\begin{tabular}{|c|c|c|c|c|c|c|c|c|c|}
\hline & Type & File & 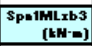 & 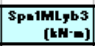 & 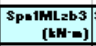 & 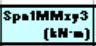 & $\begin{array}{r}\text { VoreEler } \\
\text { (0) }\end{array}$ & $\begin{array}{l}\text { ViedYri } \\
(0 / \text { sec })\end{array}$ & $\begin{array}{c}\text { Timet } \\
(\mathrm{sec})\end{array}$ \\
\hline IML $\times 53$ & nimum & out & $-3515+03$ & $4.5 E+03$ & $884 \mathrm{E}+01$ & $5.44 \mathrm{E}+03$ & & & $66+02$ \\
\hline SpnIML: & Moximum & S01.out & & +03 & & & & & $1 \mathrm{1E}+02$ \\
\hline SprimL: & Minimum & DLC1. & & & & & & & \\
\hline SppnimL & Moximum & $08.9 T_{P} \_$\$04.out & & & & & & & \\
\hline SprilML: & Minimum & $15 . \pi T_{\text {p_s }}$ So1.out & & & & & & & $2.59 \mathrm{E}+$ \\
\hline SpromL & Moximum & Hz_-12.TTP_SO3.out & & & & & & $2.61 \mathrm{E}+01$ & $2.00 \mathrm{E}+02$ \\
\hline $\mathrm{MMMx}$ & Minimum & DLC1.L0109_Ses_16.0V0_03.4Hs_10.TP_SO1.0ut & $-3.96 E+00$ & $1.82 E+00$ & $-8.72 E+01$ & $-4.00 \mathrm{E}+00$ & $-1.82 \mathrm{E}-01$ & $1.44 E+01$ & $2.28 \mathrm{E}+02$ \\
\hline onlMMxy3 & Moximum & DLC1.4_0100_SQO_ECD-R+20_02.7H?_08.9TP_SO4.OUt & $1.56 \mathrm{E}+03$ & $8.69 \mathrm{gE}+03$ & $-1.85 E+02$ & $8.83 \mathrm{E}+03$ & $-2.12 E+00$ & $1.68 \mathrm{E}+01$ & $8.26 E+01$ \\
\hline
\end{tabular}

Extreme events for LSS Main Br:

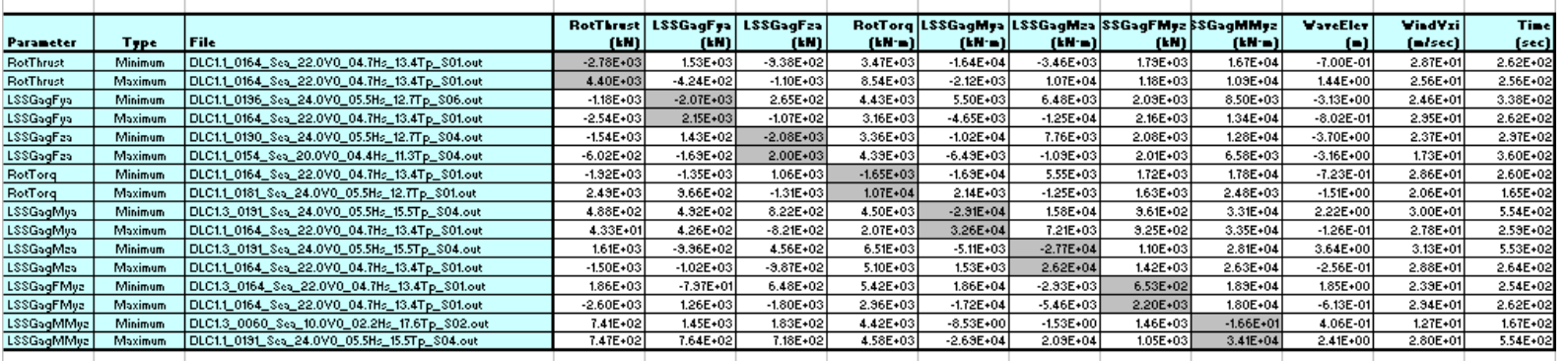

Extreme events for Yaw Bearing:

\begin{tabular}{|c|c|c|c|c|c|c|c|c|c|c|c|c|c|}
\hline Parameter & Type & File & 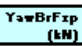 & $\begin{array}{r}a=B r F, p \\
(\text { (tw) }\end{array}$ & $\begin{array}{r}r=B \text { BrFap } \\
\text { (tw) }\end{array}$ & 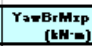 & 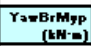 & 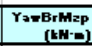 & $\begin{array}{r}\text { YasrFMxy } \\
\text { (tw) }\end{array}$ & 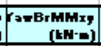 & \begin{tabular}{|r|} 
YareEler \\
(0)
\end{tabular} & $\begin{array}{c}\text { ViedYri } \\
(\theta / \mathrm{sec})\end{array}$ & 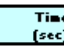 \\
\hline YowErFxp & Minimum & DLC1.L0164_Se2_22.0V0_04.7H__13.4TP_S01.out & $-7.81 \mathrm{E}+03$ & $-5.41 E+02$ & $-4.63 \mathrm{E}+03$ & $5.45 \mathrm{E}+03$ & $-2.26 \mathrm{E}+04$ & $1.28 \mathrm{E}+04$ & $7.83 \mathrm{E}+03$ & $2.32 E+04$ & $-7.14 \mathrm{E}-01$ & $2.85 \mathrm{E}+01$ & $2.62 \mathrm{E}+0 \mathrm{~F}$ \\
\hline YaWErFXP & Moximum & DLC1._0164_SEQ_22.0V0_04.7H__13.4TP_S01.out & $8.56 \mathrm{E}+03$ & $-2.12 E+02$ & $-4.56 \mathrm{E}+03$ & $9.89 \mathrm{E}+03$ & $1.18 \mathrm{E}+04$ & $9.62 \mathrm{E}+03$ & $8.57 E+03$ & $1.54 \mathrm{E}+04$ & $1.48 \mathrm{E}+00$ & $2.54 \mathrm{E}+01$ & $2.56 \mathrm{E}+0$ \\
\hline 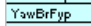 & Minimum & DLC1.L0182_Sez_24.0V0_05.5H__15.TTP_S01. Out & $3.00 \mathrm{E}+03$ & $-1.95 E+03$ & $-4.62 E+03$ & $1.07 \mathrm{E}+04$ & $1.315+04$ & $-9.98 E+03$ & $3.58 \mathrm{E}+03$ & $1.6 \mathrm{gE}+04$ & $2.64 \mathrm{E}+00$ & $2.22 E+01$ & $6.17 \mathrm{E}+0 \mathrm{Z}$ \\
\hline 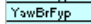 & Moximum & DLC1.L0182_SGQ_24.0V0_05.5H__15.5TP_SO1.out & $-1.16 \mathrm{E}+03$ & $1.88 \mathrm{E}+03$ & $-4.86 \mathrm{E}+03$ & $2.48 \mathrm{E}+03$ & $-1.42 E+04$ & $1.72 \mathrm{E}+03$ & $2.20 \mathrm{E}+03$ & $1.44 \mathrm{E}+04$ & $-1.13 \mathrm{E}+00$ & $2.50 \mathrm{E}+01$ & $6.111+0.2$ \\
\hline 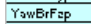 & Minimum & DLC1._0154_Sez_20.0V0_04.4H__-11.3TP_SO4. out & $-4.17 \mathrm{EE}+02$ & $-6.54 \mathrm{E}+01$ & $-6.08 \mathrm{E}+03$ & $5.55 \mathrm{E}+03$ & $6.96 \mathrm{E}+03$ & $1.01 E+03$ & $4.22 E+02$ & $8.91 E+03$ & $-3.23 \mathrm{E}+00$ & $1.77 \mathrm{TE}+01$ & $3.59 \mathrm{E}+0.2$ \\
\hline YowErfFap & Maximum & DLC1.3_0164_Sc2_22.0V0_04.7HE_13.4TP_S01.out & $8.02 \mathrm{E}+02$ & $3.79 \mathrm{E}+02$ & $-2.71 \mathrm{E}+03$ & $5.27 \mathrm{EE}+03$ & $-1.22 \mathrm{E}+04$ & $6.68 \mathrm{E}+03$ & $8.87 \mathrm{E}+02$ & $1.33 E+04$ & $5.39 \mathrm{E}-01$ & $2.03 E+01$ & $2.53 \mathrm{E}+0 \mathrm{z}$ \\
\hline$Y_{5 w E_{r} M \times p}$ & Minimum & DLC1.L0164_SCQ_22.0V0_04.7H__13.4TP_SO1.out & $-4.32 E+03$ & $-5.39 \mathrm{E}+02$ & $-4.17 \mathrm{EE}+03$ & $-2.04 \mathrm{E}+03$ & $-7.63 \mathrm{E}+03$ & $-1.90 \mathrm{E}+04$ & $4.36 \mathrm{E}+03$ & $7.89 E+03$ & $\begin{array}{c}-7.39 \mathrm{G}-01 \\
\end{array}$ & $2.915+01$ & $2.60 \mathrm{E}+0 \mathrm{Z}$ \\
\hline 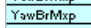 & Moximum & DLC1.L0182_SCQ_24.0V0_05.5Hs_15.5TP_SO1.0ut & $4.14 \mathrm{E}+03$ & $-1.511+03$ & $-4.91 \mathrm{E}+03$ & $1.19 E+04$ & $7.28 \mathrm{E}+03$ & $-1.52 E+03$ & $4.41 \mathrm{E}+03$ & $1.40 E+04$ & $2.52 E+00$ & $2.60 \mathrm{E}+01$ & $6.15 \mathrm{E}+0.2$ \\
\hline 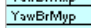 & Minimum & DLC1.L0164_SC2_22.0V0_04.7H__13.4TP_S01.0ut & 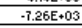 & $-8.43 \mathrm{E}+02$ & $-5.00 \mathrm{E}+03$ & $5.31 \mathrm{~s}+03$ & $-3.05 E+04$ & $7.30 \mathrm{E}+03$ & $7.31 \mathrm{E}+03$ & $3.10 \mathrm{E}+04$ & $-6.13 \mathrm{E}-0.01$ & $2.94 \mathrm{E}+01$ & $2.62 \mathrm{E}+0.2$ \\
\hline$Y_{\text {OwE }}$ & Moximum & DLC1.L019LSQ2_24.0V0_05.5Hz_15.5TP_SO4. Out & $1.44 \mathrm{E}+03$ & $2.25 E+02$ & $-4.01 E+03$ & $3.94 \mathrm{E}+03$ & $3.895+04$ & $-2.65 \mathrm{EE}+03$ & $1.46 \mathrm{E}+03$ & $3.91 E+04$ & $2.41 E+00$ & $2.80 \mathrm{E}+01$ & $5.54 \mathrm{E}+0.2$ \\
\hline YowErMzp & Minimum & DLC1.4_0026_SQ2_ECD+R_02.4HE_13.4TP_SO3.out & $7.42 E+01$ & $9.64 \mathrm{E}+01$ & $-4.63 E+03$ & $3.07 E+03$ & $-3.60 \mathrm{E}+03$ & $-2.16 \mathrm{E}+04$ & $1.22 E+02$ & $4.73 \mathrm{E}+03$ & $-8.85 \mathrm{E}-01$ & $1.19 \mathrm{EE}+01$ & $8.33 \mathrm{E}+0$ \\
\hline YowErMsp & Moximum & DLC1.L0164_SCQ_22.0V0_04.7HI_13.4TP_SO1.out & $-4.29 E+03$ & $-7.38 \mathrm{E}+01$ & $-3.78 \mathrm{E}+03$ & $7.17 \mathrm{TE}+03$ & $-2.29 E+04$ & $2.14 \mathrm{E}+04$ & $4.29 E+03$ & $2.40 \mathrm{E}+04$ & $-2.56 \mathrm{E}-01$ & $2.88 \mathrm{E}+01$ & $2.64 \mathrm{E}+0$. \\
\hline$Y_{3 w E r f M x y}$ & Minimum & 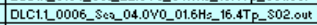 & 1.19E-01 & $-1.13 \mathrm{E}-02$ & $-5.16 \mathrm{E}+03$ & $4.17 \mathrm{EE}+02$ & $-2.36 \mathrm{E}+03$ & $-1.53 \mathrm{E}+03$ & $1.23 \mathrm{E}-01$ & $2.40 E+03$ & 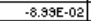 & $2.86 \mathrm{E}+00$ & $4.04 \mathrm{E}+0 \mathrm{E}$ \\
\hline YawErFMxy & Moximum & DLC1.L0164_SeQ_22.0V0_04.7HE_13.4TP_SO1.0ut & $8.56 \mathrm{E}+03$ & $-2.12 E+02$ & $-4.56 \mathrm{E}+03$ & $9.89 \mathrm{E}+03$ & $1.18 \mathrm{E}+04$ & $9.62 \mathrm{E}+03$ & $8.57 \mathrm{E}+03$ & $1.54 \mathrm{E}+04$ & $1.48 \mathrm{E}+00$ & $2.54 \mathrm{E}+01$ & $2.56 \mathrm{E}+0.0$ \\
\hline $8 \mathrm{rMMxy}$ & Minimum & 0 DLC1.3_000 & $2.15 E+02$ & $19 E+01$ & $-4.63 \mathrm{E}+03$ & $7.44 \mathrm{E}+00$ & $-9.07 E+00$ & $-1.111+03$ & $2.177+02$ & $5+00$ & $-3.12 \mathrm{E}-01$ & $3.92 E-01$ & $3.57 \mathrm{E}+0.2$ \\
\hline 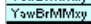 & Moximum & DLC1.L0191_Sc2_24.0V0_05.5HG_15.5TP_S04.0ut & $1.44 \mathrm{E}+03$ & $2.25 E+02$ & $-4.01 E+03$ & $3.94 \mathrm{E}+03$ & $3.89 \mathrm{E}+04$ & $-2.65 \mathrm{E}+03$ & $1.46 \mathrm{E}+03$ & $E+04$ & $2.41 E+00$ & $2.80 \mathrm{E}+01$ & 5.5 \\
\hline
\end{tabular}

Extreme events for Tomer Base:

\begin{tabular}{|c|c|c|c|c|c|c|c|c|c|c|c|c|c|}
\hline & Type & File & \begin{tabular}{r|}
$\begin{array}{r}\text { TwBsFxt } \\
(\mathbf{H})\end{array}$ \\
\end{tabular} & $\begin{array}{r}\text { TerBsFyt } \\
\text { (tw) }\end{array}$ & $\begin{array}{r}\text { TerBsFzet } \\
\text { (tw) }\end{array}$ & 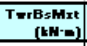 & 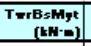 & 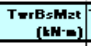 & $\begin{array}{r}\text { TerBsFMXY } \\
(\mathbf{E H}) \\
\end{array}$ & 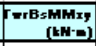 & $\begin{array}{r}\text { YareEler } \\
(\bullet) \\
\end{array}$ & $\begin{array}{l}\text { YiedVri } \\
\text { (elsece) }\end{array}$ & $\begin{array}{l}\text { Time } \\
\text { (sec) }\end{array}$ \\
\hline TwrEsFxt & Minimum & DLC1.L0164_Sce_22.0V0_04.7HE_13.4TP_S01.0ut & $-1.08 E+04$ & $-1.00 \mathrm{E}+03$ & $-1.03 E+04$ & $7.60 \mathrm{E}+04$ & $-8.55 \mathrm{E}+05$ & $1.12 E+04$ & $1.09 E+04$ & $8.59 E+05$ & & & $2.62 \mathrm{E}+02$ \\
\hline$T_{\mathrm{wrE} E \mathrm{~F} \times \mathrm{xt}}$ & Moximum & DLC1.L0164_Sc2_22.0V0_04.7HE_13.4TP_S01.0ut & $1.16 \mathrm{E}+04$ & $-2.04 \mathrm{E}+02$ & $-9.86 \mathrm{E}+03$ & $2.75 E+04$ & $9.14 \mathrm{E}+05$ & $1.24 E+04$ & $1.16 \mathrm{E}+04$ & $9.14 E+05$ & $1.55 E+00$ & $2.49 \mathrm{E}+01$ & $2.56 \mathrm{E}+02$ \\
\hline$T_{\text {wrEsfyk }}$ & Minimum & DLC1.10182_Se2_24.0V0_05.5HE_15.5TP_SO1.0ut & $3.67 \mathrm{E}+03$ & $-2.67 E+03$ & $-9.44 E+03$ & $2.15 E+05$ & $3.08 E+05$ & $-9.59 \mathrm{E}+03$ & $4.53 \mathrm{E}+03$ & $3.76 E+05$ & $2.59 E+00$ & $2.25 E+01$ & $6.17 \mathrm{E}+02$ \\
\hline$T_{\text {wrEofyt }}$ & Moximum & DLC1.L0182_SQ2_24.0V0_05.5H__15.TTP_SO1.out & $-1.38 \mathrm{E}+03$ & $2.62 E+03$ & $-9.89 E+03$ & $-1.99 E+05$ & $-\frac{-1.21 E+05}{4}$ & $5.70 \mathrm{E}+02$ & $2.96 \mathrm{E}+03$ & $2.33 E+05$ & $-1.06 \mathrm{E}+00$ & $2.41 E+01$ & $6.111+02$ \\
\hline TwrEofat & Minimum & DLC1._0154_SeQ_20.0V0_04.4HE_11.3TP_SO4.OUt & $-9.62 \mathrm{E}+02$ & $-6.34 \mathrm{E}+00$ & $-1.20 E+04$ & $\begin{array}{ll}8.95 E+03 \\
\end{array}$ & $-.5 .58 \mathrm{E}+044$ & $1.01 \mathrm{E}+03$ & $9.62 \mathrm{E}+02$ & $5.65 E+04$ & $-3.23 \mathrm{E}+00$ & $1.77 \mathrm{E}+01$ & $3.595+02$ \\
\hline TwrEsFat & Moximum & DLC1.3_0164_Se__22.0V0_04.7H__13.4TP_S01.0ut & $1.35 \mathrm{EE}+03$ & $4.43 \mathrm{E}+02$ & $-6.52 E+03$ & $-2.83 E+04$ & $1.00 \mathrm{E}+05$ & $9.38 E+03$ & $1.42 E+03$ & $1.04 E+05$ & $6.92 E-01$ & $2.15 E+01$ & $2.53 \mathrm{E}+02$ \\
\hline Eomxt & Minimum & DLC1._0182_Sce_24.0VO_05.5Hz_15.5TP_S01.out & & & & & & & & & & & \\
\hline Bemxt & Moximum & DLC1.LO & & & & & & & & & & & \\
\hline Bemyt & Minimum & DLC1.L01 & $=04$ & & & & & & & & & & \\
\hline TwrEsmyt & Moximum & DLC1._0164_ScQ_22.0VO_04.7Hs_13.4TP_SO1.out & $1.16 \mathrm{E}+04$ & $-2.04 \mathrm{E}+02$ & $\overline{2}+03$ & 04 & $9.14 E+05$ & $E+04$ & +04 & $\frac{205}{2}$ & $1.55 E+00$ & $2.49 E+01$ & $2.56 \mathrm{E}+02$ \\
\hline TwrEs:Mat & Minimum & DLC1.4_0026_SCQ_ECD+R_02.4HE_13.4TP_S03.out & $E+02$ & $8.34 \mathrm{E}+01$ & & & $2+04$ & $-2.16 \mathrm{E}+04$ & & $=04$ & $-8.85 E-01$ & $1.19 \mathrm{E}+01$ & $8.33 E+01$ \\
\hline TwrBamat & Moximum & DLC1.3_0164_SeQ_22.0V0_04.7HE_13.4TP_S01.out & & $-9.69 \mathrm{gE}+02$ & & & & $2.15 E+04$ & & .05 & & $3.09 E+01$ & \\
\hline TwESF & Minimum & DLC1.3_0063_SeQ_10.0V0_02.2HE_-17.6TP__S03.out & $-9.77 \mathrm{E}+00$ & $-1.53 \mathrm{E}+00$ & & 9.03 & 4.13 & 8.11 & $-3.34 \mathrm{E}+00$ & $9.97 \mathrm{EE}+03$ & $8.15 E-01$ & $1.21 E+01$ & $3.36 \mathrm{E}+02$ \\
\hline TwrEst & Moximum & DLC1.L_0164_Ses_22.0V0_04.7HE_13.4T P_SO1.0ut & $1.16 E+04$ & $-2.04 \mathrm{E}+02$ & -9.86 & 2.75 & 9.1. & 1.24 & 1.16 & $9.14 E+05$ & 1.55 & 2.4 & $2.56 \mathrm{E}+02$ \\
\hline TwrEsmMxy & Minimum & DLC1.5_0076_Sc2_EWSH+06.0_01.8H__08.0TP_S02.out & $-2.99 E+01$ & $1.111 E+01$ & & $-7.22 E+00$ & $-3.70 E+00$ & $-1.32 \mathrm{E}+02$ & $3.19 E+01$ & 4.64E-01 & $5.26 \mathrm{E}-01$ & $6.00 E+00$ & $3.01 E+01$ \\
\hline$T_{w r B}=M M x y$ & Moximum & DLC1.L0164_Sce_22.0V0_04.7H__13.4TP_SO1.0ut & $1.16 E+04$ & $-2.04 E+02$ & $-9.86 \mathrm{E}+03$ & $2.75 E+04$ & $9.14 E+05$ & $1.24 E+04$ & $1.16 \mathrm{E}+04$ & $9.14 E+05$ & $1.55 E+00$ & $2.49 \mathrm{E}+01$ & $2.56 \mathrm{E}+02$ \\
\hline
\end{tabular}

Extreme events for Tomer 50\%:

\begin{tabular}{|c|c|c|c|c|c|c|c|c|c|}
\hline Parameter & Type & File & 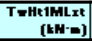 & \begin{tabular}{|r|} 
TrHelWhLt, \\
(tW-O)
\end{tabular} & 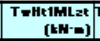 & 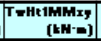 & \begin{tabular}{|c|} 
YareEler \\
$(\mathbf{0})$
\end{tabular} & 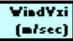 & $\begin{array}{c}\text { Tinet } \\
\text { [sec) }\end{array}$ \\
\hline $\begin{array}{c}\text { TwhelMLxt } \\
\end{array}$ & $\begin{array}{c}\text { Minimum } \\
\text { M }\end{array}$ & OLC1.L0182_SCQ_24.0V0_05.5H__15.5TP_S01.0ut & $-9.48 E+04$ & $-7.07 \mathrm{E}+04$ & $1.26 \mathrm{E}+03$ & $1.18 \mathrm{E}+05$ & $-1.09 \mathrm{E}+00$ & $2.48 \mathrm{E}+01$ & $6.11 \mathrm{E}+02$ \\
\hline TwHelMLxt & Moximum & DLC1.L0182_SeQ_24.0V0_05.5H__15.TTP_S01.out & $1.111 E+05$ & $1.64 \mathrm{E}+05$ & $-9.98 \mathrm{E}+03$ & $1.98 \mathrm{E}+05$ & $2.66 \mathrm{E}+00$ & $2.19 \mathrm{E}+01$ & $6.17 \mathrm{E}+02$ \\
\hline TwHelMLye & Minimum & DLC1.L0164_Sce_22.0V0_04.7H__13.4T P_S01.out & $3.62 E+04$ & $-4.29 E+05$ & $1.28 \mathrm{E}+04$ & $4.30 \mathrm{E}+05$ & \begin{tabular}{ll|l|}
$-7.14 E-01$ \\
\end{tabular} & $2.85 \mathrm{E}+01$ & $2.62 \mathrm{E}+02$ \\
\hline TwHelMLLt & Moximum & DLC1.L_0164_Ses_22.0V0_04.7Hz_13.4TP_SO1.out & $1.49 \mathrm{E}+04$ & $4.53 E+05$ & $1.37 \mathrm{EE}+04$ & $4.54 \mathrm{E}+05$ & $1.62 \mathrm{E}+00$ & $2.48 E+01$ & $2.56 \mathrm{E}+02$ \\
\hline TwhtimLat & Minimum & DLC1.L0164_Seq_22.0V0_04.7Ht_13.4T P_SO1.0ut & $2.27 \mathrm{E}+04$ & $-2.62 E+05$ & $-2.16 E+04$ & $2.63 \mathrm{E}+05$ & $-7.85 E-01$ & $2.91 \mathrm{E}+01$ & $2.60 \mathrm{E}+02$ \\
\hline TwHelMLizt & Moximum & DLC1.L0164_Seq_22.0V0_04.7H__13.4TP_S01.out & $9.87 \mathrm{E}+03$ & $-2.46 \mathrm{E}+05$ & $2.14 E+04$ & $2.47 \mathrm{E}+05$ & $-2.56 \mathrm{E}-01$ & $2.88 \mathrm{E}+01$ & $2.64 \mathrm{E}+02$ \\
\hline TwHelMMxy & Minimum & DLC1.5_0049_Sez_EWSW+04.0_01.6HE_08.STP_S05.out & $2.56 \mathrm{E}+00$ & $-2.41 E+00$ & $-1.02 E+01$ & $2.93 \mathrm{E}+00$ & $-9.23 \mathrm{E}-02$ & $4.00 E+00$ & $5.76 \mathrm{E}+01$ \\
\hline TwhelMMxy & Moximum & DLC1._0164_Sce_22.0V0_04.7Hs_13.4T P_SO1.0ut & $1.49 E+04$ & $4.53 E+05$ & $1.37 \mathrm{E}+04$ & $4.54 E+05$ & $1.62 \mathrm{E}+00$ & $2.48 E+01$ & $2.56 \mathrm{E}+02$ \\
\hline
\end{tabular}


Extreme events for Line 1:

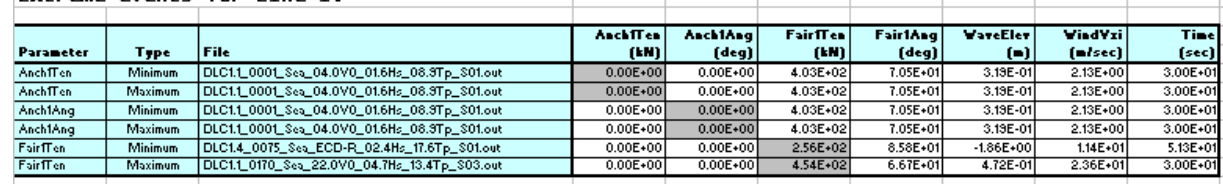

Extreme events for Line 2:

\begin{tabular}{|c|c|c|c|c|c|c|c|c|c|}
\hline ameter & Type & File & 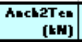 & \begin{tabular}{r|} 
Anctb2Ang \\
(deg) \\
\end{tabular} & $\begin{array}{r}\text { Fair2Tet: } \\
\text { (tW) }\end{array}$ & \begin{tabular}{r|} 
Fair2Ang \\
(deg)
\end{tabular} & $\begin{array}{r}\text { YareEler } \\
(0)\end{array}$ & $\begin{array}{l}\text { VindVxi } \\
(\omega / \sec )\end{array}$ & $\begin{array}{l}\text { Time } \\
\text { (sec) }\end{array}$ \\
\hline Anch2Ten & Minimum & DLC1.L000L_SeQ_04.0V0_01.6HE_08.9TP_S01.out & $0.00 \mathrm{E}+00$ & $0.00 \mathrm{E}+00$ & $4.08 \mathrm{E}+02$ & $6.99 \mathrm{E}+01$ & $3.19 \mathrm{E}-01$ & $2.13 E+00$ & $3.00 \mathrm{E}+01$ \\
\hline AnchêT en & Moximum & DLC1.L000LSE2_04.0V0_01.6HE_08.9TP_S01.out & t & & $8 \mathrm{E}+02$ & $6.99 \mathrm{E}+01$ & 3.19E-01 & $2.13 E+00$ & $3.00 \mathrm{E}+01$ \\
\hline$\hbar_{2} \mathrm{An}_{n}$ & Minimum & DLC1.Lo & & & & & $3.19 \mathrm{E}-01$ & $2.13 E+00$ & $3.00 \mathrm{E}+01$ \\
\hline Ang & Moximum & vet & & 0.0 & & 6. & 3.1 & $2.13 E+00$ & $3.00 \mathrm{E}+01$ \\
\hline & Minimum & DLC1.3. & & & & & $-1.63 \mathrm{E}+0$ & $1.00 \mathrm{E}+01$ & $6.15 \mathrm{E}+02$ \\
\hline & Maximum & 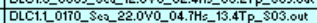 & $0.00 \mathrm{E}+00$ & $0.00 \mathrm{E}+00$ & $4.48 \mathrm{E}+02$ & $\frac{1.0 \mathrm{c}+\mathrm{TE}+01}{6.11+01}$ & $\frac{1.00500}{9.995-01}$ & $\frac{1.00 \mathrm{E}+01}{2.40+01}$ & $3.04 \mathrm{E}+01$ \\
\hline
\end{tabular}

Extreme events for Line 3 :

\begin{tabular}{|c|c|c|c|c|c|c|c|c|c|}
\hline rameter & Tgpe & File & $\begin{array}{r}\begin{array}{c}\text { Ancts3TeE } \\
\text { (tw) }\end{array} \\
\end{array}$ & \begin{tabular}{r|} 
Ancb3Ang \\
(deg) \\
\end{tabular} & \begin{tabular}{r|} 
Fair3T TeE \\
(tkH)
\end{tabular} & \begin{tabular}{r|r|} 
Fair3Ang \\
(deg)
\end{tabular} & $\begin{array}{r}\text { YareEler } \\
(0)\end{array}$ & 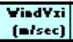 & $\begin{array}{c}T_{i=0} \\
(\text { sec })\end{array}$ \\
\hline ChITen & Minimum & DLC1.LO000L_Sez_04.0V0_01.6HE_08.9TP_S01.out & $0.00 E+00$ & $0.00 \mathrm{E}+00$ & $4.14 \mathrm{E}+02$ & $6.95 \mathrm{E}+01$ & $3.19 \mathrm{E}-01$ & $2.13 E+00$ & $3.00 \mathrm{E}+01$ \\
\hline chisten & Maximum & DLC1.L000L_Sc_04.0V0_01.6HE_08.9TP_S01.out & & $0.00 \mathrm{E}+00$ & $4.14 \mathrm{E}+02$ & $6.95 E+01$ & $3.19 \mathrm{E}-01$ & $2.13 \mathrm{E}+00$ & $3.00 \mathrm{E}+01$ \\
\hline $\mathrm{h} 3 \mathrm{~A}$ & Minimum & DLC1.LOC & & & $4.14 E+02$ & & $3.19 \mathrm{E}-01$ & $2.13 \mathrm{E}+00$ & $3.00 \mathrm{E}+01$ \\
\hline hising & Maximum & DLC1.1. & & & 02 & 6.5 & $3.19 \mathrm{E}-01$ & $2.13 E+00$ & $3.00 \mathrm{E}+01$ \\
\hline & Minimum & DLC1.3_005LSC2_08.0 & $0.00 \mathrm{E}+00$ & & $3.45 E+02$ & $7.24 \mathrm{E}+01$ & $-3.03 \mathrm{E}-01$ & $5.81 E+00$ & $1.15 \mathrm{E}+02$ \\
\hline ir $3 T$ en & Moximum & DLC1.L0079_SeQ_12.0V0_02.4Hz_09.2T P_SO3.out & $0.00 E+00$ & $0.00 \mathrm{E}+00$ & $6.12 E+02$ & $5.62 E+01$ & $9.74 E-01$ & $1.09 \mathrm{E}+01$ & $5.05 E+01$ \\
\hline
\end{tabular}

Extreme events for Line 4:

\begin{tabular}{|c|c|c|c|c|c|c|c|c|c|}
\hline Parameter & Type & File & $\begin{array}{r}\text { AachATeE } \\
\text { (tW) }\end{array}$ & $\begin{array}{r}\begin{array}{c}\text { AncliAAng } \\
\text { (deg) }\end{array} \\
\end{array}$ & $\begin{array}{r}\text { F airAT te: } \\
\text { (tEW) } \\
\end{array}$ & $\begin{array}{r}\text { Fuir4Ang } \\
\text { (deg) } \\
\end{array}$ & $\begin{array}{r}\text { YareEler } \\
(0) \\
\end{array}$ & $\begin{array}{c}\text { ViedVri } \\
(=/ s e c)\end{array}$ & $\begin{array}{l}\text { Timec } \\
(\mathrm{sec}) \\
\end{array}$ \\
\hline Anch4Ten & Minimum & 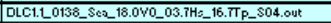 & $-1.83 \mathrm{E}-01$ & $0.00 \mathrm{E}+00$ & $6.26 \mathrm{E}+02$ & $5.50 \mathrm{E}+01$ & $-1.97 \mathrm{gE}-01$ & $1.91 E+01$ & $1.52 E+02$ \\
\hline Anch4Ten & Moximum & DLC1.4_0 & $9.11 \mathrm{~T}+02$ & $0.00 \mathrm{E}+00$ & $1.21 \mathrm{E}+03$ & $3.68 \mathrm{E}+01$ & & $1.14 \mathrm{E}+01$ & $4.65 E+01$ \\
\hline Anchising & Minimum & DLC1.1 & $0.00 \mathrm{E}+00$ & $0.00 E+00$ & $4.20 \mathrm{E}+02$ & $6.89 \mathrm{E}+01$ & $3.19 \mathrm{E}-01$ & $2.13 E+00$ & 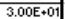 \\
\hline Anch.4Ang & Moximum & DLC1.LO00L_SeQ_04.0V0_01.6H__08.9TP_SO1.out & $0.00 \mathrm{E}+00$ & $0.00 E+00$ & 4.2 & & 3. & $2.13 E+00$ & $3.00 \mathrm{E}+0$ \\
\hline Fovir4Ten & Minimum & DLC1.5_ & $0.00 E+00$ & & $3.25 E+02$ & & $-8.02 E-01$ & $2.40 \mathrm{E}+01$ & $3.00 E+01$ \\
\hline Foirt4 en & Maximum & DLLC1.L0079_Se__12.0V0_02.4HE_099.2TP_S03.0ut & $8.52 E+02$ & $0.00 E+00$ & $1.23 E+03$ & 3.8. & $6.36 \mathrm{E}-01$ & 1.17RE+01] & $5.83 E+01$ \\
\hline
\end{tabular}

Extreme events for Line 5:

\begin{tabular}{|c|c|c|c|c|c|c|c|c|c|}
\hline Parameter & Type & File & $\begin{array}{r}\text { AncbsTe: } \\
\text { (tkW) }\end{array}$ & $\begin{array}{r}\text { Anclishng } \\
\text { (deg) }\end{array}$ & $\begin{array}{r}\text { FairsTe: } \\
\text { (tEW) }\end{array}$ & $\begin{array}{r}\text { F sir5A:g } \\
\text { (deg) }\end{array}$ & $\begin{array}{r}\text { YareEler } \\
\text { (E) }\end{array}$ & $\begin{array}{l}\text { ViedYri } \\
(=/ s e c)\end{array}$ & $\begin{array}{c}\text { Time } \\
\text { (sec) }\end{array}$ \\
\hline Anchs en & Minimum & DLC1.10103_Se__14.0V0_03.0H__08..TP__S05.out & $-2.03 E-01$ & $0.00 \mathrm{E}+00$ & $6.26 \mathrm{E}+02$ & $5.53 \mathrm{E}+01$ & $1.73 \mathrm{E}-01$ & $1.47 \mathrm{TE}+01$ & $\frac{1.36}{2.36+02}$ \\
\hline AnchTs en & Maximum & DLC1.5_029L_Se2_EW'SH+12.0_02.4HE_177.6TP_S01. out & $9.22 E+02$ & $0.00 E+00$ & $1.22 E+03$ & $3.68 \mathrm{E}+01$ & $7.04 \mathrm{E}-01$ & $1.20 E+01$ & $5.75 E+01$ \\
\hline Anchsing & Minimum & DLC1._000L_Sez_04.0V0_01.6H__08.STP_SO1.0ut & & $0.00 E+00$ & $4.18 \mathrm{E}+02$ & $6.91 E+01$ & $3.19 \mathrm{E}-01$ & $2.13 E+00$ & $3.00 \mathrm{E}+01$ \\
\hline$\frac{\text { Anchishg }}{\text { AnchSAng }}$ & $\begin{array}{l}\text { Maniminum } \\
\text { Moxinum }\end{array}$ & 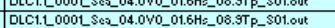 & $\frac{0.00+000}{0.00 E+00}$ & $\begin{array}{ll}0.00 \mathrm{E} E+00 \\
0.00 \mathrm{E}+00\end{array}$ & 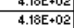 & $6 \frac{6.91+0.01}{6.911+01}$ & $\begin{array}{lll}3.19 E-0101 \\
3.19 E-01\end{array}$ & $\frac{2.13 E+100}{2.13 E+00}$ & $3.00 \mathrm{E}+01$ \\
\hline & Minimum & DLC1.5_0754_Sc2_EWSH-24.0_05.5Hs_12.TTP_S06. OUt & $0.00 E+00$ & & $3.17 \mathrm{TE}+02$ & $7.57 \mathrm{TE}+01$ & $-8.32 \mathrm{E}-01$ & $2.40 \mathrm{E}+01$ & $3.01 \mathrm{E}+01$ \\
\hline Ten & $\begin{array}{l}\text { Minimum } \\
\text { Moxinum }\end{array}$ & 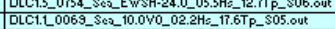 & $\frac{0.00+00}{8.92 E+02}$ & $\frac{0.00 \mathrm{E}+00}{0.00 \mathrm{E}+00}$ & 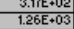 & $\frac{7.57+2+01}{3.83 E+01}$ & $\frac{-8.32 E-01}{2.19 E-01}$ & $\frac{2.40 \mathrm{E}+01}{1.11 \mathrm{E}+01}$ & 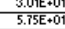 \\
\hline
\end{tabular}

Extreme events for Line 6:

\begin{tabular}{|c|c|c|c|c|c|c|c|c|c|}
\hline Parameter & Type & File & $\begin{array}{r}\text { Aactb6Ten } \\
(\text { (tw) }\end{array}$ & \begin{tabular}{|c|} 
Ancb6Ang \\
(deg)
\end{tabular} & $\begin{array}{r}\text { Fair6Tet } \\
\text { (tKH) }\end{array}$ & $\begin{array}{r}\text { F air6 Ang } \\
\text { (deg) }\end{array}$ & $\begin{array}{r}\text { VareEler } \\
(0)\end{array}$ & $\begin{array}{c}\text { VindVi } \\
(0 / \text { sec })\end{array}$ & $\begin{array}{c}\text { Tince } \\
(s \in c) \\
\end{array}$ \\
\hline AnchbTen & Minimum & DLC1.L000L_Se__04.0V0_01.6H__08.9TP_S01.out & $0.00 \mathrm{E}+00$ & $0.00 \mathrm{E}+00$ & $\frac{1.010}{4.15 E+02}$ & $\frac{1.93 \mathrm{E}+01}{61}$ & $\frac{1.01}{3.19 E-01}$ & $2.13 \mathrm{E}+00$ & $3.00 \mathrm{E}+01$ \\
\hline AncheTen & Maximum & DLC1.3_0052_SeQ_08.0V0_01.8HE_08.0TP_SO6.out & $9.915+00$ & $0.00 \mathrm{E}+00$ & $5.70 \mathrm{E}+02$ & $5.49 E+01$ & $7.60 \mathrm{E}-01$ & $7.43 E+00$ & $5.04 \mathrm{E}+01$ \\
\hline Anch6Ang & Minimum & DLC1._000L_SQ2_04.0V0_01.6HE_08.STP_SO1.out & $0.00 \mathrm{E}+00$ & $0.00 \mathrm{E}+00$ & $4.15 E+02$ & $6.93 \mathrm{E}+01$ & $3.19 E-01$ & $2.13 E+00$ & $3.00 \mathrm{E}+0$ \\
\hline Anch6Asing & Moximum & DLC1.L000L_SQQ_04.0V0_01.6H__08.STP_S01. out & $0.00 E+00$ & 0. & $4.15 E+02$ & $6.93 \mathrm{E}+01$ & 3.1. & $2.13 E+00$ & $3.00 E+01$ \\
\hline Fair6Ten & Minimum & DLC1.3. & & & & & & $2.36 \mathrm{E}+01$ & $2.50 \mathrm{E}+02$ \\
\hline Fair6Ten & Moximum & DLC1.L0069_Sez_10.0V0_02.2HE_17.6T P_S05.out & $0.00 E+00$ & 00 & $6.09 E+02$ & 001 & $2.25 \mathrm{E}-01$ & $1.11 \mathrm{E}+01$ & 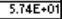 \\
\hline
\end{tabular}

Extreme events for Line 7:

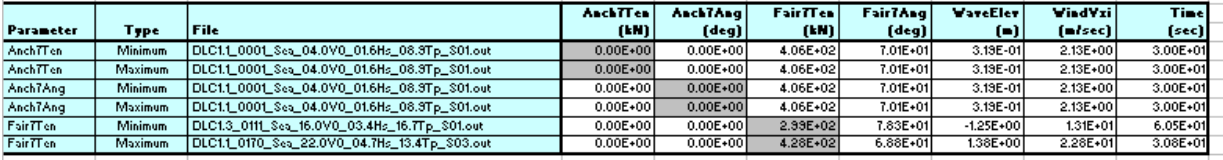

Extreme events for Line 8:

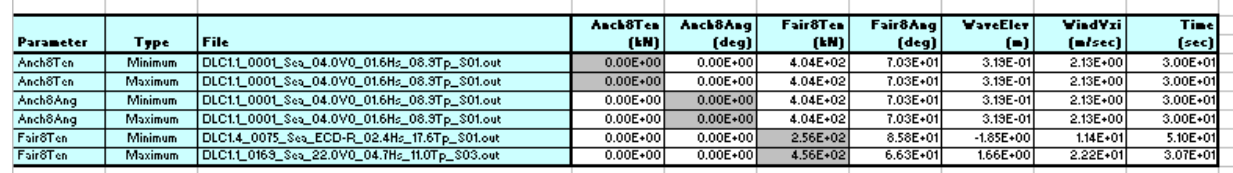

Extreme events for Rotor Perf :
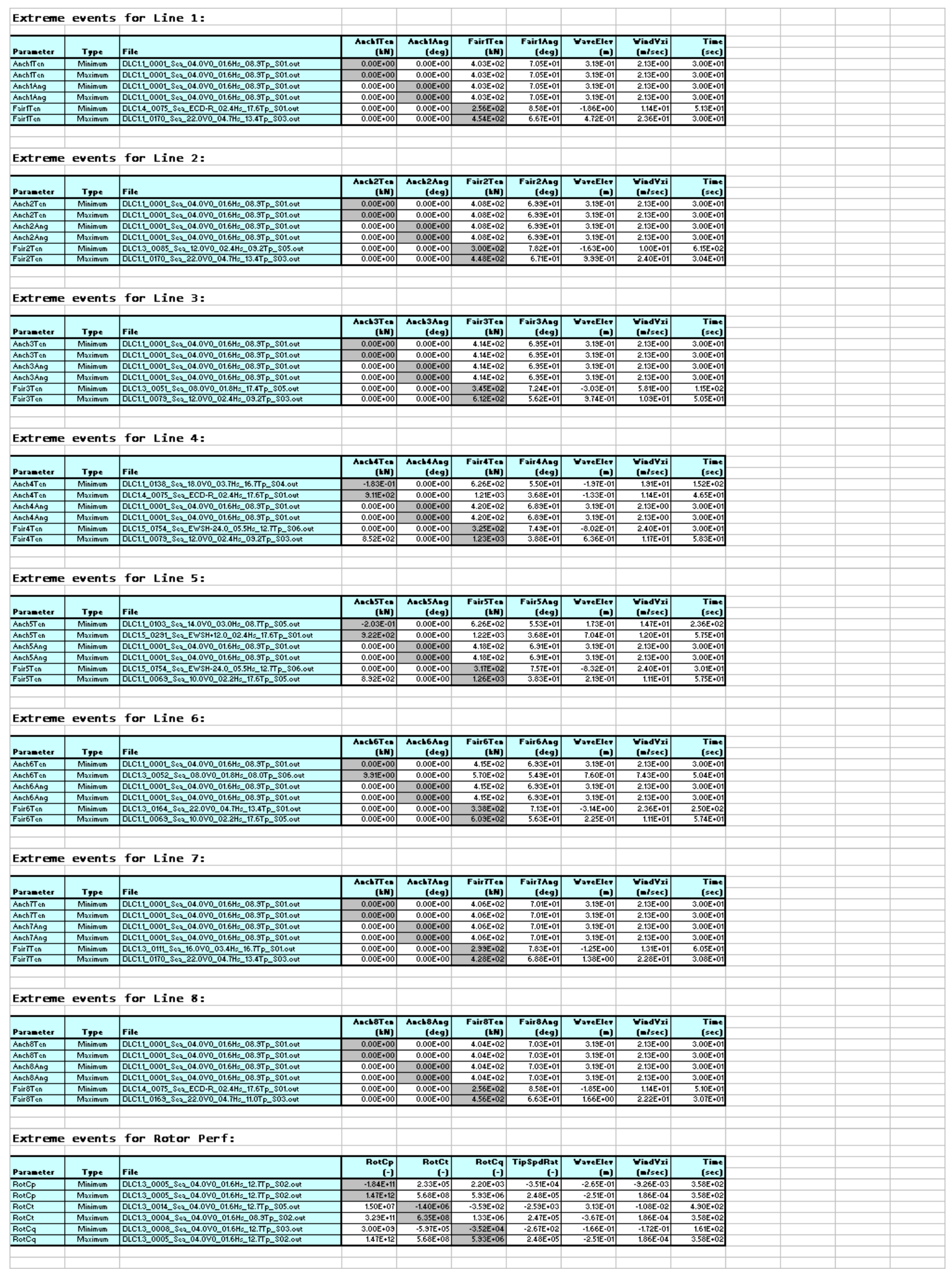


\section{REPORT DOCUMENTATION PAGE}

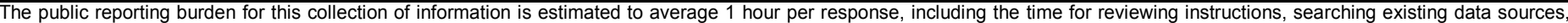

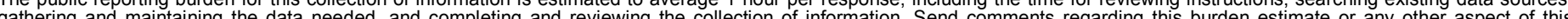

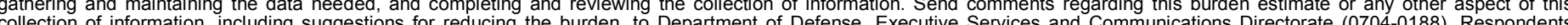

should be aware that notwithstanding any other provision of law, no person shall be subject to any penalty for failing to

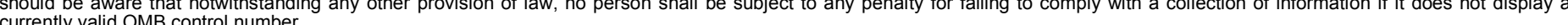

PLEASE DO NOT RETURN YOUR FORM TO THE ABOVE ORGANIZATION.
1. REPORT DATE (DD-MM-YYYY)
2. REPORT TYPE
November 2007
Technical report
3. DATES COVERED (From - To)
October 2007

4. TITLE AND SUBTITLE

Dynamics Modeling and Loads Analysis of an Offshore Floating

Wind Turbine

5a. CONTRACT NUMBER

DE-AC36-99-G010337

5b. GRANT NUMBER

5c. PROGRAM ELEMENT NUMBER

6. AUTHOR(S)

J.M. Jonkman

5d. PROJECT NUMBER

NREL/TP-500-41958

5e. TASK NUMBER

WER7.5001

5f. WORK UNIT NUMBER

7. PERFORMING ORGANIZATION NAME(S) AND ADDRESS(ES)

National Renewable Energy Laboratory

1617 Cole Blvd.

8. PERFORMING ORGANIZATION REPORT NUMBER

Golden, CO 80401-3393

NREL/TP-500-41958

9. SPONSORING/MONITORING AGENCY NAME(S) AND ADDRESS(ES)

10. SPONSOR/MONITOR'S ACRONYM(S)

NREL

11. SPONSORING/MONITORING AGENCY REPORT NUMBER

12. DISTRIBUTION AVAILABILITY STATEMENT

National Technical Information Service

U.S. Department of Commerce

5285 Port Royal Road

Springfield, VA 22161

13. SUPPLEMENTARY NOTES

14. ABSTRACT (Maximum 200 Words)

The objectives of the work described in this report are to develop a comprehensive simulation tool that can model the coupled dynamic response of offshore floating wind turbines, verify the simulation capability through model-to-model comparisons, and apply the simulation tool in an integrated loads analysis for one of the promising floating support

platform concepts.

15. SUBJECT TERMS

offshore wind energy development; modeling; wind turbine design analysis

\begin{tabular}{l}
\hline \multicolumn{3}{|l|}{ 16. SECURITY CLASSIFICATION OF: } \\
\hline \begin{tabular}{l|l|l|} 
a. REPORT & b. ABSTRACT & c. THIS PAGE \\
Unclassified & Unclassified & Unclassified \\
& & \\
\hline
\end{tabular} \\
\hline
\end{tabular}

\begin{tabular}{|c|c|}
\hline $\begin{array}{l}\text { 17. LIMITATION } \\
\text { OF ABSTRACT }\end{array}$ & $\begin{array}{l}\text { 18. } \\
\text { OF PABER } \\
\text { OF PAGES }\end{array}$ \\
\hline UL & \\
\hline
\end{tabular}

19a. NAME OF RESPONSIBLE PERSON

19b. TELEPHONE NUMBER (Include area code) 